\title{
Beitrag von Ackerbohne (Vicia faba L.), Luzerne (Medicago sativa L.) und Saatwicke (Vicia sativa L.) zur Selbstregelung der $\mathbf{N}$-Zufuhr in leguminosenbasierten Fruchtfolgen
}

\author{
Dissertation \\ zur Erlangung des Doktorgrades (Dr. sc. agr.) \\ der Fakultät für Agrarwissenschaften \\ der Georg-August-Universität Göttingen
}

vorgelegt von

Johann Anthes

aus Kelsterbach 
Institut für Pflanzenbau und Pflanzenzüchtung Institute of Agronomy and Plant Breeding der Georg-August-Universität Göttingen

D 7

1. Referent:

Prof. Dr. R. Rauber

2. Referent:

Prof. Dr. N. Claassen

Tag der mündlichen Prüfung: 03. Februar 2005 


\section{Inhaltsverzeichnis}

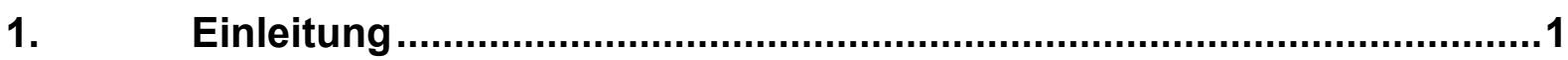

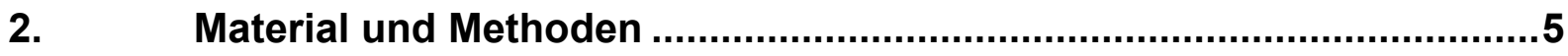

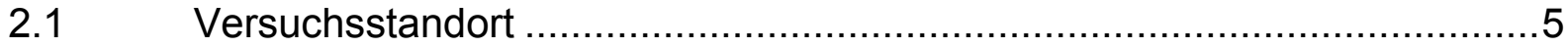

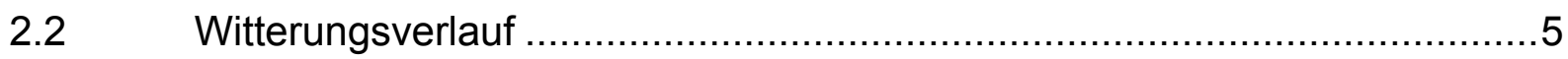

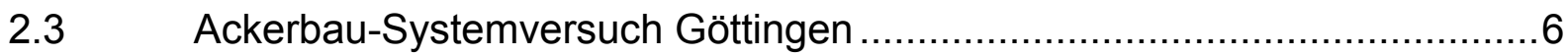

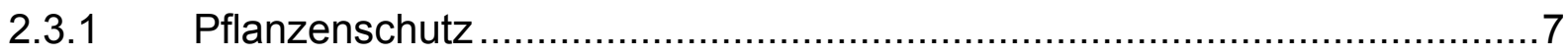

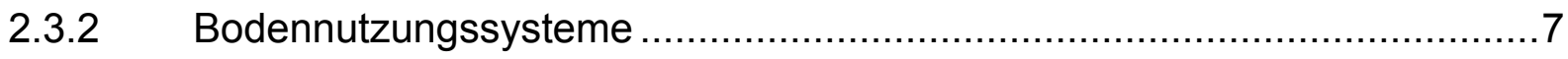

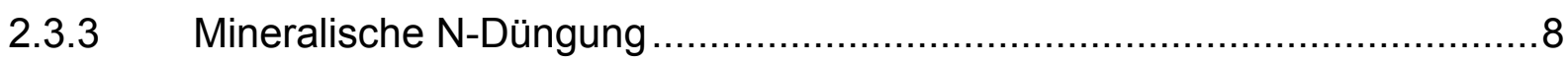

2.4 Versuchsanlage und Versuchsdurchführung .......................................

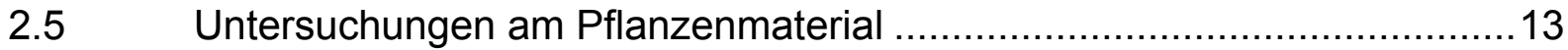

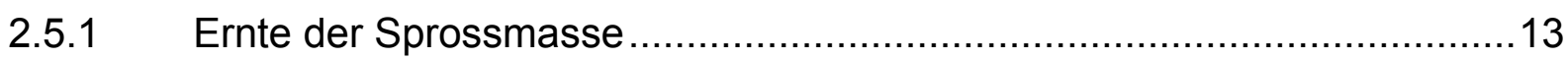

2.5.2 Entnahme der Wurzelproben .......................................................13

2.5.3 Aufbereitung der Pflanzen- und Wurzelproben .................................. 15

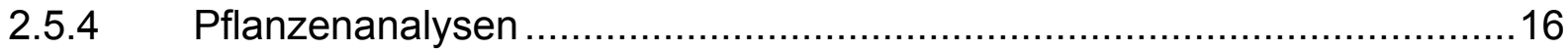

2.5.5 Schätzung der symbiotischen Stickstoff-Fixierleistung mit der

$\delta^{15} \mathrm{~N}$-Methode (Natural ${ }^{15} \mathrm{~N}$ abundance method) und erweiterten

Differenzmethode (Extended difference method) ...................................16

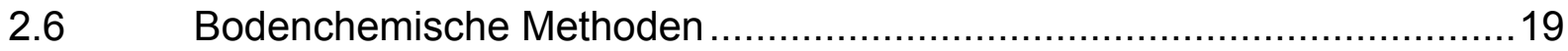

2.6.1 Bestimmung der löslichen N-Fraktionen im Boden................................ 19

2.6.2 Bestimmung des $\mathrm{N}_{\mathrm{t}}$ - und $\mathrm{C}_{\mathrm{t}}$-Gehaltes im Boden .................................19

2.6.3 Carbonatgehalt des Bodens ..............................................................20

2.6.4 Bestimmung des pflanzenverfügbaren $\mathrm{P}_{2} \mathrm{O}_{5}$ - und $\mathrm{K}_{2} \mathrm{O}-$ Gehaltes und des pH-Wertes im Boden ..........................................................20

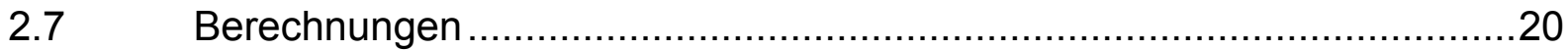

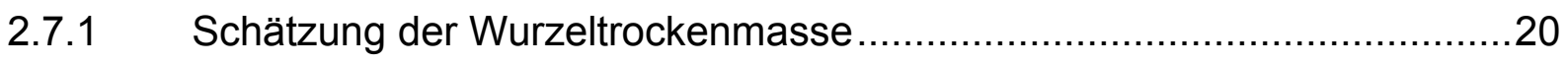

2.7.2 Berechnung der Trockenmasse-Erträge, der $\mathrm{N}_{2}$-Fixierleistung

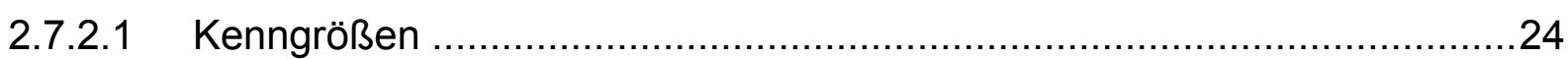

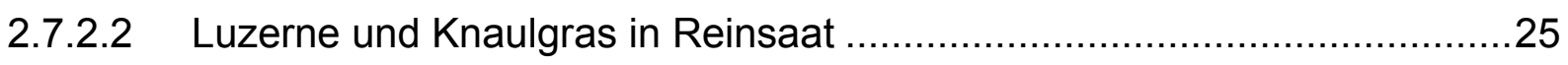

2.7.2.3 Luzerne und Knaulgras in Gemengesaat .........................................27

2.7.2.4 Ackerbohne und Hafer in Rein- und Gemengesaat...............................28

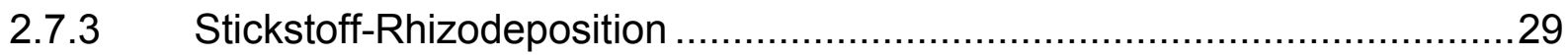

2.7.4 Erweiterter N-Flächenbilanzsaldo...............................................29

2.7.5 Transfer-Stickstoff im Gemengeanbau .............................................29 
2.8 Statistische Auswertungen 30

3. Ergebnisse 32

3.1 Ackerbohne in Rein- und Gemengesaat mit Hafer ............................. 32

3.1.1 Gesamtpflanzliche Trockenmasse-Akkumulation ................................ 32

3.1.2 Stickstofferträge und Stickstoff-Harvest-Index ................................... 36

3.1.3 N-Isotopenzusammensetzung in der gesamtpflanzlichen Biomasse ........ 40

3.1.4 Vergleich der Schätzergebnisse der symbiotisch fixierten N-Menge mittels $\delta^{15} \mathrm{~N}$ - und erweiterter Differenzmethode

3.1.5 Luft- und bodenbürtige $\mathrm{N}$-Akkumulation sowie $\mathrm{CaCl}_{2}$-extrahierbarer $\mathrm{N}_{\text {min }}$-Stickstoff im Boden

3.1.6 Transfer-Stickstoff beim Anbau von Hafer im Gemenge mit

Ackerbohne. 51

3.1.7 Einfacher und erweiterter N-Flächenbilanzsaldo 52

3.2 Luzerne in Rein- und Gemengesaat mit Knaulgras ............................. 56

3.2.1 Gesamtpflanzliche Trockenmasse-Akkumulation ............................... 56

3.2.2 Stickstofferträge und Stickstoff-Harvest-Index ................................. 61

3.2.3 N-Isotopenzusammensetzung in der gesamtpflanzlichen Biomasse ........ 68

3.2.4 Vergleich der Schätzergebnisse der symbiotisch fixierten N-Menge mittels $\delta^{15} \mathrm{~N}$ - und erweiterter Differenzmethode

3.2.5 Luft- und bodenbürtige $\mathrm{N}$-Akkumulation sowie $\mathrm{CaCl}_{2}$-extrahierbarer $\mathrm{N}_{\text {min }}$-Stickstoff im Boden

3.2.6 Transfer-Stickstoff beim Anbau von Knaulgras im Gemenge mit Luzerne.... 77

3.2.7 Einfacher und erweiterter N-Flächenbilanzsaldo .............................. 79

3.3 Saatwicke in Rein- und Gemengesaat mit Einjährigem Weidelgras ........ 82

3.3.1 Gesamtpflanzliche Trockenmasse-Akkumulation ................................ 82

3.3.2 Stickstofferträge und Stickstoff-Harvest-Index ................................. 85

3.3.3 N-Isotopenverteilung in der gesamtpflanzlichen Biomasse ....................90

3.3.4 Vergleich der Schätzergebnisse der symbiotisch fixierten N-Menge mittels der $\delta^{15} \mathrm{~N}$ - und erweiterter Differenzmethode

3.3.5 Luft- und bodenbürtige $\mathrm{N}$-Akkumulation sowie $\mathrm{CaCl}_{2}$-extrahierbarer $\mathrm{N}_{\text {min }}$-Stickstoff im Boden

3.3.6 Transfer-Stickstoff beim Anbau von Einjährigem Weidelgras im Gemenge mit Saatwicke ...................................................... 96

3.3.7 Einfacher und erweiterter N-Flächenbilanzsaldo ............................ 97

4.

Diskussion.

4.1 Methodische Aspekte zur Erhebung der Wurzelmassen 100 
4.2 Vergleich der Schätzung der symbiotisch fixierten N-Mengen mit der $\delta^{15} \mathrm{~N}$-Methode und der erweiterten Differenzmethode 104

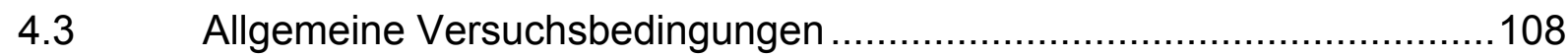

Selbstregelung der N-Zufuhr ................................................ 110

4.4.1 Gesamtpflanzliche Stickstoffakkumulation in Spross und Wurzeln .........111

4.4.2 N-Aufnahme aus dem Boden .................................................... 114

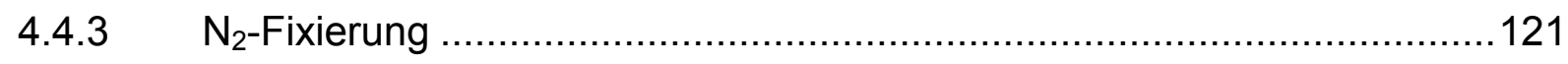

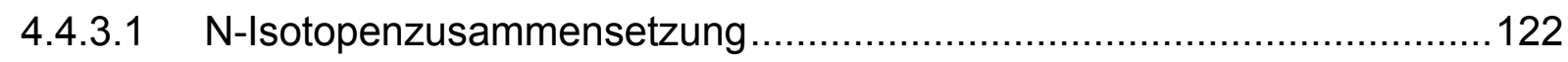

4.4.3.2 Gewichtete Anteile Stickstoff aus der Luft $\left(\mathrm{Ndfa}_{w}\right)$ und luftbürtige N-Aufnahme der Ansaatvarianten ...............................................123

4.4.3.3 Transfer-Stickstoff im Gemengeanbau .......................................... 129

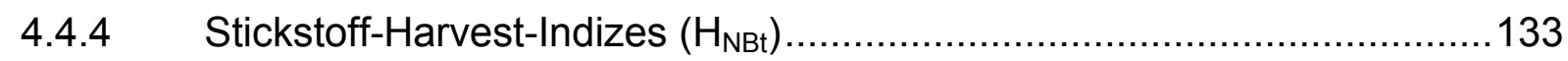

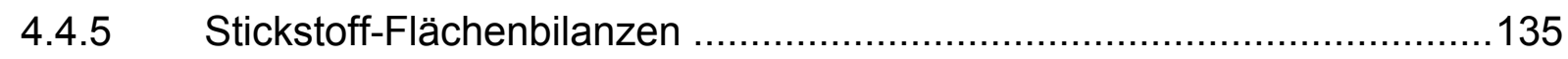

4.5 Beitrag von Ackerbohne, Luzerne und Saatwicke zur Selbstregelung

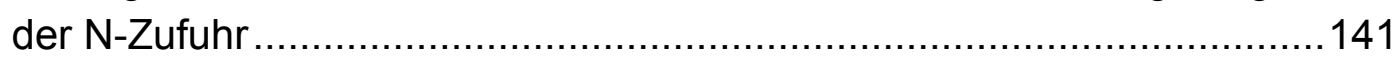

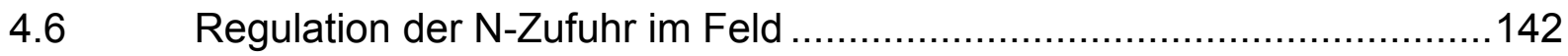

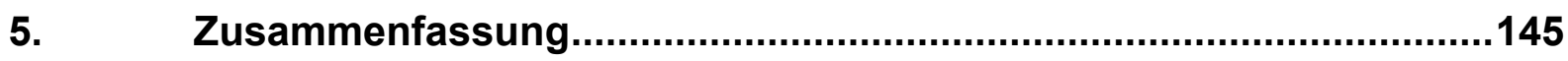

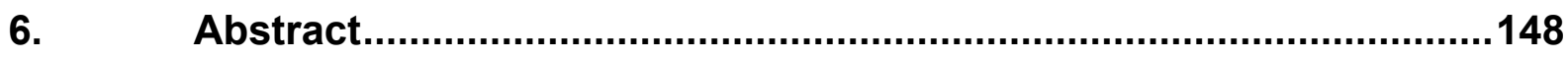

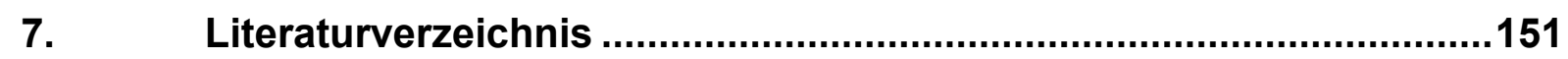

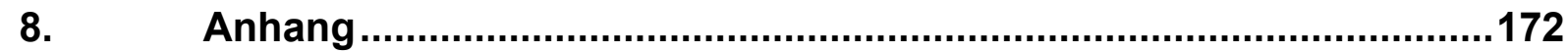




\section{Verzeichnis der Abbildungen}

Abb. 1: Monatsmitteltemperaturen (a) und monatliche Niederschlagssummen (b) im Versuchszeitraum von März 1998 bis November 1999 und im langjährigen Mittel.

Abb. 2: Lageplan der Prüfglieder im Ackerbau-Systemversuch Göttingen, Feldplan 1998

Abb. 3: Anordnung der Untersuchungsparzellen innerhalb des AckerbauSystemversuches

Abb. 4: Gesamtpflanzliche Trockenmasse-Akkumulation (dt TM ha ${ }^{-1}$; Wurzel, Stroh incl. Bestandesabfall, Korn) sowie gewichtete TrockenmasseHarvest-Indizes $\left(\mathrm{H}_{\mathrm{TMB} \text { tw }} \pm\right.$ Standardfehler $)$ beim Anbau von Ackerbohne und Hafer in Rein- und Gemengesaat zur letzten Ernte (BBCH 87) am 10.08.1998

Abb. 5: Ertragsanteile der Spross-TM beim Anbau von Ackerbohne und Hafer in Gemengesaat zur letzten Ernte (10.08.1998)

Abb. 6: Gesamtpflanzliche Trockenmasse-Akkumulation (dt TM ha ${ }^{-1}$; Wurzel, Stroh incl. Bestandesabfall, Korn) sowie gewichtete TrockenmasseHarvest-Indizes $\left(\mathrm{H}_{\mathrm{TMB} \text { tw }} \pm\right.$ Standardfehler $)$ beim Anbau von Ackerbohne und Hafer in Rein- und Gemengesaat zur letzten Ernte (BBCH 87) am 26.07.1999

Abb. 7: Ertragsanteile der Spross-TM beim Anbau von Ackerbohne und Hafer in Gemengesaat zur letzten Ernte (26.07.1999)

Abb. 8: Gesamtpflanzliche Stickstoff-Akkumulation ( $\mathrm{kg} \mathrm{N}^{-1}{ }^{-1}$; Wurzel, Stroh incl. Bestandesabfall, Korn) sowie gewichtete Stickstoff-HarvestIndizes ( $\mathrm{H}_{\mathrm{NBtw}} \pm$ Standardfehler) beim Anbau von Ackerbohne und Hafer in Rein- und Gemengesaat zur letzten Ernte (BBCH 87) am 10.08.1998

Abb. 9: Spross-Stickstoff-Ertragsanteile der Ackerbohne und des Hafers in Gemengesaat zum dritten Erntetermin (10.08.1998).

Abb. 10: Gesamtpflanzliche Stickstoff-Akkumulation ( $\mathrm{kg} \mathrm{N}^{-1}$; Wurzel, Stroh incl. Bestandesabfall, Korn) sowie gewichtete Stickstoff-HarvestIndizes $\left(\mathrm{H}_{\mathrm{NBtw}} \pm\right.$ Standardfehler) beim Anbau von Ackerbohne und Hafer in Rein- und Gemengesaat zur letzten Ernte (BBCH 87) am 26.07.1999.

Abb. 11: Spross-Stickstoff-Ertragsanteile der Ackerbohne und des Hafers in Gemengesaat zum dritten Erntetermin (26.07.1999) 
Abb. 12: Gewichtete $\delta^{15} \mathrm{~N}$-Werte (arithmetische Mittelwerte \pm Standardfehler in $\%$ ) in der gesamtpflanzlichen Biomasse (Wurzel, Stroh, Bestandesabfall, Korn) beim Anbau von Ackerbohne und Hafer in Rein- und Gemengesaat 1998

Abb. 13: Gewichtete $\delta^{15} \mathrm{~N}-$ Werte (arithmetische Mittelwerte \pm Standardfehler in $\%$ ) in der gesamtpflanzlichen Biomasse (Wurzel, Stroh, Bestandesabfall, Korn) beim Anbau von Ackerbohne und Hafer in Rein- und Gemengesaat 1999

Abb. 14: Korrelation zwischen den Ergebnissen der $\delta^{15} \mathrm{~N}$ - und erweiterten Differenzmethode zur Schätzung der gesamtpflanzlich symbiotisch fixierten N-Menge a) 1998 und b) 1999 beim Anbau von Ackerbohne in Reinsaat $(4 \mathrm{~A})$ und Gemengesaat mit Hafer $(3 \mathrm{~A} / 1 \mathrm{H}$ und $1 \mathrm{~A} / 3 \mathrm{H})$

Abb. 15: $\mathrm{CaCl}_{2}$-extrahierbarer $\mathrm{N}_{\min }$-Stickstoff im Boden $\left(\mathrm{kg} \mathrm{N}_{\min } \mathrm{ha}^{-1}\right)$, bodenund luftbürtige $\mathrm{N}$-Akkumulation in der gesamtpflanzlichen Biomasse ( $\mathrm{kg} \mathrm{N} \mathrm{ha}^{-1}$ ) und gewichtete Anteile Stickstoff aus der Luft ( $\mathrm{Ndfa}_{\mathrm{w}} \pm$ Standardfehler) beim Anbau von Ackerbohne und Hafer in Rein- und Gemengesaat 1998

Abb. 16: $\mathrm{CaCl}_{2}$-extrahierbarer $\mathrm{N}_{\min }$-Stickstoff im Boden $\left(\mathrm{kg} \mathrm{N}_{\min } \mathrm{ha}^{-1}\right.$ ), bodenund luftbürtige $\mathrm{N}$-Akkumulation in der gesamtpflanzlichen Biomasse ( $\mathrm{kg} \mathrm{N} \mathrm{ha}^{-1}$ ) und gewichtete Anteile Stickstoff aus der Luft (Ndfaw \pm Standardfehler) beim Anbau von Ackerbohne und Hafer in Rein- und Gemengesaat 1999

Abb. 17: $\quad \delta^{15} \mathrm{~N}$-Werte $(\mathrm{n}=6$, Mittelwerte \pm Standardfehler in \%o) in der HaferSprossmasse (Korn + Stroh) in Reinsaat $(4 \mathrm{H})$ und Gemengesaaten mit Ackerbohne $(1 \mathrm{~A} / 3 \mathrm{H}$ und $3 \mathrm{~A} / 1 \mathrm{H})$ am 10.08 .1998 (links) und 26.07.1999 (rechts)

Abb. 18: Einfacher (a) und erweiterter (b) N-Flächenbilanzsaldo ( $\mathrm{kg} \mathrm{N} \mathrm{ha}^{-1}$ ) Mittelwerte \pm Standardfehler) beim Anbau von Ackerbohne und Hafer in Rein- und Gemengesaat zur Körnernutzung am 10.08.1998.

Abb. 19: Einfacher (a) und erweiterter (b) N-Flächenbilanzsaldo ( $\mathrm{kg} \mathrm{N} \mathrm{ha}^{-1}$ ) Mittelwerte \pm Standardfehler) beim Anbau von Ackerbohne und Hafer in Rein- und Gemengesaat zur Körnernutzung am 26.07.1999

Abb. 20: Kumulierte gesamtpflanzliche Trockenmasse (dt TM ha ${ }^{-1}$; Wurzel, Stoppel, Schnittgut) sowie kumulierte gewichtete TrockenmasseHarvest-Indizes $\left(\mathrm{H}_{\mathrm{TMBtw}} \pm\right.$ Standardfehler) beim Anbau von Luzerne und Knaulgras in Rein- und Gemengesaat zum dritten Schnitttermin am 25.10.1998

Abb. 21: Ertragsanteile der kumulierten Spross-TM beim Anbau von Luzerne und Knaulgras in Gemengesaat bis zum dritten Schnitt 1998 
Abb. 22: Kumulierte gesamtpflanzliche Trockenmasse (dt TM ha ${ }^{-1}$; Wurzel, Stoppel, Schnittgut) sowie kumulierte gewichtete TrockenmasseHarvest-Indizes $\left(\mathrm{H}_{\mathrm{TMB}} \pm\right.$ Standardfehler) beim Anbau von Luzerne und Knaulgras in Rein- und Gemengesaat zum dritten Schnitttermin am 04.11.1999.

Abb. 23: Ertragsanteile der kumulierten Spross-TM beim Anbau von Luzerne und Knaulgras in Gemengesaat bis zum dritten Schnitt 1999

Abb. 24: Gesamtpflanzliche Stickstoff-Akkumulation $\left(\mathrm{kg} \mathrm{N} \mathrm{ha}^{-1}\right.$, Wurzel, Stoppeln, Schnittgut) sowie gewichtete Stickstoff-Harvest-Indizes $\left(\mathrm{H}_{\mathrm{NBtw}} \pm\right.$ Standardfehler) beim Anbau von Luzerne und Knaulgras in Rein- und Gemengesaat zu drei Schnitterminen im Jahr 1998 (kumulierte Werte zum zweiten und dritten Schnitttermin)

Abb. 25: Stickstoff-Ertragsanteile der kumulierten Sprosserträge von Luzerne und Knaulgras in Gemengesaat bis zum dritten Schnitt 1998

Abb. 26: Gesamtpflanzliche Stickstoff-Akkumulation $\left(\mathrm{kg} \mathrm{N}^{-1}\right.$; Wurzel, Stoppeln, Schnittgut) sowie gewichtete Stickstoff-Harvest-Indizes $\left(\mathrm{H}_{\mathrm{NBtw}} \pm\right.$ Standardfehler) beim Anbau von Luzerne und Knaulgras in Rein- und Gemengesaat zu drei Schnittterminen im Jahr 1999.

Abb. 27: Stickstoff-Ertragsanteile der kumulierten Sprosserträge von Luzerne und Knaulgras in Gemengesaat bis zum dritten Schnitt 1999

Abb. 28: Gewichtete- (erster Schnitt) bzw. kumuliert gewichtete $\delta^{15} \mathrm{~N}-W e r t e$ (zweiter und dritter Schnitt) (arithmetische Mittelwerte \pm Standardfehler in \%) in der gesamtpflanzlichen Biomasse (Erntereste + Schnittgut) beim Anbau von Luzerne und Knaulgras in Rein- und Gemengesaat im Jahr 1998.

Abb. 29: Gewichtete- (erster Schnitt) bzw. kumuliert gewichtete $\delta^{15} \mathrm{~N}$-Werte (zweiter und dritter Schnitt) (arithmetische Mittelwerte \pm Standardfehler in \%) in der gesamtpflanzlichen Biomasse (Erntereste + Schnittgut) beim Anbau von Luzerne und Knaulgras in Rein- und Gemengesaat im Jahr 1999.

Abb. 30: Korrelation zwischen den Ergebnissen der $\delta^{15} \mathrm{~N}$ - und erweiterten Differenzmethode zur Schätzung der gesamtpflanzlich symbiotisch fixierten N-Menge a) 1998 und b) 1999 beim Anbau von Luzerne in Reinsaat (4L) und Gemengesaat mit Knaulgras (3L/1K und 1L/3K).

Abb. 31: $\mathrm{CaCl}_{2}$-extrahierbarer $\mathrm{N}_{\text {min }}$-Stickstoff im Boden $\left(\mathrm{kg} \mathrm{N}_{\min } \mathrm{ha}^{-1}\right)$, bodenund luftbürtige N-Akkumulation in der gesamtpflanzlichen Biomasse $\left(\mathrm{kg} \mathrm{N} \mathrm{ha}^{-1}\right)$ und gewichtete Anteile Stickstoff aus der Luft (Ndfa \pm Standardfehler) beim Anbau von Luzerne und Knaulgras in Rein- und Gemengesaat im Jahr 1998 
Abb. 32: $\quad \mathrm{CaCl}_{2}$-extrahierbarer $\mathrm{N}_{\text {min }}$-Stickstoff im Boden $\left(\mathrm{kg} \mathrm{N}_{\min }\right.$ ha-1 ${ }^{-1}$, bodenund luftbürtige $\mathrm{N}$-Akkumulation in der gesamtpflanzlichen Biomasse $\left(\mathrm{kg} \mathrm{N} \mathrm{ha}^{-1}\right)$ und gewichtete Anteile Stickstoff aus der Luft $\left(\mathrm{Ndfa}_{\mathrm{w}} \pm\right.$ Standardfehler) beim Anbau von Luzerne und Knaulgras in Rein- und Gemengesaat im Jahr 1999

Abb. 33: Gewichtete $\delta^{15} \mathrm{~N}-$ Werte $(\mathrm{n}=6$, Mittelwerte \pm Standardfehler in \%o) in der Sprossmasse (Schnittgut + Stoppeln) des Knaulgrases in Reinsaat $(4 \mathrm{~K})$ und Gemengesaat mit Luzerne (1L/3K und $3 \mathrm{~L} / 1 \mathrm{~K}$ ) (kumulierte Werte bis 25.10.1998, links und kumulierte Werte bis 04.11.1999, rechts)

Abb. 34: Einfacher (a) und erweiterter (b) N-Flächenbilanzsaldo ( $\mathrm{kg} \mathrm{N} \mathrm{ha}^{-1}$ ) Mittelwerte \pm Standardfehler) beim Anbau von Luzerne und Knaulgras in Rein- und Gemengesaat zur Futternutzung zum dritten Schnitttermin ( $\Sigma$ Jahr) am 25.10.1998.

Abb. 35: Einfacher (a) und erweiterter (b) N-Flächenbilanzsaldo ( $\mathrm{kg} \mathrm{N} \mathrm{ha}^{-1}$, Mittelwerte \pm Standardfehler) beim Anbau von Luzerne und Knaulgras in Rein- und Gemengesaat zur Futternutzung zum dritten Schnitttermin ( $\Sigma$ Jahr) am 04.11.1999.

Abb. 36: Gesamtpflanzliche Trockenmasse (dt TM ha-1; Wurzel, Stoppel, Schnittgut) sowie gewichtete Trockenmasse-Harvest-Indizes $\left(\mathrm{H}_{\mathrm{TMBtw}}\right.$ \pm Standardfehler) beim Anbau der Saatwicke und des Einjährigen Weidelgrases in Rein- und Gemengesaat am 20.10.1998.

Abb. 37: Ertragsanteile der Spross-TM beim Anbau der Saatwicke und des Einjährigen Weidelgrases in Gemengesaat am 20.10.1998.

Abb. 38: Gesamtpflanzliche Trockenmasse (dt TM ha-1; Wurzel, Stoppel, Schnittgut) sowie gewichtete Trockenmasse-Harvest-Indizes $\left(\mathrm{H}_{\text {TMBtw }}\right.$ \pm Standardfehler) beim Anbau der Saatwicke und des Einjährigen Weidelgrases in Rein- und Gemengesaat am 17.10.1999.

Abb. 39: Ertragsanteile der Spross-TM beim Anbau der Saatwicke und des Einjährigen Weidelgrases in Gemengesaat zur Ernte (17.10.1999).

Abb. 40: Gesamtpflanzliche Stickstoff-Akkumulation $\left(\mathrm{kg} \mathrm{N} \mathrm{ha}^{-1}\right.$; Erntereste, Schnittgut) sowie gewichtete Stickstoff-Harvest-Indizes $\left(\mathrm{H}_{\mathrm{NBtw}} \quad \pm\right.$ Standardfehler) beim Anbau der Saatwicke und des Einjährigen Weidelgrases in Rein- und Gemengesaat am 20.10.1998

Abb. 41: Spross-Stickstoff-Ertragsanteile von Saatwicke und Einjährigem Weidelgras in Gemengesaat zur Ernte (20.10.1998)

Abb. 42: Gesamtpflanzliche Stickstoff-Akkumulation $\left(\mathrm{kg} \mathrm{N} \mathrm{ha}^{-1}\right.$; Erntereste, Schnittgut) sowie gewichtete Stickstoff-Harvest-Indizes $\left(\mathrm{H}_{\mathrm{NBtw}} \quad \pm\right.$ Standardfehler) beim Anbau von Saatwicke und Einjährigem Weidelgras in Rein- und Gemengesaat am 17.10.1999. 
Abb. 43: Spross-Stickstoff-Ertragsanteile der Saatwicke und des Einjährigen Weidelgrases in Gemengesaat zur Ernte am 17.10.1999

Abb. 44: Gewichtete $\delta^{15} \mathrm{~N}$-Werte (arithmetische Mittelwerte \pm Standardfehler in $\%$ ) in der gesamtpflanzlichen Biomasse (Erntereste + Schnittgut) beim Anbau von Saatwicke in Rein- und Gemengesaat mit Einjährigem Weidelgras im Jahr 1998 und 1999

Abb. 45: Korrelation zwischen den Ergebnissen der $\delta^{15} \mathrm{~N}$ - und erweiterter Differenzmethode zur Schätzung der gesamtpflanzlich symbiotisch fixierten N-Menge a) im Jahr 1998 und b) im Jahr 1999 beim Anbau von Saatwicke in Reinsaat (4S) und Gemengesaat mit Einjährigem Weidelgras (3S/1E und 1S/3E).

Abb. 46: $\mathrm{CaCl}_{2}$-extrahierbarer $\mathrm{N}_{\text {min }}$-Stickstoff im Boden $\left(\mathrm{kg} \mathrm{N}_{\min }\right.$ ha $\left.{ }^{-1}\right)$, bodenund luftbürtige $\mathrm{N}$-Akkumulation in der gesamtpflanzlichen Biomasse $\left(\mathrm{kg} \mathrm{N} \mathrm{ha}^{-1}\right)$ und gewichtete Anteile Stickstoff aus der Luft (Ndfaw \pm Standardfehler) beim Anbau der Saatwicke und des Einjährigen Weidelgrases in Rein- und Gemengesaat im Jahr 1998.

Abb. 47: $\mathrm{CaCl}_{2}$-extrahierbarer $\mathrm{N}_{\min }$-Stickstoff im Boden $\left(\mathrm{kg} \mathrm{N}_{\min } \mathrm{ha}^{-1}\right)$, bodenund luftbürtige N-Akkumulation in der gesamtpflanzlichen Biomasse $\left(\mathrm{kg} \mathrm{N} \mathrm{ha}^{-1}\right)$ und gewichtete Anteile Stickstoff aus der Luft (Ndfa \pm Standardfehler) beim Anbau der Saatwicke und des Einjährigen Weidelgrases in Rein- und Gemengesaat im Jahr 1999.

Abb. 48: $\delta^{15} \mathrm{~N}$-Werte $(\mathrm{n}=6$, Mittelwerte \pm Standardfehler in \%o $)$ in der Sprossmasse des Einjährigen Weidelgrases in Reinsaat (4E) und in Gemengesaat mit Saatwicke (1S/3E und 3S/1E) am 20.10.1998 (links) und 17.10.1999 (rechts).

Abb. 49: Einfacher (a) und erweiterter (b) N-Flächenbilanzsaldo ( $\mathrm{kg} \mathrm{N} \mathrm{ha}^{-1}$; Mittelwerte \pm Standardfehler) beim Anbau von Saatwicke und Einjährigem Weidelgras in Rein- und Gemengesaat zur Futternutzung am 20.10.1998

Abb. 50: Einfacher (a) und erweiterter (b) N-Flächenbilanzsaldo ( $\mathrm{kg} \mathrm{N} \mathrm{ha}^{-1}$; Mittelwerte \pm Standardfehler) beim Anbau der Saatwicke und des Einjährigen Weidelgrases in Rein- und Gemengesaat zur Futternutzung am 17.10.1999. 


\section{Verzeichnis der Tabellen}

Tab. 1: Merkmale der Bodennutzungssysteme (BNS). Die Abkürzungen kF und IF stehen für kurze bzw. lange Fruchtfolge ................................. 7

Tab. 2: Anzahl keimfähiger Samen $/ \mathrm{m}^{2}$ und Reihenweiten in $\mathrm{cm}$ der Reinund Gemengesaaten.

Tab. 3: $\quad \delta^{15} \mathrm{~N}_{0}$-Werte (\%o) in der Biomasse der in $\mathrm{N}$-freiem Substrat angezogenen Leguminosen

Tab. 4: Stickstoff-Anteil aus der Rhizodeposition (Ndfr) an der gesamtpflanzlichen N-Menge $\left(\mathrm{N}_{\mathrm{Bt}}\right)$ nach Schmidtke (2004).

Tab. 5: Transferierte N-Menge (Mittelwerte \pm Standardabweichung; 1998 $\mathrm{n}=5,1999 \mathrm{n}=6$ ) und Anteile aus der symbiotischen $\mathrm{N}_{2}$-Fixierung der Ackerbohne im Gemenge in der oberirdischen Sprossmasse des Hafers.

Tab. 6: Vergleich der Mittelwerte der einfachen N-Flächenbilanzsalden beim Anbau von Ackerbohne und Hafer in Rein- und Gemengesaat zur Körnernutzung 1998 und 1999

Tab. 7: Transferierte N-Menge und Anteile N im Spross des Knaulgrases (Mittelwerte \pm Standardabweichung; $n=6$ ) aus der symbiotischen $\mathrm{N}_{2}$-Fixierung der Luzerne im Gemenge.

Tab. 8: Vergleich der Mittelwerte der einfachen N-Flächenbilanzsalden beim Anbau von Luzerne und Knaulgras in Rein- und Gemengesaat zur Futternutzung 1998 und 1999.

Tab. 9: Vergleich der Mittelwerte der einfachen N-Flächenbilanzsalden beim Anbau von Saatwicke und Einjährigem Weidelgras in Reinund Gemengesaat zur Futternutzung im Jahr 1998 und 1999

Tab. 10: Einfluss der langjährigen mineralischen $\mathrm{N}$-Düngung zu den nichtlegumen Vorfrüchten $\left(\mathrm{N}_{3}\right)$ auf die Kenngrößen einer vereinfachten $\mathrm{N}$-Flächenbilanz beim Anbau von Ackerbohne, Luzerne und Saatwicke in Rein- und Gemengesaat. 


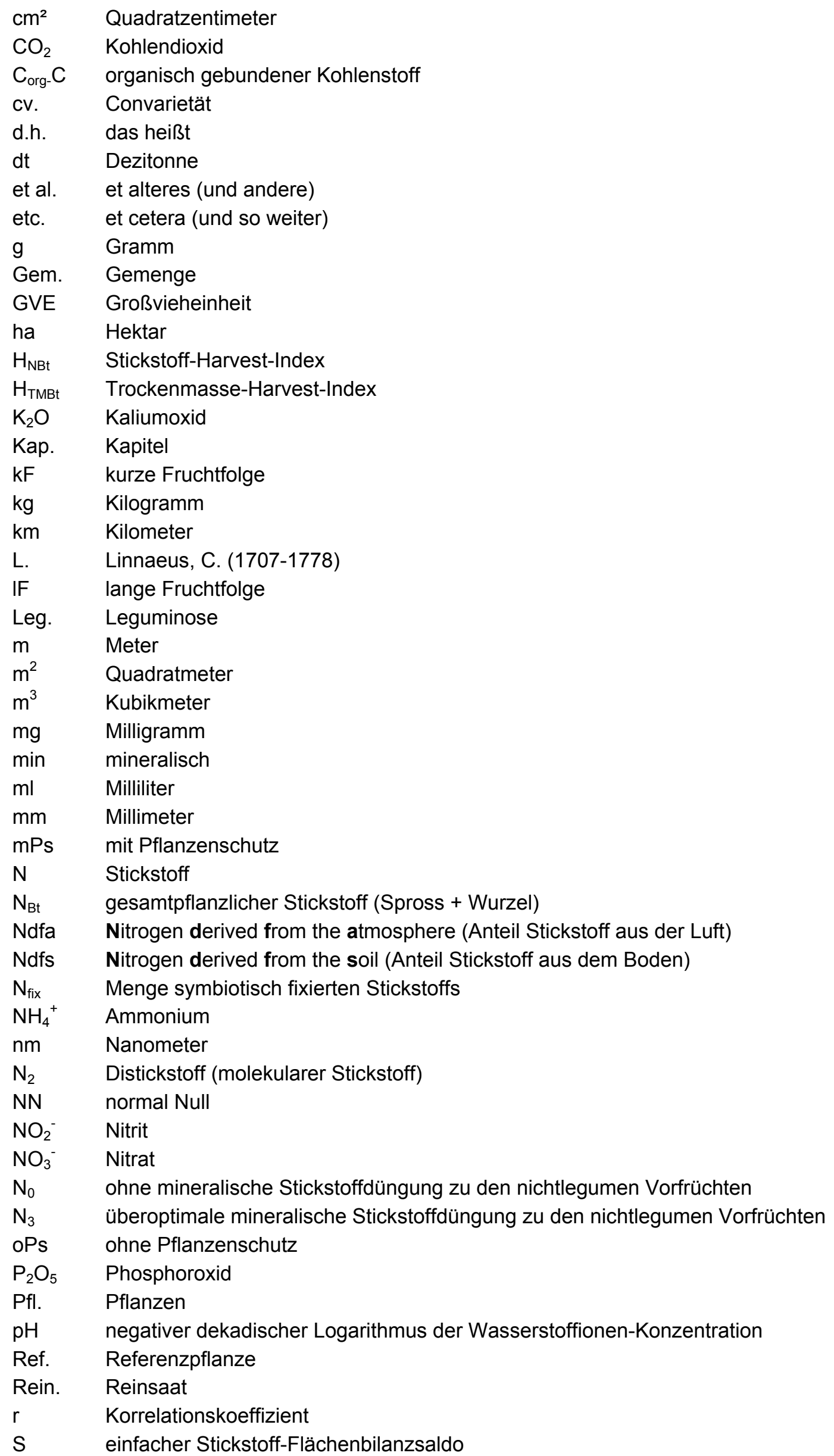


Sp. $\quad$ Spross

ssp. Subspecies

Sw erweiterter Stickstoff-Flächenbilanzsaldo

Tab. A Anhangstabelle

TM Trockenmasse

$\mathrm{TM}_{\mathrm{Bt}}$ gesamtpflanzliche Trockenmasse (Spross + Wurzel)

WDH Wiederholung

Wu. Wurzel

vgl. vergleiche

z.B. zum Beispiel 



\section{1.}

\section{Einleitung}

Selbstregelung ist ein wichtiges Wirkungsprinzip in natürlichen Ökosystemen und trägt zu einem natürlich regulierten Gleichgewicht im System bei. Charakteristisch für Ökosysteme sind Mechanismen der Rückkopplung. Negative Rückkopplungen bremsen Wachstumsprozesse, sie balancieren das System aus und halten es somit am Leben (VESTER 1978). Ursprünglich natürliche Ökosysteme werden durch Inkulturnahme über Bodenbearbeitung und Einsaat von Kulturpflanzen in Agrarökosysteme umgeformt. Selbstregelnde Mechanismen, wie zum Beispiel weit reichende Interaktionen zwischen Räuber und Beute, werden dabei teilweise außer Kraft gesetzt bzw. zerstört und müssen durch den Einsatz von Fremdenergie (z.B. Mineraldünger, Pestizide) ersetzt werden (ALTENKIRCH 1977, DIERCKS 1983). Der Grad der Fremdregulation nimmt mit steigender Intensität innerhalb des Agrarökosystems zu und kann je nach Art und Menge der eingesetzten Betriebsmittel zu unerwünschten Nebenwirkungen innerhalb und außerhalb des Ökosystems führen. Nährstoffauswaschung infolge überhöhter Düngung oder Einträge von Bioziden in benachbarte Ökosysteme sind hierfür Beispiele (AUERSWALD und HAIDER 1992, BAEUMER 1994, VAN DER PLOEG et al. 1997). Als Ursache für das Auftreten von Nebenwirkungen in fremdregulierten Agrarökosystemen sieht KNAUER (1994) das Fehlen oder falls vorhanden, die unzureichende Wirksamkeit von Rückkopplungsmechanismen.

Die bisher in der Literatur beschriebene Nutzung von Selbstregelungen in Agrarökosystemen bezieht sich nahezu ausschließlich auf die biotische Ebene. So beschreiben OHNESORGE (1994), MAHN (1994) und WOLF (1994) Regulationsmechanismen von phytophagen Schädlingen, der Populationsdynamik der Segetalflora und von bodenbürtigen Pflanzenpathogenen.

Fruchtfolgen, deren N-Zufuhr zu einem überwiegenden Anteil durch den Anbau von Leguminosen sichergestellt wird, sind in der landbaulichen Praxis insbesondere im Ökologischen Landbau verbreitet. Ein Verbot des Einsatzes von leichtlöslichen mineralischen N-Düngemitteln (EG-VO -2092/91/EWG) und eine Begrenzung der NZufuhr über andere Betriebsmittel (zugelassene organische N-Dünger, Futtermittel, Saatgut) führt dazu, dass die N-Zufuhr im Wesentlichen eine Funktion der durch die angebauten Leguminosen symbiotisch fixierten N-Menge darstellt.

Beim Anbau von Leguminosen ist bereits seit längerem bekannt, dass ein Zusammenhang zwischen dem Angebot an pflanzenverfügbarem Stickstoff im Boden und der Aufnahme von symbiotisch fixiertem Stickstoff besteht (MCAULIFFE et al. 1958). Auf Grundlage dieser physiologisch verursachten Selbstregelung, d.h. Anpassung einer Fähigkeit der Leguminose an die Standortbedingungen, leiten MöLLER (1995) und SCHMIDTKE (1997a, 1997b) ab, dass in Fruchtfolgen mit Leguminosen die Zufuhr an symbiotisch fixiertem Stickstoff über das Angebot an pflanzenverfügbarem Bodenstickstoff rückgekoppelt ist, und somit der $\mathrm{N}$-Input zumindest teilweise selbst- 
geregelt ist. Nach SCHMIDTKE (1997a) basiert die Selbstregelung der N-Zufuhr in leguminosenbasierten Fruchtfolgen auf einem direkten und einem indirekten Wirkungsmechanismus. Ein erhöhtes bodenbürtiges N-Angebot bei Leguminosen in Reinsaat kann den Anteil Stickstoff aus der Luft in der Biomasse der Pflanze direkt reduzieren. In Gemengen aus Leguminosen und Nichtleguminosen kann sich der Ertragsanteil des nichtlegumen Gemengepartners in Folge eine erhöhten N-Angebotes im Boden überproportional erhöhen und somit die symbiotisch fixierte $\mathrm{N}$ Menge der Leguminose indirekt einschränken, da die Ertragsanteile der Leguminose unter der stärkeren interspezifischen Konkurrenz durch die Nichtleguminose stark gemindert werden (VALLIS 1978).

Ob eine Selbstregelung der N-Zufuhr in leguminosenbasierten Fruchtfolgen tatsächlich vorhanden ist und welche Bedeutung dieser Regelung beizumessen ist, wurde bisher nicht systematisch in Feldversuchen untersucht.

Um unter Feldbedingungen eine Selbstregelung der N-Zufuhr in leguminosenbasierten Fruchtfolgen nachweisen und dessen Beitrag zur Steuerung des N-Haushaltes ackerbaulich genutzter Böden quantifizieren zu können, ist bei Beständen mit Leguminosen, bei denen ein Teil der Sprossmasse mit dem Erntegut abgefahren wird, die Kenntnis des mit dem Anbau von Leguminosen zu erzielenden N-Flächenbilanzsaldos (einfacher $\mathrm{N}$-Saldo $=$ fixierte $\mathrm{N}-$ Menge - Erntegut- $\mathrm{N}$ in kg ha- $\mathrm{a}^{-1}$ ) erforderlich. Wird in einer leguminosenbasierten Fruchtfolge die N-Zufuhr über Mechanismen der Selbstregelung tatsächlich gesteuert, so müsste der N-Flächenbilanzsaldo beim Anbau einer Leguminose, die bei einem geringem Vorrat an pflanzenverfügbarem Stickstoff im Boden mit dem Anbau der Leguminose zu verzeichnen ist, deutlich über dem Betrag des N-Flächenbilanzsaldos liegen, der bei einem Anbau der gleichen Leguminose bei einem erhöhtem $\mathrm{N}$-Vorrat im Boden resultiert.

Inwieweit N-Flächenbilanzsalden eine Funktion des bodenbürtiges $\mathrm{N}$-Angebotes sind, deutet sich in einer Untersuchung von SCHMIDTKE (2001) an, der den Einfluss einer Jauchedüngung auf den vereinfachten N-Flächenbilanzsaldo auf Grundlage der gesamtpflanzlichen Biomasse (Spross + Wurzeln) und auf die zur Ermittlung des NSaldos benötigten Kenngrößen untersuchte.

Im Mittelpunkt vieler Untersuchungen stand die Wirkung eines unterschiedlich hohen $\mathrm{N}$-Angebotes im Boden auf die $\mathrm{N}_{2}$-Fixierleistung von Leguminosen. Dabei wurde sowohl in Feld- als auch in Gefäßversuchen zumeist auf eine N-Düngung mit mineralischen und/oder organischen N-Düngemitteln zurückgegriffen, um ein unterschiedlich hohes N-Angebot im Boden zu erzeugen (RICHARDS und SOPER 1979, SCHERER und DANZEISEN 1980, RICHARDS und Soper 1981, ButLer und LADD 1984, JeNSEN 1986a, ButTery und Gibson 1990, ElWARAKY und HAUNOLD 1990, Cherney und DUXBERY 1994, LAMP et al. 1995, FILEK et al. 1997, GarCíA-PLAZAOLA et al. 2000, TURPIN et al. 2002). 
Lediglich LOPOTZ (1997, an Rotklee Aussaat 1990, Dauerdüngungsversuch von 1973 bis 1982, danach einheitliche Bewirtschaftung bis 1990) und BERGERSEN (1989, an Sojabohne, im Vorjahr Schwarzbrache und Anbau von Hafer) haben auch Wirkungen eines aus der Vorbewirtschaftung resultierenden unterschiedlich hohen Angebotes an Stickstoff im Boden auf das Wachstum und die $\mathrm{N}_{2}$-Fixierleistung von Leguminosen in Feldversuchen geprüft. Während aus einer mineralischen oder organischen $\mathrm{N}$ Düngung ein sprunghafter Anstieg der pflanzenverfügbaren N-Mengen vor allem im Oberboden resultiert (SCHMUDE 1991, SCHMIDTKE 2001), lassen sich Unterschiede im Vorrat an pflanzenverfügbarem Stickstoff, die aus einer längerfristigen unterschiedlichen Vorbewirtschaftung hervorgehen, sowohl im Oberboden als auch im Unterboden beobachten (SCHMUdE 1991, AlFÖLDI et al. 1992, KNUTH 1995, SCHMIDTKE 1997a). Darüber hinaus zeigen Untersuchungen von GRÖBLINGHOFF et al. (1989), KNUTH und CLAUPEIN 1990 sowie von FRANZLUEBBERS et al. (1996), dass in Abhängigkeit von der Vorbewirtschaftung eine unterschiedlich hohe Netto-N-Mineralisation organischer Boden-N-Vorräte resultieren kann, die im Gegensatz zu einer einmaligen mineralischen N-Düngergabe die gesamte Vegetationsperiode einschließt. Ergebnisse von Untersuchungen mit einem durch mineralische Düngung und einem durch langjährig unterschiedliche Vorbewirtschaftung hervorgerufenen differenzierten $\mathrm{N}$ Angebotes im Boden lassen sich hinsichtlich des Einflusses auf die $\mathrm{N}_{2}$-Fixierleistung aufgrund eines unterschiedlichen örtlichen und zeitlichen Auftretens eines erhöhten $\mathrm{N}$-Angebotes im Boden nicht direkt miteinander vergleichen.

Leguminosen werden in der landbaulichen Praxis in der Regel nicht direkt mit Stickstoff gedüngt. Somit sind in erster Linie die Wirkungen eines geänderten bodenbürtigen N-Angebotes für das Agrarökosystem von Interesse, die aus der ackerbaulichen Vorbewirtschaftung vor Ansaat der Leguminosen und nicht aus einer direkten NDüngung zu Leguminosen hervorgehen. Um die Wirkungen eines durch langjährig unterschiedliche Vorbewirtschaftung hervorgerufenen bodenbürtigen N-Angebotes auf die N-Flächenbilanz unter Feldbedingungen aufzuzeigen, wurden die Leguminosenarten Ackerbohne, Luzerne und Saatwicke in Rein- und Gemengesaat mit Gramineen auf den Dauerfeldversuchsflächen des Ackerbau-Systemversuchs Reinshof in Göttingen etabliert. Die gewählten Arten lassen aufgrund unterschiedlich langer Vegetationszeiträume, in denen sie üblicherweise angebaut werden, verbunden mit wurzelmorphologischen Unterschieden und differenzierten Nutzungsarten (Körnerund Futternutzung) sehr spezifische Reaktionen auf das bodenbürtige N-Angebot erwarten. Es sollte geprüft werden, inwieweit Unterschiede zwischen Pflanzenarten hinsichtlich einer Selbstregelung der N-Zufuhr bestehen. Die Aussaat der Prüfglieder erfolgte auf den über 15 Jahre mit geringer bzw. sehr hoher N-Düngungsintensität zu den nichtlegumen Vorfrüchten gedüngten Flächen. 
Die Ziele der vorliegenden Untersuchung waren:

(a) zu prüfen, ob und in welchem Ausmaß in leguminosenbasierten Fruchtfolgen eine Selbstregelung der N-Zufuhr vorhanden ist,

(b) festzustellen, welche Wirkungsmechanismen unter Feldbedingungen eine Selbstregelung der N-Zufuhr hervorrufen,

(c) Unterschiede zwischen Leguminosenarten und Anbauformen (Rein- und Gemengesaat) von Leguminosen im Hinblick auf eine Selbstregelung der $\mathrm{N}$-Zufuhr zu ermitteln,

(d) N-Flächenbilanzsalden für die geprüften Leguminosen zu erstellen, um den Beitrag einer Selbstregelung der N-Zufuhr zur Steuerung des NHaushaltes im Agrarökosystem quantifizieren zu können. 


\section{2.}

\section{Material und Methoden}

Die hier beschriebenen Untersuchungen wurden innerhalb des Dauerfeldversuches Ackerbau-Systemversuch Göttingen in den Jahren 1998 und 1999 mit den Leguminosenarten Saatwicke (Vicia sativa L.), Ackerbohne (Vicia faba L.) und Luzerne (Medicago sativa L.) in Rein- und Gemengesaat mit Einjährigem Weidelgras (Lolium multiflorum ssp. gaudinii), Hafer (Avena sativa) und Knaulgras (Dactylis glomerata) durchgeführt. Im Folgenden werden die für die vorliegenden Untersuchungen relevanten Teile des Versuchsaufbaus des Ackerbau-Systemversuchs Göttingen erläutert.

\section{$2.1 \quad$ Versuchsstandort}

Die Versuchsfläche liegt ca. 2 km südlich von Göttingen im Leinetalgraben, 160 bis 164 m über NN auf lössbürtigen Böden. Die Fläche weist von Süd nach Nord ein leichtes Gefälle von $2 \%$ auf. Die Mächtigkeit des Lössprofils variiert innerhalb des Versuches zwischen $70 \mathrm{~cm}$ und mehr als $170 \mathrm{~cm}$. Der Untergrund wird von Keuperton gebildet, der teilweise von periglazialen Sand- und Schotterschichten überlagert ist. Die Bodengesellschaften des Ackerbau-Systemversuchs wurden von BORNSCHEUER (1984) aufgenommen. Die Bodentypen wechseln von kolluvial überlagerten, mäßig durchschlämmter Schwarzerde im Süden (Block 1 und 2; Abb. 2) über erodierte Grieserden in den Blöcken 3 und 4 bis zu stark durchschlämmten Schwarzerden mit Grundwassereinfluss im Norden (Block 5 und 6). Die Textur im Oberboden (0 bis $35 \mathrm{~cm}$ ) schwankt zwischen einem Tongehalt von $14,9 \%$ bis $22,3 \%$, einem Gehalt an Schluff von 66,6 \% bis 73,1\% und einem Gehalt an Sand zwischen 9,9\% bis 12,2 \% (BORNSCHEUER 1984). Die chemischen Kennwerte des Bodens zu Beginn der hier vorgestellten Untersuchungen sind in Tab. A1 zusammengestellt. Die Werte zeigen eine relativ gute und gleichmäßige Versorgung des Bodens mit Stickstoff und Kohlenstoff sowie Kalium und Phosphat an.

\subsection{Witterungsverlauf}

Der Versuchsstandort gehört zur kühl gemäßigten Klimazone und ist durch ein subozeanisches Klima mit milden Sommern gekennzeichnet. Die im langjährigen Mittel (1961 bis 1990) an der Wetterstation des Deutschen Wetterdienstes in Göttingen gemessenen Jahresniederschlagssummen belaufen sich auf $648 \mathrm{~mm}$. Die Jahresmitteltemperatur beträgt im langjährigen Mittel $8,7^{\circ} \mathrm{C}$ (Abb. 1a). Die Niederschläge fallen über das Jahr gleichmäßig verteilt (Abb. 1b). Während der Vegetationsperiode 1998 (März bis November) fielen $671 \mathrm{~mm}$ Niederschlag im Vergleich zu $501 \mathrm{~mm}$ im langjährigen Mittel. Neben den Monaten April, September und November wies vor allem 
der Monat Oktober 1998 deutlich höhere Niederschläge auf (163 mm gegenüber 40 mm im langjährigen Mittel).

a) 1998

1999

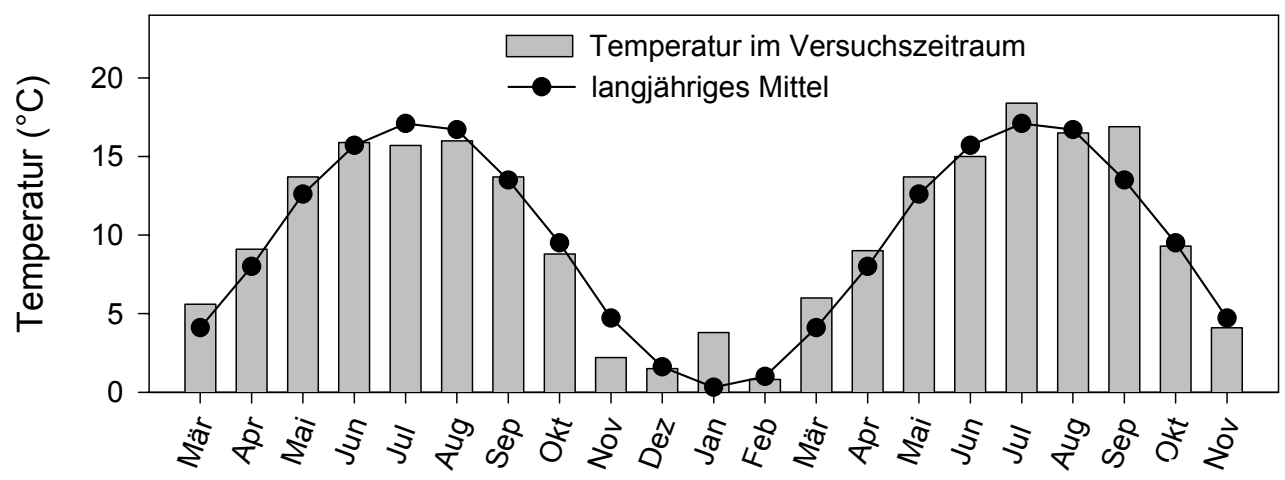

b)

1998

1999

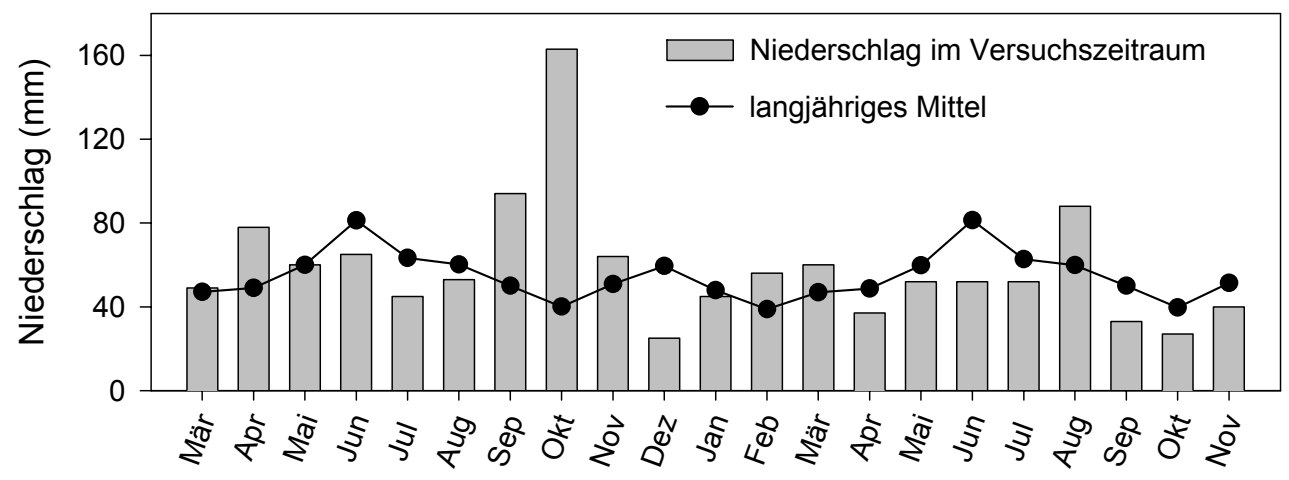

Abb. 1: Monatsmitteltemperaturen (a) und monatliche Niederschlagssummen (b) im Versuchszeitraum von März 1998 bis November 1999 und im langjährigen Mittel

Die Temperatur lag im gleichen Zeitraum mit $11,2^{\circ} \mathrm{C}$ geringfügig unter der Temperatur des langjährigen Mittels $\left(11,3^{\circ} \mathrm{C}\right)$.

In der Vegetationsperiode 1999 (März bis November) fiel mit $441 \mathrm{~mm}$ weniger Niederschlag als im langjährigen Mittel (501 mm). Die Durchschnittstemperatur im Untersuchungszeitraum (März bis November) dagegen lag mit $12,1^{\circ} \mathrm{C}$ über dem Wert des langjährigen Mittels $\left(11,3^{\circ} \mathrm{C}\right)$.

\subsection{Ackerbau-Systemversuch Göttingen}

Auf dem Versuchsgut Reinshof der Universität Göttingen wurden im Rahmen eines Dauerfeldversuches von 1981 bis 2000 in drei Wiederholungen in orthogonaler Kombination die Faktoren Pflanzenschutz, Bodennutzungssystem und der Einfluss unterschiedlicher hoher mineralischer N-Düngungsstufen untersucht. Im Folgenden wird auf den Systemversuch insofern eingegangen, als es für das Verständnis der vorlie- 
genden Untersuchung notwendig ist. Eine ausführliche Beschreibung des Ackerbau Systemversuches ist u. a. bei WILDENHAYN (1990) nachzulesen.

\subsubsection{Pflanzenschutz}

Der Faktor Pflanzenschutz wurde in zwei Stufen geprüft:

a) mit Pflanzenschutz (m Ps): Mit Ausnahme der Luzerne erfolgte in allen Kulturen ein Einsatz von chemischen Pflanzenschutzmitteln zur Bekämpfung von Unkräutern und Schaderregern.

b) ohne Pflanzenschutz (o Ps): Es wurde in allen Beständen auf chemischen Pflanzenschutz verzichtet, die Beikrautregulierung erfolgte durch Striegeln und Hacken.

\subsubsection{Bodennutzungssysteme}

Es wurden zwei Bodennutzungssysteme miteinander verglichen, die sich in der Fruchtfolgegestaltung, der Bodenbearbeitungsintensität und der organischen Düngung unterschieden.

Tab. 1: $\quad$ Merkmale der Bodennutzungssysteme (BNS). Die Abkürzungen kF und IF stehen für kurze bzw. lange Fruchtfolge

\begin{tabular}{|c|c|c|c|c|c|}
\hline \multirow[t]{2}{*}{ BNS } & \multirow[t]{2}{*}{ Kulturart } & \multirow{2}{*}{$\begin{array}{c}\text { Bodenbearbeitung } \\
\text { Tiefe, Zeitpunkt }\end{array}$} & \multirow[t]{2}{*}{ N-Stufe ${ }^{a}$} & \multicolumn{2}{|r|}{ Düngung } \\
\hline & & & & min. $\mathrm{kg} \mathrm{N} \mathrm{ha}^{-1}$ & organisch \\
\hline \multirow{6}{*}{$\mathrm{kF}$} & Zuckerrübe & Pflug & $\mathrm{N}_{0}$ & 0 & Rübenblatt \\
\hline & & $25 \mathrm{~cm}$, Oktober & $\mathrm{N}_{3}$ & $210\left(170^{b}\right)$ & Rübenblatt \\
\hline & Winterweizen & Pflug & $\mathrm{N}_{0}$ & 0 & Weizenstroh \\
\hline & & $20 \mathrm{~cm}$, September & $\mathrm{N}_{3}$ & 180 & Weizenstroh \\
\hline & Wintergerste & Pflug & $\mathrm{N}_{0}$ & 0 & Gerstenstroh + Wickengründüngung \\
\hline & & $30 \mathrm{~cm}$, November & $\mathrm{N}_{3}$ & 180 & Gerstenstroh + Wickengründüngung \\
\hline \multirow{12}{*}{ IF } & Zuckerrübe & Grubber & $\mathrm{N}_{0}$ & 0 & --------- \\
\hline & & $8 \mathrm{~cm}$, Oktober & $\mathrm{N}_{3}$ & $210\left(170^{b}\right)$ & 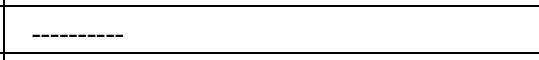 \\
\hline & Winterweizen & Pflug & $\mathrm{N}_{0}$ & 0 & Stallmist $330 \mathrm{dt} \mathrm{ha}^{-1}$ auf Stoppeln ${ }^{\mathrm{c}}$ \\
\hline & & $20 \mathrm{~cm}$, September & $\mathrm{N}_{3}$ & 180 & Stallmist $330 \mathrm{dt} \mathrm{ha}^{-1}$ auf Stoppeln ${ }^{\mathrm{c}}$ \\
\hline & Wintergerste & Pflug & $\mathrm{N}_{0}$ & 0 & Gerstenstroh $^{d}$ \\
\hline & & $30 \mathrm{~cm}$, November & $\mathrm{N}_{3}$ & 180 & Gerstenstroh $^{d}$ \\
\hline & Luzerne & Fräse & $\mathrm{N}_{0}$ & 0 & Stallmist $330 \mathrm{dt} \mathrm{ha}^{-1}$ auf Stoppeln, Herbst ${ }^{\mathrm{e}}$ \\
\hline & & $8 \mathrm{~cm}$, Oktober + April & $\mathrm{N}_{3}$ & 0 & Stallmist $330 \mathrm{dt} \mathrm{ha}^{-1}$ auf Stoppeln, Herbst ${ }^{\mathrm{e}}$ \\
\hline & Mais & Fräse & $\mathrm{N}_{0}$ & 0 & Jauche $36 \mathrm{~m}^{3}$ ha $^{-1}$ zu Mais im Frühjahr ${ }^{\mathrm{e}}$ \\
\hline & & $5 \mathrm{~cm}$, November & $\mathrm{N}_{3}$ & 120 & Jauche $36 \mathrm{~m}^{3} \mathrm{ha}^{-1}$ zu Mais im Frühjahr ${ }^{\mathrm{e}}$ \\
\hline & Ackerbohne & Pflug & $\mathrm{N}_{0}$ & 0 & Stroh der Ackerbohne \\
\hline & & $15 \mathrm{~cm}$, November & $\mathrm{N}_{3}$ & 0 & Stroh der Ackerbohne \\
\hline
\end{tabular}

${ }^{a}$ Die N-Stufen $\mathrm{N}_{1}$ und $\mathrm{N}_{2}$ sind nicht aufgeführt, da sie für die vorliegende Untersuchung nicht berücksichtigt wurden; ${ }^{\mathrm{b}}$ seit 1988 wurde die min. N-Düngung zur Zuckerrübe um $40 \mathrm{~kg} \mathrm{~N}$ ha $^{-1}$ reduziert; ${ }^{\circ} 1982-1987165 \mathrm{dt} \mathrm{ha}^{-1}$ Stallmist (ca. $80 \mathrm{~kg} \mathrm{~N} \mathrm{ha}^{-1}$ ), ab $1988330 \mathrm{dt} \mathrm{ha}^{-1}$ Stallmist (ca. $160 \mathrm{~kg} \mathrm{~N}$ ); ${ }^{d}$ bis 1988 wurde das Gerstenstroh abgefahren; ${ }^{\mathrm{e}} 1982$ bis 1987: $165 \mathrm{dt} \mathrm{ha}^{-1}$ Stallmist + $18 \mathrm{~m}^{3}$ Jauche vor Aussaat des Mais (insgesamt $120 \mathrm{~kg} \mathrm{~N}^{-1}$ ), ab 1988 Stallmist: $330 \mathrm{dt} \mathrm{ha}^{-1}$ vor Aussaat des Mais $+36 \mathrm{~m}^{3} \mathrm{Jauche}^{-1}$ bei 50 bis $60 \mathrm{~cm}$ Wuchshöhe des Mais. 
Dabei wurden eine standorttypische kurze Fruchtfolge mit Marktfrüchten und Gründüngung (kF) einer langen Fruchtfolge mit Marktfrüchten und Futterbau (IF) gegenübergestellt, die einen Betrieb mit Rinderhaltung (1 GVE ha $\left.{ }^{-1}\right)$ simuliert. Die in den Bodennutzungssystemen integrierten Fruchtarten, die unterschiedliche Verfahren und Intensität der Bodenbearbeitung, die Art und Umfang der organischen Düngung sowie die Höhe der mineralischen Dünger sind in Tab. 1 zusammengestellt.

\subsubsection{Mineralische N-Düngung}

Die Höhe der mineralischen N-Düngung wurde zu Beginn des Dauerversuches dem voraussichtlichen Bedarf der einzelnen Feldfrüchte angepasst und für die Dauer einer Fruchtfolgerotation (IF) bzw. zweier Fruchtfolgerotationen (kF) fixiert. Es wurden die mineralischen $N$-Düngestufen ohne $\left(N_{0}\right)$, suboptimal $\left(N_{1}\right)$, optimal $\left(N_{2}\right)$ und hyperoptimal $\left(N_{3}\right)$ festgelegt, um deren kumulierte Wirkung über die geprüften Fruchtfolgen und Pflanzenschutzsysteme prüfen zu können. Die Leguminosen erhielten keine mineralische N-Düngung. Die organischen Dünger wurden auf allen NStufen in gleicher Höhe ausgebracht. Um die Einflüsse langjährig unterschiedlich hoher mineralischer N-Düngung zu den nicht legumen Vorfüchten auf die angebauten Leguminosen möglichst sicher aufzeigen zu können, wurden in die vorliegende Untersuchung nur die N-Düngungsstufen ohne $\left(\mathrm{N}_{0}\right)$ und überoptimal $\left(\mathrm{N}_{3}\right)$ berücksichtigt. Die entsprechenden N-Düngemengen zu den nicht legumen Fruchtarten sind in Tab. 1 wiedergegeben. Die direkten Vorfrüchte der Leguminosen im Systemversuch sind Wintergerste vor Saatwicke (kF) und vor Luzerne (IF) sowie Mais vor Ackerbohne (IF). Die mineralischen N-Dünger zu Wintergerste wurden sowohl in der kurzen als auch in der langen Fruchtfolge in drei Gaben zu je $60 \mathrm{~kg} \mathrm{~N} \mathrm{ha}^{-1}$ zur Bestockung (BBCH 21-24), zum Schossen $(\mathrm{BBCH}$ 31) und zum Beginn des Ährenschiebens $(\mathrm{BBCH}$ 51) verabreicht. Die mineralische N-Düngung zu Mais in Höhe von $120 \mathrm{~kg} \mathrm{~N} \mathrm{ha}^{-1}$ wurde zur Keimung appliziert (BBCH 05-11). 


\section{$2.4 \quad$ Versuchsanlage und Versuchsdurchführung}

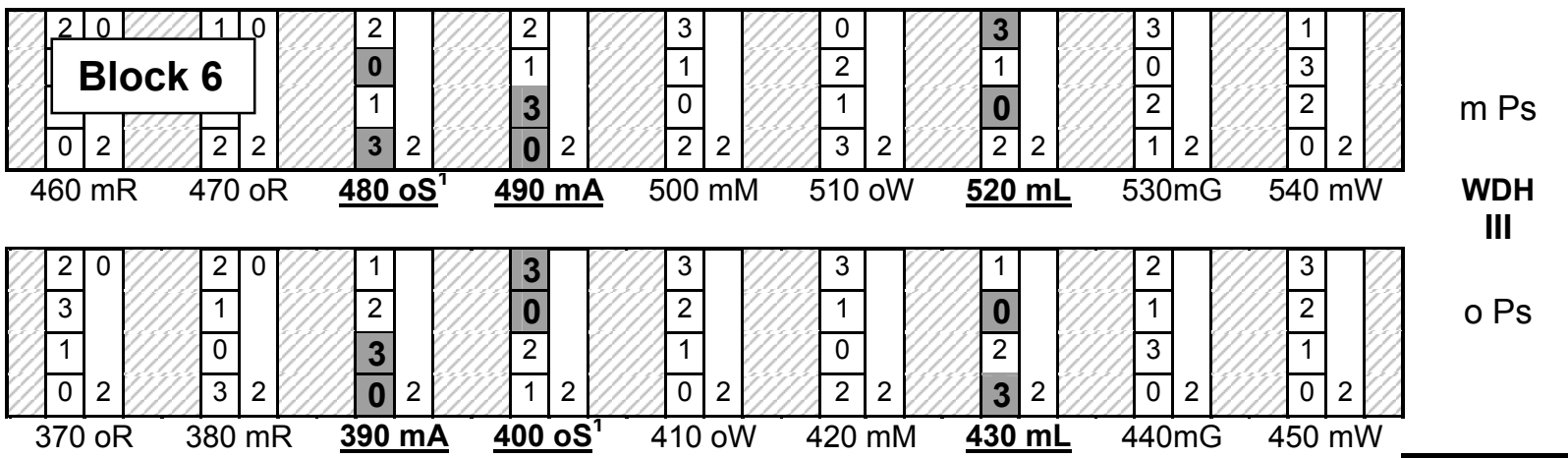

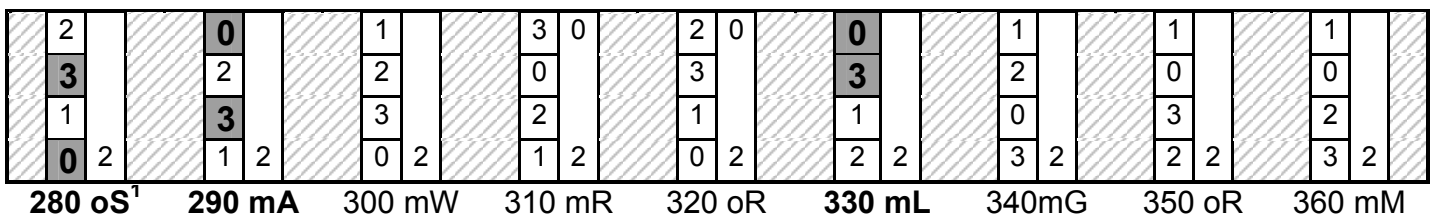

m Ps

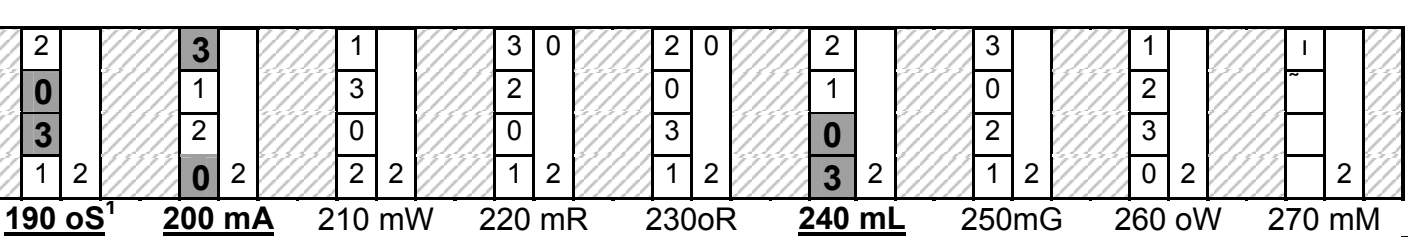

WDH

II

o Ps

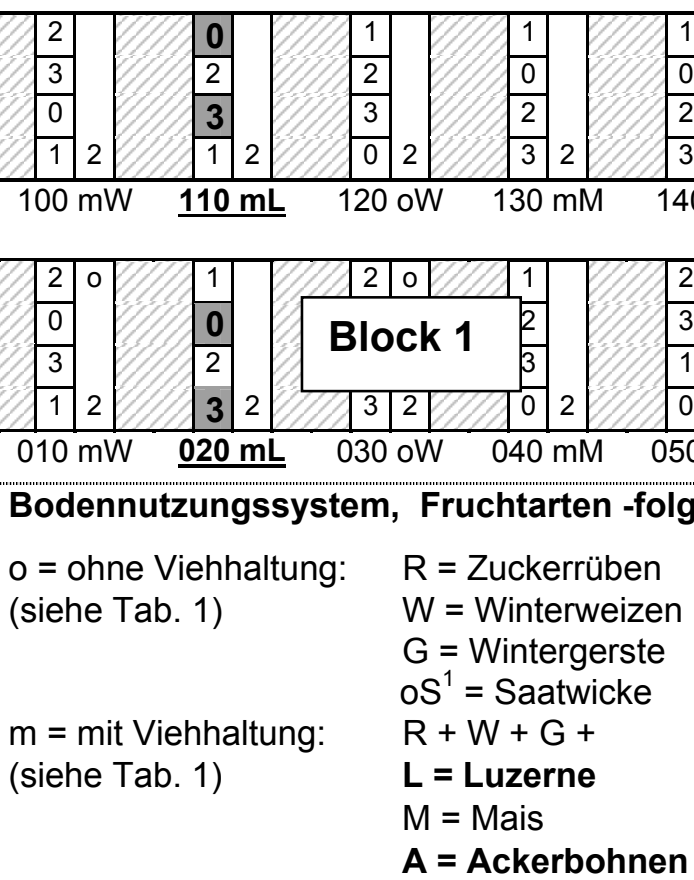

Für die vorliegende Untersuchung im Jahr 1998 genutzten Parzellen

Pflanzenschutz:

o Ps: $\quad$ ohne chemischen Pflanzenschutz; mechanische Unkrautbekämpfung

m Ps: $\quad$ umfassender chemischer Pflanzenschutz nach festem Plan

1 Hauptfrucht Wintergerste, anschließend Saatwicke als Stoppelsaat (deshalb oS statt oG)

Abb. 2: Lageplan der Prüfglieder im Ackerbau-Systemversuch Göttingen, Feldplan 1998 
In Abb. 2 ist der Lageplan der Versuchsparzellen im Ackerbau-Systemversuch Göttingen für das Untersuchungsjahr 1998 wiedergegeben. Der Lageplan für 1999 ist im Anhang abgebildet (Abb. A1). Die Aussaat erfolgte in Parzellen bei Ackerbohne/Hafer am 25.03.1998 bzw. 17.03.1999 und bei Saatwicke/Einjähriges Weidelgras am 27.07.1998 bzw. 13.07.1999 mit einer Parzellendrillmaschine nach Øyjord senkrecht (Ost-West) zur Bearbeitungsrichtung des Systemversuchs (NordSüd), in die mit grau unterlegten Mittelteilstücke (Düngungsstufen; Abb. 2) des Ackerbau-Systemversuches. Die Aussaat der Luzerne und des Knaulgrases erfolgte am 01.04.1998 bzw. 12.04.1999. Aufgrund der vorgegebenen Größe der Mittelteilstücke (7,5 m x $5 \mathrm{~m}$ ) ergaben sich Parzellengrößen von 1,5 m x $5 \mathrm{~m}$ (Abb. 3).
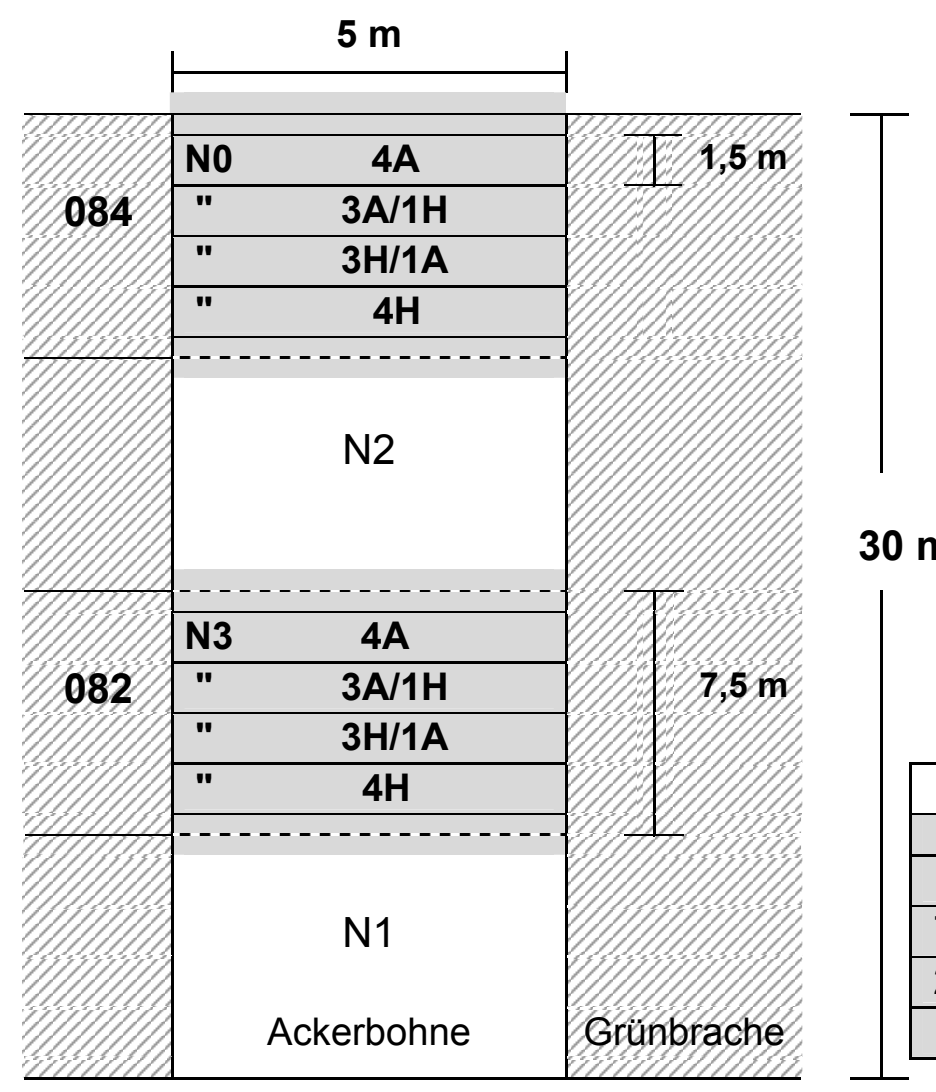

$30 \mathrm{~m}$

Parz.Nr.: 080

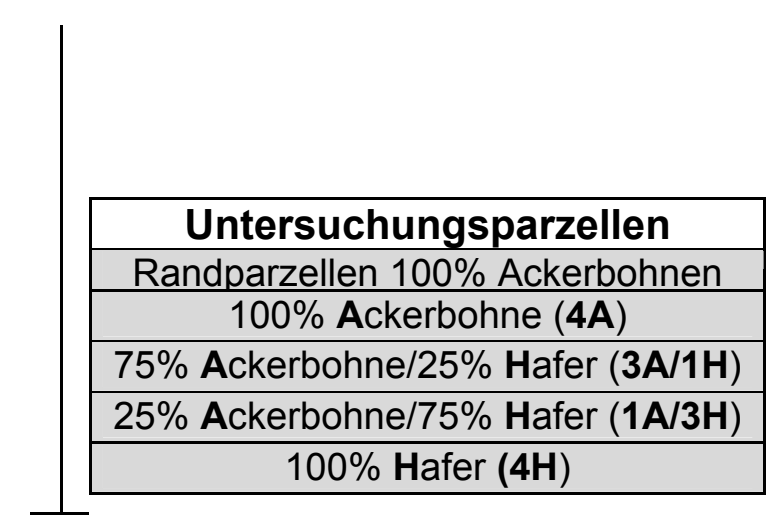

Abb. 3: Anordnung der Untersuchungsparzellen innerhalb des Ackerbau-Systemversuches

Die untersuchten Leguminosenarten Saatwicke (cv. Jaga), Ackerbohne (cv. Minica) und Luzerne (cv. Europe) entsprachen den im Systemversuch bisher angebauten Leguminosenarten und -varietäten. Die Anlage erfolgte ausschließlich in den langfristig nicht $\left(\mathrm{N}_{0}\right)$ und überoptimal $\left(\mathrm{N}_{3}\right)$ mineralisch gedüngten Dauerparzellen. Die Ansaat der Bestände erfolgte in Reinsaat und in Gemenge mit einer Nichtleguminose. Es wurde eine Verdrängungsserie nach DE WIT (1966) mit den nichtlegumen 
Reinsaaten Einjähriges Weidelgras (Lolium multiflorum ssp. gaudinii cv. Aubade) zu Saatwicke, Hafer (Avena sativa cv. Alf) zu Ackerbohne und Knaulgras (Dactylis glomerata cv. Lidaglo) zu Luzerne angelegt. Die Ansaatverhältnisse keimfähiger Samen der Leguminose zur Nichtleguminose waren $1 \mathrm{zu} 0,0,75 \mathrm{zu} 0,25$, 0,25 zu 0,75 und 0 zu 1, bemessen an der jeweiligen Reinsaatstärke der Arten. Die Ansaatvarianten sowie die Reihenabstände sind in Tab. 2 zusammengestellt.

Tab. 2: Anzahl keimfähiger Samen $/ \mathrm{m}^{2}$ und Reihenweiten in $\mathrm{cm}$ der Rein- und Gemengesaaten

\begin{tabular}{c|c|c|c|c|c|c}
\hline \multicolumn{3}{|c|}{ Reinsaat } & \multicolumn{3}{c|}{ Gemenge } & Hafer \\
\hline Abkürzung & $\begin{array}{c}\text { Acker- } \\
\text { bohne }\end{array}$ & Hafer & Abkürzung & $\begin{array}{c}\text { Acker- } \\
\text { bohne }\end{array}$ & $\begin{array}{c}\text { Reihabstand } \\
\text { in cm }\end{array}$ \\
\hline $4 \mathrm{~A}$ & 45 & 0 & $3 \mathrm{~A} / 1 \mathrm{H}$ & 34 & 50 & 28 \\
\hline $4 \mathrm{H}$ & 0 & 200 & $1 \mathrm{~A} / 3 \mathrm{H}$ & 11 & 150 & 28 \\
\hline & Luzerne & Knaulgras & & Luzerne & Knaulgras & \\
\hline $4 \mathrm{~L}$ & 400 & 0 & $3 \mathrm{~L} / 1 \mathrm{~K}$ & 300 & 133 & 18 \\
\hline $4 \mathrm{~K}$ & 0 & 530 & $1 \mathrm{~L} / 3 \mathrm{~K}$ & 100 & 398 & 18 \\
\hline & Saatwicke & $\begin{array}{c}\text { Einjähriges } \\
\text { Weidelgras }\end{array}$ & & Saatwicke & $\begin{array}{c}\text { Einjähriges } \\
\text { Weidelgras }\end{array}$ & \\
\hline $4 \mathrm{~S}$ & 160 & 0 & $3 \mathrm{~S} / 1 \mathrm{E}$ & 120 & 133 & 18 \\
\hline $4 \mathrm{E}$ & 0 & 530 & $1 \mathrm{~S} / 3 \mathrm{E}$ & 40 & 398 & 18 \\
\hline
\end{tabular}

Für die vorliegende Untersuchung ergaben sich folgende Versuchsfaktoren, die als dreifaktorielle Spaltanlage mit drei Wiederholungen angeordnet waren:

- Großteilstücke: ohne/mit chemischem Pflanzenschutz zu den Vorfrüchten (zwei Varianten),

- Mittelteilstücke: Mineral-N Düngestufen ohne $\left(\mathrm{N}_{0}\right)$ und überoptimal $\left(\mathrm{N}_{3}\right)$ zu den nichtlegumen Vorfrüchten (zwei Varianten),

- Parzellen: Ansaatvarianten (vier Varianten, vgl. Tab. 2).

Die Ansaatvarianten wurden innerhalb der Mittelteilstücke randomisiert. Die Teilstücke sind für die gesamte Versuchsdauer (18 Jahre) festgelegt, um kumulative Wirkungen der Prüffaktoren erfassen zu können. Die angebauten Früchte wandern innerhalb der Hauptteilstücke (010, 020 etc.; Abb. 2) entsprechend den Fruchtfolgen nach einem festgelegten Plan. Der Dauerversuch war so angelegt, dass jedes Prüfglied einer Fruchtart in jedem Jahr vorhanden war.

Die Saatbettbereitung erfolgte in Anlehnung an die im Systemversuch üblichen Bodenbearbeitungsverfahren. Um mit der Parzellendrillmaschine eine ausreichend tiefe Saatgutablage zu gewährleisten, wurde unter Beibehaltung einer pfluglosen Bodenbearbeitung vor der Aussaat der Ackerbohne und des Hafer die Fräse eingesetzt. Vor Aussaat der Stoppelsaaten Saatwicke und Einjährigem Weidelgras wurden 
die Stoppeln der Vorfrucht Wintergerste mit Hilfe eines Balkenmähers bodennah abgeschnitten und anschließend mit dem Stroh von den Parzellen entfernt. Durch zweimaliges Überfahren mit einer Spatenrollegge erfolgte danach die Saatbettbereitung. Die im Systemversuch übliche Vorgehensweise bei der Bestellung der Luzerne (Pflug + Kreiselegge) wurde in beiden Versuchsjahren durch eine mit dem Federzinkengrubber und der Fräse durchgeführte Saatbettbereitung ergänzt. Um den Ansprüchen der Arten an unterschiedliche Ablagetiefen gerecht zu werden, wurden die Ansaatvarianten mit Ackerbohne und Hafer bzw. Saatwicke und Einjährigem Weidelgras in zwei Arbeitsgängen ausgebracht. Während der ersten Überfahrt wurden die Ackerbohnen in einer Bodentiefe von 7 bis $8 \mathrm{~cm}$, die Saatwicken in einer Bodentiefe von 3 bis $4 \mathrm{~cm}$ Bodentiefe abgelegt. Direkt im Anschluss wurde dann der Hafer bei einer zweiten Überfahrt auf etwa $4 \mathrm{~cm}$ bzw. das Einjährige Weidelgras auf 1 bis 2 $\mathrm{cm}$ Bodentiefe gedrillt.

Aufgrund der teilweise sehr geringen Aussaatmengen, insbesondere bei den Gemengeansaaten, wurden die keimfähigen Samen der Luzerne, des Knaulgrases und des Einjährigen Weidelgrases mit einer sich zu $100 \%$ ergänzenden Menge nicht keimfähiger Samen ergänzt. Die Ansaatvariante $3 \mathrm{~L} / 1 \mathrm{~K}$ beispielsweise enthält normalerweise 0,75 und 0,25 der Reinsaatstärke der Luzerne und des Knaulgrases. Diese Mischung keimfähiger Samen wurde mit 0,25 bzw. 0,75 der Reinsaatstärke mit nicht keimfähiger Luzernesamen bzw. Knaulgrassamen ergänzt. Hiermit sollten Aussaatfehler durch Entmischung der Gemengepartner minimiert werden, um eine exaktere Saatgutverteilung innerhalb der Parzellen zu gewährleisten.

Die untersuchten Flächen wurden während dieser Untersuchung weder gedüngt noch mit Pflanzenschutzmitteln behandelt. Aufgrund eines unzureichenden Auflaufens der Ackerbohnen, was vermutlich durch das Auftreten von meist in Mischinfektionen zu beobachtenden samen- und bodenbürtigen pilzlichen sowie bakteriellen Krankheitserregern verursacht wurde, mussten einige Ackerbohnenpflanzen ergänzt werden. Zu diesem Zweck wurden ca. fünf Wochen nach der Aussaat (BBCH 12-13, MEIER 1997), sowohl im Jahr 1998 als auch im Jahr 1999 die fehlenden Pflanzen mit Hilfe von Pflanzhölzern aus den Ackerbohnen Blindparzellen der entsprechenden $\mathrm{N}$-Düngungsstufe in die Untersuchungsparzellen versetzt. Jede versetzte Pflanze wurde mit etwa $250 \mathrm{ml}$ Wasser angegossen, um ein sicheres Anwachsen zu gewährleisten. Etwa 95 \% der versetzten Pflanzen wiesen 10 Tage nach der Verpflanzung keine sichtbaren Unterschiede im Wuchs gegenüber den nicht verpflanzten Ackerbohnen auf. Nicht angewachsene Ackerbohnenpflanzen wurden von der Fläche entfernt.

Die Beikrautregulierung erfolgte per Hand sowie mit einer Roll- und Handhacke bis zum Schließen der Bestände (Tab. A3). Um Nährstoffentzüge aus den Parzellen zu vermeiden, wurde die Biomasse der Beikräuter auf der Fläche belassen. Die Untersuchungsparzellen wurden zu den in Tab. A3 angegebenen Ernteterminen 
beprobt. Jede Ernte beinhaltete die Erfassung der gesamtpflanzlichen Biomasse (Ackerbohne/Hafer: Spross und Wurzel bzw. Luzerne, Saatwicke, Knaulgras, Einjähriges Weidelgras: Schnittgut, Stoppel- und Wurzelmasse) sowie die pflanzenverfügbare $\mathrm{N}_{\min }$-Menge im Boden. Die $\mathrm{N}_{\min }$-Menge im Boden wurde vor der Aussaat je Düngungsstufe (Mittelteilstück), bei Ackerbohne und Hafer zusätzlich nach der letzten Ernte zu Vegetationsende in allen Parzellen erfasst. Darüber hinaus wurde bei der Ackerbohne mittels Blattfallkörben (Größe: $18 \times 50 \mathrm{~cm}$ ) der Bestandesabfall im Zeitraum von der zweiten Ernte bis zur Endernte erfasst.

\subsection{Untersuchungen am Pflanzenmaterial}

\subsubsection{Ernte der Sprossmasse}

Die Biomasse der Ansaaten wurde in Kleinteilflächen je Parzelle mit einer Größe von $1 \mathrm{~m}^{2}$ bei Ackerbohne/Hafer ( 3 Reihen je $1,2 \mathrm{~m}$ Länge) und $1,5 \mathrm{~m}^{2}$ bei Luzerne/Knaulgras 1998 (6 Reihen je 1,38 m Länge) bzw. 1,08 m² 1999 (6 Reihen je 1 $m$ Länge) erhoben. Die nur zu einem Zeitpunkt je Prüfjahr durchgeführte Beerntung der Bestände mit Saatwicke und Einjährigem Weidelgras erfolgte 1998 auf einer Fläche von 2,16 $\mathrm{m}^{2}$ (6 Reihen je $2 \mathrm{~m}$ Länge) und musste 1999 aufgrund von Mäusefraßschäden auf eine Fläche auf $1,08 \mathrm{~m}^{2}$ je Parzelle (6 Reihen je $1 \mathrm{~m}$ Länge) reduziert werden. Die Kleinteilflächen bei den Ackerbohne/Hafer-Beständen wurden per Hand mit Hilfe einer Säge bzw. Gartenschere direkt an der Bodenoberfläche geschnitten. Der Aufwuchs der Luzerne/Knaulgras- und Saatwicke/Einjähriges Weidelgras-Bestände wurde in die Fraktionen Schnittgutmasse und Stoppelmasse aufgeteilt. Die Beerntung der Kleinteilflächen erfolgte mit einer Gartenschere mit angeschraubtem Abstandhalter, um beim Schnitt eine Stoppelhöhe von etwa $5 \mathrm{~cm}$ zu gewährleisten. Die Stoppelmasse wurde unmittelbar im Anschluss durch Schneiden der jeweils mittleren 4 Reihen direkt an der Bodenoberfläche erfasst. Die Pflanzenproben wurden unmittelbar nach der Beerntung in einer Kühlzelle bei $4^{\circ} \mathrm{C}$ bis zur Weiterverarbeitung zwischengelagert.

\subsubsection{Entnahme der Wurzelproben}

Die Entnahme von Wurzelproben erfolgte mit der Bohrmethode (verändert nach SCHUURMAN and GOEDEWAAGEN 1971) mit Hilfe von schlaghammerbetriebenen Rammkernsonden (Fa. Stitz) mit einem Durchmesser von $8,7 \mathrm{~cm}$ in den Bodentiefen von 0 bis $25 \mathrm{~cm}$ und 25 bis $50 \mathrm{~cm}$. Der wesentliche Vorteil, der mit der in dieser Untersuchung genutzten Methode zur Wurzelprobenentnahme im Feld verbunden ist, liegt in der vergleichsweise geringen Zerstörung der Versuchsfläche sowie der Möglichkeit, mehrmals verteilt über die Parzelle Einstiche vornehmen zu können. Im Falle von Reihenkulturen ist zu berücksichtigen, dass in Abhängigkeit von den 
angebauten Kulturpflanzen die Wurzelverteilung auf der Fläche räumlich stark variiert. Im Falle von Kulturen mit ausgeprägtem allorrhizem Wurzelsystem, wie z.B. der Ackerbohne, ist für das Ergebnis nicht nur von Bedeutung, ob die Rammkernsonde zur Probenahme auf oder zwischen einer Saatreihe platziert wurde, vielmehr besteht auch ein direkter Einfluss auf die Schätzung der Wurzelmasse, ob innerhalb der Saatreihe direkt auf einer Pflanze oder zwischen zwei Pflanzen beprobt wird. In der vorliegenden Untersuchung wurde versucht, die Anzahl der Einstiche mit einer exakten Auswahl der Beprobungsorte auf ein Minimum zu reduzieren um somit mehrere Beprobungstermine innerhalb einer festgelegten Parzellengröße bzw. Versuchsfläche zu realisieren.

Die Beprobung des Wurzelraumes erfolgte auf und zwischen den Reihen. Dabei wurden in den Reinsaaten jeweils ein Einstich, in den Gemengesaaten jeweils zwei Einstiche innerhalb der Reihe und zwischen den Reihen vorgenommen. Bei der Positionierung der Rammkernsonden wurde wie folgt vorgegangen: Um sicherzustellen, dass im Hinblick auf den Bestand möglichst repräsentative Pflanzen beprobt werden, wurden die Beprobungsorte bereits vor der Beerntung der Sprossmasse festgelegt. Die Vorgehensweise beim Platzieren der Rammkernsonden, wie sie in Abhängigkeit von der Pflanzenart und Anbauform (Rein- oder Gemengeansaat) durchgeführt wurde, wird im Folgenden genauer dargestellt.

Ackerbohne in Reinsaat:

Die auf der Reihe ("a") entnommene Rammkernsonde wurde exakt auf eine Ackerbohnenpflanze gesetzt, der Bohrstock zwischen der Reihe ("z") in Nachbarschaft zur beprobten Ackerbohnenpflanze, in der Mitte des Reihenzwischenraumes.

Hafer in Reinsaat:

\section{Beprobungschema 1:}

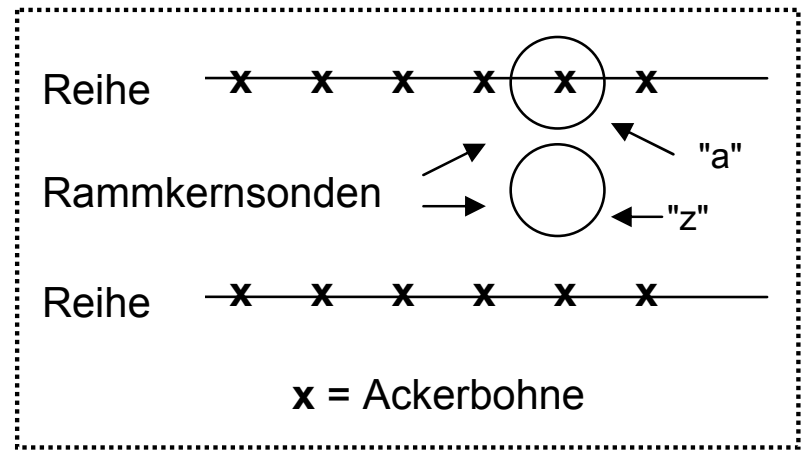

Die Rammkernsonde zur Beprobung des Bereichs auf der Reihe wurde auf mehrere, benachbarte Haferpflanzen gesetzt. Die Rammkernsonde zur Beprobung des Reihenzwischenraumes wurde zwischen zwei Reihen in Nachbarschaft zum Reihenbohrstock in der Mitte des Reihenzwischenraumes entnommen (vgl. Beprobungschema 1). Die Vorgehensweise bei der Beprobung der Reinsaaten der Luzerne, der Saatwicke, des Knaulgrases und des Einjährigen Weidelgrases erfolgte analog zur Vorgehensweise beim Hafer. 
Ackerbohne/Hafer-Gemenge:

Die erste Rammkernsonde zur Beprobung des Bereiches auf der Reihe wurde auf eine Ackerbohnenpflanze ("a1") gesetzt. Hierbei wurden gleichzeitig je nach Ansaatvariante mehrere Haferpflanzen mit erfasst. Die zweite Rammkernsonde wurde auf der Reihe ausschließlich auf Haferpflanzen ("a2") platziert. Die Rammkernsonden zur Beprobungschema 2:

Beprobung des Reihenzwischenraumes wurden wie bei den Reinsaaten beschrieben platziert ("z1" und "z2").

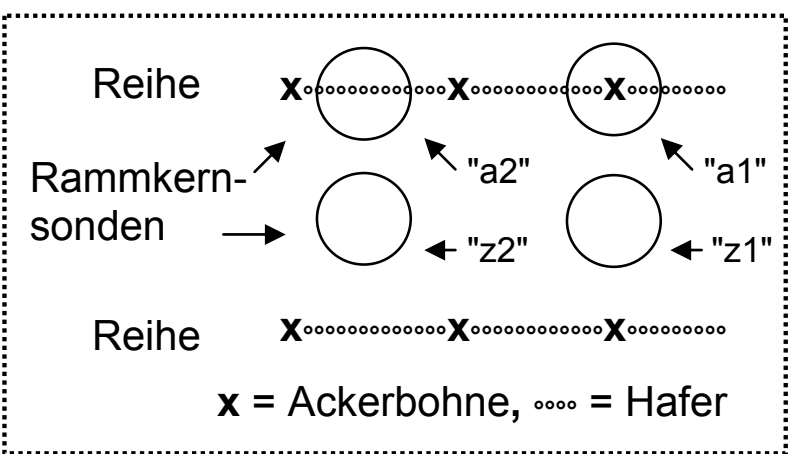

Luzerne/Knaulgras- bzw. Saatwicke/Einjähriges Weidelgras-Gemenge:

Die auf den Pflanzenreihen platzierten Rammkernsonden sollten repräsentative Individuen beider Gemengepartner enthalten. Die Rammkernsonden zur Beprobung des Reihenzwischenraumes wurden entsprechend den Reinsaaten in direkter Nachbarschaft im Reihenzwischenraum platziert. Unterschiedliche Anbaubedingungen (Pflanzenzahlen je Fläche, Reihenabstand) und deutliche Unterschiede in der Wurzelmorphologie (z.B. Ackerbohne versus Hafer) machen ein differenziertes Vorgehen bei der Berechnung der Wurzelmassen je Hektar notwendig. Eine ausführliche Beschreibung des Kalkulationsverfahrens ist in Kapitel 2.7.1 nachzulesen.

\subsubsection{Aufbereitung der Pflanzen- und Wurzelproben}

Unmittelbar nach Beerntung der Pflanzenbestände erfolgte die Weiterverarbeitung der Proben. Die Stoppelproben des Luzerne/Knaulgras- bzw. des Saatwicke/Einjähriges Weidelgras-Aufwuchses wurden in Drahtgitterkörben mit Wasser von anhaftenden Bodenpartikeln befreit. Sowohl die Stoppelproben als auch das Schnittgut wurden in die Bestandeskomponenten Leguminose und Kräuter (Luzerne- und Saatwicke-Reinsaaten) bzw. Leguminose, Gräser und Kräuter (jeweilige Gemenge) sortiert. Die Bestimmung der Frischmassen des Schnittgutes erfolgte mit einer Pflanzenwaage (Fa. Sartorius; Typ BP $340000-P \pm 0,1 \mathrm{~g}$ bis $\pm 0,5 \mathrm{~g}$ ). Anschließend wurde das Schnittgut mit einem Laborprobenhäcksler (Fa. Walter \& Wintersteiger) zerkleinert und ein Aliquot der Bestandeskomponenten für die Analyse (Stickstoffgehalt, $\delta^{15} \mathrm{~N}$-Wert) bei $60^{\circ} \mathrm{C}$ getrocknet, gewogen und in Polyethylenbeuteln zwischengelagert. Teilproben des bei $60^{\circ} \mathrm{C}$ getrockneten Pflanzenmaterials wurden zur Ermittlung des Restwassergehaltes bei $105^{\circ} \mathrm{C}$ bis zur Gewichtskonstanz nachgetrocknet. 
Der Aufwuchs der Ackerbohne und des Hafers wurde ebenfalls in die entsprechenden Ertragskomponenten aufgeteilt (Ackerbohne, Hafer, Kräuter). Nach Bestimmung der Frischmassen wurden bei der Ackerbohne die Anzahl Pflanzen je Kleinteilfläche, Hülsen je Pflanze sowie die Anzahl Körner je Hülse ermittelt. Nach dem Drusch wurden die erhaltenen Komponenten Korn und Ackerbohnenstroh (Stroh + Hülsen) sowie Haferstroh (Stroh + Rispen + Spelzen) der oben beschriebenen Weiterverarbeitung zugeführt. Die gesammelten Bestandesabfälle wurden getrennt nach Beprobungstermin in gleicher Weise verarbeitet.

Die feldfrischen Wurzelproben wurden in Polyethylenschüsseln im Gewächshaus luftgetrocknet und nach ein bis zwei Tagen im Trockenschrank bei $60^{\circ} \mathrm{C}$ bis zur Gewichtskonstanz getrocknet. Nach der Trockenmassebestimmung des Boden/WurzelGemisches erfolgte die Trennung der Wurzeln vom Boden mit Hilfe einer halbautomatischen Wurzelwaschanlage (geändert nach Delta-T, Fa. UP) über einem Sieb mit einer Maschenweite von $1 \mathrm{~mm}$. Die Wurzeln wurden manuell von Fremdpartikeln getrennt und erneut bei $60^{\circ} \mathrm{C}$ bis zur Gewichtskonstanz getrocknet. Zur Bestimmung der Trockenmassen je beernteter Fläche bzw. entnommener Bodensäulen wurden alle getrockneten Schnittgut-, Stoppel-, Körner-, Bestandesabfall- und Wurzelproben auf einer Feinwaage (Fa. Sartorius, Typ BL 310, $\pm 0,01 \mathrm{~g}$ ) ausgewogen.

\subsubsection{Pflanzenanalysen}

Die Pflanzenproben wurden mit einer Ultrazentrifugalmühle (Fa. Retsch, Typ ZM 100) auf eine Partikelgröße $\leq 0,20 \mathrm{~mm}$ vermahlen. Vor der Analyse wurden die Proben mittels einer speziellen Laborwaage (Fa. Sartorius, Typ M2P; $\pm 0,001 \mathrm{mg}$ ), in Massen von 3 bis $5 \mathrm{mg}$ in Zinnkapseln (Größe: $5 \times 9 \mathrm{~mm}$; Fa. IVA Analysentechnik) eingewogen, um dann mit einem N-Elementaranalyzer (Typ NA 1500, Fa. Carlo Erba) und nachgeschaltetem Isotopenmassenspektrometer (IRMS, Typ MAT 251, Fa. Finnigan) den Gesamtstickstoffgehalt sowie das Stickstoffisotopenverhältnis $\left({ }^{14} \mathrm{~N} /{ }^{15} \mathrm{~N}\right)$ zu ermitteln. Die Kräuterproben wurden mit einem CN-Analysator (Fa. Elementar, Typ Vario EL) auf ihren Gehalt an Stickstoff und Kohlenstoff untersucht.

\subsubsection{Schätzung der symbiotischen Stickstoff-Fixierleistung mit der $\delta^{15} \mathrm{~N}$ - Methode (Natural ${ }^{15} \mathrm{~N}$ abundance method) und erweiterten Differenz- methode (Extended difference method)}

Der Anteil Stickstoff aus der Luft in den zu prüfenden Leguminosen wurde mit Hilfe der $\delta^{15} \mathrm{~N}$-Methode (SHEARER und KOHL 1986) und der erweiterten Differenzmethode (STÜLPNAGEL 1982, HAUSER 1987) geschätzt. In der Natur kommen zwei stabile, nicht radioaktive Stickstoffisotope, das ${ }^{14} \mathrm{~N}$ - und ${ }^{15} \mathrm{~N}$-Isotop vor. Das ${ }^{15} \mathrm{~N}$-Isotop ist im Vergleich zum ${ }^{14} \mathrm{~N}$-Isotop in der Natur nur in sehr geringen Mengen vorhanden und 
kommt im molekularen Luftstickstoff zu einem vergleichsweise konstanten Anteil von 0,3663 atom $\%{ }^{15} \mathrm{~N}$ vor (JUNK und SVEC 1958). Da dieser Anteil weltweit sowohl räumlich als auch zeitlich nur durch sehr geringe Schwankungen gekennzeichnet ist (MARIOTTI 1983), wird er international als Standard genutzt. Die ${ }^{15} \mathrm{~N}$-Abweichung einer Probe vom Standard Luft wird im natürlich vorkommenden Bereich allgemein als $\delta^{15} \mathrm{~N}$ angegeben und entspricht der tausendstel atom $\%{ }^{15} \mathrm{~N}$-Abweichung vom Standard Luft und kann mit folgender Formel berechnet werden (SHEARER und KOHL 1986).

(1) $\delta^{15} \mathrm{~N}=$

$$
{ }^{15} \mathrm{~N} /{ }^{14} \mathrm{~N} \text { (Probe) }-{ }^{15} \mathrm{~N} /{ }^{14} \mathrm{~N} \text { (Standard Luft) }
$$

$$
{ }^{15} \mathrm{~N} /{ }^{14} \mathrm{~N} \text { (Standard Luft) }
$$

Um mit der $\delta^{15} \mathrm{~N}$-Methode in Leguminosen den Anteil Stickstoff aus der Luft (Nitrogen derived from the atmosphere $=\mathrm{Ndfa}$ ) bestimmen zu können, muss ein ausreichend großer Unterschied im Isotopenverhältnis zwischen molekularem Luftstickstoff und pflanzenverfügbarem Stickstoff im Boden vorhanden sein (UNKOVICH et al. 1994). Aufgrund unterschiedlicher physikalischer Eigenschaften der $\mathrm{N}$-Isotope wird bei Stickstoffumsetzungsprozessen das ${ }^{15} \mathrm{~N}$-Isotop gegenüber dem ${ }^{14} \mathrm{~N}$-Isotop diskriminiert und reichert sich so bei Prozessen des N-Umsatzes (z.B. Denitrifikation und Auswaschung) im Boden an. Wird im Feld in unmittelbarer Nachbarschaft zur Leguminose eine Nichtleguminose (Referenzpflanze) angebaut, so spiegelt der $\delta^{15} \mathrm{~N}$ Wert in der Biomasse der Nichtleguminose das Isotopenverhältnis des von der Pflanze aufgenommenen bodenbürtigen Stickstoffes wieder $\left(\delta^{15} \mathrm{~N}_{\text {Referenzpflanze }}\right)$. Mit Hilfe des $\delta^{15} \mathrm{~N}$-Wertes in der Biomasse der Nichtleguminose und der benachbart wachsenden Leguminose ( $\delta^{15} N_{\text {Leguminose }}$ ) kann der Anteil Stickstoff aus der Luft in der Biomasse der Leguminose wie folgt berechnet werden (SHEARER und KOHL 1986):

(2) $\% \mathrm{Ndfa}=$

$$
\frac{\left(\delta^{15} N_{\text {Referenzpflanze }}-\delta^{15} N_{\text {Leguminose }}\right)}{\left(\delta^{15} N_{\text {Referenzpflanze }}-\delta^{15} N_{0}\right)}
$$

In die Berechnungen zur Ermittlung der Ndfa-Werte muss der Isotopendiskriminierungsfaktor $\delta^{15} \mathrm{~N}_{0}$ einer auf $\mathrm{N}$-freiem Nährmedium gewachsenen Leguminose berücksichtigt werden. Um die Isotopendiskriminierungsfaktoren der zu prüfenden Leguminosenarten bzw. -sorten zu ermitteln, wurden alle im Feld geprüften Leguminosenarten im Gewächshaus in Gefäßen auf N-freiem Vermiculit und unter Zugabe einer N-freien Nährlösung (KESSLER 1987) angezogen. Durch Massenspektrometeranalyse und vorgeschaltete Elementaranalyse der bei $60^{\circ} \mathrm{C}$ getrockneten Sprossund Wurzelmassen wurden die $\delta^{15} \mathrm{~N}$-Werte und die gesamtpflanzliche Stickstoffmenge in der Biomasse der $\mathrm{N}$-frei angezogenen Leguminosen bestimmt. Die $\delta^{15} \mathrm{~N}$ Werte der ober- und unterirdischen Pflanzenteile sowie das in der Verrechnung zu berücksichtigende gewichtete gesamtpflanzliche Mittel der $\mathrm{N}$-frei angezogenen Pflanzen sind in Tab. 3 zusammengestellt. 
Tab. 3: $\quad \delta^{15} \mathrm{~N}_{0}$-Werte (\%o) in der Biomasse der in $\mathrm{N}$-freiem Substrat angezogenen Leguminosen

\begin{tabular}{c|c|c|c}
\hline & Ackerbohne & Luzerne & Saatwicke \\
\hline Spross & -0.3314 & -0.3449 & -0.7838 \\
\hline Wurzel & -0.2884 & -0.4047 & -0.3928 \\
\hline${ }^{a} \delta^{15} N_{0}$ & -0.3228 & -0.1941 & -0.6559 \\
\hline
\end{tabular}

${ }^{a}$ gewichteter $\delta^{15} \mathrm{~N}$-Wert in der gesamtpflanzlichen Biomasse

Da der $\delta^{15} \mathrm{~N}$-Wert der Referenzpflanze den Wert des pflanzenverfügbaren bodenbürtigen Stickstoff wiedergeben soll, kommt der Auswahl der Referenzpflanze und der räumlichen Anordnung zur Leguminose eine große Bedeutung zu, um die Genauigkeit der $\delta^{15} \mathrm{~N}$-Methode zu gewährleisten. Leguminosen und Referenzfrucht sollten Bodenstickstoff mit gleichem N-Isotopenverhältnis aufnehmen. Bei der Auswahl der Referenzpflanzen wurden Pflanzenarten berücksichtigt, die als Gemengepartner der zu prüfenden Leguminosen in der landbaulichen Praxis bedeutend sind. Die in zeitlicher und räumlicher Nachbarschaft zu den Leguminosen angebauten GramineenReinsaaten dienten jeweils als Referenzpflanzen.

Nach Ruschel et al. (1979) sowie LaRUe und PATTERSOn (1981) kann die symbiotisch fixierte N-Menge von Leguminosen, unter der Annahme gleicher Stickstoff-Aufnahme der Leguminose und einer Nichtleguminose, mit nachfolgender Rechenvorschrift (Einfache Differenzmethode, Erweiterungsstufe I) geschätzt werden:

(3) $\% \mathrm{Ndfa}=\left(\mathrm{N}_{\text {Leg }}-\mathrm{N}_{\text {Ref }}\right) / \mathrm{N}_{\text {Leg }} \times 100$

wobei $\mathrm{N}_{\text {Leg }}$ und $\mathrm{N}_{\text {Ref }}$ für die in der Sprossmasse befindliche N-Menge der Leguminose (Leg) und Referenzpflanze (Ref) steht.

STÜLPNAGEL (1982) erweiterte die Methode um die Erfassung des mineralischen Bodenstickstoffes $\left(\mathrm{N}_{\min }\right)$ zur Ernte und integrierte somit eine unterschiedlich hohe Aufnahme des mineralischen Bodenstickstoffs durch die Leguminose und Referenzpflanze in die Berechnungen (Erweiterungsstufe II):

(4) $\% \mathrm{Ndfa}=\left[\left(\mathrm{N}+\mathrm{N}_{\min }\right)_{\text {Leg }}-\left(\mathrm{N}+\mathrm{N}_{\min }\right)_{\text {Ref }} / \mathrm{N}_{\text {Leg }} \times 100\right.$

Schließlich ergänzte HAUSER (1987) die Berechnungen um den in der Wurzel, in den Knöllchen und den im Bestandesabfall befindlichen Stickstoff und leitete daraus die Erweiterungsstufen III und IV ab. Die Rechenvorschrift der Erweiterungsstufe IV lautet somit: 
(5) $\% \mathrm{Ndfa}=\left[\left(\mathrm{N}_{\mathrm{Bt}}+\mathrm{N}_{\min }\right)_{\mathrm{Leg}}-\left(\mathrm{N}_{\mathrm{Bt}}+\mathrm{N}_{\min }\right)_{\text {Ref }}\right] /\left(\mathrm{N}_{\mathrm{Bt}}\right)_{\mathrm{Leg}} \times 100$

$\mathrm{N}_{\mathrm{Bt}}$ steht hierbei für die Summe des Stickstoffs, die sich bei der Leguminose bzw. Referenzpflanze in der Spross- (einschließlich Bestandesabfall), Wurzel- und Knöllchenmasse befindet (N-Menge in der gesamtpflanzlichen Biomasse).

\subsection{Bodenchemische Methoden}

\subsubsection{Bestimmung der löslichen N-Fraktionen im Boden}

Zur Bestimmung der mineralischen Stickstofffraktionen $\left(\mathrm{NO}_{3}{ }^{-}, \mathrm{NH}_{4}{ }^{+}\right)$in der Bodenlösung wurden zu den in Tab. A3 angegebenen Beprobungsterminen Bodenproben mit Hilfe einer halbautomatischen Probenahmevorrichtung (Fa. Nietfeld) bis in eine Bodentiefe von $125 \mathrm{~cm}$ in 5 Horizonten entnommen (0 bis $25 \mathrm{~cm}, 25$ bis $50 \mathrm{~cm}, 50$ bis $75 \mathrm{~cm}, 75$ bis $100 \mathrm{~cm}, 100$ bis $125 \mathrm{~cm}$ ). Vor der Aussaat wurden die Mittelteilstücke mit jeweils sechs Einstichen je Parzelle beprobt. Die Untersuchungsparzellen wurden nach der Beerntung mit vier Einstichen (zwei auf der Reihe und zwei im Reihenzwischenraum) beprobt. Alle sechs bzw. vier Einstiche einer Variante wurden in den jeweiligen Tiefenstufen zu Mischproben vereinigt und direkt im Anschluss an die Entnahme in Kühltaschen gekühlt. Die Proben wurden noch am selben Tag mit Hilfe eines speziellen Homogenisators (Fa. Fritzmeier, Typ Weihenstephan) homogenisiert und bis zur Weiterverarbeitung bei $-20^{\circ} \mathrm{C}$ tiefgefroren.

Die Extraktion der mineralischen $\mathrm{N}-$ Verbindungen aus den Bodenproben erfolgte mit 0,01 molarer $\mathrm{CaCl}_{2}$-Lösung, indem jeweils $100 \mathrm{~g}$ einer Probe mit dem Extraktionsmittel $(250 \mathrm{ml})$ in Polyethylenflaschen eine Stunde geschüttelt wurden (VDLUFA 1991). Aus der Bodenlösung wurde über einen N-freien Faltenfilter (Fa. MachereyNagel, MN 619) ein Extrakt gewonnen und anschließend der Gehalt an mineralischem Stickstoff im Extrakt mittels luftsegmentierter Durchflussanalyse (Typ Flow solution 3, Fa. Perstorp Analytical) photometrisch detektiert. Dazu wurde $\mathrm{NO}_{3}{ }^{-}$mit Hilfe von Cadmium zu Nitrit $\left(\mathrm{NO}_{2}{ }^{-}\right)$reduziert, das mit Sulfamid unter Bildung eines Diazoniumsalzes reagiert. Der entstehende rot-violette Farbstoff wurde in der Messlösung bei $500 \mathrm{~nm}$ erfasst. Die Basis für die Bestimmung des Ammoniumgehaltes im Extrakt ist die Berthelot-Reaktion, bei der Phenole und Ammonium ein grün gefärbtes Indophenolderivat bilden, das bei $660 \mathrm{~nm}$ photometrisch detektiert wird.

\subsubsection{Bestimmung des $\mathrm{N}_{\mathrm{t}}-$ und $\mathrm{C}_{\mathrm{t}}$-Gehaltes im Boden}

Zur Bestimmung des Gesamtkohlenstoff- $\left(\mathrm{C}_{\mathrm{t}}\right)$ und Gesamtstickstoffgehaltes $\left(\mathrm{N}_{\mathrm{t}}\right)$ wurden jeweils im April 1998 und 1999 zehn Einstiche je N-Stufe (Mittelteilstück) schichtweise, aus den Tiefen 0 bis $20 \mathrm{~cm}, 20$ bis $40 \mathrm{~cm}$ und 40 bis $60 \mathrm{~cm}$ entnom- 
men und entsprechend der Tiefenstufen zu Mischproben zusammengeführt. Der zu analysierende luftrockene und gemahlene $(<2 \mathrm{~mm})$ Feinboden wurde direkt vor der Analyse bei $60^{\circ} \mathrm{C}$ getrocknet. Von jeder Probe wurde eine Doppelbestimmung durchgeführt, indem etwa 200 mg Probenmaterial in Zinnkapseln eingewogen wurden und anschließend auf Gesamtkohlenstoff und Gesamtstickstoff elementaranalytisch (vgl. Kap. 2.5.4) untersucht wurden. Parallel zur $\mathrm{N}_{t^{-}}$und $\mathrm{C}_{\mathrm{t}}$-Bestimmung wurde der Restwassergehalt des Bodens durch Trocknung bei $105^{\circ} \mathrm{C}$ bis zur Gewichtskonstanz ermittelt. Das arithmetische Mittel der Werte aus der Doppelbestimmung diente dann zur Berechnung der $\mathrm{N}_{\mathrm{t}^{-}}$und $\mathrm{C}_{\mathrm{t}}$-Gehalte des Bodens.

\subsubsection{Carbonatgehalt des Bodens}

Zur Ermittlung des $\mathrm{C}_{\text {org- }} \mathrm{C}-$ Gehaltes im Boden wurden die am CN-Elementar-Analyzer gemessenen $\mathrm{C}_{\mathrm{t}}$-Gehalte um den Carbonatkohlenstoffgehalt korrigiert. Die Bestimmung des Carbonatkohlenstoffgehaltes wurde am Kohlensäurebestimmungsgerät nach Scheibler-Finkener gemessen. Bei der Carbonatbestimmung wird das im Boden enthaltene Carbonat mit Salzsäure versetzt. Das entstehende Kohlendioxid wird volumetrisch gemessen. Aus dem gemessenen $\mathrm{CO}_{2}$-Volumen kann der $\mathrm{CaCO}_{3}-\mathrm{Ge}-$ halt berechnet werden (KRETZSCHMAR 1991).

\subsubsection{Bestimmung des pflanzenverfügbaren $\mathrm{P}_{2} \mathrm{O}_{5}$ - und $\mathrm{K}_{2} \mathrm{O}$-Gehaltes und des $\mathrm{pH}$-Wertes im Boden}

Die Analyse der Grundnährstoffe Phosphor und Kalium wurde an den im April 1998 und 1999 entnommenen Bodenproben für die Ackerkrume (0 bis $20 \mathrm{~cm}$ ) durchgeführt. Die Bestimmung erfolgte im Institut für Agrikulturchemie der Universität Göttingen mit der CAL-Methode nach ScHÜLLER (1969). Nach der Extraktion der Nährstoffe mit einer CAL-Lösung wurde die Konzentration der Nährstoffe flammenphotometrisch im Extrakt bestimmt. Die pH-Werte wurden in den Bodenschichten 0 bis $20 \mathrm{~cm}, 20$ bis $40 \mathrm{~cm}$ und 40 bis $60 \mathrm{~cm}$ in 0,01 molarer $\mathrm{CaCl}_{2}$-Lösung mittels einer pH-Elektrode bestimmt.

\subsection{Berechnungen}

\subsubsection{Schätzung der Wurzeltrockenmasse}

Um bei Reihenkulturen von den punktuell entnommenen Wurzelproben (Kap. 2.5.2) auf die je Flächeneinheit vorhandene Wurzelmasse schließen zu können, ist ein spezifisches Berechnungsverfahren abzuleiten. Das Vorgehen bei der Beprobung der Pflanzenbestände mittels Rammkernsonden macht deutlich, dass eine einfache, 
nicht spezifisch gewichtete Aufsummierung des Inhaltes der entnommenen Bodenproben zu einer nicht repräsentativen Schätzung der je Flächeneinheit tatsächlich vorhandene Wurzelmasse führen würde. Als Folge davon würden die Wurzelmengen in Reihenkulturen mit weiten Reihenabständen $(>18 \mathrm{~cm})$, wie z.B. bei Hafer in der vorliegenden Untersuchung, deutlich überschätzt werden (SMIT et. al. 2000). Noch größere Fehler würden bei Kulturen mit geringen Individuenzahlen je Flächeneinheit (z.B. Ackerbohne) und ausgeprägter räumlich inhomogen verteilter Wurzelmasse, wie sie bei allorrhizen Wurzelsystemen vorhanden ist, entstehen (VAN NooRDWIJK et al. 1985). Aus diesen Gründen wurden die erhobenen Wurzelmassen für die Kulturen Luzerne, Saatwicke, Hafer, Knaulgras und Einjähriges Weidelgras flächenbezogen gewichtet. Die Wurzelmasse der Ackerbohne wurde standraumbezogen kalkuliert.

Bei der Berechnung der Wurzelmassen wurde zunächst aus den Massen der entnommenen Bodenproben und der erhobenen Dichte des Bodens das beprobte Bodenvolumen ermittelt (Masse der entnommenen Bodenprobe dividiert durch die Dichte des Bodens). Über die Schichtdicke der beprobten Bodenschichten wurde der Flächenanteil berechnet, der der entnommenen Wurzelprobe entsprach und anschließend je Probe für die Bereiche auf der Reihe und zwischen der Reihe die Wurzelerträge in $\mathrm{dt} \mathrm{ha}^{-1}$ für jede Bodenschicht kalkuliert. Die erhaltenen Erträge wurden dann entsprechend ihrem Entnahmeort unterschiedlich gewichtet zusammengeführt.

Flächenbezogene Verrechnung:

Der Hafer wurde mit einem Reihenabstand von $28 \mathrm{~cm}$ angebaut. Das entspricht auf einer Fläche von $1 \mathrm{~m}^{2}$ einer Gesamtreihenlänge von 3,57 m. Über die Beprobung einer Reihe mit einem Bohrstockdurchmesser von $8,7 \mathrm{~cm}$ würde sich somit eine Fläche von $3106 \mathrm{~cm}^{2}\left(8,7 \times 357=3106 \mathrm{~cm}^{2}\right)$ ergeben. Von der gesamten zu beprobenden Fläche befinden sich also 31,06 \% im Reihenraum, die Restfläche $(68,94 \%)$ im Reihenzwischenraum $\left(10000 \mathrm{~cm}^{2}-3106 \mathrm{~cm}^{2}=6894 \mathrm{~cm}^{2}\right)$. Die zuvor ermittelten Flächenerträge wurden mit den hier ermittelten Korrekturfaktoren korrigiert und sowohl über die Entnahmeorte als auch Tiefenstufen addiert. Luzerne und Knaulgras sowie Saatwicke und Einjähriges Weidelgras wurden mit einem Reihenabstand von $18 \mathrm{~cm}$ angebaut. Hieraus ergeben sich Korrekturfaktoren von rund 51,6 \% (auf der Reihe) und 48,4 \% für den Reihenzwischenraum.

Standraumbezogene Verrechnung bei Ackerbohne:

Als Grundlage für die Gewichtung der Wurzelmassen je Bohrstock dient die Anzahl Pflanzen je Flächeneinheit. Der Standraum einer Pflanze wird berechnet über den mittleren Pflanzabstand innerhalb einer Reihe (a) und dem Reihenabstand $(28 \mathrm{~cm})$. Sobald der Pflanzabstand (a) den Bohrerdurchmesser von 8,7 cm übersteigt, kann der Gewichtungsfaktor einer auf einer Ackerbohnenpflanze gesetzten Rammkernsonde wie folgt berechnet werden. 
Die Berechnung des Anteils auf der Reihe in \% ergibt sich aus:

(a) $\mathrm{R} \%=$

$A_{z y}$ 100
Standraum

Fläche $=A_{z y}=(4,35 \mathrm{~cm})^{2} \times \pi$ Standraum $=a \times 28 \mathrm{~cm}$

Der Flächenanteil zwischen der Reihe ergibt sich aus:

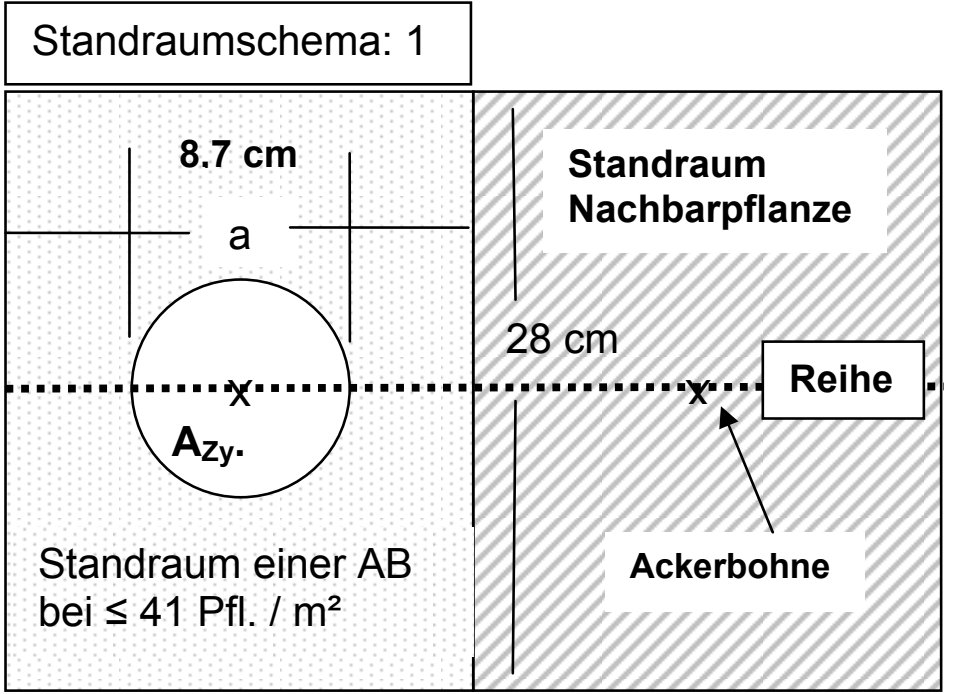

(B) $Z \%=100-R \%$

Ab einer Pflanzenzahl von 42 Einzelpflanzen je $\mathrm{m}^{2}$ überschneiden sich die Zylinderflächen bei einer theoretischen Einzelpflanzenbeprobung. Die Überschneidungsflächen müssen bei der Standraumbemessung berücksichtigt werden. Zur Berechnung der Gewichtungsfaktoren bei Pflanzenzahlen $>41$ je $\mathrm{m}^{2}$ wird die Zylinderfläche $\left(\mathrm{A}_{\mathrm{zy}}\right)$ in Gleichung a durch die Sektorenfläche $A_{\text {seg. }}$ ersetzt (Gleichung Y). Die Sektorenfläche wird mit der in Gleichung $\delta$ dargestellten Rechenvorschrift errechnet (vgl. Standraumschema: 2 ).

(v) $R \%=\frac{100 \times A_{\text {Seg. }}}{\text { Standraum }} \quad$ gilt für Pflanzenzahl $>41 / \mathrm{m}^{2}$

(ס) $A_{\text {seg. }}=$ Zylinderfläche $-2 \times\left(A_{k r .}-A_{\text {Dr. }}\right)$

$=$ Zylinderfläche $-2 \times\left[\left(r^{2} \times \arccos (1 / 2 \times a \times r)\right)-\left(1 / 2 \times a \times \sqrt{ }\left(r^{2}-(1 / 2 \times a)^{2}\right)\right)\right]$ 


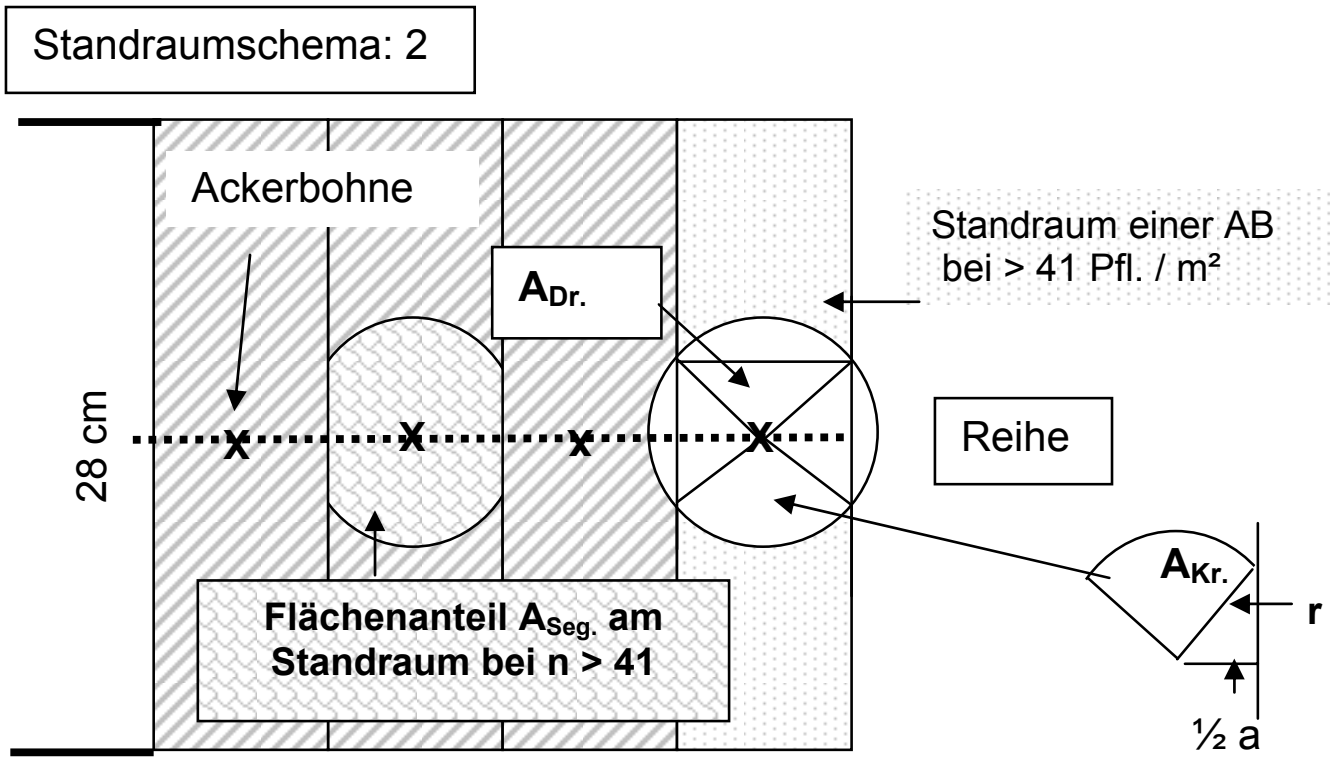

Die Gewichtungsfaktoren für die standraumbezogene Schätzung der Wurzelmassen sind in Tab. A2 zusammengestellt. Wie aus den Berechnungen hervorgeht, muss für die standraumbezogene Schätzung der Wurzelmassen die Anzahl der Pflanzen pro Fläche durch Zählungen erfasst werden.

Bei der Anwendung des hier vorgestellten Berechnungsverfahren auf im Gemenge angebaute Kulturen, müssen die Wurzelproben rechnerisch auf die Gemengepartner aufgeteilt werden, da eine manuelle Aufteilung der ausgewaschenen Wurzelproben auf die einzelnen Gemengepartner nicht möglich ist. Hierzu wurde das Spross-Wurzel-Verhältnis (e) beider Gemengepartner aus den Reinsaaten einbezogen und unterstellt, dass die Spross-Wurzel-Verhältnisse in den Gemengen gleich den der Reinsaaten sind.

Berechnung Gemengewurzeln:

(e) Faktor $\mathrm{Sp} / \mathrm{Wu}_{\text {Reinsaat }}=$

$\frac{\text { Sprossmasse Reinsaat }_{\text {Wurzelmasse Reinsaat }}}{\text { Wustat }}$

Aus dem Quotient zwischen den Sprossmassen der einzelnen Gemengepartner und den Spross-Wurzel-Verhältnissen der Reinsaaten wurden die Wurzelmassen für die jeweiligen Gemengepartner errechnet.

$$
\begin{gathered}
\text { Sprossmasse im Gemenge } \\
\text { Faktor } \mathrm{Sp} / \mathrm{W} u_{\text {Rein }}
\end{gathered}
$$

Mit Hilfe der errechneten Wurzelmassen der Gemengepartner wird der Wurzelanteil eines Gemengepartners an der gesamten Wurzelmenge im Gemenge, wie er sich 
aufgrund der Spross-Wurzel-Verhältnisse der Reinsaaten darstellen müsste, errechnet.

(g)

$$
\begin{aligned}
& \text { errechnete Wurzelmasse } \text { Gem }_{\text {Art } A} \\
& \text { (errechnete Wurzelmasse } \text { Gem Art A }+ \text { errechnete Wurzelmasse }{ }_{\text {Gem Art B) }}
\end{aligned}
$$

Die tatsächlich erhobene Wurzelmasse im Gemenge wird nun mit den entsprechenden Faktoren für den Anteil Wurzeln der Gemengepartner verrechnet und somit rechnerisch auf die Gemengepartner aufgeteilt.

$$
\begin{aligned}
& \text { Wurzelmasse }_{\text {Gem Leg }}=\text { Wurzelmasse }_{\text {Gem }} \times \text { Wurzel }_{\text {AnteilGem Leg }} \\
& \text { Wurzelmasse }_{\text {Gem Ref }}=\text { Wurzelmasse }_{\text {Gem }} \times \text { Wurzel }_{\text {AnteilGem Ref }}
\end{aligned}
$$

die Angabe erfolgt jeweils in $\mathrm{dt} \mathrm{ha}^{-1} ;{ }_{\mathrm{Gem}}=$ Gemenge $_{\text {Leg }}=$ Leguminose, ${ }_{\text {Ref }}=$ Referenzpflanze.

Die rechnerische Aufteilung der Wurzelmasse im Gemenge auf die einzelnen Gemengepartner ermöglicht nun eine Verrechnung der Wurzelmassen mit den entsprechenden Gewichtungsfaktoren (Ackerbohne mit einer standraumbezogenen Verrechnung und Hafer mit einer flächenbezogenen Verrechnung Kap. 2.7.1).

\subsubsection{Berechnung der Trockenmasse-Erträge, der $\mathrm{N}_{2}$-Fixierleistung sowie des vereinfachten $\mathrm{N}$-Flächenbilanzsaldos}

\subsubsection{Kenngrößen}

Für die nachfolgenden Berechnungen und Darstellungen werden nach SCHMIDTKE 1999 und REITER et al. 2002 erweiterte Kenngrößen und Abkürzungen verwendet:

$\mathrm{TM}_{\mathrm{Bt} 1,2,3}$ gesamtpflanzliche Trockenmasse von Luzerne und Saatwicke in Reinund Gemengesaat mit Knaulgras bzw. Einjährigem Weidelgras ( $\mathrm{dt} \mathrm{ha}^{-1}$ ) zum 1. bzW. bis zum 2. und 3. Schnitttermin ( $\mathrm{TM}_{\mathrm{Bt} 3}$ entspricht der gesamten Trockenmasseakkumulation je Jahr)

$\mathrm{TM}_{\mathrm{Bt}} \quad$ gesamtpflanzliche Trockenmasse von Ackerbohne in Rein- und Gemengesaat mit Hafer (in dt ha-1 ${ }^{-1}$ )

$\mathrm{H}_{\text {TMBt } 1,2,3} \quad$ TM-Harvest-Index $=$ Erntegut ${ }^{\star}-\mathrm{TM} / \mathrm{TM}_{\mathrm{Bt} 1,2,3}$

$\mathrm{N}_{\mathrm{Bt} 1,2,3} \quad$ Gesamt N-Menge in der Biomasse von Luzerne und Saatwicke in Reinund Gemengesaat mit Knaulgras bzw. Einjährigem Weidelgras (in kg N $\left.\mathrm{ha}^{-1}\right)$ zum 1. Schnitt bzw. bis zum 2. und 3. Schnitt $\left(\mathrm{TM}_{\mathrm{Bt} 3}\right.$ entspricht der gesamten Stickstoffakkumulation je Jahr)

$\mathrm{N}_{\mathrm{Bt}} \quad$ Gesamt N-Menge in der Biomasse Ackerbohne in Rein- und Gemengesaat mit Hafer (in kg N ha-1)

$\mathrm{H}_{\mathrm{NBt} 1,2,3} \quad \mathrm{~N}$-Harvest-Index $=$ Erntegut ${ }^{\star}-\mathrm{N} / \mathrm{N}_{\mathrm{Bt}} 1,2,3$ 
$\mathrm{H}_{\mathrm{NBtw} 1,2,3} \quad$ Mittlerer gewichteter N-Harvest-Index

Ndfa Anteil Stickstoff aus der Luft in der Leguminosenbiomasse

$\mathrm{Ndfa}_{\mathrm{Gem}} \quad$ Anteil Stickstoff aus der Luft in der Biomasse der Gemengepartner

$\mathrm{Ndfa}_{\mathrm{w}} \quad$ Mittlerer gewichteter Ndfa-Wert

$\mathrm{N}_{\mathrm{fix}} \quad$ Menge symbiotisch fixierten Stickstoffs (in $\mathrm{kg} \mathrm{N} \mathrm{ha}^{-1}$ )

Ndfs Anteil Stickstoff aus dem Boden in der Biomasse

$\mathrm{N}_{\text {Boden }} \quad$ Stickstoffmenge aus dem Boden (in $\mathrm{kg} \mathrm{N} \mathrm{ha}^{-1}$ )

$\mathrm{N}_{\text {trans }} \quad$ Im Gemenge von der Leguminose zur Nichtleguminose transferierter symbiotisch fixierter Stickstoff (in $\mathrm{kg} \mathrm{N} \mathrm{ha}^{-1}$ )

$S \quad$ einfacher N-Flächenbilanzsaldo (in $\mathrm{kg} \mathrm{ha}^{-1}$ )

Sw Erweiterter N-Flächenbilanzsaldo (in $\mathrm{kg} \mathrm{ha}^{-1}$ )

$\delta^{15} \mathrm{~N}_{\mathrm{w}} \quad$ Gewichteter $\delta^{15} \mathrm{~N}$-Wert in der gesamtpflanzlichen Biomasse

$\delta^{15} \mathrm{Nc}_{\mathrm{w}} \quad$ Gewichtete kumulierte $\delta^{15} \mathrm{~N}$ Werte des gesamtpflanzlichen Stickstoffs

Sp Spross: Futterleguminosen: oberirdische Biomasse ohne Stoppeln; Körnerleguminosen: oberirdische Biomasse

St Stoppeln

Wu Wurzeln

K Korn

$\mathrm{Ba} \quad$ Bestandesabfall

* Luzerne, Saatwicke und Gräser: Erntegut = Schnittgutmasse; Ackerbohne und Hafer: Erntegut = Kornmasse)

\subsubsection{Luzerne und Knaulgras in Reinsaat}

Die gesamtpflanzliche, vom Zeitpunkt der Aussaat bis zum ersten Schnitttermin gebildete Trockenmasse, wurde mit Gleichung (6) berechnet:

$$
\mathrm{TM}_{\mathrm{Bt} 1}=\mathrm{TM}_{\mathrm{Sp} 1}+\mathrm{TM}_{\mathrm{St} 1}+\mathrm{TM}_{\mathrm{Wu} 1} \quad \text { Angaben in } \mathrm{dt} \mathrm{ha}^{-1},{ }_{1}=\text { erster Schnitt }
$$

Durch Multiplikation der Trockenmasseerträge der einzelnen Ertragskomponenten mit den entsprechenden Gehalten an Stickstoff wurde die Stickstoffmenge in der gesamtpflanzlichen Biomasse $\left(\mathrm{N}_{\mathrm{Bt} 1}\right)$ zum ersten Schnitttermin errechnet. Der Trockenmasseertrag und die akkumulierte N-Menge bis zum zweiten und dritten Schnitttermin wurden als kumulative Erträge erfasst (SCHMIDTKE 2004). Danach resultiert die gesamtpflanzlich akkumulierte N-Menge $\mathrm{N}_{\mathrm{Bt} 2}$ und $\mathrm{N}_{\mathrm{Bt} 3}\left(\mathrm{~kg} \mathrm{~N} \mathrm{ha}^{-1}\right)$ aus den Gleichungen (7) und (8).

$$
\begin{aligned}
& N_{\mathrm{Bt} 2}=N_{\mathrm{Sp} 1}+N_{\mathrm{Sp} 2}+N_{\mathrm{St} 2}+N_{\mathrm{Wu} 2} \\
& \mathrm{~N}_{\mathrm{Bt} 3}=N_{\mathrm{Sp} 1}+N_{\mathrm{Sp} 2}+N_{\mathrm{Sp} 3}+N_{\mathrm{St} 3}+N_{\mathrm{Wu} 3} \\
& \mathrm{~N}_{\mathrm{Bt} 3}=\text { gesamtpflanzlich akkumulierte N-Menge in der Summe eines Jahres }
\end{aligned}
$$

Um den gesamtpflanzlichen N-Anteil aus der Luft (Ndfa) in den Leguminosen berechnen zu können, müssen die aus der Isotopenbestimmung erhaltenen $\delta^{15} \mathrm{~N}$-Werte 
zu einem gesamtpflanzlichen Mittelwert zusammengefasst werden. Aufgrund einer deutlichen disproportionierten N-Isotopenverteilung zwischen verschiedenen Pflanzenteilen (Schnittgut, Stoppeln, Wurzeln), wurden zur Schätzung der Ndfa-Werte das gewichtete Mittel $\left(\delta^{15} N_{w}\right)$ des $\delta^{15} \mathrm{~N}$-Wertes des gesamtpflanzlichen Stickstoffs der Leguminose und der Referenzfrucht herangezogen.

(9) $\delta^{15} \mathrm{~N}_{\mathrm{w}}=\frac{\left[\mathrm{N}_{\mathrm{Sp} 1} \times \delta^{15} \mathrm{~N}_{\mathrm{Sp} 1}+\mathrm{N}_{\mathrm{St} 1} \times \delta^{15} \mathrm{~N}_{\mathrm{St} 1}+\mathrm{N}_{\mathrm{Wu} 1} \times \delta^{15} \mathrm{~N}_{\mathrm{Wu} 1}\right]}{\left[\mathrm{N}_{\mathrm{Sp} 1}+\mathrm{N}_{\mathrm{St} 1}+\mathrm{N}_{\mathrm{Wu} 1}\right]}$

$\mathrm{N}_{\mathrm{Sp} 1}, \mathrm{~N}_{\mathrm{St} 1}, \mathrm{~N}_{\mathrm{Wu} 1}: \mathrm{N}$ im Schnittgut, in der Stoppel- und Wurzelmasse $\left(\mathrm{kg} \mathrm{N}^{-1}\right)$ zum ersten Schnitt $\delta^{15} \mathrm{~N}_{\mathrm{Sp} 1}, \delta^{15} \mathrm{~N}_{\mathrm{St} 1}, \delta^{15} \mathrm{~N}_{\mathrm{Wu} 1}: \delta^{15} \mathrm{~N}$-Wert im Schnittgut, in der Stoppel- und Wurzelmasse zum ersten Schnitt

Die gewichteten kumulierten $\delta^{15} \mathrm{~N}$ Werte des gesamtpflanzlichen Stickstoffs $\left(\delta^{15} \mathrm{Nc} \mathrm{c}_{\mathrm{w}}\right)$ bis zum zweiten bzw. dritten Schnitt wurden anhand der Gleichungen (10) und (11) errechnet.

$$
\begin{aligned}
&(10) \delta^{15} \mathrm{Nc}_{\mathrm{w}}= \frac{\left[\mathrm{N}_{\mathrm{Sp} 1} \times \delta^{15} \mathrm{~N}_{\mathrm{Sp} 1}+\mathrm{N}_{\mathrm{Sp} 2} \times \delta^{15} \mathrm{~N}_{\mathrm{Sp} 2}+\mathrm{N}_{\mathrm{St} 2} \times \delta^{15} \mathrm{~N}_{\mathrm{St} 2}+\mathrm{N}_{\mathrm{Wu} 2} \times \delta^{15} \mathrm{~N}_{\mathrm{Wu} 2}\right]}{\left[\mathrm{N}_{\mathrm{Sp} 1}+\mathrm{N}_{\mathrm{Sp} 2}+\mathrm{N}_{\mathrm{St} 2}+\mathrm{N}_{\mathrm{Wu} 2}\right]} \\
&(11) \delta^{15} \mathrm{Nc}_{\mathrm{w}}=\frac{\left[\mathrm{N}_{\mathrm{Sp} 1} \times \delta^{15} \mathrm{~N}_{\mathrm{Sp} 1}+\mathrm{N}_{\mathrm{Sp} 2} \times \delta^{15} \mathrm{~N}_{\mathrm{Sp} 2}+\mathrm{N}_{\mathrm{Sp} 3} \times \delta^{15} \mathrm{~N}_{\mathrm{Sp} 3}+\mathrm{N}_{\mathrm{St} 3} \times \delta^{15} \mathrm{~N}_{\mathrm{St} 3}+\mathrm{N}_{\mathrm{Wu} 3} \times \delta^{15} \mathrm{~N}_{\mathrm{Wu} 3}\right]}{\left[\mathrm{N}_{\mathrm{Sp} 1}+\mathrm{N}_{\mathrm{Sp} 2}+\mathrm{N}_{\mathrm{Sp} 3}+\mathrm{N}_{\mathrm{St} 3}+\mathrm{N}_{\mathrm{Wu} 3}\right]}
\end{aligned}
$$

Mit Gleichung (2) wurde der gesamtpflanzliche N-Anteil aus der Luft (Ndfa) in der Leguminose berechnet. Dazu wurden die gesamtpflanzlichen, gewichteten Mittelwerte in die Gleichung eingesetzt: Für die Berechnung der Ndfa des ersten Schnittes (siehe Gleichung 12) das gewichtete Mittel des $\delta^{15} \mathrm{~N}$ Wertes (siehe Gleichung 9), für die Berechnung der Ndfa des zweiten und dritten Schnittes die gewichteten kumulativen $\delta^{15} \mathrm{~N}$ Werte $\left(\delta^{15} \mathrm{~N} c_{w L e g}, \delta^{15} \mathrm{Nc} c_{w R e f}\right.$, siehe Gleichung 10 und 11).

$$
\% \operatorname{Ndfa}_{1}=\frac{\left(\delta^{15} N_{w R e f}-\delta^{15} N_{w L e g}\right)}{\left(\delta^{15} N_{w R e f}-\delta^{15} N_{w 0}\right)} \times 100
$$

$\delta^{15} \mathrm{~N}_{\text {wRef }}$ : gewichteter $\delta^{15} \mathrm{~N}$-Wert der gesamtpflanzlichen Biomasse der Referenzplanze

$\delta^{15} \mathrm{~N}_{\text {weg }}$ : gewichteter $\delta^{15} \mathrm{~N}$-Wert der gesamtpflanzlichen Biomasse der Leguminose (erster Schnitt vgl. Gleichung (9), bis zum zweiten und dritten Schnitt vgl. Gleichung (10) und (11)

$\delta^{15} \mathrm{~N}_{\mathrm{w} 0}$ : gewichtetes gesamtpflanzliches Mittel des $\delta^{15} \mathrm{~N}$-Wertes der auf $\mathrm{N}$-freiem Nährmedium gewachsenen Leguminose

Die Multiplikation des N-Anteils aus der Luft (Ndfa ${ }_{1,2,3}$ ) mit der N-Menge in der Biomasse $\left(N_{B t} 1,2,3\right)$ ergibt die symbiotisch fixierte $N$-Menge $\left(N_{\text {fix }}\right)$ in der Biomasse $z u$ einzelnen Schnittterminen $(1,2,3)$ bzw. der Gesamtbiomasse eines Nutzungsjahres in $\mathrm{kg} \mathrm{N} \mathrm{ha}^{-1}$ : 


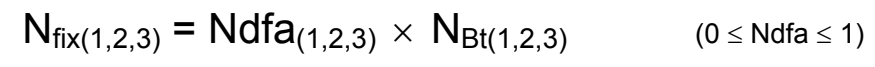

$\mathrm{N}_{\mathrm{fix} 1,2,3}=$ fixierte $\mathrm{N}$-Menge in der gesamtpflanzlichen Biomasse zum ersten Schnitt, bis zum zweiten bzw. dritten Schnitt in $\mathrm{kg} \mathrm{ha}^{-1}$ in $\mathrm{kg} \mathrm{ha}^{-1}$

Den Leguminosen stehen neben dem fixierten Luftstickstoff noch die pflanzenverfügbaren N-Anteile im Boden zur Verfügung. Der Anteil des von der Pflanze aufgenommen bodenbürtigen Stickstoff (Ndfs = Nitrogen derived from the soil) wird über den Differenzbetrag zwischen 1 und Ndfa ermittelt. Die aufgenommene bodenbürtige $\mathrm{N}$ Menge $\left(\mathrm{N}_{\text {Boden }}\right.$ in $\mathrm{kg} \mathrm{N}$ ha ${ }^{-1}$ ) wurde dann aus der Differenz zwischen $\mathrm{N}_{\mathrm{Bt}}$ und $\mathrm{N}_{\text {fix }}$ errechnet.

Mit der in Gleichung (14) wiedergegebenen Rechenvorschrift wurde der vereinfachte N-Flächenbilanzsaldo nach SCHMIDTKE (1996) berechnet. Dabei ergeben sich die in die Gleichung integrierten mittleren gewichteten Ndfa-Werte ( $\mathrm{Ndfa}_{\mathrm{w}}$ ) nicht aus den arithmetischen Mittelwerten der Wiederholungen, sondern aus dem Quotient der arithmetisch gemittelten fixierten $\mathrm{N}$-Mengen $\left(\mathrm{N}_{\mathrm{fix}}, \mathrm{kg} \mathrm{ha}^{-1}\right)$ und den arithmetisch gemittelten gesamtpflanzlich akkumulierten $\mathrm{N}-$ Mengen $\left(\mathrm{N}_{\mathrm{Bt}}, \mathrm{kg} \mathrm{ha}^{-1}\right)$. Entsprechend errechnen sich die mittleren gewichteten $\mathrm{N}$-Harvest-Indizes $\left(\mathrm{H}_{\mathrm{NBtw}}\right)$ aus dem Quotienten der arithmetisch gemittelten N-Mengen im Erntegut ( $\left.\mathrm{kg} \mathrm{N} \mathrm{ha}^{-1}\right)$ und den arithmetisch gemittelten akkumulierten $\mathrm{N}$-Mengen $\left(\mathrm{kg} \mathrm{N} \mathrm{ha}^{-1}\right)$ in der gesamtpflanzlichen Biomasse $\left(\mathrm{N}_{\mathrm{Bt}}\right)$ :

$$
\begin{array}{rlccc}
\mathbf{S} & = & {\left[\mathrm{N}_{\mathrm{Bt}} \times\left(1-\mathrm{H}_{\mathrm{NBtw}}\right) \times \mathrm{Ndfa} \mathrm{w}_{\mathrm{w}}\right]-\left[\mathrm{N}_{\mathrm{Bt}} \times\left(1-\mathrm{Ndfa}_{\mathrm{w}}\right) \times \mathrm{H}_{\mathrm{NBtw}}\right]} \\
& = & \left(\mathrm{N}_{\mathrm{Bt}} \times \mathrm{Ndfa}_{\mathrm{w}}\right) & - & \left(\mathrm{N}_{\mathrm{Bt}} \times \mathrm{H}_{\mathrm{NBtw}}\right) \\
& = & \mathrm{N}_{\mathrm{fix}} & - & \mathrm{N}_{\mathrm{Sp} \mathrm{oder} \mathrm{K}}
\end{array}
$$

$\mathrm{S}, \mathrm{N}_{\mathrm{Bt}}, \mathrm{N}_{\text {fix }}$ und $\mathrm{N}_{\mathrm{Sp} \text { oder } \mathrm{K}}$ in $\mathrm{kg} \mathrm{ha}^{-1}$

$\mathrm{Ndfa}_{\mathrm{w}}$ und $\mathrm{H}_{\mathrm{NBtw}}: 0 \leq \mathrm{Ndfa}_{\mathrm{w}} \leq 1 ; 0 \leq \mathrm{H}_{\mathrm{NBtw}} \leq 1 ;{ }^{*}=\mathrm{N}$ im Spross oder im Korn

Durch Umformung von Gleichung 14 kann gezeigt werden, dass die N-Zufuhr als symbiotisch fixierte Luft-N-Menge definiert ist, während der N-Entzug beim Anbau von Futterleguminosen über die Abfuhr des Schnittgutes erfolgt. Aus Gleichung (14) wird weiterhin deutlich, dass der vereinfachte N-Flächenbilanzsaldo maßgeblich von der gesamtpflanzlich akkumulierten N-Menge $\left(\mathrm{N}_{\mathrm{Bt}}\right)$ und dem Stickstoff-Harvest-Index $\left(\mathrm{H}_{\mathrm{NBtw}}\right)$ beeinflusst wird.

\subsubsection{Luzerne und Knaulgras in Gemengesaat}

Die Berechnung von Flächenbilanzen beim Gemengeanbau ist analog der Vorgehensweise für Reinsaaten (Gleichungen 6 bis 14). Da eine Aufteilung der Biomasse auf die Gemengepartner nur oberirdisch möglich ist, beziehen sich die Berechnungen der $\mathrm{Ndfa}_{\mathrm{Gem}}$ immer auf die Summe beider Gemengepartner. Somit muss das 
Subtraktionsglied im Zähler von Gleichung (12) durch das gewichtete Mittel des $\delta^{15} \mathrm{~N}$ Wertes des gesamtpflanzlichen Stickstoffes des Gemenges (Leguminose und Nichtleguminose, $\delta^{15} \mathrm{~N}_{\mathrm{wGem}}$ ) ersetzt werden.

$$
\% \mathrm{Ndfa}_{\mathrm{Gem}}=\frac{\left(\delta^{15} \mathrm{~N}_{\mathrm{wRef}}-\delta^{15} \mathrm{~N}_{\mathrm{wGem}}\right)}{\left(\delta^{15} \mathrm{~N}_{\mathrm{wRef}}-\delta^{15} \mathrm{~N}_{\mathrm{w}}\right)} \times 100
$$

$\delta^{15} \mathrm{~N}_{\text {wGem }}=\left[\mathrm{N}_{\text {SpGemLeg }} \times \delta^{15} \mathrm{~N}_{\text {SpGemLeg }}+\mathrm{N}_{\text {SpGemNLeg }} \times \delta^{15} \mathrm{~N}_{\text {SpGemNLeg }}+\mathrm{N}_{\text {stGemLeg }} \times \delta^{15} \mathrm{~N}_{\text {StGemLeg }}+\right.$ $\left.\mathrm{N}_{\text {stGemNLeg }} \times \delta^{15} \mathrm{~N}_{\text {StGemNLeg }}+\mathrm{N}_{\text {wuGem }} \times \delta^{15} \mathrm{~N}_{\text {WuGem }}\right] /\left[\mathrm{N}_{\text {SpGemLeg }}+\mathrm{N}_{\text {SpGemNLeg }}+\mathrm{N}_{\text {stGemLeg }}+\right.$ $\mathrm{N}_{\text {StGemNLeg }}+\mathrm{N}_{\text {WuGem] }}$

GemLeg = legumer Gemengepartner, GemNLeg = nicht legumer Gemengepartner

Der Anteil Stickstoff aus der Luft im Gemenge (Ndfa $\mathrm{Nem}_{\mathrm{G}}$ ) beinhaltet somit den luftbürtigen N-Anteil in der Leguminose und der Nichtleguminose im Gemenge (transferierter Stickstoff von der Leguminose zur Nichtleguminose vgl. Kap 0). Beziehen sich die dargestellten $\delta^{15} \mathrm{~N}$ - und Ndfa-Werte nur auf die Sprossmasse, wird darauf im Text hingewiesen.

Die Vorgehensweise bei der Berechnung des Flächenbilanzsaldos bei Saatwicke und Einjährigem Weidelgras in Rein- und Gemengesaat entspricht den Rechenvorschriften für den ersten Schnitttermin der Luzerne und des Knaulgrases.

\subsubsection{Ackerbohne und Hafer in Rein- und Gemengesaat}

Zur Erstellung des vereinfachten N-Flächenbilanzsaldos beim Anbau von Körnerleguminosen muss Gleichung (6) um die Ertragskomponenten Bestandesabfall, Korn und Stroh ergänzt werden.

$$
\begin{aligned}
& \mathrm{TM}_{\mathrm{Bt}}=\mathrm{TM}_{\mathrm{Sp}}+\mathrm{TM}_{\mathrm{Wu}} \quad \text { Angaben in } \mathrm{dt} \mathrm{ha}^{-1} \\
& \mathrm{Sp}=\text { Spross = Bestandesabfall }+ \text { Korn }+ \text { Stroh }
\end{aligned}
$$

Die gesamtpflanzlich akkumulierte N-Menge ergibt sich aus Gleichung (17).

$$
\begin{array}{ll}
N_{B t}=N_{S p}+N_{W u} & \text { Angaben in } \mathrm{kg} \mathrm{N} \mathrm{ha}^{-1} \\
N_{S p}=N_{\text {Bestandesabfall }}+N_{\text {Korn }}+N_{S t r o h} &
\end{array}
$$

Der vereinfachte N-Flächenbilanzsaldo beim Anbau von Körnerleguminosen in Reinund Gemengesaat, lässt sich schließlich ebenfalls mit Gleichung (14) errechnen (vgl. oben). Durch Umformung der Gleichung (14) lässt sich zeigen, dass sich die N-Flächenbilanz aus der Differenz zwischen $\mathrm{N}_{\mathrm{fix}}$ und Korn-N ergibt. 


\subsubsection{Stickstoff-Rhizodeposition}

Bei der Berechnung von N-Flächenbilanzen sollen möglichst alle durch die angebauten Pflanzen verursachten N-Flüsse berücksichtigt werden. Im Rahmen von erweiterten N-Flächenbilanzen wurden deshalb die Stickstoff-Rhizodeposition (Ndfr = Nitrogen deriving from rhizodeposition) in die Kalkulationen mit einbezogen (vgl. Kap. 2.7.4). Die Messung der in den Boden abgegebenen N-Mengen ist methodisch sehr aufwendig. Da Messungen der Rhizodeposition aus der eigenen Untersuchung nicht vorliegen, wurde auf Literaturangaben zurückgegriffen (Tab. 4). Die StickstoffAnteile aus der Rhizodeposition beziehen sich hierbei auf den Stickstoff zum Erntezeitpunkt in der gesamtpflanzlichen Biomasse.

Tab. 4: Stickstoff-Anteil aus der Rhizodeposition (Ndfr) an der gesamtpflanzlichen N-Menge $\left(\mathrm{N}_{\mathrm{Bt}}\right)$ nach SCHMIDTKE (2004)

\begin{tabular}{r|c|c|c}
\hline & Ackerbohne & Luzerne & Saatwicke \\
\hline Sorte & Minica & Europe & Caroline \\
SCHMIDTKE 2004 & $0,1079^{\mathrm{a}}$ & $0,089^{\mathrm{b}}$ & $0,179^{\mathrm{c}}$ \\
\hline \multicolumn{2}{|c|}{}
\end{tabular}

\subsubsection{Erweiterter N-Flächenbilanzsaldo}

Mit Hilfe der in Tab. 4 angeführten Werte für Ndfr, wurden unter Einbeziehung des vereinfachten N-Flächenbilanzsaldos aus Gleichung (14) die erweiterten N-Flächenbilanzsalden (Sw) ermittelt.

$$
\mathrm{Sw}=\mathrm{S}+\left[\mathrm{N}_{\mathrm{fix}} \times \mathrm{Ndfr}\right] \quad 0 \leq \mathrm{Ndfr} \leq 1
$$

Angaben in $\mathrm{kg} \mathrm{N}^{-1}$

\subsubsection{Transfer-Stickstoff im Gemengeanbau}

Um mit der $\delta^{15} \mathrm{~N}$-Methode $\mathrm{N}$-Transfer nachweisen zu können, muss der $\delta^{15} \mathrm{~N}-\mathrm{Wert}$ in der Biomasse des nichtlegumen Gemengepartners signifikant geringer sein als der $\delta^{15} \mathrm{~N}$-Wert der Nichtleguminose in Reinsaat. Nur unter diesen Voraussetzungen ist eine Schätzung der symbiotisch fixierten N-Menge möglich, die im Verlauf einer Vegetationsperiode an den nichtlegumen Gemengepartner transferiert wird. Mit den nachfolgenden Formeln (19) und (20) wurde der prozentuale Anteil des transferierten Stickstoffs als auch die absolute Menge in $\mathrm{kg} \mathrm{ha}^{-1} \mathrm{a}^{-1}$ berechnet: 
(19) $\% N_{\text {trans }}=\frac{\left(\delta^{15} N_{\text {ws-Ref }}-\delta^{15} N_{\text {wSpGemNLeg }}\right)}{\left(\delta^{15} N_{\text {ws-Ref }}-\delta^{15} N_{0}\right)} \times 100$

$\delta^{15} \mathrm{~N}_{\text {ws-Ref }}$ : $\quad$ gewichteter $\delta^{15} \mathrm{~N}$-Wert in der Sprossmasse der Referenzpflanze

$\delta^{15} \mathrm{~N}_{\text {wSpGemnLeg: }}$ gewichteter $\delta^{15} \mathrm{~N}$-Wert in der Sprossmasse des nichtlegumen Gemengepartners

$\delta^{15} \mathrm{~N}_{0} \quad \delta^{15} \mathrm{~N}$-Wert im Spross der in $\mathrm{N}$-freiem Substrat gewachsenen Leguminose

Die Berechnungen beziehen sich nur auf die oberirdische Biomasse, da nur hier eine Trennung der Gemengepartner in Leguminose und Nichtleguminose möglich war.

Beim Vergleich von Formel (19) und (20) ist zu erkennen, dass bei der Berechnung des $\mathrm{N}$-Transfers die Nichtleguminose im Gemenge $\left(\delta^{15} \mathrm{~N}_{\text {wSpGemNLeg }}\right)$ an Stelle der Leguminose in Formel (2) gesetzt wird und somit rechnerisch als Leguminose behandelt wird. Weiterhin wird aus dem Algorithmus deutlich, dass in allen Fällen, in denen der $\delta^{15} \mathrm{~N}$-Wert des nichtlegumen Gemengepartners geringer ist als der der Referenzpflanze aus Reinsaat, der ermittelte Wert rechnerisch einem N-Transfer zugeschrieben wird, unabhängig davon, welche Gründe zu dem niedrigeren $\delta^{15} \mathrm{~N}-$ Wert geführt haben. Deshalb wird in der Literatur auch häufig vom "scheinbaren N-Transfer" (apparent nitrogen transfer) gesprochen (HEICHEL und HENJUM 1991).

Durch Multiplikation des Transfer-N-Anteils (\% $\mathrm{N}_{\text {trans }}$ aus Gleichung 19) mit der in der Sprossmasse des nichtlegumen Gemengepartners befindlichen N-Menge $\left(\mathrm{N}_{\mathrm{SpGemNLeg}}\right)$ wurde die transferierte $\mathrm{N}-$ Menge in $\mathrm{kg} \mathrm{ha}^{-1}$ und Jahr errechnet:

$$
N_{\text {trans }}\left(\mathrm{kg} \mathrm{ha}^{-1} \mathrm{a}^{-1}\right)=\% \mathrm{~N}_{\text {trans }} \times \mathrm{N}_{\mathrm{SpGemNLeg}} / 100
$$

\subsection{Statistische Auswertungen}

Die statistische Auswertung der Daten erfolgte mit Hilfe des Programmpaketes SAS für Personalcomputer (SAS-Institute 1999, Version 8.1). Test auf Normalverteilung wurde mit dem Shapiro-Wilk-Test (SHAPIRO und WILK 1965) auf einem Niveau von $P$ $>\alpha=0,1$ durchgeführt (SAS Prozedur UNIVARIATE Option NORMAL). Die Homogenität der Varianzen (Homoskedastizität) wurde mit dem von BROWN und FORSYTHE (1974) modifizierten Levene-Test (LEVENE 1960) überprüft (SAS Prozedur LAB: HOVTEST=BF \& HOVTEST=Levene (Type=square), $\alpha=0,1$, DUFNER 1992). Waren die Daten auf dem genannten Niveau $(\alpha=0,1)$ nicht normal verteilt, wurde mittels logarithmischer Transformation, Wurzeltransformation, Transformation mit 1/Wurzeltransformation bzw. 1/x und Winkeltransformation $(\arcsin \sqrt{x})$ Normalität der Rohdaten hergestellt (MUNZERT 1992, SACHS 1993). Die statistischen Auswertungen wurden ausgehend von den Originaldaten und deren arithmetischen Mittelwerten durchgeführt. Dabei können die arithmetischen Mittelwerte geringfügig von den im Text, Abbildungen und Tabellen verwendeten gewichteten Ndfa- und $\mathrm{H}_{\mathrm{NBt}}-$ Werten (Ndfa, $\mathrm{H}_{\mathrm{NBtw}}$ ) abweichen (REITER et al. 2002). 
Die chemischen Kennwerte des Bodens, sowie die $\mathrm{N}_{\min }$-Daten vor Aussaat wurden zweifaktoriell mit den Faktoren Pflanzenschutz (mit, ohne) und Düngung $\left(\mathrm{N}_{0}, \mathrm{~N}_{3}\right)$ statistisch überprüft. Alle übrigen Ergebnisse wurden getrennt nach Jahren zunächst in einer dreifaktoriellen Spaltanlage $(n=3)$ mit den Faktoren Pflanzenschutz (fixer Faktor), Düngung (fixer Faktor) und Ansaatvariante (zufälliger Faktor) verrechnet.

Die Pflanzenschutzmaßnahmen zu den Vorfüchten führten in der vorliegenden Untersuchung zu keiner signifikanten Beeinflussung der Gesamtkohlenstoff- und Gesamtstickstoffgehalte im Boden. Gleiche Ergebnisse beobachtete CLAUPEIN (1994). Ferner beeinflussten die Pflanzenschutzmaßnahmen in den eigenen Untersuchungen weder weitere chemischen Kennwerte des Bodens noch die $\mathrm{N}_{\text {min }}$-Mengen vor Aussaat der Ansaaten signifikant (Tab. A1, Tab. A15, Tab. A34, Tab. A49). Die Regulation der Beikräuter erfolgte im Rahmen der vorliegenden Untersuchung in allen Varianten mechanisch sowie per Hand (Kap. 2.4, letzter Absatz), so dass in beiden Systemen des Pflanzenschutzes, zum Zeitpunkt der Ernte, kein signifikanter Unterschied im Hinblick auf den Beikrautbesatz in den hier untersuchten Beständen vorhanden war. Im Gegensatz zur langjährig unterschiedlichen mineralischen NDüngung zu den nichtlegumen Vorfrüchten zeigte sich beim Faktor Pflanzenschutz nur eine vergleichsweise geringe Beeinflussung der N-Flüsse in den hier vorgestellten Untersuchungen. Vor diesem Hintergrund wurden alle Ergebnisse anschließend zweifaktorielle $(n=6)$, mit den Faktoren Düngung und Ansaatvariante verrechnet und dargestellt. Auf eine dreifaktorielle Darstellung und Interpretation der Ergebnisse wurde verzichtet. Jedoch wurde in den wenigen Fällen, in denen ein Einfluss des Pflanzenschutzes zu den Vorfrüchten auf die hier untersuchten Parameter statistisch nachweisbar war, in den Ergebnistabellen anhand von Fußnoten gesondert darauf hingewiesen.

Multiple Mittelwertvergleiche wurden bei balanzierten Daten mit dem Tukey-Test (TUKEY 1953), bei unbalanzierten Daten mit Hilfe des Scheffé-Testes (KöHLER et al. 1996) ermittelt. Die Irrtumswahrscheinlichkeiten wurden berechnet für:

$$
\begin{array}{ll}
\alpha=0,05 ;\left({ }^{*}\right) & =\text { signifikant } \\
\alpha=0,01 ;\left({ }^{* *}\right) & =\text { hoch signifikant } \\
\alpha=0,001 ;\left({ }^{* *}\right) & =\text { sehr hoch signifikant } \\
\text { n.s. } & =\text { nicht signifikant }
\end{array}
$$

Mit Hilfe des Programmes SigmaPlot 2002 (Version 8.02) wurde der Zusammenhang von Merkmalen mit dem empirischen Pearsonsche Korrelationskoeffizient berechnet und dargestellt sowie alle übrigen graphischen Abbildungen hergestellt. 


\section{Ergebnisse}

\subsection{Ackerbohne in Rein- und Gemengesaat mit Hafer}

\subsubsection{Gesamtpflanzliche Trockenmasse-Akkumulation}

Die Darstellung der Ergebnisse erfolgt zunächst getrennt nach den Untersuchungsjahren 1998 und 1999. Anschließend werden die Ergebnisse beider Jahre vergleichend gegenübergestellt.

Im ersten Untersuchungsjahr 1998 unterschieden sich die gesamtpflanzlich akkumulierten Trockenmassen der Ansaatvarianten im Mittel über die Düngungsstufen (Ausnahme: Stroh-TM zur ersten Ernte) zum ersten und zweiten Erntetermin signifikant (erste Ernte: $\mathrm{TM}_{\mathrm{Bt}}$ und Wurzel-TM, zweite Ernte: $\mathrm{TM}_{\mathrm{Bt}}$, Wurzel-TM und Stroh-TM; Tab. A4). Die Ansaatvarianten der $\mathrm{N}_{3}$-Variante hatten im Mittel zum Zeitpunkt der zweiten Ernte höhere gesamtpflanzliche Trockenmassen akkumuliert als die Ansaatvarianten der $\mathrm{N}_{0}$-Variante (Tab. A4). Die gesamtpflanzlich akkumulierten Trockenmasse-Erträge zum letzten Erntetermin (10.08.1998) lagen zwischen 125,3 $\left(\mathrm{N}_{0}: 4 \mathrm{H}\right)$ und 163,1 dt TM ha ${ }^{-1}\left(\mathrm{~N}_{3}: 3 \mathrm{~A} / 1 \mathrm{H}, \mathrm{Abb}\right.$. 4). Im Mittel über die Düngungsstufen erzielten die Ansaatvarianten mit Ackerbohne in Reinsaat und im leguminosenbetonten Gemenge $(3 \mathrm{~A} / 1 \mathrm{H})$ mit 156,7 und 159,4 dt TM ha ${ }^{-1}$ signifikant höhere Gesamt-TMErträge als der Hafer in Reinsaat (136,6 dt TM ha $\left.{ }^{-1}\right)$.

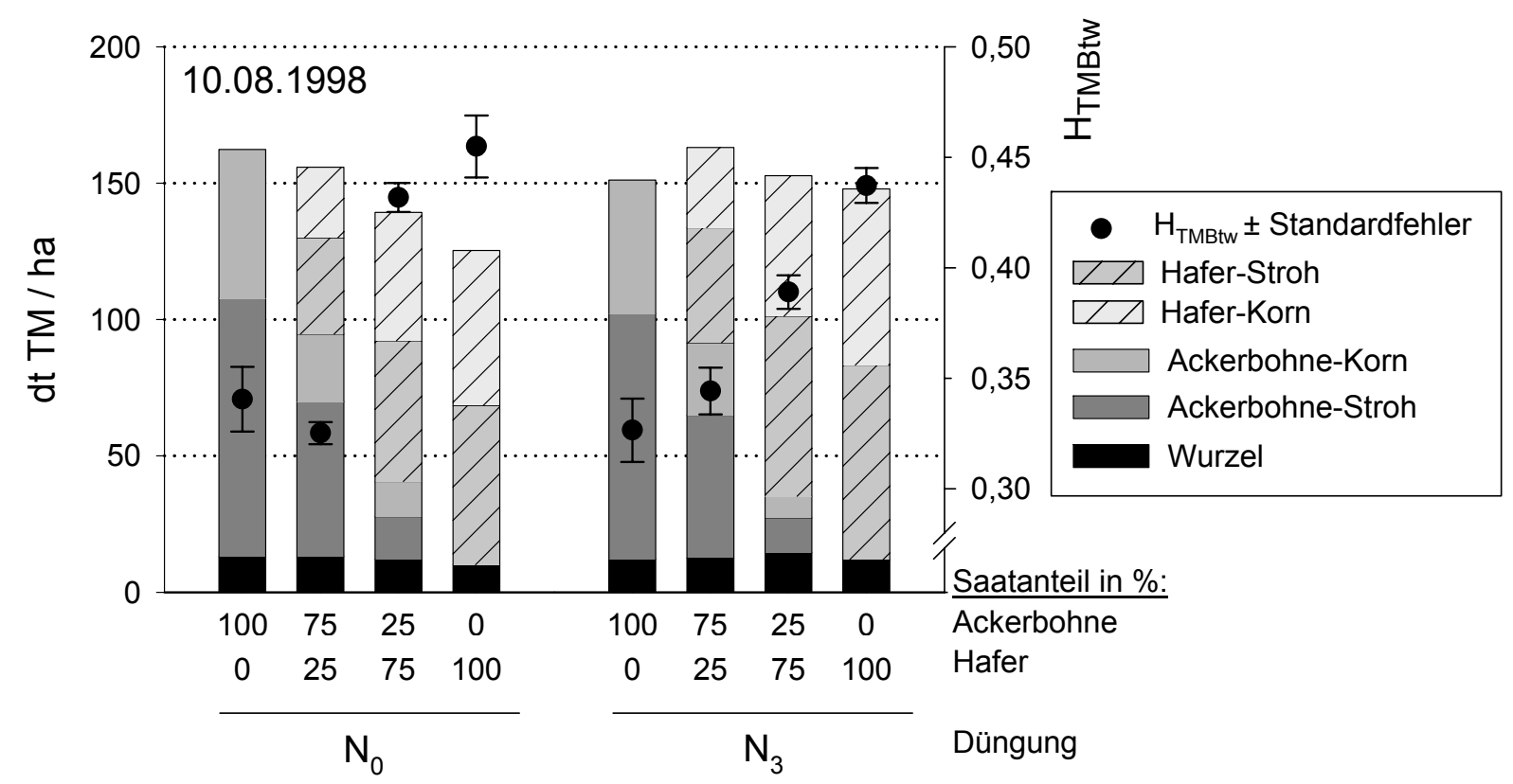

Abb. 4: Gesamtpflanzliche Trockenmasse-Akkumulation (dt TM ha ${ }^{-1}$; Wurzel, Stroh incl. Bestandesabfall, Korn) sowie gewichtete Trockenmasse-Harvest-Indizes $\left(\mathrm{H}_{\text {TMBtw }} \pm\right.$ Standardfehler) beim Anbau von Ackerbohne und Hafer in Rein- und Gemengesaat zur letzten Ernte (BBCH 87) am 10.08.1998 
Die gesamtpflanzlich akkumulierten Trockenmassen der Gemengesaaten lagen im Mittel der Düngungsstufen zu keinem Zeitpunkt über den Trockenmasseerträgen der beiden Reinsaaten.

Die Wurzelmassen der Ansaatvarianten mit Ackerbohne und Hafer lagen zum dritten Erntetermin zwischen 10,1 ( $\left.\mathrm{N}_{0}: 4 \mathrm{H}\right)$ und $14,5 \mathrm{dt} \mathrm{TM} \mathrm{ha}^{-1}\left(\mathrm{~N}_{3}: 1 \mathrm{~A} / 3 \mathrm{H}\right)$. Dabei zeigten sich keine signifikanten Unterschiede in Abhängigkeit der Ansaatvarianten und der Düngung (Tab. A5). Die Stroherträge der Ackerbohnen in Reinsaat und in Gemengesaat mit $25 \%$ Hafer $(3 \mathrm{~A} / 1 \mathrm{H})$ lagen im Mittel über die Düngerstufen zum dritten Erntetermin mit 92,4 und 93,4 dt TM ha ${ }^{-1}$ signifikant über den Stroherträgen des Hafers in Reinsaat $\left(64,9 \mathrm{dt} \mathrm{TM} \mathrm{ha}{ }^{-1}\right)$.

Die Kornerträge schwankten 1998 zwischen 49,0 ( $\left.\mathrm{N}_{3}: 4 \mathrm{~A}\right)$ und 64,6 dt TM ha ${ }^{-1}\left(\mathrm{~N}_{3}\right.$ : $4 \mathrm{H}$, Tab. A6). Ackerbohne in Reinsaat realisierte im Mittel über die Düngungsstufen mit 51,8 dt TM ha ${ }^{-1}$ signifikant geringere Kornerträge als Hafer in Reinsaat $(60,7 \mathrm{dt}$ $\mathrm{TM} \mathrm{ha}^{-1}$ ). Die Kornerträge der Ansaatvarianten $4 \mathrm{~A}$ und $1 \mathrm{~A} / 3 \mathrm{H}$ lagen in der langjährig nicht mit mineralischen $\mathrm{N}$-Düngern zu den nichtlegumen Vorfrüchten gedüngten Variante $\left(\mathrm{N}_{0}\right)$ über den Kornerträgen der $\mathrm{N}_{3}$-Variante. Die Ansaatvarianten $3 \mathrm{~A} / 1 \mathrm{H}$ und $4 \mathrm{H}$ verhielten sich umgekehrt (signifikante Wechselwirkung: Ansaatvariante $\times$ Düngung; Tab. A5, Tab. A6). Mit 9,9 (4A), 6,3 (3A/1H), 2,0 (1A/3H) und 0 dt TM ha $^{-1}(4 \mathrm{H})$ betrug der Anteil des Bestandesabfalls an der gesamtpflanzlichen TMAkkumulation 6,3 (4A), 3,9 (3A/1H) und 1,4 \% (1A/3H).

Der gesamtpflanzliche TM-Harvest-Index $\left(\mathrm{H}_{\mathrm{TMBt}}\right)$ zeigte im Mittel über die Düngungsstufen einen signifikanten Unterschied zwischen der Hafer Reinsaat $(4 \mathrm{H}: 0,445)$ bzw. dem Gemenge mit $25 \%$ Ackerbohne $(1 \mathrm{~A} / 3 \mathrm{H}: 0,409)$ und den Ackerbohnen Reinsaaten (4A: 0,330) sowie Gemengen mit 25\% Hafer $(3 \mathrm{~A} / 1 \mathrm{H}: 0,333$, Tab. A5). Mit Ausnahme der Ansaatvariante $3 \mathrm{~A} / 1 \mathrm{H}$ in der $\mathrm{N}_{0}$-Variante stiegen die gewichteten TMHarvest-Indizes mit zunehmendem Haferanteil an (Abb. 4).

In Abb. 5 sind die Trockenmasse-Ertragsanteile von Ackerbohne und Hafer in beiden Gemengesaaten zum letzten Erntetermin dargestellt. Der Ertragsanteil Hafer-Sprossmasse betrug in der Ansaatvariante mit $25 \%$ Hafer in der Ansaatmischung $(3 \mathrm{~A} / 1 \mathrm{H})$ $48,1 \%$ in der $\mathrm{N}_{3}$-Variante bzw. $43,0 \%$ in der $\mathrm{N}_{0}$-Variante. In der haferbetonten Ansaatmischung $(1 \mathrm{~A} / 3 \mathrm{H})$ betrugen die Hafer-Ertragsanteile 85,1\% $\left(\mathrm{N}_{3}\right)$ bzw. 77,6 \% $\left(\mathrm{N}_{0}\right)$. Die Unterschiede in den Ertragsanteilen der Düngungsstufen konnten im Mittel über die Ansaatvarianten 1998 statistisch nicht gesichert werden (Abb. 5). 
Abb. 5: Ertragsanteile der SprossTM beim Anbau von Ackerbohne und Hafer in Gemengesaat zur letzten Ernte (10.08.1998)

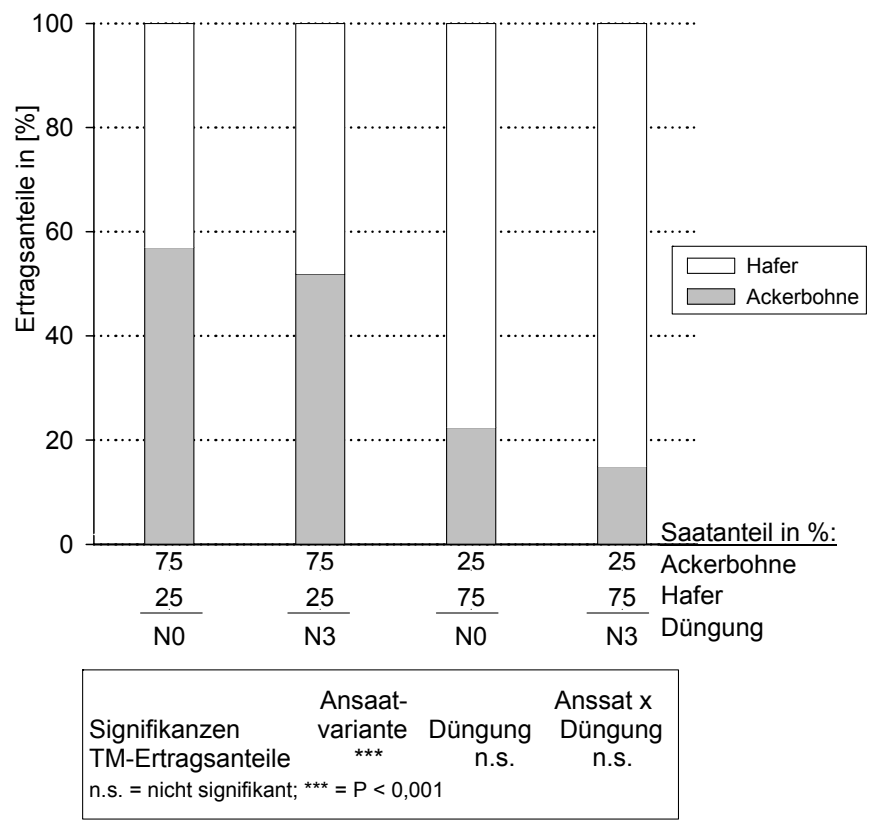

Im zweiten Untersuchungsjahr 1999 wurden in den Aufwüchsen neben den angebauten Arten Ackerbohne und Hafer auch geringe Mengen an Kräutern (Ackerbegleitflora) mit erfasst. Die Trockenmassen der Beikräuter lagen mit maximal $2 \mathrm{dt} \mathrm{ha-1}$ auf einem sehr geringen Niveau und wurden deshalb in den nachfolgenden Darstellungen und Beschreibungen nicht berücksichtigt (Tab. A7).

Die gesamtpflanzlich akkumulierten Trockenmassen der Ansaatvarianten unterschieden sich sowohl zum ersten als auch zum zweiten Erntetermin im Mittel über die Düngungsstufen signifikant (Tab. A8). Die Ansaaten mit Ackerbohne und Hafer akkumulierten zum dritten Erntetermin gesamtpflanzliche TM-Erträge zwischen 149,8 $\left(\mathrm{N}_{0}: 4 \mathrm{H}\right)$ und $175,7 \mathrm{dt} \mathrm{TM} \mathrm{ha}{ }^{-1}\left(\mathrm{~N}_{3}: 3 \mathrm{~A} / 1 \mathrm{H}\right.$, Abb. 6) und waren damit im Mittel signifikant höher als $1998\left(P_{\text {Jahr }}=0,0002\right)$.

Im Mittel über die Düngungsstufen erzielten die Gemengesaaten mit Ackerbohne und Hafer mit 173,2 $(3 \mathrm{~A} / 1 \mathrm{H})$ und 171,3 dt TM ha ${ }^{-1}(1 \mathrm{~A} / 3 \mathrm{H})$ signifikant höhere GesamtTM-Erträge als der Hafer in Reinsaat (4H: 153,6 dt TM ha ${ }^{-1}$, Tab. A9). Die Unterschiede der Wurzelmassen der Ansaatvarianten mit Ackerbohne und Hafer konnten mit Erträgen zwischen 15,1 $\left(\mathrm{N}_{0}: 4 \mathrm{H}\right)$ und 20,7 dt TM ha ${ }^{-1}\left(\mathrm{~N}_{0}: 4 \mathrm{~A}\right)$ statistisch nicht gesichert werden (Tab. A9), die Wurzelmassen lagen im Mittel aller Faktorkombinationen 1999 aber signifikant über den Wurzel-Erträgen im Jahr 1998 $\left(P_{\text {Jahr }}=0,0001\right)$. Der Strohertrag des Hafers in Reinsaat lag mit 68,6 dt TM ha-1 im Mittel der Düngungsstufen signifikant unter den Stroherträgen der Ansaaten mit Ackerbohne (Tab. A9). Mit Stroherträgen zwischen 66,5 dt TM ha ${ }^{-1}\left(\mathrm{~N}_{0}: 4 \mathrm{H}\right)$ und 92,6 
dt $\mathrm{TM} \mathrm{ha}^{-1}\left(\mathrm{~N}_{3}: 3 \mathrm{~A} / 1 \mathrm{H}\right)$ lagen die Erträge im Mittel über die Düngungsstufen auf einem mit dem Vorjahr vergleichbaren Niveau.

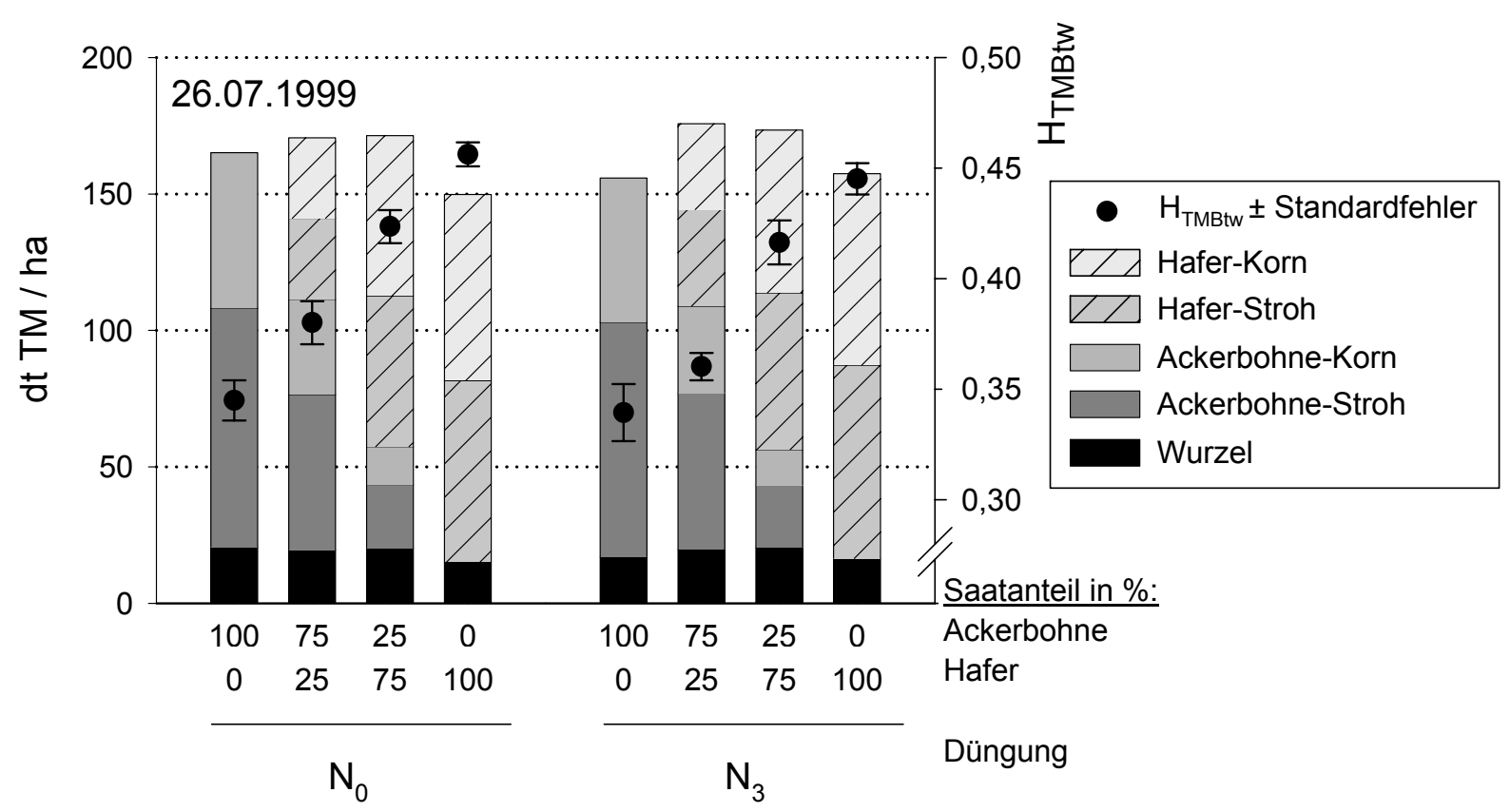

Abb. 6: Gesamtpflanzliche Trockenmasse-Akkumulation (dt TM ha ${ }^{-1}$; Wurzel, Stroh incl. Bestandesabfall, Korn) sowie gewichtete Trockenmasse-Harvest-Indizes $\left(\mathrm{H}_{\mathrm{TMB} t w} \pm\right.$ Standardfehler) beim Anbau von Ackerbohne und Hafer in Rein- und Gemengesaat zur letzten Ernte (BBCH 87) am 26.07.1999

Die Kornerträge schwankten 1999 zwischen 52,8 $\left(\mathrm{N}_{3}: 4 \mathrm{~A}\right)$ und 72,6 dt TM ha ${ }^{-1}\left(\mathrm{~N}_{3}\right.$ : 1A/3H, Tab. A6) und übertrafen damit die Kornerträge des Jahres 1998 hoch signifikant $\left(P_{\text {Jahr }}=0,0001\right)$. Die Ackerbohne in Reinsaat erzielte, wie bereits im Vorjahr, den geringsten Kornertrag (Tab. A6). Der Anstieg der Kornerträge zwischen dem ersten und zweiten Untersuchungsjahr lag zwischen +2,4 ( $\left.\mathrm{N}_{0}: 4 \mathrm{~A}\right)$ und +13,9 dt TM ha $^{-1}\left(\mathrm{~N}_{0}: 3 \mathrm{~A} / 1 \mathrm{H}\right.$, signifikante Wechselwirkung). Mit 10,2 (4A), 7,1 (3A/1H), 2,7 $(1 \mathrm{~A} / 3 \mathrm{H})$ und $0 \mathrm{dt} \mathrm{TM} \mathrm{ha}{ }^{-1}(4 \mathrm{H})$ betrug der Anteil des Bestandesabfalls an der gesamtpflanzlichen TM-Akkumulation 6,4 (4A), 4,1 (3A/1H) und 1,6 \% (1A/3H).

Der gewichtete gesamtpflanzliche TM-Harvest-Index ( $\left.\mathrm{H}_{\text {TMBtw }}\right)$ zeigte im Mittel über die Düngungsstufen mit zunehmendem Haferanteil einen signifikanten Anstieg (Abb. 6, Tab. A9). Dabei lagen die gewichteten TM-Harvest-Indizes der langjährig nicht mit mineralischen N-Düngern zu den nichtlegumen Vorfrüchten gedüngten Variante $\left(\mathrm{N}_{0}\right)$ im Mittel der Ansaatvarianten signifikant über der $\mathrm{N}_{3}$-Variante. Mit Werten zwischen 0,338 $\left(\mathrm{N}_{3}: 4 \mathrm{~A}\right)$ und 0,455 $\left(\mathrm{N}_{0}: 4 \mathrm{H}\right)$ lagen die gewichteten TM-Harvest-Indizes signifikant über den Werten des Vorjahres $\left(P_{\text {Jahr }}=0,0042\right)$.

Die Trockenmasse-Ertragsanteile von Ackerbohne und Hafer beider Gemengesaaten zum letzten Erntetermin 1999 sind in Abb. 7 dargestellt. Der Ertragsanteil der Hafer- 
Sprossmasse betrug in der Ansaatvariante mit $25 \%$ Hafer in der Ansaatmischung $(3 \mathrm{~A} / 1 \mathrm{H}) 42,6 \%$ in der $\mathrm{N}_{3}$-Variante bzw. $40,7 \%$ in der $\mathrm{N}_{0}$-Variante. Die Ansaatmischung mit $75 \%$ Hafer $(1 \mathrm{~A} / 3 \mathrm{H})$ erbrachte Hafer-Ertragsanteile von $85,1 \%\left(\mathrm{~N}_{3}\right)$ bzw. $77,6 \%\left(\mathrm{~N}_{0}\right)$. Die Unterschiede in den TM-Ertragsanteilen zwischen den Düngungsstufen konnten im Mittel über die Ansaatvarianten statistisch nicht gesichert werden (Abb. 7). Dabei lagen die Hafer-TM-Ertragsanteile im Jahr 1999 unter den Ertragsanteilen der Hafersprossmasse im Jahr $1998(P=0,0381)$.

Abb. 7: Ertragsanteile der SprossTM beim Anbau von Ackerbohne und Hafer in Gemengesaat zur letzten Ernte (26.07.1999)

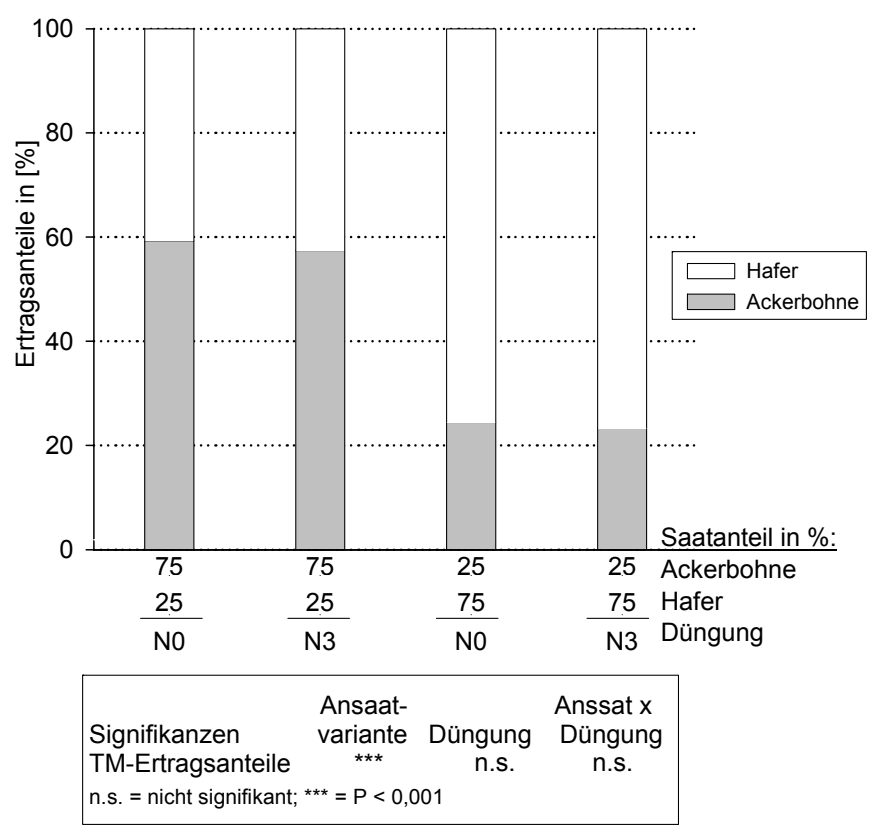

\subsubsection{Stickstofferträge und Stickstoff-Harvest-Index}

Im Folgenden werden die gesamtpflanzlich akkumulierten Stickstoff-Mengen, Stickstoff-Ertragsanteile sowie der Stickstoff-Harvest-Index getrennt nach Jahren dargestellt. Dazu sind in den Anhangstabellen Tab. A10 und Tab. A11 die Mittelwerte und Mittelwertvergleiche zu den drei Ernteterminen zusammengestellt. In den Abb. 8 und Abb. 10 werden die Stickstofferträge und die Harvest-Indizes zum jeweiligen dritten Erntetermin zusätzlich graphisch abgebildet. Schließlich sind in den Abb. 9 und Abb. 11 die Ackerbohne- und Hafer-Stickstoff-Ertragsanteile der oberirdischen Biomasse der Gemengeansaaten wiedergegeben.

Die gesamtpflanzlich akkumulierte Stickstoff-Menge $\left(\mathrm{N}_{\mathrm{Bt}}\right)$ des Hafers in Reinsaat lag im Mittel der Düngungsstufen 1998 signifikant unter den akkumulierten N-Mengen der Ansaaten mit Ackerbohne (Ausnahme: erste Ernte 1A/3H, Tab. A10). Im Vergleich der Düngungsstufen konnten im Mittel der Ansaaten bis zur Vollblüte (zweite Ernte) die Bestände der $\mathrm{N}_{3}$-Düngungsstufen signifikant höhere $\mathrm{N}$-Mengen im Stroh, in den Wurzeln und gesamtpflanzlich akkumulieren als die Bestände der $\mathrm{N}_{0}$-Variante. 
Dabei trat bei der gesamtpflanzlich akkumulierten und im Stroh akkumulierten NMenge eine signifikante Wechselwirkung zwischen den Ansaatvarianten und der Düngung auf (Tab. A10).

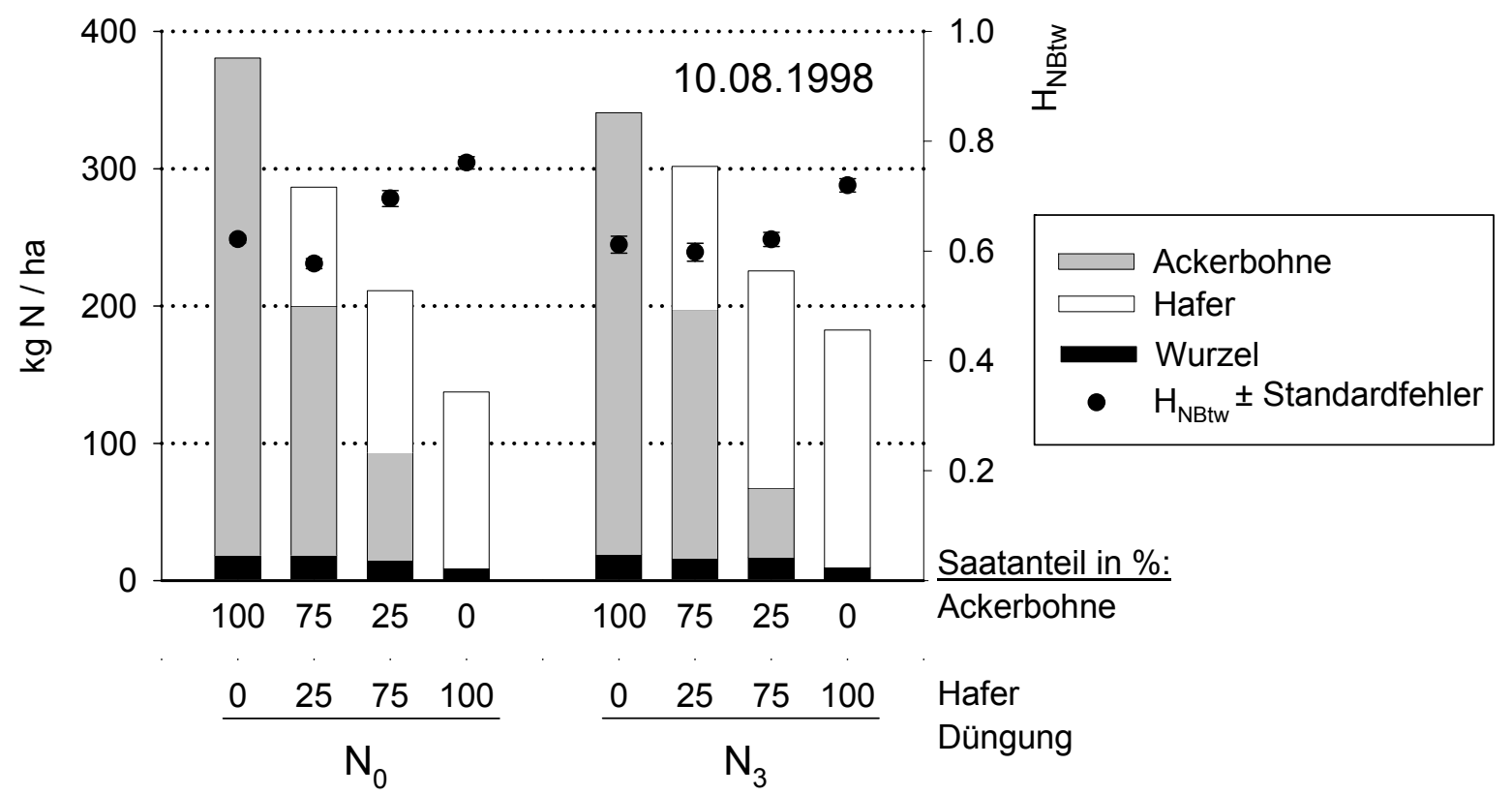

Abb. 8: Gesamtpflanzliche Stickstoff-Akkumulation (kg N ha ${ }^{-1}$; Wurzel, Stroh incl. Bestandesabfall, Korn) sowie gewichtete Stickstoff-Harvest-Indizes $\left(\mathrm{H}_{\mathrm{NBtw}}\right.$ \pm Standardfehler) beim Anbau von Ackerbohne und Hafer in Rein- und Gemengesaat zur letzten Ernte (BBCH 87) am 10.08.1998

Während in den Ansaaten mit Hafer die Bestände der $\mathrm{N}_{3}$-Variante höhere N-Mengen akkumulierten als in der $\mathrm{N}_{0}$-Variante (zweite und dritte Ernte), waren zur zweiten Ernte bei der Ackerbohne in Reinsaat etwa gleich hohe, zur dritten Ernte geringere $\mathrm{N}$-Mengen in den Pflanzen der $\mathrm{N}_{3}$-Variante festzustellen.

Bis zur Reife (BBCH 87, dritte Ernte) akkumulierten die Ansaaten mit Ackerbohne und Hafer gesamtpflanzliche Stickstoff-Mengen $\left(\mathrm{N}_{\mathrm{Bt}}\right)$ zwischen 137,2 $\left(\mathrm{N}_{0}: 4 \mathrm{H}\right)$ und 380,4 kg N ha-1 ( $\mathrm{N}_{0}$ : 4A, Abb. 8, Tab. A10). Dabei nahmen die akkumulierten N-Mengen in der Reihenfolge $4 \mathrm{~A}>3 \mathrm{~A} / 1 \mathrm{H}>1 \mathrm{~A} / 3 \mathrm{H}>4 \mathrm{H}$ im Mittel über die Düngungsstufen signifikant ab. Während die Ansaatvarianten mit Hafer in Rein- und Gemengesaat in der $\mathrm{N}_{3}$-Variante tendenziell höhere $\mathrm{N}$-Mengen akkumulierten, verhielt sich die Ackerbohne in Reinsaat (4A) entgegengesetzt (signifikante Wechselwirkung: Ansaatvariante $\times$ Düngung, Tab. A10, Abb. 8). Mit 227,7 kg N ha ${ }^{-1}$ akkumulierte die Ackerbohne in Reinsaat (4A), im Mittel über die Düngungsstufen, signifikant höhere KornN-Mengen im Vergleich zu den Ansaaten mit Hafer (Tab. A10). Sowohl bei den Korn$\mathrm{N}$ - als auch bei den Stroh-N-Mengen trat eine signifikante Wechselwirkung zwischen der Ansaatvariante und der Düngung auf. 
Mit Stickstoffmengen zwischen $0(4 \mathrm{H})$ und $36 \mathrm{~kg} \mathrm{~N} \mathrm{ha}^{-1}\left(\mathrm{~N}_{0}: 4 \mathrm{~A}\right)$ zum dritten Erntetermin akkumulierten die Ansaaten durchschnittlich 6,2\% des gesamtpflanzlichen Stickstoffs im Bestandesabfall. Der Anteil Wurzel-N am gesamtpflanzlich akkumulierten Stickstoff sank im Mittel aller Varianten von 9,4\% zur Blüte (2. Ernte) auf $5,9 \%$ bis zur Reife (dritte Ernte) ab und lag damit im Durchschnitt auf dem Niveau des Stickstoffs im Bestandesabfall (Tab. A10).

Im Mittel über die Düngungsstufen wies der Hafer in Reinsaat den höchsten gewichteten Stickstoff-Harvest-Index auf $\left(\mathrm{H}_{\mathrm{NB} \text { tw }}\right.$; Abb. 8, Tab. A10). Im Mittel der Ansaaten lagen die gewichteten $\mathrm{N}$-Harvest-Indizes der $\mathrm{N}_{3}$-Variante unter denen der $\mathrm{N}_{0}$-Variante. Dabei unterschieden sich die Harvest-Indizes gleicher Ansaaten zwischen den Düngungsstufen bei Hafer in Reinsaat $(4 \mathrm{H})$ und im Gemenge mit $25 \%$ Ackerbohnen $(1 \mathrm{~A} / 3 \mathrm{H})$ deutlicher als bei Ackerbohne in Reinsaat (4A) und im Gemenge mit $25 \%$ Hafer $(3 \mathrm{~A} / 1 \mathrm{H}$; signifikante Wechselwirkung: Ansaatvariante $\times$ Düngung; Tab. A10).

In Abb. 9 sind die Stickstoff-Ertragsanteile der Ackerbohne und des Hafers in beiden Gemengesaaten zum letzten Erntetermin abgebildet. Der N-Ertragsanteil der Hafer-Sprossmasse am gesamtpflanzlich akkumulierten Stickstoff im Spross betrug in der leguminosenbetonten Ansaatvariante $(3 \mathrm{~A} / 1 \mathrm{H}) \quad 36,8 \% \quad\left(\mathrm{~N}_{3}\right)$ bzw. $32,4 \%$ in der $\mathrm{N}_{0}$-Variante. In der haferbetonten Ansaatmischung $(1 \mathrm{~A} / 3 \mathrm{H})$ betrugen die Hafer-Ertragsanteile $75,8 \% \quad\left(\mathrm{~N}_{3}\right)$ bzw. $60,0 \%$ in der $\mathrm{N}_{0}$-Variante. Im Mittel der Ansaatvarianten lagen somit die N-Ertragsanteile des Hafer-Sprosses der $\mathrm{N}_{3}$-Variante signifikant über den $\mathrm{N}$-Ertragsanteilen der $\mathrm{N}_{0}$-Variante (Abb. 9).

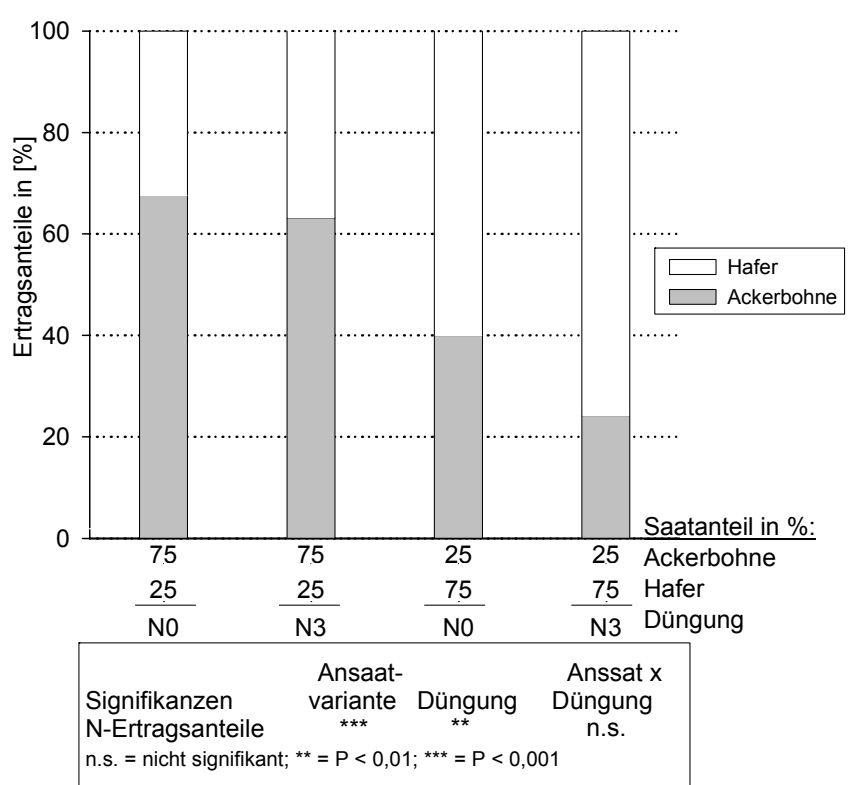

Abb. 9: Spross-Stickstoff-Ertragsanteile der Ackerbohne und des Hafers in Gemengesaat zum dritten Erntetermin (10.08.1998)

Im zweiten Untersuchungsjahr 1999 wurden in den Aufwüchsen, neben den angebauten Arten Ackerbohne und Hafer, auch geringe Mengen an Kräutern mit erfasst. Die Kräuter akkumulierten oberirdisch mit maximal $2,8 \mathrm{~kg} \mathrm{~N}^{-1}$ nur sehr geringe Mengen an Stickstoff, die im Folgenden nicht weiter berücksichtigt wurden (Tab. A7).

Weder die gesamtpflanzliche Stickstoffakkumulation noch die N-Akkumulation der Ertragskomponenten wurden durch die langjährig unterschiedlich hohe mineralische $\mathrm{N}$-Düngung zu den nichtlegumen Vorfrüchten signifikant beeinflusst. Die Ansaaten 
mit Ackerbohne $(4 \mathrm{~A}, 3 \mathrm{~A} / 1 \mathrm{H}, 1 \mathrm{~A} / 3 \mathrm{H})$ akkumulierten zu allen drei Ernteterminen gesamtpflanzlich signifikant mehr Stickstoff im Vergleich zur Hafer-Reinsaat $(4 \mathrm{H}, \mathrm{Tab}$. A11). Mit Werten zwischen 141,3 $\left(\mathrm{N}_{0}: 4 \mathrm{H}\right)$ und $369,5 \mathrm{~kg} \mathrm{~N} \mathrm{ha}^{-1}\left(\mathrm{~N}_{0}: 4 \mathrm{~A}\right)$ lagen die gesamtpflanzlich akkumulierten $\mathrm{N}$-Mengen $\left(\mathrm{N}_{\mathrm{Bt}}\right)$ ebenso wie die Korn-N-Erträge mit Werten zwischen 107,9 ( $\left.\mathrm{N}_{0}: 4 \mathrm{H}\right)$ und $234,8 \mathrm{~kg} \mathrm{~N} \mathrm{ha}^{-1}\left(\mathrm{~N}_{0}: 4 \mathrm{~A}\right)$ auf einem vergleichbaren Niveau wie im Vorjahr. Dabei nahmen die gesamtpflanzlich akkumulierten $\mathrm{N}$ Mengen sowie die Korn-N-Erträge in der Reihenfolge $4 \mathrm{~A}>3 \mathrm{~A} / 1 \mathrm{H}>1 \mathrm{~A} / 3 \mathrm{H}>4 \mathrm{H}$ signifikant ab. Im Jahr 1999 akkumulierten die Ansaaten im Bestandesabfall StickstoffMengen zwischen $0(4 \mathrm{H})$ und $32 \mathrm{~kg} \mathrm{~N}^{-1}\left(\mathrm{~N}_{0}: 4 \mathrm{~A}\right)$. Damit waren im Mittel der Ansaaten $6,0 \%$ des gesamtpflanzlichen Stickstoffs im Bestandesabfall enthalten. Die in den Wurzeln akkumulierte N-Menge lag im Jahr 1999 zwischen 9,1 (1. Ernte $\mathrm{N}_{0}: 4 \mathrm{H}$ ) und 46,7 kg N ha ${ }^{-1}$ (2. Ernte $\mathrm{N}_{0}: 3 \mathrm{~A} / 1 \mathrm{H}$, Tab. A11). Die Wurzel-N-Erträge übertrafen damit zu allen drei Ernteterminen die entsprechenden Erträge der Bestände zu den Ernteterminen des Vorjahres ( $P_{\text {Jahr }}=0,0001$ zu allen Ernteterminen).

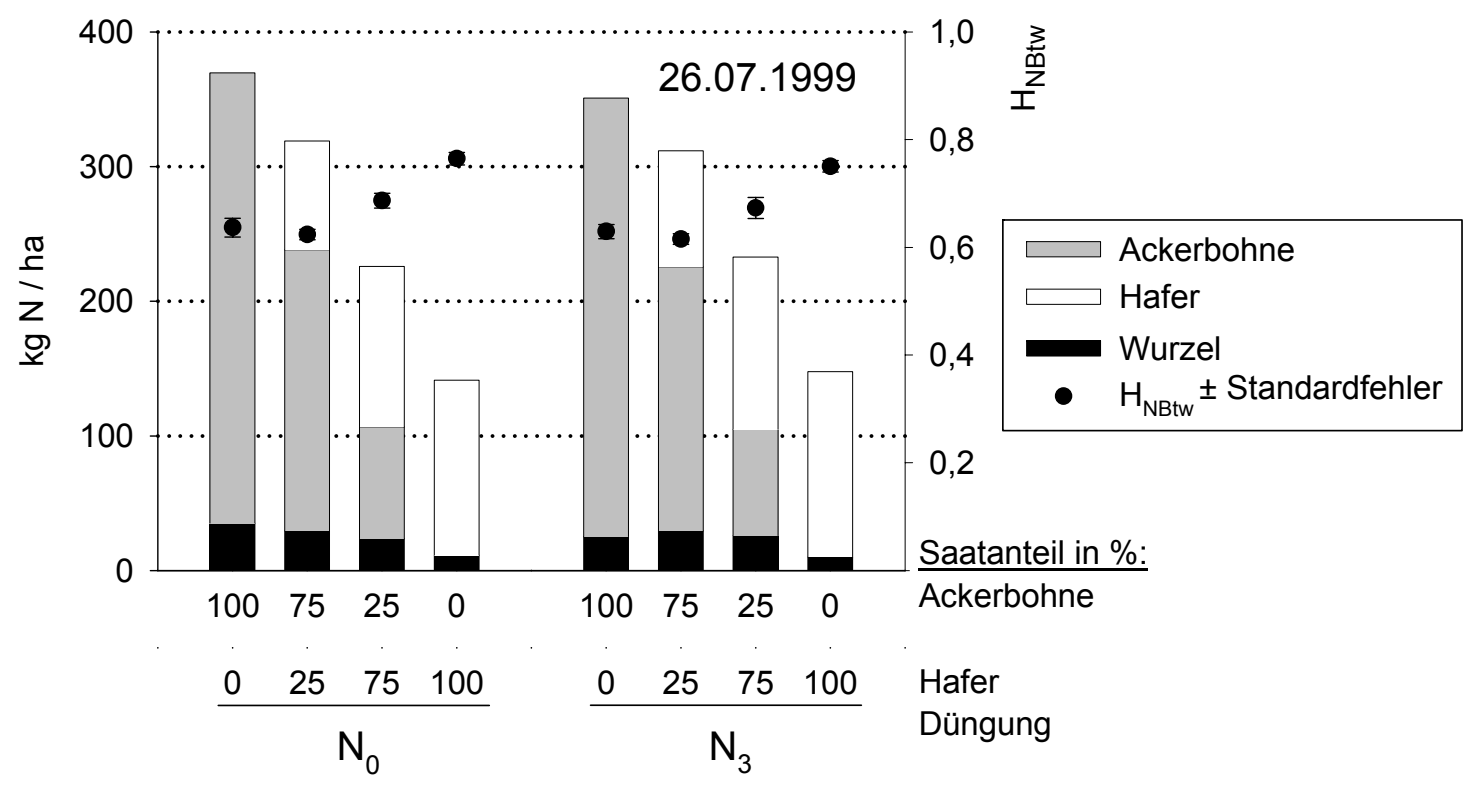

Abb. 10: Gesamtpflanzliche Stickstoff-Akkumulation (kg N ha ${ }^{-1}$; Wurzel, Stroh incl. Bestandesabfall, Korn) sowie gewichtete Stickstoff-Harvest-Indizes $\left(\mathrm{H}_{\mathrm{NBtw}}\right.$ \pm Standardfehler) beim Anbau von Ackerbohne und Hafer in Rein- und Gemengesaat zur letzten Ernte (BBCH 87) am 26.07.1999

Der Anteil Wurzel-N am gesamtpflanzlich akkumulierten Stickstoff lag im Mittel aller Varianten zum Zeitpunkt der Blüte (zweite Ernte) bei 17,6 \% und sank bis zur Reife (dritte Ernte) auf 9,0\% ab.

Im Mittel über die Düngungsstufen wies Hafer in Reinsaat $(4 \mathrm{H})$ den höchsten gewichteten N-Harvest-Index auf (Abb. 10, Tab. A11). Im Mittel aller Varianten lagen 
die gewichteten N-Harvest-Indizes 1999 signifikant über den Werten von 1998 ( $P_{\text {Jahr }}$ $=0,0031)$.

In Abb. 11 sind die Stickstoff-Ertragsanteile der Ackerbohne und des Hafer in Gemengesaat zum letzten Erntetermin 1999 abgebildet. Der N-Ertragsanteil der Hafer-Sprossmasse am gesamtpflanzlich akkumulierten Stickstoff in der GemengeSprossmasse betrug in der leguminosenbetonten Ansaatvariante $(3 \mathrm{~A} / 1 \mathrm{H}) 30,9 \%$ $\left(\mathrm{N}_{3}\right)$ bzw. 27,9\% in der $\mathrm{N}_{0}$-Variante. In der haferbetonten Ansaatmischung $(1 \mathrm{~A} / 3 \mathrm{H})$ betrugen die Hafer-N-Ertragsanteile 61,5\% ( $\left.\mathrm{N}_{3}\right)$ bzw. 59,1\% in der $\mathrm{N}_{0}$-Variante. Im Mittel der Ansaatvarianten wurden die N-Ertragsanteile der Hafer-Sprossmasse am gesamtpflanzlich akkumulierten Stickstoff in der Gemenge-Sprossmasse nicht durch die Düngungsvarianten beeinflusst. (Abb. 11). Im Mittel lagen die Ertragsanteile unter denen des Vorjahres.

Abb. 11: Spross-Stickstoff-Ertragsanteile der Ackerbohne und des Hafers in Gemengesaat zum dritten Erntetermin (26.07.1999)

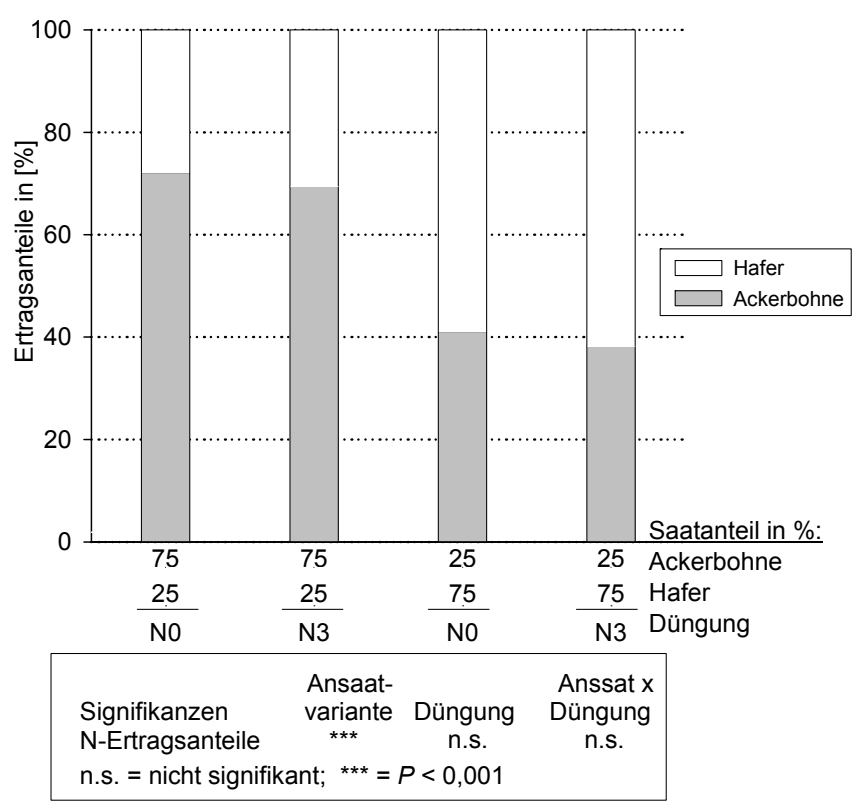

\subsubsection{N-Isotopenzusammensetzung in der gesamtpflanzlichen Biomasse}

Um mit der $\delta^{15} \mathrm{~N}$-Methode in Leguminosen und Leguminosen-Gras-Gemengen den Anteil Stickstoff aus der Luft bestimmen zu können, muss ein ausreichend großer Unterschied im Isotopenverhältnis zwischen molekularem Luftstickstoff und pflanzenverfügbarem Bodenstickstoff vorhanden sein (Kap. 2.5.5). In Abb. 12 sind die arithmetischen Mittelwerte der gewichteten gesamtpflanzlichen $\delta^{15} \mathrm{~N}-$ Werte der Ackerbohne und des Hafers in Rein- und Gemengesaat zu drei Ernteterminen für das Jahr 1998 dargestellt. Hierbei ist zu berücksichtigen, dass sich die gesamtpflanzlichen $\delta^{15} \mathrm{~N}$-Werte im Gemenge nur für die Summe des N-Anteils beider Ge- 
mengepartner darstellen lassen, da eine Trennung der unterirdischen N-Anteile nicht möglich ist (vgl. Kap. 2.7.2.3). Die $\delta^{15} \mathrm{~N}$-Werte in der Sprossmasse von Ackerbohne und Hafer in Rein- und Gemengesaat sind im Anhang der Tab. A12 zu entnehmen.

Die $\delta^{15} \mathrm{~N}$-Werte des Hafers in Reinsaat lagen im Mittel der Düngungsstufen mit Werten von 3,77 \%o (erste Ernte), 4,77\%o (zweite Ernte) und 3,60 \%o (dritte Ernte) zu allen drei Ernteterminen signifikant über den Werten der Ackerbohnen in Reinsaat (erste Ernte: 2,35\%; zweite Ernte: 1,22\%; dritte Ernte: 0,97\%o; Tab. A13). Dabei stiegen die gesamtpflanzlichen $\delta^{15} \mathrm{~N}$-Werte zu allen drei Ernteterminen mit zunehmendem Haferanteil signifikant an (Ausnahme: erste Ernte $3 \mathrm{~A} / 1 \mathrm{H}, 1 \mathrm{~A} / 3 \mathrm{H}$ ). Die Differenzen in den $\delta^{15} \mathrm{~N}$-Werten zwischen Ackerbohne und Hafer in Reinsaat lagen im Jahr $1998 \mathrm{im}$ Minimum bei 1,31\%o (erste Ernte, $\mathrm{N}_{3}$ ) und maximal bei 4,15\%o (zweite Ernte, $\mathrm{N}_{3}$ ).

Im Mittel der Ansaatvarianten führte die langjährige mineralische N-Düngung $\left(\mathrm{N}_{3}\right) \mathrm{zu}$ den nichtlegumen Vorfrüchten zum zweiten und dritten Erntetermin zu einem signifikanten Anstieg im $\delta^{15} \mathrm{~N}$-Wert der gesamtpflanzlichen Biomasse im Vergleich zu der $\mathrm{N}_{0}$-Variante (Tab. A13).

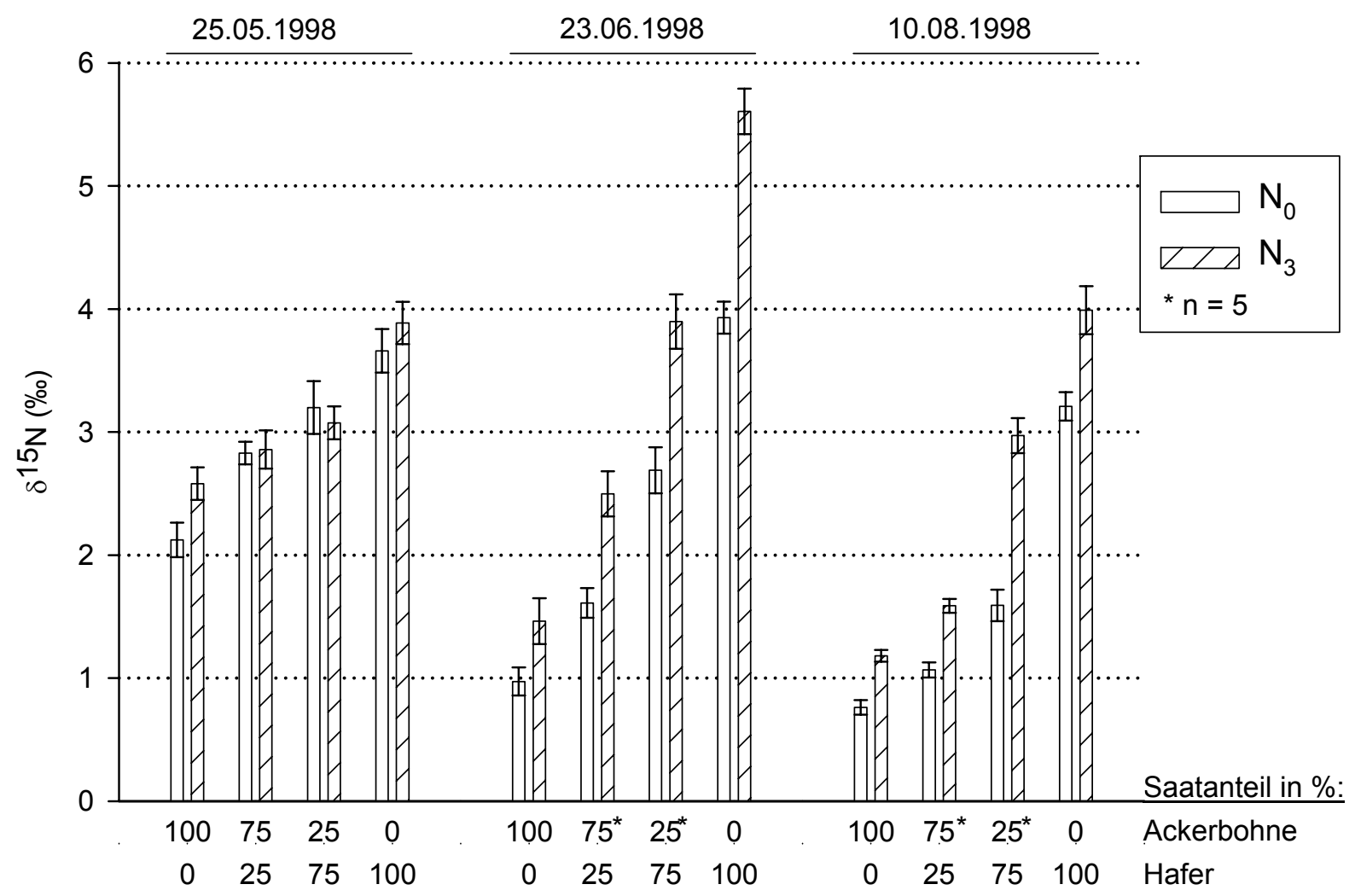

Abb. 12: Gewichtete $\delta^{15} \mathrm{~N}$-Werte (arithmetische Mittelwerte \pm Standardfehler in \%o) in der gesamtpflanzlichen Biomasse (Wurzel, Stroh, Bestandesabfall, Korn) beim Anbau von Ackerbohne und Hafer in Rein- und Gemengesaat 1998 
Zur letzten Ernte am 10.08.1998 trat zwischen den Ansaatvarianten und Düngungsstufen eine signifikante Wechselwirkung auf (Tab. A13). So war die Differenz im $\delta^{15} \mathrm{~N}$-Wert der Bestände aus den $\mathrm{N}_{0^{-}}$und $\mathrm{N}_{3}$-Parzellen in der Ansaatvariante mit $25 \%$ Ackerbohne $(1 \mathrm{~A} / 3 \mathrm{H})$ größer als in der Reinsaat Ackerbohne (4A).

Die gewichteten gesamtpflanzlichen $\delta^{15} \mathrm{~N}$-Werte von Ackerbohne und Hafer in Reinund Gemengesaat zu drei Ernteterminen des zweiten Untersuchungsjahres 1999 sind in Abb. 13 wiedergegeben.

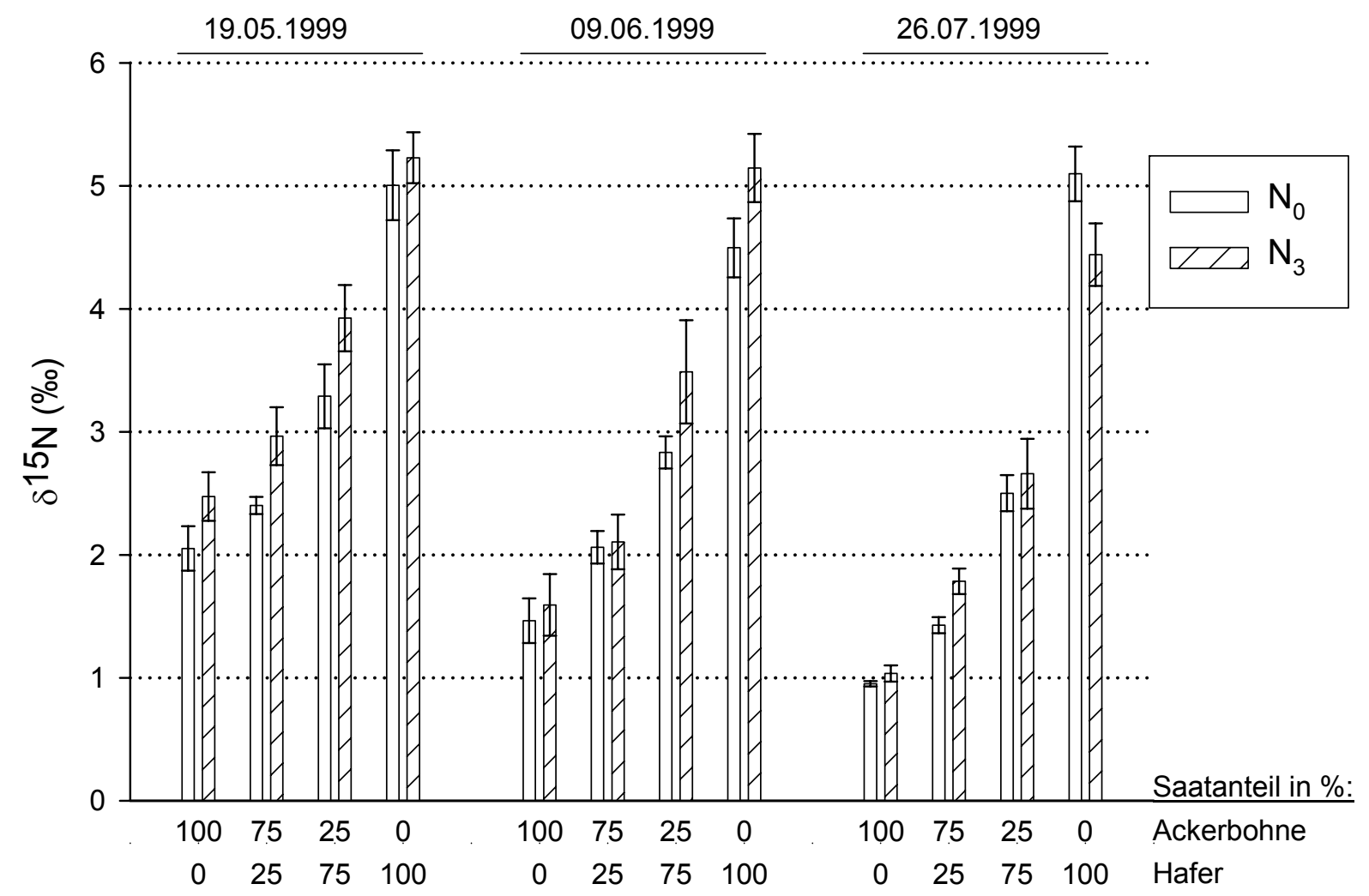

Abb. 13: Gewichtete $\delta^{15} \mathrm{~N}$-Werte (arithmetische Mittelwerte \pm Standardfehler in $\%$ ) in der gesamtpflanzlichen Biomasse (Wurzel, Stroh, Bestandesabfall, Korn) beim Anbau von Ackerbohne und Hafer in Rein- und Gemengesaat 1999

Auch 1999 lagen die $\delta^{15} \mathrm{~N}$-Werte des Hafers in Reinsaat im Mittel der Düngungsstufen mit Werten von 5,12\%o (erste Ernte), 4,83\%o (zweite Ernte) und 4,77\%o (dritte Ernte) zu allen drei Ernteterminen signifikant über den Werten der Ackerbohne in Reinsaat (erste Ernte: 2,26 \%; zweite Ernte: 1,53\%; dritte Ernte: 0,99\%; Tab. A13). Die gesamtpflanzlichen $\delta^{15} \mathrm{~N}$-Werte stiegen zu allen drei Ernteterminen mit zunehmendem Haferanteil signifikant an. Die Differenzen der $\delta^{15} \mathrm{~N}-$ Werte zwischen Ackerbohne und Hafer in Reinsaat lagen 1999 im Minimum bei 2,76 \%o (erste Ernte, $\mathrm{N}_{3}$ ) und maximal bei 4,15\% (dritte Ernte, $\mathrm{N}_{0}$ ). Eine Beeinflussung der $\delta^{15} \mathrm{~N}-$ Werte durch die Düngung zu den nichtlegumen Vorfüchten konnte im Jahr 1999 nur zum ersten Erntetermin nachgewiesen werden (Tab. A13). Zur letzten Ernte am 26.07.1999 trat zwischen den Ansaatvarianten und Düngungsstufen eine signifikante Wechselwir- 
kung auf. Während in der Hafer-Reinsaat aus der $\mathrm{N}_{3}$-Variante ein geringerer $\delta^{15} \mathrm{~N}$ Wert vorlag als in der Biomasse aus der $\mathrm{N}_{0}$-Variante, lagen bei den anderen Beständen die $\delta^{15} \mathrm{~N}$-Werte in der Biomasse aus der $\mathrm{N}_{0}$-Variante unter denen aus der $\mathrm{N}_{3}$-Variante.

Lagen die gemessenen $\delta^{15} \mathrm{~N}$-Werte zu den ersten beiden Ernteterminen 1999 noch auf einem im Vergleich zum Vorjahr ähnlich hohem Niveau, waren die $\delta^{15} \mathrm{~N}$-Werte zum dritten Erntetermin im Jahr 1999 hoch signifikant über den Werten zum gleichen Erntetermin des Vorjahres $\left(P_{\mathrm{Jahr}}=0,0004\right)$. Dabei waren sowohl zwischen den Faktoren Düngung und Jahr, Ansaatvariante und Jahr als auch Ansaatvariante und Düngung und Jahr jeweils signifikante Wechselwirkungen zu beobachten.

\subsubsection{Vergleich der Schätzergebnisse der symbiotisch fixierten N-Menge mittels $\delta^{15} \mathrm{~N}$ - und erweiterter Differenzmethode}

Die in Kapitel 3.1.3 dargestellten gesamtpflanzlichen $\delta^{15} \mathrm{~N}$-Werte dienten zur Ermittlung des Anteils Stickstoff aus der Luft in der Biomasse der Ackerbohne und des Ackerbohne/Hafer-Gemenges. Dazu wurden die gewichteten gesamtpflanzlichen $\delta^{15} \mathrm{~N}$-Werte des Hafers in Reinsaat, der zu prüfenden Ansaaten mit Ackerbohne bzw. Ackerbohne-Hafer-Gemenge und von auf $\mathrm{N}$-freiem Nährmedium angezogenen Ackerbohnen (Tab. 3) in Gleichung 2 (Kap. 2.5.5) eingesetzt und der Anteil Stickstoff aus der Luft ermittelt (Ndfa). Durch Multiplikation mit der gesamtpflanzlich akkumulierten $\mathrm{N}$-Menge $\left(\mathrm{N}_{\mathrm{Bt}}\right)$ mit dem Anteil $\mathrm{N}$ aus der Luft wurde die symbiotisch fixierte $\mathrm{N}$ Menge errechnet (Gleichung 13; Kap. 2.7.2.2).

Die in der gesamtpflanzlichen Biomasse akkumulierten luftbürtigen N-Mengen wurden neben der $\delta^{15} \mathrm{~N}$ - Methode auch mit der erweiterten Differenzmethode geschätzt. In Abb. 14 sind die Korrelationskoeffizienten zwischen den Ergebnissen der beiden Schätzmethoden zur Quantifizierung der luftbürtigen N-Mengen beim Anbau von Ackerbohne in Rein- und Gemengesaat mit Hafer für die Untersuchungsjahre 1998 (a) und 1999 (b) dargestellt. Die Abbildungen beinhalten alle Schätzergebnisse (inklusive negative Werte und Werte größer 100 \% Ndfa) die an den Ernteterminen über alle Faktorkombinationen ermittelt wurden.

Die Korrelationskoeffizienten für den Vergleich der Ergebnisse zur Schätzung der gesamtpflanzlich fixierten N-Menge betrugen bei den Ansaatvarianten mit Ackerbohne 1998 0,93 und 1999 0,92 (Abb. 14a und b). Alle Vergleiche erbrachten sehr enge Korrelationen mit hohem Signifikanzniveau $(P=0,0001)$. 


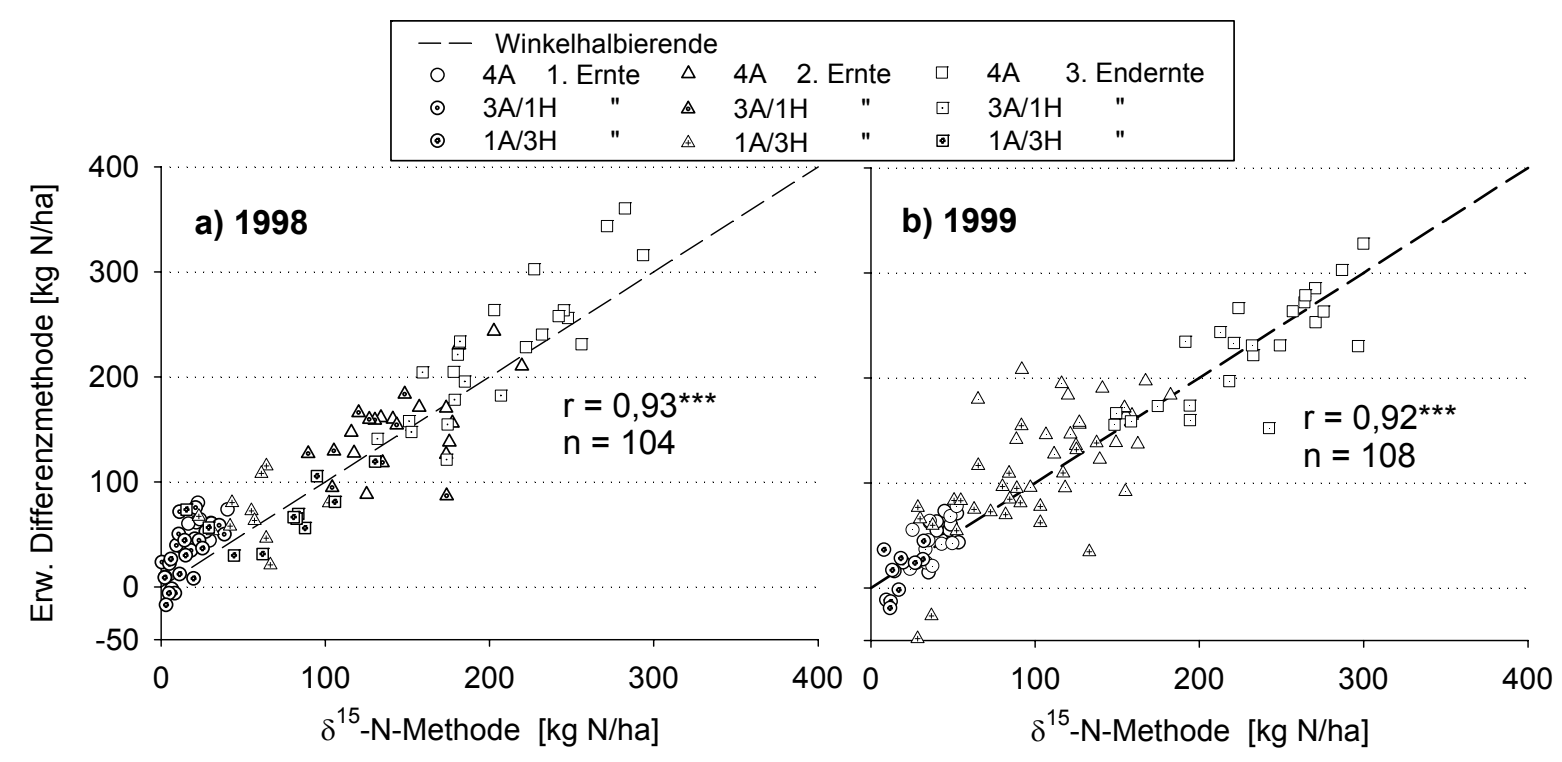

Abb. 14: Korrelation zwischen den Ergebnissen der $\delta^{15} \mathrm{~N}$ - und erweiterten Differenzmethode zur Schätzung der gesamtpflanzlich symbiotisch fixierten $\mathrm{N}$ Menge a) 1998 und b) 1999 beim Anbau von Ackerbohne in Reinsaat (4A) und Gemengesaat mit Hafer $(3 \mathrm{~A} / 1 \mathrm{H}$ und $1 \mathrm{~A} / 3 \mathrm{H})$

Im Mittel eines Jahres lagen die mittels erweiterter Differenzmethode geschätzten fixierten Stickstoffmengen mit 13,6 kg N ha-1 1998 und 6,3 $\mathrm{kg} \mathrm{N}$ ha $^{-1} 1999$ geringfügig über den luftbürtigen $\mathrm{N}$-Mengen, die mit der $\delta^{15} \mathrm{~N}$-Methode ermittelt wurden. Die Differenzen der einzelnen Schätzwerte, die mittels der beiden Methoden errechnet wurden, schwankten dagegen sehr. Im Jahr 1998 lagen die Schätzwerte der erweiterten Differenzmethode maximal bei $60,3 \mathrm{~kg} \mathrm{~N} \mathrm{ha}^{-1}$ über bzw. $87,1 \mathrm{~kg} \mathrm{~N}^{-1}$ unter den Werten, die mittels $\delta^{15} \mathrm{~N}$-Methode ermittelt wurden (Abb. 14a). Im zweiten Untersuchungsjahr 1999 wurden die Schätzergebnisse der $\delta^{15} \mathrm{~N}$-Methode durch die mit der erweiterten Differenzmethode ermittelten Werte bis maximal $116,1 \mathrm{~kg} \mathrm{~N} \mathrm{ha}^{-1}$ über- bzw. bis maximal $98,6 \mathrm{~kg} \mathrm{~N}^{-1}$ unterschätzt (Abb. 14b).

In der vorliegenden Untersuchung wurden im Jahr 1998 an vier Einzelschätzungen zur ersten Ernte mit der erweiterten N-Differenzmethode rechnerisch "negative" Ndfa-Werte gefunden. In zwei Fällen wurden Ndfa-Werte über $100 \%$ ermittelt. Im Jahr 1999 wiesen vier Schätzungen zur ersten Ernte bzw. zwei Schätzungen zur zweiten Ernte "negative" Ndfa-Werte auf.

Die Korrelationen zwischen den Schätzergebnissen der beiden Methoden, die zu jeweils einem Beprobungstermin vorlagen, sind in Tab. A14 im Anhang enthalten. 


\subsubsection{Luft- und bodenbürtige $\mathrm{N}$-Akkumulation sowie $\mathrm{CaCl}_{2}$-extrahierbarer $\mathbf{N}_{\text {min }}$-Stickstoff im Boden}

Die im ersten Untersuchungsjahr 1998 vorgefundenen $\mathrm{CaCl}_{2}$-extrahierbaren $\mathrm{N}_{\text {min }}$ Mengen im Boden (Nitrat- und Ammonium-N, Kap. 2.6.1) werden in den Abbildungen über die gesamte Profiltiefe, aufgeteilt in die 5 beprobten Horizonte, wiedergegeben. Im Rahmen der statistischen Verrechnungen wurden die $\mathrm{N}_{\min }$-Stickstoff-Mengen als Summe über die gesamten Profiltiefe $\left(0\right.$ bis $125 \mathrm{~cm}$ ) sowie einer Aufteilung der $\mathrm{N}_{\text {min }}{ }^{-}$ Stickstoff-Mengen in Ober- $\left(\sum_{0}\right.$ bis $\left.50 \mathrm{~cm}\right)$ und Unterboden $\left(\sum_{50}\right.$ bis $\left.125 \mathrm{~cm}\right)$ gegenübergestellt.

Vor Aussaat der Bestände mit Ackerbohne und Hafer wurde das Angebot an pflanzenverfügbarem Stickstoff im Boden erfasst. Mit $\mathrm{N}_{\text {min }}$-Mengen in Höhe von 92,2 $\mathrm{kg} \mathrm{N}$ ha $^{-1}$ in 0 bis $125 \mathrm{~cm}$ Bodentiefe wiesen die langjährig nicht mit mineralischen $\mathrm{N}$ Düngern zu den nichtlegumen Vorfrüchten gedüngten Parzellen $\left(\mathrm{N}_{0}\right)$ signifikant niedrigere $\mathrm{N}_{\text {min }}$-Mengen im Vergleich zu der $\mathrm{N}_{3}$-Variante $\left(172,3 \mathrm{~kg} \mathrm{~N} \mathrm{ha}^{-1}\right.$ ) auf (Abb. 15; Tab. A15a). Dabei wird bei einer Aufteilung der Profile in Ober- und Unterboden deutlich, dass sich signifikante Unterschiede im Vergleich der Düngungsstufen auf den Unterboden (50 bis $125 \mathrm{~cm}$ ) beschränken (Tab. A15a). Die Vorräte an pflanzenverfügbarem Stickstoff im Boden wurden von den Ansaaten mit Ackerbohne und Hafer im ersten Untersuchungsjahr 1998 in unterschiedlichem Maße genutzt. Die residualen $\mathrm{N}_{\text {min }}$-Mengen im Boden, die zur letzten Ernte unter den Ansaaten vorgefunden wurden, nahmen in der Reihenfolge $4 \mathrm{~A}>3 \mathrm{~A} / 1 \mathrm{H}>1 \mathrm{~A} / 3 \mathrm{H}$ signifikant bzw. $>4 \mathrm{H}$ tendenziell über die gesamte Profiltiefe ( 0 bis 125,0 bis 50 und 50 bis 125 $\mathrm{cm})$ ab (Tab. A16, Abb. 15). Die Ansaaten mit langjährig mineralischer N-Düngung zu den nichtlegumen Vorfrüchten $\left(\mathrm{N}_{3}\right)$ wiesen signifikant höhere residuale $\mathrm{N}_{\text {min- }}$ Mengen im Vergleich zur $\mathrm{N}_{0}$-Variante auf. Hierbei zeigen sich bei einer differenzierteren Betrachtungsweise die Unterschiede vor allem im Unterboden. Die Unterschiede der $\mathrm{N}_{\text {min }}$-Mengen gleicher Ansaaten zwischen $\mathrm{N}_{0}$ - und $\mathrm{N}_{3}$-Varianten nahmen mit zunehmendem Haferanteil im Bestand ab (signifikante Wechselwirkung: Ansaatvariante $\times$ Düngung in 0 bis $125 \mathrm{~cm}$ und 50 bis $125 \mathrm{~cm}$, Tab. A16).

Die höheren $\mathrm{N}_{\min }$-Mengen unter den Ansaatvarianten nach Vorfrüchten mit mineralischer N-Düngung ( 0 bis $125 \mathrm{~cm}$ ) ergaben sich bereits zum ersten und zweiten Erntetermin, wobei sich signifikante Unterschiede vor allem im Unterboden (50 bis 125 $\mathrm{cm}$ ) zeigten. Die höheren $\mathrm{N}_{\min }$-Mengen im Boden unter Ackerbohne in Reinsaat (4A) und im Gemenge mit $25 \%$ Hafer $(3 \mathrm{~A} / 1 \mathrm{H})$ konnten im Mittel über die Düngungsstufen zum ersten Erntetermin (zweiter $\mathrm{N}_{\text {min }}$-Probenahmetermin) nur im Oberboden ( 0 bis $50 \mathrm{~cm}$ ) und zur zweiten Ernte nur im Unterboden (50 bis $125 \mathrm{~cm}$; dritter $\mathrm{N}_{\min }$-Probenahmetermin) statistisch gesichert werden. Einen Einfluss der Düngung auf die Höhe der $\mathrm{N}_{\text {min }}$-Mengen im Oberboden konnte zu keinem der drei Erntetermine festgestellt werden (Tab. A16). 


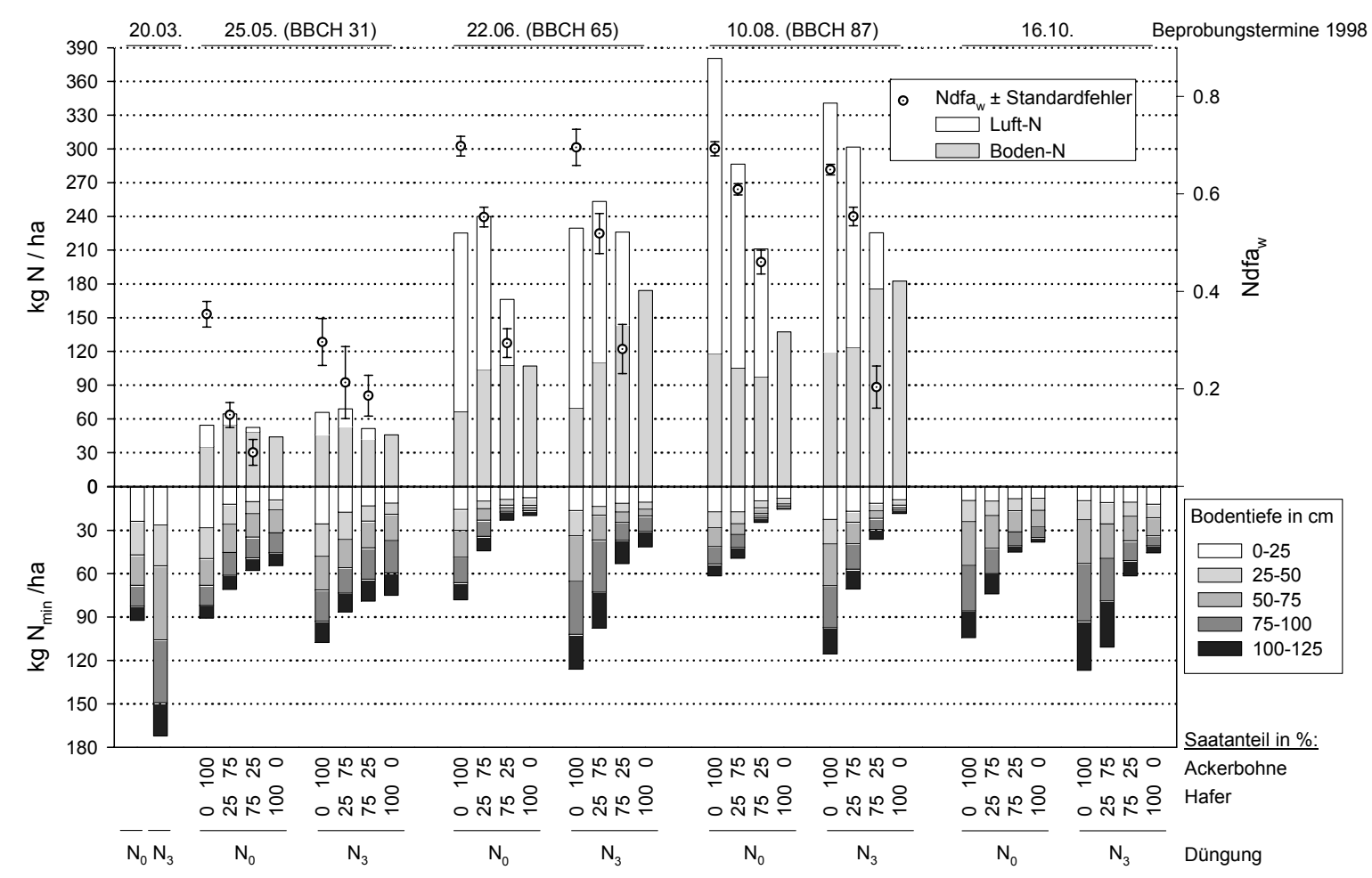

Abb. 15: $\mathrm{CaCl}_{2}$-extrahierbarer $\mathrm{N}_{\text {min }}$-Stickstoff im Boden $\left(\mathrm{kg} \mathrm{N}_{\min } \mathrm{ha}^{-1}\right)$, boden- und luftbürtige N-Akkumulation in der gesamtpflanzlichen Biomasse $\left(\mathrm{kg} \mathrm{N}^{-1}\right)$ und gewichtete Anteile Stickstoff aus der Luft $\left(\mathrm{Ndfa}_{w} \pm\right.$ Standardfehler) beim Anbau von Ackerbohne und Hafer in Rein- und Gemengesaat 1998

Um eine Schätzung der potentiellen Auswaschungsgefährdung der residualen $\mathrm{N}_{\text {min- }}$ Menge in Abhängigkeit der Ansaaten und Düngungsstufen genauer beurteilen zu können, wurden die Stickstoffvorräte im Boden zusätzlich vor Winter am 16.10.1998 bestimmt (Abb. 15). Im Mittel der Düngungsstufen traten in 0 bis $125 \mathrm{~cm}$ Bodentiefe signifikant höhere $\mathrm{N}_{\min }$-Mengen auf den vormals mit Ackerbohnen-Reinsaat bestellten Parzellen im Vergleich mit den Ansaaten mit Hafer in Rein- und Gemengesaat auf. Alle Parzellen, die zuvor Gemengebewuchs $(3 \mathrm{~A} / 1 \mathrm{H} ; 1 \mathrm{~A} / 3 \mathrm{H})$ aufwiesen, zeigten signifikant höhere $\mathrm{N}_{\text {min }}$-Mengen im Vergleich zu den Flächen mit Hafer-Reinsaat (Tab. A16). Die Unterschiede beschränkten sich dabei im Mittel über die Düngungsstufen auf den Unterboden. Ein Einfluss der langjährig unterschiedlich hohen Düngungsintensität zu den nichtlegumen Vorfrüchten $\left(\mathrm{N}_{\min } \mathrm{N}_{3}>\mathrm{N}_{\min } \mathrm{N}_{0}\right)$ konnte im Mittel der Ansaatvarianten im Oberboden ( 0 bis $50 \mathrm{~cm}$ Bodentiefe) und auf der gesamten Profiltiefe ( 0 bis $125 \mathrm{~cm}$ Bodentiefe) statistisch gesichert werden (Tab. A16).

Ackerbohnen sind in der Lage, sich neben dem fixierten Luftstickstoff auch pflanzenverfügbaren Stickstoff aus dem Boden anzueignen. Über den Anteil Luftstickstoff (Ndfa) lässt sich der Anteil Bodenstickstoff (Ndfs) in der Biomasse errechnen (Kap. 2.7.2.1; Gleichung 13 und folgender Text). Die im Folgenden dargestellten Ergeb- 
nisse der luft- und bodenbürtigen N-Akkumulation basieren auf den Schätzergebnissen des Anteils Stickstoff aus der Luft, die mittels der $\delta^{15} \mathrm{~N}$-Methode ermittelt wurden. Hierbei ist zu beachten, dass sich die gewichteten Anteile Stickstoff aus der Luft $\left(\mathrm{Ndfa}_{\mathrm{w}}\right)$ bei den Ansaaten mit Ackerbohne und Hafer im Gemenge (3A/1H und $1 \mathrm{~A} / 3 \mathrm{H}$ ) nur für die Summe des Stickstoffs in beiden Gemengepartnern (inkl. Wurzeln) darstellen lassen (Kap. 2.7.2.3). Während die gewichteten Anteile Stickstoff aus der Luft im Spross der Ackerbohne mit zunehmendem Haferanteil deutlich anstiegen (Tab. A12), zeigten sich bei einer gesamtpflanzlichen Betrachtungsweise, ab dem Zeitpunkt der Blüte (22.06.1998), signifikant höhere gewichtete Anteile Stickstoff aus der Luft bei den Ackerbohnen in Reinsaat (4A) gegenüber den gewichteten Anteilen Stickstoff aus der Luft in den Gemengeansaaten (Tab. A17). Im Mittel der Ansaatvarianten mit Ackerbohne wies die Variante ohne langjährige mineralische NDüngung zu den nichtlegumen Vorfrüchten $\left(\mathrm{N}_{0}\right)$ zum dritten Erntetermin signifikant höhere gewichtete Anteile Stickstoff aus der Luft in der Biomasse im Vergleich zu der $\mathrm{N}_{3}$-Variante auf. Dabei stiegen die Unterschiede zwischen den Düngungsvarianten gleicher Ansaaten mit zunehmendem Haferanteil an (10.08.1998: 4A 4\%-Punkte; 3A/1H 5,1 \%-Punkte; 1A/3H 25,7 \%-Punkte; signifikante Wechselwirkung: Ansaatvariante $\times$ Düngung, Tab. A17).

Zu allen drei Ernteterminen eigneten sich die Ansaaten mit Ackerbohne und Hafer den pflanzenverfügbaren Bodenstickstoff in Abhängigkeit von den Düngungsstufen in unterschiedlichem Maße an (zwischen Ansaatvariante $\times$ Düngung waren signifikante Wechselwirkungen zu allen drei Ernteterminen nachweisbar; Tab. A17, Abb. 15). Während die Ackerbohne in Reinsaat in der $\mathrm{N}_{0}$ - und $\mathrm{N}_{3}$-Variante nahezu gleiche $\mathrm{N}$ Mengen aus dem Boden aufnahm, nahmen die Bestände mit Hafer in der $\mathrm{N}_{3}$-Variante mehr Stickstoff aus dem Boden auf als in der $\mathrm{N}_{0}$-Variante. Mit 182,5 bzw. 137,3 $\mathrm{kg} \mathrm{N}^{-1}\left(4 \mathrm{H} \mathrm{N}_{3}\right.$ bzw. $\left.\mathrm{N}_{0}\right)$ akkumulierte der Hafer in Reinsaat zur dritten Ernte signifikant mehr bodenbürtigen Stickstoff als die Ackerbohne in Reinsaat und die Ackerbohne im Gemenge mit 25 \% Hafer (4A: 119,5 bzw. 118,2 und 3A/1H: 123,7 bzw. $103,9 \mathrm{~kg} \mathrm{~N} \mathrm{ha}^{-1}$ ). Im Mittel über die Düngungsstufen nahmen die symbiotisch fixierten Stickstoffmengen zum dritten Erntetermin in der Reihenfolge $4 \mathrm{~A}>3 \mathrm{~A} / 1 \mathrm{H}>1 \mathrm{~A} / 3 \mathrm{H}$ signifikant ab (Tab. A17). Dabei fixierten die Ansaaten mit Ackerbohne in der langjährig ohne mineralische $\mathrm{N}$-Düngung $\mathrm{zu}$ den nichtlegumen Vorfrüchten gedüngten Variante $\left(\mathrm{N}_{0}\right)$ signifikant mehr Stickstoff aus der Luft als in der $\mathrm{N}_{3}$-Variante $\left(\mathrm{N}_{0}, \mathrm{~N}_{3}\right.$, 4A 262,2, 221,2; 3A/1H 182,6, 178,1; 1A/3H 113,4, 49,8).

Die Menge an pflanzenverfügbarem Stickstoff im Boden vor Aussaat der Ackerbohne und des Hafers wurde im zweiten Untersuchungsjahr am 15.03.1999 erhoben. Mit $\mathrm{N}_{\text {min }}$-Vorräten von $102,7 \mathrm{~kg} \mathrm{~N}^{-1}$ in 0 bis $125 \mathrm{~cm}$ Bodentiefe wiesen die langjährig mit mineralischen $\mathrm{N}$-Düngern zu den nichtlegumen Vorfrüchten gedüngten Parzellen $\left(\mathrm{N}_{3}\right)$ eine signifikant höhere $\mathrm{N}_{\text {min }}$-Menge im Vergleich zu der $\mathrm{N}_{0}$-Variante $(72,3 \mathrm{~kg} \mathrm{~N}$ $\mathrm{ha}^{-1}$ ) auf (Abb. 16; Tab. A15b). Dabei war ein signifikanter Einfluss der Düngungsstu- 
fen auf die $\mathrm{N}_{\text {min }}$-Menge im Boden sowohl im Ober- als auch im Unterboden nachweisbar.

Die $\mathrm{N}_{\min }$-Mengen lagen im Jahr 1999 vor Aussaat in allen Bodenschichten hoch signifikant bzw. signifikant unter den $\mathrm{N}_{\text {min }}$-Mengen vor der Aussaat im Jahr 1998 $\left(P_{0 \text { bis } 125 \mathrm{~cm}}=0,0007 ; P_{0 \text { bis } 50 \mathrm{~cm}}=0,0013 ; P_{50 \text { bis } 125 \mathrm{~cm}}=0,0156\right)$. Dabei wirkte sich die langjährig unterschiedlich hohe mineralische N-Düngung zu den nichtlegumen Vorfrüchten im Vergleich der Jahre unterschiedlich auf die $\mathrm{N}_{\text {min }}$-Menge im Unterboden aus (signifikante Wechselwirkung: Düngung $\times$ Jahr; $P_{50 \text { bis } 125 \mathrm{~cm}}=0,036$ ). Während die $\mathrm{N}_{\text {min }}$-Menge in der $\mathrm{N}_{0}$-Variante zwischen den Jahren nur wenig differierte, lag die $\mathrm{N}_{\text {min }}$-Menge in der $\mathrm{N}_{3}$-Variante im Jahr 1998 um 51,2 kg N $\mathrm{Nin}_{\text {min }}$ hä höher als im Jahr 1999.

Auch im zweiten Untersuchungsjahr nutzten die Ansaaten der Ackerbohne und des Hafers in Rein- und Gemengesaat die Vorräte an pflanzenverfügbarem Stickstoff im Boden unterschiedlich stark aus. Mit Werten von 64,7 bzw. $73,6 \mathrm{~kg} \mathrm{~N}^{-1}$ (4A: $\mathrm{N}_{0}$ bzw. $\mathrm{N}_{3}$ ) in 0 bis $125 \mathrm{~cm}$ Bodentiefe hinterließen die Ackerbohnen-Reinsaaten im Mittel über die Düngungsstufen zur letzten Ernte 1999 signifikant höhere $\mathrm{N}_{\mathrm{min}}$-Mengen im Boden im Vergleich zu den Ansaaten mit Hafer (Tab. A18). Mit zunehmendem Haferanteil nahm die residuale $\mathrm{N}_{\text {min }}$-Menge im Boden ab, wobei die Unterschiede zwischen Hafer in Reinsaat $(4 \mathrm{H})$ und im Gemenge mit $25 \%$ Ackerbohne $(1 \mathrm{~A} / 3 \mathrm{H})$ statistisch nicht zu sichern waren. Die zur dritten Ernte vorgefundenen Abstufungen der $\mathrm{N}_{\min }$-Mengen im Boden in Abhängigkeit von den Ansaatvarianten konnten im Oberboden bereits zur ersten und zweiten Ernte (zweite und dritte $\mathrm{N}_{\text {min }}{ }^{-}$ Probenahmetermin) beobachtet werden (Tab. A18). Signifikant höhere $\mathrm{N}_{\text {min }}-$ Mengen im Unterboden unter den Reinsaaten der Ackerbohne (4A) und Gemengen mit $25 \%$ Hafer $(3 \mathrm{~A} / 1 \mathrm{H})$ konnten zur zweiten Ernte festgestellt werden. Höhere $\mathrm{N}_{\min }$-Vorräte unter der $\mathrm{N}_{3}$-Variante wurden im Jahr 1999 nur zur zweiten Ernte in 0 bis $125 \mathrm{~cm}$ Bodentiefe bzw. 0 bis $50 \mathrm{~cm}$ beobachtet. (Tab. A18, dritter Probenahmetermin).

Die Ansaaten mit Hafer in Reinsaat $(4 \mathrm{H})$ und im Gemenge mit $25 \%$ Ackerbohne $(1 \mathrm{~A} / 3 \mathrm{H})$ waren in beiden Jahren in der Lage, die $\mathrm{N}_{\text {min }}$-Menge in 0 bis $125 \mathrm{~cm}$ Bodentiefe weitestgehend zu nutzen (maximale Menge im Jahr 1998: $1 \mathrm{~A} / 3 \mathrm{H} \mathrm{N} 336,2 \mathrm{~kg} \mathrm{~N}$ $\mathrm{ha}^{-1}$ und minimale Menge im Jahr 1998: $4 \mathrm{H} \mathrm{N} \mathrm{N}_{0} 15,5 \mathrm{~kg} \mathrm{~N} \mathrm{ha}^{-1}$ in 0 bis125 cm zur dritten Ernte). Im Mittel über die Ansaatvarianten und Düngungsstufen lagen die residualen $\mathrm{N}_{\text {min }}$-Mengen zur dritten Ernte im Jahr 1998 signifikant über denen des Jahres $1999(P=0,0108)$. Dabei traten sowohl zwischen den Ansaatvarianten und den Jahren $(P=0,0022)$ als auch zwischen den Düngungsstufen und Jahren $(P=$ $0,0009)$ hoch signifikante Wechselwirkung auf. 


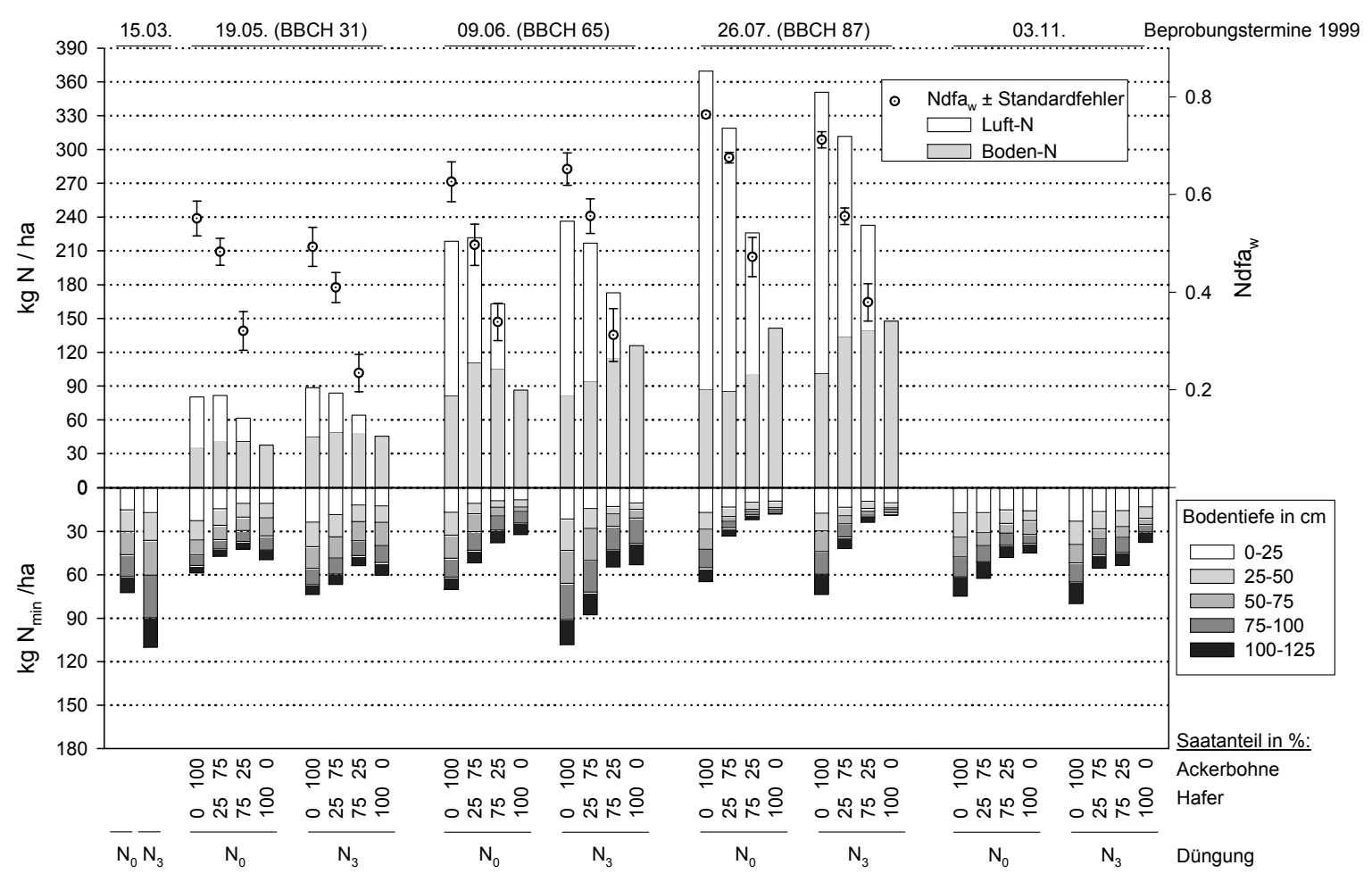

Abb. 16: $\mathrm{CaCl}_{2}$-extrahierbarer $\mathrm{N}_{\text {min }}$-Stickstoff im Boden $\left(\mathrm{kg} \mathrm{N}_{\min }\right.$ ha ${ }^{-1}$ ), boden- und luftbürtige $\mathrm{N}$-Akkumulation in der gesamtpflanzlichen Biomasse ( $\left.\mathrm{kg} \mathrm{N} \mathrm{ha}^{-1}\right)$ und gewichtete Anteile Stickstoff aus der Luft (Ndfaw \pm Standardfehler) beim Anbau von Ackerbohne und Hafer in Rein- und Gemengesaat 1999

Die $\mathrm{N}_{\text {min }}$-Beprobung vor Winter am 03.11.1999 erbrachte mit $\mathrm{N}_{\min }$-Mengen in 0 bis $125 \mathrm{~cm}$ Bodentiefe in Höhe von $37,7\left(4 \mathrm{H}, \mathrm{N}_{3}\right)$ bis $80,0 \mathrm{~kg} \mathrm{~N} \mathrm{ha}^{-1}\left(4 \mathrm{~A}, \mathrm{~N}_{3}\right)$ im Mittel der Düngungsstufen hoch signifikant geringere $\mathrm{N}_{\text {min }}$-Mengen im Vergleich zum Vorjahr $(P$ $=0,0005)$. Die vormals mit Ackerbohnen-Reinsaat (4A) bestellten Parzellen wiesen im Vergleich mit Hafer in Rein- und Gemengesaat mit $25 \%$ Ackerbohne (4H und $1 \mathrm{~A} / 3 \mathrm{H}$ ) im Mittel über die Düngungsstufen signifikant höhere $\mathrm{N}_{\min }$-Mengen auf (Tab. A18; Abb. 16). Im Mittel der Ansaatvarianten beeinflussten die langjährig unterschiedlich hohen $N$-Düngungsintensitäten $\left(N_{0}\right.$ und $\left.N_{3}\right)$ zu den nichtlegumen Vorfrüchten die $\mathrm{N}_{\min }$-Vorräte in 0 bis $125 \mathrm{~cm}$ Bodentiefe signifikant. Die $\mathrm{N}_{\text {min }}$-Vorräte im Oberboden (0 bis $50 \mathrm{~cm}$ Bodentiefe) verdeutlichen, dass sich die Düngungsstufen $\left(\mathrm{N}_{0}\right.$ und $\left.\mathrm{N}_{3}\right)$ in Abhängigkeit der Ansaatvarianten unterschiedlich auf die Höhe der Vorräte auswirkten (signifikante Wechselwirkung: $4 \mathrm{~A}$ und $1 \mathrm{~A} / 3 \mathrm{H}: \mathrm{N}_{\min } \mathrm{N}_{3}>\mathrm{N}_{\min } \mathrm{N}_{0}$; $3 \mathrm{~A} / 1 \mathrm{H}$ und $4 \mathrm{H}: \mathrm{N}_{\min } \mathrm{N}_{0}>\mathrm{N}_{\min } \mathrm{N}_{3}$; Tab. A18).

Während auch 1999 die gewichteten Anteile Stickstoff aus der Luft (Ndfa ${ }_{w}$ ) im Spross von Ackerbohne mit zunehmendem Haferanteil deutlich anstiegen (Tab. A12), lagen bei einer gesamtpflanzlichen Betrachtungsweise die gewichteten Anteile Stickstoff 
aus der Luft von Ackerbohnen in Reinsaat (4A) im Mittel über die Düngungsstufen zu allen drei Ernteterminen signifikant über den Anteilen der Gemengeansaaten (Tab. A19, Abb. 16). Die Variante ohne langjährige mineralische $N$-Düngung zu den nichtlegumen Vorfüchten $\left(\mathrm{N}_{0}\right)$ wies im Vergleich zur $\mathrm{N}_{3}$-Variante zum dritten Erntetermin tendenziell höhere gewichtete Anteile Stickstoff aus der Luft in der Biomasse auf. Die gewichteten Anteile Stickstoff aus der Luft in der Biomasse der Bestände überstiegen im Jahr 1999 die Werte des Jahres 1998 sowohl zur ersten als auch zur dritten Ernte signifikant, wobei die Unterschiede zur ersten Ernte am deutlichsten ausfielen $\left(P_{\text {Jahr 1.Ernte }}=0,0001 ; P_{\text {Jahr } 3 . E r n t e}=0,0013\right)$.

Bis zur letzten Ernte hatten die Ackerbohnen in Reinsaat (4A) mit 87,3 $\left(\mathrm{N}_{0}\right)$ und $101,3 \mathrm{~kg} \mathrm{~N} \mathrm{ha}^{-1}\left(\mathrm{~N}_{3}\right)$ signifikant geringere bodenbürtige Stickstoffmengen in der Biomasse akkumuliert als die Ansaaten mit Hafer in Rein- oder Gemengesaat $(4 \mathrm{H}$, $3 \mathrm{~A} / 1 \mathrm{H}, 1 \mathrm{~A} / 3 \mathrm{H}$, Tab. A19). Dabei traten Unterschiede zwischen den Ansaatvarianten erst ab dem Entwicklungsstadium Blüte der Ackerbohne auf (zweite Ernte: $1 \mathrm{~A} / 3 \mathrm{H}$ signifikant höhere bodenbürtige N-Mengen in der Biomasse im Vergleich zu 4A). Die Variante mit langjähriger mineralischer N-Düngung zu den nichtlegumen Vorfrüchten $\left(\mathrm{N}_{3}\right)$ wies im Vergleich zur $\mathrm{N}_{0}$-Variante zum dritten Erntetermin signifikant höhere $\mathrm{N}$ Mengen in der Biomasse aus bodenbürtiger N-Quelle auf.

Im Mittel aller Varianten nahmen die Ansaaten im Jahr 1999 im Vergleich zum Vorjahr geringere Mengen an bodenbürtigem Stickstoff auf $(P=0,0038)$, wobei eine signifikante Wechselwirkung zwischen den Jahren, den Ansaatvarianten und der Düngung zu verzeichnen war $(P=0,0105)$.

Im Mittel über die Düngungsstufen nahmen die fixierten Stickstoffmengen, analog zum Vorjahr, zum dritten Erntetermin in der Reihenfolge $4 \mathrm{~A}>3 \mathrm{~A} / 1 \mathrm{H}>1 \mathrm{~A} / 3 \mathrm{H}$ signifikant ab (Tab. A19). Ein Einfluss der langjährigen mineralischen N-Düngung zu den nichtlegumen Vorfrüchten auf die fixierten N-Mengen konnte im zweiten Untersuchungsjahr zu keinem Zeitpunkt statistisch gesichert werden. Gleichwohl hatten die Ansaaten mit Ackerbohnen $(4 \mathrm{~A}, 3 \mathrm{~A} / 1 \mathrm{H}, 1 \mathrm{~A} / 3 \mathrm{H})$ der Variante ohne langjährig mineralische $\mathrm{N}$-Düngung zu den nichtlegumen Vorfüchten $\left(\mathrm{N}_{0}\right)$ zur letzten Ernte im Vergleich zu der $\mathrm{N}_{3}$-Variante tendenziell höhere Mengen an luftbürtigem Stickstoff in der Biomasse akkumuliert (Differenzen: 4A $\mathrm{N}_{0}:+32,7 ; 3 \mathrm{~A} / 1 \mathrm{H} \mathrm{N}_{0}:+55,8$ bzw. $1 \mathrm{~A} / 3 \mathrm{H} \mathrm{N}_{0}$ : $+31,8 \mathrm{~kg} \mathrm{~N}^{-1}$; Tab. A19).

Die fixierten luftbürtigen Stickstoffmengen übertrafen 1999 zum ersten und dritten bzw. unterschritten zum zweiten Schnitttermin die Stickstoffmengen des Vorjahres signifikant $\left(P_{\text {Jahr 1.Ernte }}=0,0001 ; P_{\text {Jahr 2.Ernte }}=0,0346 ; P_{\text {Jahr 3.Ernte }}=0,0022\right)$. 


\subsubsection{Transfer-Stickstoff beim Anbau von Hafer im Gemenge mit Acker- bohne}

Beim Anbau von Körner- und Futterleguminosen im Gemenge mit Gräsern kann im Verlauf der Vegetationsperiode ein Teil des von der Leguminose symbiotisch fixierten Stickstoffs an den nichtlegumen Gemengepartner transferiert werden. Liegen die $\delta^{15} \mathrm{~N}$-Werte einer im Gemenge angebauten Nichtleguminose signifikant unter denen einer in unmittelbarer Nachbarschaft angebauten Nichtleguminose in Reinsaat, können mit der $\delta^{15} \mathrm{~N}$-Methode die Anteile des Transfer- $\mathrm{N}$ an der in der oberirdischen Biomasse der Nichtleguminose akkumulierten N-Menge ermittelt werden (Kap. 2.7.5). In Abb. 17 sind die $\delta^{15} \mathrm{~N}$-Werte im Spross des Hafers in Rein- und Gemengesaat $(4 \mathrm{H}, 1 \mathrm{~A} / 3 \mathrm{H}, 3 \mathrm{~A} / 1 \mathrm{H})$ zur jeweils letzten Ernte der Jahre 1998 und 1999 in Abhängigkeit von der langjährig unterschiedlich hohen N-Düngung zu den nichtlegumen Vorfrüchten $\left(\mathrm{N}_{0}\right.$ und $\left.\mathrm{N}_{3}\right)$ dargestellt.

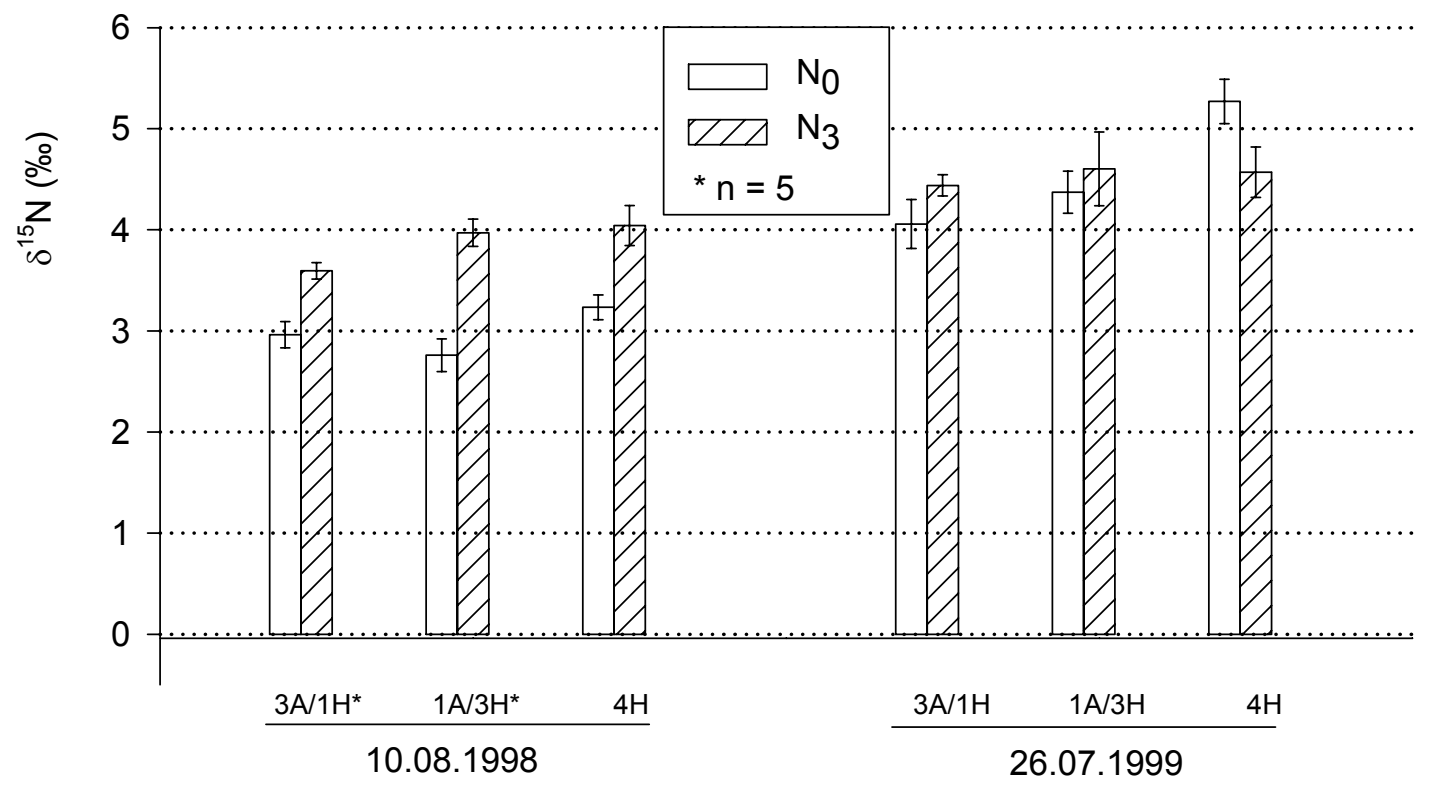

Abb. 17: $\delta^{15} \mathrm{~N}-$ Werte $(\mathrm{n}=6$, Mittelwerte \pm Standardfehler in $\%$ ) in der Hafer-Sprossmasse (Korn + Stroh) in Reinsaat $(4 \mathrm{H})$ und Gemengesaaten mit Ackerbohne (1A/3H und 3A/1H) am 10.08.1998 (links) und 26.07.1999 (rechts)

In beiden Untersuchungsjahren konnten signifikante Unterschiede zwischen den $\delta^{15} \mathrm{~N}$-Werten des Hafers in Reinsaat $(4 \mathrm{H})$ und den $\delta^{15} \mathrm{~N}$-Werten des Hafers in Gemengesaat $(1 \mathrm{~A} / 3 \mathrm{H}$ und $3 \mathrm{~A} / 1 \mathrm{H})$ nachgewiesen werden (Tab. A20). Der $\delta^{15} \mathrm{~N}-$ Wert des Hafers in Reinsaat (4H) war im Jahr 1998 im Mittel der Düngungsstufen mit 3,64 \%o signifikant höher als die Werte des Hafers im Gemenge mit der Ackerbohne (1A/3H und $3 \mathrm{~A} / 1 \mathrm{H}$ jeweils $3,31 \%$ ). Im zweiten Untersuchungsjahr wurde lediglich zwischen dem $\delta{ }^{15} \mathrm{~N}$-Wert des Hafers in Reinsaat $(4 \mathrm{H}=4,92 \%$ ) und des Hafers im Gemenge 
mit $75 \%$ Ackerbohne $(3 \mathrm{~A} / 1 \mathrm{H}=4,25 \%)$ ein signifikanter Unterschied festgestellt. Die $\delta^{15} \mathrm{~N}$-Werte des Hafers in der langjährig nicht mit mineralischen N-Düngern zu den nichtlegumen Vorfüüchten gedüngten Variante $\left(\mathrm{N}_{0}\right)$ lagen 1998 stets unter den $\delta^{15} \mathrm{~N}$ Werten im Hafer aus der $\mathrm{N}_{3}$-Variante $\left(\mathrm{N}_{0}: 4 \mathrm{H}=3,23,3 \mathrm{H} / 1 \mathrm{~A}=2,76,1 \mathrm{H} / 3 \mathrm{~A}=2,96 \%\right.$; $\mathrm{N}_{3}: 4 \mathrm{H}=4,04,3 \mathrm{H} / 1 \mathrm{~A}=3,97,1 \mathrm{H} / 3 \mathrm{~A}=3,59 \%$ ). Dabei waren die Unterschiede zwischen den Düngungsstufen im Mittel über die Ansaatvarianten signifikant (Tab. A20). Dagegen konnte im Jahr 1999 kein Einfluss der Düngung auf den $\delta^{15} \mathrm{~N}$-Wert des Hafers im Mittel der Ansaatvarianten nachgewiesen werden. Allerdings wurde in beiden Untersuchungsjahren eine signifikante Wechselwirkung zwischen der Düngung und den Ansaatvarianten ermittelt (Tab. A20). Die Unterschiede in den $\delta^{15} \mathrm{~N}$ Werten zwischen den Jahren waren hoch signifikant. Zudem trat eine signifikante Wechselwirkung zwischen den Jahren und den Ansaatvarianten auf.

Die in den Gemengen aus Ackerbohne und Hafer geschätzte Transfer-N-Menge lag im Mittel im Jahr 1998 zwischen 3,6 und 16,0 kg ha-1 sowie im Jahr 1999 zwischen 3,7 und 17,9 kg ha- (Tab. 5). Der Anteil des Transfer-N an der Gesamt-N-Menge in der Hafer Sprossmasse schwankte damit zwischen 2,2\% (1998: $1 \mathrm{~A} / 3 \mathrm{H} \mathrm{N} \mathrm{N}_{3}$ ) und 21,8 \% (1999: 3A/1H No). Im Jahr 1999 war in beiden Gemengeansaaten sowohl die transferierte N-Menge als auch der Anteil des Transfer- $\mathrm{N}$ an der Gesamt-N-Menge der Hafer-Sprossmasse in der $\mathrm{N}_{0}$-Düngungsstufe signifikant höher als in der $\mathrm{N}_{3}$ Düngungsstufe (Tab. A21).

Tab. 5: Transferierte N-Menge (Mittelwerte \pm Standardabweichung; $1998 n=5$, $1999 \mathrm{n}=6$ ) und Anteile aus der symbiotischen $\mathrm{N}_{2}$-Fixierung der Ackerbohne im Gemenge in der oberirdischen Sprossmasse des Hafers.

\begin{tabular}{|c|c|c|c|c|}
\hline & \multicolumn{2}{|c|}{${ }^{1} \mathrm{~N}_{\text {trans }}$ in $\mathrm{kg} \mathrm{ha}^{-1}$} & \multicolumn{2}{|c|}{${ }^{2}$ Anteil $\mathrm{N}_{\text {trans }}$ in $\%$} \\
\hline Ansaatvariante & $\mathrm{N}_{0}$ & $\mathrm{~N}_{3}$ & $\mathrm{~N}_{0}$ & $\mathrm{~N}_{3}$ \\
\hline 1998 & & & & \\
\hline $3 \mathrm{~A} / 1 \mathrm{H}$ & $7,3 \pm 2,2$ & $10,3 \pm 9,3$ & 8,6 & 9,6 \\
\hline $1 \mathrm{~A} / 3 \mathrm{H}$ & $16,0 \pm 10,1$ & $3,6 \pm 5,3$ & 13,5 & 2,2 \\
\hline 1999 & & & & \\
\hline $3 \mathrm{~A} / 1 \mathrm{H}$ & $17,9 \pm 5,9$ & $3,7 \pm 5,1$ & 21,8 & 4,5 \\
\hline $1 \mathrm{~A} / 3 \mathrm{H}$ & $17,7 \pm 12,1$ & $5,5 \pm 6,8$ & 15,4 & 5,0 \\
\hline
\end{tabular}

${ }^{1}$ transferierte N-Menge in kg ha ${ }^{-1},{ }^{2}$ Anteil des Transfer-Stickstoffes an der Gesamt-N-Menge der Hafer Sprossmasse

\subsubsection{Einfacher und erweiterter N-Flächenbilanzsaldo}

Um den Beitrag von Leguminosen zu einer Selbstregelung in Agrarökosystemen quantifizieren zu können, ist bei geernteten Kulturen der N-Flächenbilanzsaldo die maßgebliche Bewertungsgröße. Mit den bereits dargestellten Kenngrößen gesamtpflanzlich akkumulierte Stickstoff-Menge $\left(\mathrm{N}_{B \mathrm{~B}}\right)$, Stickstoff-Harvest-Index $\left(\mathrm{H}_{\mathrm{NBt}}\right.$, beide Kap. 3.1.2) und Anteil Stickstoff aus der Luft (Ndfa, Kap. 3.1.5) wurde mit der in 
Gleichung 14 (Kap. 2.7.2.2) vorgegebenen Rechenvorschrift der vereinfachte N-Flächenbilanzsaldo errechnet.

Die einfachen N-Flächenbilanzsalden beim Anbau von Ackerbohne in Rein- und Gemengesaat mit Hafer lagen im Jahr 1998 mit Werten zwischen +26,3 (4A, $\left.N_{0}\right)$ und $-131,2 \mathrm{~kg} \mathrm{~N} \mathrm{ha}^{-1}\left(4 \mathrm{H}, \mathrm{N}_{3}\right.$; Abb. 18a) signifikant unter den Salden im Jahr $1999(P=$ 0,0065), die zwischen $+47,5\left(4 \mathrm{~A}, \mathrm{~N}_{0}\right)$ und $-110,9 \mathrm{~kg} \mathrm{~N} \mathrm{ha}^{-1}\left(4 \mathrm{H}, \mathrm{N}_{3}\right.$; Abb. 19a) lagen. Dabei verringerten sich in beiden Jahren die einfachen $\mathrm{N}$-Flächenbilanzsalden in der Reihenfolge $4 \mathrm{~A}>3 \mathrm{~A} / 1 \mathrm{H}>1 \mathrm{~A} / 3 \mathrm{H}>\mathrm{H}$ signifikant (Tab. 6).

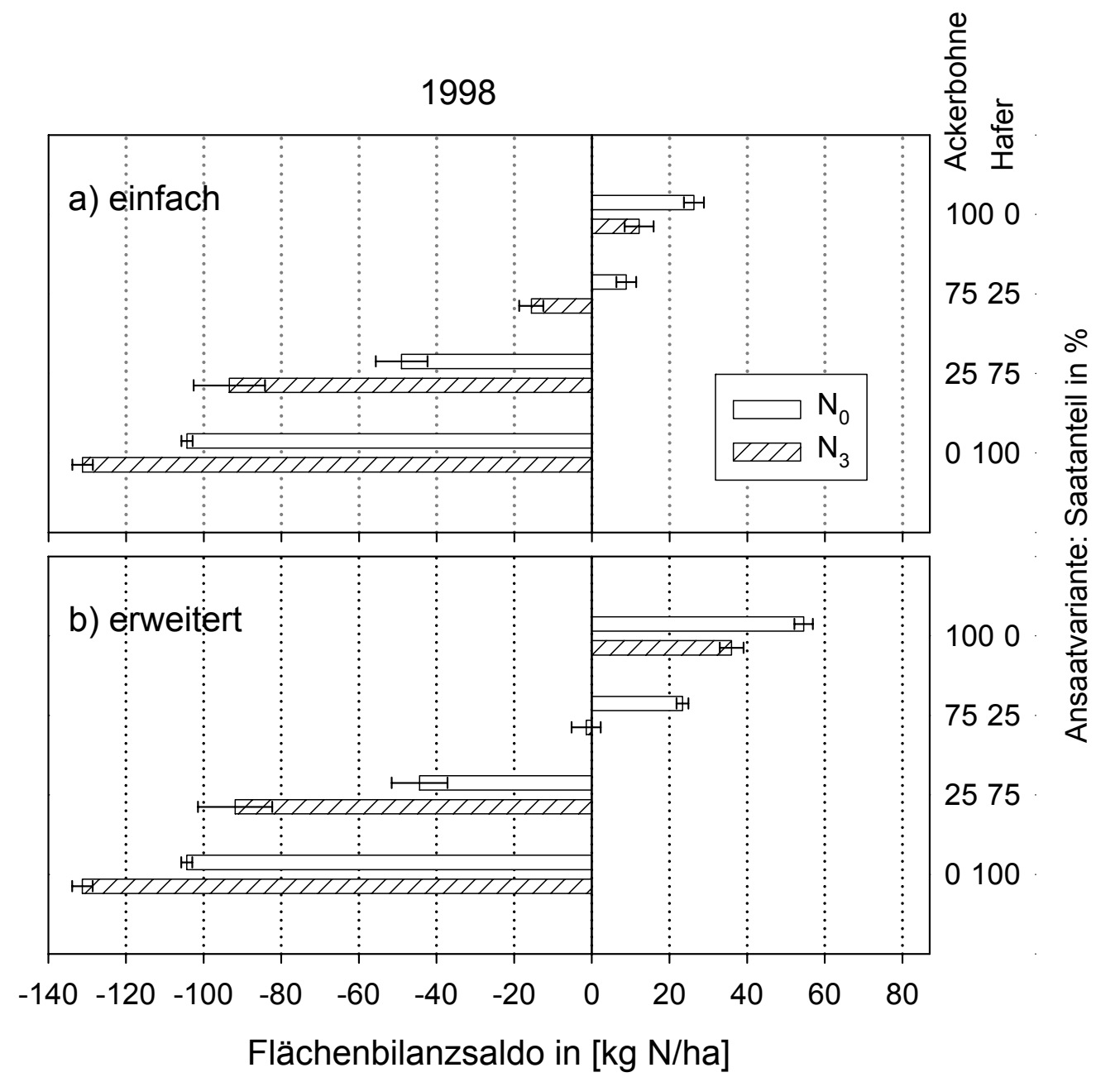

Abb. 18: Einfacher (a) und erweiterter (b) N-Flächenbilanzsaldo ( $\mathrm{kg} \mathrm{N} \mathrm{ha}^{-1}$ ) Mittelwerte \pm Standardfehler) beim Anbau von Ackerbohne und Hafer in Reinund Gemengesaat zur Körnernutzung am 10.08.1998

Im Mittel über die Ansaatvarianten lag der N-Flächenbilanzsaldo nach Vorfrüchten, die langfristig nicht mit mineralischen $\mathrm{N}$-Düngern versorgt wurden $\left(\mathrm{N}_{0}\right)$, in allen geprüften Ansaaten im Jahr 1998 und auch im Jahr 1999 stets über dem Saldo nach langfristig überoptimal versorgten Vorfrüchten $\left(\mathrm{N}_{3}, \mathrm{Tab} .6\right)$. 
Tab. 6: Vergleich der Mittelwerte der einfachen N-Flächenbilanzsalden beim Anbau von Ackerbohne und Hafer in Rein- und Gemengesaat zur Körnernutzung 1998 und 1999

\begin{tabular}{|c|c|c|c|c|c|c|}
\hline & \multicolumn{4}{|c|}{ Ansaatvariante } & \multirow[b]{2}{*}{ Düngung } & \multirow{2}{*}{$\begin{array}{l}\text { Wechsel- } \\
\text { wirkungen }\end{array}$} \\
\hline 1998 & $4 \mathrm{~A}$ & $3 \mathrm{~A} / 1 \mathrm{H}$ & $1 \mathrm{~A} / 3 \mathrm{H}$ & $4 \mathrm{H}$ & & \\
\hline einfacher Bilanzsaldo & $a^{s}$ & $b^{2}$ & $c^{2}$ & $\mathrm{~d}^{* *}$ & * & n.s. \\
\hline 1999 & & & & & & \\
\hline einfacher Bilanzsaldo & $a^{\top}$ & $\mathrm{b}$ & C & $d^{* *}$ & * & n.s. \\
\hline
\end{tabular}

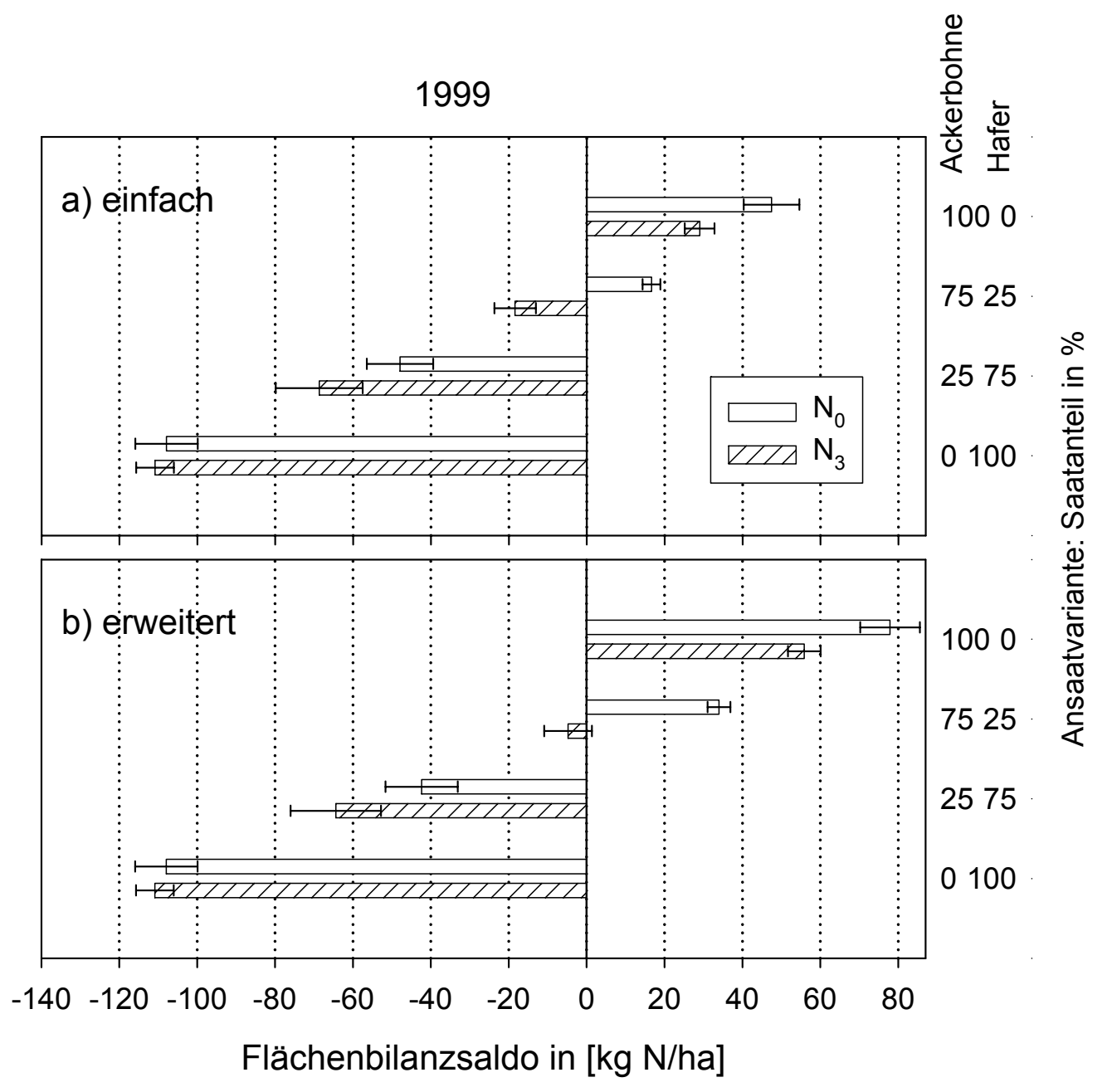

Abb. 19: Einfacher (a) und erweiterter (b) N-Flächenbilanzsaldo ( $\mathrm{kg} \mathrm{N} \mathrm{ha}^{-1}$ ) Mittelwerte \pm Standardfehler) beim Anbau von Ackerbohne und Hafer in Reinund Gemengesaat zur Körnernutzung am 26.07.1999

Die Differenzen im Bilanzsaldo zwischen $\mathrm{N}_{0}$ - und $\mathrm{N}_{3}$-Variante lagen 1998 mit Werten von $14,1(4 \mathrm{~A}), 24,4(3 \mathrm{~A} / 1 \mathrm{H}), 44,4(1 \mathrm{~A} / 3 \mathrm{H})$ und $26,9 \mathrm{~kg} \mathrm{~N}^{-1}(4 \mathrm{H})$ bei den Ansaaten 
mit Hafer über den Differenzen des zweiten Untersuchungsjahres (4A: 18,5; 3A/1H: 35; 1A/3H: 20,8; 4H: 3 kg N ha-1).

Unter Einbeziehung der Werte für die Rhizodeposition beim Anbau von Ackerbohnen (Kap. 2.7.3) wurden mit Gleichung 18 die erweiterten N-Flächenbilanzsalden errechnet (Kap. 2.7.4). Dabei wurden zwei Annahmen getroffen:

1) Ackerbohne gibt im Gemenge mit Hafer den gleichen Anteil Stickstoff über Rhizodeposition an den Boden ab wie Ackerbohne in Reinsaat.

2) Der Anteil Stickstoff aus der Luft (Ndfa) im Stickstoff, der über Rhizodeposition abgegeben wird entspricht dem Anteil Stickstoff aus der Luft (Ndfa) in der gesamtpflanzlichen Biomasse.

Durch Berücksichtigung der fixierten N-Menge des Stickstoffs aus der Rhizodeposition (Tab. A22) erhöhen sich die N-Flächenbilanzsalden auf maximal $+54,5 \mathrm{~kg} \mathrm{~N} \mathrm{ha}^{-1}$ im Jahr 1998 (4A, No ; Abb. 18b) und +70,9 kg N ha-1 im Jahr 1999 (4A, No; Abb. 19b). 


\subsection{Luzerne in Rein- und Gemengesaat mit Knaulgras}

\subsubsection{Gesamtpflanzliche Trockenmasse-Akkumulation}

Im Folgenden werden die Ergebnisse der Trockenmasse-Akkumulation von Luzerne und Knaulgras in Rein- und Gemengesaat getrennt nach Jahren dargestellt. Die Mittelwerte der gesamtpflanzlichen Trockenmasse-Akkumulation, der TrockenmasseErträge der Wurzeln, Stoppeln und des Schnittgutes, der gewichteten Trockenmasse-Harvest-Indizes sowie die Ergebnisse der Varianzanalyse sind für die ersten beide Schnitte in den Anhangstabellen Tab. A24 für das Versuchsjahr 1998 und Tab. A27 für das zweite Untersuchungsjahr 1999 dargestellt. Die Mittelwerte und Mittelwertvergleiche zum jeweils dritten Schnittermin sind den Anhangstabellen Tab. A25 und Tab. A28 zu entnehmen und zusätzlich graphisch in den Abb. 20 (1998) und Abb. 22 (1999) wiedergegeben. Die oberirdischen TM-Ertragsanteile der Luzerne und des Knaulgrases in den Gemengeansaaten sind den Abb. 21 und Abb. 23 zu entnehmen. Die Wurzel-TM wurde einzeln und zusammen mit der Stoppel-TM als Ernterest-TM verrechnet. Die Ertragsdaten des jeweiligen zweiten und dritten Schnittes beider Jahre stellen die kumulierten Erträge bis zum zweiten bzw. dritten Schnittermin dar (vgl. Kap. 2.7.2.2 Gleichungen 7 und 8). Im Jahr 1998 war kein Bestandesabfall messbar. Im Jahr 1999 wurde der am 24.10. messbare Bestandesabfall zu den Ernteresten des dritten Schnittes addiert (Tab. A28).

Neben Luzerne und Knaulgras wurden im Jahr 1998 auch geringe Mengen an Kräutern geerntet. Die Kräuter-Trockenmassen lagen mit Werten von maximal 1,1 dt TM ha $^{-1}$ auf einem sehr niedrigen Niveau und wurden daher in den folgenden Beschreibungen und Darstellungen der gesamtpflanzlichen Trockenmasse-Akkumulation nicht näher berücksichtigt (Tab. A23).

Die Ansaaten der Luzerne und des Knaulgrases in Rein- und Gemengesaat akkumulierten zwischen 26,5 ( $\left.\mathrm{N}_{0}: 4 \mathrm{~K}\right)$ und 34,6 dt TM ha- ${ }^{-1}\left(\mathrm{~N}_{0}\right.$ : $\left.3 \mathrm{~L} / 1 \mathrm{~K}\right)$ zum ersten Schnittermin im Jahr 1998 geringe Mengen gesamtpflanzliche Trockenmasse. Dabei unterschieden sich die TM-Erträge des Schnittgutes und der Erntereste zwischen den Ansaaten im Mittel über die Düngungsstufen signifikant (Tab. A24). Die Wurzel-TM der langjährig nicht mit mineralischen $\mathrm{N}$-Düngemitteln zu den nichtlegumen Vorfrüchten gedüngten Variante $\left(\mathrm{N}_{0}\right)$ lag bei der Luzerne-Reinsaat $(4 \mathrm{~L})$ und dem leguminosenbetonten Gemenge (3L/1K) über, beim Knaulgras in Reinsaat (4K) und dem grasbetonten Gemenge (1L/3K) unter den Werten der $\mathrm{N}_{3}$-Variante (signifikante Wechselwirkung: Ansaatvariante $\times$ Düngung; Tab. A24). Die gesamtpflanzlich gewichteten TM-Harvest-Indizes ( $\left.\mathrm{H}_{\text {TMBtw }}\right)$ nahmen zum Zeitpunkt des ersten Schnittes der Luzerne Reinsaat (4L) mit zunehmendem Knaulgras-Anteil im Mittel über die Düngungsstufen signifikant ab (Tab. A24). Ein Einfluss der Düngungsstufen konnte zum ersten Schnitttermin nicht nachgewiesen werden. 
Bis zum zweiten Schnitttermin unterschied sich die kumulierte, gesamtpflanzliche Trockenmasse, Ernterest-TM und Schnittgut-TM signifikant zwischen den Ansaatvarianten (Tab. A24). Die Schnittgut-TM und Ernterest-TM waren in Abhängigkeit von der Düngung zu den nichtlegumen Vorfrüchten im Mittel der Ansaatvarianten signifikant verschieden. Dabei lagen die Ernterest-Trockenmassen der langjährig nicht mit mineralischen N-Düngemitteln zu den nichtlegumen Vorfrüchten gedüngten Variante $\left(\mathrm{N}_{0}\right)$ über den Ernterest-Trockenmassen der $\mathrm{N}_{3}$-Variante (Tab. A24). Die Ansaatvarianten mit Luzerne der $\mathrm{N}_{0}$-Variante akkumulierten im Mittel signifikant mehr Wurzel-TM im Vergleich zu den Ansaaten der $\mathrm{N}_{3}$-Variante. Dagegen akkumulierte das Knaulgras in Reinsaat (4K) mehr Wurzel-TM in der $\mathrm{N}_{3}$-Variante, so dass eine signifikante Wechselwirkung zwischen Ansaatvarianten und der Düngung bestand (Tab. A24).

Die kumulierten gesamtpflanzlich gewichteten TM-Harvest-Indizes $\left(\mathrm{H}_{\text {TMBtw }}\right)$ nahmen zum Zeitpunkt des zweiten Schnittes ausgehend von Luzerne Reinsaat (4L) mit zunehmendem Knaulgras-Anteil im Mittel über die Düngungsstufen signifikant ab, wobei die Ansaatvarianten der $\mathrm{N}_{3}$-Variante signifikant höhere Werte aufwiesen als die Ansaaten der $\mathrm{N}_{0}$-Variante (Tab. A24).

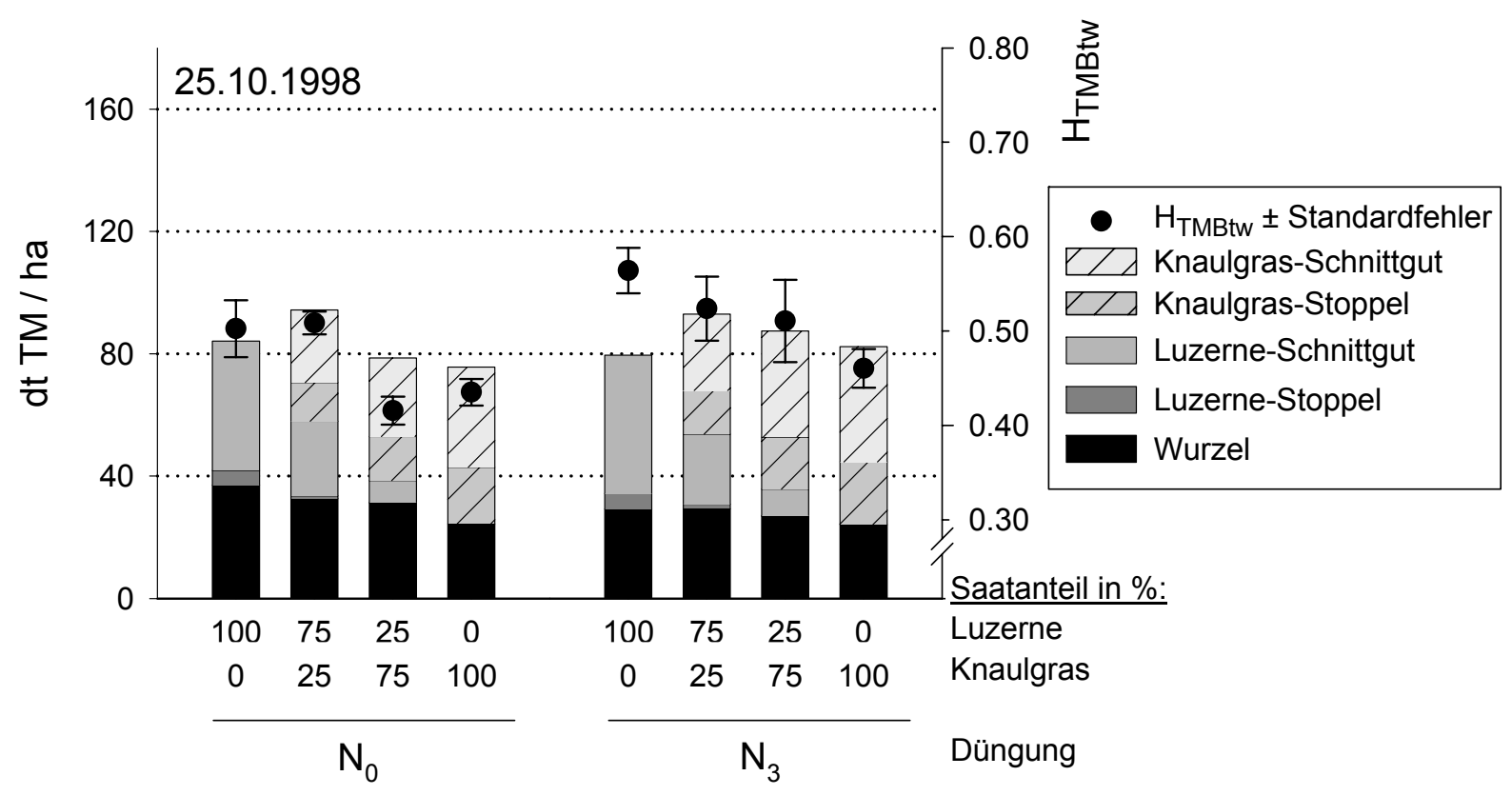

Abb. 20: Kumulierte gesamtpflanzliche Trockenmasse (dt TM ha ${ }^{-1}$; Wurzel, Stoppel, Schnittgut) sowie kumulierte gewichtete Trockenmasse-Harvest-Indizes $\left(\mathrm{H}_{\mathrm{TMBtw}} \pm\right.$ Standardfehler) beim Anbau von Luzerne und Knaulgras in Reinund Gemengesaat zum dritten Schnitttermin am 25.10.1998

Die bis zum Zeitpunkt des dritten Schnittes kumulierten gesamtpflanzlichen Trockenmassen $\left(=\sum 1998\right)$ lagen zwischen 75,6 $\left(4 \mathrm{~K} \mathrm{~N}_{0}\right)$ und 94,3 dt TM ha ${ }^{-1}\left(3 \mathrm{~L} / 1 \mathrm{~K} \mathrm{~N}_{0}\right)$ und damit auch in der Summe des Jahres auf einem vergleichsweise niedrigen 
Niveau (Abb. 20). Im Mittel über die Düngungsstufen konnten Ertragsunterschiede nur zwischen den Ansaatvarianten Knaulgras Reinsaat (4K) und dem leguminosenbetonten Gemenge (3L/1K) statistisch gesichert werden (Tab. A25). Das Gemenge mit $75 \%$ Luzerne (3L/1K) akkumulierte sehr hoch signifikant mehr Schnittgut-TM als Knaulgras in Reinsaat (4K) und im Gemenge mit $25 \%$ Luzerne (1L/3K). Im Mittel akkumulierten die Ansaatvarianten in der langjährig mit mineralischen N-Düngern zu den nichtlegumen Vorfüchten gedüngten Variante $\left(\mathrm{N}_{3}\right)$ signifikant mehr SchnittgutTM als in der $\mathrm{N}_{0}$-Variante (Tab. A25). Die Ernterest-TM unterschieden sich weder in Abhängigkeit von den Ansaatvarianten noch der Düngung. Die höchsten Wurzel-TM wurden von der Luzerne in Reinsaat (4L) akkumuliert. Dabei konnten allerdings nur die Unterschiede im Vergleich zum Knaulgras in Reinsaat (4K) statistisch gesichert werden (Tab. A25). Die gesamtpflanzlich gewichteten TM-Harvest-Indizes $\left(\mathrm{H}_{\text {TMBtw }}\right)$ nahmen zum Zeitpunkt des dritten Schnittes von der Luzerne Reinsaat (4L) mit zunehmendem Knaulgras-Anteil in der Ansaatmischung im Mittel ab, ohne durch die Düngungsstufen signifikant beeinflusst zu sein (Abb. 20; Tab. A25).

In Abb. 21 sind die bis zum Zeitpunkt des dritten Schnitttermins kumulierten oberirdischen Trockenmasse-Ertragsanteile der Luzerne und des Knaulgrases in beiden Gemengesaaten dargestellt. Der Ertragsanteil KnaulgrasSprossmasse (Schnittgut + Stoppeln) betrug in der Ansaatvariante mit $25 \%$ Knaulgras in der Ansaatmischung (3L/1K) $61,6 \%\left(\mathrm{~N}_{3}\right)$ bzw. $58,1 \%$ in der $\mathrm{N}_{0}$-Variante. In der grasbetonten Ansaatmischung (1L/3K) betrugen die KnaulgrasErtragsanteile $85,5 \% \quad\left(\mathrm{~N}_{3}\right)$ bzw. $84,4 \%\left(N_{0}\right)$. Die Unterschiede in den oberirdischen Ertragsanteilen zwischen den Düngungsstufen konnten im Mittel über die Ansaatvarianten im Jahr 1998 statistisch nicht gesichert werden (Abb. 21).

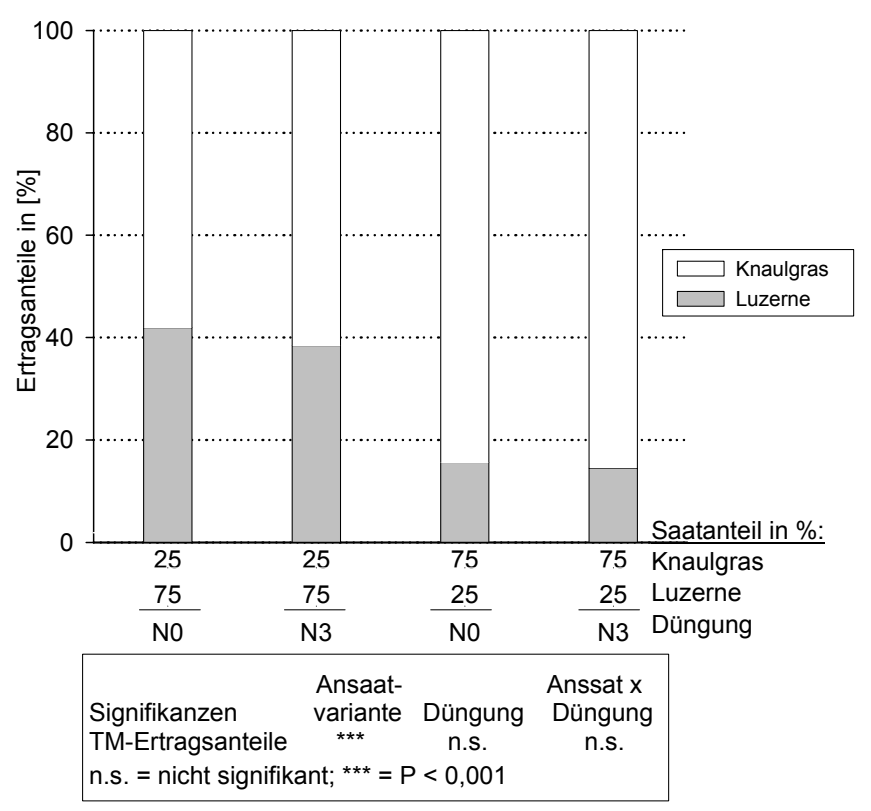

Abb. 21: Ertragsanteile der kumulierten Spross-TM beim Anbau von Luzerne und Knaulgras in Gemengesaat bis zum dritten Schnitt 1998

Wie aus Tab. A26 hervorgeht, lagen die Kräuter-Trockenmassen auch im zweiten Untersuchungsjahr 1999 mit maximal 3,8 dt TM ha ${ }^{-1}$ je Schnitttermin auf einem vergleichsweise niedrigen Niveau und wurden daher in den weiteren Beschreibungen nicht näher berücksichtigt. 
Die Ansaatvarianten mit Luzerne und Knaulgras akkumulierten zwischen 56,4 ( $\mathrm{N}_{0}$ : 4K) und 85,2 dt TM ha ${ }^{-1}$ ( $\mathrm{N}_{0}:$ 4L) zum ersten Schnittermin im Jahr 1999 und bildeten damit deutlich mehr gesamtpflanzliche Trockenmasse als im Vorjahr $\left(P_{\text {Jahr }}=0,0001\right)$. Dabei lagen die akkumulierten Trockenmassen in der langjährig ohne mineralische $\mathrm{N}$-Düngung zu den nichtlegumen Vorfrüchten gedüngten Variante $\left(\mathrm{N}_{0}\right)$ bei der Luzerne in Reinsaat (4L) und im Gemenge mit $25 \%$ Knaulgras (3L/1K) über bzw. bei Knaulgras in Reinsaat (4K) und im Gemenge mit $25 \%$ Luzerne (1L/3K) unter den Trockenmassen der $\mathrm{N}_{3}$-Variante (signifikante Wechselwirkung: Ansaatvariante $\times$ Düngung; Tab. A27). Während im Jahr 1999 die gesamtpflanzlich akkumulierte Trockenmasse mit zunehmendem Knaulgras-Anteil sehr hoch signifikant abnahm (Tab. A27), wies im Jahr 1998 das leguminosenbetonte Gemenge (3L/1K) im Mittel die höchste TM-Akkumulation auf (Wechselwirkung: Ansaatvariante $\times$ Jahr; $P$ $=0,0001$ ). Eine im Mittel signifikante Abnahme der Trockenmasse mit zunehmenden Anteil nichtlegumen Gemengepartner wurde auch für die TM-Erträge des Schnittgutes und der Wurzeln (Ausnahme: Wurzel-TM $1 \mathrm{~L} / 3 \mathrm{~K}<4 \mathrm{~K}$ ) festgestellt (Tab. A27). Dabei traten sowohl bei der Schnittgut-TM als auch bei den Ernterest-TM signifikante Wechselwirkungen zwischen der Ansaatvariante und der Düngung auf (4L: TM$\mathrm{N}_{0}>\mathrm{TM}-\mathrm{N}_{3} ; 4 \mathrm{~K}: \mathrm{TM}-\mathrm{N}_{0}<\mathrm{TM}-\mathrm{N}_{3} ; \mathrm{Tab}$. A27). Die gesamtpflanzlich gewichteten TMHarvest-Indizes $\left(\mathrm{H}_{\mathrm{TMBtw}}\right)$ der Ansaaten von Luzerne und Knaulgras im Gemenge lagen zum Zeitpunkt des ersten Schnittes im Mittel der Düngungsstufen über den TM-Harvest-Indizes der Reinsaaten ( $\mathrm{H}_{\text {TMBtw: }}$ : $\mathrm{L} / 1 \mathrm{~K}>4 \mathrm{~K} ; 1 \mathrm{~L} / 3 \mathrm{~K}>4 \mathrm{~L}$; Tab. A27).

Auch bis zum zweiten Schnitttermin lagen die kumulierten gesamtpflanzlichen Trockenmassen mit Werten zwischen 80,3 ( $\mathrm{N}_{0}$ : 4K) und 148,6 dt TM ha ${ }^{-1}\left(\mathrm{~N}_{0}: 4 \mathrm{~L}\right)$ sehr deutlich über den Mengen, die im Jahr 1998 ermittelt wurden $(P=0,0001)$. Wie bereits zum ersten Schnitt traten auch hier sehr hoch signifikante Wechselwirkungen zwischen Ansaatvariante und Jahr auf $(P=0,0001)$. Die kumulierte gesamtpflanzliche Trockenmasse, die kumulierten Schnittgut-TM, die kumulierten Wurzel-TM und die Ernterest-TM nahmen mit zunehmendem Anteil der Nichtleguminose signifikant ab (Tab. A27). Die gesamtpflanzliche- und die Schnittgut-TM-Akkumulation zeigte bei den Ansaatvarianten eine unterschiedliche Reaktion in Abhängigkeit von den Düngungsstufen (signifikante Wechselwirkung: Ansaatvariante $\times$ Düngung). Im Mittel der Ansaatvarianten akkumulierten die Ansaaten der $\mathrm{N}_{0}$-Variante signifikant mehr Wurzel-TM als in der $\mathrm{N}_{3}$-Variante (Tab. A27).

Die kumulierten, gesamtpflanzlich gewichteten TM-Harvest-Indizes $\left(\mathrm{H}_{\text {TMBtw }}\right)$ zeigten im Mittel der Düngungsstufen signifikante Unterschiede in Abhängigkeit von den Ansaatvarianten und lagen mit Werten zwischen 0,48 $\left(\mathrm{N}_{3}: 4 \mathrm{~K}\right)$ und $0,63\left(\mathrm{~N}_{3}: 3 \mathrm{~L} / 1 \mathrm{~K}\right)$ deutlich über den Indizes zum zweiten Schnitt im Jahr $1998(P=0,0002)$. 


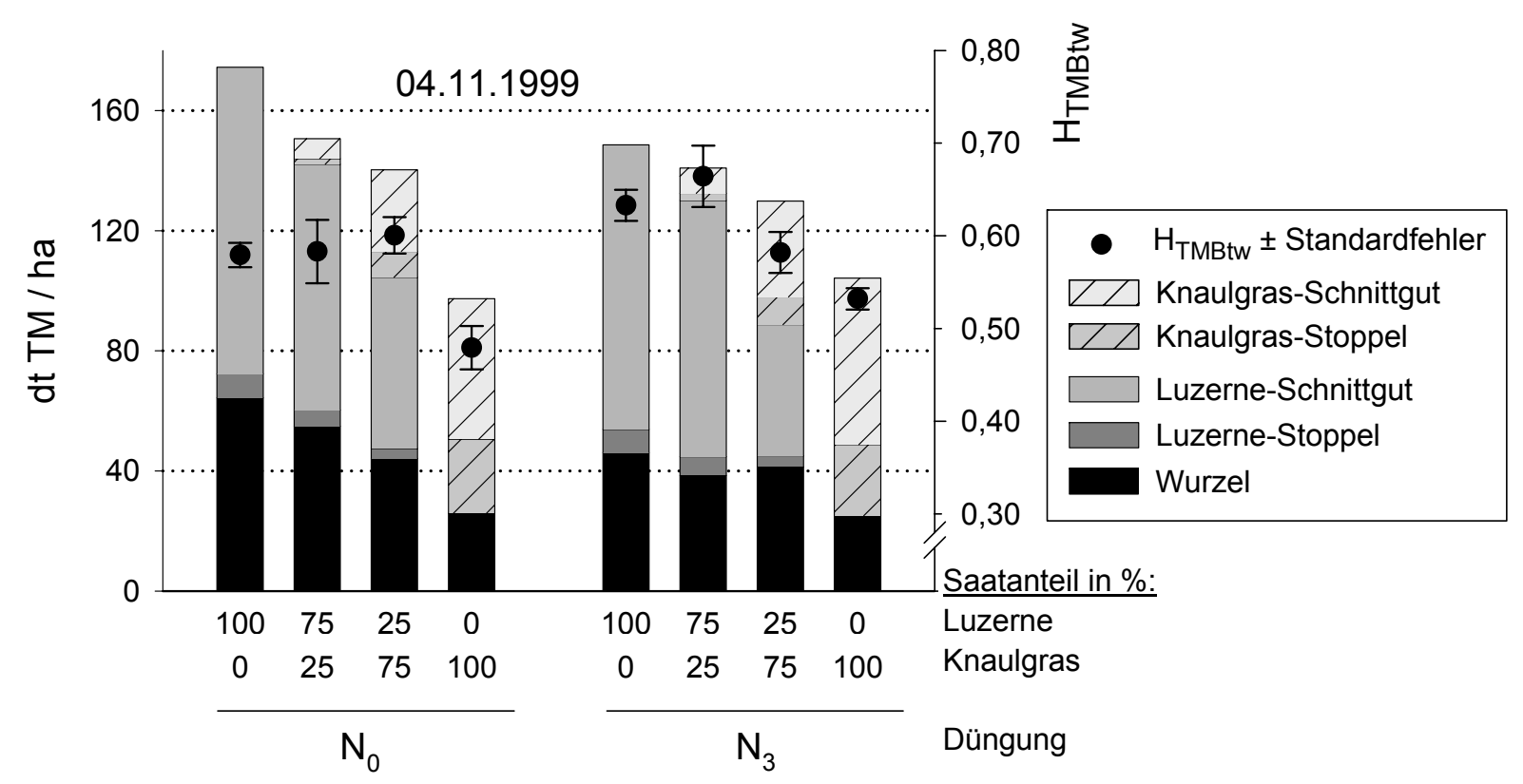

Abb. 22: Kumulierte gesamtpflanzliche Trockenmasse (dt TM ha ${ }^{-1}$; Wurzel, Stoppel, Schnittgut) sowie kumulierte gewichtete Trockenmasse-Harvest-Indizes $\left(\mathrm{H}_{\text {TMBtw }} \pm\right.$ Standardfehler) beim Anbau von Luzerne und Knaulgras in Reinund Gemengesaat zum dritten Schnitttermin am 04.11.1999

Die bis zum Zeitpunkt des dritten Schnittes kumulierten gesamtpflanzlichen Trockenmassen $\left(=\Sigma\right.$ 1999) lagen mit Werten zwischen 97,4 $\left(\mathrm{N}_{0}\right.$ : 4K) und 176,6 dt TM ha $^{-1}$ ( $\mathrm{N}_{0}$ : 4L) ebenfalls sehr deutlich über den Erträgen des Vorjahres $\left(P_{\mathrm{Jahr}}=\right.$ $0,0001)$. Analoges gilt für die kumulierten TM-Erträge der Schnittgut-, Ernterest- und Wurzel-TM sowie für die kumulierten gewichteten gesamtpflanzlichen TM-HarvestIndizes ( $P_{\text {Jahr: }}$ Schnittgut, Ernterest, Wurzel, HTMBtw $\left.=0,0001\right)$. Allerdings akkumulierte im Jahr 1999, im Gegensatz zu 1998, die Luzerne in Reinsaat (4L) die höchsten Trockenmassen (Wechselwirkung: Ansaatvariante $\times$ Jahr; $P=0,0001$ ). Im Mittel über die Düngungsstufen akkumulierte das Knaulgras in Reinsaat (4K) die geringsten gesamtpflanzlichen-, Wurzel- und Schnittgut-TM (Ausnahme: Wurzel-TM 1L/3K = 4K; Tab. A28). Die Ansaatvarianten mit Luzerne (4L, 3L/1K, 1L/3K) akkumulierten in den langjährig nicht mit mineralischen N-Düngemitteln zu der nichtlegumen Vorfrüchten gedüngten Variante $\left(\mathrm{N}_{0}\right)$ signifikant mehr, Knaulgras in Reinsaat (4K) signifikant weniger Trockenmasse im Vergleich zur $\mathrm{N}_{3}$-Variante (Wechselwirkung: Ansaatvariante $\times$ Düngung; Tab. A28, Abb. 22). Auch die Akkumulation an Schnittgut-TM zeigte eine unterschiedliche Reaktion der Ansaaten auf die Düngung (Wechselwirkung: Ansaatvariante $\times$ Düngung). Alle Ansaatvarianten akkumulierten in der langjährig nicht mit mineralischen N-Düngemitteln zu den nichtlegumen Vorfüchten gedüngten Variante $\left(\mathrm{N}_{0}\right)$ signifikant mehr Wurzel- und Ernterest-TM als in der $\mathrm{N}_{3}$-Variante (Tab. A28). Zu allen Ernteterminen im Jahr 1998 und 1999 nahm mit zunehmendem Knaulgras Anteil die Wurzel-TM in der Regel ab und die Stoppel-TM tendenziell zu. 
Der kumulierte gesamtpflanzlich gewichtete TM-Harvest-Index ( $\left.\mathrm{H}_{\text {TMBtw }}\right)$ von Knaulgras in Reinsaat (4K) lag bis zum Zeitpunkt des dritten Schnittes signifikant unter den Indizes von Luzerne in Reinsaat (4L) bzw. dem Gemenge mit $25 \%$ Knaulgras $(3 \mathrm{~L} / 1 \mathrm{~K})$. Im Mittel der Ansaatvarianten wies die $\mathrm{N}_{0}$-Variante niedrigere Indizes auf als die $\mathrm{N}_{3}$-Variante (Tab. A28, Abb. 22).

In Abb. 23 sind die kumulierten oberirdischen Trockenmasse-Ertragsanteile von Luzerne und Knaulgras in beiden Gemengesaaten bis zum Zeitpunkt des dritten Schnitttermins dargestellt. Der Ertragsanteil KnaulgrasSprossmasse betrug in der Ansaatvariante mit $25 \%$ Knaulgras $(3 \mathrm{~L} / 1 \mathrm{~K}) 10,7 \%\left(\mathrm{~N}_{3}\right)$ bzw. 8,9 \% in der $\mathrm{N}_{0}$-Variante. In der grasbetonten Ansaatmischung (1L/3K) betrugen die Knaulgras-Ertragsanteile 46,7\% $\left(\mathrm{N}_{3}\right)$ bzw. 36,9\% $\left(\mathrm{N}_{0}\right)$. Die Unterschiede in den oberirdischen Ertragsanteilen des Knaulgrases zwischen den Düngungsstufen konnten im Mittel über die Ansaatvarianten 1999 statistisch nicht gesichert werden (Abb. 23) lagen aber deutlich unter den Anteilen im Vorjahr $\left(P_{\text {Jahr }}=0,0001\right)$.

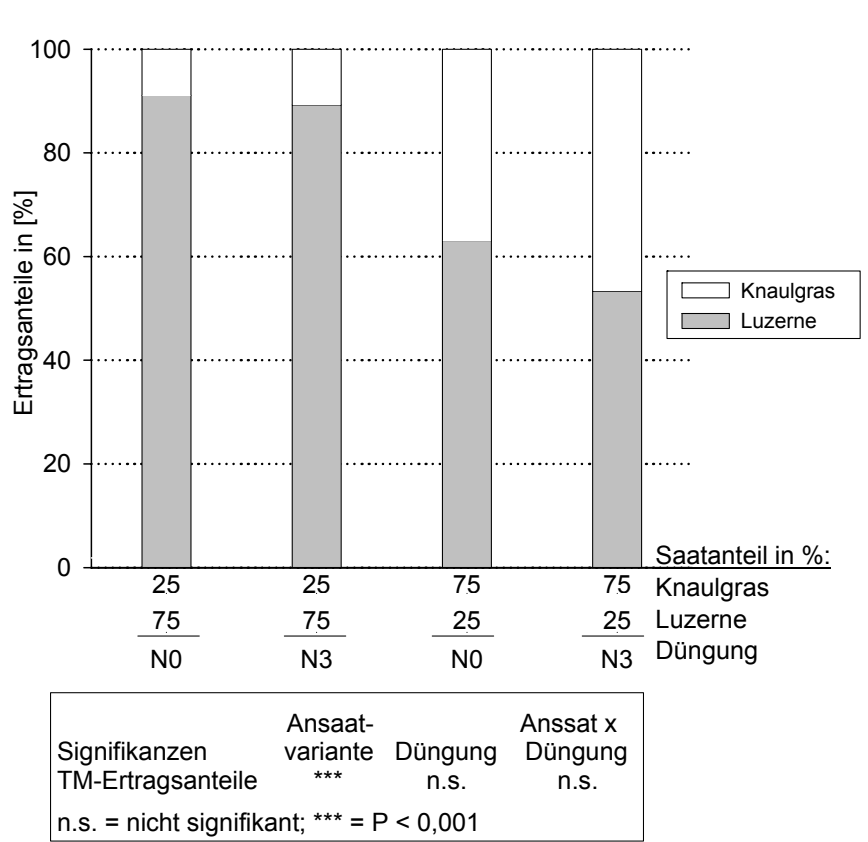

Abb. 23: Ertragsanteile der kumulierten Spross-TM beim Anbau von Luzerne und Knaulgras in Gemengesaat bis zum dritten Schnitt 1999

\subsubsection{Stickstofferträge und Stickstoff-Harvest-Index}

Im Folgenden werden die gesamtpflanzlich akkumulierten Stickstoff-Mengen, Stickstoff-Erträge der Pflanzenbestandteile sowie die Stickstoff-Harvest-Indizes getrennt nach Jahren dargestellt. In den Abb. 24 und Abb. 26 sind die Stickstofferträge und die gewichteten Harvest-Indizes graphisch abgebildet. In den Anhangstabellen Tab. A29 und Tab. A30 sind zusätzlich die Mittelwerte und Mittelwertvergleiche zu den drei Ernteterminen abgetragen. Schließlich sind in den Abb. 25 und

Abb. 27 die Luzerne- und Knaulgras-Stickstoff-Ertragsanteile der oberirdischen Biomasse der Gemengeansaaten wiedergegeben. 
Im ersten Untersuchungsjahr wurden im Maximum 3,9 $\mathrm{kg} \mathrm{N} \mathrm{ha}^{-1}$ (Tab. A23), im Jahr $19998,9 \mathrm{~kg} \mathrm{~N} \mathrm{ha}^{-1}$ (Tab. A26) im Spross von Kräutern akkumuliert. Auf diese Stickstoff-Mengen werden in den weiteren Beschreibungen und Darstellungen nicht näher eingegangen, da nur vergleichsweise geringe N-Mengen in der Kräutersprossmasse akkumuliert wurden.

Im Jahr 1998 akkumulierten die Ansaaten der Luzerne und des Knaulgrases in Reinund Gemengesaat zum ersten Schnitt gesamtpflanzlich ( $\mathrm{N}_{\mathrm{Bt}}$ ) zwischen 40,7 ( $\mathrm{N}_{0}$ : 4K) und 93,6 kg N ha-1 ( $\mathrm{N}_{3}: 3 \mathrm{~L} / 1 \mathrm{~K}$; Abb. 24 und Tab. A29). Im Mittel der Düngungsstufen lagen die gesamtpflanzlich akkumulierten N-Mengen des Knaulgrases in Reinsaat (4K) signifikant unter den akkumulierten N-Mengen der Ansaaten mit Luzerne (4L, $3 \mathrm{~L} / 1 \mathrm{~K}, 1 \mathrm{~L} / 3 \mathrm{~K}$ ). Während Luzerne in Reinsaat (4L) und im Gemenge mit $25 \%$ Knaulgras $(3 \mathrm{~L} / 1 \mathrm{~K})$ in der langjährig nicht mit mineralischen N-Düngern zu den nichtlegumen Vorfüchten gedüngten Variante $\left(\mathrm{N}_{0}\right)$ mehr Stickstoff gesamtpflanzlich akkumulierte als in der $\mathrm{N}_{3}$-Variante, verhielten sich Knaulgras in Reinsaat (4K) und im Gemenge mit $25 \%$ Luzerne (1L/3K) entgegengesetzt (signifikante Wechselwirkung: Ansaatvariante $\times$ Düngung; Tab. A29). Die Schnittgut-N-Mengen unterschieden sich zwischen den Ansaaten analog der gesamtpflanzlichen N-Mengen signifikant. Dagegen konnten für die Ernterest-N-Mengen (Wurzel-N + Stoppel-N) keine gesicherten Unterschiede zwischen den Ansaaten festgestellt werden. Allerdings reagierten die Ansaatvarianten in gleicher Weise wie die gesamtpflanzlich akkumulierten N-Mengen differenziert auf die Düngung (signifikante Wechselwirkung: Ansaatvariante $\times$ Düngung, Tab. A29). Luzerne in Reinsaat (4L) akkumulierte im Mittel über die Düngungsstufen signifikant mehr Stickstoff in den Wurzeln als Knaulgras in Reinsaat $(4 \mathrm{~K})$ und dem grasbetonten Gemenge (1L/3K). Dabei zeigte sich eine deutliche Wechselwirkung zwischen der Ansaatvariante und der Düngung (Tab. A29). Im Mittel der Düngungsstufen lagen die gewichteten gesamtpflanzlichen NHarvest-Indizes der Luzerne in Reinsaat (4L) und im Gemenge mit $25 \%$ Knaulgras (3L/1K) signifikant über den Werten der übrigen Ansaatvarianten (1L/3K und 4K).

Die bis zum zweiten Schnitttermin kumulierten gesamtpflanzlichen Stickstoff-Mengen nahmen mit Werten zwischen 194,5 ( $\left.\mathrm{N}_{0}: 4 \mathrm{~L}\right)$ und $80,1 \mathrm{~kg} \mathrm{~N}^{-1}\left(\mathrm{~N}_{0}: 4 \mathrm{~K}\right)$ von der Luzerne in Reinsaat (4L) mit zunehmendem nichtlegumen Gemengepartner im Bestand signifikant ab (Abb. 24 und Tab. A29). Dabei akkumulierte die Luzerne in Reinsaat (4L) und im leguminosenbetonten Gemenge (3L/1K) in der $\mathrm{N}_{0}$-Variante mehr Stickstoff als in der $\mathrm{N}_{3}$-Variante. Das Knaulgras in Reinsaat (4K) und das grasbetonte Gemenge (1L/3K) verhielten sich entgegengesetzt (signifikante Wechselwirkung: Ansaatvariante $\times$ Düngung; Tab. A29). Die N-Erträge im Schnittgut, in den Ernteresten und den Wurzeln wiesen zum zweiten Schnitttermin im Mittel über die Düngungsstufen signifikant abnehmende Stickstoff-Mengen mit zunehmendem Grasanteil auf (Tab. A29). Die N-Erträge wurden durch die Düngungsstufen signifikant beeinflusst. Während die Ansaaten mit Luzerne in der $\mathrm{N}_{0}$-Variante signifikant mehr Ernterest- bzw. Wurzel-N-Mengen akkumulierten als in der $\mathrm{N}_{3}$-Variante, akku- 
mulierte das Knaulgras in Reinsaat (4K) mehr Ernterest- bzw. Wurzel-Stickstoff in der $\mathrm{N}_{3}$-Variante (signifikante Wechselwirkung: Ansaatvariante $\times$ Düngung; Tab. A29). Weniger deutlich waren die Unterschiede im Schnittgut-N-Ertrag in Abhängigkeit der Düngungsstufen. So wurde nur in der Ansaatvariante mit $75 \%$ Luzerne $(3 \mathrm{~L} / 1 \mathrm{~K})$ in der $\mathrm{N}_{0}$-Variante mehr Stickstoff im Vergleich zur $\mathrm{N}_{3}$-Variante akkumuliert (signifikante Wechselwirkung: Ansaatvariante $\times$ Düngung; Tab. A29). Im Mittel der Düngungsstufen konnten bei den gewichteten gesamtpflanzlichen $\mathrm{N}$-Harvest-Indizes keine gesicherten Unterschiede in Abhängigkeit von der Ansaatvariante festgestellt werden. Allerdings lagen im Mittel der Ansaatvarianten die N-Harvest-Indizes der Bestände aus der $\mathrm{N}_{3}$-Variante signifikant über den $\mathrm{N}$-Harvest-Indizes der $\mathrm{N}_{0}$-Variante (Abb. 24 und Tab. A29).

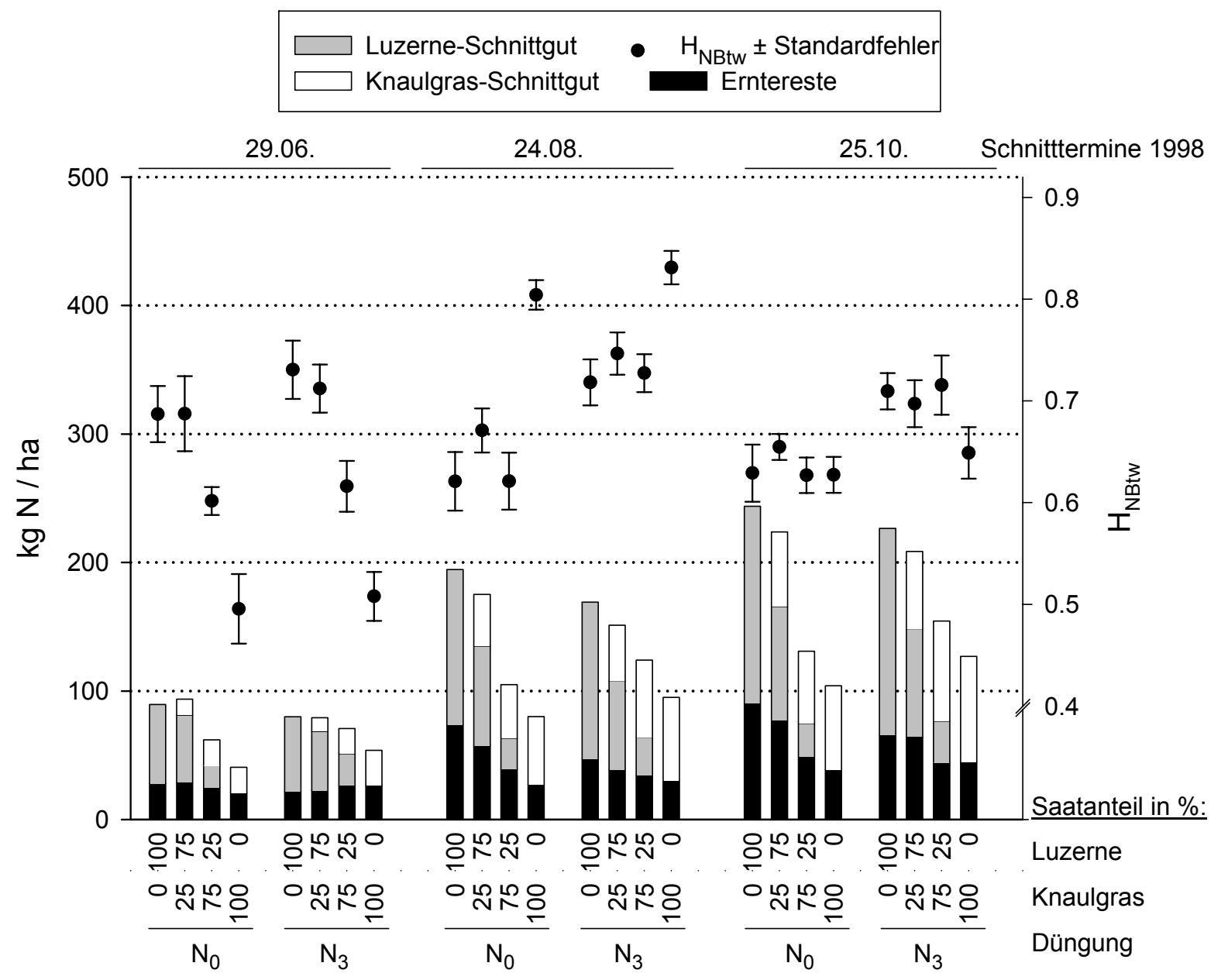

Abb. 24: Gesamtpflanzliche Stickstoff-Akkumulation (kg N ha ${ }^{-1}$, Wurzel, Stoppeln, Schnittgut) sowie gewichtete Stickstoff-Harvest-Indizes $\left(\mathrm{H}_{\mathrm{NBtw}} \pm\right.$ Standardfehler) beim Anbau von Luzerne und Knaulgras in Rein- und Gemengesaat zu drei Schnittterminen im Jahr 1998 (kumulierte Werte zum zweiten und dritten Schnitttermin) 
Die bis zum dritten Schnittermin kumulierten N-Mengen entsprechen der Jahressumme an Stickstoff, die von den Pflanzenbeständen akkumuliert wurden. Die bis zum dritten Schnitttermin kumulierten gesamtpflanzlichen Stickstoff-Mengen lagen mit Werten zwischen 243,8 $\left(\mathrm{N}_{0}: 4 \mathrm{~L}\right)$ und $104,1 \mathrm{~kg} \mathrm{~N}^{-1}\left(\mathrm{~N}_{0}: 4 \mathrm{~K}\right)$ auf einem vergleichsweise niedrigen Niveau (Abb. 24). Die im Schnittgut und gesamtpflanzlich akkumulierten N-Mengen nahmen mit zunehmendem Anteil an Knaulgras signifikant ab (Tab. A29). Dabei akkumulierte die Luzerne in Reinsaat (4L) und im leguminosenbetonten Gemenge (3L/1K) gesamtpflanzlich mehr Stickstoff in der $\mathrm{N}_{0}$-Variante als in der $\mathrm{N}_{3}$-Variante. Das Knaulgras in Reinsaat (4K) und im Gemenge mit $25 \%$ Luzerne (1L/3K) verhielt sich entgegengesetzt (signifikante Wechselwirkung: Ansaatvariante $\times$ Düngung; Tab. A29). Im Mittel der Ansaaten akkumulierten die Pflanzen signifikant mehr Stickstoff im Schnittgut der $\mathrm{N}_{3}$-Variante im Vergleich zur $\mathrm{N}_{0}$-Variante. Die Luzerne in Reinsaat (4L) und im Gemenge mit $25 \%$ Knaulgras (3L/1K) akkumulierte im Mittel der Düngungsstufen signifikant mehr Wurzel-N bzW. mehr Ernterest-N im Vergleich zum Knaulgras in Reinsaat (4K) und im Gemenge mit $25 \%$ Luzerne (1L/3K; Tab. A29). Im Vergleich der Düngungsstufen akkumulierten die Ansaatvarianten mit Luzerne mehr, Knaulgras in Reinsaat (4K) dagegen weniger Wurzel-N in der langjährig nicht mit mineralischen N-Düngern zu den nichtlegumen Vorfrüchten gedüngten Variante $\left(\mathrm{N}_{0}\right.$; signifikante Wechselwirkung: Ansaatvariante $\times$ Düngung; Tab. A29). Auch zum dritten Schnittermin konnten im Mittel der Düngungsstufen bei den kumulierten, gewichteten gesamtpflanzlichen N-HarvestIndizes keine gesicherten Unterschiede in Abhängigkeit von der Bestandeszusammensetzung festgestellt werden. Allerdings lagen im Mittel der Ansaatvarianten die $\mathrm{N}$-Harvest-Indizes der Bestände aus der $\mathrm{N}_{3}$-Variante signifikant über den $\mathrm{N}$-HarvestIndizes der $\mathrm{N}_{0}$-Variante (Abb. 24 und Tab. A29). Während die Wurzel-N-Mengen mit zunehmendem Anteil Knaulgras abnahmen, lagen die Stoppel-N-Mengen bei den Ansaaten mit Knaulgras tendenziell über den Werten der Luzerne Reinsaat (4L; Tab. A29).

In Abb. 25 sind die kumulierten Stickstoff-Ertragsanteile der Luzerne und des Knaulgrases in beiden Gemengesaaten als kumulierte Werte bis zum letzten Schnitttermin abgebildet. Der N-Ertragsanteil der Knaulgras-Sprossmasse am gesamten Spross-N-Ertrag im Gemenge betrug in der leguminosenbetonten Ansaatvariante (3L/1K) 48,5\% $\left(\mathrm{N}_{3}\right)$ bzw. 46,6 \% $\left(\mathrm{N}_{0}\right)$. In der grasbetonten Ansaatmischung (1L/3K) betrugen die Knaulgras-Ertragsanteile 75,9\% ( $\left.\mathrm{N}_{3}\right)$ bzw. 73,7\% $\left(\mathrm{N}_{0}\right)$. Im Mittel der Ansaatvarianten konnten keine Unterschiede in den $\mathrm{N}$-Ertragsanteilen zwischen den Düngungsstufen festgestellt werden (Abb. 25). 
Abb. 25: Stickstoff-Ertragsanteile der kumulierten Sprosserträge von Luzerne und Knaulgras in Gemengesaat bis zum dritten Schnitt 1998

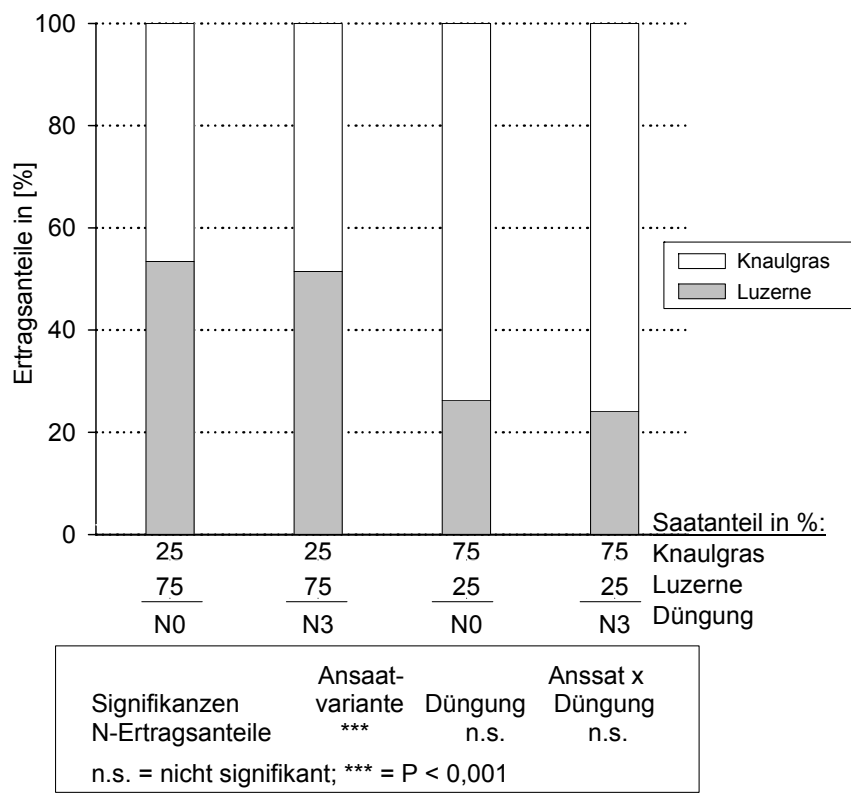

Die zum ersten Schnitt des zweiten Untersuchungsjahres gesamtpflanzlich akkumulierten Stickstoff-Mengen lagen zwischen 229,1 ( $\left.\mathrm{N}_{0}: 4 \mathrm{~L}\right)$ und 79,6 $\mathrm{kg} \mathrm{N}^{-1}\left(\mathrm{~N}_{0}: 4 \mathrm{~K}\right)$ und übertrafen damit die N-Mengen des ersten Schnittes des Vorjahres hoch signifikant $\left(P_{\mathrm{Jahr}}=0,0001\right)$. Während die Unterschiede der gesamtpflanzlich und im Schnittgut akkumulierten N-Mengen zwischen der Luzerne in Reinsaat (4L) und im Gemenge mit $25 \%$ Knaulgras $(3 \mathrm{~L} / 1 \mathrm{~K})$ statistisch nicht gesichert werden konnten, nahmen die N-Mengen mit zunehmenden Knaulgras-Anteil signifikant ab (Tab. A30). Hierbei akkumulierten die Ansaatvarianten mit Luzerne in der $\mathrm{N}_{0}$-Variante mehr Stickstoff als in der $\mathrm{N}_{3}$-Variante. Das Knaulgras in Reinsaat $(4 \mathrm{~K})$ verhielt sich entgegengesetzt (signifikante Wechselwirkungen: Ansaatvariante $\times$ Düngung bei $N_{B t}$ und Schnittgut-N; Tab. A30). Im Mittel der Düngungsstufen akkumulierten die Ansaaten mit Luzerne in Reinsaat (4L) und im Gemenge mit $25 \%$ Knaulgras (3L/1K) signifikant mehr Ernterest- bzw. Wurzel-N als die Ansaaten mit Knaulgras in Reinsaat (4K) und im Gemenge mit $25 \%$ Luzerne (1L/3K; Tab. A30). Die gewichteten gesamtpflanzlichen Stickstoff-Harvest-Indizes unterschieden sich weder in Abhängigkeit der Ansaatvarianten noch in Abhängigkeit der Düngungsstufen (Tab. A30).

Die bis zum zweiten Schnitttermin kumulierten gesamtpflanzlichen Stickstoff-Mengen lagen mit Werten zwischen 377,0 ( $\left.\mathrm{N}_{0}: 4 \mathrm{~L}\right)$ und 103,7 $\mathrm{kg} \mathrm{N} \mathrm{ha}^{-1}\left(\mathrm{~N}_{0}: 4 \mathrm{~K}\right)$ hoch signifikant über den $\mathrm{N}$-Mengen des Vorjahres $(P=0,0001)$. Dabei steigerte die Luzerne in Reinsaat (4L) im Mittel den N-Ertrag im Vergleich zum ersten Schnitt im Jahr 1998 um 163,8 kg N ha ${ }^{-1}$, während beim Knaulgras in Reinsaat (4K) sich der gesamtpflanzliche N-Ertrag nur um $25,0 \mathrm{~kg} \mathrm{~N}^{-1}$ erhöhte (signifikante Wechselwirkung: Ansaatvariante $\times$ Jahr). Die gesamtpflanzlich akkumulierte N-Menge und die NMenge in den einzelnen Pflanzenbestandteilen nahm von Luzerne in Reinsaat (4L) 
mit zunehmenden nichtlegumen Gemengepartner im Bestand signifikant ab (Abb. 26, Tab. A30). Während bei den gesamtpflanzlichen und im Schnittgut akkumulierten $\mathrm{N}$ Mengen signifikante Wechselwirkungen zwischen Ansaatvariante und Düngung auftraten, lagen die kumulierten Ernterest-N-Erträge in der $\mathrm{N}_{0}$-Variante im Mittel der Ansaaten über den Erträgen der Ansaaten aus der $\mathrm{N}_{3}$-Variante (Tab. A30). Im Mittel der Ansaatvarianten lagen die Wurzel-N-Mengen der $\mathrm{N}_{0}$-Variante signifikant über den $\mathrm{N}$-Erträgen der $\mathrm{N}_{3}$-Variante. Dabei nahmen die Differenzen gleicher Ansaaten zwischen den Düngungsstufen von Luzerne in Reinsaat (4L; $37,3 \mathrm{~kg}$ ) mit zunehmendem Knaulgras Anteil ab (3L/1K: 22,3 kg; 1L/3K: 15,2 kg; 4K: 0,5 kg; Wechselwirkung: Ansaatvariante $\times$ Düngung; Tab. A30). Die gewichteten kumulierten gesamtpflanzlichen Stickstoff-Harvest-Indizes unterschieden sich auch zum zweiten Schnitttermin weder in Abhängigkeit der Ansaatvariante noch in Abhängigkeit der Düngungsstufe (Tab. A30) signifikant und lagen auf dem gleichen Niveau des Vorjahres.

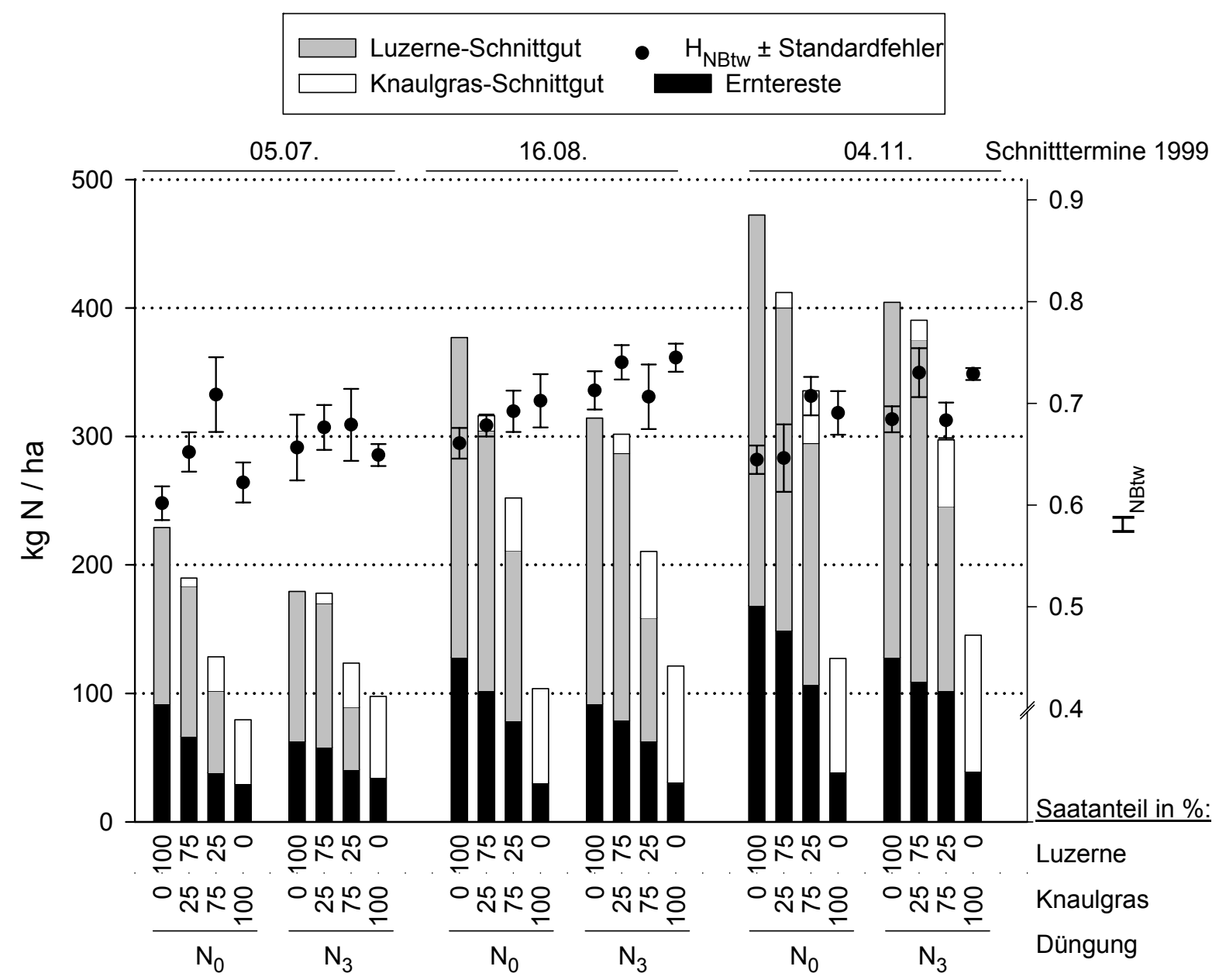

Abb. 26: Gesamtpflanzliche Stickstoff-Akkumulation ( $\mathrm{kg} \mathrm{N} \mathrm{ha}^{-1}$; Wurzel, Stoppeln, Schnittgut) sowie gewichtete Stickstoff-Harvest-Indizes $\left(\mathrm{H}_{\mathrm{NBtw}} \pm\right.$ Standardfehler) beim Anbau von Luzerne und Knaulgras in Rein- und Gemengesaat zu drei Schnittterminen im Jahr 1999 
Die bis zum dritten Schnitttermin 1999 kumulierten N-Mengen übertrafen mit Werten zwischen 472,4 ( $\left.\mathrm{N}_{0}: 4 \mathrm{~L}\right)$ und 127,2 $\mathrm{kg} \mathrm{N}^{-1}\left(\mathrm{~N}_{0}: 4 \mathrm{~K}\right)$ die Erträge des Vorjahres deutlich $\left(P_{\text {Jahr }}=0,0001\right)$. Dabei sanken die N-Ertragszuwächse der Ansaaten im Vergleich der Jahre mit zunehmendem Knaulgras-Anteil im Bestand (sehr hoch signifikante Wechselwirkung: Ansaatvariante $\times$ Jahr). Die gesamtpflanzlichen und im Schnittgut akkumulierten Stickstoff-Mengen nahmen im Mittel über die Düngungsstufen signifikant mit Zunahme des Ertragsanteiles Knaulgrases ab. Die Ansaaten mit Luzerne akkumulierten in der $\mathrm{N}_{0}$-Variante gesamtpflanzlich signifikant mehr Stickstoff im Vergleich zu der Luzerne der $\mathrm{N}_{3}$-Variante. Das Knaulgras in Reinsaat (4K) verhielt sich entgegengesetzt (signifikante Wechselwirkung: Ansaatvariante $\times$ Düngung; Tab. A30). Die Schnittgut-N-Mengen sanken entsprechend der Abstufung bei den gesamtpflanzlichen N-Mengen. Auch hier trat eine Wechselwirkung zwischen der Ansaatvariante und der Düngung auf (Tab. A30). Die signifikant geringsten Ernterest- und Wurzel-N-Mengen wies Knaulgras in Reinsaat (4K) auf. Dabei lagen die N-Mengen im Mittel der Ansaatvarianten in der $\mathrm{N}_{0}$-Variante signifikant über den Erträgen aus der $\mathrm{N}_{3}$-Variante (Tab. A30).

Im Bestandesabfall wurden mit Werten zwischen 7,0 ( $\left.\mathrm{N}_{0}: 4 \mathrm{~L}\right)$ und $0 \mathrm{~kg} \mathrm{~N} \mathrm{ha}^{-1}(4 \mathrm{~K})$ nur geringe N-Mengen akkumuliert. Auch zum dritten Schnitttermin konnten im Mittel der Düngungsstufen bei den gewichteten gesamtpflanzlichen $\mathrm{N}$-Harvest-Indizes keine gesicherten Unterschiede in Abhängigkeit der Ansaatvariante festgestellt werden. Im Mittel lagen die kumulierten N-Harvest-Indizes signifikant über den Indizes des Vorjahres $\left(P_{\mathrm{Jahr}}=0,0259\right)$. Im Mittel der Ansaatvarianten lagen die N-Harvest-Indizes der Bestände aus der $\mathrm{N}_{3}$-Variante signifikant über den Indizes der $\mathrm{N}_{0}$ Variante (Abb. 26 und Tab. A30).

In Abb. 27 sind die kumulierten Stickstoff-Ertragsanteile der Luzerne und des Knaulgrases in beiden Gemengesaaten zum letzten Schnitttermin abgebildet. Der NErtragsanteil der Knaulgras-Sprossmasse am gesamtpflanzlich akkumulierten Stickstoff im Spross betrug in der leguminosenbetonten Ansaatvariante (3L/1K) 8,4 \% $\left(\mathrm{N}_{3}\right)$ bzw. 7,3 \% in der $\mathrm{N}_{0}$-Variante. In der grasbetonten Ansaatmischung (1L/3K) betrugen die Knaulgras-Ertragsanteile an Stickstoff 35,3\% $\left(\mathrm{N}_{3}\right)$ bzw. 27,8 \% $\left(\mathrm{N}_{0}\right)$. Im Mittel der Ansaatvarianten konnten somit keine Unterschiede in den N-Ertragsanteilen zwischen den Düngungsstufen festgestellt werden (Abb. 27). Die Ansaatvariante hatte jedoch einen signifikanten Einfluss auf den Anteil des Knaulgrases an der insgesamt im Gemenge akkumulierten Spross-N-Menge. 
Abb. 27: Stickstoff-Ertragsanteile der kumulierten Sprosserträge von Luzerne und Knaulgras in Gemengesaat bis zum dritten Schnitt 1999

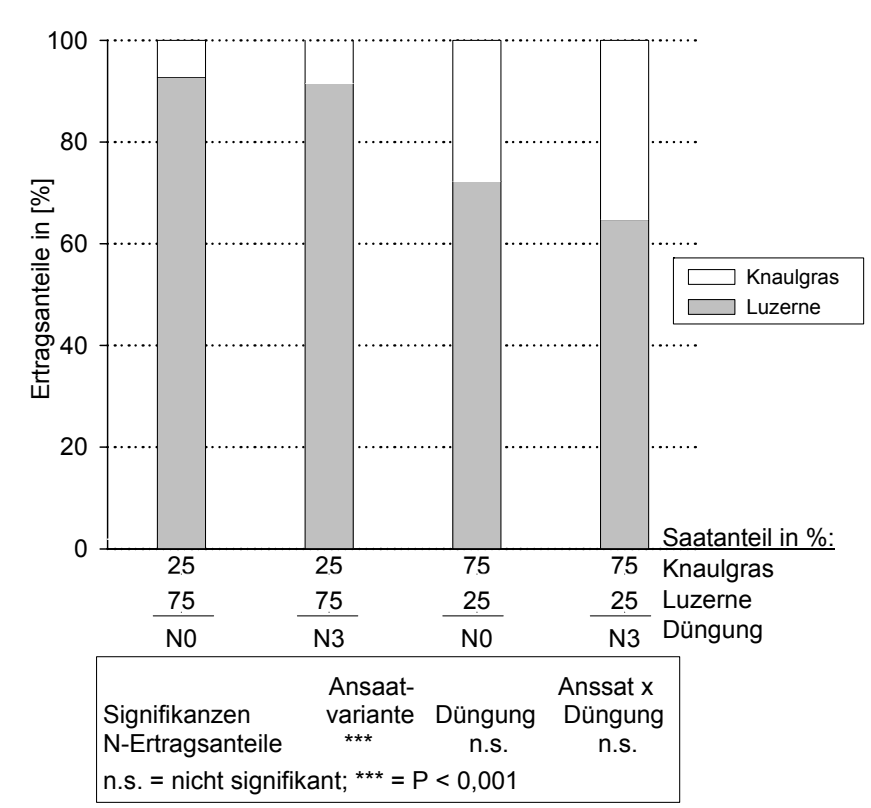

\subsubsection{N-Isotopenzusammensetzung in der gesamtpflanzlichen Biomasse}

In Abb. 28 sind die arithmetischen Mittelwerte $(n=6)$ der gewichteten gesamtpflanzlichen $\delta^{15} \mathrm{~N}$-Werte der Luzerne und des Knaulgrases in Rein- und Gemengesaat zu drei Schnitterminen für das Jahr 1998 dargestellt. Die $\delta^{15} \mathrm{~N}$-Werte des zweiten- und dritten Schnittes sind als arithmetische Mittelwerte der kumulierten gewichteten $\delta^{15} \mathrm{~N}$ Werte dargestellt. Die $\delta^{15} \mathrm{~N}$-Werte in der Sprossmasse von Luzerne und Knaulgras in Rein- und Gemengesaat sind der Tab. A31 zu entnehmen.

Die $\delta^{15} \mathrm{~N}$-Werte des Knaulgrases in Reinsaat (4K) lagen im Mittel der Düngungsstufen mit Werten von 5,97\%o (erster Schnitt), 4,86\%o (zweiter Schnitt) und 4,42\%o (dritter Schnitt) zu allen drei Schnittterminen signifikant über den Werten von Luzerne in Reinsaat (4L; erster Schnitt: 2,73\%o; zweiter Schnitt: 1,94\%; dritter Schnitt: $1,85 \%$; Tab. A32a). Hierbei ist im Mittel der Düngungsstufen eine tendenzielle Abnahme der $\delta^{15} \mathrm{~N}$-Werte des Knaulgrases in Reinsaat (4K) im Jahresverlauf erkennbar. Während sich die $\delta^{15} \mathrm{~N}$-Werte der Luzerne in Reinsaat (4L) und im Gemenge mit $25 \%$ Knaulgras (3L/1K) statistisch nicht gesichert unterschieden, stiegen die gesamtpflanzlichen $\delta^{15} \mathrm{~N}$-Werte mit zunehmendem Knaulgras-Anteil signifikant an (Ausnahme: erster Schnitt: 3L/1K und 1L/3K; Tab. A32a). Die Unterschiede im $\delta^{15} \mathrm{~N}$ Wert in der Biomasse zwischen den Düngungsvarianten $\mathrm{N}_{0}$ und $\mathrm{N}_{3}$ gleicher Ansaaten waren zum zweiten und dritten Schnitt bei Knaulgras in Reinsaat (4K) größer als bei den Ansaaten mit Luzerne (signifikante Wechselwirkung: Ansaatvariante $\times$ Düngung; Tab. A32a). 


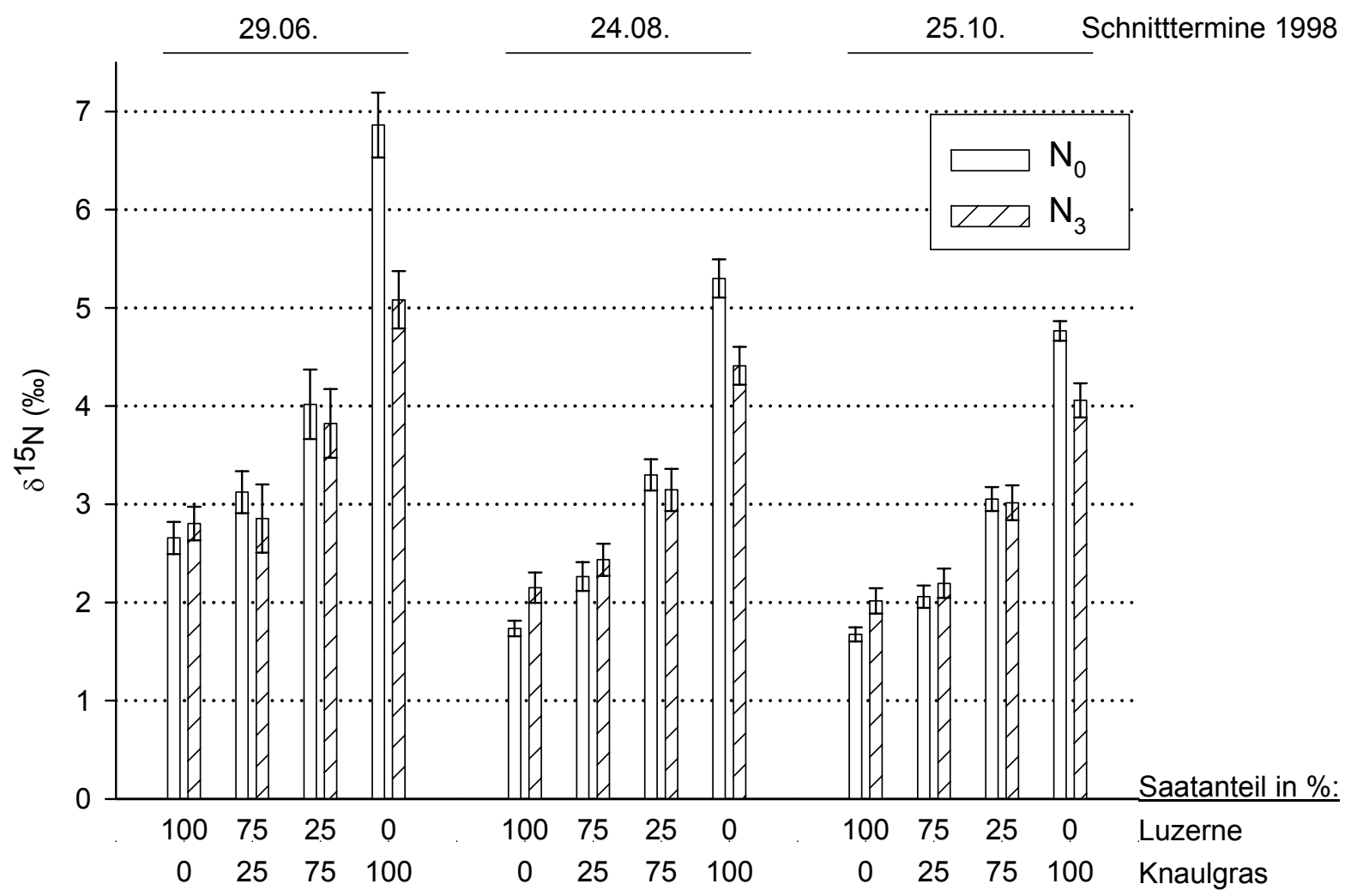

Abb. 28: Gewichtete- (erster Schnitt) bzw. kumuliert gewichtete $\delta^{15} \mathrm{~N}$-Werte (zweiter und dritter Schnitt) (arithmetische Mittelwerte \pm Standardfehler in \%o) in der gesamtpflanzlichen Biomasse (Erntereste + Schnittgut) beim Anbau von Luzerne und Knaulgras in Rein- und Gemengesaat im Jahr 1998

Die Differenzen in den $\delta^{15} \mathrm{~N}$-Werten zwischen Luzerne und Knaulgras in Reinsaat (4L und 4K) lagen im Jahr 1998 im Minimum bei 2,04 \%o (dritter Schnitt, $\mathrm{N}_{3}$ ) und maximal bei 4,2\%o (erster Schnitt, $\mathrm{N}_{0}$ ). Im Mittel der Ansaatvarianten konnte kein gesicherter Einfluss der Düngungsstufen $\mathrm{N}_{0}$ und $\mathrm{N}_{3}$ auf die Höhe der $\delta^{15} \mathrm{~N}$-Werte in der Biomasse der Pflanzen festgestellt werden (Tab. A32a).

Die gewichteten gesamtpflanzlichen $\delta^{15} \mathrm{~N}$-Werte der Luzerne und des Knaulgrases in Rein- und Gemengesaat zu drei Schnitterminen des zweiten Untersuchungsjahres 1999 sind in Abb. 29 wiedergegeben.

Auch im zweiten Untersuchungsjahr (1999) lagen die $\delta^{15} \mathrm{~N}$-Werte des Knaulgrases in Reinsaat (4K) im Mittel der Düngungsstufen mit Werten von 4,44 \%o (erster Schnitt), 4,14\%o (zweiter Schnitt) und 3,92\%o (dritter Schnitt) zu allen drei Schnittterminen signifikant über den Werten der Luzerne in Reinsaat (4L; erster Schnitt: 1,29\%; zweiter Schnitt: 0,97\%; dritter Schnitt: 0,88\%; Tab. A32b). Hierbei nahmen die $\delta^{15} \mathrm{~N}-$ Werte des Knaulgrases in Reinsaat (4K) im Jahresverlauf tendenziell ab. Während zu allen drei Schnittterminen im Mittel der Düngungsstufen keine gesicherten 
Unterschiede zwischen den $\delta^{15} \mathrm{~N}$-Werten der Luzerne in Reinsaat (4L) und im Gemenge mit $25 \%$ Knaulgras $(3 \mathrm{~L} / 1 \mathrm{~K})$ festgestellt werden konnten, lagen die $\delta^{15} \mathrm{~N}$ Werte im Vergleich zum Gemenge mit $25 \%$ Luzerne / $75 \%$ Knaulgras und Knaulgras in Reinsaat (4K) signifikant darunter (Tab. A32b). Dabei lagen die $\delta^{15} \mathrm{~N}-$ Werte der Bestände aus der $\mathrm{N}_{0}-$ Variante in den Ansaaten mit Luzerne unter, bei Knaulgras in Reinsaat (4K) über den $\delta^{15} \mathrm{~N}$-Werten der Bestände aus der $\mathrm{N}_{3}$-Variante (signifikante Wechselwirkung: Ansaatvariante $\times$ Düngung).

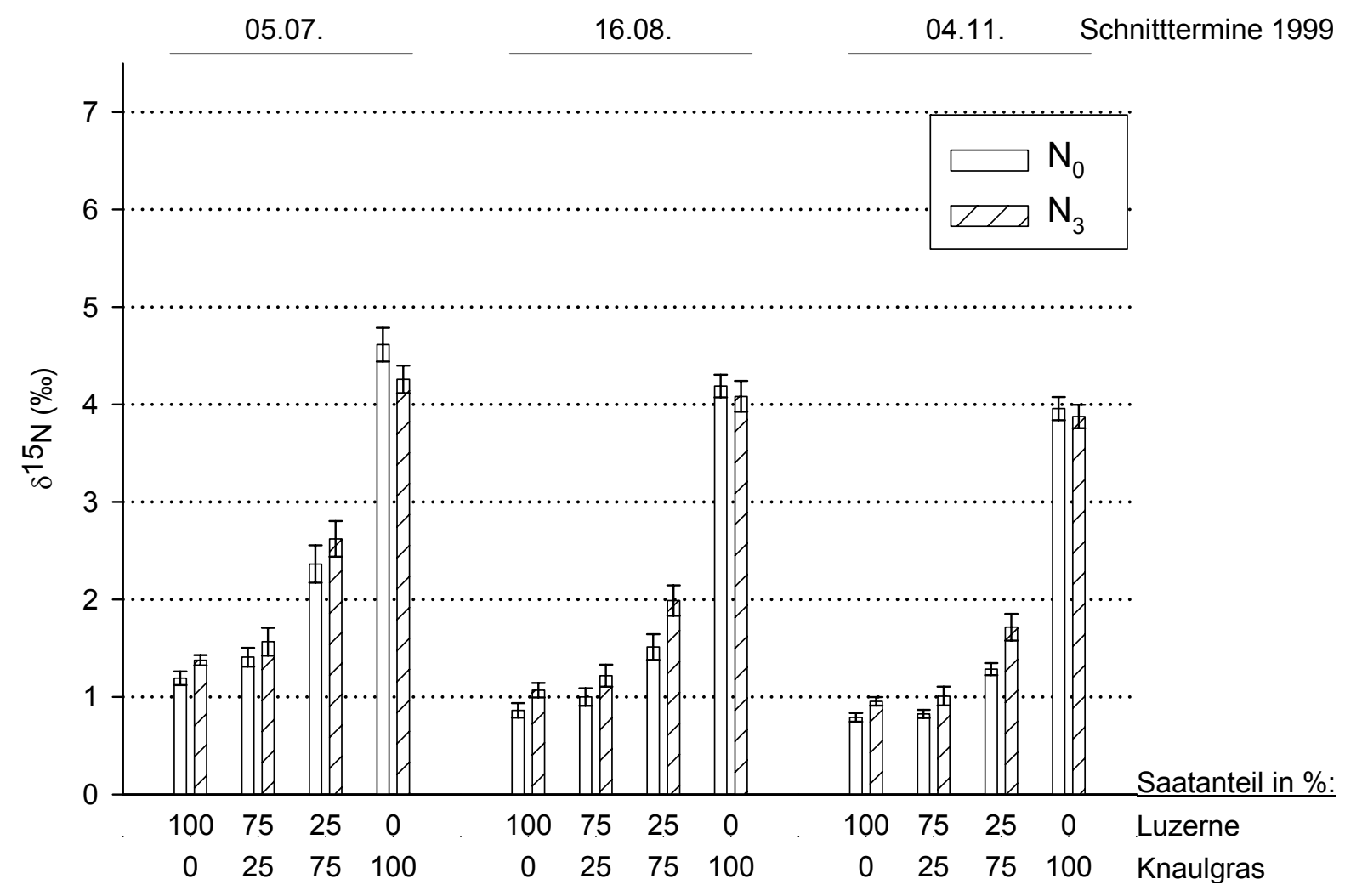

Abb. 29: Gewichtete- (erster Schnitt) bzw. kumuliert gewichtete $\delta^{15} \mathrm{~N}$-Werte (zweiter und dritter Schnitt) (arithmetische Mittelwerte \pm Standardfehler in \%o) in der gesamtpflanzlichen Biomasse (Erntereste + Schnittgut) beim Anbau von Luzerne und Knaulgras in Rein- und Gemengesaat im Jahr 1999

Die Differenzen in den $\delta^{15} \mathrm{~N}$-Werten zwischen Luzerne und Knaulgras in Reinsaat (4L und 4K) lagen im Jahr $1999 \mathrm{im}$ Minimum bei 2,88 \%o (erster Schnitt, $\mathrm{N}_{3}$ ) und maximal bei 3,42 \%o (erster Schnitt, $\mathrm{N}_{0}$ ). Auch im zweiten Untersuchungsjahr konnte im Mittel der Ansaatvarianten kein gesicherter Einfluss der Düngungsstufe $\left(\mathrm{N}_{0}\right.$ und $\left.\mathrm{N}_{3}\right)$ auf die Höhe der $\delta^{15} \mathrm{~N}$-Werte festgestellt werden (Tab. A32b). Zu allen drei Schnittterminen lagen die $\delta^{15} \mathrm{~N}$-Werte in der Biomasse der Bestände im Mittel im Jahr 1999 signifikant unter den $\delta^{15} \mathrm{~N}$-Werten der gleichen Schnittermine des Vorjahres $\left(P_{\text {Jahr }}=0,0001\right.$ alle drei Schnitttermine). Zum zweiten und dritten Schnittermin waren zwischen den 
Faktoren Ansaatvariante $\times$ Jahr, sowie Ansaatvariante $\times$ Jahr $\times$ Düngung signifikante Wechselwirkungen zu beobachten.

\subsubsection{Vergleich der Schätzergebnisse der symbiotisch fixierten N-Menge mittels $\delta{ }^{15} \mathrm{~N}$ - und erweiterter Differenzmethode}

In Abb. 30 sind die Korrelationskoeffizienten zwischen den Ergebnissen der beiden Schätzmethoden zur Quantifizierung der luftbürtigen N-Mengen beim Anbau von Luzerne in Rein- und Gemengesaat mit Knaulgras für die Untersuchungsjahre 1998 (a) und 1999 (b) dargestellt. Die Abbildungen beinhalten alle Schätzergebnisse (inklusive "negative" Werte, vgl. unten) die an den Ernteterminen über alle Faktorkombinationen ermittelt wurden.

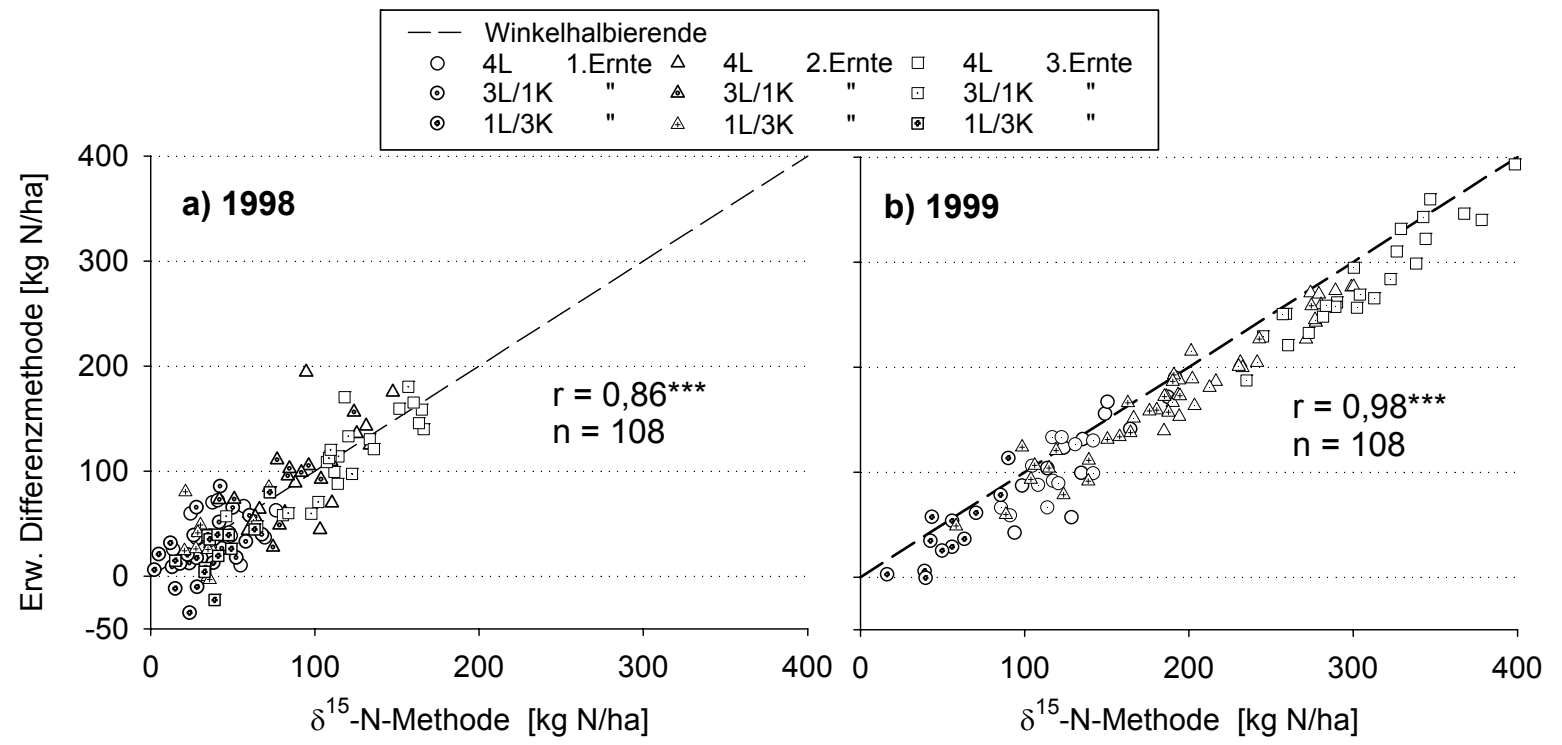

Abb. 30: Korrelation zwischen den Ergebnissen der $\delta^{15} \mathrm{~N}$ - und erweiterten Differenzmethode zur Schätzung der gesamtpflanzlich symbiotisch fixierten $\mathrm{N}$ Menge a) 1998 und b) 1999 beim Anbau von Luzerne in Reinsaat (4L) und Gemengesaat mit Knaulgras (3L/1K und 1L/3K).

Der Korrelationskoeffizient für den Vergleich der Ergebnisse zur Schätzung der gesamtpflanzlich fixierten N-Menge lag im Jahr 1999 mit 0,98 über dem Koeffizienten, der für das Jahr 1998 ermittelt wurde (0,86; Abb. 30). Alle Vergleiche erbrachten sehr enge Korrelationen mit hohem Signifikanzniveau zwischen den Schätzergebnissen $(P=0,0001)$. Im Mittel eines Jahres lagen die mittels erweiterter Differenzmethode geschätzten fixierten Stickstoffmengen mit 3,3 $\mathrm{kg} \mathrm{N} \mathrm{ha}^{-1} 1998$ und 19,5 kg N ha $^{-1} 1999$ geringfügig unter der luftbürtigen N-Menge, die mit der $\delta^{15} \mathrm{~N}$-Methode geschätzt wurde. Im Jahr 1998 lagen die Schätzwerte der erweiterten Differenzmethode maximal bei $99,7 \mathrm{~kg} \mathrm{~N}^{-1}$ über bzw. $61,5 \mathrm{~kg} \mathrm{~N}^{-1}$ unter den Werten, die 
mittels $\delta^{15} \mathrm{~N}$-Methode ermittelt wurden (Abb. 30a). Im zweiten Untersuchungsjahr 1999 wurden die Schätzergebnisse der $\delta^{15} \mathrm{~N}$-Methode durch die mit der erweiterten Differenzmethode ermittelten Werte bis maximal $25,2 \mathrm{~kg} \mathrm{~N}^{-1}$ über bzw. 71,3 $\mathrm{kg} \mathrm{N} \mathrm{ha}^{-1}$ unterschätzt (Abb. 30b). Die Korrelationskoeffizienten zwischen den Schätzergebnissen, die sich zu den einzelnen Schnittterminen ergaben, sind in Tab. A33 enthalten.

Im Jahr 1998 waren bei der erweiterten Differenzmethode zum ersten Schnitttermin in drei Fällen, zum zweiten und dritten Schnitttermin jeweils in einem Fall und im Jahr 1999 in einem Fall zum ersten Schnitttermin ein "negativer" Schätzwert für die symbiotisch fixierte N-Menge zu verzeichnen. Alle negativen Werte traten in der grasbetonten Gemengevariante (1L/3K) auf.

\subsubsection{Luft- und bodenbürtige $\mathrm{N}$-Akkumulation sowie $\mathrm{CaCl}_{2}$-extrahierbarer $\mathbf{N}_{\text {min }}$-Stickstoff im Boden}

Die vor der Aussaat der Luzerne und des Knaulgrases am 24.03.1998 vorgefundenen Mengen an pflanzenverfügbarem Stickstoff im Boden sind in Abb. 31 abgebildet und im Anhang der Tab. A34 zu entnehmen. Mit $\mathrm{N}_{\text {min }}$-Mengen in Höhe von 60,7 kg N ha $^{-1}(0$ bis $125 \mathrm{~cm}$ ) wiesen die langjährig nicht mit mineralischen N-Düngern zu den nichtlegumen Vorfü̈chten gedüngten Parzellen $\left(\mathrm{N}_{0}\right)$ signifikant niedrigere $\mathrm{N}_{\min }$-Mengen im Vergleich zu der $\mathrm{N}_{3}$-Variante $\left(81,9 \mathrm{~kg} \mathrm{~N}^{-1}\right)$ auf. Signifikante Unterschiede im Vergleich der Düngungsstufen waren auf den Unterboden (50 bis $125 \mathrm{~cm}$ ) beschränkt (Tab. A34a).

Die deutlich unterschiedliche Ausnutzung des pflanzenverfügbaren Stickstoffs im Boden, wie sie für die Ansaaten der Ackerbohne und des Hafer beschrieben wurden, konnten für die Ansaaten mit Luzerne in Rein- und Gemengesaat mit Knaulgras nicht beobachtet werden. Die residualen $\mathrm{N}_{\text {min }}$-Mengen im Boden, die zum dritten Schnitt 1998 unter den Ansaaten vorgefunden wurden, lagen mit Werten zwischen 20,9 $\left(\mathrm{N}_{3}\right.$ : $4 \mathrm{~L}$ ) und $13,9 \mathrm{~kg} \mathrm{~N}^{-1}\left(\mathrm{~N}_{0}: 3 \mathrm{~L} / 1 \mathrm{~K}\right.$ und $\left.1 \mathrm{~L} / 3 \mathrm{~K}\right)$ in 0 bis $125 \mathrm{~cm}$ Bodentiefe auf einem vergleichsweise niedrigen Niveau (Abb. 31, Tab. A35). Lediglich die leicht erhöhten residualen $\mathrm{N}_{\text {min }}$-Mengen unter der Luzerne in Reinsaat (4L; 0 bis $125 \mathrm{~cm}$ ), konnten im Mittel der Düngungsstufen gegenüber der Gemengeansaat mit $75 \%$ Knaulgras (1L/3K) statistisch gesichert werden (Tab. A35). Die Aufteilung in Ober- und Unterboden verdeutlicht, dass die Unterschiede durch eine signifikant höhere $\mathrm{N}_{\min }$-Menge im Oberboden der Luzerne in Reinsaat (4L) resultieren. Die Düngungsstufen $\left(\mathrm{N}_{0}, \mathrm{~N}_{3}\right)$ beeinflussten die residualen $\mathrm{N}_{\text {min }}$-Mengen im Boden nach Anbau von Luzerne und Knaulgras nicht signifikant (Tab. A35). Die unterschiedliche Aneignung von Bodenstickstoff bis zum dritten Schnitt wurde in gleicher Abstufung (in 0 bis $125 \mathrm{~cm}$ und 0 bis $50 \mathrm{~cm}$ ) bereits zum ersten Schnitttermin beobachtet. Allerdings lag zu diesem Zeitpunkt die $\mathrm{N}_{\text {min }}$-Menge in 0 bis $125 \mathrm{~cm}$ Bodentiefe der $\mathrm{N}_{3}$-Variante im Mittel signifikant über der $\mathrm{N}_{\text {min- }}$-Menge der $\mathrm{N}_{0}$-Variante (Tab. A35). Im Mittel der 
Ansaatvarianten wurde zum zweiten Schnittermin in 0 bis $125 \mathrm{~cm}$ Bodentiefe signifikant mehr $\mathrm{N}_{\text {min }}$-Stickstoff in der $\mathrm{N}_{0}$-Variante im Vergleich zu der $\mathrm{N}_{3}$-Variante festgestellt. Der signifikante Einfluss der N-Düngung zu den nichtlegumen Vorfrüchten beschränkte sich allerdings auf den Unterboden (Tab. A35). Unter der Luzerne in Reinsaat (4L) befand sich im Mittel der Düngungsstufen zum Zeitpunkt des zweiten Schnittes in 0 bis $50 \mathrm{~cm}$ Bodentiefe signifikant mehr $\mathrm{N}_{\min }$-Stickstoff, in 50 bis $125 \mathrm{~cm}$ Bodentiefe signifikant weniger $\mathrm{N}_{\min }$-Stickstoff im Vergleich zu Knaulgras in Reinsaat (4K). Hieraus resultieren bei einer Betrachtungsweise auf die gesamte Profiltiefe keine statistisch gesicherten Unterschiede im Mittel der Ansaatvarianten (Tab. A35).

Die im Folgenden dargestellten Ergebnisse der luft- und bodenbürtigen N-Akkumulation basieren auf den Schätzergebnissen des Anteils Stickstoff aus der Luft, die mit der $\delta^{15} \mathrm{~N}$-Methode ermittelt wurden. Auch hierbei ist zu berücksichtigen, dass sich die gewichteten Anteile Stickstoff aus der Luft (Ndfa $\left.{ }_{w}\right)$ bei den Ansaaten mit Luzerne und Knaulgras im Gemenge (3L/1K und $1 \mathrm{~L} / 3 \mathrm{~K})$ nur für die Summe des Stickstoffs aus beiden Gemengepartnern (inkl. Wurzeln) darstellen lassen (Kap. 2.7.2.3). Die gewichteten Anteile Stickstoff aus der Luft im Spross von Luzerne in Rein- und Gemengesaat sind im Anhang der Tab. A31 zu entnehmen. Wie aus den Daten hervorgeht, steigen mit zunehmendem Knaulgrasanteil die gewichteten Anteile Stickstoff aus der Luft $\left(\mathrm{Ndfa}_{\mathrm{w}}\right)$ deutlich an. Die gewichteten Anteile Stickstoff aus der Luft $\left(\mathrm{Ndfa}_{\mathrm{w}}\right)$ in den Beständen mit Luzerne sanken in der gesamtpflanzlichen Biomasse im Mittel über die Düngungsstufen ab dem Zeitpunkt des ersten Schnittes mit zunehmendem Anteil Knaulgras signifikant ab (Abb. 31; Ausnahme: erster Schnitt: 4L und 3L/1K; Tab. A36). Im Mittel der Ansaatvarianten mit Luzerne wies die Variante ohne langjährige mineralische $\mathrm{N}$-Düngung zu den nichtlegumen Vorfrüchten $\left(\mathrm{N}_{0}\right)$ zu allen Schnittterminen signifikant höhere Anteile Stickstoff aus der Luft in der Biomasse im Vergleich zu den Beständen aus der $\mathrm{N}_{3}$-Variante auf (Tab. A36).

Eine höhere Aufnahme an pflanzenverfügbarem Bodenstickstoff durch das Knaulgras in Reinsaat $(4 \mathrm{~K})$ gegenüber den Ansaaten mit Luzerne konnte im Mittel über die Düngungsstufen nur zum ersten Schnitttermin nachgewiesen werden. Sowohl zum zweiten als auch zum dritten Schnitttermin konnten keine Unterschiede in der Aneignung von Bodenstickstoff durch die Ansaatvarianten festgestellt werden (Tab. A36). Allerdings partizipierten die Ansaatvarianten in der langjährig nicht mit mineralischen N-Düngern zu den nichtlegumen Vorfrüchten gedüngten Variante $\left(\mathrm{N}_{0}\right)$ zu allen drei Schnittterminen signifikant geringer am pflanzenverfügbaren Bodenstickstoff als die Bestände der $\mathrm{N}_{3}$-Variante (Tab. A36). Luzerne in Reinsaat (4L) fixierte mit Werten zwischen 35,0 ( $\mathrm{N}_{3}: 4 \mathrm{~L}$ 1. Schnitt) und $152,0 \mathrm{~kg} \mathrm{~N}^{-1}\left(\mathrm{~N}_{0}: 4 \mathrm{~L} 3\right.$. Schnitt) mit Ausnahme des ersten Schnitttermins im Mittel über die Düngungsstufen die höchste Menge an luftbürtigem Stickstoff, wobei die fixierten N-Mengen mit zunehmendem Anteil Knaulgras im Gemenge signifikant abnahmen (Tab. A36). Die Ansaatvarianten fixierten zu allen Schnittterminen in der $\mathrm{N}_{0}$-Variante signifikant mehr 
luftbürtigen Stickstoff als die Pflanzen der $\mathrm{N}_{3}$-Variante. Die Differenzen der fixierten $\mathrm{N}$-Menge gleicher Ansaaten zwischen $\mathrm{N}_{0}$ - und $\mathrm{N}_{3}$-Variante nahmen mit zunehmendem Anteil Knaulgras zum zweiten und dritten Schnitttermin signifikant ab (Wechselwirkung: Ansaatvariante $\times$ Düngung; Tab. A36). Während bei Luzerne in Reinsaat (4L) die Differenz in der symbiotisch fixierten $\mathrm{N}$-Menge zwischen der $\mathrm{N}_{0^{-}}$und $\mathrm{N}_{3}$ Variante von $41,2 \mathrm{~kg} \mathrm{~N}^{-1}$ lag, betrug diese in der grasbetonten Ansaat (1L/3K) lediglich bei $5,7 \mathrm{~kg} \mathrm{~N} \mathrm{ha}^{-1}$.

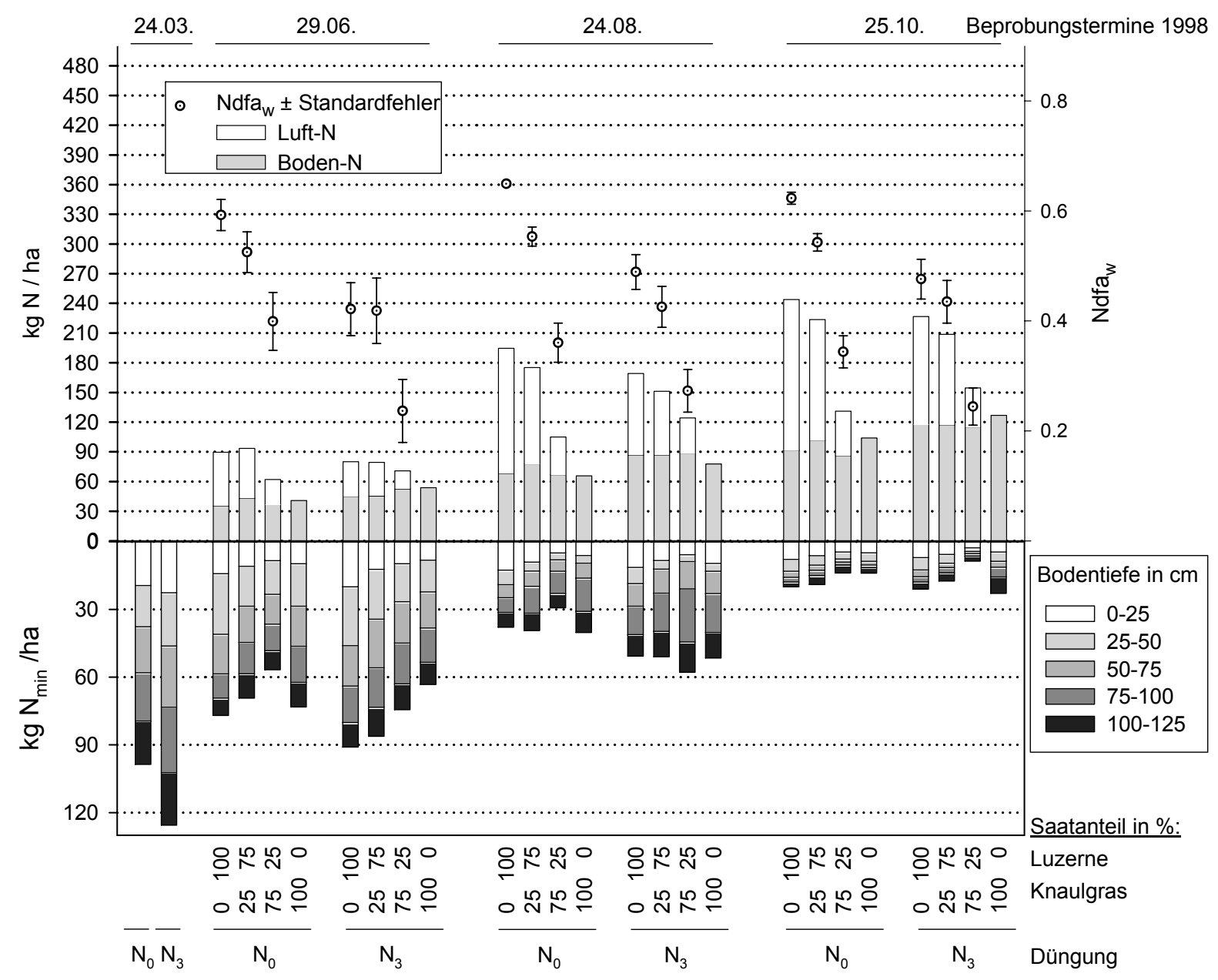

Abb. 31: $\mathrm{CaCl}_{2}$-extrahierbarer $\mathrm{N}_{\min }$-Stickstoff im Boden $\left(\mathrm{kg} \mathrm{N}_{\min } \mathrm{ha}^{-1}\right)$, boden- und luftbürtige N-Akkumulation in der gesamtpflanzlichen Biomasse $\left(\mathrm{kg} \mathrm{N} \mathrm{ha}^{-1}\right)$ und gewichtete Anteile Stickstoff aus der Luft ( $\mathrm{Ndfa}_{w} \pm$ Standardfehler) beim Anbau von Luzerne und Knaulgras in Rein- und Gemengesaat im Jahr 1998

Die im zweiten Untersuchungsjahr vor der Aussaat der Luzerne und des Knaulgrases am 06.04.1999 vorgefundenen Mengen an pflanzenverfügbarem Stickstoff im Boden sind in Abb. 32 abgebildet und im Anhang der Tab. A34b zu entnehmen. Mit $\mathrm{N}_{\text {min }}{ }^{-}$ Mengen in Höhe von 70,3 und $82,3 \mathrm{~kg} \mathrm{~N} \mathrm{ha}^{-1}\left(\mathrm{~N}_{0}\right.$ und $\mathrm{N}_{3}$; 0 bis $125 \mathrm{~cm}$ ) wiesen die 
Parzellen weder im Ober- noch im Unterboden Unterschiede in Abhängigkeit der langjährigen unterschiedlichen N-Düngung auf und lagen damit signifikant über den $\mathrm{N}_{\text {min }}$-Mengen des Vorjahres ( $P_{\mathrm{Jahr}}=0,0146$; Tab. A34b). Während sich die $\mathrm{N}_{\text {min }}{ }^{-}$ Mengen im Boden in der $\mathrm{N}_{3}$-Variante zwischen den Jahren kaum unterschieden, lagen sie in der $\mathrm{N}_{0}$-Variante im Jahr 1999 um $12 \mathrm{~kg} \mathrm{~N}_{\min }$ ha $^{-1}$ über denen des Vorjahres (signifikante Wechselwirkung: Jahr $\times$ Ansaatvariante: $P_{0 \text { bis } 125 \mathrm{~cm}}=0,0179$ ).

Die residualen $\mathrm{N}_{\text {min }}$-Mengen im Boden zum letzten Schnitttermin im Jahr 1999 lagen mit Werten zwischen 20,5 $\left(\mathrm{N}_{3}: 3 \mathrm{~L} / 1 \mathrm{~K}\right)$ und $15,3 \mathrm{~kg} \mathrm{~N}^{-1}\left(\mathrm{~N}_{3}: 4 \mathrm{~K}\right)$ in 0 bis $125 \mathrm{~cm}$ Bodentiefe auf einem vergleichbaren Niveau zum Vorjahr (Abb. 32 und Tab. A37). Im Mittel der Düngungsstufen konnten weder über die gesamte Profiltiefe (0 bis $125 \mathrm{~cm}$ ) noch im Unterboden (50 bis $125 \mathrm{~cm}$ ) gesicherte Unterschiede in Abhängigkeit von den Ansaaten beobachtet werden. Lediglich leicht geringere residuale $\mathrm{N}_{\text {min }}$-Mengen unter der Knaulgras-Reinsaat $(4 \mathrm{~K})$ im Oberboden $(0$ bis $50 \mathrm{~cm}$ ) konnten im Mittel der Düngungsstufen gegenüber den Ansaaten mit Luzerne (4L, 3L/1K, $1 \mathrm{~L} / 3 \mathrm{~K}$ ) als signifikant gesichert werden (Tab. A37). Zu keinem der drei Schnitttermine konnte eine Beeinflussung der $\mathrm{N}_{\text {min }}$-Menge im Boden durch die Düngung statistisch gesichert werden. Auch zum ersten und zweiten Schnitttermin wurden die $\mathrm{N}_{\text {min }}$-Mengen im Boden durch die Ansaatvarianten nur sehr gering beeinflusst, lagen aber signifikant über den $\mathrm{N}_{\text {min }}$-Mengen zum jeweiligen ersten und zweiten Schnitttermin des Vorjahres (beide Schnitte: $P_{\text {Jahr }}=0,0001$ ). Die im Mittel der Düngungsstufen zum ersten Schnitt signifikant geringere $\mathrm{N}_{\text {min }}$-Menge im Boden unter Knaulgras in Reinsaat (4K) im Vergleich zu Luzerne in Reinsaat (4L) in 0 bis $125 \mathrm{~cm}$ Bodentiefe resultierten vor allem aus den signifikant geringeren $\mathrm{N}_{\text {min }}$-Mengen im Oberboden (Tab. A37). Zum zweiten Schnitttermin wurden unter Knaulgras in Reinsaat (4K) im Oberboden signifikant geringere, im Unterboden signifikant höhere $\mathrm{N}_{\min }$-Stickstoff-Mengen im Vergleich zu Luzerne in Reinsaat (4L) und im Gemenge mit $25 \%$ Knaulgras (3L/1K) festgestellt (Tab. A37).

Die gewichteten Anteile Stickstoff aus der Luft (Ndfa $)_{w}$ im Spross von Luzerne in Rein- und Gemengesaat für 1999 sind im Anhang der Tab. A31 zu entnehmen. Der leichte Anstieg der gewichteten Anteile Stickstoff aus der Luft mit zunehmendem Knaulgrasanteil konnte statistisch nicht gesichert werden. Die gewichteten Anteile Stickstoff aus der Luft $\left(\mathrm{Ndfa}_{\mathrm{w}}\right)$ der Ansaaten mit Luzerne in der gesamtpflanzlichen Biomasse lagen mit Werten zwischen 0,76 (erster Schnitt: $N_{3} 1 \mathrm{~L} / 3 \mathrm{~K}$ ) und 0,38 (zweiter und dritter Schnitt: $\mathrm{N}_{0} 4 \mathrm{~L}$ ) zu allen drei Schnittterminen signifikant über den Anteilen, die im Jahr 1998 ermittelt wurden (alle Schnitte: $P_{\text {Jahr }}=0,0001$ ). Während die Gemengeansaaten mit $25 \%$ Luzerne $(1 \mathrm{~L} / 3 \mathrm{~K})$ zu allen Schnittterminen die signifikant niedrigsten Anteile Stickstoff aus der Luft aufwiesen, konnten keine gesicherten Unterschiede zwischen Luzerne in Reinsaat (4L) und dem Gemenge mit $25 \%$ Knaulgras (3L/1K) festgestellt werden (Abb. 32 und Tab. A38). Im Mittel der 
Ansaatvarianten lagen die gewichteten Anteile Stickstoff aus der Luft in der Biomasse der Bestände der $\mathrm{N}_{0}$-Variante zu allen drei Schnitten signifikant über den Anteilen der Bestände aus der $\mathrm{N}_{3}$-Variante (Tab. A38).

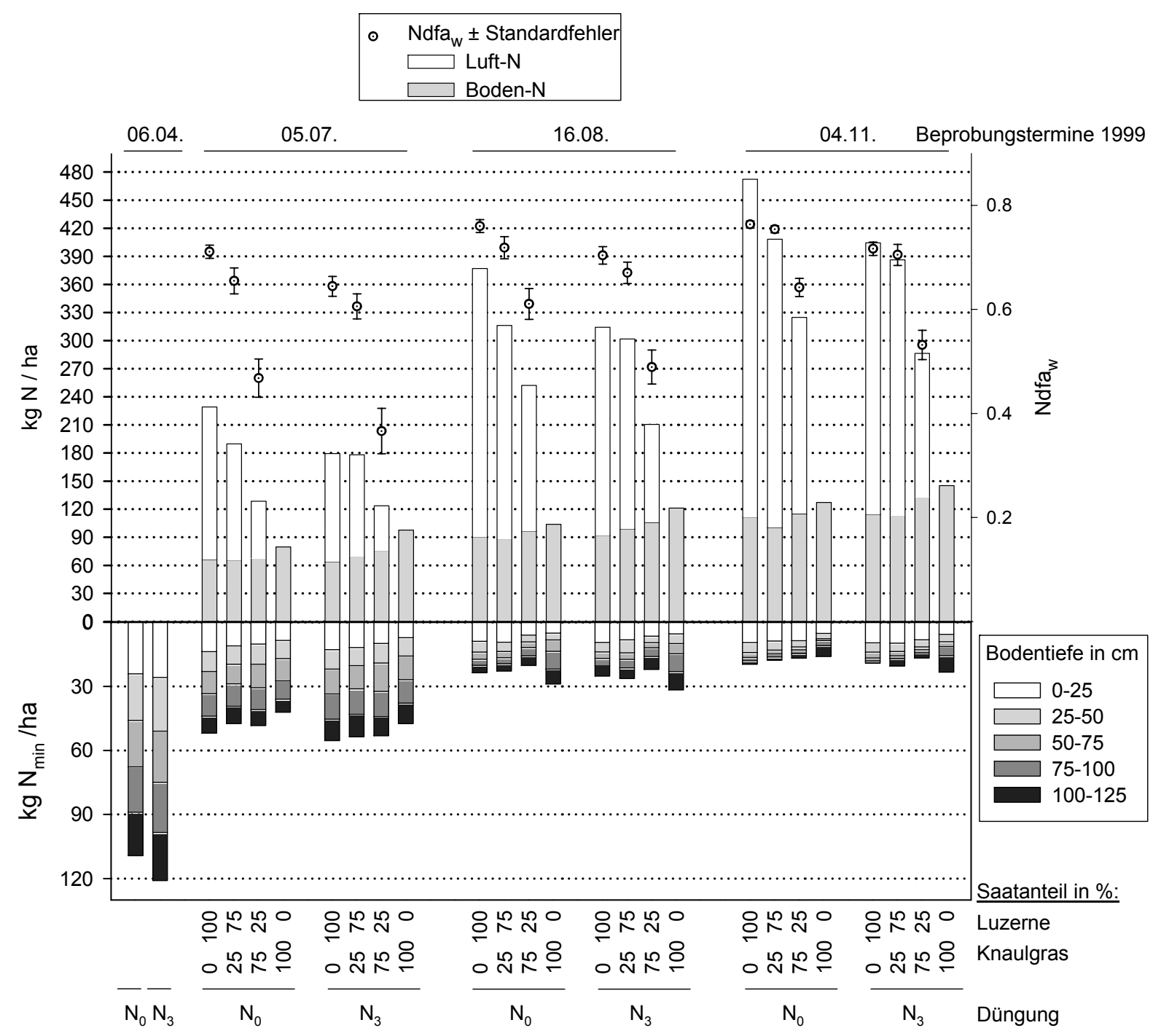

Abb. 32: $\mathrm{CaCl}_{2}$-extrahierbarer $\mathrm{N}_{\min }$-Stickstoff im Boden $\left(\mathrm{kg} \mathrm{N}_{\min } \mathrm{ha}^{-1}\right)$, boden- und luftbürtige N-Akkumulation in der gesamtpflanzlichen Biomasse $\left(\mathrm{kg} \mathrm{N} \mathrm{ha}^{-1}\right)$ und gewichtete Anteile Stickstoff aus der Luft $\left(\mathrm{Ndfa}_{w} \pm\right.$ Standardfehler) beim Anbau von Luzerne und Knaulgras in Rein- und Gemengesaat im Jahr 1999

Eine erhöhte Aufnahme von pflanzenverfügbarem Bodenstickstoff durch das Knaulgras in Reinsaat (4K) gegenüber den Ansaaten mit Luzerne konnte im Mittel über die Düngungsstufen zum ersten und zweiten Schnitttermin nachgewiesen werden. Zum dritten Schnitttermin konnte die erhöhte bodenbürtige N-Aufnahme des Knaulgrases in Reinsaat nur noch im Vergleich zur Luzerne in Reinsaat (4L) und im Gemenge mit $75 \%$ Luzerne (3L/1K) statistisch gesichert werden (Tab. A38). Die im 
Mittel der Ansaatvarianten tendenziell erhöhte Aufnahme von Bodenstickstoff in der $\mathrm{N}_{3}$-Variante konnte zu keinem Termin statistisch gesichert werden. Im Mittel akkumulierten die Ansaatvarianten zu allen drei Schnittterminen 1999 mehr bodenbürtigen Stickstoff in der Biomasse im Vergleich zum Vorjahr (erster und zweiter Schnitt: $P_{\text {Jahr }}$ $=0,0001$; dritter Schnitt: $\left.P_{\text {Jahr }}=0,0045\right)$. Dabei erhöhten die Ansaaten mit Knaulgras in Reinsaat (4K) im Mittel die bodenbürtige Stickstoffaufnahme gegenüber Luzerne in Reinsaat (4L) überproportional (signifikante Wechselwirkung: Ansaatvariante $\times$ Jahr: $P=0,0038)$. Die Ansaaten mit Luzerne $(4 \mathrm{~L}, 3 \mathrm{~L} / 1 \mathrm{~K}, 1 \mathrm{~L} / 3 \mathrm{~K})$ fixierten mit Werten bis maximal 360,7 kg N ha-1 ( $\mathrm{N}_{0}: 4 \mathrm{~L}$ 3. Schnitt) zu allen drei Schnitterminen mehr luftbürtigen Stickstoff im Vergleich zum Vorjahr (alle Schnitttermine: $P_{\text {Jahr }}=0,0001$ ). Im Mittel der Düngungsstufen nahmen die fixierten N-Mengen der Luzerne in Reinsaat (4L) mit zunehmendem Knaulgras Anteil signifikant ab (Ausnahme: dritter Schnitt 4L = 3L/1K; Tab. A38). An allen drei Schnittterminen wiesen die Ansaatvarianten in den langjährig nicht mit mineralischen N-Düngern zu den nichtlegumen Vorfüchten gedüngten Variante $\left(\mathrm{N}_{0}\right)$ signifikant höhere gewichtete Anteile Stickstoff aus der Luft in der Biomasse im Vergleich zur $\mathrm{N}_{3}$-Variante auf (Tab. A38).

\subsubsection{Transfer-Stickstoff beim Anbau von Knaulgras im Gemenge mit Luzerne}

In Abb. 33 sind die kumulierten $\delta^{15} \mathrm{~N}$-Werte im Spross (Schnittgut + Stoppeln) des Knaulgrases in Rein- und Gemengesaat zum dritten Schnitttermin im Jahr 1998 und 1999 in Abhängigkeit einer langjährig unterschiedlich hohen N-Düngung zu den nichtlegumen Vorfrüchten $\left(\mathrm{N}_{0}\right.$ und $\mathrm{N}_{3}$ ) dargestellt. In beiden Untersuchungsjahren konnten signifikante Unterschiede zwischen dem $\delta^{15} \mathrm{~N}$-Wert im Spross des Knaulgrases in Reinsaat $(4 \mathrm{~K})$ und dem $\delta^{15} \mathrm{~N}$-Wert im Spross des Knaulgrases in Gemengesaat (3L/1K und $1 \mathrm{~L} / 3 \mathrm{~K})$ nachgewiesen werden (Tab. A39).

Der $\delta^{15} \mathrm{~N}$-Wert des Knaulgrases in Reinsaat (4K) war sowohl im Jahr 1998 als auch im Jahr 1999 im Mittel über die Düngestufen mit 4,64 \%o bzw. 4,17\%o signifikant höher als der $\delta^{15} \mathrm{~N}$-Wert des Knaulgrases in den jeweiligen Gemengen mit Luzerne (1998: $1 \mathrm{~L} / 3 \mathrm{~K}=4,01 \%$ o, 3L/1K = 3,29 \%; 1999: $1 \mathrm{~L} / 3 \mathrm{~K}=3,49 \%$, 3L/1K = 2,74 \%o). Der $\delta^{15} \mathrm{~N}$-Wert des Knaulgrases in den langjährig nicht mit mineralischen N-Düngern zu den nichtlegumen Vorfrüchten gedüngten Variante $\left(\mathrm{N}_{0}\right)$ lag im Jahr $1998 \mathrm{im}$ Mittel der Ansaatvarianten signifikant über dem $\delta^{15} \mathrm{~N}$-Wert im Knaulgras aus der $\mathrm{N}_{3}$-Variante (Tab. A39). Demgegenüber konnte 1999 kein Einfluss der Düngung zu den Vorfrüchten auf den $\delta^{15} \mathrm{~N}$-Wert des Knaulgrases nachgewiesen werden. Die Unterschiede im $\delta^{15} \mathrm{~N}$-Wert des Knaulgrases zwischen den Untersuchungsjahren waren signifikant und es wurden signifikante Wechselwirkungen zwischen dem Faktor Jahr und N-Düngung zu den nichtlegumen Vorfrüchten festgestellt. 


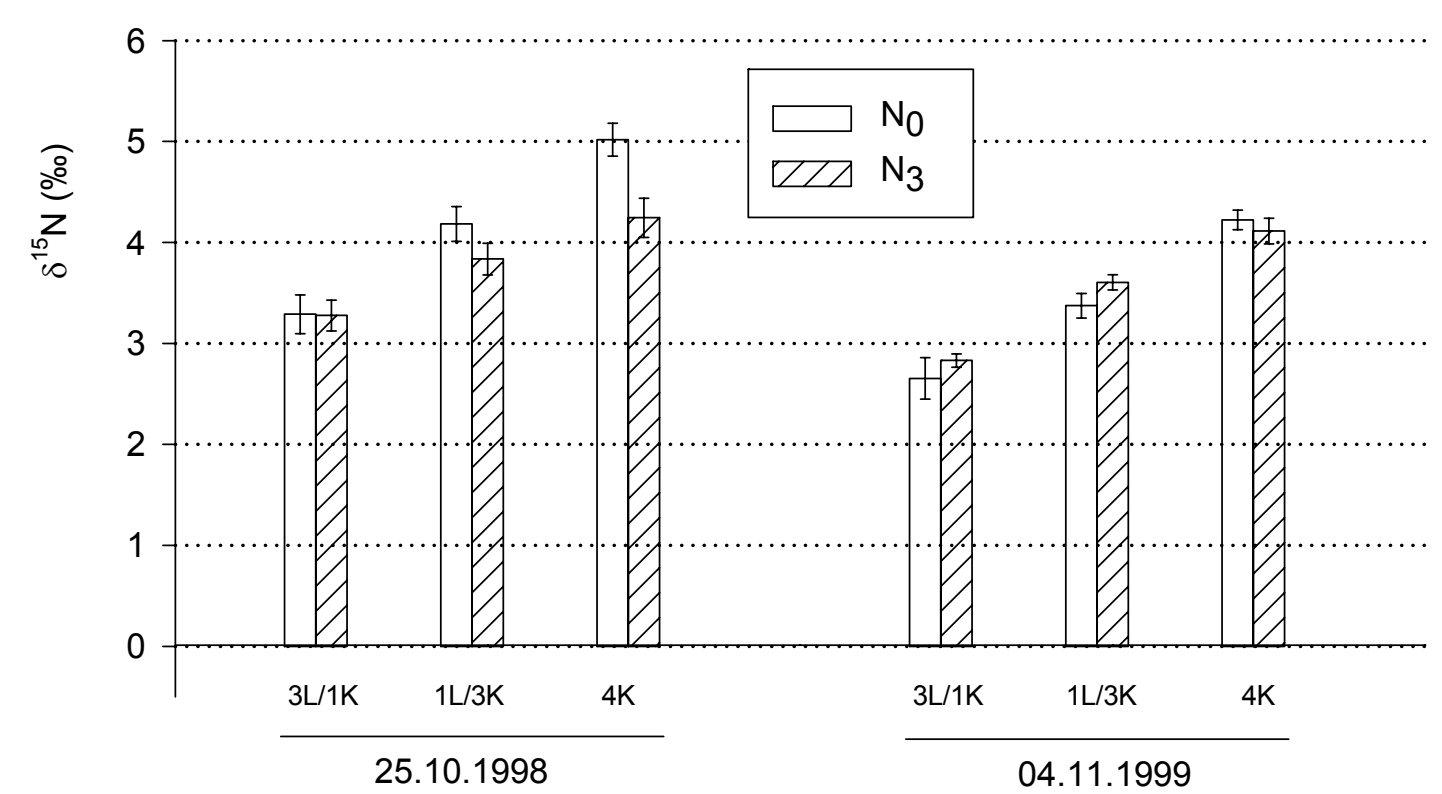

Abb. 33: Gewichtete $\delta^{15} \mathrm{~N}$-Werte $(\mathrm{n}=6$, Mittelwerte \pm Standardfehler in $\%$ ) in der Sprossmasse (Schnittgut + Stoppeln) des Knaulgrases in Reinsaat (4K) und Gemengesaat mit Luzerne (1L/3K und $3 \mathrm{~L} / 1 \mathrm{~K}$ ) (kumulierte Werte bis 25.10.1998, links und kumulierte Werte bis 04.11.1999, rechts)

Die in den Gemengen aus Luzerne und Knaulgras geschätzte Transfer-N-Menge lag im Jahr 1998 zwischen 9,9 und 31,2 $\mathrm{kg} \mathrm{ha}^{-1}$ sowie im Jahr 1999 zwischen 8,5 und 16,6 $\mathrm{kg} \mathrm{ha}^{-1}$ (Tab. 7). Dabei traten hoch signifikante Wechselwirkungen zwischen dem Jahr und der Ansaatvariante auf. So wurde im Jahr 1998 in der luzernedominierten Ansaat (3L/1K) mehr Stickstoff transferiert als in der knaulgrasdominierten Ansaat (1L/3K), während die Ansaaten im Jahr 1999 ein diametrales Verhalten in der Höhe der transferierten N-Menge zeigten. Der Anteil des Transfer-Stickstoffes an der Gesamt-N-Menge in der Sprossmasse des Knaulgrases schwankte zwischen 8,0 \% (1998 1L/3K N 3 ) und 34,6 \% (1999 3L/1K No).

Tab. 7: Transferierte N-Menge und Anteile $\mathrm{N}$ im Spross des Knaulgrases (Mittelwerte \pm Standardabweichung; $n=6$ ) aus der symbiotischen $\mathrm{N}_{2}$-Fixierung der Luzerne im Gemenge

\begin{tabular}{l|c|c|c|c}
\hline & ${ }^{1} \mathrm{~N}_{\text {trans }}$ in $\mathrm{kg} \mathrm{ha}^{-1}$ & ${ }^{2}$ Anteil $\mathrm{N}_{\text {trans }}$ in $\%$ \\
\hline Ansaatvariante & $\mathrm{N}_{0}$ & $\mathrm{~N}_{3}$ & $\mathrm{~N}_{0}$ & $\mathrm{~N}_{3}$ \\
\hline 1998 & $31,2 \pm 14,2$ & $19,6 \pm 12,3$ & 32,0 & 20,2 \\
\hline $3 \mathrm{~L} / 1 \mathrm{~K}$ & $15,0 \pm 9,7$ & $9,9 \pm 8,9$ & 15,0 & 8,0 \\
\hline $1 \mathrm{~L} / 3 \mathrm{~K}$ & \multicolumn{4}{|l}{} \\
\hline 1999 & $8,5 \pm 1,1$ & $9,0 \pm 3,1$ & 34,6 & 28,6 \\
\hline $3 \mathrm{~L} / 1 \mathrm{~K}$ & $16,6 \pm 10,6$ & $9,4 \pm 7,5$ & 18,4 & 9,3 \\
\hline $1 \mathrm{~L} / 3 \mathrm{~K}$ & $\begin{array}{l}|l| \\
1\end{array}$ transferierte N-Menge in $\mathrm{kg} \mathrm{ha}^{-1},{ }^{2}$ Anteil des Transfer-Stickstoffes an der Gesamt-N-Menge in der \\
Knaulgras-Sprossmasse
\end{tabular}


Im Jahr 1998 waren sowohl die transferierte N-Menge als auch der Anteil des Transfer-Stickstoffes an der Gesamt-N-Menge in der Knaulgras-Sprossmasse im Mittel über die Ansaatvarianten in der $\mathrm{N}_{0}$-Variante signifikant höher als in der $\mathrm{N}_{3^{-}}$ Variante (Tab. A40). Im zweiten Untersuchungsjahr 1999 war kein Einfluss der langjährigen unterschiedlich hohen $\mathrm{N}$-Düngung $\left(\mathrm{N}_{0}\right.$ und $\left.\mathrm{N}_{3}\right)$ zu den nichtlegumen Vorfrüchten auf die transferierte N-Menge nachweisbar.

\subsubsection{Einfacher und erweiterter N-Flächenbilanzsaldo}

Um den Beitrag von Futterleguminosen zu einer Selbstregelung in Agrarökosystemen quantifizieren zu können, sind im Folgenden die sich aus den Kenngrößen kumulierte gesamtpflanzliche Stickstoff-Menge $\left(\mathrm{N}_{\mathrm{Bt}}\right)$, dem kumulierten gewichteten Stickstoff-Harvest-Index $\left(\mathrm{H}_{\mathrm{NBtw}}\right.$, beide Kap. 3.2.2) und dem kumulierten gewichteten Anteil Stickstoff aus der Luft (Ndfa ${ }_{w}$, Kap. 3.2.5) ergebenden N-Flächenbilanzsalden (Kap. 2.7.2.2; Gleichung 14) als Summe je Jahr dargestellt.

Die einfachen N-Flächenbilanzsalden beim Anbau von Luzerne in Rein- und Gemengesaat mit Knaulgras lagen im Jahr 1998 mit Werten zwischen -1,7 ( $\mathrm{N}_{0}:$ 4L) und $82,2 \mathrm{~kg} \mathrm{~N} \mathrm{ha}^{-1}\left(\mathrm{~N}_{3}: 4 \mathrm{~K}\right.$; Abb. 34a) im Mittel der Ansaatvarianten und Düngungsstufen signifikant unter den Salden im Jahr $1999\left(P_{\text {Jahr }}=0,0001\right)$, die zwischen $+56,7\left(\mathrm{~N}_{0}\right.$ : $4 \mathrm{~L})$ und $-105,9 \mathrm{~kg} \mathrm{~N}$ ha ${ }^{-1}\left(\mathrm{~N}_{3}\right.$ : 4K; Abb. 35a) lagen. Während sich im Vergleich der Jahre die Bilanzsalden bei Luzerne in Reinsaat (4L) im Mittel um +62,9 kg erhöhten, fielen diese bei Knaulgras in Reinsaat (4K) im Mittel um -23,4 $\mathrm{kg} \mathrm{N}^{-1}$ ab (Wechselwirkung: Ansaatvariante $\times$ Jahr, $P=0,0001)$. Die einfachen $\mathrm{N}$-Flächenbilanzsalden verringerten sich in beiden Jahren in der Reihenfolge $4 \mathrm{~L}>3 \mathrm{~L} / 1 \mathrm{~K}>1 \mathrm{~L} / 3 \mathrm{~K}>4 \mathrm{~K}$, allerdings konnten nicht alle Unterschiede statistisch gesichert werden (Tab. 8).

In beiden Untersuchungsjahren lagen im Mittel über die Ansaatvarianten die N-Flächenbilanzsalden nach Vorfrüchten die langfristig nicht mit mineralischen N-Düngern versorgt wurden $\left(\mathrm{N}_{0}\right)$ in allen geprüften Ansaaten stets signifikant über den Salden nach langfristig überoptimal versorgten Vorfrüchten $\left(\mathrm{N}_{3}, \mathrm{Tab} .8\right)$.

Die Differenz im Bilanzsaldo zwischen der $\mathrm{N}_{0}$ - und der $\mathrm{N}_{3}$-Variante lag im Jahr 1998 bei $50,3(4 \mathrm{~L}), 28,5(3 \mathrm{~L} / 1 \mathrm{~K}), 33,7$ (1L/3K) und $16,9 \mathrm{~kg} \mathrm{~N}^{-1}(4 \mathrm{~K})$ und im Jahr 1999 bei 43,3 (4L), 53,2 (3L/1K), 24,3 (1L/3K) und $17,5 \mathrm{~kg} \mathrm{~N}^{-1}(4 \mathrm{~K})$. 


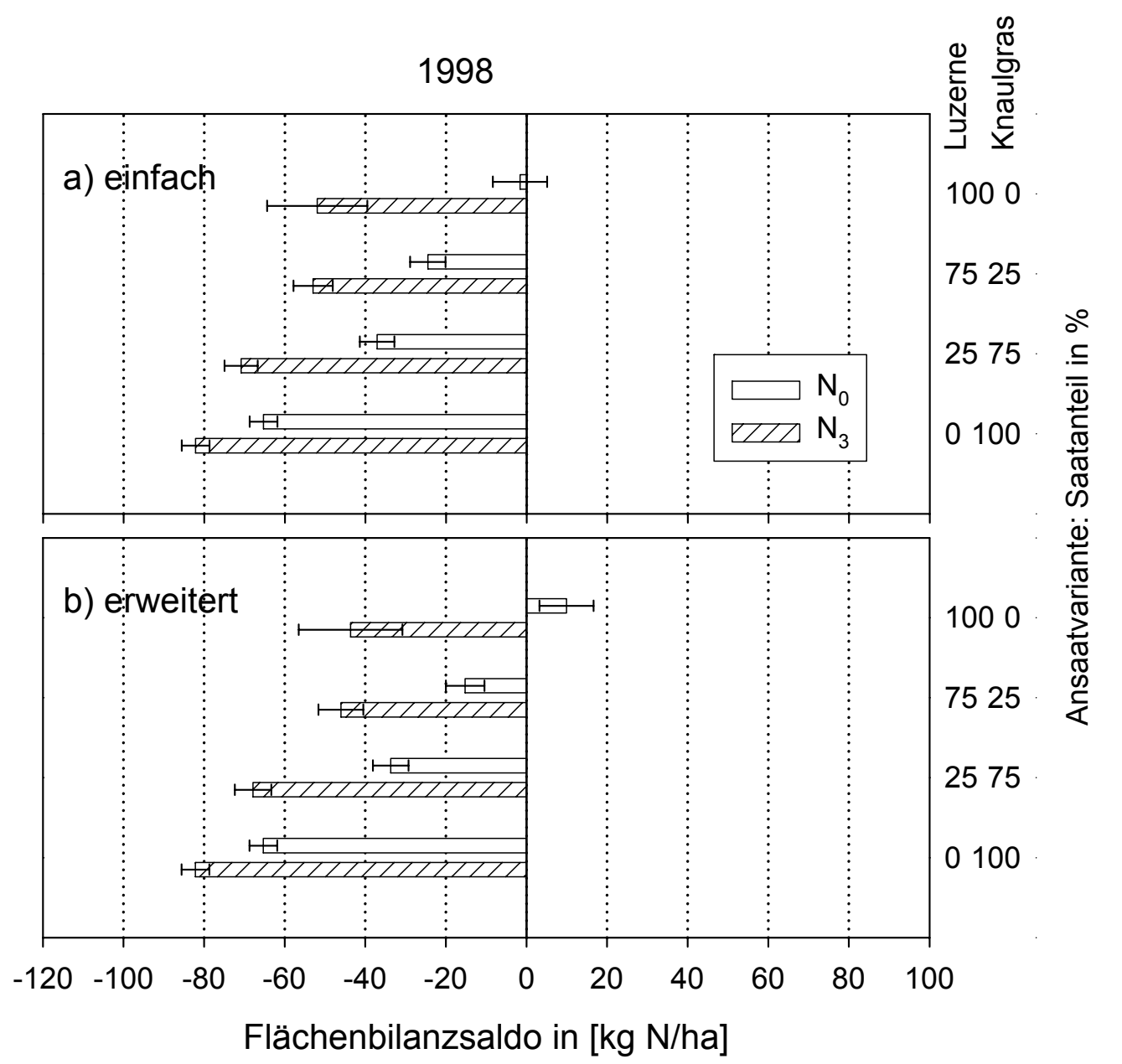

Abb. 34: Einfacher (a) und erweiterter (b) N-Flächenbilanzsaldo $\left(\mathrm{kg} \mathrm{N} \mathrm{ha}^{-1}\right)$ Mittelwerte \pm Standardfehler) beim Anbau von Luzerne und Knaulgras in Reinund Gemengesaat zur Futternutzung zum dritten Schnittermin $(\Sigma$ Jahr $)$ am 25.10 .1998

Tab. 8: Vergleich der Mittelwerte der einfachen N-Flächenbilanzsalden beim Anbau von Luzerne und Knaulgras in Rein- und Gemengesaat zur Futternutzung 1998 und 1999

\begin{tabular}{|c|c|c|c|c|c|c|}
\hline & \multicolumn{4}{|c|}{ Ansaatvariante } & \multirow[b]{2}{*}{ Düngung } & \multirow{2}{*}{$\begin{array}{l}\text { Wechsel- } \\
\text { wirkungen }\end{array}$} \\
\hline 1998 & $4 \mathrm{~L}$ & $3 \mathrm{~L} / 1 \mathrm{~K}$ & 1L/3K & $4 \mathrm{~K}$ & & \\
\hline einfacher Bilanzsaldo & A & $a b$ & $\mathrm{bc}$ & $\mathrm{C}^{*}$ & $* * *$ & n.s. \\
\hline 1999 & & & & & & \\
\hline einfacher Bilanzsaldo & $A$ & $a$ & $\mathrm{~b}$ & $\mathrm{C}^{* * *}$ & $* * *$ & n.s. \\
\hline
\end{tabular}

Unter Einbeziehung der Werte für die N-Rhizodeposition wurden erweiterte N-Flächenbilanzsalden für die Bestände mit Luzerne (4L, 3L/1K, 1L/3K) errechnet. Dabei wurden die unter Kap. 3.1.7 gemachten Annahmen zur Einbeziehung der Anteile Rhizodeposition, auch für die Ansaaten mit Luzerne, zugrunde gelegt. 
Durch Berücksichtigung der fixierten N-Menge des Stickstoffs aus der Rhizodeposition (Tab. A41) erhöhen sich die N-Flächenbilanzsalden im ersten Jahr auf maximal $+9,9 \mathrm{~kg} \mathrm{~N}$ ha ${ }^{-1}\left(\mathrm{~N}_{0}: 4 \mathrm{~L} ;\right.$ Abb. 34b) und +84,2 $\mathrm{kg} \mathrm{N}$ ha $^{-1} \mathrm{im}$ Jahr 1999 ( $\mathrm{N}_{0}: 4 \mathrm{~L} ; A b b$. $35 b)$.

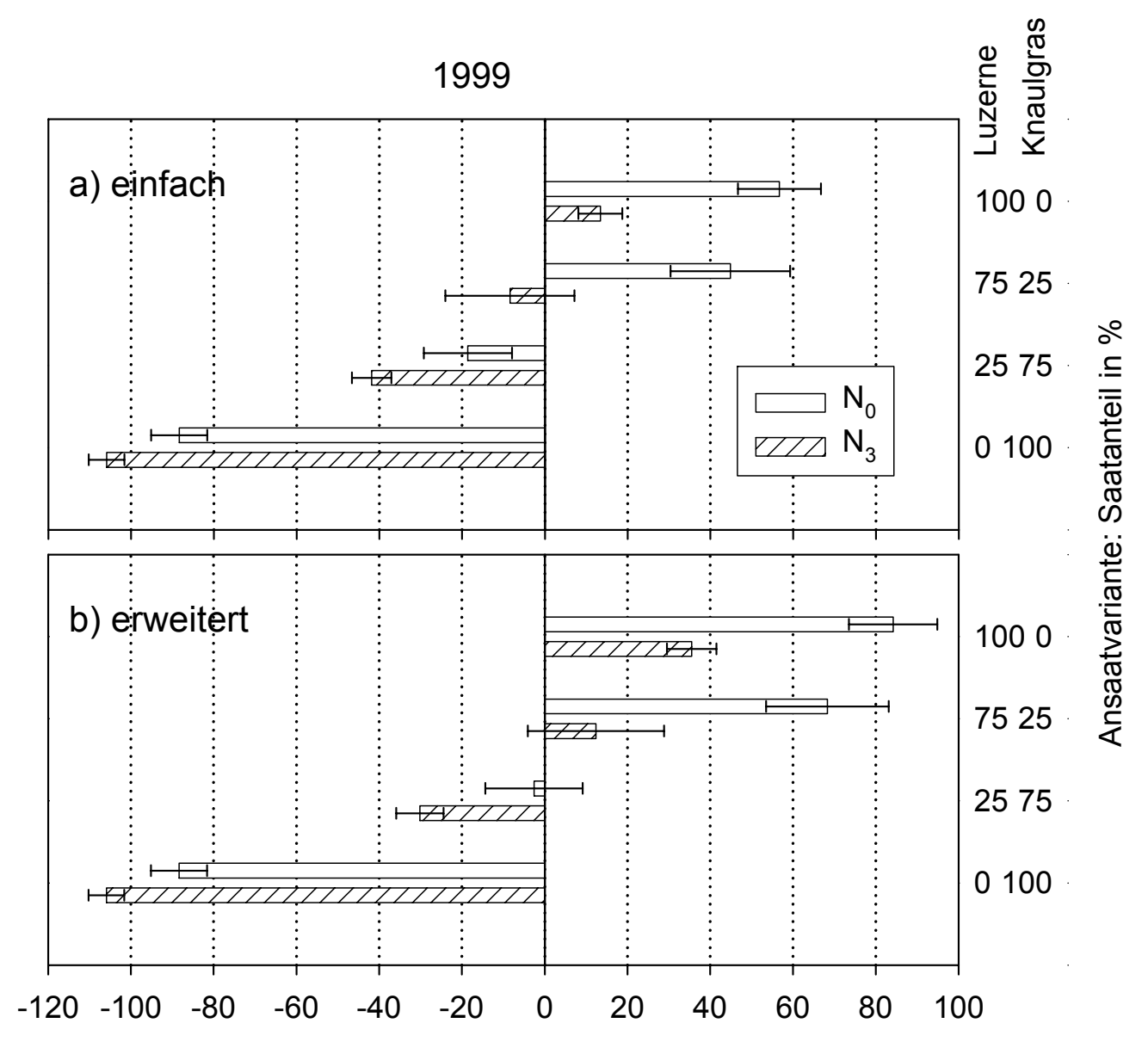

Flächenbilanzsaldo in [kg N/ha]

Abb. 35: Einfacher (a) und erweiterter (b) N-Flächenbilanzsaldo (kg N ha ${ }^{-1}$, Mittelwerte \pm Standardfehler) beim Anbau von Luzerne und Knaulgras in Reinund Gemengesaat zur Futternutzung zum dritten Schnitttermin ( $\Sigma$ Jahr) am 04.11.1999 


\subsection{Saatwicke in Rein- und Gemengesaat mit Einjährigem Weidelgras}

\subsubsection{Gesamtpflanzliche Trockenmasse-Akkumulation}

Die Ergebnisse der Trockenmasse-Akkumulation der Saatwicke und des Einjährigen Weidelgrases in Rein- und Gemengesaat werden nachfolgend getrennt nach Jahren dargestellt. Die gesamtpflanzliche Trockenmasse-Akkumulation, die TrockenmasseErträge der Wurzel-, Stoppel-, Schnittgutmasse sowie der gewichteten Trockenmasse-Harvest-Indizes sind den Tab. A43 (1998) und Tab. A45 (1999) im Anhang zu entnehmen und zusätzlich graphisch in den Abb. 36 und Abb. 38 wiedergegeben. Die Wurzel-TM wurde einzeln und zusammen mit der Stoppel-TM als Ernterest-TM verrechnet. Die oberirdischen TM-Ertragsanteile der Saatwicke und des Einjährigen Weidelgrases in den Gemengeansaaten sind in den Abb. 37 und Abb. 39 dargestellt.

Beim Anbau der Saatwicke und des Einjährigen Weidelgras wurden im Jahr 1998 und 1999 geringe Mengen (maximal 2,1 bzw. 0,7 dt TM ha-1) an Kräuter-Sprossmasse geerntet, die in den folgenden Beschreibungen und Darstellungen der gesamtpflanzlichen Trockenmasse-Akkumulation nicht berücksichtigt wurden (Tab. A42).

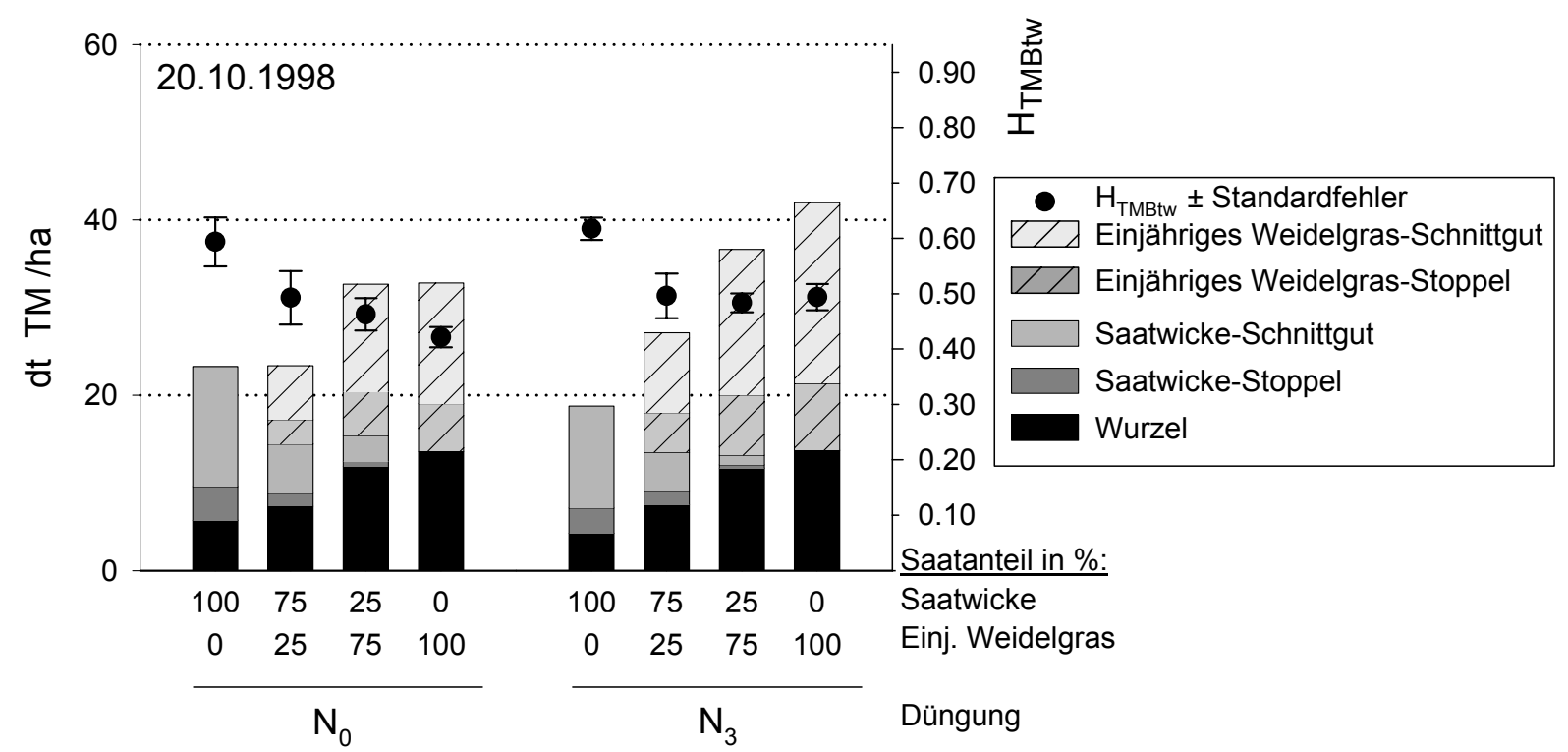

Abb. 36: Gesamtpflanzliche Trockenmasse (dt TM ha ${ }^{-1}$; Wurzel, Stoppel, Schnittgut) sowie gewichtete Trockenmasse-Harvest-Indizes $\left(\mathrm{H}_{\mathrm{TMB}} \pm\right.$ Standardfehler) beim Anbau der Saatwicke und des Einjährigen Weidelgrases in Rein- und Gemengesaat am 20.10.1998

Im ersten Untersuchungsjahr akkumulierten die Ansaaten der Saatwicke und des Einjährigen Weidelgras bis 86 Tage nach der Saat zwischen 18,8 $\left(\mathrm{N}_{3}: 4 S\right)$ und 41,9 dt TM ha ${ }^{-1}\left(\mathrm{~N}_{3}\right.$ : 4E; Abb. 36). Einjähriges Weidelgras in Reinsaat (4E) akkumulierte 
sowohl gesamtpflanzlich als auch beim Ertrag an Schnittgut, Ernterest und Wurzeln im Mittel der Düngungsstufen signifikant höhere Trockenmassen im Vergleich zur Saatwicke in Reinsaat (4S) und im Gemenge mit $25 \%$ Einjährigem Weidelgras (3S/1E; Tab. A43). Während die Saatwicke in Reinsaat (4S) in der $\mathrm{N}_{0}$-Variante signifikant mehr gesamtpflanzliche Trockenmasse akkumulierte als in der $\mathrm{N}_{3}$-Variante, verhielten sich die Ansaaten mit Einjährigem Weidelgras entgegengesetzt (signifikante Wechselwirkung Ansaatvariante $\times$ Düngung; Tab. A43). Auch auf die Schnittgut-TM wirkte sich der Faktor N-Düngung zu den nichtlegumen Vorfrüchten $\left(\mathrm{N}_{0}\right.$ und $\mathrm{N}_{3}$ ) in gleicher Weise differenziert aus (hoch signifikante Wechselwirkung). Während der Ertrag an Wurzel- und Stoppel-TM mit zunehmendem Anteil an Einjährigem Weidelgras im Bestand im Mittel über die Düngungsstufen anstieg, nahmen die gesamtpflanzlich gewichteten TM-Harvest-Indizes $\left(\mathrm{H}_{\mathrm{TMBtw}}\right)$ mit zunehmendem Anteil Einjährigem Weidelgras im Bestand im Mittel über die Düngungsstufen ab (Abb. 36 und Tab. A43).

In Abb. 37 sind die oberirdischen Trockenmasse-Ertragsanteile der Saatwicke und des Einjährigen Weidelgras in beiden Gemengesaaten zur Ernte dargestellt. Der Ertragsanteil Sprossmasse (Schnittgut + Stoppeln) an Einjährigem Weidelgras betrug in der Ansaatvariante mit $25 \%$ Einjährigem Weidelgras in der Ansaatmischung $70,4 \%$ in der $\mathrm{N}_{3}$-Variante bzw. 59,0 \% in der $\mathrm{N}_{0}$-Variante. In der grasbetonten Ansaatmischung (1S/3E) betrugen die TM-Ertragsanteile des Einjährigen Weidelgrases $94,0 \% \quad\left(\mathrm{~N}_{3}\right)$ bzw. $84,2 \%\left(N_{0}\right)$. Die Unterschiede in den oberirdischen Ertragsanteilen zwischen den Düngungsstufen konnten im Mittel über die Ansaat-

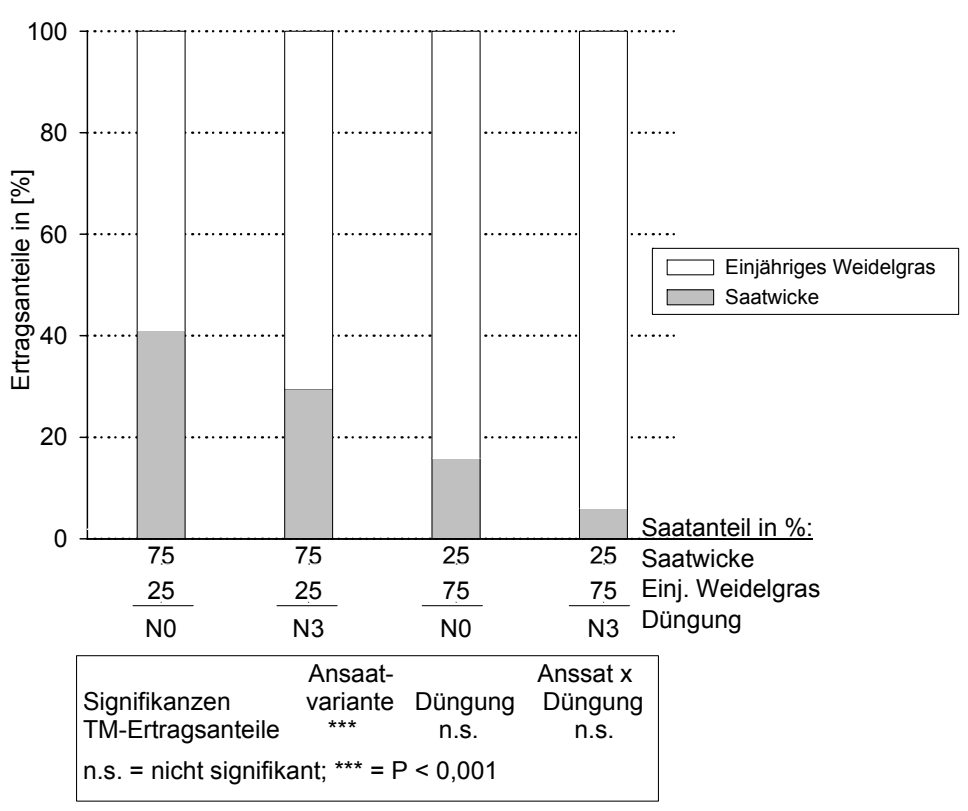

Abb. 37: Ertragsanteile der Spross-TM beim Anbau der Saatwicke und des Einjährigen Weidelgrases in Gemengesaat am 20.10.1998

varianten im Jahr 1998 statistisch nicht gesichert werden (Abb. 37).

Im zweiten Untersuchungsjahr (1999) akkumulierten die Ansaaten der Saatwicke und des Einjährigen Weidelgrases in Rein- und Gemengesaat bis 96 Tage nach der Aussaat mit Werten zwischen 32,5 ( $\mathrm{N}_{0}$ : 4E) und 57,5 dt TM ha ${ }^{-1}\left(\mathrm{~N}_{0}\right.$ : 4S; Abb. 38) im Mittel deutlich mehr gesamtpflanzliche Trockenmasse im Vergleich zum Vorjahr $\left(P_{\text {Jahr }}=0,0001\right)$. Während die Ansaaten mit der Saatwicke deutlich an gesamtpflanz- 
licher Trockenmasse im zweiten Unersuchungsjahr hinzu gewannen, lagen die Erträge des Einjährigen Weidelgrases in Reinsaat (4E) auf etwa gleichem Niveau wie im Jahr 1998 (signifikante Wechselwirkung: Ansaatvariante $\times$ Jahr). Zusätzlich wurde eine Wechselwirkung zwischen Ansaatvariante und Düngung sowie Ansaatvariante, Düngung und Jahr beobachtet. Einjähriges Weidelgras in Reinsaat (4E) akkumulierte im Jahr 1999 im Vergleich zu den Ansaaten mit Saatwicke im Mittel der Düngungsvarianten signifikant geringere gesamtpflanzliche und Schnittgut-TM-Erträge und höhere Ernterest- bzw. Wurzel-Trockenmassen (Ausnahme: Unterschied im TMBt von $1 \mathrm{~S} / 3 \mathrm{E}$ und 4E nicht gesichert; Tab. A44). Lagen die Schnittgut-Erträge im Jahr 1999 deutlich über den Erträgen des Vorjahres $\left(P_{\text {Jahr }}=0,0001\right)$, zeigten sich bei den Ernteresten bzw. Wurzelmassen deutlich geringere Erträge $1999\left(P_{\text {Jahr }}\right.$ jeweils $=$ 0,0001). Bei den TM-Erträgen der Wurzeln, Stoppeln und des Ernterestes traten signifikante Wechselwirkung zwischen den Ansaatvarianten und dem Jahr auf. Während sich die Schnittgut-Erträge der Ansaaten mit der Saatwicke deutlich erhöhten, fielen die Erträge des Einjährigen Weidelgrases in Reinsaat (4E) im Mittel zwischen den zwei Untersuchungsjahren etwa gleich aus.

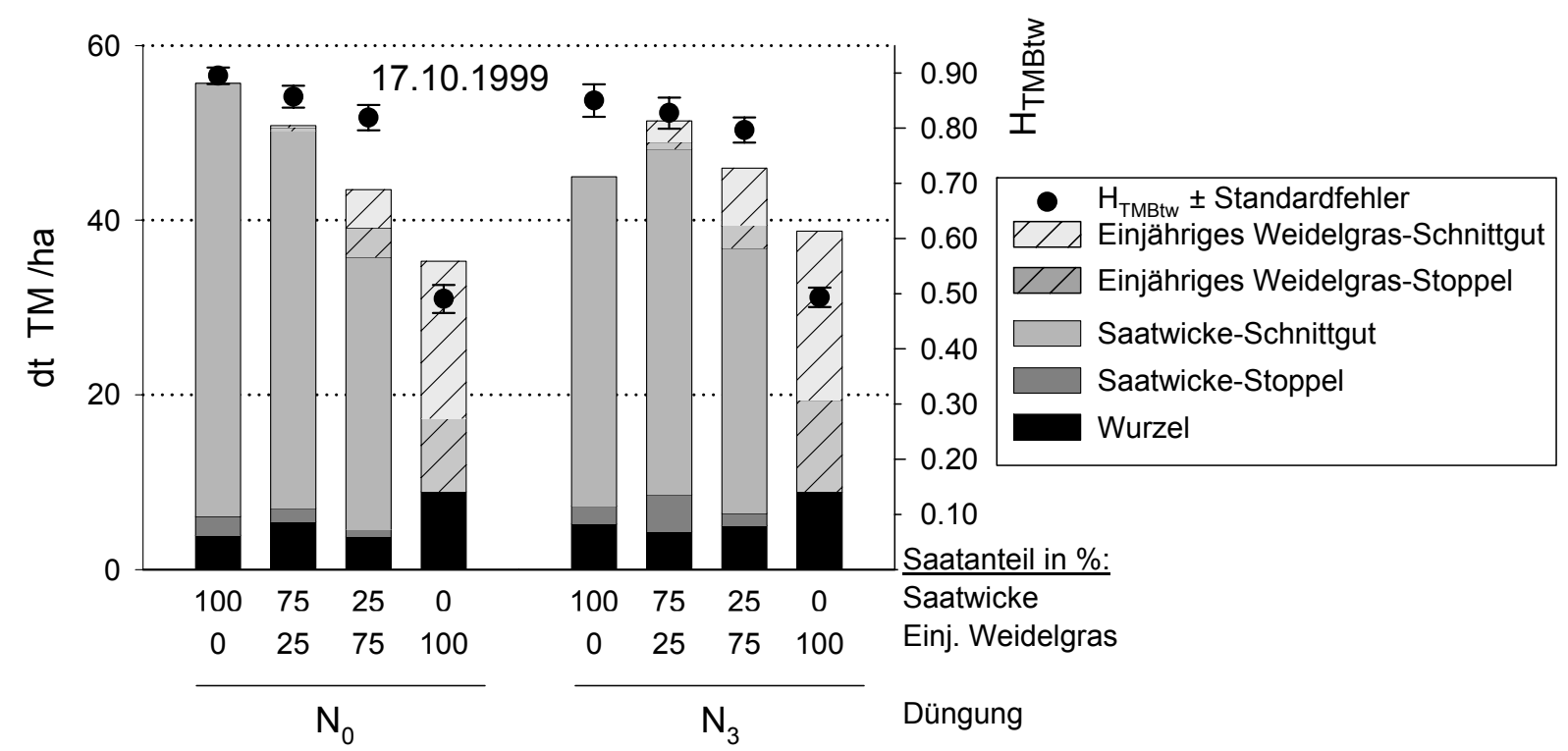

Abb. 38: Gesamtpflanzliche Trockenmasse (dt TM ha ${ }^{-1}$; Wurzel, Stoppel, Schnittgut) sowie gewichtete Trockenmasse-Harvest-Indizes $\left(\mathrm{H}_{\mathrm{TMB} \text { tw }} \pm\right.$ Standardfehler) beim Anbau der Saatwicke und des Einjährigen Weidelgrases in Rein- und Gemengesaat am 17.10.1999

Die gesamtpflanzlich gewichteten TM-Harvest-Indizes $\left(\mathrm{H}_{\text {TMBtw }}\right)$ wurden bei den Ansaaten mit Saatwicke auf einem gleich hohen Niveau und damit hoch signifikant über den Werten der Reinsaat des Einjährigen Weidelgras (4E) ermittelt. Lagen die Indizes des Einjährigen Weidelgrases in Reinsaat (4E) auf einem ähnlich hohen Niveau wie im Vorjahr, überstiegen die Indizes der Ansaaten mit Saatwicke die 
Werte des Jahres 1998 deutlich $\left(P_{\text {Jahr }}=0,0001\right.$; signifikante Wechselwirkung: Ansaatvariante $\times$ Jahr). Die langjährig unterschiedliche mineralische $N$-Düngung zu den nichtlegumen Vorfüchten $\left(\mathrm{N}_{0}\right.$ und $\left.\mathrm{N}_{3}\right)$ hatte im Jahr 1999 keinen gesicherten Einfluss auf die Höhe der TM-Akkumulation der Ansaaten (Tab. A44).

In Abb. 39 sind die oberirdischen Trockenmasse-Ertragsanteile der Saatwicke und des Einjährigen Weidelgrases in beiden Gemengesaaten zur Ernte am 17.10.1999 dargestellt. Der Ertragsanteil Sprossmasse (Schnittgut + Stoppeln) an Einjährigem Weidelgras betrug in der Ansaatvariante mit $25 \%$ Einjährigem Weidelgras in der Ansaatmischung 7,2\% in der $\mathrm{N}_{3}$-Variante bzw. 1,8 \% in der $\mathrm{N}_{0}$-Variante. In der grasbetonten Ansaatmischung betrugen die TM-Ertragsanteile des Einjährigen Weidelgrases 23,0 \% $\left(\mathrm{N}_{3}\right)$ bzw. 21,5\% $\left(\mathrm{N}_{0}\right)$. Die oberirdischen Ertragsanteile des Einjährigen Weidelgrases im Gemenge wurden auch im zweiten Untersuchungsjahr nicht gesichert durch die Düngungsstufen beeinflusst (Abb. 39), lagen aber signifikant unter den TM-Ertragsanteilen des Vorjahres $\left(P_{\text {Jahr }}=0,0001\right)$.

Abb. 39: Ertragsanteile der Spross-TM beim Anbau der Saatwicke und des Einjährigen Weidelgrases in Gemengesaat zur Ernte (17.10.1999)

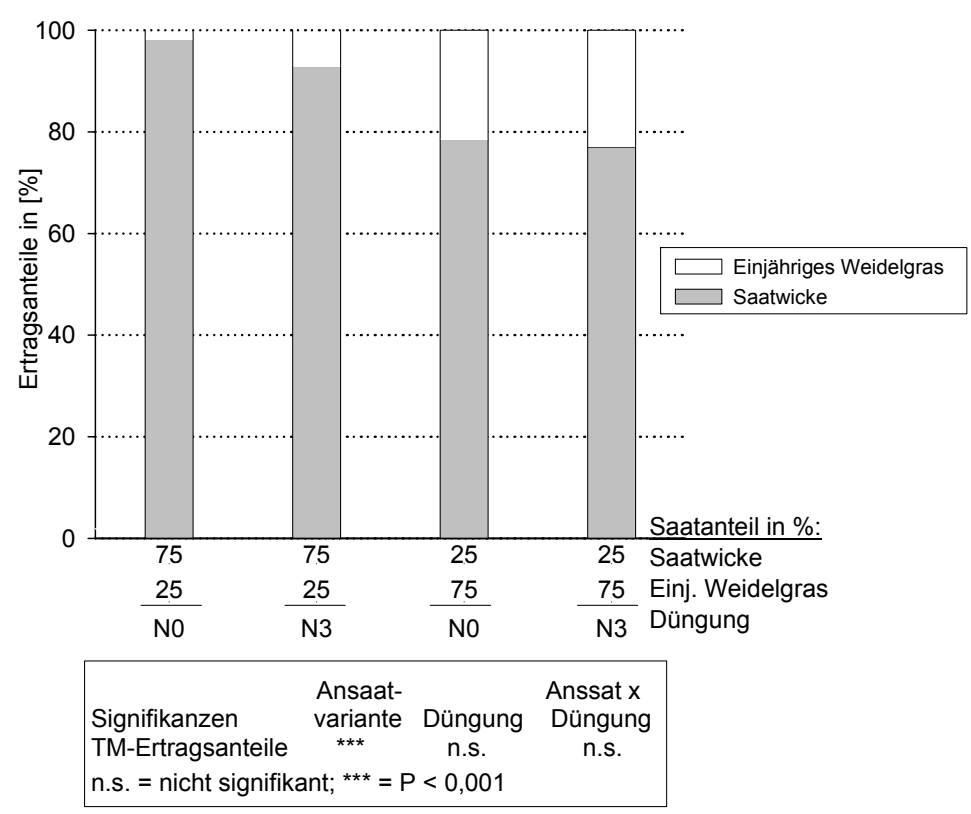

\subsubsection{Stickstofferträge und Stickstoff-Harvest-Index}

Im vorliegenden Kapitel werden die gesamtpflanzlich akkumulierten Stickstoff-Mengen $\left(\mathrm{N}_{\mathrm{Bt}}\right)$, Stickstoff-Erträge in Spross und Wurzel sowie die gewichteten StickstoffHarvest-Indizes $\left(\mathrm{H}_{\mathrm{NBtw}}\right)$ getrennt nach Jahren dargestellt. In den Abb. 40 und Abb. 42 sind die Stickstofferträge und die gewichteten N-Harvest-Indizes graphisch abgebildet. In den Anhangstabellen Tab. A45 und Tab. A46 sind die Mittelwerte und Mittelwertvergleiche zusammengestellt. Schließlich sind in den Abb. 41 und Abb. 43 
die Stickstoff-Ertragsanteile der oberirdischen Biomasse der Gemengeansaaten dargestellt.

Im ersten Untersuchungsjahr wurden im Maximum 6,5 $\mathrm{kg} \mathrm{N}$ ha $^{-1}$, im Jahr 1999 maximal $1,8 \mathrm{~kg} \mathrm{~N} \mathrm{ha}^{-1}$ (Tab. A42) im Spross der Kräuter akkumuliert. Diese StickstoffMengen werden in den weiteren Beschreibungen und Darstellungen nicht weiter berücksichtigt.

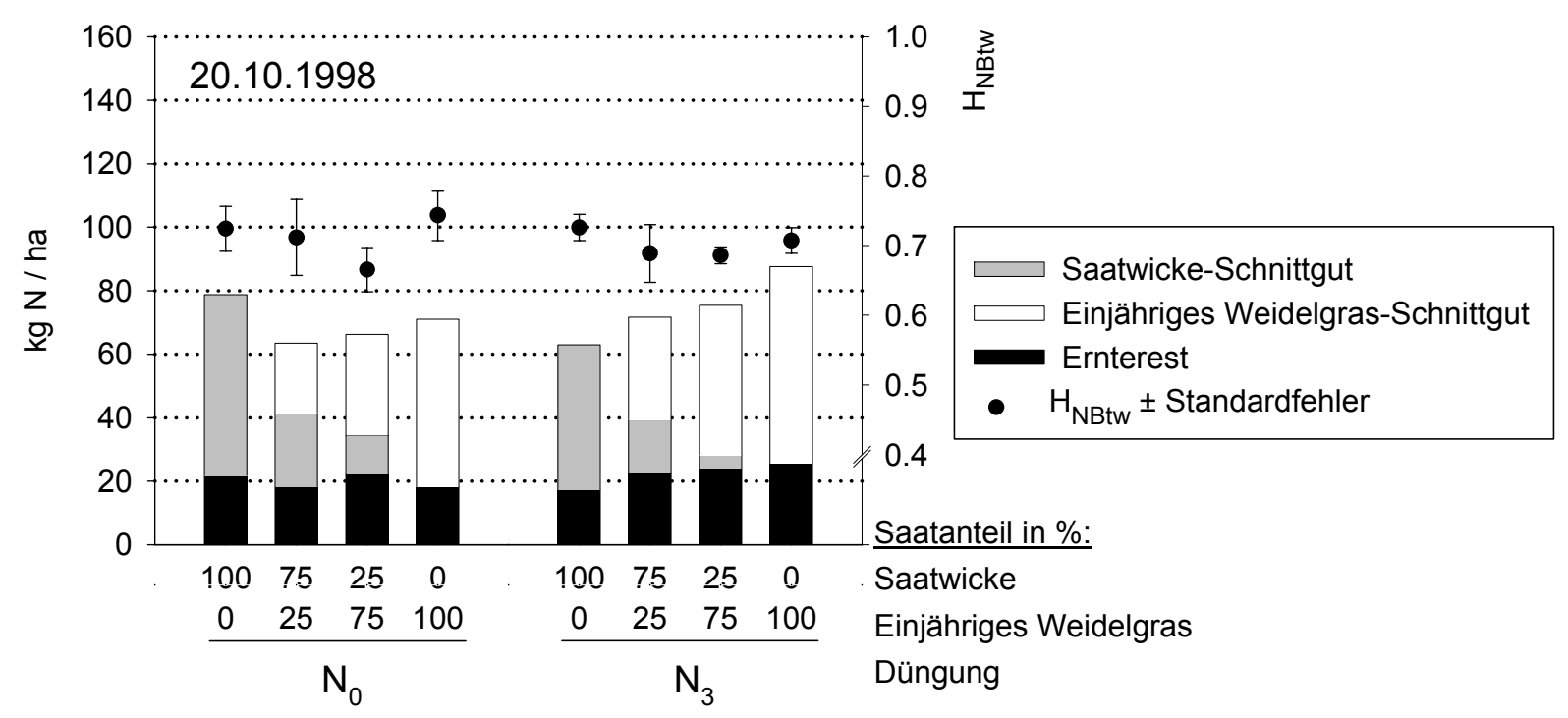

Abb. 40: Gesamtpflanzliche Stickstoff-Akkumulation ( $\mathrm{kg} \mathrm{N} \mathrm{ha}^{-1}$; Erntereste, Schnittgut) sowie gewichtete Stickstoff-Harvest-Indizes $\left(\mathrm{H}_{\mathrm{NBtw}} \pm\right.$ Standardfehler $)$ beim Anbau der Saatwicke und des Einjährigen Weidelgrases in Rein- und Gemengesaat am 20.10.1998

Die gesamtpflanzlichen N-Erträge $\left(\mathrm{N}_{\mathrm{Bt}}\right)$ der Stoppelsaaten der Saatwicke in Reinund Gemengesaat mit Einjährigem Weidelgras erreichten $1998 \mathrm{~N}$-Erträge zwischen 63,0 ( $\left.\mathrm{N}_{3}: 4 \mathrm{~S}\right)$ und 87,6 $\mathrm{kg} \mathrm{N}$ ha- $^{-1}\left(\mathrm{~N}_{3}: 4 \mathrm{E}\right.$; Abb. 40 und Tab. A45). Im Mittel der Düngungsstufen zu den nichtlegumen Vorfrüchten konnten zwischen den Ansaatvarianten keine gesicherten Unterschiede in der gesamtpflanzlichen N-Akkumulation und in den N-Erträgen im Ernterest und Schnittgut beobachtet werden. Ein gesicherter Einfluss der langjährig unterschiedlich hohen mineralischen N-Düngung zu den nichtlegumen Vorfrüchten $\left(\mathrm{N}_{0}\right.$ und $\left.\mathrm{N}_{3}\right)$ konnte lediglich beim Stickstoff der Erntereste festgestellt werden (Tab. A45). Während die N-Mengen im Ernterest der Saatwicke in Reinsaat (4S) in der ungedüngten Variante $\left(\mathrm{N}_{0}\right)$ über den Werten der gedüngten Variante $\left(\mathrm{N}_{3}\right)$ lagen, verhielten sich die Ansaaten mit Einjährigem Weidelgras entgegengesetzt (hoch signifikante Wechselwirkung: Ansaatvariante $\times$ Düngung; Tab. A45). Im Jahr 1998 wurden im Mittel $28 \%$ (4S), $30 \%$ (3S/1E), $32 \%$ (1S/3E) und $27 \%$ (4E) des gesamtpflanzlichen Stickstoffs in den Ernteresten akkumuliert. Die gewichteten gesamtpflanzlichen N-Harvest-Indizes der Saatwicke in Rein- und 
Gemengesaat mit Einjährigem Weidelgras unterschieden sich weder in Abhängigkeit von der Ansaatvariante noch der N-Düngung zu den nichtlegumen Vorfrüchten (Tab. A45).

In Abb. 41 sind die Stickstoff-Ertragsanteile der Saatwicke und des Einjährigen Weidelgrases in beiden Gemengesaaten zur Ernte abgebildet. Der N-Ertragsanteil der Sprossmasse des Einjährigen Weidelgrases am gesamtpflanzlich akkumulierten Stickstoff im Spross betrug in der leguminosenbetonten Ansaatvariante (3S/1E) $68,0 \%$ in der $\mathrm{N}_{3}$-Variante bzw. 53,9 \% in der $\mathrm{N}_{0}$-Variante. In der grasbetonten Ansaatmischung (1S/3E) betrugen die N-Ertragsanteile des Einjährigen Weidelgrases 91,3 \% $\left(\mathrm{N}_{3}\right)$ bzw. 76,1 \% in der $\mathrm{N}_{0}$-Variante. Im Mittel der Ansaatvarianten konnten keine Unterschiede in den N-Ertragsanteilen zwischen den Düngungsstufen festgestellt werden (Abb. 41)

Abb. 41: Spross-Stickstoff-Ertragsanteile von Saatwicke und Einjährigem Weidelgras in Gemengesaat zur Ernte (20.10.1998)

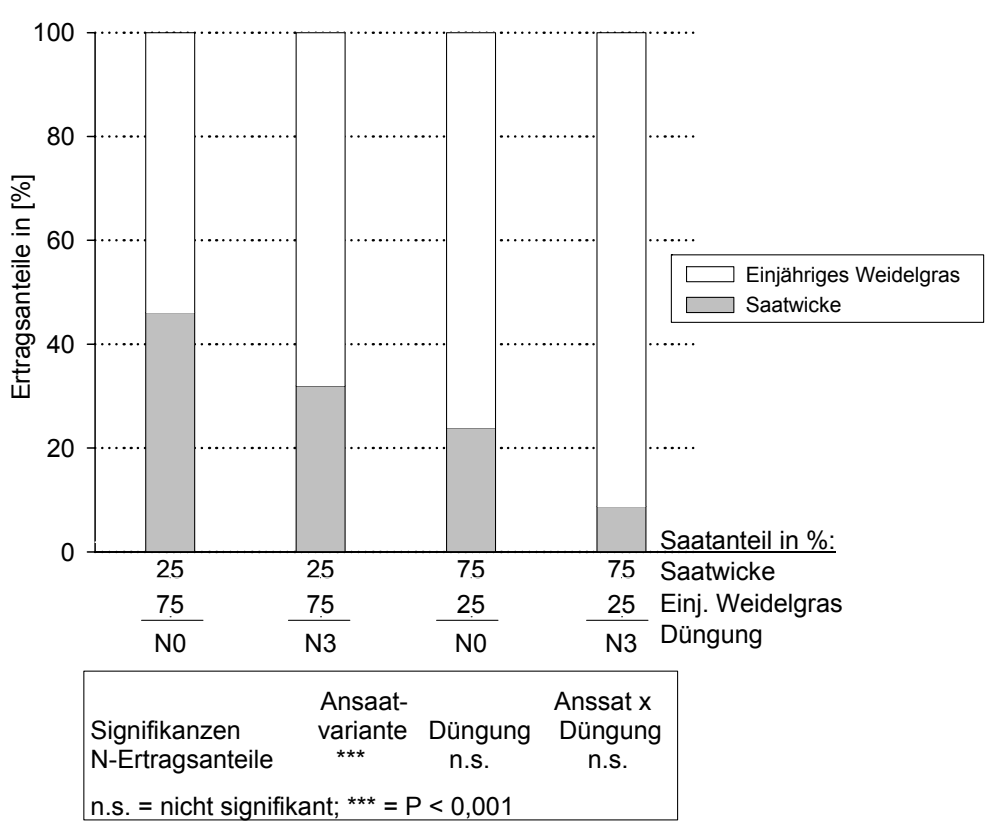

Im zweiten Untersuchungsjahr (1999) stiegen die gesamtpflanzlichen N-Erträge $\left(\mathrm{N}_{\mathrm{Bt}}\right)$ der Saatwicke in Rein- und Gemengesaat mit Einjährigem Weidelgras auf N-Mengen zwischen 50,8 ( $\left.\mathrm{N}_{0}: 4 \mathrm{E}\right)$ und $155,5 \mathrm{~kg} \mathrm{~N}^{-1}\left(\mathrm{~N}_{0}: 4 \mathrm{~S}\right)$ und lagen damit deutlich höher als im Vorjahr ( $P_{\text {Jahr }}=0,0001$; Abb. 42 und Tab. A46). Während sich die Erträge der Ansaaten mit Saatwicke deutlich erhöhten, nahmen die N-Erträge des Einjährigen Weidelgrases in Reinsaat (4E) leicht ab (sehr hoch signifikante Wechselwirkung zwischen Ansaatvariante und Jahr). Damit lagen sowohl die gesamtpflanzlichen als auch die im Schnittgut akkumulierten N-Mengen des Einjährigen Weidelgrases in Reinsaat (4E) signifikant unter den Erträgen der Ansaaten mit Saatwicke (Tab. A46). Die Ernterest-N-Mengen lagen im Jahr 1999 signifikant unter den Ernterest-N-Mengen des Vorjahres $\left(P_{\text {Jahr }}=0,0001\right)$. Damit wurde im Jahr 1999 in den Ernteresten der Ansaaten mit Saatwicke mit im Mittel $10 \%$ (4S), $11 \%$ (3S/1E), 12 \% (1S/3E) deutlich 
weniger und in den Ernteresten des Einjährigen Weidelgrases in Reinsaat (4E: $32 \%$ ) deutlich mehr Stickstoff akkumuliert. Hieraus ergeben sich im Mittel der Düngungsstufen im Vergleich zum Vorjahr deutlich gestiegene gewichtete gesamtpflanzliche NHarvest-Indizes der Ansaaten mit Saatwicke und signifikant niedrigere N-HarvestIndizes beim Einjährigen Weidelgras in Reinsaat (4E; Tab. A46; $P_{\text {Jahr }}=0,0001$; signifikante Wechselwirkung zwischen Ansaatvariante und Jahr). Weder die gesamtpflanzliche Stickstoffakkumulation noch die Stickstoffakkumulation im Schnittgut oder den Ernteresten wurde durch die N-Düngung zu den nichtlegumen Vorfrüchten ( $\mathrm{N}_{0}$ und $\mathrm{N}_{3}$ ) nachweisbar beeinflusst (Tab. A46).

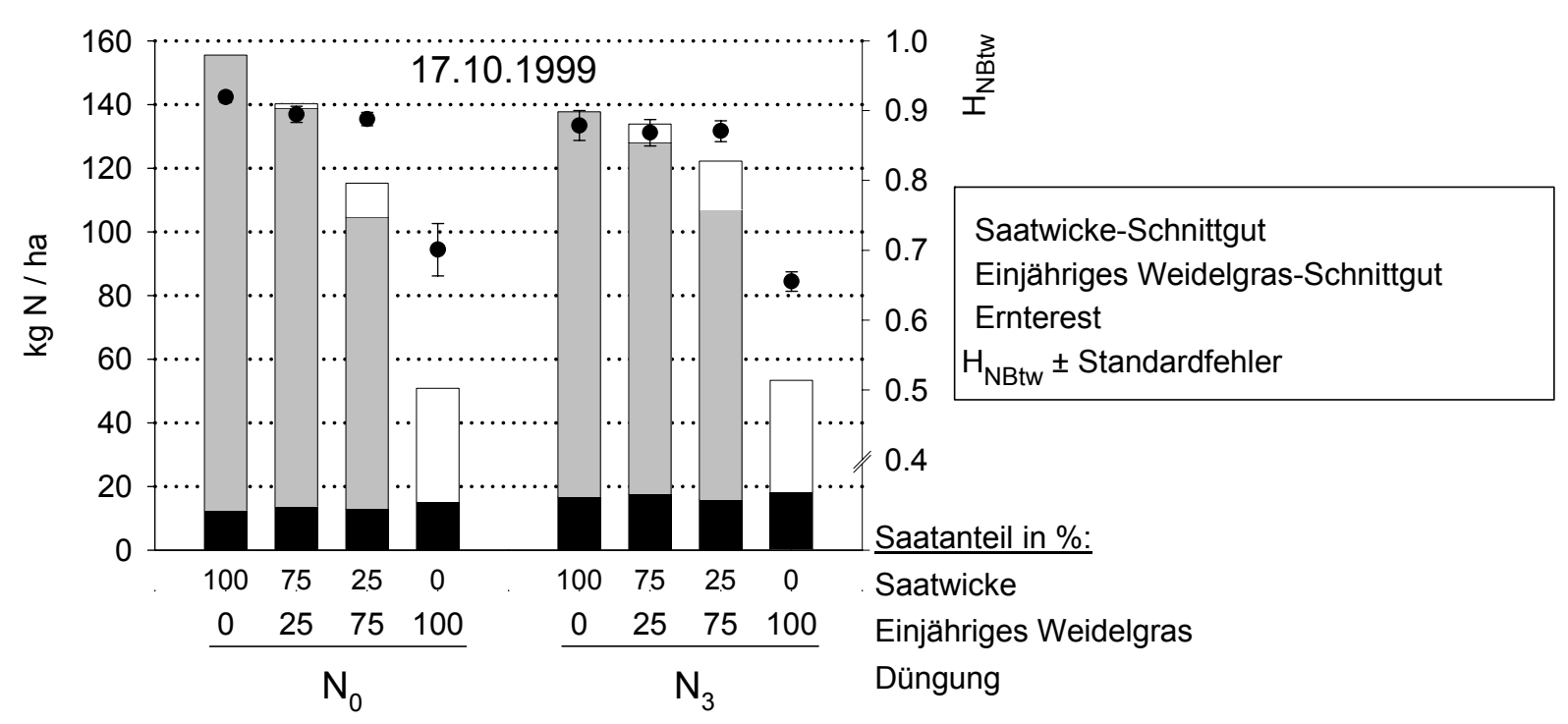

Abb. 42: Gesamtpflanzliche Stickstoff-Akkumulation ( $\mathrm{kg} \mathrm{N} \mathrm{ha}^{-1}$; Erntereste, Schnittgut) sowie gewichtete Stickstoff-Harvest-Indizes $\left(\mathrm{H}_{\mathrm{NBtw}} \pm\right.$ Standardfehler) beim Anbau von Saatwicke und Einjährigem Weidelgras in Rein- und Gemengesaat am 17.10.1999

In Abb. 43 sind die Stickstoff-Ertragsanteile der Saatwicke und des Einjährigen Weidelgrases in beiden Gemengesaaten zur Ernte abgebildet. Der N-Ertragsanteil der Sprossmasse des Einjährigen Weidelgrases am gesamtpflanzlich akkumulierten Stickstoff im Spross betrug in der leguminosenbetonten Ansaatvariante (3S/1E) $6,3 \%\left(\mathrm{~N}_{3}\right)$ bzw. $1,6 \%$ in der $\mathrm{N}_{0}$-Variante. In der grasbetonten Ansaatmischung (1S/3E) betrugen die $\mathrm{N}$-Ertragsanteile des Einjährigen Weidelgrases 19,3\% $\left(\mathrm{N}_{3}\right)$ bzw. $14,4 \%$ in der $\mathrm{N}_{0}$-Variante. Im Mittel der Ansaatvarianten wurden die N-Ertragsanteile auch im zweiten Jahr nicht gesichert durch die N-Düngung zu den nichtlegumen Vorfrüchten beeinflusst (Abb. 43). Sie lagen im Mittel deutlich unter den NErtragsanteilen des Vorjahres $\left(P_{\mathrm{Jahr}}=0,0001\right)$. 
Abb. 43: Spross-Stickstoff-Ertragsanteile der Saatwicke und des Einjährigen Weidelgrases in Gemengesaat zur Ernte am 17.10.1999

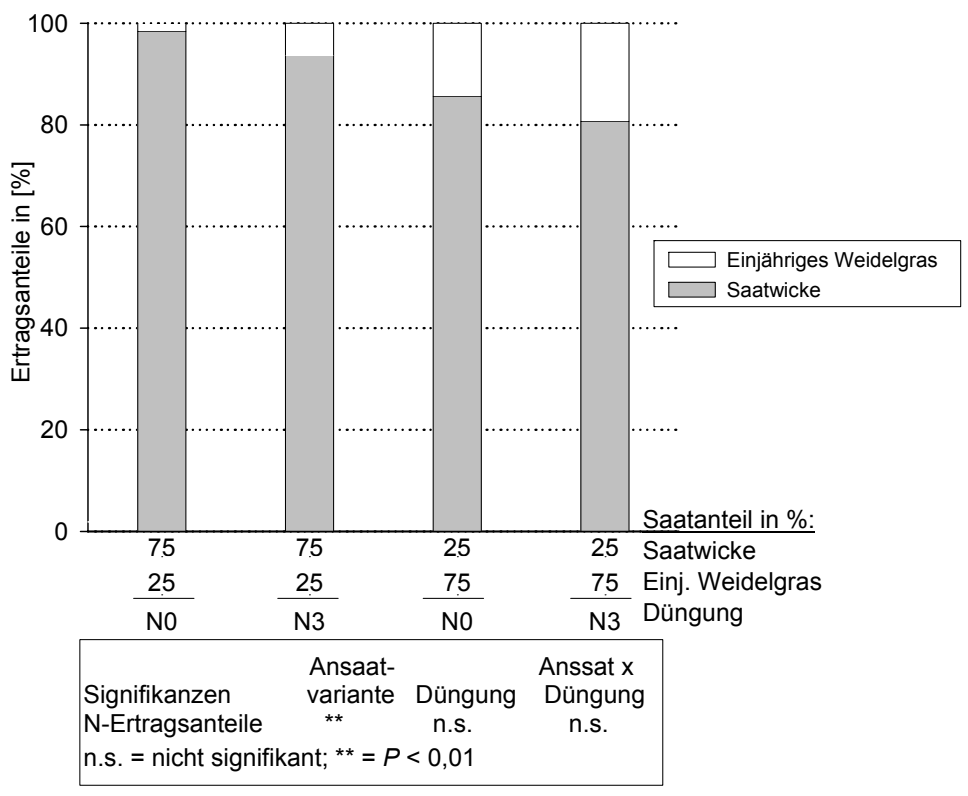




\subsubsection{N-Isotopenverteilung in der gesamtpflanzlichen Biomasse}

In Abb. 44 sind die arithmetischen Mittelwerte der gewichteten gesamtpflanzlichen $\delta^{15} \mathrm{~N}$-Werte, in Tab. A47 die gewichteten $\delta^{15} \mathrm{~N}$-Werte in der Sprossmasse, der Saatwicke und des Einjährigen Weidelgrases in Rein- und Gemengesaat für die Jahre 1998 und 1999 dargestellt. Die gewichteten gesamtpflanzlichen $\delta^{15} \mathrm{~N}$-Werte des Einjährigen Weidelgrases in Reinsaat (4E) lagen im Mittel der Düngungsstufen in beiden Jahren mit Werten von 3,73\%o (1998) und 2,77\%o (1999) hoch bzw. sehr hoch signifikant über den Werten von Saatwicke in Reinsaat (4S; 1998: 1,95\%; 1999: 0,60 \%; Tab. A48). Während im Jahr 1998 die gesamtpflanzlichen $\delta^{15} \mathrm{~N}$-Werte von der Saatwicke in Reinsaat (4S) mit zunehmenden Anteil Einjährigen Weidelgrases im Bestand signifikant anstiegen, unterschieden sich die gesamtpflanzlichen $\delta^{15} \mathrm{~N}$-Werte der Ansaaten mit Saatwicke im Jahr 1999 nicht bzw. im Vergleich mit Einjährigem Weidelgras in Reinsaat (4E) deutlich (Tab. A48).

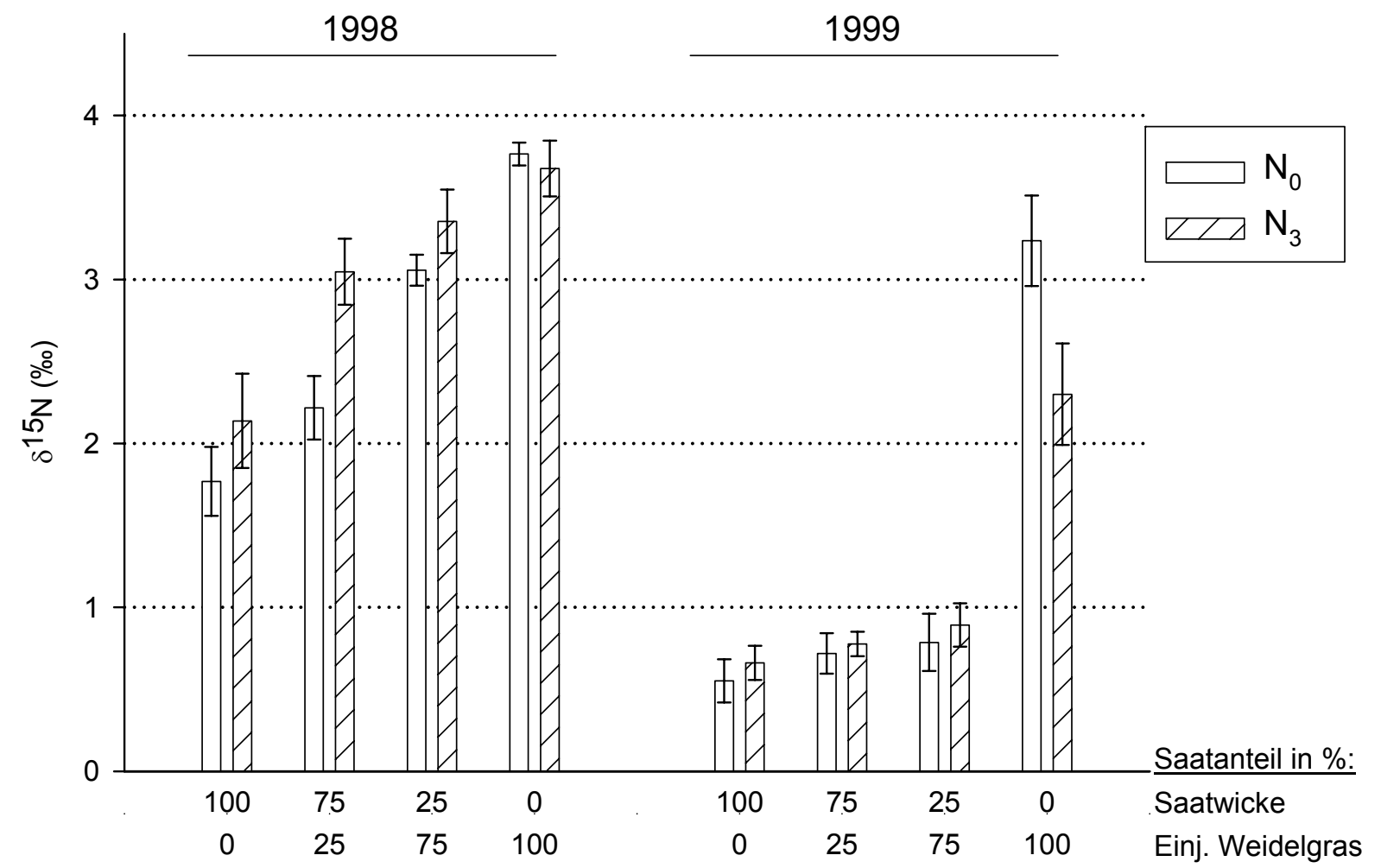

Abb. 44: Gewichtete $\delta^{15} \mathrm{~N}$-Werte (arithmetische Mittelwerte \pm Standardfehler in $\%$ ) in der gesamtpflanzlichen Biomasse (Erntereste + Schnittgut) beim Anbau von Saatwicke in Rein- und Gemengesaat mit Einjährigem Weidelgras im Jahr 1998 und 1999

Die $\delta^{15} \mathrm{~N}$-Werte der langfristig nicht mit mineralischen $\mathrm{N}$-Düngern zu den nichtlegumen Vorfü̈chten gedüngten Variante $\left(N_{0}\right)$ lagen im Jahr 1998 bei den Ansaaten mit 
Saatwicke unter, bei Einjährigem Weidelgras in Reinsaat (4E) über den $\delta^{15} \mathrm{~N}$-Werten der gedüngten Variante (signifikante Wechselwirkung: Ansaatvariante $\times$ Düngung; Tab. A48). Im Vergleich zu 1998 lagen die $\delta^{15} \mathrm{~N}$-Werte im Jahr 1999 bei den Ansaaten mit Saatwicke und Einjährigem Weidelgras um 1,35 (4S), 1,9 (3S/1E), 2,4 (1S/3E) und 0,9\%o-Punkte (4E) unter den $\delta^{15} \mathrm{~N}$-Werten in der Biomasse des Vorjahres (sehr hoch signifikante Wechselwirkung: Ansaatvariante $\times$ Jahr).

\subsubsection{Vergleich der Schätzergebnisse der symbiotisch fixierten N-Menge mittels der $\delta^{15} \mathrm{~N}$ - und erweiterter Differenzmethode}

In Abb. 45 sind die Korrelationen zwischen den Ergebnissen der beiden Schätzmethoden zur Quantifizierung der luftbürtigen N-Menge beim Anbau von Saatwicke in Rein- und Gemengesaat mit Einjährigem Weidelgras für die Untersuchungsjahre 1998 (a) und 1999 (b) dargestellt. Die Abbildungen beinhalten alle Schätzergebnisse inklusive der ermittelten "negativen" Werte.

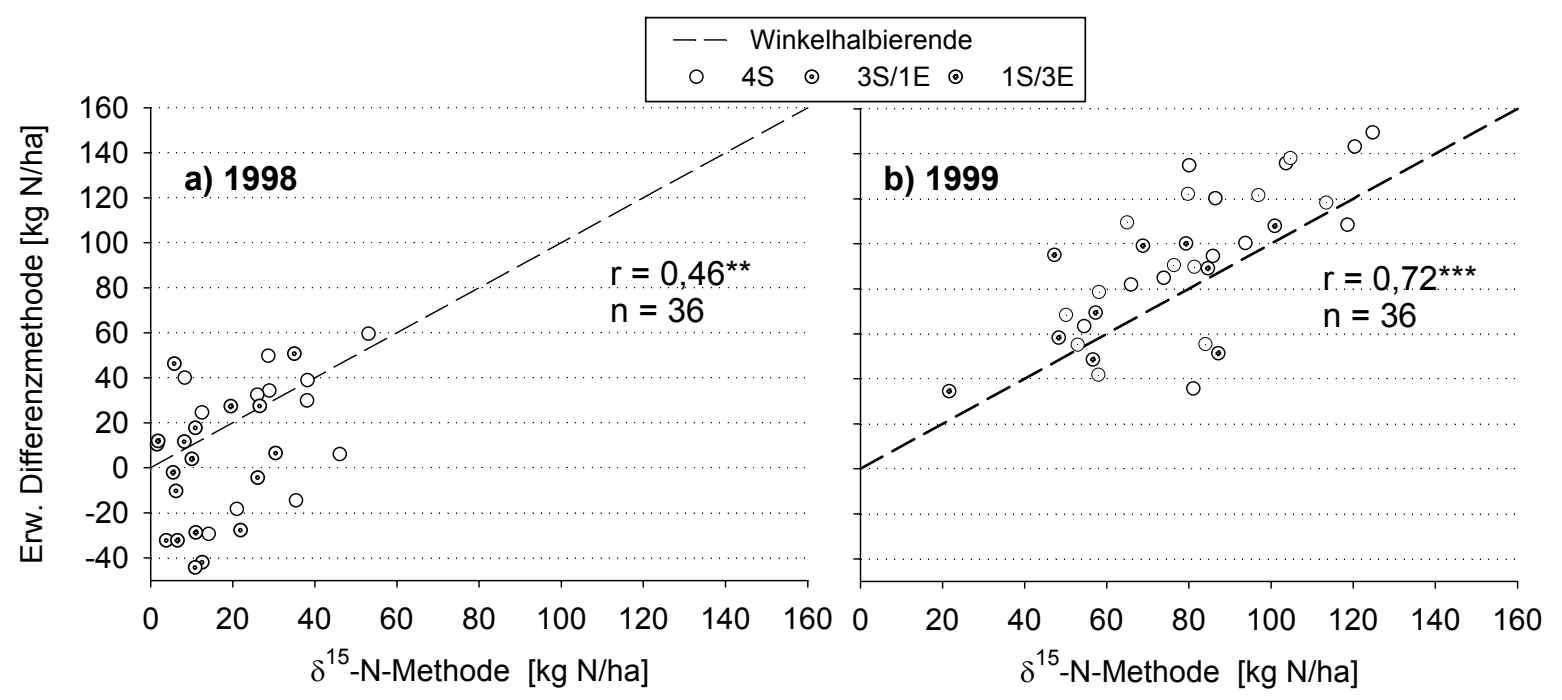

Abb. 45: Korrelation zwischen den Ergebnissen der $\delta^{15} \mathrm{~N}$ - und erweiterter Differenzmethode zur Schätzung der gesamtpflanzlich symbiotisch fixierten $\mathrm{N}$ Menge a) im Jahr 1998 und b) im Jahr 1999 beim Anbau von Saatwicke in Reinsaat (4S) und Gemengesaat mit Einjährigem Weidelgras (3S/1E und 1S/3E).

Der Korrelationskoeffizient für den Vergleich der Ergebnisse zur Schätzung der gesamtpflanzlich fixierten N-Menge lag im Jahr 1999 mit 0,72 deutlich über dem Korrelationskoeffizient, der im Jahr 1998 mit 0,46 ermittelt wurde (Abb. 45). Das Signifikanzniveau war im Jahr 1998 weniger hoch $(P=0,005)$ als im Jahr $1999(P=$ $0,0001)$. Die Schätzwerte, die mittels erweiterter Differenzmethode berechnet wur- 
den, lagen im Mittel aller Varianten 12,6 kg N ha-1 (1998) unter bzw. 12,3 kg N ha-1 (1999) über der luftbürtigen N-Menge, die mit der $\delta^{15} \mathrm{~N}$-Methode geschätzt wurde. In 1998 lagen die Schätzwerte die mittels erweiterter Differenzmethode ermittelt wurden bis maximal $40,5 \mathrm{~kg} \mathrm{~N}^{-1} a^{-1}$ über bzw. $55,2 \mathrm{~kg} \mathrm{~N}^{-1}$ unter den Werten, die mittels $\delta^{15} \mathrm{~N}$-Methode geschätzt wurden (Abb. 45a). Im zweiten Untersuchungsjahr (1999) wurden die Schätzergebnisse der $\delta^{15} \mathrm{~N}$-Methode durch die mit der erweiterten Differenzmethode ermittelten Werte bis maximal $54,8 \mathrm{~kg} \mathrm{~N}^{-1}$ über- bzw. bis $45,4 \mathrm{~kg} \mathrm{~N}$ ha $^{-1}$ unterschätzt (Abb. 45b). Rechnerisch "negative" Fixierleistungen wurden ausschließlich im Jahr 1998 bei 17 von 36 Schätzwerten, die mittels der Differenzmethode ermittelt wurden, festgestellt. Dabei wiesen drei Werte der Reinsaatvariante (4S) sowie jeweils sieben Werte der Gemengevarianten (3S/1E und 1S/3E) "negative" Werte aus.

\subsubsection{Luft- und bodenbürtige N-Akkumulation sowie $\mathrm{CaCl}_{2}$-extrahierbarer $\mathbf{N}_{\text {min }}$-Stickstoff im Boden}

Die vor der Aussaat der Saatwicke und des Einjährigen Weidelgrases am 21.07.1998 vorgefundenen Mengen an pflanzenverfügbarem Stickstoff im Boden sind der Abb. 46 und im Anhang der Tab. A49 a zu entnehmen. Mit $\mathrm{N}_{\text {min }}$-Mengen in Höhe von 28,8 $\mathrm{kg} \mathrm{N}$ ha $^{-1}(0$ bis $125 \mathrm{~cm}$ ) wiesen die langjährig nicht mit mineralischen N-Düngern zu den nichtlegumen Vorfüchten gedüngten Parzellen $\left(\mathrm{N}_{0}\right)$ signifikant niedrigere $\mathrm{N}_{\text {min }}{ }^{-}$ Mengen im Vergleich zu der gedüngten Variante auf $\left(\mathrm{N}_{3} ; 76,2 \mathrm{~kg} \mathrm{~N}^{-1}\right)$. Während sich die Düngungsstufen im Oberboden in der $\mathrm{N}_{\text {min }}$-Menge um 44,6 kg unterschieden, waren es im Unterboden nur 2,8 $\mathrm{kg} \mathrm{N}^{-1}$. Allerdings waren die Unterschiede sowohl im Ober- als auch im Unterboden signifikant verschieden (Tab. A49a).

Die residualen $\mathrm{N}_{\text {min }}$-Mengen im Boden, die zur Ernte am 20.10.1998 unter den Ansaaten vorgefunden wurden, lagen in 0 bis $125 \mathrm{~cm}$ Bodentiefe zwischen 22,6 ( $\mathrm{N}_{0}$ : 4E) und $73,7 \mathrm{~kg} \mathrm{~N} \mathrm{ha}^{-1}\left(\mathrm{~N}_{3}: 4 \mathrm{~S}\right)$. Im Mittel der Düngungsstufen lagen die residualen $\mathrm{N}_{\text {min }}$-Mengen unter Einjährigem Weidelgras in Reinsaat (4E) sowohl im Unter- als auch im Oberboden signifikant unter den residualen $\mathrm{N}_{\min }$-Mengen im Boden nach Saatwicke in Reinsaat (4S) und im Gemenge mit $25 \%$ Einjährigem Weidelgras (3S/1E; Tab. A50). Die im Mittel der Ansaatvarianten signifikant höheren $\mathrm{N}_{\min }$-Mengen unter der langjährig gedüngten Variante $\left(N_{3}\right)$, gehen insbesondere auf deutliche Unterschiede im Unterboden zwischen den Düngungsstufen zurück (Tab. A50). Zum Zeitpunkt des ersten $\mathrm{N}_{\text {min }}$-Probenahmetermins nach Bestandesetablierung am 03.09.1998 lagen die $\mathrm{N}_{\min }$-Mengen unter Einjährigem Weidelgras in Reinsaat (4E) und im Gemenge mit $25 \%$ Saatwicke (1S/3E) bereits signifikant unter den $\mathrm{N}_{\text {min- }}$ Mengen im Boden unter Saatwicke in Reinsaat (4S) und im Gemenge mit $25 \%$ Einjährigem Weidelgras (3S/1E). Im Zeitraum vom ersten $\mathrm{N}_{\text {min }}$-Probenahmetermin bis zur Ernte nahmen die $\mathrm{N}_{\text {min }}$-Mengen im Mittel im Oberboden tendenziell ab, im Unterboden tendenziell zu. 


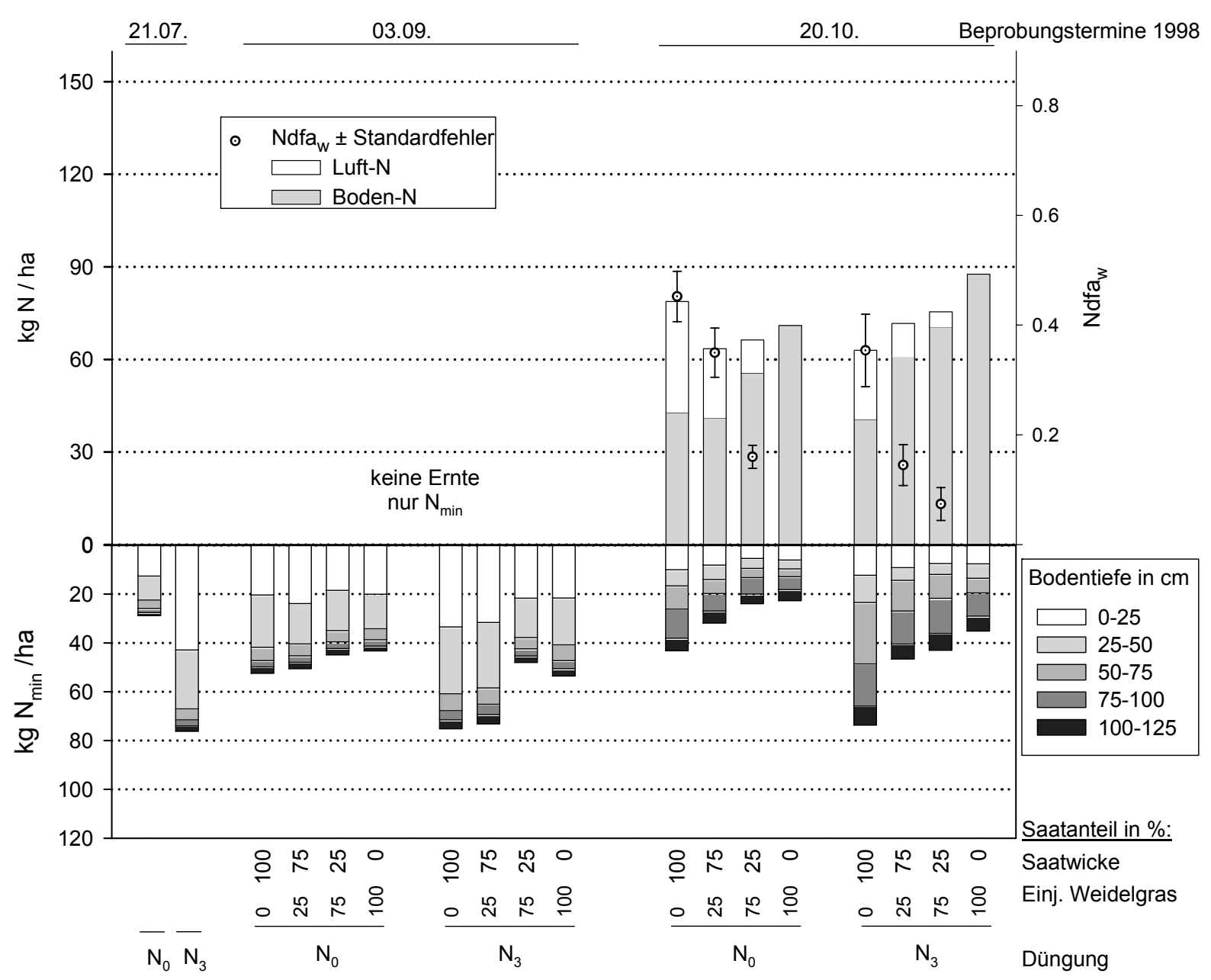

Abb. 46: $\mathrm{CaCl}_{2}$-extrahierbarer $\mathrm{N}_{\min }$-Stickstoff im Boden $\left(\mathrm{kg} \mathrm{N}_{\min } \mathrm{ha}^{-1}\right)$, boden- und luftbürtige N-Akkumulation in der gesamtpflanzlichen Biomasse $\left(\mathrm{kg} \mathrm{N} \mathrm{ha}^{-1}\right)$ und gewichtete Anteile Stickstoff aus der Luft $\left(\mathrm{Ndfa}_{w} \pm\right.$ Standardfehler) beim Anbau der Saatwicke und des Einjährigen Weidelgrases in Rein- und Gemengesaat im Jahr 1998

Analog zur Vorgehensweise bei Ackerbohne und Luzerne beziehen sich die Anteile Stickstoff aus der Luft (Ndfaw) bei den Ansaaten mit Saatwicke und Einjährigem Weidelgras im Gemenge (3S/1E und 1S/3E) auf die Summe des Stickstoffs (inkl. Wurzeln) in beiden Gemengepartnern (Kap. 2.7.2.3). Die Anteile Stickstoff aus der Luft im Spross von Saatwicke in Rein- und Gemengesaat sind im Anhang der Tab. A47 zu entnehmen. Hierbei stiegen die Anteile Stickstoff aus der Luft im Mittel über die Düngungsstufen von Saatwicke in Reinsaat (4S) zur Saatwicke im Gemenge mit 25\% Einjährigem Weidelgras (3S/1E) tendenziell, zur Saatwicke im Gemenge mit $75 \%$ Einjährigem Weidelgras (1S/3E) signifikant an.

Die gewichteten Anteile Stickstoff aus der Luft in der gesamtpflanzlichen Biomasse der Bestände mit Saatwicke sanken mit zunehmendem Anteil von Einjährigem Wei- 
delgras im Bestand signifikant ab (Tab. A51). Dabei lagen die gewichteten Anteile Stickstoff aus der Luft in der $\mathrm{N}_{0}$-Variante signifikant über den Anteilen in den Beständen der $\mathrm{N}_{3}$-Variante. Einjähriges Weidelgras in Reinsaat (4E) akkumulierte im Vergleich zu den Ansaaten mit Saatwicke mit 71,0 und 87,6 kg N ha- ${ }^{-1}$ (4E: $\mathrm{N}_{0}$ und $\mathrm{N}_{3}$ ) im Mittel der Düngungsstufen signifikant mehr Bodenstickstoff (Tab. A51). Mit 35,9 und 22,4 $\mathrm{kg} \mathrm{N} \mathrm{ha}^{-1}$ (4S: $\mathrm{N}_{0}$ und $\mathrm{N}_{3}$ ) fixierte die Saatwicke in Reinsaat (4S) signifikant mehr Stickstoff aus der Luft als im Gemenge mit $75 \%$ Einjährigem Weidelgras (1S/3E N $N_{0}$ : $10,7 \mathrm{~kg} \mathrm{~N} \mathrm{ha}^{-1}$ bzw. $\mathrm{N}_{3}: 4,9 \mathrm{~kg} \mathrm{~N} \mathrm{ha}^{-1}$ ).

Im zweiten Untersuchungsjahr beliefen sich die vor Aussaat der Saatwicke und des Einjährigen Weidelgrases am 09.07.1999 vorgefundenen Mengen an pflanzenverfügbarem Stickstoff im Boden auf 25,5 bzw. $36,8 \mathrm{~kg} \mathrm{~N} \mathrm{ha}^{-1}\left(\mathrm{~N}_{0}\right.$ bzw. $\mathrm{N}_{3}$ : 0 bis $125 \mathrm{~cm}$ ) und lagen damit signifikant unter den $\mathrm{N}_{\text {min }}$-Mengen vor Aussaat im Vorjahr $\left(P_{\mathrm{Jahr}}=\right.$ 0,0118). Die Unterschiede zwischen den Düngungsstufen $\left(N_{0}\right.$ und $\left.N_{3}\right)$ konnten weder im Ober- noch im Unterboden statistisch gesichert werden (Tab. A49b).

Die residualen $\mathrm{N}_{\text {min }}$-Mengen im Boden, die bis zur Ernte von den Ansaaten nicht genutzt wurden, beliefen sich im Jahr 1999 in 0 bis $125 \mathrm{~cm}$ Bodentiefe auf Werte zwischen 15,2 ( $\left.\mathrm{N}_{0}: 4 \mathrm{E}\right)$ und $39,3 \mathrm{~kg} \mathrm{~N}^{-1}\left(\mathrm{~N}_{3}: 3 \mathrm{~S} / 1 \mathrm{E}\right)$ und lagen somit unter den residualen $\mathrm{N}_{\text {min }}$-Mengen im Jahr $1998\left(P_{\text {Jahr }}=0,0214\right)$. Während sich die residualen $\mathrm{N}_{\text {min }}$-Mengen im Mittel bei Saatwicke in Reinsaat (4S) um 25,5 kg N ha- im Vergleich zum Vorjahr verringerten, waren es bei Einjährigem Weidelgras in Reinsaat (4E) nur $10,5 \mathrm{~kg} \mathrm{~N} \mathrm{ha}^{-1}$ (signifikante Wechselwirkung: Ansaatvariante $\times$ Jahr). Im Mittel der Düngungsstufen lagen die residualen $\mathrm{N}_{\text {min }}$-Mengen unter Einjährigem Weidelgras in Reinsaat (4E) sowohl im Oberboden als auch über die gesamte Profiltiefe signifikant unter den residualen $\mathrm{N}_{\text {min }}$-Mengen im Boden nach Saatwicke in Reinsaat (4S) und im Gemenge mit $25 \%$ Einjährigem Weidelgras (3S/1E; Tab. A52). Zum Zeitpunkt des ersten $\mathrm{N}_{\min }$-Probenahmetermins (24.08.1999) wurden im Mittel der Düngungsstufen keine Unterschiede zwischen den Ansaatvarianten festgestellt. Im Mittel der Ansaatvarianten lagen die an beiden Probenahmeterminen (24.08. und 17.10.1999) ermittelten $\mathrm{N}_{\text {min }}$-Mengen der $\mathrm{N}_{3}$-Variante über die gesamte Profiltiefe signifikant über den Werten der $\mathrm{N}_{0}$-Variante (Tab. A52). Im Zeitraum vom ersten $\mathrm{N}_{\text {min }}$-Probenahmetermin bis zur Ernte reduzierten die Ansaaten die $\mathrm{N}_{\text {min }}$-Mengen im Boden vor allem im Oberboden.

Die Anteile Stickstoff aus der Luft im Spross von Saatwicke in Rein- und Gemengesaat 1999 sind im Anhang der Tab. A47 zu entnehmen. Während die Abnahme der Anteile Stickstoff aus der Luft im Mittel über die Düngungsstufen von Saatwicke in Reinsaat (4S) zur Saatwicke im Gemenge mit 25\% Einjährigem Weidelgras (3S/1E) statistisch nicht gesichert werden konnte, war der Anstieg zur Saatwicke im Gemenge mit $75 \%$ Einjährigem Weidelgras (1S/3E) signifikant. 


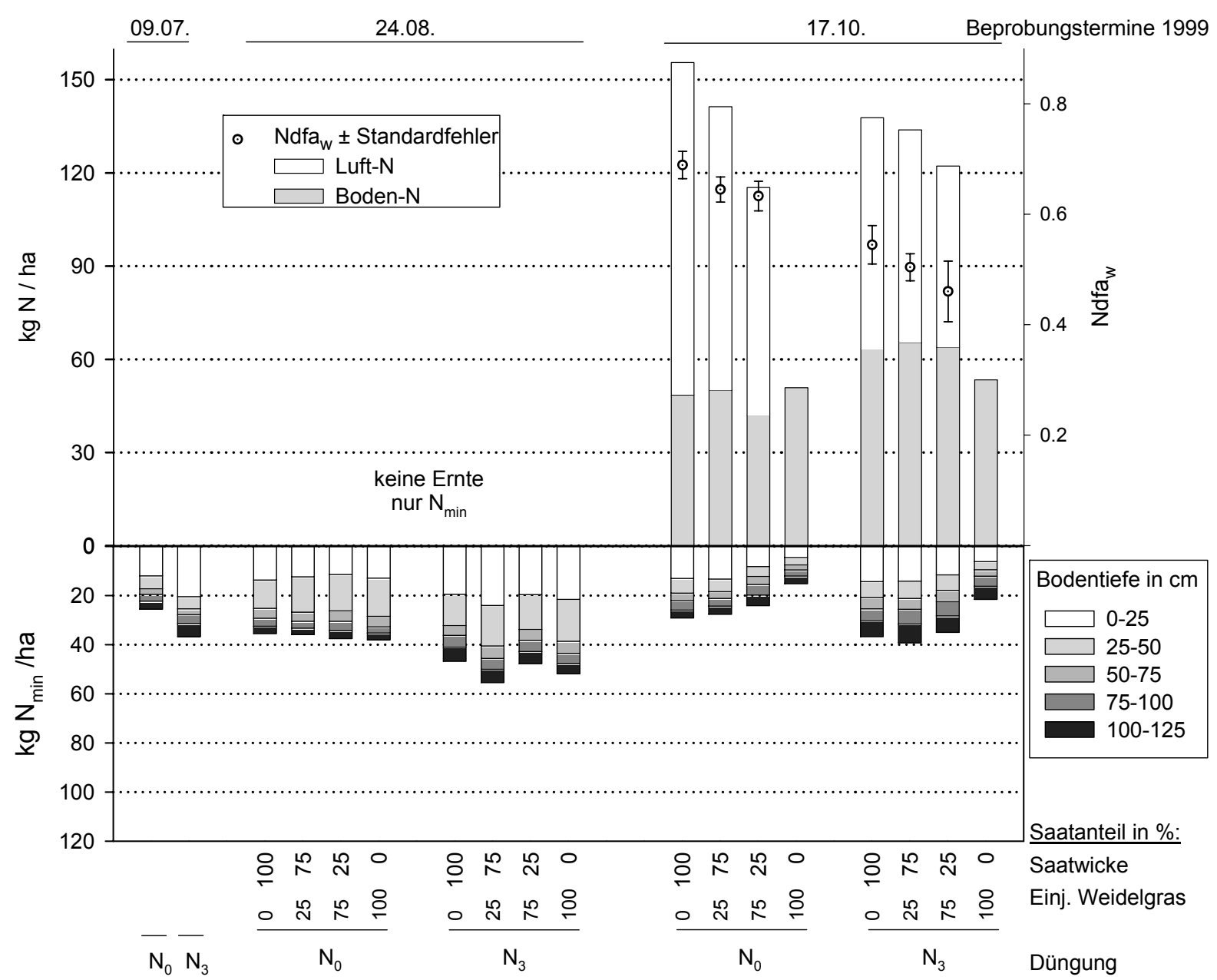

Abb. 47: $\mathrm{CaCl}_{2}$-extrahierbarer $\mathrm{N}_{\min }$-Stickstoff im Boden $\left(\mathrm{kg} \mathrm{N}_{\min } \mathrm{ha}^{-1}\right)$, boden- und luftbürtige N-Akkumulation in der gesamtpflanzlichen Biomasse $\left(\mathrm{kg} \mathrm{N}^{-1}\right)$ und gewichtete Anteile Stickstoff aus der Luft $\left(\mathrm{Ndfa}_{w} \pm\right.$ Standardfehler) beim Anbau der Saatwicke und des Einjährigen Weidelgrases in Rein- und Gemengesaat im Jahr 1999

Die gewichteten Anteile Stickstoff aus der Luft (Ndfaw) in der gesamtpflanzlichen Biomasse wiesen im Mittel der Ansaatvarianten mit Saatwicke keine gesicherten Unterschiede auf, lagen aber in der $\mathrm{N}_{0}$-Variante signifikant über den Anteilen in der Biomasse der $\mathrm{N}_{3}$-Variante (Tab. A53). Die gewichteten Anteile Stickstoff aus der Luft $\left(\mathrm{Ndfa}_{\mathrm{w}}\right)$ lagen im Jahr $1999 \mathrm{im}$ Mittel deutlich über den Werten des Jahres 1998 ( $P_{\text {Jahr }}$ $=0,0001)$. Während die gewichteten Anteile Stickstoff aus der Luft $\left(\mathrm{Ndfa}_{w}\right)$ der Gemengeansaaten (3S/1E und 1S/3E) im Mittel um 33 bzw. 54,8 Prozentpunkte im Vergleich zum Vorjahr anstiegen, waren es bei Saatwicke in Reinsaat (4S) 20,5 Prozentpunkte (signifikante Wechselwirkung: Ansaatvariante $\times$ Jahr). Während sich die akkumulierten bodenbürtigen N-Mengen weder zwischen den Ansaatvarianten noch zwischen den Beständen unterschieden, fixierten die Ansaaten in der $\mathrm{N}_{0}$-Vari- 
ante signifikant mehr Stickstoff aus der Luft als die Ansaaten der $\mathrm{N}_{3}$-Variante (Tab. A53). Konnten im Mittel der Ansaatvarianten und Düngungsstufen keine gesicherten Unterschiede an aufgenommenen Mengen an Bodenstickstoff im Vergleich der Jahre festgestellt werden, zeigten sich bei den Ansaatvarianten mit Saatwicke im Mittel leicht erhöhte bzw. bei Einjährigem Weidelgras in Reinsaat (4E) deutlich geringere Boden-N-Mengen, die von den Pflanzen aufgenommen wurden (signifikante Wechselwirkung: Ansaatvariante $\times$ Jahr). Mit 107,0 und $74,4 \mathrm{~kg} \mathrm{~N}^{-1}\left(4 \mathrm{~S}: \mathrm{N}_{0}\right.$ und $\mathrm{N}_{3}$ ) fixierte die Saatwicke in Reinsaat signifikant mehr Stickstoff aus der Luft als im Gemenge mit $75 \%$ Einjährigem Weidelgras (1S/3E, $\mathrm{N}_{0}: 73,3$ und $\mathrm{N}_{3}: 58,3 \mathrm{~kg} \mathrm{~N} \mathrm{ha}^{-1}$; Tab. A53). Damit lagen auch die fixierten N-Mengen im Mittel der Ansaaten über den Werten des Vorjahres $\left(P_{\text {Jahr }}=0,0001\right)$.

\subsubsection{Transfer-Stickstoff beim Anbau von Einjährigem Weidelgras im Gemenge mit Saatwicke}

Weder im Jahr 1998 noch im Jahr 1999 konnten signifikante Unterschiede zwischen den $\delta^{15} \mathrm{~N}$-Werten in der Sprossmasse des Einjährigen Weidelgrases aus Reinsaat (4E) und aus Gemengesaat mit Saatwicke (3S/1E und 1S/3E) festgestellt werden (Abb. 48 und Tab. A54). Die Unterschiede im $\delta^{15} \mathrm{~N}$-Wert zwischen den Jahren waren signifikant und es traten signifikante Wechselwirkungen zwischen den Jahren und der Düngung sowie zwischen der Düngung und den Ansaatvarianten auf.

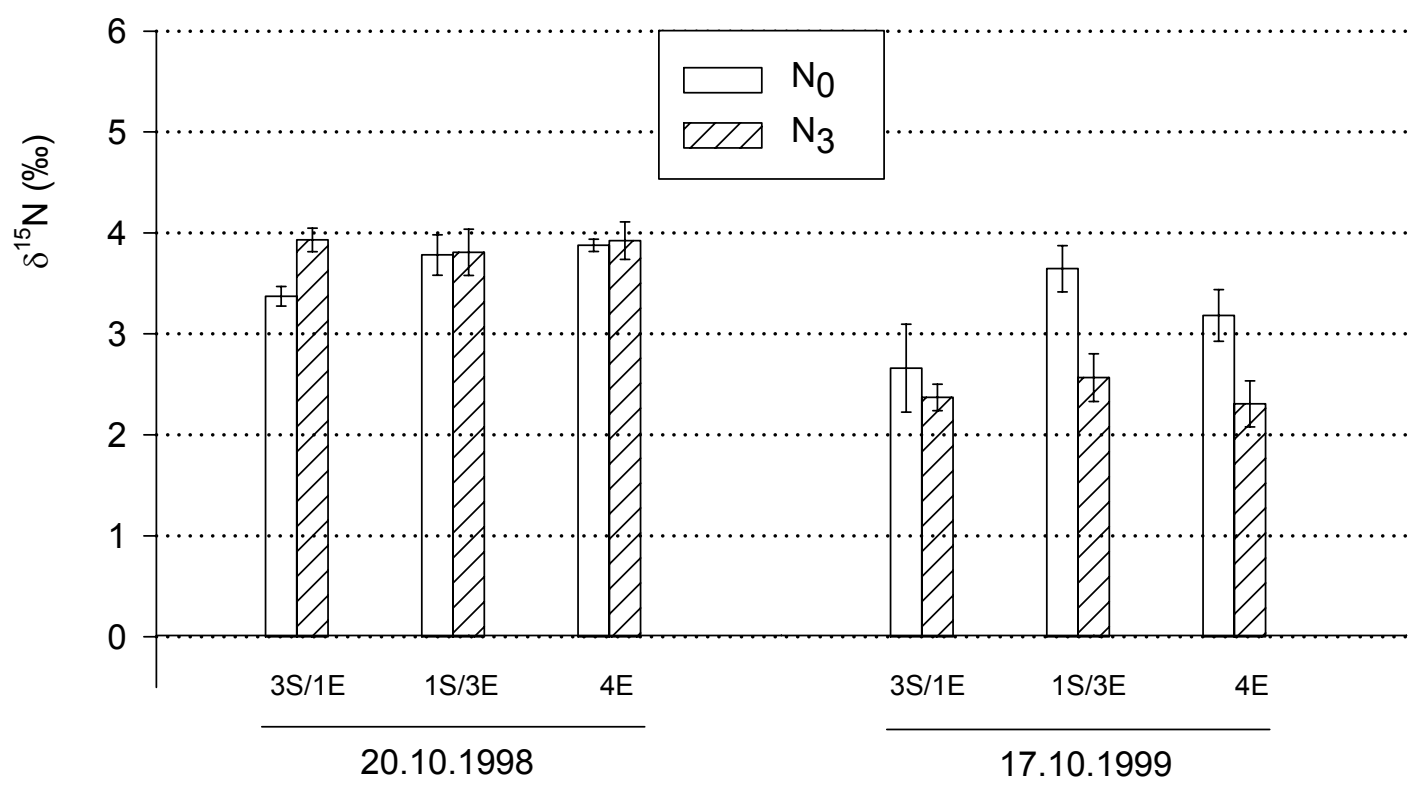

Abb. 48: $\delta^{15} \mathrm{~N}$-Werte $(\mathrm{n}=6$, Mittelwerte \pm Standardfehler in \%o) in der Sprossmasse des Einjährigen Weidelgrases in Reinsaat (4E) und in Gemengesaat mit Saatwicke (1S/3E und 3S/1E) am 20.10.1998 (links) und 17.10.1999 (rechts). 
In der vorliegenden Untersuchung war es nicht möglich, mit der $\delta^{15} \mathrm{~N}$-Methode einen Stickstofftransfer von der Saatwicke zum Einjährigen Weidelgras nachzuweisen.

\subsubsection{Einfacher und erweiterter N-Flächenbilanzsaldo}

Die einfachen N-Flächenbilanzsalden beim Anbau der Saatwicke in Rein- und Gemengesaat mit Einjährigem Weidelgras lagen im Jahr 1998 mit Werten zwischen $-21,0\left(\mathrm{~N}_{0}: 4 \mathrm{~S}\right)$ und $-62,0 \mathrm{~kg} \mathrm{~N}$ ha ${ }^{-1}\left(\mathrm{~N}_{3}\right.$ : 4E; Abb. 49a) auf einem ähnlichen Niveau wie im Jahr 1999. Im Jahr 1999 betrugen die Salden zwischen -29,1 ( $\mathrm{N}_{0}:$ 1S/3E) und $-48,0 \mathrm{~kg} \mathrm{~N} \mathrm{ha}^{-1}\left(\mathrm{~N}_{3}:\right.$ 1S/3E; Abb. 50a).

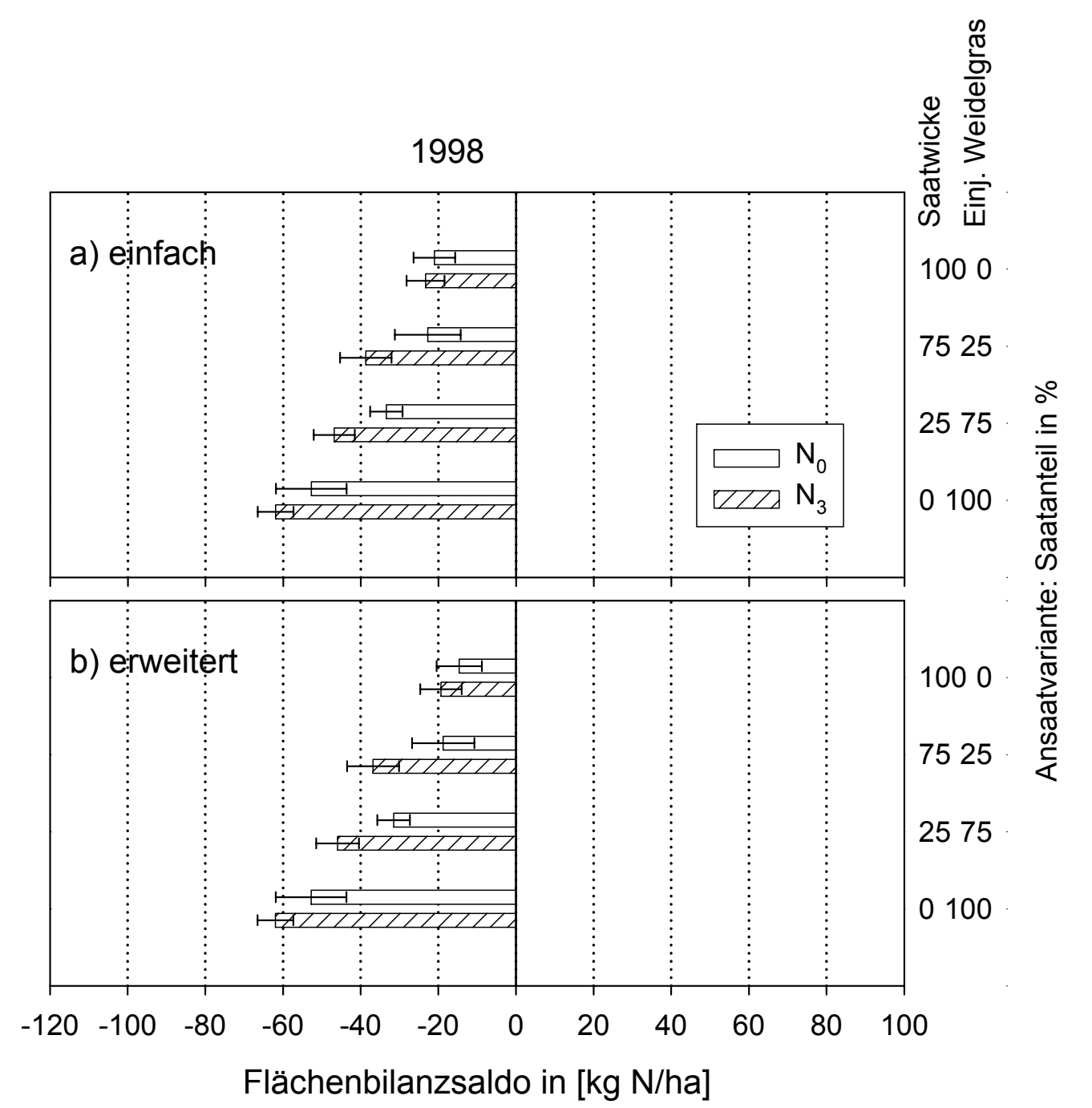

Abb. 49: Einfacher (a) und erweiterter (b) N-Flächenbilanzsaldo ( $\mathrm{kg} \mathrm{N} \mathrm{ha}^{-1}$; Mittelwerte \pm Standardfehler) beim Anbau von Saatwicke und Einjährigem Weidelgras in Rein- und Gemengesaat zur Futternutzung am 20.10.1998 
Während im Vergleich der Jahre die Bilanzsalden bei Saatwicke in Reinsaat (4S) im Mittel um -19,1 kg N ha ${ }^{-1}$ im zweiten Jahr sanken, stiegen diese bei Einjährigem Weidelgras in Reinsaat (4K) im Mittel um 22,1 kg N ha-1 an (signifikante Wechselwirkung: Ansaatvariante $\times$ Jahr). Die einfachen $\mathrm{N}$-Flächenbilanzsalden verringerten sich im Jahr 1998 in der Reihenfolge 4S > 3S/1E > 1S/3E > 4E, allerdings konnten nur die Unterschiede im Bilanzsaldo zwischen dem Einjährigen Weidelgras in Reinsaat (4E) und der Saatwicke in Rein- und Gemengesaat mit $25 \%$ Einjährigem Weidelgras (3S/1E) statistisch gesichert werden (Tab. 9).

Im zweiten Untersuchungsjahr sanken die N-Flächenbilanzsalden tendenziell von 4S $>3 \mathrm{~S} / 1 \mathrm{E}$, um dann mit zunehmenden Anteil an Einjährigem Weidelgras tendenziell wieder anzusteigen (3S/1E $<1 \mathrm{~S} / 3 \mathrm{E}<4 \mathrm{E})$.

Tab. 9: Vergleich der Mittelwerte der einfachen N-Flächenbilanzsalden beim Anbau von Saatwicke und Einjährigem Weidelgras in Rein- und Gemengesaat zur Futternutzung im Jahr 1998 und 1999

\begin{tabular}{|c|c|c|c|c|c|c|}
\hline & \multicolumn{4}{|c|}{ Ansaatvariante } & \multirow[b]{2}{*}{ Düngung } & \multirow{2}{*}{$\begin{array}{l}\text { Ansaatvariante } \\
\text { x Düngung }\end{array}$} \\
\hline 1998 & $4 S$ & $3 S / 1 E$ & $1 \mathrm{~S} / 3 \mathrm{E}$ & $4 \mathrm{E}$ & & \\
\hline einfacher Bilanzsaldo & a & a & $a b$ & $\mathrm{~b}^{* * *}$ & n.s. & n.s. \\
\hline 1999 & & & & & & \\
\hline einfacher Bilanzsaldo & a & a & a & a & n.s. & n.s. \\
\hline
\end{tabular}

In beiden Untersuchungsjahren lagen im Mittel über die Ansaatvarianten die N-Flächenbilanzsalden der Bestände aus der $\mathrm{N}_{0}$-Variante in allen geprüften Ansaaten auf einem vergleichbaren Niveau mit den $\mathrm{N}$-Salden der Bestände aus der $\mathrm{N}_{3}$-Variante. Im Mittel über die Jahre lagen die N-Salden der langfristig nicht mit mineralischen NDüngern versorgten Variante $\left(\mathrm{N}_{0}\right)$ über den Salden nach langfristig überoptimal mit mineralischen $\mathrm{N}$-Düngemitteln versorgten Vorfrüchten $\left(\mathrm{N}_{3}, P_{\text {Düngung }}=0,0062\right)$. Die Differenzen im Bilanzsaldo zwischen der $\mathrm{N}_{0}$ - und der $\mathrm{N}_{3}$-Variante lagen im Jahr 1998 bei 2,3 (4S), 15,9 (3S/1E), 13,4 (1S/3E) und 9,2 $\mathrm{kg} \mathrm{N}$ ha $^{-1}(4 \mathrm{E})$ und 1999 bei 10,6 (4S), 12,7 (3S/1E), 19,0 (1S/3E) und 0,7 kg N ha ${ }^{-1}(4 \mathrm{E})$.

Da auch für die Saatwicke Angaben zur Höhe der N-Rhizodeposition vorliegen, kann für die Bestände mit der Saatwicke ein erweiterter N-Flächenbilanzsaldo errechnet werden. Dabei wurden die unter Kap. 3.1.7 beschriebenen Annahmen zur Einbeziehung der Anteile Rhizodeposition für Saatwicke und Einjährigem Weidelgras angewendet. 


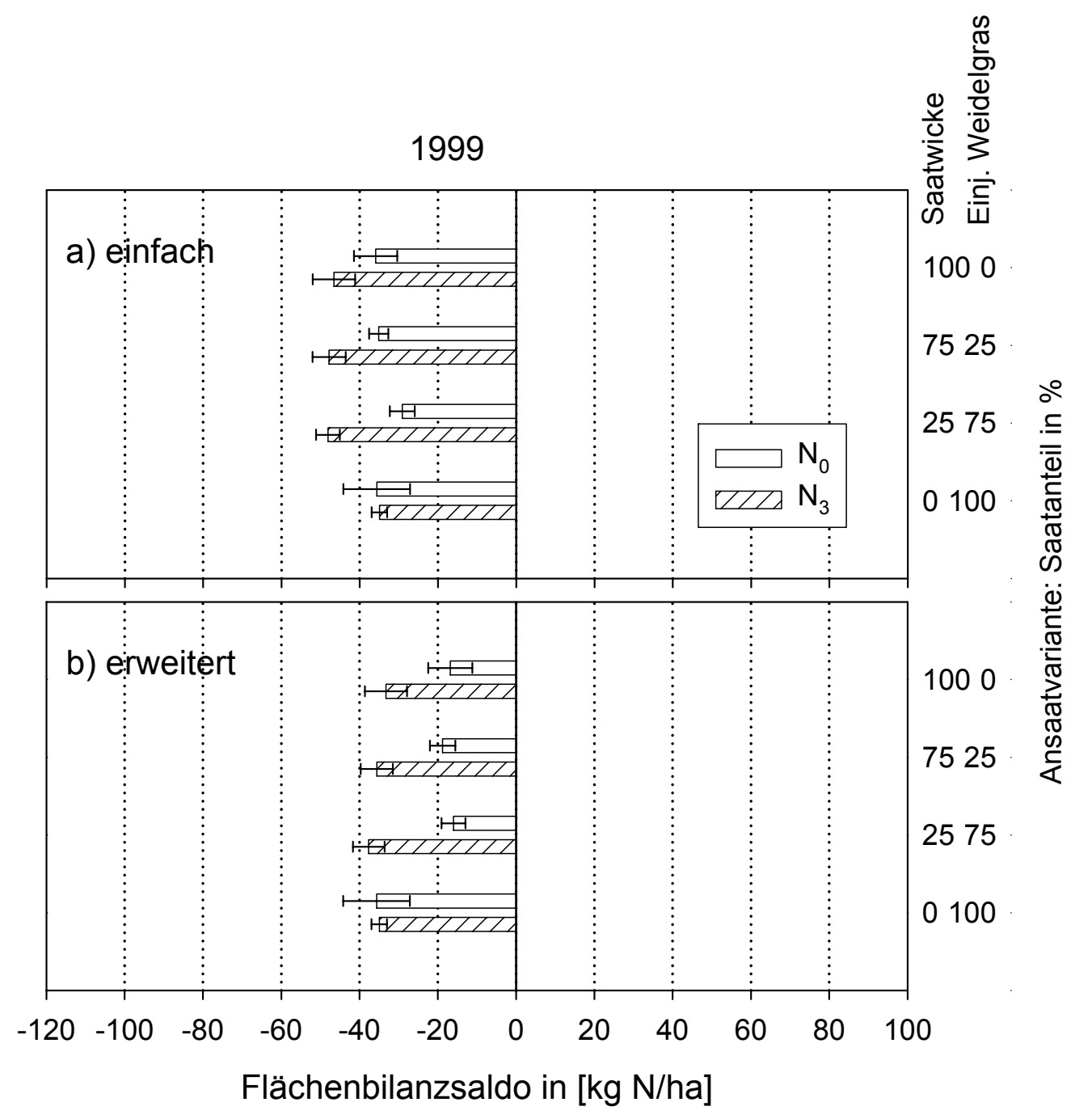

Abb. 50: Einfacher (a) und erweiterter (b) N-Flächenbilanzsaldo ( $\mathrm{kg} \mathrm{N} \mathrm{ha}^{-1}$; Mittelwerte \pm Standardfehler) beim Anbau der Saatwicke und des Einjährigen Weidelgrases in Rein- und Gemengesaat zur Futternutzung am 17.10.1999

Durch Berücksichtigung der fixierten N-Menge des Stickstoffs aus der Rhizodeposition (Tab. A55) erhöhen sich die N-Flächenbilanzsalden im ersten Jahr auf maximal $14,6 \mathrm{~kg} \mathrm{~N} \mathrm{ha}^{-1}\left(\mathrm{~N}_{0}\right.$ : 4S; Abb. 49b) und $-16,0 \mathrm{~kg} \mathrm{~N} \mathrm{ha}^{-1} \mathrm{im}$ Jahr 1999 ( $\mathrm{N}_{0}: 1 \mathrm{~S} / 3 \mathrm{E}$; Abb. $50 \mathrm{~b})$. Damit lagen die erweiterten N-Flächenbilanzsalden in beiden Jahren auf vergleichbarem Niveau. 


\section{Diskussion}

Im ersten Teil der Diskussion werden zunächst einige wichtige Aspekte der im Rahmen dieser Untersuchung angewandten Methoden beleuchtet. Im zweiten Teil wird der Beitrag der angebauten Leguminosen in Rein- und Gemengesaat zur Selbstregelung der $\mathrm{N}$-Zufuhr anhand der ermittelten Ergebnisse diskutiert.

\subsection{Methodische Aspekte zur Erhebung der Wurzelmassen}

Werden Ertragsdaten vor dem Hintergrund einer Flächenbilanzierung beurteilt, sind die geernteten (exportierten) TM- und N-Mengen sowie die auf der Fläche verbleibenden Erntereste von Bedeutung. Bei den Ansaaten mit Futterleguminosen umfassen die Erntereste die Summe von Wurzeln, Stoppeln und Bestandesabfall. Die Erntereste von Ackerbohne und Hafer ergeben sich aus der Summe von Wurzeln, Stroh, Hülsen bzw. Spelzen und Rispen sowie dem Bestandesabfall (vgl. Kap. 2.5.3). Während die oberirdischen Anteile der Biomasse an den Ernterückständen relativ einfach erhoben werden können, ist die Bestimmung der Wurzelmasse vergleichsweise aufwendig und unsicher. Aus diesem Grund werden daher zunächst methodische Aspekte der Wurzelprobenahme diskutiert, um die hier ermittelten Wurzelmassen besser einordnen zu können.

Die Wurzelverteilung in Böden am natürlichen Standort ist heterogen. Neben morphologischen Einflussgrößen, die sich aus dem Anbau unterschiedlicher Pflanzenarten ergeben (monokotyle oder dikotyle Pflanzen; homorrhizes oder allorrhizes Wurzelsystem), gehören auch die spezifischen Standortverhältnisse, variierende Anbautechniken (Breitsaat, Reihensaat, Einzelkornsaat) sowie die unterschiedlichen Bodenbearbeitungsverfahren zu den Faktoren, die die Wurzelverteilung im Boden beeinflussen können (MOHR 1978, BÖHM 1979, EHLERS et al. 1981, GEISLER 1983, STEINECK 1983, VAN NORDWIJK et al. 1985).

Für die Beprobung der Wurzeln im Felde stehen verschiedene Methoden zur Verfügung (SCHUURMAN und GoEdEWAageN 1971). Die Eignung einer Methode hängt vor allem davon ab, welche Fragestellung bzw. welche Wurzelparameter zur Beantwortung der Versuchsfragen notwendig sind. Eine für alle Versuchsfragen geeignete Methode steht nicht zur Verfügung (BöHM 1978). Ein Ziel der vorliegenden Untersuchung war es, den Beitrag von Ackerbohne, Luzerne und Saatwicke zur Selbstregelung der Stickstoffzufuhr in leguminosenbasierten Fruchtfolgen zu quantifizieren. Hierzu wurden vereinfachte N-Flächenbilanzsalden ermittelt, die möglichst die gesamtpflanzliche Biomasse der Leguminosen berücksichtigen sollten. Die quantitative Aufnahme des Wurzelsystems (Wurzelmasse in $\mathrm{dt} \mathrm{ha}^{-1}$ ) und der darin gebundenen $\mathrm{N}$-Mengen ist daher erforderlich. Die am weitesten verbreiteten Methoden zur Erfas- 
sung der Wurzelmasse sind die Ausgrabungs-, Monolith- und Bohrmethode (BÖHM 1979). Der Aufwand der Probenahme und der destruktive Charakter der Methoden nehmen in genannter Reihenfolge deutlich ab. Aufgrund dieser Tatsachen kann für die Probenahme innerhalb eines laufenden Dauerfeldversuches nur die Bohrmethode in Frage kommen. Sie ermöglicht es, eine große Anzahl von Pflanzen zu beproben, ohne die Flächen vollständig zu zerstören.

Da bei der Beprobung der Wurzeln mittels Rammkernsonden nur ein kleiner Teil der tatsächlich vorhandenen Wurzeln erfasst wird, kommt sowohl dem Stichprobenumfang als auch dem Ort der Beprobung große Bedeutung zu (SCHUURMAN und GoEdeWAAGEN 1971, BÖHM 1979; KÖPKE 1979, VAN NORDWIJK et al. 1985). Eine einheitliche Vorgehensweise bei der Wurzelbeprobung mittels Bohrkernen ist aus der Literatur nicht erkennbar. So variiert neben dem Stichprobenumfang und dem Ort der Beprobung auch der Bohrstockdurchmesser. VAN NORDWIJK et al. (1985) erreichten einen Variationskoeffizienten von $40 \%$ mit $n \geq 25$ Einstichen bei der Beprobung von Grünland. Allerdings wurden die Bohrstöcke mit einem Durchmesser von $7 \mathrm{~cm}$ auf der Fläche zufällig verteilt (VAN NORDWIJK et al. 1985). VEPRASKAS und HOYT (1988) benutzten zur Beprobung von Nicotiana tabacum und Zea mays Bohrkerne von 5,5 bzw. 7,6 cm Durchmesser bei einem Stichprobenumfang von $\mathrm{n}=5$ bzw. $\mathrm{n}=3$. Bei BöHM et al. (1977) variierten die Bohrstockdurchmesser von 5,0 bis 10,2 cm bei der Beprobung von Glycine max. Dabei wurden zu den ersten beiden Probenahmeterminen jeweils ein Bohrstock auf und zwischen der Reihe, zum dritten Probenahmetermin jeweils ein Bohrstock auf und zwei Bohrstöcke zwischen den Reihen mit unterschiedlichen Abständen zur Reihe beprobt. Über den Beprobungsort auf der Reihe wurden keine Angaben gemacht. PROFFITT et al. 1985 beprobten Sommer-Weizen mit jeweils zwei Bohrstöcken (Durchmesser $6 \mathrm{~cm}$ ) auf und zwischen den Reihen und rechneten die erstellte Mischprobe auf die Fläche hoch. SMIT et al. (2000) und VAN NoRDWIJK et al. (1985) weisen darauf hin, dass bei Getreide eine einfache Hochrechnung der Wurzeltrockengewichte der Bohrstöcke auf und zwischen der Reihe in Abhängigkeit der Reihenabstände zu einer Überschätzung der Wurzelmassen um mehr als 30 \% führen kann. Bei Pflanzen mit ausgeprägtem allorrhizem Wurzelsystem und geringen Individuenzahlen pro Fläche (weite Reihenabstände) sind noch deutlichere Abweichungen in Abhängigkeit des Beprobungsortes zu erwarten.

Ein Vergleich von Wurzeldaten ist verursacht durch unterschiedliche Beprobungssysteme sehr schwierig. Zudem werden die Wurzeldaten durch unterschiedliche Probenlagerungen und Probenaufbereitungen in hohem Maße beeinträchtigt und die Variation noch erhöht (KöPKE 1979, VAN NORDWIJK und FLORIS 1979, AMATO und PARDO 1994). So werden für den Prozess der Wurzelauswaschung Siebe verwendet, deren Maschenweiten von 0,2 bis über $2 \mathrm{~mm}$ variieren (BÖHM 1977, АMATO und PARDO 1994). AMATO und PARDO (1994) ermittelten bei Ackerbohne und Weizen nach Auswaschung mit einem $1 \mathrm{~mm}$ Sieb nur $75 \%$ der Wurzelmasse im Vergleich zur Auswaschung mit einem Sieb mit 0,2 mm Maschenweite. Der Anteil der WurzelLängen-Dichte betrug sogar nur $34 \%$. Bei der Lagerung und insbesondere bei der 
Auswaschung von Wurzeln können unter ungünstigen Bedingungen somit Trockenmasse-Verluste bis zu $40 \%$ auftreten (VAN NORDWIJK und FLORIS 1979, AMATO und PARDO 1994). Die genannten Untersuchungen deuten darauf hin, dass Pflanzen mit unterschiedlichen Wurzelsystemen (homorrhiz - allorrhiz) bzw. Feinwurzelanteilen sehr differenziert auf unterschiedliche Probenbehandlungen reagieren können. Probenahmeserien im Verlaufe einer Vegetationsperiode zeigen zudem, dass Auswaschungsverluste bzw. Verluste durch Probenlagerung im Verlaufe der Vegetation, d.h. mit zunehmendem physiologischen Alter der Pflanzen, sinken und mit zunehmendem Anteil an Feinwurzeln ansteigen (VAN NORDWIJK und FLORIS 1979, AMATO und PARDO 1994).

In der vorliegenden Untersuchung wurden in den Reinsaaten zwei und in den Gemengeansaaten vier Einstiche pro Parzelle gezielt auf den Reihen bzw. in den Reihenzwischenräumen platziert (vgl. Kap. 2.5.2). Die Bohrstöcke hatten einen Durchmesser von $8,7 \mathrm{~cm}$. Die Maschenweite betrug $1 \mathrm{~mm}$. Die Aufbereitung der Wurzelproben erfolgte nach der in Kap. 2.5.3 beschriebenen Vorgehensweise. Die Wurzeldaten wurden standraumbezogen bei Ackerbohne und flächenbezogen bei Hafer, Luzerne, Knaulgras, Saatwicke und Einjährigem Weidelgras verrechnet (Kap. 2.7.1). Hierdurch wurden sowohl die Anzahl der Pflanzen (Ackerbohne) als auch die Reihenweiten bei der Berechnung der Gewichtungsfaktoren mit einbezogen. Bei einer Hochrechnung der erhaltenen Wurzeltrockenmassen der Bohrkerne in gleichem Verhältnis (50/50), würden die Wurzelmassen im Vergleich mit einer standraum- oder flächenbezogenen Verrechnung überschätzt. Während bei den Kulturen mit $18 \mathrm{~cm}$ Reihenweiten (Luzerne, Knaulgras, Saatwicke, Einjähriges Weidelgras) die Wurzelmassen um bis zu $5 \%$ überschätzt würden, errechnet sich für Hafer als Reihenkultur, bei einer Reihenweite von $28 \mathrm{~cm}$, bis zu $40 \%$ höhere Werte im Vergleich zu einer flächenbezogenen Verrechnung. Die Bohrkerne auf den Reihen der Ansaaten mit Ackerbohnen wurden gezielt auf nur eine Ackerbohnenpflanze platziert. Eine Hochrechnung der Wurzeltrockenmassen im Verhältnis 50/50 mit den Wurzeltrockenmassen der Bohrkerne zwischen den Reihen, würde somit durch das Beprobungsmuster bedingt in den Reinsaaten die Wurzelmassen im Vergleich zu einer standraumbezogenen Verrechnung um bis zu $80 \%$ überschätzen. Bei abnehmender Anzahl Ackerbohnen pro Fläche (z.B. im Gemenge) könnten die Wurzelmassen bis über $240 \%$ überschätzt werden. Ein direkter Vergleich der ermittelten Variationskoeffizienten mit Werten aus der Literatur ist nicht sinnvoll, da in vorliegender Untersuchung keine echten Wiederholungen innerhalb einer Fläche vorgenommen wurden. Die Variationskoeffizienten der ermittelten Wurzelmassen sind Tab. A56 dem Anhang zu entnehmen. Die u.a. von VAN NORDWIJK et al. 1985 beobachtete Zunahme der Streuung der Werte mit zunehmender Bodentiefe lassen sich auch bei den Variationskoeffizienten in dieser Untersuchung beobachten. Darüber hinaus können hohe Variationskoeffizienten auch eine Folge deutlich abnehmender Wurzelmengen sein und somit ein Erklärungsansatz für die im Mittel höheren Koeffizienten der Ansaaten mit Saatwicke und Einjährigem Weidelgras. 
Die Beprobung der Wurzelmassen erfolgte bis in eine Tiefe von $50 \mathrm{~cm}$. Eine Zusammenschau von Literaturangaben verdeutlicht, dass die untersuchten Pflanzenarten zwar in der Lage sind, den Boden bis in größere Bodentiefen zu durchwurzeln. Bei einer Beprobung bis in $50 \mathrm{~cm}$ Bodentiefe werden in Abhängigkeit von den Pflanzenarten allerdings über $95 \%$ der Wurzelmassen bei Ackerbohne, Hafer, Saatwicke, Einjährigem Weidelgras und Knaulgras erfasst, bei Luzerne über 90 \% (KÖNEKAMP 1952, KöneKAMP und VetTer 1953, BAKER und GarwoOd 1959, KutscheRA 1960, KÖPKE 1979, EhLERS et al. 1983, LOHMANN 1984, MüLler 1984, De WiLLIGeN und VAN NoRdWIJK 1987, Hauser 1987, KöNINGs 1987, GRegory 1988, MANZKE 1990, KÖRSCHENS 1992, HEERAMAN und JUMA 1993).

Ein spezifisches Problem stellt die Wurzelbeprobung von Gemengen dar. Die in der Literatur beschriebenen Verfahren bei der Beprobung von Wurzelgemengen sind zum Teil sehr ungenau bzw. sehr teuer und aufwendig (BAKER und GARWOOD 1959, HauggaARd-Nielsen et al. 2001). HauggaARd-Nielsen et al. (2001) konnten beim Anbau von Pisum sativum und Hordeum vulgare in Rein- und Gemengesaat mit markiertem ${ }^{32}$ Phosphor zeigen, dass sich die Wurzelverteilung der Arten im Gemenge durch interspezifische Konkurrenz von der Wurzelverteilung der Arten in Reinsaat unterscheidet. Allerdings werden keine absoluten Angaben der Wurzelmassen der Gemengeansaaten im Vergleich zu den Reinsaaten gemacht. Da die Gemengewurzeln nach dem Auswaschungsprozess visuell nicht getrennt werden können, wurde in der vorliegenden Untersuchung eine rechnerische Aufteilung vorgenommen (vgl. Kap. 2.7.1). Hierbei ist zu berücksichtigen, dass von der Wurzelverteilung der Arten im Gemenge abweichende Wurzelverteilungen der Arten in Reinsaat nicht mit erfasst werden. Allerdings ist es eher unwahrscheinlich, dass sich in der vorliegenden Untersuchung, aufgrund von interspezifischer Konkurrenz im Gemenge eine Veränderung der absoluten Wurzelmassen der Bestandespartner ergeben. Gleichwohl fehlen soweit ersichtlich die experimentellen Belege für das quantitative Verhalten der Wurzeln einzelner Gemengepartner im Vergleich zur Reinsaat. Fehler wären vor allem dann zu erwarten, wenn die veränderte Tiefenverteilung der Wurzeln im Gemenge sich bis weit unterhalb der Beprobungstiefe von 50 $\mathrm{cm}$ auswirkt. Hierzu liegen in der Literatur, soweit erkennbar, keine Angaben vor.

Die beschriebenen Einflüsse auf die gewonnenen Wurzeldaten verdeutlichen, dass ein Vergleich von Wurzeldaten teilweise sehr problematisch sein kann. Vor diesem Hintergrund wurden die eigenen Wurzeldaten nur mit Untersuchungen verglichen, die auch am Standort Reinshof, allerdings zum Teil mit abweichender Methodik, ermittelt wurden. Trotz der beschriebenen methodischen Probleme bei der Wurzelbeprobung wurden die erhobenen Wurzeldaten in Abhängigkeit der Ansaatvarianten und Düngungsstufen statistisch verrechnet, um insbesondere den Einfluss der Düngungsvarianten auf die Höhe der Wurzelmassen ableiten zu können. 


\subsection{Vergleich der Schätzung der symbiotisch fixierten N-Mengen mit der $\delta^{15} \mathrm{~N}$-Methode und der erweiterten Differenzmethode}

Bisher stehen keine Feldmethoden zur direkten Messung der symbiotisch fixierten NMenge zur Verfügung, so dass eine Validierung der Schätzergebnisse, die in der vorliegenden Arbeit mit der $\delta^{15} \mathrm{~N}$-Methode und der erweiterten Differenzmethode ermittelt wurden, nicht möglich ist. Vor diesem Hintergrund wird im Rahmen dieser Arbeit von Schätzungen der $\mathrm{N}_{2}$-Fixierleistung gesprochen (HAUSER 1987).

Um mit der $\delta^{15} \mathrm{~N}$-Methode den Anteil Stickstoff aus der Luft einer Leguminose schätzen zu können, ist der Anbau einer möglichst in Nachbarschaft zur Leguminose wachsenden nicht nodulierenden Referenzpflanze erforderlich (BERGERSEN et al. 1985, SHEARER und KOHL 1986). Voraussetzung für möglichst treffgenaue Schätzergebnisse ist, dass Leguminose und Referenzpflanze bodenbürtigen Stickstoff mit gleicher Isotopenzusammensetzung $\left({ }^{14} \mathrm{~N}\right.$ und ${ }^{15} \mathrm{~N}$ ) aufnehmen (FRIED und BROESHART 1975). Die Isotopenzusammensetzung des pflanzenverfügbaren Bodenstickstoffs kann allerdings sowohl räumlich (MARIOTTI et al. 1980, BERGERSEN et al. 1985) als auch zeitlich (TURNER et al. 1987) stark schwanken. SHEARER und KOHL (1986) empfehlen daher den Anbau mehrerer Referenzfruchtarten, um den $\delta^{15} \mathrm{~N}$-Wert des aufgenommenen Boden- $\mathrm{N}$ auch unter wechselnden Bedingungen treffgenauer bestimmen zu können.

In der vorliegenden Untersuchung konnte der Anbau verschiedenartiger Referenzpflanzen nicht realisiert werden, da die dafür benötigte Fläche innerhalb der vorgegebenen Versuchsanlage des Dauerfeldversuchs nicht zur Verfügung stand. Aus diesem Grund wurden jeweils nur eine Referenzpflanzenart genutzt, die sich bereits in vorherigen Untersuchungen bewährt hatte. Als Referenzpflanze für Ackerbohne diente Hafer, der auch von HAUSER (1987), HAUNZ et al. (1988), MAIDL et al. (1996), SCHMIDTKE (1996) und SCHMIDTKE (2001) verwendet wurde, für Luzerne wurde Knaulgras wie bei WEST und WEDIN (1985) und TA und FARIS (1987a) sowie für Saatwicke Einjähriges Weidelgras (BECKMANN 1998) eingesetzt. Die Referenzpflanzen wurden zeitgleich und in unmittelbarer Nachbarschaft zu den Ansaaten mit Leguminosen angebaut (vgl. Abb. 3). Um die Effekte räumlicher Schwankungen im $\delta^{15} \mathrm{~N}$-Wert des bodenbürtigen Stickstoffs in der Fläche zu minimieren, wurde der Anteil Stickstoff aus der Luft mit der jeweils benachbarten Referenzpflanze innerhalb des Mittelteilstücks der Versuchsanlage geschätzt.

Da die $\delta^{15} \mathrm{~N}$-Werte der einzelnen Bodenhorizonte auf den Versuchsparzellen nicht direkt bestimmt wurden, lassen sich keine Aussagen über mögliche vertikale Gradienten in den hier beprobten Bodenprofilen machen. Vertikale Gradienten der $\delta^{15} \mathrm{~N}$ Werte wurden u.a. von BROARDBENT et al. (1980) festgestellt. Des Weiteren ermittelten LEDGARD et al. (1985a) unterschiedliche Aufnahmeraten von spezifisch gebunde- 
nem Ammonium bei monokotylen (Lolium perenne) und dikotylen (Trifolium subterraneum) Pflanzen. Eine Ammoniumfixierung kann zur Veränderung der Isotopenzusammensetzung im Boden-N führen (KARAMANOS und ReNNIE 1978). Da monokotyle Pflanzen (z.B. Lolium perenne) in der Lage sind, vermehrt spezifisch gebundenes Ammonium aufzunehmen, ist deren Eignung umstritten. KILIAN (1994) schätzte den Anteil Stickstoff aus der Luft bei verschiedenen Ackerbohnensorten mit Lolium perenne und Brassica oleracea var. medullosa auf Parabraunerde aus Löss und konnte keine Unterschiede in den $\delta^{15} \mathrm{~N}$-Werten zwischen den Sprossmassen der Referenzpflanzen feststellen. Die Befunde von KILIAN (1994) deuten darauf hin, dass entweder keine unterschiedlichen Isotopenzusammensetzungen vorlagen, oder das das spezifisch gebundene Ammonium mit gleichen Anteilen aufgenommen wurde. Unterschiedliche Isotopenverteilungen beeinflussen die Schätzgenauigkeit der $\mathrm{N}_{2}$-Fixierung nur dann, wenn Leguminose und Referenzpflanze gleichzeitig Boden-N räumlich und/oder zeitlich in unterschiedlichen Mengen aufnehmen.

Eine signifikant unterschiedliche Aufnahme von pflanzenverfügbarem Bodenstickstoff aus dem Unterboden wurde zwischen Ackerbohne und Hafer in beiden Jahren (Tab. A16 und Tab. A18) und bei Saatwicke und Einjährigem Weidelgras im ersten Untersuchungsjahr 1998 festgestellt (Tab. A50). Inwieweit diese unterschiedliche Aufnahme an mineralischem Stickstoff aus dem Unterboden durch eventuelle vertikale Gradienten des Isotopenverhältnisses die Schätzergebnisse in der vorliegenden Untersuchung tatsächlich beeinträchtigt haben, lässt sich abschließend nicht sicher beurteilen.

Der für die Schätzung des Anteils Stickstoff aus der Luft in Leguminosen mit der $\delta^{15} \mathrm{~N}$-Methode benötigte Unterschied im Isotopenverhältnis zwischen molekularem Luftstickstoff (entspricht dem $\delta^{15} \mathrm{~N}_{0}$-Wert der Leguminose) und pflanzenverfügbaren Stickstoff im Boden (entspricht $\delta^{15} \mathrm{~N}$-Wert der Referenzpflanze) war bei allen Ansaatvarianten mit Leguminosen signifikant (Differenz Luzerne/Knaulgras $=5,40 \%$, Ackerbohne/Hafer $=4,51 \%$, Saatwicke/Einjähriges Weidelgras $=3,91 \%$ ). Nach BREMER und VAN KESSEL (1990) nimmt die Genauigkeit der Schätzung der symbiotischen $\mathrm{N}_{2}$-Fixierleistung mit steigender Differenz zwischen dem $\delta^{15} \mathrm{~N}$-Wert der Referenzpflanze und dem $\delta^{15} \mathrm{~N}$-Wert des molekularen Luftstickstoffs zu. Dementsprechend nimmt die Genauigkeit der Schätzung der symbiotischen $\mathrm{N}_{2}$-Fixierleistung in der hier vorgestellten Untersuchung in der Reihenfolge Luzerne - Ackerbohne Saatwicke ab. Dies ist auch eine Folge des Einflusses des Messfehlers auf die Schätzergebnisse. Die $\delta^{15} \mathrm{~N}$-Werte der Pflanzenproben wurden mittels eines Massenspektrometers mit einer Reproduzierbarkeit von $\pm 0,1$ bis $\pm 0,15 \% \delta^{15} \mathrm{~N}$ gemessen. Wird jeweils eine maximale, durch die Messgenauigkeit des Massenspektrometers bedingte Abweichung in der Messung unterstellt, würde beispielsweise der Ndfa-Wert im Schnittgut der Saatwicke in Reinsaat der $\mathrm{N}_{3}$-Variante $1998(0,32) \mathrm{um}$ bis zu $\pm 0,062$ abweichen. Die Abweichung des Ndfa-Wertes im Schnittgut der Saat- 
wicke in Reinsaat der $N_{0}$-Variante $1999(0,70)$ würde bei einem entsprechenden Messfehler um $\pm 0,077$ schwanken. Das bedeutet, dass der relative Fehler mit steigendem Ndfa-Wert (entspricht steigender Differenz im $\delta^{15} \mathrm{~N}$-Werte zwischen Leguminose und Referenzpflanze) deutlich abnimmt. Zu berücksichtigen ist hierbei, dass die Ndfa-Werte jeweils aus den gewichteten gesamtpflanzlichen $\delta^{15} \mathrm{~N}$-Werten berechnet wurden. Diese ergeben sich aus mehreren Einzelmessungen der einzelnen Komponenten der pflanzlichen Biomasse mit unterschiedlichen N-Anteilen an der gesamtpflanzlichen Biomasse (Kap. 2.7.2.2, Text 3. Absatz, Gleichung (12)). Das bedeutet, im ungünstigsten Fall könnte der allein durch den Messfehler bedingte Schätzfehler noch etwas größer werden.

Detaillierte Zusammenstellungen der Einflussgrößen auf die Schätzgenauigkeit der $\delta^{15} \mathrm{~N}$-Methode finden sich u.a. bei SHEARER und KOHL (1986) sowie SCHMIDTKE (1997a).

Die im Rahmen dieser Untersuchung erhobenen Daten ermöglichten auch die Schätzung des Anteils Stickstoff aus der Luft mit der erweiterten Differenzmethode und somit einen Vergleich mit den Schätzergebnissen der $\delta^{15} \mathrm{~N}$-Methode.

Die beim Vergleich der beiden Methoden ermittelten Korrelationskoeffizienten variierten von 0,46 bis 0,98. Der Vergleich der ermittelten Schätzwerte und der Korrelationskoeffizienten verdeutlicht die zum Teil erheblichen Unterschiede der Methoden (vgl. Kap. 3.1.4, 3.2.4 und 3.3.4). Die höchste Übereinstimmung der Methoden wurde bei den Ansaaten mit Luzerne 1999, der geringste Zusammenhang bei den Ansaaten mit Saatwicke 1998 ermittelt. Während bei den Ansaaten mit Luzerne in Rein- und Gemengesaat mit Knaulgras die mit der erweiterten Differenzmethode geschätzten gewichteten Anteile Stickstoff aus der Luft unter den Werten der $\delta^{15} \mathrm{~N}$-Methode lagen, verhielten sich die mit den beiden Methoden ermittelten Ergebnisse bei den Ansaaten mit Ackerbohne in Rein- und Gemengesaat mit Hafer entgegengesetzt. Die Fixierleistungen der Ansaaten mit Saatwicken wurden mit der erweiterten Differenzmethode im ersten Jahr unter- bzw. im zweiten Jahr im Vergleich zu den mit der $\delta^{15} \mathrm{~N}$-Methode ermittelten Werten überschätzt. Gute Übereinstimmungen zwischen beiden Methoden werden insbesondere bei hohen Anteilen Stickstoff aus der Luft und/oder niedrigen $\mathrm{N}_{\min }$-Werten erzielt. Hierfür dürfte auch die Variation, die bei der Messung der pflanzenverfügbaren $\mathrm{N}_{\min }$-Mengen im Boden auftreten kann (AUFHAMmER et al. 1989), verantwortlich sein.

Biologisch nicht mögliche "negative" Fixierleistungen wurden in der vorliegenden Untersuchung mit der erweiterten Differenzmethode vor allem zu frühen Vegetationsstadien (z.B. Ackerbohne erste und zweite Ernte, vgl. Kap. 3.1.4), bei sehr niedrigen Anteilen Stickstoff aus der Luft (Saatwicke 1998, Tab. A51) und/oder sehr hohen Angebot an pflanzenverfügbaren mineralischen Bodenstickstoff rechnerisch ermittelt (HEUWINCKEL und GUTSER 1997). Schätzwerte "negativer" Fixierleistungen ermittelten 
auch StüLPNAGEL (1982), HeUWINCKEL und GUTSER (1997) sowie SCHMIDTKE (2001) mit der erweiterten Differenzmethode. Wie aus Gleichung (5) in Kapitel 2.5.5 hervorgeht, werden "negative" Ndfa-Werte geschätzt, wenn $\left(N_{B t}+N_{\min }\right)_{\text {Leg }}<\left(N_{B t}+N_{\text {min }}\right)_{\text {Ref }}$ wird. Sowohl hohe $\mathrm{N}_{\min }$-Werte als auch ein sehr gutes Wachstum der Referenzpflanzen (STÜLPNAGEL 1982) können hierfür die Ursache sein. Wird die Differenz $\left(N_{B t}+N_{\text {min }}\right)_{\text {Leg }}-\left(N_{B t}+N_{\text {min }}\right)_{\text {Ref }}$ dagegen größer als der $\left(N_{B t}\right)_{\text {Leg }}$, ergeben sich rechnerisch Ndfa-Werte $>100 \%$. Sowohl für die "negativen" Ndfa-Werte (hohe $\mathrm{N}_{\mathrm{min}}$-Werte unter der Referenzpflanze) als auch für die Ndfa-Werte $>100 \%$ sind in der vorgestellten Untersuchung hohe $\mathrm{N}_{\text {min }}$-Werte verantwortlich. In zwei Fällen führten höhere $\mathrm{N}_{\text {min }}$-Mengen im Boden unter der Ackerbohne in Reinsaat im Jahr 1998 zur ersten Ernte $\left(\mathrm{N}_{\text {minLeg }}=118,4\right.$ bzw. $105,8 \mathrm{~kg} \mathrm{~N} \mathrm{Na}^{-1}$ ) gegenüber $\mathrm{N}_{\min }$-Mengen unter Hafer in Reinsaat in Höhe von 63,3 bzw. 56,1 kg N ha ${ }^{-1}$ zu einem rechnerischen Ndfa-Wert von über $100 \%$. Hohe $\mathrm{N}_{\min }$-Werte unter Leguminosen im Vergleich zu Nichtleguminosen könnten zum Teil auch eine Folge unterschiedlicher Mineralisations- und Immobilisationsbedingungen im Boden sein und somit schließlich zu einer Überschätzung der gewichteten Anteile Stickstoff aus der Luft mit der erweiterten Differenzmethode führen.

In Gemengen aus Leguminosen und Nichtleguminosen kann fixierter Stickstoff von der Leguminose an den nichtlegumen Gemengepartner transferiert werden (vgl. 3.1.6 und 3.2.6). Mit der erweiterten Differenzmethode werden transferierte N-Mengen zwar mit erfasst, deren Höhe kann aber nicht geschätzt werden (HEUWINCKEL und GUTSER 1997). Somit werden bei der erweiterten Differenzmethode die transferierten Anteile Stickstoff aus der Luft rechnerisch der luftbürtigen N-Aufnahme der Leguminose zugeschrieben (die Boden-N-Aufnahme der Leguminose wird also unterschätzt).

Auch wenn im Mittel zum Teil gute Übereinstimmungen zwischen den Schätzwerten beider Methoden zu verzeichnen waren (Abb. 30), ergaben sich beim Vergleich einzelner Werte sehr hohe Abweichungen bis hin zu Bereichen, in denen eine Schätzung der symbiotisch fixierten N-Menge mittels erweiterter Differenzmethode nicht möglich war. Somit wird deutlich, dass auch die Schätzgenauigkeit der erweiterten Differenzmethode Grenzen hat und v.a. bei sehr geringen Anteilen Stickstoff aus der Luft, im Gegensatz zur $\delta^{15} \mathrm{~N}$-Methode, eine Schätzung der wahrscheinlicheren $\mathrm{N}$ Flüsse in Gemengen aus Leguminosen und Nichtleguminosen nur unzureichend möglich ist.

Aus den genannten Gründen wurden die N-Flächenbilanzsalden auf Grundlage der mittels $\delta^{15} \mathrm{~N}$-Methode geschätzten gewichteten Anteile Stickstoff aus Luft und Boden berechnet und bilden im Folgenden die Grundlage der diskutierten Ergebnisse. 


\subsection{Allgemeine Versuchsbedingungen}

Wie aus den $\mathrm{N}_{\min }$-Vorräten im Boden vor Untersuchungsbeginn hervorgeht, resultiert aus der langjährigen unterschiedlichen mineralischen N-Düngung zu den nichtlegumen Vorfrüchten im Vergleich der Düngungsvarianten zum Teil höhere $\mathrm{N}_{\text {min }}$-Mengen in der $\mathrm{N}_{3}$-Variante (Tab. A15, Tab. A34, Tab. A49). Demgegenüber lassen sich keine signifikanten Unterschiede zwischen Varianten der langjährig unterschiedlichen mineralischen N-Düngung im Gesamtkohlenstoff- und Gesamtstickstoffgehalt des Bodens vor Beginn der eigenen Untersuchung feststellen (Tab. A1). Nach einer vereinfachten Stickstoffbilanz (Zufuhr durch Düngung und symbiotische $\mathrm{N}_{2}$-Fixierung minus Entzug durch Erntegut in $\mathrm{kg} \mathrm{ha}^{-1}$ ) über die gesamte Fruchtfolge ermittelte KNUTH (1995) Differenzen im Bilanzsaldo zwischen der $\mathrm{N}_{0}$ - und $\mathrm{N}_{3}$-Variante in Höhe von $230 \mathrm{~N} \mathrm{ha}^{-1}$ in der langen Fruchtfolge (im Zeitraum von 6 Jahren) bzw. $336 \mathrm{~kg} \mathrm{~N}$ ha $^{-1}$ in der kurzen Fruchtfolge (im Zeitraum von 3 Jahren). Es ist bekannt, dass die im Boden organisch gebundenen Mengen an Kohlenstoff und Stickstoff nach einer Änderung der Bewirtschaftung, insbesondere bei Böden mit hoher Bonität, nur sehr langfristig mit Gehaltsänderungen reagieren (BECK 1990). Dagegen können sich in mikrobiellen Kenngrößen auch kurzfristig Wirkungen der Bewirtschaftung widerspiegeln. Die unterschiedliche Stickstoffdüngung $\left(\mathrm{N}_{0}, \mathrm{~N}_{3}\right)$ führte nach Untersuchungen von MANZKE (1993) zu keiner deutlichen Unterscheidung bei Parametern, die den Stickstoffstatus des Bodens charakterisieren (Heißwasserlöslicher Stickstoff, Proteaseaktivität, Arginin-Ammonifikation). Offenbar sind die Unterschiede zwischen den Düngungsvarianten zu gering, um im betrachteten Zeitraum von 16 Jahren auf diesem Standort deutliche Änderungen in den $\mathrm{N}_{\mathrm{t}}$-Gehalten des Bodens hervorzubringen. Darüber hinaus zeigen Untersuchungen von KNUTH (1990) im Durchschnitt erhöhte Nettomineralisationsraten an organisch gebundenem Stickstoff in der $\mathrm{N}_{3}$ Variante. Offensichtlich stellen die insgesamt $\mathrm{N}$-reicheren Ernterückstände der $\mathrm{N}_{3}$ Variante eine vergleichsweise leichter mineralisierbare organische $\mathrm{N}$-Fraktion dar, die zu hohen Nettomineralisationsraten beitragen. Die mineralischen Düngermengen in der $\mathrm{N}_{3}$-Variante sowie der über Mineralisation frei werdende $\mathrm{N}_{\text {min }}$-Stickstoff, die nicht vollständig durch die angebauten Pflanzen entzogen wurden, führten offenbar nicht zu einer verstärkten $\mathrm{N}$-Immobilisation im Boden und Anhebung der N-Mengen im Humusvorrat des Bodens. Untersuchungen von KNUTH (1995) deuten an, dass ein beachtlicher Teil der erhöhten $\mathrm{N}_{\text {min }}$-Mengen vor allem in der $\mathrm{N}_{3}$-Variante vorrangig durch eine erhöhte Auswaschung aus dem Boden verloren gingen.

Bei der Bewertung der Ergebnisse der geprüften Arten muss berücksichtigt werden, dass die Arten nicht unter ceteris paribus Bedingungen verglichen wurden. Die Ansaaten mit Ackerbohne und Luzerne wurden in der Fruchtfolge mit Einsatz von Wirtschaftsdüngemitteln nach Mais bzw. nach Wintergerste angebaut, die Ansaaten mit Saatwicke in der kurzen Fruchtfolge ohne Einsatz von Wirtschaftsdüngern. Deshalb erfolgte keine statistische Analyse mit dem Faktor Pflanzenart (Vergleich der Ansaaten mit Saatwicke mit den Ansaaten der Ackerbohne und der Luzerne). 
Als Bodentypen des Versuchsstandortes finden sich Schwarzerden verschiedener Degradierungsstufen, die teilweise kolluvial überlagert wurden, sowie unterschiedlich ausgeprägte Grieserden, die mehr oder weniger erodiert sind. Mit Bodenwertzahlen von etwa 80 Bodenpunkten kann dieser Standort als vergleichsweise fruchtbar angesehen werden. Der Witterungsverlauf im Untersuchungszeitraum zeigt $1998 \mathrm{im}$ Vergleich zum langjährigen Mittel deutlich höhere Niederschläge im Zeitraum März bis November (+170 mm). Allerdings fielen diese Niederschläge vor allem gegen Ende der Vegetationsperiode (September und Oktober, Abb. 1). Die Temperaturverläufe ließen in beiden Versuchsjahren keine Besonderheiten gegenüber dem langjährigen Mittel erkennen. Die Boden- und Witterungsbedingungen wiesen für das Pflanzenwachstum im Versuchszeitraum relativ günstige Gegebenheiten auf. Wie aus den wichtigsten chemischen Bodenkennwerten am Versuchsstandort hervorgeht, war das Wachstum der Pflanzen nicht durch die verfügbaren Mengen an $\mathrm{P}$ und $\mathrm{K}$ im Boden limitiert (Tab. A1). Die Daten zeigen keine deutlichen Unterschiede zwischen den Düngungsstufen auf.

Die relativ guten Wachstumsbedingungen am Standort kommen auch in den vergleichsweise hohen TM-Erträgen der Bestände zum Ausdruck. Die gesamtpflanzlichen TM-Erträge von Ackerbohne in Reinsaat lagen im Mittel der Düngungsstufen mit Werten von 159,1 bzw. 160,5 dt TM ha ${ }^{-1}$ in beiden Jahren auf einem sehr hohen Niveau (Abb. 4 und Abb. 6). SchMIDTKE (1999) ermittelte bei der Ackerbohne (cv. Minica) maximal 118,3, HAUSER (1987) mit der gleichen Sorte 144,6 dt TM ha-1. Auch die Kornerträge der Hafer-Reinsaaten deuten mit Werten bis zu 70,2 dt TM ha ${ }^{-1}$ auf die im Mittel guten Standortbedingungen hin (Tab. A6). Die gesamtpflanzlich akkumulierte Trockenmasse bei der Luzerne wies im Vergleich der Jahre deutliche Unterschiede auf. In 1998 lagen die Erträge im Mittel der Düngungsstufen mit 81,8 dt TM ha $^{-1}$ (Tab. A25) unter, im zweiten Jahr mit Werten von im Mittel 163,3 dt TM ha ${ }^{-1}$ (Tab. A28) deutlich über den von ScHMIDTKE (1999) bei Luzerne (cv. Europe) im Ansaatjahr ermittelten Erträgen (129,4 bzw. 127,3 dt TM ha $\left.{ }^{-1}\right)$. Während der Feldaufgang der meisten Ansaaten gut bis sehr gut verlief, lag der Feldaufgang der Ansaaten mit Luzerne und Knaulgras im ersten Untersuchungsjahr nur bei $25 \%$ der ausgesäten keimfähigen Samen. Überdurchschnittlich hohe Niederschläge im April 1998 dürften eine zügige Entwicklung der Bestände und eine Kompensation der geringen Bestandesdichten über eine Bestockung während der sehr langsamen Jugendentwicklung weitgehend verhindert haben (HEUSER 1931). Hierin dürften die Gründe für die vergleichsweise niedrigen Erträge der Ansaaten mit Luzerne und Knaulgras im ersten Untersuchungsjahr zu suchen sein. Die bei den Reinsaaten der Saatwicke ermittelten gesamtpflanzlichen TM-Erträge lagen im Jahr $1999 \mathrm{im}$ Mittel der Düngungsstufen mit 53 dt TM ha ${ }^{-1}$ (Tab. A44) deutlich über den Erträgen in 1998 $\left(21,1 \mathrm{dt} \mathrm{TM} \mathrm{ha}{ }^{-1}\right.$, Tab. A43) und damit auch über den von BECKMANN (1998) ermittelten TM-Erträgen, die in der Sprossmasse der Saatwicke erfasst wurden (max. 45,5 dt $\mathrm{TM}_{\mathrm{ha}}{ }^{-1}$ ). Die deutlichen Unterschiede im Sprossertrag der Saatwicke zwischen den 
beiden Jahren dürften vor allem in dem im zweiten Versuchsjahr um 13 Tage früheren Aussaattermin begründet sein. Nach einer späteren Aussaat (01.08.) ermittelte BECKMANN (1998) nur noch 25,6 dt TM ha ${ }^{-1}$ in der Sprossmasse der Saatwicke gegenüber $37,3 \mathrm{dt} \mathrm{TM}$ ha $^{-1}$ bei früherer Aussaat. Sowohl RENIUS und LÜTKE ENTRUP (1985) als auch NÖSBERGER und OPITZ Von BobeRfELD (1986) weisen auf die Bedeutung eines frühen Aussaattermines für die Ertragsleistung von Stoppelsaaten hin. Der Einfluss der langjährig unterschiedlichen N-Düngung zu den nichtlegumen Vorfrüchten auf die TM-Erträge und die gesamtpflanzliche Stickstoffakkumulation wird im Abschnitt 4.4.1 diskutiert.

\subsection{Selbstregelung der $\mathbf{N}-$ Zufuhr}

Der Beitrag von Ackerbohne, Luzerne und Saatwicke zur Selbstregelung der NZufuhr kann aus dem vereinfachten $\mathrm{N}$-Flächenbilanzsaldo abgeleitet werden. Wie aus Gleichung (14) hervorgeht (vgl. Kap. 2.7.2.2) ist die Höhe des vereinfachten NFlächenbilanzsaldos eine Funktion der gesamtpflanzlich akkumulierten N-Menge $\left(\mathrm{N}_{\mathrm{Bt}}\right)$, des Stickstoff-Harvest-Index $\left(\mathrm{H}_{\mathrm{NBt}}\right)$ und des Anteils Stickstoff aus der Luft (Ndfa) bzw. des Anteils Stickstoff aus bodenbürtiger Quelle (Ndfs) in der Biomasse der Pflanzen. In Tab. A57 im Anhang sind Angaben zu N-Flächenbilanzsalden beim Anbau von Ackerbohne, Luzerne und Saatwicke aus der Literatur zusammengestellt. Aus diesen Ergebnissen zu vereinfachten N-Flächenbilanzsalden geht hervor, dass alle drei zur Berechnung des einfachen N-Flächenbilanzsaldos benötigten Kenngrößen, bei Ackerbohne und Luzerne unter Feldbedingungen innerhalb einer Art stark schwanken können. Untersuchungen von HAUSER (1987) bzw. SCHMIDTKE (1999) an Ackerbohnen (cv. Minica, Kristall bzw. Minica) am Standort Reinshof zeigen, dass die Stickstoff-Harvest-Indizes an einem Standort mit Werten zwischen 0,72 bis 0,75 vergleichsweise wenig schwankt. Werden zusätzlich mehrere Standorte und Genotypen mit in den Vergleich einbezogen, unterscheiden sich die Stickstoff-HarvestIndizes deutlicher (z.B. Jost 2003, 0,42 bis 0,78). Die gleichen Tendenzen lassen sich grundsätzlich auch für Ansaaten mit Luzerne belegen, was aus dem Vergleich der Stickstoff-Harvest-Indizes aus den Untersuchungen von SCHMIDTKE (1999, Reinshof, 0,76 bis 0,80) und JUNG (2003, Reinshof Göttingen, Oederquart, 0,54 bis 0,83 ) hervorgeht (vgl. Kap. 4.4.4). Sowohl die gesamtpflanzlich akkumulierten NMengen $\left(\mathrm{N}_{\mathrm{Bt}}\right)$ als auch die Anteile Stickstoff aus der Luft (Ndfa) in der Biomasse der Pflanzen sind an einem Standort stärkeren Schwankungen unterworfen. Bei HAUSER (1987) und SCHMIDTKE (1999) lagen die $\mathrm{N}_{\mathrm{Bt}}$-Werte zwischen 230 und $418 \mathrm{~kg} \mathrm{~N} \mathrm{ha}^{-1}$, die Ndfa-Werte zwischen 0,69 und 0,92. Jost (2003) ermittelte an vier verschiedenen Standorten in Niedersachsen $\mathrm{N}_{\mathrm{Bt}}$-Werte bei der Ackerbohne in Höhe von 140,9 bis $320 \mathrm{~kg} \mathrm{~N} \mathrm{ha}^{-1}$ und Ndfa-Werte von 0,44 bis 0,87 . Bei Luzerne zeigen sich in der Untersuchung von JUNG (2003, ohne Dasselsbruch) erhebliche Unterschiede bei den $\mathrm{N}_{\mathrm{Bt}}$-Werten (126,7 bis 598,6 kg N ha-1) und den Anteilen Stickstoff aus der Luft $(0,34$ 
bis 0,80$)$. In dieser Untersuchung wurden die Ansaaten mit Luzerne allerdings überjährig genutzt (1. und 2. Hauptnutzungsjahr).

Bei Saatwicke lassen sich bisher keine Aussagen über die Schwankungsbreite dieser Kenngrößen machen, da soweit ersichtlich hier nur eine Untersuchung vorliegt, aus der die Kenngrößen zur Berechnung einer N-Flächenbilanz abgeleitet werden können (Tab. A57). Im Folgenden werden die Kenngrößen zur Ermittlung eines einfachen N-Flächenbilanzsaldos über alle zu prüfenden Ansaaten in Abhängigkeit eines unterschiedlichen bodenbürtigen $\mathrm{N}$-Angebotes gegenübergestellt, um den Beitrag der angebauten Leguminosenarten und Anbauformen zur Selbstregelung der N-Zufuhr darzustellen.

\subsubsection{Gesamtpflanzliche Stickstoffakkumulation in Spross und Wurzeln}

Um im Rahmen einer N-Flächenbilanzierung eine möglichst vollständige Erfassung der durch die Pflanzen verursachten N-Flüsse zu erreichen, müssen neben den oberirdischen N-Mengen auch die in den Wurzeln akkumulierten N-Mengen quantifiziert werden. Hierbei sollten die in Kapitel 4.1 diskutierten Besonderheiten bei der Erfassung der unterirdischen Biomasse der Pflanzen mit berücksichtigt werden. Vergleiche zu Wurzeldaten, die andere Autoren erfasst haben, sollten zumindest mit Daten von vergleichbaren Standorten durchgeführt und unter Vorbehalt betrachtet werden.

Die in der eigenen Untersuchung gemessenen unterirdischen N-Mengen beim Anbau von Ackerbohne und Hafer stimmen gut mit Werten von HAUSER (1987) überein, der die unterirdische Biomasse bis zur maximalen Durchwurzelungstiefe mit Hilfe der Monolithmethode am Standort Reinshof in Göttingen erfasste. In der genannten Untersuchung betrugen die maximalen unterirdischen N-Mengen von Ackerbohne (cv. Minica) und Hafer (cv. Flämings Silber) 37,8 kg (29,4 kg Wurzel-N + 8,4 kg Knöllchen-N) bzw. 18,8 kg N ha ${ }^{-1}$ von Hafer. Die höchsten Wurzel-N-Mengen der eigenen Untersuchung wurden 1999 zur zweiten Ernte gemessen und beliefen sich auf $41,4 \mathrm{~kg} \mathrm{~N}^{-1}$ bei Ackerbohne und 18,2 $\mathrm{kg} \mathrm{N}^{-1}$ bei Hafer (Tab. A11). MANZKE (1990) ermittelte in Nachbarschaft zum Systemversuch am Reinshof bei Luzerne (cv. Europe) die Wurzelmassen mit der Monolithmethode bis in $90 \mathrm{~cm}$ Bodentiefe. Am Ende des ersten Hauptnutzungsjahres (vier Schnitte, überjähriger Bestand) betrugen die Wurzel-N-Mengen $141 \mathrm{~kg} \mathrm{ha}{ }^{-1}$ und lagen damit auf vergleichbarem Niveau mit den maximal ermittelten N-Mengen in der Wurzel der Luzerne in Reinsaat der eigenen Untersuchung (1999: 4L No: 143,6 kg ha ${ }^{-1}$, Tab. A30). Nach KÖPKE (1979) liefert die Monolithmethode die treffgenausten Ergebnisse. Vor diesem Hintergrund müssen die hier ermittelten Wurzel-N-Mengen als vergleichsweise hoch angesehen werden. Quantitative Aussagen über die methodisch bedingten Abweichungen lassen sich nach heutigem Kenntnisstand in Abhängigkeit verschiedener Pflanzenarten nicht 
machen. Standort- und durch die Pflanzenarten bedingte Einflüsse auf die Wurzelmassen bzw. Wurzel-N-Mengen lassen sich daher nur schwer quantifizieren. Die dargestellten Vergleiche mit Ergebnissen ermittelt mit der Monolithmethode und der hier modifizierten Probenahme mittels Rammkernsonden deuten an, dass die ermittelten Daten zur Wurzelmasse offenbar gut vergleichbar sind. Die dargestellten Ergebnisse deuten an, dass eine systematische Wurzelbeprobung mittels Rammkernsonden offenbar die Möglichkeit bietet, in Dauerfeldversuchen und in umfangreichen faktoriellen Versuchen (JOST 2003, JUNG 2003) die Wurzelmassen mit relativ hoher Treffgenauigkeit und vertretbarem Aufwand zu quantifizieren. Eine wichtige Voraussetzung für den Vergleich von Wurzeldaten, die mit verschiedenen Beprobungsmethoden ermittelt wurden, ist neben der Berücksichtigung der Beprobungstiefe auch die Verwendung möglichst gleicher Aufbereitungsverfahren. Auf die Bedeutung der Erfassung der gesamtpflanzlichen Biomasse (ober- und unterirdische Biomasse) wird später im Rahmen der N-Flächenbilanzsalden eingegangen (Kap. 4.4.5).

Die in der gesamtpflanzlichen Biomasse (Spross + Wurzel) akkumulierten N-Mengen der eigenen Untersuchungen erreichten bei den Ansaaten mit Ackerbohne, Luzerne und Saatwicke maximal 380,4 (1998 No 4A, Tab. A10), 472,4 (1999 No 4L, Tab. A30) und 155,5 (1999 $\mathrm{N}_{0} 4 \mathrm{~S}$, Tab. A46) kg N ha-1. Die von SCHMIDTKE (1999) am Reinshof in Göttingen ermittelten gesamtpflanzlichen N-Mengen lagen bei Ackerbohne (cv. Minica) auf gleicher Höhe $\left(383,7 \mathrm{~kg} \mathrm{~N} \mathrm{ha}^{-1}\right)$, bei Luzerne (cv. Europe) mit 363,1 kg N $\mathrm{ha}^{-1}$ im Ansaatjahr unter den Mengen, die im Rahmen der hier vorgestellten Untersuchungen ermittelt wurden (Tab. A10, Tab. A11, Tab. A29, Tab. A30). In Versuchen von JUNG (2003) erreichte die Luzerne am selben Untersuchungsstandort eine gesamtpflanzliche N-Akkumulation zwischen 408 und $598 \mathrm{~kg} \mathrm{~N}^{-1}$ je Hauptnutzungsjahr (jeweils vier Schnitte). In einer methodisch gut vergleichbaren Untersuchung von Jost (2003), in der u.a. drei verschiedene Ackerbohnen Genotypen über drei Jahre auf vier Standorten in Niedersachsen geprüft wurden, akkumulierte Ackerbohne (cv. Alfred) am Reinshof maximal $370 \mathrm{~kg} \mathrm{~N} \mathrm{ha}^{-1}$. Die in der Literatur angegebenen akkumulierten N-Mengen bei Saatwicke als Stoppelansaat schwanken je nach Aussaattermin und Vegetationsdauer deutlich. In einer Untersuchung von MANZKE (1990) akkumulierte Saatwicke zwischen 69 (105 Tage) und $100 \mathrm{~kg} \mathrm{~N} \mathrm{ha}^{-1}$ (110 Tage) in Spross und Wurzel. BECKMANN (1998) ermittelte nach einer frühen Aussaat (30.06.) der Saatwicke in einem Sortengemisch (cv. Prussia + Lolita) nach 96 Tagen $167 \mathrm{~kg}$ $\mathrm{N} \mathrm{ha}^{-1}$ in der Sprossmasse. Bei späterer Aussaat am 01.08. akkumulierte die Saatwicke nach 63 Tagen lediglich $71 \mathrm{~kg} \mathrm{~N} \mathrm{ha}^{-1}$. Auf die Bedeutung des Aussaattermins für die Ergebnisse in der eigenen Untersuchung wurde bereits in Kap. 4.3 verwiesen. Die maximalen gesamtpflanzlich akkumulierten N-Mengen der hier geprüften Ansaaten lagen wie oben dargestellt, bei Ackerbohne auf einem mit Literaturangaben vergleichbaren, bei Luzerne im Ansaatjahr und Saatwicke auf hohen Niveau. 
Die Leguminosen in Reinsaat akkumulierten in der $\mathrm{N}_{0}$-Variante stets mehr Stickstoff als in der $\mathrm{N}_{3}$-Variante (Tab. A10, Tab. A11, Tab. A29, Tab. A30, Tab. A45, Tab. A46). Signifikant war der Einfluss der Düngung zu den Vorfrüchten im Mittel der Ansaatvarianten allerdings nur bei den Ansaaten mit Luzerne im Jahr 1999. Während sich die Nichtleguminosen in Reinsaat umgekehrt verhielten (bei Hafer 1998, sowie Knaulgras 1998 und 1999 signifikante Wechselwirkung zwischen Ansaatvariante x Düngung), zeigten die Gemenge keine gleichgerichtete Reaktion in der Höhe der gesamtpflanzlichen N-Akkumulation in Abhängigkeit von der langjährig unterschiedlichen mineralischen N-Düngung zu den nichtlegumen Vorfrüchten. Die unterschiedlich hohen $\mathrm{N}_{\text {min }}$-Mengen im Vergleich der Düngungsstufen führten bei den Leguminosen Reinsaaten sehr wahrscheinlich zu einer mehr oder weniger starken Hemmung der Knöllchenbildung (HARPER und GIBSON 1984) und damit zu einer Verminderung der symbiotischen $\mathrm{N}_{2}$-Fixierleistung (HEINZMANN 1981, StREETER 1988). Hierdurch wurde die Infektion der Leguminosenwurzeln durch Knöllchenbakterien vermutlich bereits zu einem frühen Zeitpunkt in der Vegetation eingeschränkt. Hierauf deuten die schon zur ersten Ernte tendenziell erhöhten Fixierleistungen der Ackerbohne in der $\mathrm{N}_{0}$-Variante und die deutlich höhere Fixierleistung bei Luzerne hin (Tab. A17, Tab. A19, Tab. A36, Tab. A38, vgl. Kap. 4.4.3.2).

Die Fixierung von molekularem Stickstoff ist für die Pflanze ein relativ energieaufwendiger Prozess. Nach ScHULz et al. (1999) veratmet Ackerbohne beispielsweise etwa 2,87 mg C/mg N $\mathrm{m}_{\text {fix }}$. Um $200 \mathrm{~kg} \mathrm{~N}$ ha $^{-1} \mathrm{im}$ Jahr zu fixieren, errechnet sich hieraus ein zusätzlicher Bedarf an Kohlenstoff von etwa $574 \mathrm{~kg} \mathrm{C} \mathrm{bzw.} \mathrm{1,44} \mathrm{t} \mathrm{Trockenmasse.}$ Unter der Voraussetzung dass am Standort sonstige Wachstumsfaktoren (Einstrahlung, Wasser, ph-Wert, Mikro- und Makronährstoffe) ausreichend zur Verfügung stehen, sind nodulierende Ackerbohnen in der Lage, ihre Photosyntheseleistung an den zusätzlichen Kohlenstoffbedarf anzupassen. In Untersuchungen von MERBACH (1982) wiesen nodulierende Pflanzen deutlich höhere photosynthetische Aktivität auf als nichtnodulierende Pflanzen der gleichen Art.

Auch unter den vorliegenden Standortbedingungen waren die Ackerbohnen offensichtlich in der Lage, den höheren Energiebedarf für die leicht erhöhten Fixierungsleistungen der Bestände aus der $\mathrm{N}_{0}$-Variante bereitzustellen und damit vermehrt Stickstoff zu assimilieren und in der Biomasse zu akkumulieren. Andererseits waren die Ackerbohnen vor allem in der $\mathrm{N}_{3}$-Variante offenbar nicht in der Lage, den $\mathrm{N}_{\text {min }}{ }^{-}$ Stickstoff im Boden stärker zu nutzen, woraus schließlich eine etwas geringere gesamtpflanzlich akkumulierte N-Menge resultierte. Auch andere Autoren weisen darauf hin, dass die Ackerbohne auf erhöhte $\mathrm{N}_{\text {min }}$-Mengen im Boden in der Regel nicht mit einem Ertragszuwachs reagiert und infolgedessen die Fixierleistung sinkt (MARTIN 1990, Scherer und DANZEISEN 1980).

Bei Luzerne resultierten aus den geringeren $\mathrm{N}_{\text {min }}$-Mengen in der $\mathrm{N}_{0}$-Variante im Vergleich zu der $\mathrm{N}_{3}$-Variante (Tab. A35 und Tab. A37) neben einer höheren $\mathrm{N}_{2}-$ Fixierleistung auch eine höhere Wurzelmasse (Tab. A25, Tab. A28) und Wurzel NMengen (Tab. A29, Tab. A30). Von höheren Wurzelmassen bei Luzerne infolge 
niedriger Gehalte an pflanzenverfügbarem Stickstoff im Boden berichten auch FISHBECK und PHILLIPS (1981) sowie BÉLANGER und RICHARDS (2000). Seit langem ist bekannt, dass eine N-Düngung, vor allem bei sehr niedrigen Gehalten an mineralischem Stickstoff im Boden, zu einem Ertragszuwachs bei Leguminosen führen kann, was KUNELIUS (1974) und TRIMBLE et al. (1987) insbesondere im Ansaatjahr beobachteten. Gleichzeitig wurden durch erhöhte $\mathrm{N}_{\text {min }}$-Mengen im Boden infolge einer N-Düngung das Spross-Wurzel-Verhältnis erweitert und die Wurzelmasse der Luzerne eingeschränkt.

Die Luzerne akkumulierte im ersten Untersuchungsjahr in den hier dargestellten Versuchen vergleichsweise wenig Biomasse-N (Tab. A29). Die im Verhältnis zur gebildeten Biomasse relativ hohen $\mathrm{N}_{\text {min }}$-Werte im Boden führten im ersten Untersuchungsjahr bei Luzerne in der $\mathrm{N}_{0}$-Variante zu leicht erhöhten gesamtpflanzlich akkumulierten N-Mengen im Vergleich zur $\mathrm{N}_{3}$-Variante (Tab. A29). Im zweiten Versuchsjahr verminderten die sich besser entwickelten Ansaaten mit Luzerne die $\mathrm{N}_{\min }$-Mengen im Boden in beiden Düngungsvarianten $\left(N_{0}\right.$ und $\left.N_{3}\right)$ zu einem früheren Vegetationszeitpunkt (Tab. A37). Die resultierenden deutlich erhöhten $\mathrm{N}_{2}$-Fixierleistungen führten zu deutlich höheren Wurzel-N-Mengen in der $\mathrm{N}_{0}$-Variante, infolgedessen sich auch die Schnittgut-N-Mengen und damit der $\mathrm{N}_{\mathrm{Bt}}$ erhöhte (Tab. A38, Tab. A30). Die Ansaaten der $\mathrm{N}_{3}$-Variante waren offensichtlich nicht in der Lage die vermutlich bereits zu Vegetationsbeginn stärker eingeschränkte $\mathrm{N}_{2}$-Fixierleistung nach weitgehender Erschöpfung des bodenbürtigen $\mathrm{N}$-Angebotes an die $\mathrm{N}_{2}$-Fixierleistung der $\mathrm{Be}$ stände aus der $\mathrm{N}_{0}$-Variante anzugleichen. Insbesondere nach einer Schnittnutzung erfolgt der Wiederaustrieb der Luzernepflanzen zu einem überwiegenden Anteil aus $\mathrm{N}$-Reservestoffen der Pfahlwurzeln und aus dem $\mathrm{N}_{\min }$-Angebot des Bodens (TA et al. 1990, LemAIRE et al. 1992). Aus den in beiden Varianten gleich niedrigen $\mathrm{N}_{\min }-\mathrm{Men}-$ gen im Boden, gleichzeitig aber deutlich höheren gespeicherten Wurzel-N-Mengen der Luzerne, die in der $\mathrm{N}_{0}$-Variante gewachsen ist, lassen sich für die Luzerne der $\mathrm{N}_{3}$-Variante schlechtere Wachstumsbedingungen ableiten, die schließlich eine deutlich niedrigere gesamtpflanzliche N-Akkumulation zur Folge hatte.

\subsubsection{N-Aufnahme aus dem Boden}

Die vor Aussaat der Ansaaten mit Ackerbohne, Luzerne und Saatwicke vorhandenen $\mathrm{N}_{\text {min }}$-Mengen im Boden ließen deutliche Unterschiede zwischen den Varianten $\mathrm{N}_{0}$ und $\mathrm{N}_{3}$ sowohl über die gesamte Profiltiefe als auch in der Aufteilung auf Ober- und Unterboden erkennen. Im ersten Untersuchungsjahr 1998 wies der Boden der langjährig mineralisch gedüngten Variante $\left(\mathrm{N}_{3}\right)$ deutlich höhere $\mathrm{N}_{\text {min }}$-Vorräte auf als der Boden der $\mathrm{N}_{0}$-Variante. Dabei beschränkten sich signifikante Unterschiede zwischen den Varianten vor dem Anbau von Ackerbohne und Luzerne jeweils auf den Unterboden (Tab. A15, Tab. A34 und Tab. A49). Auch im zweiten Untersuchungsjahr lagen die $\mathrm{N}_{\text {min }}$-Mengen im Boden der $\mathrm{N}_{3}$-Variante über den Mengen im Boden der ungedüngten Variante $\left(\mathrm{N}_{0}\right)$, konnten aber nur vor Aussaat der Ansaaten mit Acker- 
bohne statistisch gesichert werden. Beim Vergleich der $\mathrm{N}_{\min }$-Mengen im Boden vor Aussaat muss die Stellung der einzelnen Früchte in der Fruchtfolge der im AckerbauSystemversuch Göttingen verglichenen Bodennutzungssysteme berücksichtigt werden. Während die Ackerbohne in der Fruchtfolge mit Einsatz von Wirtschaftsdüngemitteln nach Mais, die Luzerne nach Wintergerste im darauf folgenden Frühjahr angebaut wurde, erfolgte der Anbau der Saatwicke in der Fruchtfolge ohne Einsatz von Wirtschaftsdüngemitteln als Stoppelsaat nach Wintergerste (vgl. Tab. 1).

KNUTH (1995) ermittelte in einer Untersuchung zur Quantifizierung von Stickstoffauswaschungsverlusten im Systemversuch am Standort Reinshof im Zeitraum von 1988 bis 1993 u.a. die $\mathrm{N}_{\min }$-Mengen in 0 bis $90 \mathrm{~cm}$ Bodentiefe jeweils im Herbst und im Frühjahr. Im Mittel der Untersuchungsjahre lagen die $\mathrm{N}_{\min }$-Mengen vor Ackerbohne bei 102 bzw. $135 \mathrm{~kg} \mathrm{~N}$ ha $^{-1}$ in der $\mathrm{N}_{0}$ - bzw. $\mathrm{N}_{3}$-Variante. Dabei traten insbesondere in den tieferen Bodenschichten hohe $\mathrm{N}_{\min }$-Mengen auf. Während die $\mathrm{N}_{\min }$-Mengen mit 43 bzw. $42 \mathrm{~kg} \mathrm{~N} \mathrm{ha}^{-1}$ im Oberboden nahezu auf einem Niveau lagen, erhöhten sich die $\mathrm{N}_{\text {min }}$-Mengen in tieferen Bodenschichten deutlicher in der $\mathrm{N}_{3}$-Variante (30-90 cm $92 \mathrm{~kg} \mathrm{~N} \mathrm{ha}^{-1}$ in der $\mathrm{N}_{3}$-Variante bzw. $60 \mathrm{~kg} \mathrm{~N} \mathrm{ha}^{-1}$ in der $\mathrm{N}_{0}$-Variante). Die Wintergerste war in der Lage, die $\mathrm{N}_{\text {min-Mengen }}$ im Boden weitgehend $z u$ nutzen und die $\mathrm{N}_{\text {min }}$-Vorräte vor der Aussaat der Luzerne in beiden Düngungsstufen auf niedrige Werte von 36 und $39 \mathrm{~kg} \mathrm{~N}$ ha $^{-1} \mathrm{zu}$ reduzieren. Anschließend stiegen die Werte über Winter unter Luzerne bis auf ca. $45 \mathrm{~kg} \mathrm{~N}$ ha $^{-1}$ nur leicht an (KNUTH 1995). In der vorliegenden Untersuchung erhöhten sich die $\mathrm{N}_{\text {min }}$-Mengen unter der Schwarzbrache über Winter durch die Mineralisation offensichtlich stärker, sodass etwas höhere $\mathrm{N}_{\text {min }}$-Mengen im Frühjahr vor der Aussaat der Luzerne vorlagen (Tab. A34, abzüglich der Bodenschicht $100-125 \mathrm{~cm}$ resultieren in 0 - $100 \mathrm{~cm}$ 1998: 63,6 bzw. 71,4 kg N ha $^{-1}$, 1999: 60,2 bzw. 70,7 $\mathrm{kg} \mathrm{N}$ ha $^{-1}$ in der $\mathrm{N}_{0}$ - bzw. $\mathrm{N}_{3}$-Variante). Vermutlich wurden die höheren $\mathrm{N}_{\text {min }}$-Mengen im Unterboden durch eine Verlagerung von Nitrat über Winter in tiefere Bodenschichten verursacht. KNUTH (1995) ermittelte in der Fruchtfolge ohne Einsatz von Wirtschaftsdüngemitteln $\mathrm{N}_{\text {min }}$-Mengen im Herbst nach Saatwicke von 40 und $50 \mathrm{~kg} \mathrm{~N}$ ha ${ }^{-1}$ in der $\mathrm{N}_{0}$-und $\mathrm{N}_{3}$-Variante. In dieser Untersuchung wurde nach Wintergerste vor der Aussaat der Saatwicke keine $N_{\text {min }}$-Beprobung durchgeführt. Aus den $\mathrm{N}_{\min }$-Daten der Wintergerste der Fruchtfolge mit Einsatz von Wirtschaftsdüngemittel geht hervor, dass die Bestände in beiden Düngungsvarianten in der Lage waren, die $\mathrm{N}_{\text {min }}$-Mengen im Profil auf Werte unter $40 \mathrm{~kg} \mathrm{~N} \mathrm{ha}^{-1} \mathrm{zu}$ senken. Für die mit Wintergerste bestellten Flächen der Fruchtfolge ohne Wirtschaftsdüngemittel ist anzunehmen, dass sie tendenziell noch niedrigere $\mathrm{N}_{\text {min }}$-Mengen im Boden aufwiesen, da die Parzellen der $\mathrm{N}_{3}$-Variante deutlich höhere Mineralisationsraten aufwiesen und die Wintergerstenbestände der beiden Fruchtfolgen nahezu gleiche N-Mengen akkumulierten (KNUTH 1990, CLAUPEIN 1994). Hierauf deuten auch die $\mathrm{N}_{\text {min }}$-Mengen im Frühjahr 1991 unter Wintergerste von etwa 30 bzw. $40 \mathrm{~kg} \mathrm{~N}^{-1}$ in der $\mathrm{N}_{0}$ - bzW. $\mathrm{N}_{3}$-Variante der Fruchtfolge ohne Einsatz von Wirtschaftsdüngemittel hin (KNUTH 1995). Die in der eigenen Untersuchung vor Aussaat von Saatwicke erhöhten $\mathrm{N}_{\text {min }}$-Mengen im Oberboden (Tab. A49) könnten zum Teil durch eine ver- 
stärkte N-Mineralisation in der $\mathrm{N}_{3}$-Variante verursacht worden sein (KNUTH 1990). Die Tatsache, dass vor Aussaat der Saatwicke die oberirdische Biomasse der Wintergerste (Korn + Stroh + Stoppel) von der Fläche abgefahren wurde, könnte mit zu diesem Befund beigetragen haben. Durch Inkorporation von anorganischem Stickstoff in die organische Substanz infolge einer Strohdüngung können die $\mathrm{N}_{\text {min }}$-Gehalte im Boden deutlich gesenkt werden (SCHMEER und MENGEL 1985).

Die Ansaaten mit Luzerne und alle Nichtleguminosen waren in beiden Jahren in der Lage, den $\mathrm{N}_{\min }$-Vorrat im Boden nahezu vollständig auszunutzen. Der Saatwicke in Reinsaat gelang dies nur im zweiten Untersuchungsjahr, der Ackerbohne in Reinsaat weder im Jahr 1998 noch im Jahr 1999 (Tab. A16, Tab. A18, Tab. A35, Tab. A37, Tab. A50 und Tab. A52).

\section{Ackerbohne}

Die Ackerbohne in Reinsaat zeigte trotz einer relativ hohen durchschnittlichen $\mathrm{N}$ Aufnahme aus dem Boden von 106,6 kg N ha-1 (Tab. A17 und Tab. A19) offenbar eine deutliche $\mathrm{N}$-Sparsamkeit und hinterließ zur Ernte residuale $\mathrm{N}_{\min }$-Mengen zwischen 61,5 und $115,5 \mathrm{~kg} \mathrm{~N}^{-1}\left(\mathrm{~N}_{0}\right.$ und $\mathrm{N}_{3}$ 1998). Damit lag die Boden-N-Aufnahme der Ackerbohne in Reinsaat etwas über den von SCHMIDTKE (2001) bei Ackerbohne ermittelten Werten (Sorte Minica, Standort Reinshof, im Durchschnitt 94,8 kg N ha-1). Des Weiteren ist eine Abnahme der residualen $\mathrm{N}_{\text {min }}$-Mengen im Boden zur Ernte im Mittel über die Düngungsstufen mit zunehmendem Anteil Hafer in beiden Untersuchungsjahren zu erkennen (Ausnahme: 4H = 3H/1A 1998 und 1999).

Beim Vergleich der Ansaatvarianten wird deutlich, dass die Ackerbohne offensichtlich nur sehr eingeschränkt in der Lage ist, nennenswerte Stickstoffmengen aus dem Unterboden aufzunehmen. Dies deutet sich auch beim Vergleich der Untersuchungsjahre der Ackerbohnen in Reinsaat an. Während die Ackerbohne in Reinsaat im Jahr 1998 unabhängig von der Düngungsstufe sich nahezu gleiche Mengen an Boden-N aneignete, übertraf im Jahr 1999 die Aufnahme aus dem Boden in der $\mathrm{N}_{3}-$ Variante die der $\mathrm{N}$-Aufnahme der Bestände aus der $\mathrm{N}_{0}$-Variante. Dabei waren im Jahr 1998 die Unterschiede im $\mathrm{N}_{\min }$-Vorrat des Bodens zu allen drei Ernteterminen nur im Unterboden, im Jahr 1999 lediglich zur zweiten Ernte im Oberboden festzustellen. Die Ackerbohne wies bis zur zweiten Ernte im Jahr 1999 keine unterschiedlichen Boden-N-Aufnahmeraten in Abhängigkeit der langjährig unterschiedlichen $\mathrm{N}$ Düngung zu den nichtlegumen Vorfrüchten auf (Tab. A19). Gleichzeitig konnte ein Einfluss der langjährig unterschiedlich hohen mineralischen N-Düngung zu den nichtlegumen Vorfrüchten auf die $\mathrm{N}_{\text {min }}$-Mengen im Boden nur zur zweiten Ernte im Oberboden nachgewiesen werden (Tab. A18). Die unterschiedlichen Boden-NAufnahmeraten der Ackerbohne zur Endernte im Jahr 1999 sind vermutlich auf eine vermehrte $\mathrm{N}$-Aufnahme der nach der zweiten Ernte erhöhten $\mathrm{N}_{\text {min }}$-Menge im Oberboden der $\mathrm{N}_{3}$-Variante zurückzuführen (Tab. A19). Der Hafer-Reinsaat war es dagegen möglich, mit $\mathrm{N}$-Aufnahmen zwischen 137,3 und $182,5 \mathrm{~kg} \mathrm{~N} \mathrm{ha}^{-1}\left(\mathrm{~N}_{0}\right.$ und $\mathrm{N}_{3} 1998$; Tab. A17) sich deutlich mehr Boden-N, vor allem auch aus dem Unterboden, 
anzueignen als die Ackerbohne in Reinsaat und die residualen $\mathrm{N}_{\text {min }}$-Mengen in der Summe über die gesamte Profiltiefe $(0$ bis $125 \mathrm{~cm}$ ) bis auf Werte zwischen 15,5 und $19,0 \mathrm{~kg} \mathrm{~N} \mathrm{ha}{ }^{-1}\left(\mathrm{~N}_{0} 1998\right.$ und $\mathrm{N}_{3}$ 1999) zu reduzieren (Tab. A16 und Tab. A18). Im Mittel eigneten sich die Pflanzenbestände in der Variante mit mineralischer $\mathrm{N}$ Düngung zu den nichtlegumen Vorfrüchten $\left(\mathrm{N}_{3}\right)$ in beiden Jahren mehr bodenbürtigen Stickstoff an als die Ansaaten in der $\mathrm{N}_{0}$-Variante. Während im Jahr 1999 alle Ansaatvarianten mit im Mittel gleichen gesteigerten Boden-N-Aufnahme in den $\mathrm{N}_{3}$ Variante auf das erhöhte N-Angebot reagierten, reagierten im Jahr 1998 nur die Ansaaten mit Hafer, im Gegensatz zu Ackerbohne in Reinsaat, deutlich auf das erhöhte $\mathrm{N}_{\min }$-Angebot im Unterboden.

Über hohe residuale $\mathrm{N}_{\min }$-Mengen beim Anbau von Ackerbohnen wurde in mehreren Untersuchungen berichtet (z.B.: STÜLPNAGEL 1982, HAUSER 1987, KöNINGS 1987, KÖPKE 1987, ENGEL und MANGSTL 1988, HuBER 1988, LÜTKE-ENTRUP et al. 1989, LÜTKE-ENTRUP und StemANN 1990, StülpNAGEL 1990, LÜTKE-ENTRUP et al. 1993, AufHAMmER et al. 1994, JUSTUS und KÖPKE 1995). Nach KAGE (1992) sind für die hohen residualen $\mathrm{N}_{\text {min }}$-Mengen nach Ackerbohne in erster Linie die geringe Durchwurzelungsintensität und das sich daraus ableitende geringe Nitrataneignungsvermögen der Ackerbohne verantwortlich. Die Ackerbohne bildet ein allorrhizes Wurzelsystem aus, das im Gegensatz zum homorrhizen Wurzelsystem des Hafers durch sehr geringe Wurzellängen charakterisiert ist und vor allem den Unterboden nur wenig durchwurzelt (KUTSCHERA 1960, VetTer und Scharafat 1964, MeYER 1984, MÜLLER 1984, SCHMIDTKE 2001). Offenbar nutzte die Ackerbohne auch in der vorliegenden Untersuchung vor allem den Stickstoff im Oberboden. Die großen Unterschiede im Unterboden in der Höhe der residualen $\mathrm{N}_{\min }$-Menge zwischen den Düngungsstufen bei den Ansaaten mit Ackerbohne deuten dies an. Demgegenüber nahmen in beiden Untersuchungsjahren die Differenzen der residualen $\mathrm{N}_{\text {min }}$-Menge im Unterboden zwischen den Düngungsstufen mit zunehmendem Haferanteil im Bestand deutlich ab, was auf eine Zunahme der Boden-N-Aufnahme durch den Hafer insbesondere aus dem Unterboden hinweist (Tab. A16 und Tab. A18). Eine deutliche Zunahme der Wurzellängendichten durch den Anbau der Ackerbohne im Gemenge mit Hafer und eine daraus resultierende deutliche Abnahme der residualen $\mathrm{N}_{\text {min }}{ }^{-}$ Menge im Boden konnten JUsTus und KÖPKE (1995) nachweisen.

Nach CHALK et al. (1993) und TURPIN et al. (2002) wird unter N-Sparsamkeit ("N sparing effect") die geringere Nutzung des $\mathrm{N}$-Vorrates im Boden einer nodulierenden Leguminose im Vergleich zu einer nicht nodulierenden Leguminose oder einer Nichtleguminose verstanden. Die in Tab. A58 abgetragenen Differenzen zwischen den residualen $\mathrm{N}_{\min }$-Mengen (v.a. Nitrat) der Ansaaten mit Leguminosen und den Reinsaaten der Referenzpflanzen weisen auf nennenswerte Differenzen in beiden Jahren vor allem bei den Ansaaten mit Ackerbohne und bei der Reinsaat von Saatwicke im zweiten Untersuchungsjahr hin. Über die Herkunft des Nitrates zum Erntezeitpunkt lassen sich unterschiedliche Auffassungen finden (UNKOVICH et al. 1997). 
Nach UNKOVICH und PATE (2000) stammt ein beachtlicher Anteil des Nitrates aus der Mineralisation von organischen N-Verbindungen, die zuvor durch eine N-Rhizodeposition der Leguminose in den Boden abgegeben werden. Vor diesem Hintergrund sprechen UNKOVICH et al. (1997) von einem "scheinbaren" N-Sparsamkeits-Effekt.

Auf Grundlage der von SCHMIDTKE (2004) ermittelten Anteile der Rhizodeposition an der gesamtpflanzlichen Biomasse (vgl. Tab. 4) ergibt sich für die hier angebauten Ansaaten mit Ackerbohne eine N-Rhizodeposition in Höhe von 1,5 bis $30,5 \mathrm{~kg} \mathrm{~N}^{-1}$ (Tab. A22), für die Ansaaten mit Luzerne Werte zwischen 3,0 bis $27,5 \mathrm{~kg} \mathrm{~N} \mathrm{ha}^{-1}$ (Tab. A41) und für die Ansaaten mit Saatwicke geschätzte Mengen an N-Rhizodeposition zwischen 0,9 bis $19,2 \mathrm{~kg} \mathrm{~N}^{-1}$ (Tab. A55), die aus der Fixierung stammen. Die in der vorliegenden Untersuchung ermittelten transferierten N-Mengen der Ackerbohne zu Hafer in Gemengesaat (Tab. 5) deuten darauf hin, dass ein beachtlicher Anteil der über Rhizodeposition abgegebenen organischen $\mathrm{N}$-Verbindungen im Verlauf der Vegetationsperiode offenbar mineralisiert wurde und als mineralischer Stickstoff vor allem im Oberboden verfügbar wurde. Eine weitere $\mathrm{N}$-Quelle stellen die in den $\mathrm{Be}-$ standesabfällen akkumulierten N-Mengen der Ackerbohne und Luzerne dar (Tab. A10, Tab. A11 und Tab. A30). In einem Labor-Bebrütungsversuch testeten SCHMIDTKE und VABITSCH (2001) die Höhe der Stickstoffmineralisierung aus Bestandesabfällen der Ackerbohnensorte Minica. Dabei waren nach 84 Tagen Bebrütungsdauer rund $18 \%$ des organischen Stickstoffs im fein vermahlenen und mit dem Boden gut vermischten Bestandesabfall mineralisiert. Bei Ackerbohne in Reinsaat dürften somit über die Mineralisation von auf die Bodenoberfläche fallenden Bestandesabfällen zum Zeitpunkt der Ernte dem Oberboden nicht mehr als $5 \mathrm{~kg} \mathrm{~N} \mathrm{ha}^{-1}$ zugeführt worden sein, zumal den Bestandesabfällen nur etwa 7 Wochen zur Mineralisation zur Verfügung standen (Bestandesabfall war in beiden Jahren ab der Blüte messbar; Zeitraum von zweiter Ernte bis Endernte: 199849 Tage, 199947 Tage).

Sowohl die durch Mineralisierung von über Rhizodeposition in den Boden gelangten organischen N-Verbindungen als auch über Mineralisierung der über Bestandesabfall zugeführten N-Mengen werden zum überwiegenden Teil dem Oberboden zugeführt. Die über Rhizodeposition und Bestandesabfall dem Oberboden zugeführten N-Mengen lassen vermuten, dass es sich bei den residualen $\mathrm{N}_{\text {min }}$-Mengen zur Ernte im Oberboden, insbesondere bei Ackerbohne in Reinsaat, zu einem Großteil um von der Pflanze abgegebenen Stickstoff handelt und hier von einem zum Teil "scheinbaren" N-Sparsamkeits-Effekt gesprochen werden kann.

STÜLPNAGEL (1990) geht hingegen davon aus, dass es sich bei dem im durchwurzelten Bodenraum verbleibenden Nitratstickstoff vor allem um nicht aufgenommenes bodenbürtiges Nitrat handelt, d.h. die Menge an Restnitratstickstoff fällt um so niedriger aus, je geringer die $\mathrm{N}_{\min }$-Mengen vor Aussaat und die während der Vegetationsperiode mineralisierten Stickstoff-Mengen sind. Er konnte diesen Zusammenhang empirisch belegen, indem er einen engen Zusammenhang zwischen der $N_{\min }-M e n g e$ 
im Boden vor Aussaat und der $\mathrm{N}_{\mathrm{min}}$-Menge im Boden zur Ernte der Ackerbohne in Reinsaat feststellte. Dabei stellte er in 0 bis $30 \mathrm{~cm}$ Bodentiefe den geringsten, in 30 bis $90 \mathrm{~cm}$ Bodentiefe den engsten Zusammenhang zwischen der Nitratmenge im Boden im Frühjahr und der Nitratmenge im Boden zur Ernte fest. Auch in der vorliegenden Untersuchung zeigt sich eine deutliche N-Sparsamkeit, die sich bei der

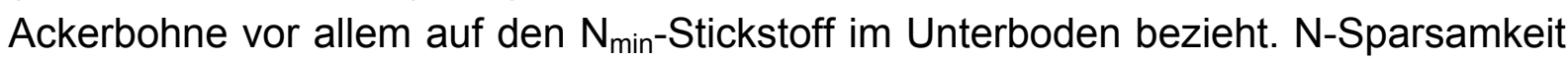
kann offenbar vor allem auch im Unterboden dann auftreten, wenn durch eine eingeschränkte Durchwurzelungsintensität einer Leguminose (Ackerbohne) pflanzenverfügbarer Stickstoff nicht erschlossen werden kann. Die hohen residualen $\mathrm{N}_{\min }-\mathrm{Men}-$ gen nach Anbau der Ackerbohne in Reinsaat traten in der vorliegenden Untersuchung im Mittel zu rund $60 \%$ im Unterboden auf (Tab. A16, Tab. A18). Vor diesem Hintergrund dürften weit mehr als $50 \%$ der insgesamt im Profil vorgefundenen residualen $\mathrm{N}_{\text {min }}$-Mengen nach Ackerbohne auf einen $\mathrm{N}$-Sparsamkeits-Effekt zurückzuführen sein und nur ein geringerer Anteil aus der Mineralisation von organischen $\mathrm{N}$-Verbindungen aus Bestandesabfällen und Rhizodeposition stammen.

Der Anbau der Ackerbohne führt nicht immer zu hohen residualen $\mathrm{N}_{\text {min }}$-Mengen im Boden zur Ernte, wie u.a. aus einer umfassenden Studie von JosT (2003) hervorgeht. In einer dreijährigen Untersuchung mit Körnerleguminosen wurden auch von Ackerbohnen der Sorten Alfred, Caspar und Scirocco auf vier unterschiedlichen Standorten in Niedersachsen die N-Flächenbilanzsalden ermittelt. Dabei variierten die residualen $\mathrm{N}_{\text {min }}$-Mengen zwischen 15 und $65 \mathrm{~kg} \mathrm{~N}^{-1}$ in 0 bis $125 \mathrm{~cm}$ Bodentiefe. Die $\mathrm{N}_{\text {min }}-$ Mengen zur Aussaat lagen zwischen 32,5 bis $73,3 \mathrm{~kg} \mathrm{~N}^{-1}$, Die deutlichste Varianz der residualen $\mathrm{N}_{\text {min }}$-Mengen wurde durch die Standorte verursacht, gefolgt von den Jahres- und Sorteneffekten.

\section{Luzerne}

Die Boden-N-Aufnahme der Luzerne in Reinsaat lag mit $109 \mathrm{~kg} \mathrm{~N} \mathrm{ha}^{-1}$ im Mittel der Düngungsstufen und Jahre auf vergleichbarem Niveau der von KELNER et al. (1997) gefundenen Werte im Ansaatjahr (100 kg N ha ${ }^{-1}$ ) bzw. unter den von WALLEY et al. (1996) bzw. von SCHMIDTKE (2001) geschätzten Werten (143,8 bzw. 131,5 kg N ha-1). Die Bestände der $\mathrm{N}_{3}$-Variante akkumulierten im Jahr 1998 mehr bodenbürtigen $\mathrm{N}_{\text {min }}$ Stickstoff als die Bestände der $\mathrm{N}_{0}$-Variante und reagierten somit auf die erhöhten $\mathrm{N}_{\text {min }}$-Mengen im Unterboden (Tab. A34). Dabei deutet sich an, dass die Luzerne schneller in der Lage war, sich den Stickstoff im Unterboden anzueignen als das Knaulgras in Reinsaat (2. Ernte Tab. A35 und Tab. A37). Wie aus den in Tab. A58 im Anhang abgetragenen Differenzen zwischen der residualen $\mathrm{N}_{\min }$-Menge im Boden der Ansaaten mit Luzerne und den Reinsaaten des Knaulgrases hervorgeht, zeigt sich unter den hier vorgefundenen Versuchsbedingungen in beiden Jahren nur ein geringer N-Sparsamkeits-Effekt in den Ansaaten mit Luzerne. Die Differenzen in der Höhe der residualen $\mathrm{N}_{\text {min }}$-Menge im Ober- und Unterboden zwischen den Reinsaaten der Luzerne und des Knaulgrases deuten an, dass die Luzerne in Reinsaat im Ver- 
gleich zum Knaulgras in Reinsaat tendenziell eine höhere bodenbürtige Stickstoffmenge aus dem Unterboden aufnimmt und die Ansaaten mit zunehmendem Knaulgrasanteil stärker am Stickstoff des Oberbodens partizipieren. Die bereits im Ansaatjahr verstärkte Aufnahme des Stickstoffs aus dem Unterboden durch die Luzerne bestätigen auch Untersuchungen von SCHMIDTKE (2001), der im Mittel für Luzerne ( $c v$. Europe) die doppelte Gesamtwurzellänge $\left(2480 \mathrm{~m} / \mathrm{m}^{2}\right)$ im Vergleich zu Ackerbohne ( $c v$. Minica, $1240 \mathrm{~m} / \mathrm{m}^{2}$ ) ermittelte. Dabei wies Luzerne vor allem im Unterboden (50 bis $100 \mathrm{~cm}$ ) deutlich höhere Wurzellängen auf als die Ackerbohne. Diese Beobachtungen bestätigen auch Ergebnisse zur Nitrat-Aufnahme aus dem Unterboden bei mehrjährigen Luzerneansaaten (MATHERS et al. 1975, BLUMENTHAL und Russelle 1996, Blumenthal et al. 1999). Die Herkunft des residualen $\mathrm{N}_{\text {min- }}$ Stickstoffes zur Ernte kann auch bei den Ansaaten mit Luzerne nicht eindeutig geklärt werden. Die aus Tab. 7 hervorgehenden transferierten N-Mengen im Spross des Knaulgrases deuten an, dass auch hier ein beachtlicher Anteil des von Luzerne über Rhizodeposition an den Boden abgegebenen, aus der Symbiose stammenden $\mathrm{N}$-Menge (Tab. A41) im Verlaufe der Vegetation mineralisiert wurde. Hierdurch kann vermutlich auch teilweise die residuale $\mathrm{N}_{\text {min }}$-Menge im Boden unter Luzerne in Reinsaat verursacht worden sein. Somit müsste auch hier von einem zum Teil "scheinbaren" N-Sparsamkeits-Effekt gesprochen werden. Wie aus Tab. A29 und Tab. A30 im Anhang hervorgeht, traten Bestandesabfälle der Luzernebestände nur im Jahr 1999 im nennenswertem Umfang auf. Ein Beitrag von mineralisiertem organischem Stickstoff aus Bestandesabfällen am residualen $\mathrm{N}_{\text {min }}$-Stickstoff zur Ernte kann daher weitgehend ausgeschlossen werden.

\section{Saatwicke}

MANZKE (1990) fand am Ende zweier Vegetationsperioden nach Saatwicke (104 und 107 Wachstumstage) residuale $\mathrm{N}_{\text {min }}$-Mengen im Boden in Höhe von 33,7 bis $89,7 \mathrm{~kg}$ $\mathrm{N}^{-1} \mathrm{a}^{-1}$ und schätzte die Boden-N-Aufnahme der Saatwicke auf im Mittel 24,8 bis 42,8 $\mathrm{kg} \mathrm{N} \mathrm{ha}^{-1}$. Angaben über die $\mathrm{N}_{\min }$-Mengen zur Aussaat waren in dieser Arbeit nicht vorhanden. Im Vergleich dazu lag die residuale $\mathrm{N}_{\text {min }}$-Menge im Boden unter Saatwicke in Reinsaat in der vorliegenden Untersuchung geringfügig niedriger (Tab. A50 und Tab. A52), die Aufnahme von bodenbürtigem Stickstoff mit Werten zwischen 40,6 bis 63,6 kg N ha-1 ( $\mathrm{N}_{3} 1998$ und 1999) lag höher als die von MANZKE (1990) ermittelten Beträge (vgl. Kap. 3.3.5). Wie aus den Beschreibungen in Kapitel 3.3.6 hervorgeht, konnte weder im Jahr 1998 noch im Jahr 1999 ein N-Transfer von der Saatwicke zum Einjährigen Weidelgras mit der $\delta^{15} \mathrm{~N}$-Methode nachgewiesen werden. Aus Tab. A55 im Anhang gehen die geschätzten, über Rhizodeposition an den Boden abgegebenen N-Mengen hervor. Offensichtlich wurden innerhalb der relativ kurzen Vegetationszeit der Saatwicke und des Einjährigen Weidelgrases nur so geringe, aus der Rhizodeposition und der Symbiose stammende Mengen Stickstoff mineralisiert, dass sie mit der hier genutzten $\delta^{15} \mathrm{~N}$-Methode nicht nachgewiesen werden konnten. Insofern muss davon ausgegangen werden, dass die residualen $\mathrm{N}_{\text {min }}$-Mengen zu einem überwiegenden Anteil auf einen N-Sparsamkeits-Effekt der 
Saatwicke zurückzuführen ist und nur ein geringer Anteil aus der Mineralisation von organischem Stickstoff der Saatwicke stammt. Wie aus den Untersuchungen von MANZKE (1990) hervorgeht, bildete die Saatwicke auf dem untersuchten Standort im Mittel $98 \%$ ihrer Wurzelmasse in der Bodenschicht 0 bis $60 \mathrm{~cm}$ bzw. $93 \%$ der Gesamtwurzelmasse in 0 bis $30 \mathrm{~cm}$ Bodentiefe aus. Die Erhebungen in der eigenen Untersuchung erbrachten für die Saatwicke in Reinsaat im Jahr 1998 im Mittel 89 \% und im Jahr $199972 \%$ der ermittelten Wurzelmasse in der Bodenschicht 0 bis $25 \mathrm{~cm}$ Bodentiefe. Die im Verlaufe der Vegetationsperiode im Unterboden mineralisierten NMengen konnten im Jahr 1998 von den im Vergleich zu 1999 schwach entwickelten Ansaaten mit Saatwicke offensichtlich nicht genutzt werden, so dass hier von einer $\mathrm{N}$-Sparsamkeit der Saatwicke auszugehen ist. Im zweiten Untersuchungsjahr waren die Ansaaten mit Saatwicke offenbar besser in der Lage, insbesondere in der $\mathrm{N}_{3}$ Variante Stickstoff auch aus dem Unterboden aufzunehmen. Eine verminderte Mineralisation im Boden als Ursache der geringeren residualen $\mathrm{N}_{\min }$-Menge (Tab. A52) scheidet in der vorliegenden Untersuchung aus, da trotz geringerer $\mathrm{N}_{\text {min }}$-Menge zur Aussaat mehr Stickstoff im Verlaufe der Vegetation aus dem Boden akkumuliert wurde als im Vorjahr (Boden-N-Aufnahme: Tab. A51 und Tab. A53).

Die langjährig unterschiedliche N-Düngung zu den nichtlegumen Vorfrüchten hat deutlich höhere $\mathrm{N}_{\text {min }}$-Mengen vor Aussaat der Ansaaten mit Ackerbohne und Luzerne im Unterboden der $\mathrm{N}_{3}$-Variante zur Folge. Während die Ackerbohne in Reinsaat nur auf die höheren $\mathrm{N}_{\text {min }}$-Mengen im Oberboden der $\mathrm{N}_{3}$-Variante mit erhöhten $\mathrm{N}$-Aufnahmen aus dem Boden reagierte, waren die Ansaaten mit Luzerne in der Lage, die $\mathrm{N}$-Aufnahme aus dem gesamten Bodenprofil zu steigern, und somit auf die erhöhten bodenbürtigen $\mathrm{N}$-Mengen der $\mathrm{N}_{3}$-Variante im Unterboden zu reagieren (Tab. A35 und Tab. A37). Gleiches gilt für die Gemenge mit Ackerbohne und Hafer. Die Ansaaten mit Saatwicke waren nur im Jahr 1999 in der Lage, nennenswerte Mengen Stickstoff aus dem Unterboden zu akkumulieren.

\subsection{3 $\quad \mathrm{N}_{2}$-Fixierung}

Verschiedene Bakterienarten unterschiedlicher Gattungen sind in der Lage, in Symbiose mit Leguminosen, den molekularen Luftstickstoff in organische Verbindungen zu überführen. Diesen Prozess, bei dem molekularer Stickstoff $\left(\mathrm{N}_{2}\right)$ zu Ammoniak $\left(\mathrm{NH}_{3}\right)$ reduziert wird, bezeichnet man als $\mathrm{N}_{2}$-Fixierung (MENGEL und KIRKBY 1987). Dabei sind die in Symbiose mit Leguminosen lebenden $\mathrm{N}_{2}$-bindenden Bakterien der Gattung Rhizobium bei landwirtschaftlich genutzten Kulturpflanzen am weitesten verbreitet. Für die $\mathrm{N}_{2}$-Fixierleistung wurde eine sehr weite Spannbreite von ca. 20 bis $670 \mathrm{~kg} \mathrm{ha}^{-1}$ und Jahr ermittelt (WERNER 1987). Eine wichtige Voraussetzung für hohe Fixierleistungen ist eine effektive Symbiose zwischen Bakterium und Wirtspflanze (Schlegel 1981, HeInZMAnN 1981, HaRdarson 1993). Da die $\mathrm{N}_{2}$-Fixierung energielimitiert ist, erhöhen alle Standortfaktoren, welche die Photosynthese und das 
Wachstum des Wirtes positiv beeinflussen, die $\mathrm{N}_{2}$-Bindungsfähigkeit des Symbionten (ETTLINGER 1983).

Innerhalb des Systemversuchs Reinshof wurden bereits seit 1981 die in dieser Untersuchung geprüften Leguminosen angebaut (WILDENHAYN 1990). Daher kann von einer ausreichenden Besiedlung mit artspezifischen Rhizobien ausgegangen werden (HeINZMANN 1981, HARDARSON 1993).

\subsubsection{N-Isotopenzusammensetzung}

Wie aus den Ausführungen in den Kapiteln 3.1.3, 3.2.3 und 3.3.3 im Ergebnisteil hervorgeht, lagen die $\delta^{15} \mathrm{~N}-$ Werte der Referenzpflanzen im Mittel der Düngungsvarianten deutlich über den $\delta^{15} \mathrm{~N}$-Werten der Leguminosen und damit auch deutlich über den $\delta^{15} \mathrm{~N}$-Werten des molekularem Luftstickstoff (entspricht dem $\delta^{15} \mathrm{~N}_{0}$-Wert der Leguminose). Damit war es möglich innerhalb dieses Dauerfeldversuchs den Anteil Stickstoff aus der Luft mittels $\delta^{15} \mathrm{~N}$-Methode zu schätzen. Soweit erkennbar wurden damit leguminosenbedingte N-Flüsse erstmals in einem Dauerfeldversuch mit Hilfe $\operatorname{der} \delta^{15} \mathrm{~N}$-Methode quantifiziert.

Die langfristige unterschiedliche $N$-Düngungsintensität $\left(\mathrm{N}_{0}\right.$ und $\left.\mathrm{N}_{3}\right)$ führte nur bei den Ansaaten mit Hafer in Rein- und Gemengesaat mit Ackerbohne zu einem deutlichen Unterschied in den $\delta^{15} \mathrm{~N}$-Werten der gesamtpflanzlichen Biomasse. Lediglich zur zweiten und dritten Ernte im Jahr 1998 sowie zur ersten Ernte im Jahr 1999 ließen sich in der gesamtpflanzlichen Biomasse der Pflanzen aus der $\mathrm{N}_{3}$-Variante höhere $\delta^{15} \mathrm{~N}$-Werte feststellen als in der pflanzlichen Biomasse, die in der $\mathrm{N}_{0}$-Variante gewachsen war (Tab. A13). SCHMIDTKE (2001) ermittelte sowohl bei Ackerbohne als auch bei Luzerne auf den mit Jauche gedüngten Flächen stets höhere $\delta^{15} \mathrm{~N}-W e r t e$ in der pflanzlichen Biomasse als auf der ungedüngten Variante. Allerdings wurden hier die Flächen vor der Aussaat direkt mit einer Jauche gedüngt $\left(80 \mathrm{~kg} \mathrm{~N} \mathrm{ha}^{-1}\right)$, die einen $\delta^{15} \mathrm{~N}$-Wert von $21,7 \%$ aufwies.

Von einer Absenkung der $\delta^{15} \mathrm{~N}-$ Werte innerhalb der Vegetationsperiode in der Biomasse der Referenzpflanzen, wie sie zum Teil auch in der hier vorgestellten Untersuchung beobachtet wurde, ist auch von anderen Autoren berichtet worden (BERGERSEN et al. 1985, HøGH-JENSEN und SCHJOERRING 1994). Als Ursache wird die Aufnahme von pflanzenverfügbaren Stickstoffverbindungen mit einem niedrigeren $\delta^{15} \mathrm{~N}$-Wert aus tieferen Bodenschichten diskutiert (BREMER und VAN KESSEL 1990). 


\subsubsection{Gewichtete Anteile Stickstoff aus der Luft (Ndfa $\left.{ }_{w}\right)$ und luftbürtige N- Aufnahme der Ansaatvarianten}

Die Anteile Stickstoff aus der Luft (Ndfa) in Leguminosen werden durch den pflanzenverfügbaren $\mathrm{N}_{\min }$-Stickstoff im Boden beeinflusst. Verschiedene Prozesse der Symbiose der Leguminose und Knöllchenbakterien sind davon betroffen. Aus vielen Untersuchungen ist die hemmende Wirkung von $\mathrm{N}_{\min }$-Stickstoff auf die symbiotische $\mathrm{N}_{2}$-Fixierung bekannt (z.B. WeRnER 1987, STREETER 1988). Bereits die Bildung der Wurzelknöllchen wird bei hohen Gehalten an Nitratstickstoff in der Wurzelzone gehemmt (HARPER und GIBSON 1984). Vorhandene Knöllchen können in ihrem Wachstum durch Ammonium-N, in stärkerem Maße aber durch Nitrat-N gehemmt werden (IMSANDE 1986, STREETER 1988). Hohe Nitratkonzentrationen können schließlich eine frühzeitige Seneszenz der Knöllchen induzieren (CHEN und PHILLIPS 1977). Nach Bethlenfalvay et al. 1978 sowie StREeter 1985a und 1985b besteht die Hauptwirkung des Nitrates in der Hemmung der Stickstofffixierungsaktivität der Knöllchen durch Inhibierung der Nitrogenase, des Schlüsselenzyms der Stickstoffbindung. Ein geringer Gehalt an $\mathrm{N}_{\text {min }}$-Stickstoff im Boden kann in der Jugendentwicklung das Wachstum und die Stickstofffixierung bei vielen Leguminosenarten auch fördern (STREETER 1988).

Über die innerpflanzlichen Regulationsmechanismen der symbiotischen $\mathrm{N}_{2}$-Fixierung gibt es in der Literatur gegenwärtig drei konkurrierende Hypothesen. Eine Möglichkeit geht von der Regulation über die Kohlenstoffversorgung aus. Die symbiotische $\mathrm{N}_{2}$ Fixierung ist ein sehr energieaufwendiger Prozess, der damit große Mengen an Assimilaten benötigt. Die Nitratreduktion in den Knöllchen zu Ammoniak ist ebenso auf eine Assimilatversorgung angewiesen und konkurriert somit mit der Stickstofffixierung um die Assimilat- und Energieversorgung (BETHLENFALVAY und PHILLIPS 1977, BetHlenfalvaY et al. 1978, HouWAARD 1980). Die Regulation über die Sauerstoffdiffusion in die Knöllchen wird als zweite Theorie diskutiert. Die Nitrogenase besteht aus zwei Proteinkomponenten, dem kleineren Fe-Protein und dem größeren Mo-Fe-Protein (KIM und REES 1994). Beide Komponenten sind essentiell für die Nitrogenaseaktivität. Das Fe-Protein ist extrem sauerstoffempfindlich. In der Leguminosen-Symbiose übernimmt deshalb das Pigment Leghämoglobin eine Schutzfunktion. Es bindet und transportiert den Luftsauerstoff in den Knöllchen. Dadurch wird sichergestellt, dass der Sauerstoffpartialdruck auf ein für die oxidative Phosphorylierung notwendiges Niveau gesenkt wird und für die Nitrogenase einen Schutz vor Inaktivierung bietet (WERNER und KROTZKY 1983). Durch Reduktion von Nitrat-N kann innerhalb des Knöllchengewebes u.a. auch Nitrit entstehen, das in der Lage ist, Leghämoglobin zu oxidieren und somit den kontrollierten Sauerstofftransport in die Knöllchen zu stören (STREETER 1988). Die dritte Theorie (N-"feedback"-Regulation) geht von einer Regulation der $\mathrm{N}_{2}$-Fixierung durch ein bisher nicht zweifelsfrei identifiziertes Produkt der Stickstoffixierung aus (SCHULZE 2004). Bisher liegen in der Literatur keine Hinweise dafür vor, dass sich die physiologisch bedingten Regulati- 
onsmechanismen bei verschiedenen Leguminosenarten grundsätzlich unterscheiden.

Inwieweit eine negative Rückkopplung der symbiotischen $\mathrm{N}_{2}$-Fixierleistung von verschiedenen legumen Genotypen auf das Angebot an $\mathrm{N}_{\text {min }}$-Stickstoff im Boden tatsächlich zu einer Selbstregelung der N-Zufuhr beiträgt, ist von verschiedenen Faktoren abhängig. Dazu gehören neben der genetisch verankerten Fähigkeit einer Leguminose, bodenbürtigen Stickstoff aufzunehmen, alle Standortfaktoren, die das Wachstum der Leguminosen beeinflussen, das zeitliche und räumliche Auftreten von pflanzenverfügbarem $\mathrm{N}_{\min }$-Stickstoff im Boden und die Interaktion zwischen Umwelt und Leguminose hinsichtlich der luft- und bodenbürtigen N-Akkumulation der Pflanze (SCHMIDTKE 1997a). Somit sind Durchwurzelungsintensität, Wachstumsdauer und NAkkumulationsvermögen wichtige, das Reaktionsvermögen auf das bodenbürtige NAngebot beeinflussende Kenngrößen. Zudem ist auch eine enge physiologisch bedingte negative Rückkopplung zwischen der Nitrataufnahme und der $\mathrm{N}_{2}$-Fixierung der Pflanze notwendig.

In der hier vorgestellten Untersuchung wurden neben den Leguminosen in Reinsaat auch die Ndfa-Werte in Gemengen aus Leguminosen und Nichtleguminosen auf Grundlage der gesamtpflanzlichen Biomasse (Spross + Wurzel) geschätzt. Die Darstellung der Ndfa-Werte in der gesamtpflanzlichen Biomasse der Leguminosen im Gemenge lassen sich nur für die Summe beider Gemengepartner darstellen, da eine Zuordnung der Wurzeln im Gemenge zu den Bestandespartnern nicht möglich ist. In der vorliegenden Untersuchung sollen die gesamtpflanzlichen N-Flüsse zur Ermittlung vereinfachter N-Flächenbilanzsalden genutzt werden. Diese lassen sich bei einer gesamtpflanzlichen Bilanzierung nur über den Ndfa-Wert ermitteln, der sich auf den gesamtpflanzlichen Stickstoff im Gemenge bezieht. Werden nur die oberirdischen Anteile der Biomasse berücksichtigt, können die vereinfachten N-Flächenbilanzsalden sowohl über die Ndfa-Werte berechnet werden, die sich auf den legumen Stickstoff im Gemenge beziehen, als auch über die Ndfa-Werte, die sich auf den Stickstoff beider Gemengepartner (Leguminose und Nichtleguminose) beziehen. SCHMIDTKE et al. (2004) schätzten die boden- und luftbürtige N-Akkumulation in der Sprossmasse von Lens culinaris Medik. in Rein- und Gemengesaat mit Hordeum vulgare ssp. nudum $\mathrm{L}$. mit der $\delta^{15} \mathrm{~N}$-Methode. Wurden die gewichteten Ndfa-Werte im Gemenge aus Linse und Gerste auf den Stickstoff der Linse im Gemenge bezogen, stiegen die gewichteten Ndfa-Werte im Vergleich zur Linse in Reinsaat tendenziell an. Werden die gewichteten Ndfa-Werte auf die gesamte Stickstoffmenge im Gemenge (Linse + Gerste) bezogen, sinken die gewichteten Ndfa Werte im Vergleich zur Reinsaat deutlich ab.

Die tatsächlich im Feld ablaufenden N-Flüsse werden bei einer gesamtpflanzlichen Betrachtungsweise, mit der hier angewandten Methodik, nur über die gewichteten Ndfa-Werte, die sich auf den gesamtpflanzlichen Stickstoff beider Gemengepartner 
beziehen, richtig geschätzt und dienen somit als Grundlage für die Berechnungen der Anteile Stickstoff aus Boden und Luft.

Die gewichteten Anteile Stickstoff aus der Luft $\left(\mathrm{Ndfa}_{\mathrm{w}}\right)$ in der gesamtpflanzlichen Biomasse nahmen bei allen Ansaaten mit Leguminosen im Mittel der Düngungsvarianten mit zunehmenden nichtlegumen Gemengepartner, bis auf wenige Ausnahmen (Ndfa ${ }_{w}: 4 \mathrm{~L}=3 \mathrm{~L} / 1 \mathrm{~K}$ und $4 \mathrm{~S}=3 \mathrm{~S} / 1 \mathrm{E}=1 \mathrm{~S} / 3 \mathrm{E}$, Tab. A38, Tab. A53), ab (Tab. A17, Tab. A19, Tab. A36, Tab. A51). Dagegen stiegen die gewichteten Anteile Stickstoff aus der Luft in der Sprossmasse der Ackerbohne in beiden Düngungsvarianten in gleicher Richtung an (Tab. A12). Sowohl die Ackerbohne als auch die Luzerne wiesen mit einem Anteil Stickstoff aus der Luft von rund 0,76 jeweils in den Reinsaaten der $\mathrm{N}_{0}$-Variante die höchsten $\mathrm{Ndfa}_{\mathrm{w}}$-Werte in der gesamtpflanzlichen Biomasse auf. Damit lagen die gewichteten Ndfa-Werte auf vergleichbarem Niveau mit Werten, wie sie in Versuchen von KILIAN (1994), WALLEY et al. (1996), TURPIN et al. (2002) sowie Jost (2003) mit der $\delta^{15} \mathrm{~N}$-Methode erhoben wurden (Tab. A57). Über einen Anstieg der Ndfa-Werte in der Sprossmasse der Ackerbohne im Gemenge gegenüber den Anteilen aus der Luft in der Sprossmasse der Ackerbohnen in Reinsaat berichten DANSO et al. (1987). Die im Vergleich zur Ackerbohne in Reinsaat erhöhten Ndfa-Werte im Spross der Ackerbohne im Gemenge lagen in der vorliegenden Untersuchung mit 0,83 bis 0,95 (Tab. A12) auf einem mit den Werten von DANso et al. (1987) vergleichbaren Niveau $(0,85$ bis 0,96$)$.

Ein Anstieg der $\mathrm{Ndfa}_{w}$-Werte im Spross der Luzerne in Gemengesaat gegenüber den $\mathrm{Ndfa}_{\mathrm{w}}$-Werten im Spross der Luzerne in Reinsaat lässt sich nur im ersten Untersuchungsjahr zwischen der Luzerne in Reinsaat und im Gemenge mit 75 \% Knaulgras nachweisen (1998, $\mathrm{N}_{0}$ bzw. $\mathrm{N}_{3}$, 4L: 0,56 bzw. 0,44 < 1L/3K: 0,67 bzw. 0,59, Tab. A31). Dieser Befund deckt sich gut mit Ergebnissen von BURITY et al. (1989), die den Anteil Stickstoff aus der Luft und den Anteil Transfer-N beim Anbau von Medicago sativa in Rein- und Gemengesaat mit Phleum pratense und Bromus inermis untersuchten und ebenfalls nur zum Teil einen Anstieg des Anteils Stickstoffs aus der Luft in der Leguminosen aus Gemengebau gegenüber der Leguminose aus Reinsaat nachweisen konnten.

Für Saatwicke liegen in der Literatur nur wenige Untersuchungen über die Fixierleistung vor (MANZKE 1990, BECKMANN 1998). MANZKE (1990) schätzte in unmittelbarer Nachbarschaft des Dauerfeldversuchs am Reinshof in Göttingen Anteile Stickstoff aus der Luft in der Biomasse der Saatwicke mit der erweiterten Differenzmethode (Referenzfrucht: Welsches Weidelgras) mit Werten zwischen 0,39 bis 0,56. Die eigenen Werte der Saatwicke in Reinsaat lagen zwischen 0,36 bis 0,69 (Tab. A51 und Tab. A53). Ein direkter Vergleich der Daten erscheint allerdings aufgrund der geringen Übereinstimmung der Ergebnisse beider Methoden insbesondere bei Saatwicke nicht plausibel (vgl. Kap. 4.2). Ein Anstieg der gewichteten Ndfa-Werte in der Sprossmasse der Saatwicke im Vergleich zur Reinsaat war nur im Jahr 1998 
nachweisbar (Tab. A47). Demgegenüber konnte BECKMANN (1998) keine erhöhten Anteile Stickstoff aus der Luft von Vicia sativa im Gemenge mit Lolium multiflorum ermitteln.

Die Bestände mit Leguminosen wiesen am Ende der Vegetation in der langjährig ohne mineralische $\mathrm{N}$-Düngemittel $\mathrm{zu}$ den nichtlegumen Vorfüchten gedüngten Variante $\left(\mathrm{N}_{0}\right)$ stets höhere gewichtete Ndfa-Werte in der gesamtpflanzlichen Biomasse auf als die Bestände, die in der $\mathrm{N}_{3}$-Variante gewachsen waren (Tab. A17, Tab. A36, Tab. A38, Tab. A51 und Tab. A53, Ausnahme: Ackerbohne 1999, tendenziell höhere Anteile $\mathrm{N}$-Luft in der $\mathrm{N}_{0}$ - gegenüber der $\mathrm{N}_{3}$-Variante, Tab. A19). Analog zu den gewichteten Ndfa-Werten verhielt sich die symbiotisch fixierte $\mathrm{N}$-Menge (Ausnahme: Saatwicke 1998, tendenziell höhere fixierte $\mathrm{N}$-Mengen in der $\mathrm{N}_{0}$ - gegenüber der $\mathrm{N}_{3}$-Variante Tab. A51). Die Ansaaten mit Leguminosen akkumulierten jeweils in den Reinsaaten der $\mathrm{N}_{0}$-Variante im zweiten Untersuchungsjahr die höchsten fixierten N-Mengen (4L: 360,7; 4A: 282,2; 4S: $107,0 \mathrm{~kg} \mathrm{~N} \mathrm{ha}^{-1}$ ).

Bei allen geprüften Genotypen scheint eine negative Rückkopplung des mineralischen Bodenstickstoffs auf den Anteil des symbiotisch fixierten Stickstoffs wirksam geworden zu sein. Die Ansaaten mit Ackerbohne wiesen in beiden Versuchsjahren, die Ansaaten mit Saatwicke im Jahr 1998 während der gesamten Vegetationszeit deutlich höhere $\mathrm{N}_{\min }$-Mengen auf als die Ansaaten mit Luzerne. Leguminosen unterscheiden sich deutlich in ihrer Fähigkeit den bodenbürtigen Stickstoff aufzunehmen. Die Effizienz, mit der Leguminosen Stickstoff aufnehmen, setzt sich aus der Aufnahmeeffizienz für boden- und luftbürtigen Stickstoff zusammen (SCHMIDTKE und RAUBER 2000). Die Aufnahmeeffizienz für bodenbürtigen Stickstoff einer Leguminose errechnet sich aus dem Quotienten der Stickstoffaufnahme aus dem Boden (Ndfs) und dem am Standort pflanzenverfügbaren Stickstoffangebot im Boden (Ns). In erster Näherung ergibt sich das pflanzenverfügbare Stickstoffangebot im Boden aus der gesamtpflanzlich akkumulierten N-Menge $\left(\mathrm{N}_{\mathrm{Bt}}\right)$ einer zeitgleich angebauten Referenzpflanze (SCHMIDTKE und RAUBER 2000). Die im Mittel der Düngungsvarianten niedrigeren Anteile Stickstoff aus der Luft im ersten Untersuchungsjahr der Ackerbohne in Reinsaat (Tab. A17, Tab. A19), korrespondieren mit einer höheren Aufnahmeeffizienz für bodenbürtigen Stickstoff der Ackerbohne in Jahr 1998 (75,5\%) im Vergleich zu 1999 (65,0 \%). Während sich die Ackerbohne im Jahr 1998 bis zu 86,0 \% des bodenbürtigen Stickstoffes in der $\mathrm{N}_{0}$-Variante aneignete, lag die Aufnahmeeffizienz für bodenbürtigen Stickstoff in der $\mathrm{N}_{3}$-Variante lediglich bei $65,0 \%$. Hieraus lässt sich schließen, dass eine negative Rückkopplung der zumeist im Unterboden vorhandenen höheren $\mathrm{N}_{\text {min }}$-Mengen in der $\mathrm{N}_{3}$-Variante im ersten Untersuchungsjahr auf die $\mathrm{N}_{2}$ Fixierleistung insbesondere bei Ackerbohne nur wenig wirksam werden konnte (vgl. Kap. 4.3). Nach KAGE (1992) ist eine enge negative Rückkopplung der $\mathrm{N}_{2}$-Fixierleistung der Ackerbohne auf das Angebot an pflanzenverfügbarem Stickstoff eines Bodens im Feld nur dort gegeben, wo sowohl die Durchwurzelungsintensität als auch die Transportraten des bodenbürtigen Stickstoffs zur Leguminosenwurzel ausrei- 
chend hoch sind. Sowohl bei der Ackerbohne als auch bei der Saatwicke waren diese Bedingungen offensichtlich nur zum Teil gegeben.

Die Differenz zwischen den $\mathrm{Ndfa}_{\mathrm{w}}$-Werten in der Biomasse der Bestände der Düngungsvarianten ( $\mathrm{N}_{0}$ und $\mathrm{N}_{3}$ ) wurden bei den Ansaaten mit Ackerbohne zur Endernte im Jahr 1998 mit zunehmendem Haferanteil erhöht $(\Delta 4 \mathrm{~A}=0,04, \Delta 3 \mathrm{~A} / 1 \mathrm{H}=0,05, \Delta$ $1 \mathrm{~A} / 3 \mathrm{H}=0,32$, Tab. A17). Wie oben (Kap. 4.3) bereits dargelegt, ist der Hafer aufgrund seines fein verteilten homorrhizen Wurzelsystems in der Lage, Nitrat-N mit hoher Rate auch aus dem Unterboden aufzunehmen (KAGE 1992). Hafer ist damit in der Konkurrenz um bodenbürtigen Stickstoff im Gemenge der Ackerbohne überlegen. Ein Vergleich der boden- und luftbürtigen N-Aufnahmen im Spross der Ackerbohne in Reinsaat und im Gemenge sowie der Spross-N-Ertragsanteile des Hafers verdeutlichen dies. Die N-Ertragsanteile der Hafer-Sprossmasse in den Gemengesaaten mit Ackerbohne der $\mathrm{N}_{3}$-Variante wiesen im Jahr 1998 mit Werten von 36,8 bzw. 75,8 \% in der Ansaatvariante mit 25 bzw. $75 \%$ Hafer deutlich höhere NErtragsanteile in der Hafer-Sprossmasse auf als in der Hafer-Sprossmasse der $\mathrm{N}_{0}$ Variante (3A/1H: 32,4 \%, 1A/3H: $60 \%$, Abb. 9). Die Boden-N-Aufnahme in der Sprossmasse der Ackerbohne im Gemenge in Relation zur N-Aufnahme aus dem Boden der Ackerbohne in Reinsaat (relative Boden-N-Aufnahme) lag im Mittel der Gemenge im Jahr 1998 bei 0,10 ( $\left.\mathrm{N}_{0}\right)$ bzw. 0,15 $\left(\mathrm{N}_{3}\right)$ und im Jahr 1999 bei 0,23 $\left(\mathrm{N}_{0}\right.$, $\left.\mathrm{N}_{3}\right)$. Mit Werten von 0,47 $\left(\mathrm{N}_{0}, \mathrm{~N}_{3}\right)$ in 1998 und 0,50 $\left(\mathrm{N}_{0}, \mathrm{~N}_{3}\right)$ im Jahr 1999 lag das Verhältnis zwischen der N-Aufnahme aus der Luft der Ackerbohne im Gemenge und der Ackerbohne in Reinsaat deutlich über der relativen Boden-N-Aufnahme. Hieraus lässt sich ableiten, dass die Konkurrenz des Hafers, weitgehend unbeeinflusst durch das Angebot an pflanzenverfügbarem Stickstoff im Boden $\left(\mathrm{N}_{0}, \mathrm{~N}_{3}\right)$, deutlicher die bodenbürtige $\mathrm{N}$-Aufnahme als die Höhe der $\mathrm{N}_{2}$-Fixierung der Ackerbohne einschränkt. Zu vergleichbaren Ergebnissen kommen JENSEN (1996a) beim Anbau von Erbsen in Rein- und Gemengesaat mit Gerste sowie ScHMIDTKE et al. (2004) beim Anbau von Lens culinaris Medik. in Rein- und Gemengesaat mit Hordeum vulgare ssp. nudum L. Die verstärkte Konkurrenz des nichtlegumen Gemengepartners um den Boden-Stickstoff im Gemenge kann schließlich einen Anstieg der gewichteten Ndfa-Werte der Leguminose im Gemenge bewirken (CRAIGH et al. 1981, DANSo et al. 1987, MALLARINO et al. 1990).

In der hier vorgestellten Versuchsreihe reagierten die Ansaaten mit Leguminosen auf die höheren pflanzenverfügbaren Stickstoffmengen aus Vorräten des Bodens in der langjährig gedüngten Variante $\left(\mathrm{N}_{3}\right)$ mit einer mehr oder weniger deutlichen Abnahme der symbiotisch fixierten Stickstoffmenge. Die zunehmenden Anteile der Nichtleguminose im Gemenge führten zum Teil zu ansteigenden gewichteten Ndfa-Werten in der Sprossmasse der Leguminose im Gemenge. Gleichzeitig nahmen die Ertragsanteile der Leguminosen im Gemenge ab und reduzierten die fixierten N-Mengen im Vergleich zu den Reinsaaten. Hieraus resultieren abnehmende fixierte N-Mengen in der gesamtpflanzlichen Biomasse der Gemenge mit zunehmendem nichtlegumen 
Gemengepartner. Die Differenz der fixierten N-Menge der Bestände aus $\mathrm{N}_{0^{-}}$und $\mathrm{N}_{3^{-}}$ Variante war bei der Luzerne im Vergleich aller geprüften Bestände mit $71 \mathrm{~kg}$ im zweiten Versuchsjahr am größten.

SCHMIDTKE (2001) ermittelte bei Ackerbohne, sowohl bei den Ndfa-Werten als auch bei den fixierten $\mathrm{N}$-Mengen, deutlich höhere Differenzen zwischen nicht gedüngten bzw. mit Jauche gedüngten Ackerbohnen $\left(80 \mathrm{~kg} \mathrm{~N}^{-1}\right)$ im Vergleich zu Luzerne. Wie aus den von SCHMIDTKE (2001) ermittelten $\mathrm{N}_{\text {min }}$-Mengen ersichtlich wird, werden durch die applizierte Jauche vor allem die $\mathrm{N}_{\min }$-Mengen des Oberbodens erhöht. Die Jauchedüngung führte hier offensichtlich bei der Ackerbohne zu einer deutlich stärkeren negativen Rückkopplung auf die $\mathrm{N}_{2}$-Fixierleistung als bei der tief wurzelnden Luzerne. In den eigenen Versuchen waren die Ansaaten mit Luzerne am stärksten in der Lage, auf die vor allem im Unterboden auftretenden erhöhten N-Mengen zu reagieren. Hierbei wird deutlich, dass offenbar der Art der Düngung bei wurzelmorphologisch sehr verschiedenen Leguminosenarten im Hinblick auf das Ausmaß einer negativen Rückkopplung der $\mathrm{N}_{2}$-Fixierung auf das bodenbürtige $\mathrm{N}$-Angebot im Verlaufe der Vegetation große Bedeutung zukommt. Wie oben bereits dargestellt reagiert die Ackerbohne in Reinsaat in den eigenen Untersuchungen insbesondere im ersten Untersuchungsjahr mit einer deutlichen Abnahme der Aufnahmeeffizienz für bodenbürtigen Stickstoff in der $\mathrm{N}_{3}$-Variante im Vergleich zur $\mathrm{N}_{0}$-Variante. Im Mittel beider Jahre lag die Aufnahmeeffizienz in der $\mathrm{N}_{0}$-Variante mit $74 \%$ deutlich über der Aufnahmeeffizienz der $\mathrm{N}_{3}$-Variante $(66 \%)$. Die Jauchedüngung in der von SCHMIDTKE (2001) durchgeführten Untersuchung führte im Mittel der Jahre bei Ackerbohne zu einer Aufnahmeeffizienz von $85 \%$ im Vergleich zu $86 \%$ in der ungedüngten Variante. Die Ackerbohne reagierte offensichtlich auf die vor allem im Oberboden erhöhten $\mathrm{N}_{\text {min }}$-Mengen durch eine Düngung zur Ackerbohne, mit einer dem Hafer vergleichbaren Steigerung der bodenbürtigen N-Aufnahme. KAGE (1992) konnte in einem Nährlösungsversuch zeigen, dass die Ackerbohne Nitrat-N mit hoher Influxrate aufnehmen kann und infolgedessen die $\mathrm{N}_{2}$-Fixierung drastisch sinkt. Die von SCHMIDTKE (2001) geprüfte Ackerbohne verringerte die Anteile Stickstoff aus der Luft infolge der Düngung von 0,76 auf 0,64 und die luftbürtigen N-Mengen im Mittel der Jahre von 295 auf $217 \mathrm{~kg} \mathrm{~N}^{-1}$ deutlich (Referenzpflanze: Hafer). Die langjährige unterschiedliche Düngung in der eigenen Untersuchung $\left(\mathrm{N}_{0}, \mathrm{~N}_{3}\right)$, die offensichtlich verstärkt auch im Unterboden wirksam wurde, verminderte den Anteil Stickstoff aus der Luft in der Biomasse der Ackerbohne im Mittel der Jahre lediglich um vier Prozentpunkte (von 0,72 auf 0,68). Ebenso wurde mit $37 \mathrm{~kg} \mathrm{~N}^{-1}$ (von 272 auf 235 $\mathrm{kg} \mathrm{N} \mathrm{ha}{ }^{-1}$ ) auch eine deutlich geringere Abnahme der luftbürtigen $\mathrm{N}$-Mengen im Vergleich zur Untersuchung von SCHMIDTKE (2001) beobachtet. Eine deutliche Abnahme der Anteile Stickstoff aus der Luft in Folge einer mineralischen N-Düngung wurde aus vielen Untersuchungen mit Ackerbohnen beobachtet. RICHARDS und SOPER (1981) ermittelten über mehrere Standorte infolge einer Applikation von mineralischen N-Düngern (90 kg N ha-1) im Mittel eine Abnahme der Ndfa-Werte von 0,68 auf 0,41 . JENSEN (1986a) schätzte die Reduktion des Anteils Stickstoff aus der 
Luft nach einer mineralischen Düngerapplikation (50 $\mathrm{kg} \mathrm{N}^{-1}$ ) zur Aussaat auf 15 Prozentpunkte bei Ackerbohne bzw. auf bis zu 20 Prozentpunkte bei der Erbse.

Die von SCHMIDTKE (2001) ermittelten Anteile Stickstoff aus der Luft und die luftbürtigen N-Mengen beim Anbau von Luzerne reagierten weniger deutlich im Vergleich zur Ackerbohne auf die Jauchedüngung. Die Anteile Stickstoff aus der Luft und die luftbürtigen N-Mengen reduzierten sich von 0,75 auf 0,73 und von 253 auf $203 \mathrm{~kg} \mathrm{~N}$ ha $^{-1}$ (Referenzpflanze: Deutsches Weidelgras). Die Abnahme des Anteils Stickstoff aus der Luft und der luftbürtigen N-Mengen der Luzerne in Reinsaat infolge der langjährigen unterschiedlichen Bewirtschaftung der eigenen Untersuchungen $\left(\mathrm{N}_{0}, \mathrm{~N}_{3}\right)$ war im Mittel der Jahre deutlicher im Vergleich zur Ackerbohne. Die Ndfa-Werte verminderten sich von 0,69 auf 0,60 und die luftbürtige N-Menge von 256 auf $199 \mathrm{~kg}$ $\mathrm{N}$ ha ${ }^{-1}$.

Die dargestellten Ergebnisse der Anteile Stickstoff aus der Luft deuten an, dass die Art der Düngung in Abhängigkeit der untersuchten Leguminosenart einen Einfluss auf leguminosenbedingte N-Flüsse am Standort haben kann. Insbesondere Arten, die den Boden weniger intensiv und tiefgehend durchwurzeln, scheinen differenzierter auf das Zeit-Tiefen-Profil des pflanzenverfügbaren Bodenstickstoffs zu reagieren. Vor diesem Hintergrund kann offenbar von Ergebnissen zur Wirkung eines durch Düngung hervorgerufenen $\mathrm{N}$-Angebot im Boden auf die $\mathrm{N}_{2}$-Fixierleistung von Leguminosen nicht auf die Reaktion der Pflanze geschlossen werden, die mittels eines durch Vorbewirtschaftung geänderten N-Angebot im Boden verursacht werden.

\subsubsection{Transfer-Stickstoff im Gemengeanbau}

Beim Anbau von Leguminosen im Gemenge mit Nichtleguminosen kann im Verlauf der Vegetationsperiode symbiotisch fixierter Stickstoff von der Leguminose zum nichtlegumen Gemengepartner übertragen werden. Von ToMm et al. (1994), Tомм et al. (1995) sowie HøGH-JENSEN und SCHJOERRING (2000) wurde auch ein Transfer von der Nichtleguminose zur Leguminose nachgewiesen. Diese Prozesse werden im Allgemeinen als Stickstoff-Transfer bezeichnet. Der Transfer von Stickstoff, der sowohl auf ober- als auch auf unterirdischem Wege stattfinden kann, vollzieht sich überwiegend auf indirektem Weg durch die Mineralisation von abgestorbenen Pflanzenbestandteilen (Knöllchen, Wurzeln, Bestandesabfall) und anschließender Aufnahme durch den Gemengepartner (TA und FARIS 1987a, TA und FARIS 1987b, TA und FARIS 1988, HARDARSON et al. 1988, HEICHEL und HENJUM 1991). Weitere Wege des Stickstoff-Transfers sind eine direkte Übertragung von Stoffen über die Wurzeln durch Mykorrhiza-Pilze, Auswaschung von $\mathrm{N}$ aus dem oberirdischen Pflanzenbestand durch Niederschläge und durch gasförmige Stickstoffverluste aus dem Spross der Leguminose, die von der Nichtleguminose absorbiert werden (BURITY et 
al. 1989, HAMEL et al. 1991, DenMEAD et al. 1976). VALLIS (1978) und LEDGARD (1991) berichten auch von $\mathrm{N}$-Transfer durch Exkremente von Weidetieren.

Die absolute Höhe der transferierten N-Menge ist von vielen Faktoren abhängig. Die Gemengepartner, die Nutzungsart (Körner-, Schnitt- oder Weidenutzung), die Nutzungsdauer und vor allem die Höhe des Ertrages sind als wichtige Einflussgrößen zu nennen (HAYNES 1980). Nach BROPHY et al. (1987) steigt der Anteil des transferierten Stickstoffs in Gemengen aus Futterleguminosen und Gras mit zunehmendem Ertragsanteil der Leguminose an. In einigen Untersuchungen konnte kein N-Transfer nachgewiesen werden (z.B. DANSO et al. 1987, LEDGARD et al. 1985b, JENSEN 1996a, Jost 2003). Die Schätzung der transferierten $\mathrm{N}$-Mengen mittels $\delta^{15} \mathrm{~N}$-Methode sind nur dann möglich, wenn die $\delta^{15} \mathrm{~N}$-Werte des nichtlegumen Gemengepartners signifikant niedriger sind als die $\delta^{15} \mathrm{~N}$-Werte der gleichen Art in Reinsaat (Kap. 2.7.5). Die für treffgenaue Schätzergebnisse benötigten Unterschiede der $\delta^{15} \mathrm{~N}-$ Werte in der Biomasse der Nichtleguminosen aus Rein- gegenüber Gemengesaat, wie sie für die $\delta^{15} \mathrm{~N}$-Methode gefordert werden (vgl. Kap. 4.2), sind zwischen den Gräsern in Reinsaat und Gemengesaat häufig nicht vorhanden (z.B. SCHMIDTKE 1997a, JOST 2003). Vor diesem Hintergrund dürfte zum Beispiel die Messgenauigkeit bei der Bestimmung des $\delta^{15} \mathrm{~N}$-Wertes in der Biomasse der Nichtleguminose aus Rein- und Gemengesaat die Genauigkeit bei der Schätzung des N-Transfers erheblich beeinflussen (vgl. Kap. 4.2).

Unter Leguminosen kann organischer Stickstoff im Boden verstärkt mineralisiert und vom Gemengepartner aufgenommen werden (JENSEN und SøRENSEN 1988). Da dieser Stickstoff nicht vom Transfer-Stickstoff unterscheidbar ist, kann es zu einer Überschätzung der transferierten N-Mengen kommen. Einige Autoren sprechen daher vom "scheinbaren" N-Transfer (z.B. HEICHEL und HENJUM 1991).

Die Tab. 5 und Tab. 7 zeigen die transferierten N-Mengen zu den nichtlegumen Gemengepartnern der Ackerbohne und der Luzerne. Mit transferierten N-Mengen zwischen 3,6 und 17,9 $\mathrm{kg} \mathrm{N}$ ha $^{-1}$ deckte der Hafer im Gemenge zwischen 2,2 und $21,8 \%$ seiner Stickstoffmenge in der Sprossmasse aus dem Transfer-Stickstoff. Während zwischen den Ansaatvarianten mit Ackerbohne und Hafer keine Unterschiede bestanden, wurden im Jahr 1999 in der $\mathrm{N}_{0}$-Variante, im Vergleich zur $\mathrm{N}_{3}$ Variante, mehr Stickstoff transferiert (Tab. A21).

Untersuchungen mit vergleichbaren Ergebnissen beim Anbau der Ackerbohne liegen, soweit erkennbar, in der Literatur nicht vor. COCHRAN und SCHLENTNER (1995) fanden lediglich geringe Hinweise für einen N-Transfer zwischen Ackerbohne und Hafer. Zu ähnlichen Befunden kamen IZAURRALDE et al. (1992) bei Pisum sativa und Hordeum vulgare sowie VAN KESSEL und ROSKOSKI (1988) bei Vigna unguiculata und Zea mays. EAGLESHAM et al. (1981) konnten beim Anbau von Vigna unguiculata und 
Zea mays steigende Mengen Transfer- $\mathrm{N}$ bei abnehmenden Gehalten an mineralischem Bodenstickstoff beobachten.

Die transferierten $\mathrm{N}$-Mengen beim Anbau der Luzerne und des Knaulgrases im Gemenge sowie die Anteile an Transfer-Stickstoff in der Sprossmasse des Knaulgrases lagen mit Werten bis zu 31,2 $\mathrm{kg} \mathrm{N}$ ha $^{-1}$ bzw. bis zu einem Anteil $\mathrm{N}$ im Knaulgrasspross von 34,6 \% über den Werten, die im Haferspross ermittelt wurden. Die Anteile des Transfer-Stickstoffs an der N-Menge in der Knaulgras-Sprossmasse lagen in beiden Jahren in der luzernebetonten Ansaatmischung über den Anteilen, die im knaulgrasbetonten Gemenge ermittelt wurden. Die Menge an transferiertem Stickstoff lag nur im Jahr 1998 im luzernebetonten Gemenge höher (Tab. A40). Knaulgras deckte im Jahr 1998 in der $\mathrm{N}_{0}$-Variante seinen Stickstoffbedarf zu einem höheren Anteil aus dem Transfer-Stickstoff im Vergleich zur $\mathrm{N}_{3}$-Variante.

WALLEY et al. (1996) trennten Gemengewurzel von Luzerne und Bromus riparius, nach Extraktion der Wurzeln aus dem Boden mit Wasser, bis in $15 \mathrm{~cm}$ Bodentiefe anhand der anhaftenden Stoppeln. Die Wurzeln im Sieb (2 mm) aus 0 bis $15 \mathrm{~cm}$ und 15 bis $30 \mathrm{~cm}$ Bodentiefe konnten den Arten nicht zugeordnet werden. Die im Ansaatjahr ermittelte transferierte $\mathrm{N}$-Menge (Spross und ein Teil der Wurzeln) von Luzerne zu Bromus riparius lag mit rund $8 \mathrm{~kg} \mathrm{~N} \mathrm{ha}^{-1}$ deutlich unter der Menge, die in den eigenen Untersuchungen gefunden wurden (nur Spross). Die höchsten Anteile Transfer-Stickstoff im Gemenge mit Luzerne im Ansaatjahr ermittelten BROPHY et al. (1987) mit $68 \%$ in der oberirdischen Biomasse von Phalaris arundinaceae. Die Pflanzen wurden hier allerdings erst nach der Anzucht im Gewächshaus per Hand in Freiland versetzt. Die transferierten N-Mengen steigen in der Regel mit der Nutzungsdauer an (BROPHY et al. 1987, TA und FARIS 1987b, HARDARSON et al. 1988, HeICHEL und HenJum 1991, WALley et al. 1996). Die höchste Transfer-N-Menge, die in einer Untersuchung von JUNG (2003) in einem Gemenge aus Luzerne und Wiesenschwingel am Standort Reinshof registriert wurde, betrug im zweiten Hauptnutzungsjahr lediglich $12 \mathrm{~kg} \mathrm{~N} \mathrm{ha}{ }^{-1}$.

Im Vergleich mit den Angaben anderer Autoren dürfte die ermittelte transferierte $\mathrm{N}$ Menge von der Luzerne zum Knaulgras der eigenen Untersuchung als vergleichsweise hoch angesehen werden, zumal diese nur im Ansaatjahr geschätzt wurden. Dies gilt umso mehr, da die transferierte N-Menge, die sich in den Ernteresten befand, hier methodisch bedingt nicht mit erfasst wurde. Transfer von Stickstoff zum nichtlegumen Gemengepartner vollzieht sich offenbar mit höherer Wahrscheinlichkeit beim Anbau von Futterleguminosen im Vergleich zu Körnerleguminosen. Wie von einigen Autoren berichtet, findet Stickstoff-Transfer überwiegend auf indirektem Wege statt, indem abgestorbene Pflanzenbestandteile wie Knöllchen und Wurzeln über Mineralisation wieder pflanzenverfügbar werden (TA und FARIS 1987a, HEICHEL und HENJUM 1991). Insofern scheint es nicht verwunderlich, dass die Transfer-Mengen meistens im Verlaufe der Vegetation ansteigen. Weiterhin gibt es Belege, das ausgelöst durch eine Schnittnutzung verstärkt Knöllchen und Wurzelgewebe abge- 
geben werden können (z.B. TA und FARIS 1987a). Während die Ackerbohnen und der Hafer im Gemenge eine Vegetationsdauer von 139 bzw. 132 Tagen aufwiesen, wuchsen die Ansaaten mit Luzerne und Knaulgras rund 70 Tage länger und wurden dreimal geschnitten (208 bzw. 206 Tage). Sowohl die Schnittnutzung der Bestände mit Luzerne als auch die Länge der Vegetationsdauer haben vermutlich maßgeblich zu den höheren Transfermengen beigetragen. Untermauert wird dies durch die Tatsache, dass beim Anbau der Saatwicke mit Einjährigem Weidelgras in der vorliegenden Untersuchung kein N-Transfer nachweisbar war (86 bzw. 97 Tage Wachstumsdauer).

Aus der langjährigen mineralischen N-Düngung zu den nichtlegumen Vorfrüchten $\left(\mathrm{N}_{3}\right)$ resultiert zum Teil deutlich geringere transferierte N-Menge im Vergleich zu den Beständen aus der $\mathrm{N}_{0}$-Variante. Inwieweit diese erhöhte transferierte $\mathrm{N}$-Menge bei niedrigeren Gehalten an mineralischem Stickstoff $\left(\mathrm{N}_{0}\right)$ auf eine vermehrte Abgabe von $\mathrm{N}$ über Rhizodeposition beim Anbau von Leguminosen zurückzuführen ist, lässt sich weder aus den eigenen Untersuchungen noch aus Literaturangaben direkt ableiten. Bei den erhöhten geschätzten Mengen an N-Rhizodeposition der $\mathrm{N}_{0}$-Variante der eigenen Untersuchung muss berücksichtigt werden, dass sich die rechnerisch ermittelten Mengen an N-Rhizodeposition nur auf den Anteil Stickstoff beziehen, der aus der Fixierung stammt und nicht auf die insgesamt über Rhizodeposition abgegebene N-Menge. Des Weiteren wurde unterstellt, dass die abgegebenen Anteile Stickstoff aus der Rhizodeposition (Tab. 4) in beiden Düngungsvarianten ( $N_{0}$, $\mathrm{N}_{3}$ ) gleich sind. Somit resultieren aus den höheren fixierten $\mathrm{N}-$ Mengen der $\mathrm{N}_{0}-\mathrm{Vari}-$ ante höhere geschätzte N-Mengen, die aus der Fixierung stammen und über Rhizodeposition an den Boden abgegeben wurden (Tab. A22, Tab. A41, Tab. A55). HøGHJENSEN und SCHJOERRING (2001) ermittelten in einem Feldversuch die N-Rhizodeposition von Trifolium pratense, Trifolium repens und Lolium perenne in Rein- und Gemengesaat über zwei Jahre auf einem lehmigen Sand. Die Versuchsfläche wurde zuvor über 30 Jahre hauptsächlich mit Getreide genutzt, die oberirdische Biomasse von der Fläche abgefahren und weder mit Kalium noch mit Phosphor gedüngt. Die Fläche war somit durch sehr geringe Mengen an mikrobieller Biomasse, organischer Substanz und Gesamtstickstoff $\left(\mathrm{N}_{\mathrm{t}}\right)$ charakterisiert. Unter diesen Umständen vermuten die Autoren einen besonders hohen Anteil der Rhizodeposition am N-Umsatz im Boden landwirtschaftlich genutzter Böden. Mit einem geschätzten Anteil der N-Rhizodeposition (inklusive Feinwurzeln) am gesamtpflanzlich akkumulierten Stickstoff von $70 \%$ lagen die ermittelten Werte auf einem sehr hohen Niveau. JENSEN (1987 und 1996b) schließt aus seinen Untersuchungen, dass die über Rhizodeposition der Erbse abgegebenen N-Mengen für die im Gemenge angebaute Sommergerste vor allem bei niedrigen $\mathrm{N}$-Düngermengen eine signifikante Stickstoffquelle darstellt. Sowohl die Untersuchungen von HøGH-JENSEN und SCHJOERRING (2001) als auch von JENSEN (1996b) deuten an, dass die über Rhizodeposition abgegebenen NMengen bei niedrigen Gehalten an mineralischem Bodenstickstoff offenbar an Bedeutung zunehmen. Über Rhizodeposition an den Boden abgegebene N-Mengen 
können schließlich nach deren Umbau (Immobilisation und Remineralisation) wieder als mineralischer Stickstoff rezykliert werden und somit auch zum verstärkten $\mathrm{N}$ Transfer beitragen (EAGLESHAM et al. 1981).

\subsubsection{Stickstoff-Harvest-Indizes $\left(\mathrm{H}_{\mathrm{NBt}}\right)$}

Der Stickstoff-Harvest-Index errechnet sich aus dem Quotienten des Stickstoffs im Erntegut und dem Stickstoff in der gesamtpflanzlichen Biomasse. Auch im Rahmen einer $\mathrm{N}$-Flächenbilanzierung beschreibt der $\mathrm{H}_{\mathrm{NBt}}$ den Anteil an Stickstoff der durch das Erntegut (Körner oder Schnittgut) von der Fläche exportiert wird. Werden vor allem die oberirdischen vegetativen Pflanzenteile geerntet, wird der Harvest-Index maßgeblich vom Spross-Wurzel-Verhältnis bestimmt (Spross abzüglich Stoppelanteil). Das Spross-Wurzel-Verhältnis ist für jede Kulturpflanze und Sorte, für jedes Entwicklungsstadium und für jede Kombination von Standortfaktoren eine relativ konstante Größe (BAEUMER 1992). Dies zeigen auch die hier vorgelegten Ergebnisse der gewichteten N-Harvest-Indizes bei Luzerne in Reinsaat im Vergleich der Jahre. Obwohl im Jahr 1999 nahezu doppelt so hohe gesamtpflanzliche N-Mengen $\left(N_{B t}\right)$ im Vergleich zum Vorjahr akkumuliert wurden, lagen die gewichteten $\mathrm{H}_{\mathrm{NBt}}-$ Werte nahezu auf gleichem Niveau (1998: 0,63 bzw. 0,71; 1999: 0,64 bzw. 0,68 in $\mathrm{N}_{0}$ bzw. $\mathrm{N}_{3}$; Tab. A29, Tab. A30). Die Variation der in der Literatur ermittelten $\mathrm{N}$-Harvest-Indizes ist ebenfalls relativ gering, wenn nicht verschiedene Standorte und/oder Sorten miteinander verglichen werden (z.B. HAUSER 1987, RÄTZ 1998, SCHMIDTKE 1999, MANZKE 1990, Tab. A57).

Die eigenen Ergebnisse der Ansaaten mit Ackerbohnen und Luzerne stimmen mit gewichteten $\mathrm{H}_{\mathrm{NBt}}-$ Werten von 0,61 bis 0,71 gut mit Ergebnissen anderer Autoren überein (Tab. A57). Die Reinsaaten mit Saatwicke wiesen mit gewichteten $\mathrm{H}_{\mathrm{NBt}^{-}}$ Werten von 0,72 bis 0,92 deutlich höhere Indizes auf als von MANZKE (1990, Sorte: "türkische Wicke") ermittelt. Inwieweit diese Unterschiede durch die Sorten bedingt sind, lässt sich nicht eindeutig klären. Eine weitere Ursache könnte in der unterschiedlichen Methode zur Bestimmung der Wurzelmasse begründet sein. Die von MANZKE (1990) angewandte Monolithmethode gilt als eine der Methoden mit der höchsten Treffgenauigkeit (KÖPKE 1979). Die von MANZKE (1990) ermittelten unterir-

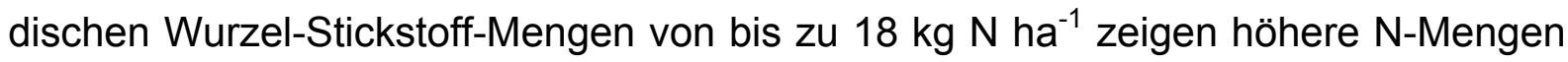
im Vergleich zu den Ergebnissen der vorliegenden Untersuchung (8,4 bis 13,2 kg N $\mathrm{ha}^{-1}$, Tab. A45 und Tab. A46). Hieraus resultieren deutlich engere Spross-WurzelVerhältnisse und damit niedrigere $\mathrm{H}_{\mathrm{NBt}}$-Werte. Weiterhin könnten die deutlichen Unterschiede der gewichteten $\mathrm{H}_{\mathrm{NBt}}$-Werte im Vergleich der Jahre zum Teil auch eine Folge der zum Erntezeitpunkt physiologisch etwas älteren Pflanzen im zweiten Untersuchungsjahr sein. Die Saatwicke erreicht ihre maximale Wurzelmasse zur Blüte. Die Blüte hemmt sowohl das Wachstum der Wurzeln als auch der Knöllchen (PATE 1958). Somit kann angenommen werden, dass bis zur Ernte im Jahr 1999 ein 
größerer Teil der Wurzelmasse im Vergleich zum Vorjahr bereits wieder abgebaut war, und daraus ein deutlich höherer gewichteter N-Harvest-Index zum Erntezeitpunkt resultiert.

Der Einfluss der Düngungsvarianten auf die gewichteten $\mathrm{H}_{\mathrm{NBt}}$-Werte im Vergleich der geprüften Arten war deutlich unterschiedlich. Die gewichteten $\mathrm{H}_{\mathrm{NBt}}$-Werte der Ansaaten mit Saatwicke ließen keine Beeinflussung durch die Düngung erkennen. Die Ansaaten mit Ackerbohne und Hafer wiesen im Mittel der Ansaatvarianten in der $\mathrm{N}_{0}$ Variante im Jahr 1998 höhere gewichtete $\mathrm{H}_{\mathrm{NBt}}$-Werte im Vergleich zur $\mathrm{N}_{3}$-Variante auf, im Jahr 1999 waren sie nur tendenziell höher. Die gewichteten N-Harvest-Indizes der Ansaaten mit Luzerne der $\mathrm{N}_{3}$-Variante lagen dagegen in beiden Jahren deutlich über den Werten der $\mathrm{N}_{0}$-Variante. Vergleichbare Ergebnisse beobachtete auch SCHMIDTKE (1999) bei Ackerbohne und Luzerne. In der mit Jauche gedüngten Variante wies die Ackerbohne etwas geringere, Luzerne höhere $\mathrm{N}$-Harvest-Indizes auf.

Eine mögliche Erklärung für die höheren gewichteten N-Harvest-Indizes der Ansaaten mit Luzerne der $\mathrm{N}_{3}$-Variante könnte eine Änderung des Spross-Wurzel-Verhältnisses infolge einer sich ändernden innerpflanzlichen Assimilatverteilung sein. Die Luzerne aus der $\mathrm{N}_{0}$-Variante wies in beiden Jahren einen deutlich höheren Anteil Stickstoff aus der Luft im Vergleich zu der Luzerne aus der $\mathrm{N}_{3}$-Variante auf. Hohe Fixierleistungen sind nur bei ausreichender Energieversorgung möglich. Somit müssten in der $\mathrm{N}_{0}$-Variante vermehrt Assimilate aus dem Spross über das Phloem in die Wurzeln und Knöllchen transferiert werden. Daraus kann neue Wurzel- und Knöllchenmasse gebildet, sowie vorhandene Knöllchen mit Energie versorgt werden. Folge ist ein Anstieg der Wurzelmasse in der $\mathrm{N}_{0}$-Variante gegenüber der Luzerne aus der $\mathrm{N}_{3}$-Variante (Tab. A25 und Tab. A28), so dass sich das Spross-WurzelVerhältnis der Luzerne der $\mathrm{N}_{0}$-Variante reduziert (1998 Spross/Wurzel = 1,3 bzw. 1,7; 1999: 1,8 bzw. 2,3 in $N_{0}$ bzw. $N_{3}$ ). Letztendlich könnten somit aus der stärkeren relativen Zunahme der Wurzelmasse und stärkeren relativen Abnahme der Sprossmasse in der $\mathrm{N}_{0}$-Variante niedrigere $\mathrm{N}$-Harvest-Indizes resultieren.

Warum die Ackerbohne in der eigenen Untersuchung nicht in gleicher Weise reagiert, könnte mit auf der Tatsache beruhen, dass eine negative Rückkopplung der symbiotischen $\mathrm{N}_{2}$-Fixierleistung auf das Angebot an pflanzenverfügbaren $\mathrm{N}$-Mengen im Boden bei der Ackerbohne im Vergleich zu der Luzerne nur schwächer wirksam wurde. Die Luzerne wies in beiden Jahren in der $\mathrm{N}_{0}$-Variante deutlich höhere gewichtete Ndfa-Werte und höhere fixierte $\mathrm{N}$-Mengen im Vergleich zur $\mathrm{N}_{3}$-Variante auf. Die Differenzen im $\mathrm{N}_{\min }$-Vorrat des Bodens vor Vegetationsbeginn zwischen den Düngungsvarianten waren geringer als vor der Aussaat der Ansaaten mit Ackerbohne. Die Ackerbohne reagierte im Jahr 1998 zwar auch mit erhöhten gewichteten Ndfa-Werten und erhöhten Fixierleistungen in der $\mathrm{N}_{0}$-Variante, konnte die bodenbürtige $\mathrm{N}$-Aufnahme in der $\mathrm{N}_{3}$-Variante, trotz hoher $\mathrm{N}_{\text {min }}$-Mengen im Boden im Vergleich zur $\mathrm{N}_{3}$-Variante allerdings nicht erhöhen (Tab. A17). 
Zusätzlich könnten die differenzierten Sink-Source-Verhältnisse innerhalb des Stoffwechsels der Ackerbohne zu einer von der Luzerne abweichenden Reaktion beigetragen haben. Ab dem zweiten Erntetermin ist bei der Ackerbohne kein Netto-Zuwachs an Wurzel-N mehr zu verzeichnen. Zwischen den vegetativen und generativen Pflanzenteilen besteht ein Konkurrenzverhältnis um Assimilate (JAQUIÉRY und KELLER 1978). Die Blüten, Hülsen und die Triebspitze könnten in der Konkurrenz um Assimilate dem Wurzelwachstum überlegen sein. Hierauf deuten auch Ergebnisse von HAUSER (1987) hin, der beim Anbau von Ackerbohne (cv. Minica und Kristall) die Senkenkapazität veränderte, in dem er jeweils alle Blüten bzw. Hülsen mit Ausnahme der ersten bzw. ersten beiden hülsentragenden Nodien entfernte. Die Teilenthülsung bewirkte im Mittel beider Jahre u.a. eine Verringerung des Spross-Wurzel-Verhältnisses zur Ernte, welches in drei von vier Fällen durch eine deutliche Zunahme der Wurzelmasse begründet war.

\subsubsection{Stickstoff-Flächenbilanzen}

Die Erstellung von Nährstoffsalden für landwirtschaftlich genutzte Flächen kann dazu beitragen, unterschiedliche Anbausysteme im Hinblick auf ihre Nachhaltigkeit und Umweltverträglichkeit zu beurteilen. Die Ermittlung umfassender Stickstoff-Flächenbilanzsalden zur Erfassung aller N-Flüsse ist aufgrund der komplexen Umbau- und Abbauprozesse organischer N-Verbindungen in Boden und Pflanze sowie durch das Auftreten von Stickstoff-Verlusten in mineralischer oder gasförmiger Form methodisch sehr schwierig und aufwendig. In leguminosenbasierten Fruchtfolgen kommt die Eigenschaft der Leguminosen hinzu, Luftstickstoff über die Symbiose mit Knöllchenbakterien zu assimilieren und in den pflanzlichen N-Kreislauf zu integrieren. Im Rahmen der vorliegenden Untersuchung wurden vereinfachte und erweiterte $\mathrm{N}$ Flächenbilanzsalden ermittelt, deren Grundlage die wichtigsten durch die pflanzliche Biomasse beeinflussten Bilanzposten berücksichtigt. Werden die zu prüfenden Leguminosen nicht zusätzlich mit Stickstoff gedüngt, ergibt sich der vereinfachte $\mathrm{N}$ Flächenbilanzsaldo aus der Differenz der symbiotisch fixierten Stickstoff-Menge und des mit dem Erntegut (Schnittgut oder Körner) abgefahrenen Stickstoffs. Nach Umformung von Gleichung (14) zur Berechnung des vereinfachten N-Flächenbilanzsaldos wird der unmittelbare Zusammenhang der Kenngrößen der Bilanzgleichung ersichtlich (Kap. 2.7.2.2): $\mathrm{S}=\mathrm{N}_{\mathrm{Bt}}$ * $\left(\mathrm{Ndfa}-\mathrm{H}_{\mathrm{NBt}}\right)$

Diese vereinfachte Beziehung macht deutlich, dass ein positiver Saldo zu verzeichnen ist, wenn in Bezug auf den gesamtpflanzlichen Stickstoff der Anteil des fixierten Stickstoffs (Ndfa) höher ist als der Anteil des Stickstoffs im Erntegut $\left(\mathrm{H}_{\mathrm{NBt}}\right)$.

Neben den in dieser Untersuchung berücksichtigten N-Bilanzgrößen müssten für eine umfassende Stickstoffbilanzierung in Agrarökosystemen weitere Stickstoffflüsse 
bilanziert werden. Hierzu zählen Stickstoff-Zuflüsse über asymbiotische Stickstofffixierung und atmosphärischer N-Eintrag über trockene und nasse Deposition sowie Stickstoff-Abflüsse durch Bodenabtrag, gasförmige Entbindung und Auswaschungsverluste (ISERMANN und ISERMANN 1998). Aufgrund von methodischen Schwierigkeiten bei der Erfassung dieser N-Flüsse soll im Folgenden zumindest eine Bewertung dieser Größen im Hinblick auf eine N-Flächenbilanzierung am untersuchten Standort vorgenommen werden.

Die wichtigsten frei lebenden $\mathrm{N}_{2}$-bindende Mikroorganismen (asymbiotisch) gehören den Bakteriengattungen Beijerinckia, Azotobakter, Enterobacter und Spirillum sowie verschiedenen Cyanobakterien an und leben bevorzugt in der Rhizosphäre höherer Pflanzen. Hier finden sie die als Nahrung dienenden leicht abbaubaren Kohlenhydrate in Form von Wurzelausscheidungen, abgestoßenen Wurzelzellen und abgestorbenen Wurzelhaaren. Nach MENGEL und KIRKBY (1987) belaufen sich die asymbiotisch fixierten N-Mengen im gemäßigten Klimabereich auf 5 bis $10 \mathrm{~kg} \mathrm{~N} \mathrm{ha}^{-1}$ und Jahr. Stickstoffeinträge über atmosphärische Deposition erfolgen durch Aerosole und N-haltige Gase sowie über das Niederschlagswasser (NIEDER und SCHOLLMEYER 1988). BÖTTCHER (1983) und BACH (1987) beziffern die so eingetragenen N-Mengen mit durchschnittlich 15 bis $20 \mathrm{~kg} \mathrm{~N}$ ha $^{-1}$. NIEDER und SCHOLLMEYER (1988) rechnen mit Einträgen von über $60 \mathrm{~kg} \mathrm{~N} \mathrm{ha}^{-1}$ in Abhängigkeit der Exposition zu Industrieanlagen.

Stickstoffverluste durch Erosion (Bodenabtrag) sind aufgrund der Geländeeigenschaften auf den untersuchten Flächen kaum zu erwarten (CLAUPEIN 1994). Wird Boden über Erosion tatsächlich abgetragen, wird vermutlich in beiden Düngungsvarianten in gleicher Höhe Stickstoff verlagert, da die Gesamtstickstoffgehalte $\left(\mathrm{N}_{\mathrm{t}}\right)$ des Oberbodens keine signifikanten Unterschiede aufwiesen (Tab. A1).

Infolge von Ammoniakemission, Denitrifikation und Nitrifikation können gasförmige Stickstoffverluste verursacht werden. Zu gasförmigen Verlusten durch AmmoniakVerflüchtigung kann es vor allem bei der Anwendung organischer Dünger kommen, die allerdings während des Versuchszeitraumes nicht zur Anwendung kamen. Denitrifikation und Nitrifikation finden im Boden unter gesättigten und ungesättigten Bedingungen gleichzeitig statt und können zu gasförmigen N-Verlusten (v.a. NO, $\mathrm{N}_{2} \mathrm{O}$ ) führen (Russow et al. 2000). Das Verhältnis der beiden Prozesse ist vom Wassergehalt abhängig. KAISER et al. (1998) quantifizierten die gasförmigen Stickstoff-Verluste in einem dreijährigen Feldversuch bei Grünfutterpflanzen und anschließender Sommergerste auf einem sandigen Lehm in Niedersachsen. Die höchsten Erträge und niedrigsten $\mathrm{N}_{2} \mathrm{O}$-Emissionen $\left(1,45 \mathrm{~kg} \mathrm{~N}_{2} \mathrm{O}-\mathrm{N}\right.$ ha- $\left.{ }^{-1} \mathrm{a}^{-1}\right)$ wurden im Mittel der Jahre bei Trifolium pratense ohne jegliche N-Düngung ermittelt. Höhere $\mathrm{N}_{2} \mathrm{O}$-Emission wurde mit im Mittel 2,1 $\mathrm{kg} \mathrm{N}_{2} \mathrm{O}-\mathrm{N} \mathrm{ha}^{-1} \mathrm{a}^{-1}$ bei Lolium perenne mit einer

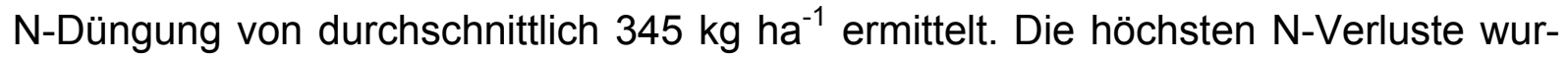
den nach Einarbeitung der Pflanzenreste bei Sommergerste von bis zu 6,1 kg N${ }_{2} \mathrm{O}-\mathrm{N}$ 
$\mathrm{ha}^{-1} \mathrm{a}^{-1}$ gemessen. Grundsätzlich stiegen die emittierten $\mathrm{N}_{2} \mathrm{O}$-Mengen mit steigender $\mathrm{NO}_{3}{ }^{-}$-Konzentration in der Bodenlösung an. Die Ergebnisse deuten an, dass auch in den eigenen Untersuchungen unterschiedlich hohe gasförmige N-Verluste in Abhängigkeit der Düngungsstufen wahrscheinlich sind, da in der $\mathrm{N}_{3}$-Variante teilweise deutlich höhere $\mathrm{N}_{\min }$-Mengen im Vergleich zur $\mathrm{N}_{0}$-Variante vorlagen (z.B. Tab. A16). Die absoluten $\mathrm{N}$-Mengen dürften sich allerdings, wie die dargestellten Zahlen der emittierten N-Mengen andeuten, auf einem niedrigen Niveau bewegen und damit die $\mathrm{N}$-Bilanzen in Abhängigkeit der Düngungsvarianten nur wenig beeinflussen. Allerdings werden $\mathrm{N}$-Verluste durch Denitrifikation im gemäßigten Klimabereich auf landwirtschaftlich genutzten Flächen mit bis zu $30 \mathrm{~kg} \mathrm{~N}$ ha $^{-1}$ angegeben (NIEDER und SCHOLLMEYER 1988).

Verluste durch Nitratauswaschung werden durch die Nitratkonzentration in der Bodenlösung und der Grundwasserneubildung im Verlaufe des Jahres bestimmt (ROHMANN und SONTHEIMER 1985). Trotz hoher nutzbarer Feldkapazität des Bodens am Untersuchungsstandort (175 bis $210 \mathrm{~mm}$ in 0 bis $1 \mathrm{~m}$ Bodentiefe, BORNSCHEUER 1984) konnten in Abhängigkeit der Niederschlagsverhältnisse insbesondere ab Herbst Nitratverlagerungen aus dem durchwurzelten Bodenraum beobachtet werden (KNUTH 1995). Dabei ermittelte KNUTH (1995) in der $\mathrm{N}_{3}$-Variante deutlich höhere ausgetragene $\mathrm{N}$-Mengen im Vergleich zur $\mathrm{N}_{0}$-Variante. Eine Nitratverlagerung im Boden innerhalb des Zeitraumes, der in der vorliegenden Untersuchung betrachtet wurde, könnte zu höheren Nitrat-Verlusten in der $\mathrm{N}_{3}$-Variante im Vergleich zur $\mathrm{N}_{0}$ Variante geführt haben (z.B. $\mathrm{N}_{\min }$ am 20.03.1998 vor Aussaat der Ackerbohnen in $\mathrm{N}_{3}$ $=117,7 \mathrm{~kg} \mathrm{~N}^{-1}$ in 50 bis $125 \mathrm{~cm}$ Bodentiefe Tab. A15, $\mathrm{N}_{\min }$ am 25.05.1998 zur 1 . Ernte in $\mathrm{N}_{3}=39,2$ bis $59,5 \mathrm{~kg} \mathrm{~N} \mathrm{ha}^{-1} \mathrm{Tab}$. A16).

\section{Vereinfachte N-Flächenbilanzen}

Die zur Berechnung vereinfachter N-Flächenbilanzsalden benötigten Kenngrößen wurden auf Grundlage der gesamtpflanzlichen Biomasse (Spross + Wurzel) ermittelt. Anhand zweier Rechenbeispiele soll die Diskrepanz zwischen den N-Salden mit Berücksichtigung der gesamtpflanzlichen akkumulierten N-Mengen bzw. nur der oberirdisch akkumulierten N-Mengen aufgezeigt werden. Für die Ackerbohne in Reinsaat (1999) errechnet sich, mit Berücksichtigung des Stickstoff in den Wurzeln, ein vereinfachter $\mathrm{N}$-Flächenbilanzsaldo in der $\mathrm{N}_{0}$-Variante in Höhe von $+47,4 \mathrm{~kg} \mathrm{~N}$ ha $^{-1}$, ohne Wurzel-N ergibt sich ein $\mathrm{N}$-Bilanzsaldo von $+25,9 \mathrm{~kg} \mathrm{~N}^{-1}$. Für die Luzerne in Reinsaat (1999) errechnet sich, mit Berücksichtigung des Stickstoffs in den Wurzeln ein vereinfachter N-Flächenbilanzsaldo in der $\mathrm{N}_{3}$-Variante in Höhe von $+13,4 \mathrm{~kg} \mathrm{~N}$ ha $^{-1}$, ohne Wurzel-N ergibt sich ein vereinfachter $\mathrm{N}$-Bilanzsaldo von - 73,4kg N ha ${ }^{-1}$. In der Regel führt eine unterlassene Erfassung der unterirdischen $\mathrm{N}$ Mengen zu einer Unterschätzung der N-Salden, da sich sowohl die fixierte N-Menge (Import-N) erniedrigt als auch der N-Harvest-Index erhöht. Mit steigenden unterirdischen N-Mengen an der gesamtpflanzlichen $\mathrm{N}$-Menge vergrößert sich demzufolge die Unterschätzung des N-Flächenbilanzsaldo (PEOPLES 2001, PEOPLES et al. 2001). 
Eine Zusammenstellung von Kennwerten des pflanzlichen $\mathrm{N}$-Haushaltes und vereinfachter N-Flächenbilanzsalden aus Literaturangaben ist im Anhang der Tab. A57 zu entnehmen. Die Angaben beziehen sich auf die gesamtpflanzliche Biomasse der Reinsaaten von Ackerbohne, Luzerne und Saatwicke.

Die vereinfachten N-Flächenbilanzsalden der Leguminosen in Reinsaat der eigenen Untersuchungen lagen zwischen $+56,7 \mathrm{~kg} \mathrm{~N} \mathrm{ha}^{-1}$ (1999 $\left.\mathrm{N}_{0} 4 \mathrm{~L}\right)$ und - 46,6 (1999 $\mathrm{N}_{3}$ 4S). Bei den Gemengen schwankten die vereinfachten N-Flächenbilanzsalden zwischen + 47,6 (1999 $\left.\mathrm{N}_{0} 3 \mathrm{~L} / 1 \mathrm{~K}\right)$ und $-93,4 \mathrm{~kg} \mathrm{~N}^{-1}$ (1998 $\mathrm{N}_{3}$ 1A/3H). Die vereinfachten N-Flächenbilanzsalden der Ackerbohne in Reinsaat stimmen gut mit N-Salden ermittelt von SCHMIDTKE (1999, cv. Minica, + 7,5 und +46,8 $\mathrm{kg} \mathrm{N}^{-1}$, Referenz: Hafer) überein (Abb. 18, Abb. 19). Während die N-Flächenbilanzsalden der Luzerne in Reinsaat in 1998 gut mit den von SCHMIDTKE (1999, cv. Europe, - 16,6 und - 61,3 $\mathrm{kg} \mathrm{N} \mathrm{ha}{ }^{-1}$, Referenz: Deutsches Weidelgras) ermittelten Salden übereinstimmen, lagen die N-Salden der eigenen Untersuchung im zweiten Untersuchungsjahr deutlich darüber $\left(\mathrm{N}_{0}:+56,7, \mathrm{~N}_{3}:+13,4\right.$, Abb. 34, Abb. 35). JUNG (2003) ermittelte 1999 und 2000 jeweils im ersten Hauptnutzungsjahr vereinfachte $\mathrm{N}$-Salden der Luzerne in Reinsaat (cv. Europe) in Höhe von $+95,4$ bzw. $+114,8 \mathrm{~kg} \mathrm{~N} \mathrm{ha}^{-1}$ am Standort Reinshof in Göttingen. Direkt vergleichbare Untersuchungen mit Gemengen anderer Autoren liegen soweit erkennbar nicht vor. JUNG (2003) ermittelte beim Anbau der Luzerne (cv. Europe) im Gemenge (jeweils 50\% der Reinsaatstärke) mit Wiesenschwingel (cv. Cosmos) am Standort Reinshof im Mittel zweier Jahre vereinfachte $\mathrm{N}$-Salden von $+21,3 \mathrm{~kg} \mathrm{ha}^{-1}$ im ersten Hauptnutzungsjahr (vier Schnitte). Beim Anbau der Ackerbohne (cv. Scirocco) im Gemenge (Saatstärke 80\% bzw. 20\% der Reinsaatstärke bei Ackerbohne und Hafer) mit Hafer (cv. Lutz) ermittelte Jost (2003) am gleichen Standort vereinfachte $\mathrm{N}-S a l d e n$ zwischen $+0,6$ bis $+35,1 \mathrm{~kg} \mathrm{ha}^{-1}$. Dabei sanken die N-Flächenbilanzsalden mit zunehmendem N-Ertragsanteile des Hafers im Gemenge ab. Die N-Salden der Bestände, die in den eigenen Untersuchungen ermittelt wurden, verhielten sich analog. Lediglich bei Gemengen mit sehr geringen $\mathrm{N}$-Ertragsanteilen der Nichtleguminosen, wie in der vorliegenden Untersuchung im Vergleich der Reinsaaten von Luzerne mit 3L/1K in beiden Jahren bzw. den Reinsaaten von Saatwicke mit 1S/3E und 3S/1E in beiden Jahren, können keine signifikanten Unterschiede der N-Salden im Mittel der Düngungsstufen zwischen Gemengen und Reinsaaten festgestellt werden (Tab. 8, Tab. 9).

Die N-Flächenbilanzsalden lagen nach Vorfüchten die nicht mit mineralischen NDüngemitteln versorgt wurden $\left(\mathrm{N}_{0}\right)$ in allen geprüften Ansaaten stets über den Salden nach langfristig überoptimal $\left(\mathrm{N}_{3}\right)$ versorgten Vorfüchte. Luzerne in Reinsaat führte in beiden Jahren zu einer deutlich höheren Differenz im N-Bilanzsaldo zwischen $N_{0}$ und $N_{3}$ (im Mittel 42,6 kg ha-1) als Knaulgras in Reinsaat (im Mittel 17,2 kg $\left.h^{-1}\right)$. Ähnlich hohe Differenzen im Bilanzsaldo konnten bei Ackerbohne und Saat- 
wicke gegenüber den entsprechenden Nichtleguminosen in Reinsaat nicht beobachtet werden.

\section{Erweiterte N-Flächenbilanzsalden}

Negative N-Flächenbilanzsalden, wie sie zum Teil sowohl bei Körner- als auch bei Futterleguminosen in Rein- und Gemengesaat ermittelt werden, stehen in Widerspruch zu den oft beobachteten positiven N-Vorfruchteffekten nach Anbau von Leguminosen (z.B. HEINZMANN 1981, KÖPKE 1987). Ursache hierfür ist häufig die fehlende Berücksichtigung der im Verlaufe der Vegetation über Rhizodeposition an den Boden abgegebenen N-Verbindungen. Im Allgemeinen wird unter Rhizodeposition die Abgabe von organischem Material über die Pflanzenwurzel an den Boden verstanden. Während die Abgabe von Kohlenstoff gut erforscht ist (MARSCHNER 1995), liegen über die Abgabe organischer $N$-Verbindungen vergleichsweise wenige Untersuchungen vor (JANZEN und BRUINSMA 1989, LORY et al. 1992, DUBACH und Russell 1994, JeNSEN 1996b, Høgh-JeNSEN und SchJOERRING 2001, KHAN et al. 2002, MAYER et al. 2003a, MAYER et al. 2003b). Die über Rhizodeposition an den Boden abgegebenen N-Mengen sind abhängig von den angebauten Leguminosenarten und den spezifischen Standortbedingungen. Weiterhin muss beachtet werden, dass die Höhe der über Rhizodeposition abgegebenen N-Mengen auch aufgrund methodischer Unterschiede schwanken können (KAHN et al. 2002). MAYER et al. 2003b ermittelte N-Mengen die über Rhizodeposition bei der Ackerbohne (cv. Scirocco) an den Boden abgegeben wurden mit der Docht-Methode in Höhe von $15,5 \%$ des gesamtpflanzlichen Stickstoffs. Mit der gleichen Methodik wies SCHMIDTKE (2004) bei Ackerbohne (cv. Minica) N-Rhizodepositionsmengen von $10,8 \%$ des sich in der gesamtpflanzlichen Biomasse befindlichen Stickstoffs (Grünreife) nach. Beim Anbau von Futterleguminosen können insbesondere bei mehrjährigem Anbau erhebliche Mengen Stickstoff über Rhizodeposition an den Boden abgegeben werden und zum Teil auch während der Vegetation wieder mineralisiert werden (HøGH-JENSEN und SCHJOERRING 2001). Für die in der vorliegenden Untersuchung genutzten Leguminosen wurden die von SCHMIDTKE (2004) ermittelten Stickstoff-Anteile aus der Rhizodeposition zur Ermittlung der erweiterten N-Flächenbilanzsalden genutzt (vgl. Tab. 4). Dabei wurde unterstellt, dass unabhängig von den Ansaatvarianten (Rein- oder Gemengesaat) und Düngungsstufen gleiche Anteile Stickstoff über Rhizodeposition mit gleichen Anteilen Stickstoff aus der Luft (im Vergleich zum gesamtpflanzlichen $\mathrm{N}$ ) abgegeben wurden. Die erweiterten $\mathrm{N}$ Flächenbilanzsalden erhöhen sich um die Beträge an Stickstoff, die aus der Rhizodeposition und der symbiotischen $\mathrm{N}_{2}$-Fixierung stammen (Tab. A22, Tab. A41 und Tab. A55). Damit liegen die erweiterten N-Flächenbilanzsalden der Leguminosen in Reinsaat der eigenen Untersuchungen zwischen + 84,2 (1999 $\left.\mathrm{N}_{0} 4 \mathrm{~L}\right)$ und - 33,3 kg N $\mathrm{ha}^{-1}$ (1999 $\mathrm{N}_{3} 4 \mathrm{~S}$ ). Bei den Gemengen schwankten die erweiterten N-Flächenbilanzsalden zwischen $+71,3\left(1999 \mathrm{~N}_{0} 3 \mathrm{~L} / 1 \mathrm{~K}\right)$ und $-91,9 \mathrm{~kg} \mathrm{~N} \mathrm{ha}^{-1}\left(1998 \mathrm{~N}_{3} 1 \mathrm{~A} / 3 \mathrm{H}\right)$. 
Die Unterschiede im N-Flächenbilanzsaldo gleicher Ansaaten zwischen $\mathrm{N}_{0^{-}}$und $\mathrm{N}_{3^{-}}$ Varianten ergaben sich einerseits aus einer geänderten Boden- $\mathrm{N}$ - und Luft-N-Aufnahme (vgl. Kap. 4.4.2 und 4.4.3.2) und andererseits ganz wesentlich aufgrund einer geänderten Gesamt-N-Akkumulation (Kap. 4.4.1) und eines modifizierten N-HarvestIndex (Kap. 4.4.4).

In Tab. 10 ist der Einfluss der langjährigen mineralischen N-Düngung zu den nichtlegumen Vorfrüchten $\left(\mathrm{N}_{0}, \mathrm{~N}_{3}\right)$ auf die Kenngrößen einer vereinfachten N-Flächenbilanz beim Anbau von Ackerbohne, Luzerne und Saatwicke in Rein- und Gemengesaat mit Hafer, Knaulgras und Einjährigem Weidelgras für beide Versuchsjahre zusammengestellt. Unter den hier vorgefundenen Standortbedingungen zeigt sich die deutlichste Reaktion der Ansaaten mit Ackerbohne und Luzerne auf die langjährige mineralische N-Düngung zu den nichtlegumen Vorfüchten $\left(\mathrm{N}_{0}, \mathrm{~N}_{3}\right)$ im Mittel der Jahre beim N-Flächenbilanzsaldo (S). Dagegen zeigt sich bei Saatwicke die deutlichste Reaktion der Düngungsstufen beim Anteil Stickstoff aus der Luft (Tab. 10).

Tab. 10: Einfluss der langjährigen mineralischen N-Düngung zu den nichtlegumen Vorfrüchten $\left(\mathrm{N}_{3}\right)$ auf die Kenngrößen einer vereinfachten N-Flächenbilanz beim Anbau von Ackerbohne, Luzerne und Saatwicke in Rein- und Gemengesaat.

\begin{tabular}{|c|c|c|c|c|c|c|c|c|c|c|}
\hline \multicolumn{6}{|c|}{1998} & \multicolumn{5}{|c|}{1999} \\
\hline Ackerbohne & $4 \mathrm{~A}$ & $3 \mathrm{~A} / 1 \mathrm{H}$ & $1 \mathrm{~A} / 3 \mathrm{H}$ & $4 \mathrm{H}$ & & $4 \mathrm{~A}$ & $3 \mathrm{~A} / 1 \mathrm{H}$ & $1 \mathrm{~A} / 3 \mathrm{H}$ & $4 \mathrm{H}$ & \\
\hline $\mathrm{N}_{\mathrm{Bt}}$ & $<1$ & $>^{2}$ & $>$ & $>$ & n.s. & $<$ & $<$ & $>$ & $>$ & n.s. \\
\hline $\mathrm{H}_{\mathrm{NBt}}$ & $<$ & $>$ & $<$ & $>$ & * & $<$ & $=$ & $<$ & $<$ & n.s. \\
\hline Ndfa & $<$ & $<$ & $<$ & & ** & $<$ & $<$ & $<$ & & n.s. \\
\hline $\mathrm{S}$ & $<$ & $<$ & $<$ & $<$ & * & $<$ & $<$ & $<$ & $<$ & * \\
\hline Luzerne & $4 \mathrm{~L}$ & $3 \mathrm{~L} / 1 \mathrm{~K}$ & $1 \mathrm{~L} / 3 \mathrm{~K}$ & $4 \mathrm{~K}$ & & $4 \mathrm{~L}$ & $3 \mathrm{~L} / 1 \mathrm{~K}$ & 1L/3K & $4 \mathrm{~K}$ & \\
\hline $\mathrm{N}_{\mathrm{Bt}}$ & $<$ & $<$ & $>$ & $>$ & n.s. & $<$ & $<$ & $<$ & $>$ & * \\
\hline $\mathrm{H}_{\mathrm{NBt}}$ & $>$ & $>$ & $>$ & $>$ & ** & $>$ & $>$ & $<$ & $>$ & ** \\
\hline Ndfa & $<$ & $<$ & $<$ & & ** & $<$ & $<$ & $<$ & & ** \\
\hline$S$ & $<$ & $<$ & $<$ & $<$ & $* * *$ & $<$ & $<$ & $<$ & $<$ & $* * *$ \\
\hline Saatwicke & $4 S$ & $3 \mathrm{~S} / 1 \mathrm{E}$ & $1 \mathrm{~S} / 3 \mathrm{E}$ & $4 \mathrm{E}$ & & $4 S$ & $3 \mathrm{~S} / 1 \mathrm{E}$ & $1 \mathrm{~S} / 3 \mathrm{E}$ & $4 \mathrm{E}$ & \\
\hline $\mathrm{N}_{\mathrm{Bt}}$ & $<$ & $>$ & $>$ & $>$ & n.s. & $<$ & $<$ & $>$ & $>$ & n.s. \\
\hline $\mathrm{H}_{\mathrm{NBt}}$ & $>$ & $<$ & $>$ & $<$ & n.s. & $<$ & $<$ & $<$ & $<$ & n.s. \\
\hline $\mathrm{Ndfa}$ & $<$ & $<$ & $<$ & & * & $<$ & $<$ & $<$ & & $* *$ \\
\hline $\mathrm{S}$ & $<$ & $<$ & $<$ & $<$ & n.s. & $<$ & $<$ & $<$ & $>$ & n.s. \\
\hline
\end{tabular}

${ }^{1}<\mathrm{N}_{3}$ kleiner als $\mathrm{N}_{0},{ }^{2}>\mathrm{N}_{3}$ größer als $\mathrm{N}_{0}$, n.s. = nicht signifikant; ${ }^{*}=P<0,05 ;{ }^{* *}=P<0,01 ;{ }^{* * *}=P<0,001$

Allerdings kann die leguminosenbedingte Zufuhr und Abfuhr von Stickstoff auf bzw. von der Fläche nicht hinreichend durch die Quantifizierung der $\mathrm{N}_{2}$-Fixierleistung beim Anbau von Leguminosen geschätzt werden. Bei der Saatwicke in Reinsaat beispielsweise, steigt die fixierte N-Menge im Vergleich der Jahre von 35,9 auf 107,0 kg $\mathrm{N}$ ha $^{-1}$ an ( $\mathrm{N}_{0} 1998$ und 1999 Tab. A51, Tab. A53). Gleichzeitig erhöhen sich die gesamtpflanzliche N-Akkumulation $\left(\mathrm{N}_{\mathrm{Bt}}\right)$ und der gewichtete Stickstoff-Harvest-Index $\left(\mathrm{H}_{\mathrm{NBtw}}\right)$ von 78,7 auf 155,5 $\mathrm{kg} \mathrm{N}^{-1}$ bzw. von 0,72 auf 0,92 ( $\mathrm{N}_{0} 1998$ und 1999, Tab. A45, Tab. A46). Der sich aus den Kenngrößen $\mathrm{N}_{\mathrm{B} t}, \mathrm{H}_{\mathrm{NBtw}}$ und $\mathrm{Ndfa}_{\mathrm{w}}$ errechnete 
vereinfachte N-Flächenbilanzsaldo (Gleichung (14), Kap. 2.7.2.2) verringert sich dagegen von - 21,0 auf $-35,9 \mathrm{~kg} \mathrm{~N}^{-1} \mathrm{~N}^{-1} \mathrm{~N}_{0} 1998$ und 1999, Abb. 49, Abb. 50). Es wird deutlich, dass erst eine Gesamtbetrachtung der Kenngrößen in Form des NFlächenbilanzsaldos die leguminosenbedingte $\mathrm{N}$-Flüsse näherungsweise $\mathrm{zu}$ beschreiben vermag. Zu gleichem Ergebnis kommt JUNG (2003) in einer umfassenden Studie beim Anbau von Luzerne, Rotklee und Perserklee in Rein- und Gemengesaat mit Poaceen.

\subsection{Beitrag von Ackerbohne, Luzerne und Saatwicke zur Selbstregelung der N-Zufuhr}

Die dargestellten Ergebnisse machen deutlich, dass in leguminosenbasierten Fruchtfolgen eine Selbstregelung der N-Zufuhr wirksam wird. Dabei sind Leguminosenarten am untersuchten Standort in unterschiedlichem Maße in der Lage, einen Beitrag zur Selbstregelung der N-Zufuhr zu leisten. Es zeigt sich, dass die langjährige unterschiedlich hohe mineralische N-Düngung zu den nichtlegumen Vorfüchten einen deutlichen Einfluss auf die Zeit-Tiefen-Funktion des bodenbürtigen Stickstoffes und damit insbesondere auf die Höhe der $\mathrm{N}_{\min }$-Mengen im Unterboden hat. Inwieweit die geprüften Leguminosenarten auf das differenzierte $\mathrm{N}_{\text {min }}$-Angebot im Ober- und Unterboden reagieren, ist offenbar in hohem Maße eine Funktion der artspezifischen Wurzeltiefe und Durchwurzelungsintensität und der Länge der Vegetationsdauer der Leguminose.

Die N-Flächenbilanzsalden der $\mathrm{N}_{0}$-Variante der Ansaaten mit Ackerbohne und Luzerne in Rein- und Gemengesaat mit Hafer und Knaulgras lagen in beiden Jahren deutlich, die N-Salden der Ansaaten mit Saatwicke und Einjährigem Weidelgras lediglich tendenziell, über den Salden der Bestände aus der $\mathrm{N}_{3}$-Variante. Während die Unterschiede im N-Flächenbilanzsaldo gleicher Ansaaten zwischen der $\mathrm{N}_{0^{-}}$und $\mathrm{N}_{3}$-Variante bei den Ansaaten mit Ackerbohne und Saatwicke deutlich geringer als die zu Vegetationsbeginn vorgefundenen Mengen an $\mathrm{N}_{\text {min }}$-Stickstoff im Boden waren, lagen die Unterschiede im N-Bilanzsaldo bei Luzerne deutlich darüber. Die Luzerne

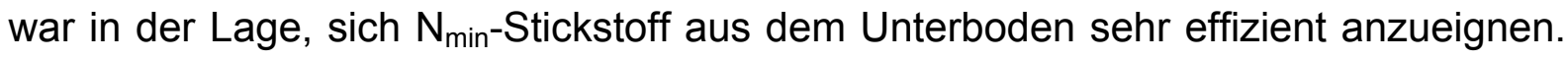
Somit trägt vor allem bei den Ansaaten mit Luzerne auch der Stickstoff aus dem Unterboden zur Reduktion der N-Flächenbilanzsalden und damit zur Reduktion der $\mathrm{N}$-Zufuhr auf die Fläche bei.

Weiterhin zeigen die Ergebnisse, dass Mechanismen der Selbstregelung direkt und indirekt, in Abhängigkeit der Leguminosenart und Anbauform, auf die Höhe der symbiotisch fixierten N-Menge (SCHMIDTKE 1997a und 1997b) und damit auf die NFlächenbilanzsalden wirken. In Gemengen aus Leguminosen und Nichtleguminosen wurden unter dem Einfluss einer mineralischen N-Düngung zu den Gemengen häufig steigende Ertragsanteile des nichtlegumen Gemengepartners beobachtet (z.B. 
BUtLeR und LADD 1985, JeNSEN 1986b, FRANKOW-LINDBERg 1987, SCHMUde 1991, COCHRAN und SCHLENTNER 1995, BESCHOW et al. 2000). Wie aus den vorliegenden Untersuchungen hervorgeht, führte lediglich im ersten Untersuchungsjahr die langjährige mineralische N-Düngung zu den nichtlegumen Vorfüchten $\left(\mathrm{N}_{3}\right)$ im Mittel der Ansaatvarianten zu höheren N-Ertragsanteilen des Hafers im Gemenge mit Ackerbohne. Infolgedessen erhöhte sich die Differenz der $\mathrm{Ndfa}_{w}$-Werte zwischen den Düngungsvarianten $\left(\mathrm{N}_{0}\right.$ und $\mathrm{N}_{3}$ ) bei den Ansaaten mit Ackerbohne mit zunehmendem Haferanteil (vgl. Kap. 4.4.3.2, signifikante Wechselwirkung Düngung $x$ Ansaatvariante, Tab. A17). Allerdings wiesen mit Ausnahme der Ansaaten der Ackerbohne in Rein- und Gemengesaat mit Hafer im Jahr 1999 alle Ansaatvarianten mit Leguminosen höhere Fixierleistungen in der $\mathrm{N}_{0}$-Variante im Vergleich zu der $\mathrm{N}_{3}$-Variante auf. Hieraus lässt sich ableiten, dass eine Verminderung der symbiotischen Stickstofffixierleistung offensichtlich vorrangig durch die direkte Einwirkung des mineralischen Stickstoffs im Boden auf die Leguminosen erfolgte. Die von SCHMIDTKE (1997a und 1997b) beschriebene überproportionale Förderung der Nichtleguminose bei erhöhtem mineralischen Stickstoffangebotes im Boden in Gemengen aus Leguminosen und Nichtleguminosen wurde in der vorliegenden Untersuchung nur wenig wirksam. Die Wirkung einer langjährigen unterschiedlichen Vorbewirtschaftung auf die Stickstofffixierleistung und den N-Flächenbilanzsaldo beim Anbau von Leguminosen in Rein- und Gemengesaat mit Nichtleguminosen stimmen nach den hier ermittelten Ergebnissen in der Wirkung nicht mit Ergebnissen aus Versuchen überein, in denen mineralische $\mathrm{N}$-Düngemittel direkt zu den Leguminosen appliziert wurden. Eine mineralische N-Düngung bewirkt eine starke Anhebung des pflanzenverfügbaren Stickstoffs im Oberboden, der im Leguminosen-Nichtleguminosen-Gemenge offenbar zu einer überproportionalen Förderung der Nichtleguminose führt. In dessen Folge geht die symbiotische $\mathrm{N}_{2}$-Fixierleistung der Leguminose zurück. Ein durch die Vorfrucht bedingtes erhöhtes Angebot an Stickstoff im Boden befindet sich offenbar, so zeigen die hier vorgelegten Untersuchungsergebnisse (z.B. Tab. A34a), in vielen Fällen auch im Unterboden. Dieser Stickstoff wird offenbar zum Teil erst zeitlich verzögert vom Nichtleguminosen-Gemengepartner aufgenommen und führt dann nicht mehr zu einer überproportionalen Förderung des Wachstums der Nichtleguminose. Die Folge ist, dass die Leguminose im Gemenge weniger stark durch die Konkurrenz des Nichtleguminosen-Gemengepartners im Wachstum und in der symbiotischen $\mathrm{N}_{2}$-Fixierleistung eingeschränkt wird.

\subsection{Regulation der N-Zufuhr im Feld}

Leguminosen werden in der landbaulichen Praxis für die Erzeugung von Proteinen für die tierische und menschliche Ernährung, zur Auflockerung getreidereicher Fruchtfolgen und zur Versorgung von Fruchtfolgen mit symbiotisch fixiertem Stickstoff angebaut. Insbesondere in leguminosenbasierten Fruchtfolgen, in denen der fixierte Stickstoff über Leguminosen als alleinige N-Quelle zur Verfügung steht, ist 
auch die Ertragsleistung der Nichtleguminosen eng mit der Fixierleistung der angebauten Leguminosen verknüpft (MÖLLER 1995). Über die positive Wirkung beim Anbau der Leguminosen auf die Erträge der nichtlegumen Folgefrüchte wurde von mehreren Autoren berichtet (z.B: HEINZMANN 1981, KÖPKE 1987). Eine umweltverträgliche Nährstoffversorgung durch den Anbau von Leguminosen setzt voraus, dass $\mathrm{N}$-Verluste und damit die Belastung von Umweltgütern minimiert werden. Sowohl in den hier vorgestellten Untersuchungen als auch in der Arbeit von SCHMIDTKE (1997a) zeigt sich eine deutliche negative Rückkopplung der symbiotischen $\mathrm{N}_{2}$-Fixierleistung auf die mineralischen N-Mengen im Boden beim Anbau verschiedener Leguminosenarten. Die eigenen Ergebnisse belegen darüber hinaus auch die Wirkung einer negativen Rückkopplung des Angebotes an pflanzenverfügbarem Stickstoff im Boden auf den N-Flächenbilanzsaldo.

Sowohl SteVEnson et al. (1995) als auch SchmidTKE (1997a) folgern aus ihren Untersuchungen mit Erbse und Rotklee, dass der Ertrag und die $\mathrm{N}_{2}$-Fixierleistung der angebauten Leguminosen mit hoher Wahrscheinlichkeit vor allem durch den Faktor Wasser bestimmt werden. STEVENSON et al. (1995) ermittelten bis in $60 \mathrm{~cm}$ Bodentiefe innerhalb heterogener Flächen deutlich Unterschiede im Wassergehalt, die schließlich die N-Mineralisierung und somit die $\mathrm{N}_{\text {min }}$-Mengen im Boden deutlich beeinflussten. Der Anteil Stickstoff aus der Luft in der Sprossmasse der angebauten Erbse variierte dabei zwischen 72 und $84 \%\left(\delta^{15} \mathrm{~N}\right.$-Methode). Die Autoren folgern aus ihren Beobachtungen, dass das Angebot an mineralischem Bodenstickstoff erst dann zum bestimmenden Faktor für die Höhe der $\mathrm{N}_{2}$-Fixierleistung wird, wenn eine ausreichende Wasserversorgung zur Verfügung steht. Inwieweit teilflächenspezifisch unterschiedlich hohe Vorräte an mineralischem Stickstoff im Boden die N-Flächenbilanzsalden verändern, lässt sich aus den Daten nicht sicher ableiten. Aus einem niedrigeren Gehalt an mineralischem Stickstoff im Boden resultieren höhere Fixierleistungen, höhere Trockenmasse-Harvest-Indizes und etwas niedrigere gesamtpflanzlich akkumulierte Trockenmassen (STEVENSON et al. 1995). Somit deuten sich auch unter diesen heterogenen Feldbedingungen eine Regulation der N-Zufuhr an. Die Fähigkeit auf Standortfaktoren mit einer Anpassung der Fixierleistung und anderer, für die Ermittlung von N-Flächenbilanzsalden benötigten Kenngrößen zu reagieren, wurde u.a. von REICHARDT (1990) und RÄTZ (1998) für Ackerbohne sowie von REICHARDT et al. (1987) für Luzerne nachgewiesen.

Eine negative Rückkopplung der $\mathrm{N}_{2}$-Fixierleistung auf das Angebot an mineralischem Bodenstickstoff wird in der Fläche offenbar aufgrund kleinräumiger Standortunterschiede wirksam (STEVENSON und VAN KESSEL 1996). Wie die eigenen Untersuchungen belegen, werden bei tief wurzelnden Leguminosen, hier Luzerne, auch die $\mathrm{N}_{\text {min }}{ }^{-}$ Mengen im Unterboden bei der Verminderung der $\mathrm{N}_{2}$-Fixierleistung und damit bei der Verminderung des N-Flächenbilanzsaldos wirksam. Diese negative Rückkopplung auf den $\mathrm{N}_{\text {min }}$-Stickstoff im Unterboden dürfte mit zu einer Verminderung des Nitrat-N im Unterboden beitragen, der in hohem Maße auswaschungsgefährdet ist. Dies 
deuten auch die höheren Nitratkonzentrationen in der Bodenlösung in $170 \mathrm{~cm}$ Bodentiefe unter Ackerbohne im Vergleich zu Luzerne im Ackerbau-Systemversuch Göttingen an, die von KNUTH (1995) ermittelt wurden. Durch den Anbau von Ackerbohne im Gemenge mit Hafer können auch die hohen $\mathrm{N}_{\text {min }}$-Mengen im Unterboden, die in der vorliegenden Untersuchung bereits vor der Aussaat vorlagen, zur Senkung des N-Flächenbilanzsaldos beitragen. Insbesondere im ersten Untersuchungsjahr führte der $\mathrm{N}_{\text {min }}$-Stickstoff sowohl zu einer direkten Verminderung der Fixierleistung der Ackerbohne als auch zu einer indirekten Einschränkung der Fixierleistung und damit zu einer deutlichen Abnahme der N-Flächenbilanzsalden (Abb. 18). Gleichzeitig erhöhte sich die Differenz im Flächenbilanzsaldo gleicher Ansaaten zwischen der $\mathrm{N}_{0}$ - und $\mathrm{N}_{3}$-Variante mit zunehmendem Anteil Hafer im Gemenge und trägt somit wirksam zur Verminderung des N-Bilanzüberschuss auf der Fläche bei. Im Vergleich zur Ackerbohne in Reinsaat konnte so der Anteil des $\mathrm{N}_{\text {min }}$-Stickstoffs vor allem im Unterboden vor Winter stark reduziert werden (Tab. A16).

Die vorliegende Untersuchung verdeutlicht, dass Selbstregulationsprozesse nicht nur auf der biotischen Ebene wirksam werden, sondern in beachtlichem Maße auch durch Interaktionen zwischen abiotischen und biotischen Bestandteilen - z.B. die Zufuhr von Stickstoff - wirksam begrenzen können (MöLLER 1995, SCHMIDTKE 1997a). 


\section{Zusammenfassung}

In einem zweijährigen Feldversuch wurde der Beitrag von Ackerbohne, Luzerne und Saatwicke in Rein- und Gemengesaat mit Hafer, Knaulgras und Einjährigem Weidelgras zur Selbstregelung der $\mathrm{N}$-Zufuhr in leguminosenbasierten Fruchtfolgen quantifiziert. Dabei sollte die Wirkung eines aus der Vorbewirtschaftung resultierenden unterschiedlich hohen Angebotes an Stickstoff im Boden auf den vereinfachten $\mathrm{N}$-Flächenbilanzsaldo von Leguminosen unter Freilandbedingungen ermittelt werden. Die Leguminosenarten wurden hierzu 1998 und 1999 in einem Dauerfeldversuch auf einem Lösslehmstandort (Ackerbau-Systemversuch Göttingen) nach 15-jährig unterschiedlicher mineralischer $\mathrm{N}$-Düngungsintensität $\left(\mathrm{N}_{0}, \mathrm{~N}_{3}\right)$ zu den nichtlegumen Vorfrüchten angebaut und die Trockenmasse- und Stickstofferträge der gesamtpflanzlichen Biomasse erfasst. Die Höhe des mineralischen Bodenstickstoffs wurde mittels $\mathrm{N}_{\text {min }}$-Methode an mehreren Probenahmeterminen gemessen. Die Ansaat der Arten erfolgte in einer substitutiven Verdrängungsserie. Die luft- und bodenbürtige N-Aufnahme der Leguminosen wurde unter Nutzung stabiler Isotope mit der $\delta^{15} \mathrm{~N}$-Methode und mit der erweiterten Differenzmethode geschätzt.

- Die $\delta^{15} \mathrm{~N}$-Werte des Hafers in Reinsaat lagen zwischen 3,60 und 5,12 \%o (1998: Endernte und 1999: 1. Ernte), bei Knaulgras zwischen 3,92 und 5,97 \%o (1999: 3. Schnitt und 1998: 1. Schnitt) und bei Einjährigem Weidelgras bei 2,77 bzw. 3,73 \%o (1999 bzw. 1998). Damit lagen die $\delta^{15} \mathrm{~N}$-Werte der Referenzpflanzen in Reinsaat zu allen Ernteterminen signifikant über den Werten der Leguminosen in Reinsaat und signifikant über den $\delta^{15} \mathrm{~N}$-Werten des molekularen Luftstickstoffs $\left(\delta^{15} \mathrm{~N}_{0}\right)$. Somit konnten mit Hilfe der $\delta^{15} \mathrm{~N}$-Methode leguminosenbedingte $\mathrm{N}$-Flüsse im Dauerfeldversuch quantifiziert werden.

- Aus der langjährigen unterschiedlichen mineralischen N-Düngung zu den nichtlegumen Vorfrüchten $\left(\mathrm{N}_{0}, \mathrm{~N}_{3}\right)$ resultieren vor Aussaat der Ansaaten mit Ackerbohnen $\mathrm{N}_{\text {min }}$-Mengen in 0 bis $125 \mathrm{~cm}$ Bodentiefe von 72,3 bis $172,3 \mathrm{~kg} \mathrm{~N}^{-1}$ ha 15.03 .1999 $\mathrm{N}_{0}$, 20.03.1998 $\mathrm{N}_{3}$ ), vor den Ansaaten mit Luzerne $\mathrm{N}_{\text {min }}$-Mengen zwischen 60,7 bis $82,3 \mathrm{~kg} \mathrm{~N} \mathrm{ha}^{-1}$ (24.03.1998 $\mathrm{N}_{0}, 06.04 .1999 \mathrm{~N}_{3}$ ) und vor der Aussaat der Ansaaten mit Saatwicke $\mathrm{N}_{\text {min }}$-Mengen von 25,5 bis 76,2 $\mathrm{kg} \mathrm{N}^{-1}\left(09.07 .1999 \mathrm{~N}_{0}, 21.07 .1998 \mathrm{~N}_{3}\right.$ ). Signifikante Unterschiede zwischen den $\mathrm{N}_{\min }$-Mengen der Düngungsstufen beschränkten sich im Jahr 1998 vor Aussaat der Ansaaten mit Ackerbohne und Luzerne auf den Unterboden (50 bis $125 \mathrm{~cm}$ ), höhere $\mathrm{N}_{\min }$-Mengen in der $\mathrm{N}_{3}$-Variante über die gesamte Profiltiefe wurden vor Ackerbohne 1999 und vor Saatwicke 1998 festgestellt.

- Aufgrund signifikanter Unterschiede der $\delta^{15} \mathrm{~N}$-Werte zwischen der Referenzpflanze in Reinsaat und der gleichen Art im Gemenge konnte mittels $\delta^{15} \mathrm{~N}$-Methode Transfer- 
$\mathrm{N}$ von der Ackerbohne zum Hafer und von der Luzerne zum Knaulgras nachgewiesen werden. Hafer und Knaulgras im Gemenge mit Ackerbohne und Luzerne akkumulierten transferierte $\mathrm{N}-$ Mengen bis zu 17,9 und $31,2 \mathrm{~kg} \mathrm{~N} \mathrm{ha}^{-1}$ (1999: $3 \mathrm{~A} / 1 \mathrm{H} \mathrm{N} \mathrm{N}_{0}$ und 1998: $3 \mathrm{~L} / 1 \mathrm{~K} \mathrm{~N} \mathrm{~N}_{0}$ ) und damit bis zu 21,8 und 34,6 \% (1999: 3A/1H $\mathrm{N}_{0}$ und 1999: 3L/1K $\mathrm{N}_{0}$ ) ihrer gesamtpflanzlich akkumulierten $\mathrm{N}-M e n g e$. Sowohl die transferierten $\mathrm{N}$ Mengen als auch die Anteile Transfer- $\mathrm{N}$ lagen in der $\mathrm{N}_{0}$-Variante bei Hafer im Jahr 1999 und bei Knaulgras im Jahr 1998 im Mittel der Gemengeansaaten deutlich über den Werten der $\mathrm{N}_{3}$-Variante.

- Im Hinblick auf eine Selbstregelung der N-Zufuhr am Standort ergaben sich die Unterschiede im N-Flächenbilanzsaldo gleicher Ansaaten zwischen der $\mathrm{N}_{0}$ - und $\mathrm{N}_{3^{-}}$ Variante einerseits aus einer geänderten Boden- $\mathrm{N}-$ bzw. Luft-N-Aufnahme und andererseits ganz wesentlich aufgrund einer geänderten Gesamt-N-Akkumulation und eines modifizierten N-Harvest-Indexes.

- Die N-Aufnahme aus dem Boden betrug bei den Reinsaaten der Ackerbohne zwischen 87,3 und 119,5 $\mathrm{kg} \mathrm{N}$ ha $^{-1}$ (1999: $\mathrm{N}_{0}, 1998: \mathrm{N}_{3}$ ), der Luzerne zwischen 91,8 und 117,9 kg N ha- ${ }^{-1}$ (1998: $\mathrm{N}_{0}, \mathrm{~N}_{3}$ ) und der Saatwicke zwischen 40,6 und 63,3 kg N ha $^{-1}$ (1998: $N_{3}, 1999: N_{3}$ ). Die Ansaaten im Gemenge von Ackerbohne und Hafer akkumulierten zwischen 85,5 und 175,6 $\mathrm{kg} \mathrm{N} \mathrm{ha}^{-1}$ (1999: 3A/1H No, 1998: $1 \mathrm{~A} / 3 \mathrm{H} \mathrm{N} \mathrm{N}_{3}$ ), von Luzerne und Knaulgras zwischen 86,1 und $138 \mathrm{~kg} \mathrm{~N}^{-1}$ (1998: 1L/3K N $\mathrm{N}_{0}$, 1999: $1 \mathrm{~L} / 3 \mathrm{~K} \mathrm{~N}_{3}$ ) und von Saatwicke und Einjährigem Weidelgras zwischen 41,1 und 70,5 $\mathrm{kg} \mathrm{N}^{-1} \mathrm{~A}^{-1}$ (1998: 3S/1E No, 1S/3E N 3 ) Stickstoff aus dem Boden. Signifikant höhere $\mathrm{N}$-Mengen aus dem Boden der $\mathrm{N}_{3}$-Variante akkumulierten die Ansaaten mit Ackerbohne in beiden Jahren, die Ansaaten mit Luzerne im Jahr 1998.

- Die Schätzungen der symbiotischen $\mathrm{N}_{2}$-Fixierleistung der Reinsaaten ergaben bei Ackerbohne N-Mengen zwischen 221,2 und 282,2 $\mathrm{kg} \mathrm{N} \mathrm{ha}^{-1}$ (1998: $\mathrm{N}_{3}, 1999: \mathrm{N}_{0}$ ), bei Luzerne zwischen 108,8 und 360,7 kg N ha-1 (1998: $\mathrm{N}_{3}, 1999: \mathrm{N}_{0}$ ) und bei Saatwicke zwischen 22,4 und 107,0 $\mathrm{kg} \mathrm{N}^{-1}$ (1998: $\mathrm{N}_{3}, 1999$ : $\mathrm{N}_{0}$ ). Die fixierten $\mathrm{N}-M e n g e n$ der $\mathrm{N}_{0}$-Variante lag bei allen Ansaaten mit Leguminosen über den fixierten $\mathrm{N}$-Mengen der $\mathrm{N}_{3}$-Variante (signifikant: Ackerbohne 1998, Luzerne 1998, 1999).

- Die gesamtpflanzlich (Wurzel + Spross + Bestandesabfall) akkumulierten N-Mengen der Ansaaten mit Ackerbohne und Hafer lagen zwischen 137,2 und 380,4 kg N ha $^{-1}$ (1998: $4 \mathrm{H} \mathrm{N} \mathrm{N}_{0}$ und 4A $\mathrm{N}_{0}$ ), der Ansaaten mit Luzerne und Knaulgras zwischen 104,1 und 472,4 $\mathrm{kg} \mathrm{N}^{-1}$ (1998: $4 \mathrm{~K} \mathrm{~N}$ 0, 1999: 4L No) und der Ansaaten mit Saatwicke und Einjährigem Weidelgras zwischen 50,8 und 155,5 kg N N $^{-1}$ (1999: 4E No und 4S $\mathrm{N}_{0}$ ). Die Leguminosen in Reinsaat akkumulierten im Vergleich der Düngungsvarianten tendenziell höhere N-Mengen, bei Luzerne im Jahr 1999 signifikant höhere NMengen in der $\mathrm{N}_{0}$-Variante. 
- Während die N-Harvest-Indizes der Ansaaten mit Ackerbohne in der $\mathrm{N}_{3}$-Variante signifikant bzw. tendenziell unter den Werten der $\mathrm{N}_{0}$-Variante lagen, übertrafen die $\mathrm{N}$ Harvest-Indizes der Ansaaten mit Luzerne in der $\mathrm{N}_{3}$-Variante die der $\mathrm{N}_{0}$-Variante hoch signifikant.

- Die vereinfachten N-Flächenbilanzsalden der Reinsaaten lagen bei der Ackerbohne zwischen $+12,2$ und $+47,5 \mathrm{~kg} \mathrm{~N}^{-1}$ (1998: $\mathrm{N}_{3}$, 1999: $\mathrm{N}_{0}$ ), der Luzerne zwischen - 51,9 und + 56,7 kg N ha ${ }^{-1}$ (1998: $\mathrm{N}_{3}, 1999: \mathrm{N}_{0}$ ) und der Saatwicke zwischen - 21,0 und - 46,6 kg N ha ${ }^{-1}$ (1998: $\mathrm{N}_{0}, 1999: \mathrm{N}_{3}$ ). Innerhalb der Ansaaten nahm der Saldo mit zunehmendem Nichtleguminosenanteil ab (Ausnahme Saatwicke im Jahr 1999). Die N-Flächenbilanzsalden lagen in der $\mathrm{N}_{0}$-Variante, in allen geprüften Ansaaten stets über den Salden der $\mathrm{N}_{3}$-Variante.

- Luzerne in Reinsaat führte in beiden Jahren zu einer deutlich höheren Differenz im $\mathrm{N}$-Bilanzsaldo zwischen $\mathrm{N}_{0}$ und $\mathrm{N}_{3}$ (im Mittel 42,7 $\mathrm{kg} \mathrm{ha}^{-1}$ ) als Knaulgras in Reinsaat (im Mittel 17,2 kg ha-1). Ähnlich hohe Differenzen im Bilanzsaldo konnten bei Ackerbohne und Saatwicke gegenüber den entsprechenden Nichtleguminosen in Reinsaat nicht beobachtet werden. Luzerne und alle Nichtleguminosen waren in beiden Jahren in der Lage den $\mathrm{N}_{\text {min }}$-Vorrat im Boden nahezu vollständig auszunutzen, Ackerbohne und Saatwicke dagegen nicht. Im Vergleich der geprüften Leguminosen leistet Luzerne, unter den hier vorliegenden Bedingungen, den größten Beitrag zur Selbstregelung der $\mathrm{N}$-Zufuhr.

- Die Ergebnisse belegen eine in Teilen selbst geregelte N-Zufuhr in leguminosenbasierten Fruchtfolgen. Darüber hinaus verdeutlichen sie, dass Selbstregulationsprozesse in Agrarökosystemen nicht nur auf biotischer Ebene, sondern auch auf abiotischer Ebene wirksam werden. 


\section{Abstract}

The contributions of faba bean, alfalfa and vetch as sole crops (M) and intercropped (I) with oat, orchard grass and annual ryegrass to the self-regulation of $\mathrm{N}$ input in legume-based cropping systems was examined in a 2-year field trial. The nonlegume crops were influenced by long-term different mineral $\mathrm{N}$-fertilization regimes $\left(\mathrm{N}_{0}, \mathrm{~N}_{3}\right)$. The field study was carried out on a loess soil near Goettingen (Germany) in 1998 and 1999 as part of a wider long-term field experiment which started in 1981. The dry matter and $\mathrm{N}$ yield (root and shoot) were investigated as well as the mineral nitrogen from the soil at several sampling dates. The experiments were carried out following a substitutive replacement principle. Soil $\mathrm{N}$ uptake and symbiotic $\mathrm{N}_{2}$ fixation of the legumes were estimated using the natural ${ }^{15} \mathrm{~N}$ abundance method and the extended difference method.

- The weighted $\delta^{15} \mathrm{~N}$ values of oat as sole crop ranged from 3.60 to $5.12 \%$ (1998: final harvest and 1999: first harvest), of orchard grass from 3.92 to $5.97 \%$ (1999: third cut and 1998: first cut) and of annual ryegrass from 2.77 to $3.73 \%$ (1999 and 1998). The $\delta^{15} \mathrm{~N}$ values of the reference plants in the sole crop significantly exceeded the $\delta^{15} \mathrm{~N}$ values of the legumes in the sole crop and the $\delta^{15} \mathrm{~N}$ value of atmospheric $\mathrm{N}_{2}$ $\left(\delta^{15} \mathrm{~N}_{0}\right)$. Therefore it was possible to use the natural ${ }^{15} \mathrm{~N}$ abundance method to estimate legume based nitrogen flow in a long-term field experiment.

- Before sowing fababean as a sole crop or intercropped the mineral nitrogen content ranged from 72.3 to $172.3 \mathrm{~kg} \mathrm{~N} \mathrm{ha}^{-1}\left(15.03 .1999 \mathrm{~N}_{0}, 20.03 .1998 \mathrm{~N}_{3}\right)$, before alfalfa from 60.7 to $82.3 \mathrm{~kg} \mathrm{~N} \mathrm{ha}^{-1}\left(24.03 .1998 \mathrm{~N}_{0}, 06.04 .1999 \mathrm{~N}_{3}\right)$ and before vetch from 25.5 to $76.2 \mathrm{~kg} \mathrm{~N} \mathrm{ha}^{-1}\left(09.07 .1999 \mathrm{~N}_{0}, 21.07 .1998 \mathrm{~N}_{3}\right)$. Significant differences in the mineral nitrogen between the nitrogen levels $\left(\mathrm{N}_{0}, \mathrm{~N}_{3}\right)$ before sowing fababean and alfalfa occurred only at deeper soil layers (50 to $125 \mathrm{~cm}$ depth) in 1998. Higher mineral nitrogen content at the $\mathrm{N}_{3}$-level occurred before sowing fababean in 1999 and vetch in 1998 at all measured depths $(0$ to $125 \mathrm{~cm})$.

- The transfer of $\mathrm{N}$ from fababean and alfalfa to oat and orchard grass was estimated by using the natural ${ }^{15} \mathrm{~N}$ abundance method because of the differences in the weighted $\delta^{15} \mathrm{~N}$ values of reference plants as sole crops and in mixtures. Nitrogen transfer from fababean and alfalfa to associated oat and orchard grass was 17.9 and $31.2 \mathrm{~kg} \mathrm{~N} \mathrm{ha}^{-1}$ (1999: $25 \%$ oat $\mathrm{N}_{0}$ and 1998: $25 \%$ orchard grass, $\mathrm{N}_{0}$ ) and contributed from 21.8 to $34.6 \%$ of the total annual $\mathrm{N}$ yield (1999: $25 \%$ oat $\mathrm{N}_{0}$ and 1999 : $25 \%$ orchard grass $\mathrm{N}_{0}$ ). Both the proportion and the absolute amounts of transferred $\mathrm{N}$ at $\mathrm{N}_{0}$-level exceeded the values at $\mathrm{N}_{3}$-level for oat in 1999 and orchard grass in 1998. 
- With regard to the self-regulation of $\mathrm{N}$ input at this site: differences in $\mathrm{N}$ balances due to cropping method, i.e. single crop or intercropped, occurred because of differences in soil $\mathrm{N}$ uptake and $\mathrm{N}_{2}$ fixation, and also due to different $\mathrm{N}$ yields and a modified $\mathrm{N}$ harvest index.

- The soil $\mathrm{N}$ uptake in the pure stands of fababean ranged from 87.3 to $119.5 \mathrm{~kg} \mathrm{~N}$ $\mathrm{ha}^{-1}$ (1999: $\mathrm{N}_{0}, 1998: \mathrm{N}_{3}$ ), of alfalfa from 91.8 to $117.9 \mathrm{~kg} \mathrm{~N} \mathrm{ha}^{-1}\left(1998: \mathrm{N}_{0}, \mathrm{~N}_{3}\right.$ ) and of vetch from 40.6 to $63.3 \mathrm{~kg} \mathrm{~N}$ ha $^{-1}$ (1998: $\mathrm{N}_{3}, 1999: \mathrm{N}_{3}$ ). In mixtures soil $\mathrm{N}$ uptake in fababean and oat reached from 85.5 to $175.6 \mathrm{~kg} \mathrm{~N} \mathrm{ha}^{-1}$, in alfalfa and orchard grass from 86.1 to $138 \mathrm{~kg} \mathrm{~N} \mathrm{ha}^{-1}$ and in vetch and annual ryegrass from 41.1 to $70.5 \mathrm{~kg} \mathrm{~N}$ $\mathrm{ha}^{-1}$. Fababean in mixtures took up higher rates of $\mathrm{N}$ at $\mathrm{N}_{3}$-level in both years, alfalfa only in 1998.

- The amount of symbiotically fixed nitrogen in fababean ranged from 221.2 to 282.2 kg N ha ${ }^{-1}$ (1998: $N_{3}, 1999: N_{0}$ ), in alfalfa from 108.8 to $360.7 \mathrm{~kg} \mathrm{~N}^{-1}$ (1998: $\mathrm{N}_{3}$, 1999: $\mathrm{N}_{0}$ ) and in vetch from 22.4 to $107.0 \mathrm{~kg} \mathrm{~N} \mathrm{ha}^{-1}$ (1998: $\mathrm{N}_{3}, 1999: \mathrm{N}_{0}$ ) when intercropped. The amount of symbiotically fixed nitrogen at the $\mathrm{N}_{0}$-level exceeded the amount of symbiotically fixed nitrogen at the $\mathrm{N}_{3}$-level (significant: fababean 1998 , alfalfa 1998 and 1999).

- The nitrogen yield (root + shoot + litter) for fababean and oat ranged from 137.2 to $380.4 \mathrm{~kg} \mathrm{~N} \mathrm{ha}^{-1}$ (1998: oat $\mathrm{N}_{\mathrm{M}}$ and faba bean $\mathrm{N}_{\mathrm{M}}$ ), for alfalfa and orchard grass from 104.1 and $472.4 \mathrm{~kg} \mathrm{~N}^{-1}$ (1998: orchard grass $\mathrm{N}_{0}$, 1999: alfalfa $\mathrm{N}_{\mathrm{M}}$ ) and for vetch and annual ryegrass from 50.8 to $155.5 \mathrm{~kg} \mathrm{~N}^{-1}$ (1999: ryegrass $\mathrm{N}_{0}$ and vetch $\mathrm{N}_{\mathrm{M}}$ ). The $\mathrm{N}$ yield of legumes at the $\mathrm{N}_{0}$-level exceeded the values at the $\mathrm{N}_{3}$-levels in pure stands, and of alfalfa in 1999 significantly.

- While the $\mathrm{N}$ harvest index at the $\mathrm{N}_{3}$-level for fababean plantings tended to exceed the values at the $\mathrm{N}_{0}$-level, the harvest index in alfalfa plantings at $\mathrm{N}_{3}$-levels was significantly higher compared to $\mathrm{N}_{0}$-levels.

- The simplified $\mathrm{N}$ balance of monocropped fababean range from +12.2 to $+47.5 \mathrm{~kg}$ $\mathrm{N}^{-1}$ (1998: $\mathrm{N}_{3}, 1999: \mathrm{N}_{0}$ ), of monocropped alfalfa from -51.9 to $+56.7 \mathrm{~kg} \mathrm{~N}^{-1}$ (1998: $\mathrm{N}_{3}, 1999: \mathrm{N}_{0}$ ) and of monocropped vetch from - 21.0 to - $46.6 \mathrm{~kg} \mathrm{~N} \mathrm{ha}^{-1}$ (1998: $N_{0}, 1999: N_{3}$ ). The $N$ balance declined with an increasing proportion of nonlegumes. The simplified $\mathrm{N}$ balances at $\mathrm{N}_{0}$-level exceeded the values at $\mathrm{N}_{3}$-level.

- The differences in $\mathrm{N}$ balance between the different $\mathrm{N}$ levels for monocropped alfalfa (average $42.7 \mathrm{~kg} \mathrm{ha}^{-1}$ ) exceeded the differences for monocropped orchard grass (average $17.2 \mathrm{~kg} \mathrm{ha}^{-1}$ ) in both years. Similar differences in $\mathrm{N}$ balances with faba bean, vetch and reference crops were not found. Alfalfa and the nonlegumes took up 
soil $\mathrm{N}$ at low levels, fababean and vetch did not. At this site, alfalfa made the strongest contribution to the self-regulation of $\mathrm{N}$ input.

- The results indicated, in part, a self-regulation in legume based cropping systems. Beyond this it showed that processes of self-regulation in agro ecosystems did not only occur at biotic levels, but also at abiotic levels. 


\section{Literaturverzeichnis}

Alföldi, T., MÄder, P., Schachenmann, O., Niggli, U., Besson, J.-M., 1992: DoKVersuch: Vergleichende Langzeituntersuchungen in den drei Anbausystemen biologisch-dynamisch, Organisch-biologisch und Konventionell. III. Boden: $\mathrm{N}_{\mathrm{min}}{ }^{-}$ Untersuchungen, 1. und 2. Fruchtfolgeperiode. Schweiz. Landw. Fo. 32, Heft 1/1992, 59-82.

AlteNKIRCH, W., 1977: Ökologie. Sauerländer, Aarau, Frankfurt am Main.

AmATO, M., PARDO, A., 1994: Root length and biomass losses during sample preparation with different screen mesh sizes. Plant and Soil, 161: 299-303.

Auerswald, K., HAIDER, J., 1992: Eintrag von Agrochemikalien in Oberflächengewässer durch Bodenerosion. Z. f. Kulturtechnik und Landentwicklung 33, 222229.

Aufhammer, W., Federwolf, K.-G., Kempf, H., KüBler, E., Stützel, H., 1989: Variabilitätsursachen und Aussagemöglichkeit der $\mathrm{N}_{\text {min }}$-Methode. Landwirtschaftliche Forschung 42, H.4, 281-292

Aufhammer, W., Fiegenbaum, A., KüBler, E., 1994: Zur Problematik der Stickstoffrückstände von Ackerbohnen (Vicia faba L.). Teil 3: Nutzung und Sicherung von Nitratstickstoff im Boden nach Ackerbohnen durch Untersaaten. Die Bodenkultur, Band 46, Heft 1.

BACH, M., 1987: Die potentielle Nitratbelastung des Sickerwassers durch die Landwirtschaft in der Bundesrepublik Deutschland. Göttinger Bodenkundliche Berichte 93, 1-186.

BAeumer, K., 1992: Allgemeiner Pflanzenbau. 3. Aufl., Ulmer Verlag, Stuttgart.

BAEUMER, K., 1994: Möglichkeiten und Grenzen der Gestaltung von Bodennutzungssystemen im Hinblick auf eine vermehrte Nutzung von Selbstregulationsmechanismen im Agrarökosystem. Berichte über Landwirtschaft, 209. Sonderheft, Band 7, 102-122.

BAKER, H.K., GARWOOD, E.A., 1959: Studies on the root development of herbage plants. Journal of Brit. Grassl. 94-104.

BECK, T., 1990: Der Einfluss langjähriger Bewirtschaftungsweise auf bodenmikrobiologische Eigenschaften. Kali-Briefe 20, 17-19.

BeCKMAnN, E., 1998: Zum Wert von Vicia sativa L. und Trifolium resupinatum L. unter variierenden Bedingungen im Zwischenfruchtanbau. Diss. Gießen.

BÉlANGER, G., Richards, J. E., 2000: Dynamics of biomass and N accumulation of alfalfa under three $\mathrm{N}$ fertilization rates. Plant and Soil, 219, 177-185.

Bergersen, F .J., Brockwell, J., Gault, R. R. Morthorpe, L., Peoples, M. B., Tur NER, G. L., 1989: Effects of available soil nitrogen and rates of inoculation on nitrogen fixation by irrigated soybeans and evaluation of $\delta^{15} \mathrm{~N}$ methods for measurement. Aust. J. Agric. Res. 40, 763-780. 
Bergersen, F.J., Turner, G. L., Gault, R. R. Chase, D.L., Brockwell, J., 1985: The natural abundance of ${ }^{15} \mathrm{~N}$ in an irrigate soybean crop and its use for calculation of nitrogen fixation. Aust. J. Agric. Res. 36, 411-423.

Beschow, H., Schulze, J., Merbach, W., 2000: Transfer of symbiotically fixed nitrogen in an alfalfa-grass mixture studied through isotope dilution in a pot experiment. Isotopes Environ. Health Stud., 2000. VOL, 36, pp. 21-33.

BethlenfalvaY, G.I., ABu-ShakRA, S.S., PhILliPS, D.A., 1978: Independence of nitrogen nutrition and photosynthesis in Pisum sativum L. I. Effect of combined nitrogen on symbiotic nitrogen fixation and photosynthesis. PI. Physiol. 62, 127-130.

Bethlenfalvay, G.I., PHiLliPS, D.A., 1977: Effect of light intensity on efficiency of carbon dioxide and nitrogen reduction in Pisum sativum L. PI. Physiol. 60, 868871.

BLUMENTHAL, J.M., RusSelLe, M.P., 1996: Subsoil nitrate uptake and symbiotic dinitrogen fixation by alfalfa. Agron. J., 88, 909-915

BLUMENTHAL, J.M., RusSELLE, M.P., LAMB, J.F.S., 1999: Subsoil nitrate and bromide uptake by contrasting alfalfa entries. Agron. J. 91, 269-275.

BöHM, W., 1978: Die Bestimmung des Wurzelsystems am natürlichen Standort. KaliBriefe (Büntehof) 14, (2), 91-101.

BöHM, W., 1979: Methods of Studying Root Systems. Springer-Verlag, Berlin, Heidelberg, New York.

BÖHM, W., MADUAKOR, H, TAYLOR, H.M., 1977: Comparison of five methods for characterizing Soybean rooting density and development. Agron. J., Vol. 69, MayJune 1977.

BORNSCHEUER, H., 1984: Die Bodengesellschaft auf dem Systemversuch Reinshof. Diplomarbeit am Institut für Pflanzenbau und Pflanzenzüchtung der Georg-August Universität in Göttingen.

BÖTTCHER, J., 1983: Bioelementbilanzen und -transport in Löss- und Sand-Lysimetern bei unterschiedlichen Grundwasserständen. Göttinger Bodenkundliche Berichte 76, 1-205.

BREMER, E., VAN KESSEL, C., 1990: Appraisal of the nitrogen-15 natural abundance method for quantifying dinitrogen fixation. Soil Sci. Soc. Am. J. 54, 404-411.

BROAdBent, F.E., RAuschKolb, R.S., LeWIS, K.A., Chang, G.Y., 1980: Spatial Variability of Nitrogen-15 and Total Nitrogen in Some Virgin and Cultivated Soils. Soil Sci. Soc. Am. J. 44, 524-527.

BROPHY, L.S., HEICHEL, G.H., RUSSELLE, M.P., 1987: Nitrogen Transfer from Forage Legumes to Grass in a Systematic Planting Design. Crop Sci. 27, 753-758.

Brown, M.B., Forsythe, A.B., 1974: Robust Tests fort he Equality of Variance. J. Americ. Statist. Assoc., 69, 364-367.

BURITY, H.A., TA, T.C., FARIS, M.A., COULMAN, B.E., 1989: Estimation of nitrogen fixation and transfer from alfalfa to associated grasses in mixed swards under field conditions. Plant and Soil 114, 249-255. 
BUTLER, J. H. A., LADD, J. N., 1985: Symbiotically-fixed and soil derived nitrogen in legumes grown in pots in soils with different amounts of available nitrate. Soil Biol. Biochem. Vol. 17, No. 1, 47-55.

Buttery, B. R., GiBson, A. H., 1990: The effect of nitrate on the time course of nitrogen fixation and growth in Pisum sativum and Vicia faba. Plant and Soil 127, 143-146.

Chalk, P.M., Smith, C.J., Hamilton, S.D., Hopmans, P., 1993: Characterization of the $\mathrm{N}$ benefit of a grain legume (Lupinus angustifolius L.) to a cereal (Hordeum vulgare L.) by an in situ ${ }^{15} \mathrm{~N}$ isotope dilution technique. Biol. Fert. Soils 15, 39-44.

CHEN, P.-C., PHILLIPS, D.A., 1977: Induction of root nodule senescence by combined nitrogen in Pisum sativum L. Plant Physiol. 59, 440-442.

Cherney, J. H., DuXBuRY, J. M., 1994: Inorganic nitrogen supply and symbiotic dinitrogen fixation in alfalfa. J. Plant Nutr., 2053-2067.

ClaupeIN, W., 1994: Möglichkeiten und Grenzen der Extensivierung im Ackerbau. Wirkungen der Bewirtschaftungsintensität auf die langfristige Produktivität und Stabilität von Agrarökosystemen und deren Umweltwirkungen. Habilitationsschrift, Triade-Verlag Erika Claupein, Göttingen.

CochRAN, V.L., SchlentNER, S.F. 1995: Intercropped oat and fababean in Alaska. Dry matter production, dinitrogen fixation, nitrogen transfer, and nitrogen fertilizer response. Agron. J., 87, 420-424.

Craigh, L.A., Wiebold, W.J., McIntosh, M.S., 1981: Nitrogen Fixation Rates of Alfalfa and Red Clover Grown in Mixture with Grasses. Agron. J. 73, 996-998.

DANSO, S.K.A., ZAPATA, F., HARDARSON, G., 1987: Nitrogen fixation in fababeans as affected by plant population density in sole or intercropped systems with barley. Soil Biol. Biochem. Vol. 19, No. 4, 411-415.

De Willigen, P., Van NoordViJk, M., 1987: Roots, Plant Production and Nutrient Use Efficiency. Ph. D. thesis Agricultural University Wageningen, NL, 282 p.

DE WitT, C. T., Tow, P. G., ENNIK, G. C., 1966: Competition between legumes and grasses. Versl. Landbouwk Onderz., 687.

DenmeAd, O.T., FRENEY, J.R., Simpson, J.R., 1976: A closed ammonia cycle within a plant canopy. Soil Biol. Biochem. 8, 161-164.

DIERCKS, R., 1983: Alternativen im Landbau. Verlag Eugen Ulmer, Stuttgart.

DuBACH, M., RusselL, M.P., 1994: Forage Legume Roots and Nodules and their Role in Nitrogen Transfer. Agron. J. 86: 259-266.

Dufner, J., Jensen, U., Schumacher, E., 1992: Statistik mit SAS. B.G. Teubner, Stuttgart.

Eaglesham, A.R.J., Ayanaba, A., Ranga Rao, V., Eskew, D.L., 1981: Improving the nitrogen nutrition of maize by intercropping with cowpea. Soil Biol. Biochem. 13, 169-171. 
EG-Vo -2092/91/EWG, 1991: Verordnung (EWG) Nr. 2092/91 des Rates vom 24. Juni 1991 über den ökologischen Landbau und die entsprechende Kennzeichnung der landwirtschaftlichen Erzeugnisse und Lebensmittel. In: Amtsblatt der Europäischen Gemeinschaft L 198, Jg. 34, 22.07.1991.

EhleRs, W., Khosla, B.K. KöPKe, R., StülpNAgel, R., BöHm, W., BAeUmer, K., 1981: Tillage effects on root development, water uptake and growth of oats. Soil and Tillage Research, 1, 19-34.

EhleRs, W., KÖPKE, U., HeSSE, F., BÖHM, W., 1983: Penetration resistance and root growth of oats in tilled and untilled loess soil. Soil and Tillage Research, 3, 261275.

Elwaraky, M. K., HaUnOLD, E., 1990: Use of ${ }^{15} \mathrm{~N}$ to determine $\mathrm{N}_{2}$-fixation by legumes, utilization of fertilizer $\mathrm{N}$ and residual effects on a subsequent wheat crop. International Atomic Energy Agency Vienna.

Engel, T., MAngstL, A., 1988: Entwicklung des $\mathrm{N}_{\min }$-Gehaltes im Herbst und Winter auf Praxisschlägen. Mitt. Ges. Pflanzenbauwiss., 1, 12-15.

ETTLINGER, L., 1983: Möglichkeiten der Beeinflussung der biologischen Stickstoffbindung. Die Bodenkultur 34, 365-375.

FILEK, W., KosCIELNIAK, J., GRZESIAK, S., 1997: The effect of nitrogen fertilization and population density of the fied bean (Vicia faba L. minor) of indeterminate and determinate growth habit on the symbiosis with root nodule bacteria and on the seed yield. J. Agron. Crop Sci., 179, 171-177.

FISHBECK, K.A., PHILLIPS, D.A., 1981: Combined nitrogen and vegetative regrowth of symbiotically-grown alfalfa. Agron. J. 73, 975-978.

FRANKOW-LINDBERG, B.E., 1987: Lucerne-grass swards with different nitrogen application and grass components. Swedish J. agric. Res., 17, 193-197.

Franzluebbers, A. J., Hons, F. M., Zuberer, D. A., 1996: Seasonal dynamics of active soil carbon and nitrogen pools under intensive cropping in conventional and no tillage. Z. Pflanzenernähr. Bodenk. 159, 343-349.

FRied, M., BroeshaRT, H., 1975: An independent measurement of the amount of nitrogen fixed by a legume crop. Plant and Soil 43, 707-711.

Gárcia-Plazaola, J. I., Hernandez, A., Becerril, J. M., ArRese-Igor, C., 2000:

Long-term effects of nitrate on lucerne (Medicago sativa $L$.) nitrogen fixation are not influenced by the denitrification status of the microsymbiont. Plant and Soil 216: 139-145.

GEISLER, G., 1983: Untersuchungen zur Ökologie der Wurzelsysteme Landwirtschaftlicher Kulturpflanzen (Mais, Ackerbohne, Gerste). Internationales Symposium vom 27.-29. September 1982 veranstaltet von der Bundesanstalt für alpenländische Landwirtschaft, Gumpenstein, S. 173-183.

GREGORY, P.J., 1988: Root growth of chickpea, faba bean, lentil, and pea and effects of water and salt stresses. World Crops: cool season food legumes, Kluwer Academic Publishers. 
GRÖBlINGHOFF, F. F., HAIDER, K., BECK, T., 1989: Einfluss unterschiedlicher Bewirtschaftungssysteme auf biochemische Stoffumsetzungen. VDLUFA-Schriftenreihe 28, Kongressband 1988, 893-908.

Hamel, C., Nesser, C., BarRantes-Cartin, U., Smith, D.L., 1991: Endomycorrhizal fungal species mediate ${ }^{15} \mathrm{~N}$ transfer from soybean to maize in non-fumigated soil. Plant and Soil 138, 41-47.

HARDARSON, G., 1993: Methods for enhancing symbiotic nitrogen fixation. Plant and Soil 152, 1-17.

HARDARSON, G., DANSO, S.K.A. ZAPATA, F., 1988: Dinitrogen Fixation Measurements in Alfalfa-Ryegrass Swards Using Nitrogen-15 and Influence of the Reference Crop. Crop Sci. 28, 101-105.

HARPER, J.E., GIBSON, A.H., 1984: Differential nodulation tolerance to nitrate among legume species. Crop Sci. 24, 797-801.

HauggaARd-Nielsen, H., JenSEN, E.S., 2001: Evaluating pea and barley cultivars for complementarity in intercropping at different levels of soil $\mathrm{N}$ availability. Field Crops Res., 72, 185-196.

HAUnZ, F.-X., MAIDL, F.-X., FISChBECK, G., 1988: Stickstoff-Fixierung von Körnerleguminosen und deren Bedeutung für den N-Umsatz einer Fruchtfolge. Mitt. Ges. Pflanzenbauwiss., 1, 22-24.

HAUSER, S., 1987: Schätzung der symbiotisch fixierten Stickstoffmenge von Ackerbohnen (Vicia faba L.) mit erweiterten Differenzmethoden. Diss. (agr.) Universität Göttingen.

HAYNES, R.J., 1980: Competitive Aspects of the Grass-Legume Association. Advanc. Agron. 33, 227-261.

HeERAmAN, D.A., JUMA, N.G., 1993: A comparison of minirhizotron, core and monolith methods for quantifying barley (Hordeum vulgare L.) and fababean (Vicia faba L.) root distribution. Plant and Soil 148, 29-41.

Heichel, G. H., Henuum, K. I. 1991: Dinitrogen fixation, Nitrogen Transfer and Productivity of Forage Legume-Grass Communities. Crop Sci. 31, 202-208.

HeInZmanN, F., 1981: Assimilation von Luftstickstoff durch verschiedene Leguminosenarten und dessen Verwertung durch Getreidenachfrüchte. Diss. Hohenheim.

Heuser, O., 1931: Die Luzerne. Paul Parey Verlag, Berlin.

HEUWINKEL, H., GUTSER, R., 1997: Kritische Bewertung der Feldmethoden zur Abschätzung von $\mathrm{N}_{2}$-Bindung und $\mathrm{N}$-Flüssen in Klee-Luzerne-Gras Beständen. Mitt. Ges. Pflanzenbauwiss. 10, 65-66.

Høgh-JeNSEN, H. SCHJOERRING, J.K., 1994: Measurement of biological dinitrogen fixation in grassland: Comparison of the enriched ${ }^{15} \mathrm{~N}$ dilution and natural ${ }^{15} \mathrm{~N}$ abundance methods at different nitrogen application rates and defoliation frequencies. Plant and Soil 166, 153-163. 
HøGH-JENSEN, H., SCHJOERRING, J.K., 2000: Below-ground nitrogen transfer between different grassland species: Direct quantification by ${ }^{15} \mathrm{~N}$ leaf feeding compared with indirect dilution of soil ${ }^{15} \mathrm{~N}$. Plant and Soil 227, 171-183.

HøGH-JENSEN, H. SCHJOERRING, J.K., 2001: Rhizodeposition of nitrogen by red clover, white clover and ryegrass leys, Soil Biol. Biochem. 33, 439-448.

HOUWAARD, F., 1980: Influence of ammonium and nitrate on nitrogenase activity of pea plants as affected bv light intensity and sugar addition. Plant and Soil 54, 271-282.

HUBER, R. 1988: Biologische N-Fixierung der Ackerbohnen und deren Auswirkungen auf den N-Haushalt des Bodens im Rahmen getreidebetonter Fruchtfolgen. Diss ETH Zürich.

IMSANDE, J., 1986: Nitrate-ammonium ratio for ph homeostasis in hydroponically grown soybean. L. Exp. Bot. 37, 341-346.

ISERMANN, K., ISERMANN, R., 1998: Food production and consumption in Germany: N flows and $\mathrm{N}$ emissions. Nutr. Cyc. Agroecosys. 52, 289-301.

IZAURRALDE, R.A., McGILL, W.A., JUMA, N.G., 1992: Nitrogen fixation efficiency, interspecific $\mathrm{N}$ transfer, and root growth in barley-field pea intercrop on a Black Chernozemic soil. Biol. Fert. Soil 13, 11-16.

JANZEN, H.H., BRUINSMA, Y., 1989: Methodology for the quantification of root and rhizosphere nitrogen dynamics by exposure of shoots to N-labelled ammonia. Soil Biol. Biochem., 21, No. 2, 189-196.

JAQUIÉRY, R., KELLER, E.R., 1978: Beeinflussung des Fruchtansatzes bei der Ackerbohne (Vicia faba L.) durch die Verteilung der Assimilate. Teil I: Angew. Botanik 52, 261-276, 1978.

JENSEN, E.S., 1986a: Symbiotic $N_{2}$ fixation in pea and field bean estimated by $15 \mathrm{~N}$ fertilizer dilution in field experiments with barley as a reference crop. Plant and Soil, 92, 3-13.

JENSEN, E.S., 1986b: Intercropping field bean with spring wheat. Vortr. Pflanzenzüchtg. 11, 67-75.

JENSEN, E.S., 1987: Seasonal patterns of growth and nitrogen fixation in field grown pea. Plant and Soil 101, 29-37.

JENSEN, E.S., 1996a: Grain yield, symbiotic $\mathrm{N}_{2}$ fixation and interspecific competition for inorganic $\mathrm{N}$ in pea-barley intercrops. Plant and Soil 182, 25-38.

JENSEN, E.S., 1996b: Barley uptake of $\mathrm{N}$ deposited in the rhizosphere of associated field pea. Soil Biol. Biochem. 28, 159-168.

JENSEN, E.S., SøRENSEN, L.H., 1988: Uptake of soil nitrogen by soybean as influenced by symbiotic $\mathrm{N}_{2}$-fixation or fertilizer nitrogen supply. Soil Biol. Biochem. 20, 921-925.

Jost, B., 2003: Untersuchungen und Kalkulationstabellen zur Schätzung der $\mathrm{N}_{2}$ Fixierleistung und der N-Flächenbilanz beim Anbau von Lupinus albus und Lupinus luteus in Reinsaat und von Vicia faba und Pisum sativum in Reinsaat und im Gemenge mit Avena sativa. Diss. (agr.) Universität Göttingen. 
JUNG, R., 2003: Stickstoff-Fixierleistung von Luzerne (Medicago sativa L.), Rotklee (Trifolium pratense L.) und Persischem Klee (Trifolium resupinatum L.) in Reinsaat und Gemenge mit Poaceen. Diss. (agr.) Universität Göttingen.

JUNK, G., SVEC, H. V., 1958: The absolute abundance of the nitrogen isotopes in the atmosphere and compressed gas from various sources. Geochim. et Cosmochim. Acta 14, 234-243.

JUSTUS, M., KÖPKE, U., 1995: Reduzierung von Nitratverlusten beim Anbau von Ackerbohnen. In Landwirtschaftliche Fakultät der Universität Bonn (Hrsg.) Abschlußbericht MURL-Projekt. Forschungsberichte des Lehr- und Forschungsschwerpunktes „Unmweltverträgliche und standortgerechte Landwirtschaft“, Heft 23, ISSN 0943-9684.

KAGE, H., 1992: Zu den Ursachen hoher Restnitratmengen beim Anbau von Ackerbohnen. Triade Verlag Göttingen, 1-186.

KaISer, E.A., KoHRS, K., KÜCKE, M., Schnug, E., Munch, J.C., HeInemeyer, O.,1998: Nitrous oxide release from arable soil: importance of perennial forage crops. Biol. Fertil. Soils 28, 36-43.

KaRmANOS, R.E., RENNIE, D.A., 1978: Nitrogen isotope fractionation during ammonium exchange reactions with soil clay. Can. J. Soil Sci. 58, 53-60.

KELNER, D.J., VeSSEY, K., ENTZ, M.H., 1997: The nitrogen dynamics of 1-, 2- and 3year stands of alfalfa in a cropping system. Agric., Ecosys. and Environments. 64, 1-10.

KESSLER, W., 1987: Einfluss des Lichtangebotes und der Temperatur auf Ertragsbildung und biologische Stickstoff-Fixierung von Weißklee (Trifolium repens L.). Diss., ETH Zürich.

Khan, D.F., Peoples, M.B., Chalk, P.M., HeRRidge, D.F., 2002: Quantifying belowground nitrogen of legumes. 2. A comparison of ${ }^{15} \mathrm{~N}$ and non isotopic methods. Plant and Soil 239, 277-289.

KILIAN, S., 1994: Stickstoffkreislauf in einem Vicia faba Feld. Diss. Universität Marburg.

KIM, J., REES, D.C., 1994: Nitrogenase and biological nitrogen fixation. Biochemistry 33 (1994) 2, 389-397.

KNAUER, N., 1994: Folgen der Fremdregulation von Acker-Ökosystemen auf verschiedene Bereiche der Kulturlandschaft. Berichte über Landwirtschaft, 209. Sonderheft, Band 7, 9-21.

KNUTH, F., 1990: Untersuchungen zur Schätzung der Nettomineralisationsrate von Bodenstickstoff. Beobachtungen am Ackerbau-Systemversuch Reinshof. Diplomarbeit, Universität Göttingen.

KNUTH, F., 1995: Quantifizierung von Stickstoffverlusten durch Auswaschung bei unterschiedlicher Bewirtschaftungsintensität. Diss (agr.) Universität Göttingen, Cuvillier Verlag Göttingen.

KNUTH, F., CLAUPEIN, W., 1990: Mineralisierung von Bodenstickstoff bei unterschiedlicher Bewirtschaftungsintensität. Beobachtungen am Ackerbau-Systemversuch Reinshof. Mitt. Ges. Pflanzenbauwiss. 3, 297-300.

KöHLER, W., SchaChtel, G., Voleske, P., 1996: Biostatistik. 2. Auflage. Springer Verlag, Berlin, Heidelberg, New York. 
KöHNLEIN, A.H., VetTeR, H., 1953: Ernterückstände und Wurzelbild. Paul Parey Verlag, Hamburg und Berlin.

KöNEKAMP, A.H., 1952: Teilergebnisse und Wurzeluntersuchungen. Z. f. Pflanzenernährung, Düngung und Bodenkunde, 60, (105) Band, Heft 2.

KÖNINGS, I., 1984: Durchwurzelung des Bodens von Hafer, Erbsen und Ackerbohnen und im Boden verbleibende Nitratmengen -Beobachtungen an einem Feldversuch 1986-. Diplomarbeit, Universität Göttingen.

KÖPKE, U., 1979: Ein Vergleich von Feldmethoden zur Bestimmung des Wurzelwachstums landwirtschaftlicher Kulturpflanzen. Diss (agr.) Universität Göttingen.

KÖPKE, U., 1987: Symbiotische Stickstoff-Fixierung und Vorfruchtwirkung von Ackerbohnen (Vicia faba L.). Habilitationsschrift, Göttingen.

KÖRSCHENS, M., 1992: Simulationsmodelle für den Umsatz und die Reproduktion der organischen Substanz im Boden. Ber. Über Landw. 206, 140-154.

KRETZSCHMAR, R., 1991: Kulturtechnisch-Bodenkundliches Praktikum. Ausgewählte Laboratoriumsmethoden - Eine Anleitung zum selbstständigen Arbeiten an Böden. 7. Auflage Kiel.

KUNELIUS, H.T., 1974: Effect of weed control and fertilization at establishment on the growth and nodulation of alfalfa. Agron. J. 66, 806-808.

KUTSCHERA, L., 1960: Wurzelatlas mitteleuropäischer Ackerunkräuter und Kulturpflanzen. DLG-Verlags-GmbH, Frankfurt a.M.

Lamb, J.F.S., Barnes, D.K., Russelle, M.P., Vance, C.P., Heichel, G.H., Henjum, K. I., 1995: Ineffectively and effectively nodulated alfalfas demonstrate biological nitrogen fixation continues with high nitrogen fertilization. Crop Sci. 35, 153-157.

La Rue, T.A., Patterson, T.G., 1981: How much nitrogen do legumes fix? Adv. Agron. 34, 15-38.

LEDGARD, S.F., 1991: Transfer of fixed nitrogen from white clover to associated grass in swards grazed by dairy cows, estimated using ${ }^{15} \mathrm{~N}$ method. Plant and Soil 131, 215-223.

LEDGARD, S.F., FRENEY, J.R., SIMPSON, J.R., 1985b: Assessing nitrogen transfer from legumes to associated grasses. Soil Biol. Biochem. 17, 575-577.

Ledgard, S.F., Simpson, J.R., FreneY, J.R., Bergersen, F.J., Morton, R., 1985a: Assessment of the relative uptake of added and indigenous soil nitrogen by nodulated legumes and reference plants in the ${ }^{15} \mathrm{~N}$ dilution measurement of $\mathrm{N}_{2}$ fixation: Glasshouse application of method. Soil Biol. Biochem. 17, 323-328.

Lemaire, G., Khaity, M., Onillon, B., AlliRAnd, J.M., Chartier, M., Gosse, G., 1992: Dynamics of accumulation and partitioning of $\mathrm{N}$ in leaves, stems and roots of lucerne (Medicago sativa L.) in a dense canopy. Ann. Bot. 70, 429-435.

LeVENE, H., 1960: Robust Test for Equality of Variances. In: Olkin,l. et al. (eds.): Contribution to Probability and Statistics: Essays in Honor of Harold Hotelling. Stanford University Press, 278-292. 
LoHMANN, E., 1984: Kennwerte der Wurzeldichte von Hafer und Ackerbohnen - Ein Vergleich der Profilwand- mit der Monolithmethode. Diplomarbeit, Institut f. Pflanzenbau u. Pflanzenzü. Univ. Göttingen.

LOPOTZ, H.-W., 1997: Biologische $\mathrm{N}_{2}$-Fixierung von Klee-Reinbeständen und KleeGras-Gemengen unter besonderer Berücksichtigung des Einflusses der NNachlieferung des Bodens. Diss. (agr.) Universität Bonn.

LORY, J.A., RusselL, M.P. HEICHEL, G.H., 1992: Quantification of Symbiotically Fixed Nitrogen in Soil Surrounding Alfalfa Roots and Nodules. Agron. J. 84, 10331040.

LÜtKe-Entrup, N., Blome, K.H., StemanN, G., 1989: Körnerleguminosen ohne Nitratprobleme anbauen. DLG-Mitteilungen, 4, 182, 191.

LÜTKE-ENTRUP, N., GRÖBlinghOFF, F.-F., StemANN, G., 1993: Untersuchungen zur Effizienz von Gras-Untersaaten in Ackerbohnen. Gesunde Pflanzen, 45. Jahrg., Heft 5, 178-182.

LÜTKE-Entrup, N., StemanN, G., 1990: Ackerbohnen. Untersaaten lösen Nitratprobleme. DLG-Mitteilungen, 6, 276-277.

MAHN, E.-G., 1994: Regulationsmechanismen bei der Populationsdynamik von Segetalpflanzen. Berichte über Landwirtschaft, 209. Sonderheft, Band 7, 68-84.

MaIDL, F.X., HAunZ, F.X., PANSE, A., FISCHBECK, G., 1996: Transfer of grain legume nitrogen within a crop rotation containing winter wheat and winter barley. J. Agron. Crop Sci., 176, 47-57.

Mallarino, A.P., Wedin, W.F., Goyenola, R.S., Perdomo, C.H.; West, C.P., 1990:

Legume Species and Proportion Effects on Symbiotic Dinitrogen Fixation in Legume-Grass Mixtures. Agron. J. 82, 785-789.

MANZKE, F., 1990: Schätzung der symbiotisch fixierten Stickstoffmenge von Luzerne (Medicago Media Pers.) und Saatwicke (Vicia Sativa L.) mit der erweiterten Differenzierungsmethode. Diplomarbeit, Universität Göttingen.

MARIOTTI, A., 1983: Atmospheric nitrogen is a reliable standard for natural ${ }^{15} \mathrm{~N}$ abundance measurements. Nature 303, 685-687.

Mariotti, A., Pierre, D., Vedy, J.C., Bruckert, S., Gulllemont, J., 1980: The abundance of natural nitrogen 15 in the organic matter of soils along an altitudinal gradient. Catena, 7, 293-300.

MARTIN, P., 1990: Einfluss von Mineralstoffen auf das symbiotische $\mathrm{N}_{2}$-Bindungssystem bei Leguminosen. Kali-Briefe (Büntehof) 20, 93-110.

MARSCHNER, H., 1995: Mineral nutrition of higher plants. Second Edition, Academic Press, London, San Diego, New York, Boston, Sydney, Tokyo, Toronto.

MATHERS, A.C., SteWART, B.A., BlaiR, B., 1975: Nitrat-nitrogen removal from soil profiles by alfalfa. J. Environ. Qual. 4, 403-405.

Mayer, J., Buegger, F., Jensen, E.S., Schloter, M., Hess, J., 2003a: Residual nitrogen contribution from grain legumes to succeeding wheat and rape and related microbial process. Plant and Soil 255, 541-554. 
Mayer, J., Buegger, F., Jensen, E.S., Schloter, M., Hess, J., 2003b: Estimating N rhizodeposition of grain legumes using a ${ }^{15} \mathrm{~N}$ in situ stem labelling method. Soil Biol. Biochem. 35, 21-28.

McAuliffe, C., Chamblee, D.S., URibe-Arango, H., Woodhouse, W.W., 1958:

Influence of inorganic nitrogen on nitrogen fixation by legumes as revealed by ${ }^{15}$ N. Agron. J. 50, 334-337.

MeIER, U., 1997: Growth stages of mono- and dicotyledonous plants. Blackwell Wissenschafts-Verlag, Berlin, Wien.

Mengel, K., KIRKBY, E.A., 1987: Principles of plant nutrition. International Potash Institute, Bern.

MERBACH, W., 1982: Untersuchung über Stickstoffumsatz und symbiotische $\mathrm{N}_{2}$ Fixierung bei Körnerleguminosen. Diss. B. Uni. Halle-Wittenberg.

MEYER, C., 1984: Zum Einfluss des Wasserhaushaltes auf die Ertragsbildung bei Ackerbohne und Hafer. Diss (agr.) Universität Göttingen.

MoHR, H.D., 1978: Die Durchwurzelung von Böden in Abhängigkeit von wichtigen Bodeneigenschaften. KALI-BRIEFE (Büntehof) 14, (2), 102-113.

MÖLLER, D. 1995: ECosiM - Ein dynamisches Systemsimulationsmodell für Betriebe des ökologischen Landbaus. Gießener Schriften zur Agrar- und Ernährungswirtschaft 23, 1-183, DLG-Verlag, Frankfurt/Main.

MÜLLER, U., 1984: Wasserhaushalt von Ackerbohne und Hafer auf Löss-Parabraunerde. Diss (agr.) Universität Göttingen.

MUNZERT, M., 1992: Einführung in das pflanzenbauliche Versuchswesen: Grundlagen und Praxis des Versuchswesens im landwirtschaftlichen, gärtnerischen und forstwirtschaftlichen, gärtnerischen und forstwirtschaftlichen Pflanzenbau. Berlin, Hamburg.

Nieder, R., Schollmayer, G., 1988: Gemeindebezogene Stickstoffbilanzen in NW zur Abschätzung der potentiellen Nitratbelastung des Grundwassers durch landwirtschaftliche Nutzung. Mittl. Dt. Bodenk. Ges. 57, 83-88.

NöSBERger, J., OpITZ Von Boberfeld, W., 1986: Grundfutterproduktion. Verlag Paul Parey, Berlin und Hamburg.

OHNESORGE, B., 1994: Regulationsmechanismen bei der Populationsdynamik von phytophagen Schädlingen. Berichte über Landwirtschaft, 209. Sonderheft, Band 7, 54-67.

PATE, J.S., 1958: Nodulation studies in legumes. Aust. J. of Biol. Agric., Vol 11, 496515.

PEOPLES, M.B., 2001: Legume root nitrogen in cropping system nitrogen cycling. GRAIN Legumes No. $33-3^{\text {rd }}$ quarter 2001. 
Peoples, M.B., Bowman, A.M., Gault, R.R., Herridge, D.F., McCallum, M.H., McC ORMiCK, K.M., Norton, R.M., Rochester, I.J., ScAmmelL, G.J., SchWEnKe, G.D. 2001: Factors regulating the contribution of fixed nitrogen by pasture and crop legumes to different farming systems of eastern Australia. Plant and Soil 228, 29-41.

Proffitt, A.P.B., Berliner, P.K., OOsterhuis, D.M., 1985: A comparative study of roots distribution and water extraction efficiency by wheat grown under highand low-frequency irrigation. Agron. J. 77: 655-662.

RÄTZ, D., 1998: Räumliche Variabilität pflanzlicher Kenngrößen der N-Flächenbilanz beim Anbau von Leguminosen. Untersuchung mit Hilfe der $\delta^{15} \mathrm{~N}$-Methode in Ackerschlägen. Dipl. (agr.) Universität Göttingen.

REICHARDT, K., 1990: Soil spatial variability and symbiotic nitrogen fixation by legumes. Soil Science, Vol. 150, No 3, USA.

Reichardt, K., Hardarson, G, Zapata, F. Kirda, C. Danso, S.K.A., 1987: Site variability effect on field measuremant of symbiotic nitrogen fixation using the ${ }^{15} \mathrm{~N}$ isotope dilution method. Soil Biol. Biochem. 19, 405-409.

ReITER, K., SchmidTKE, K., RAUBER, R., 2002: Estimation of symbiotic $\mathrm{N}_{2}$ fixation by a low-level, large-scale ${ }^{15} \mathrm{~N}$-application technique. Soil Biol. Biochem. 34, 303314.

RENIUS, W., LÜTKE-ENTRUP, E., 1985: Zwischenfruchtbau zur Futtergewinnung und Gründüngung. DLG-Verlag, Frankfurt.

RICHARDS, J.E., SOPER, R. J., 1979: Effect of N fertilizer on yield, protein content, and symbiotic $\mathrm{N}$ fixation in fababeans. Agron. J. 71, 807-811.

RICHARDS, J. E., SOPER, R. J., 1981: N fertilization of field-grown faba beans in Manitoba. Can. J. Soil Sci., 62, 21-30.

ROHMANN, U., SONTHEIMER, H., 1985: Nitrat im Grundwasser (Ursachen - Bedeutung - Lösungswege). DVWG - Forschungsstelle am Engler-Bunte Institut, Karlsruhe.

Ruschel, A.P., Vose, P.B., Victoria, R.L. SAlati, E., 1979: Comparisons of isotope techniques and non-nodulating isolines to study the effect of ammonium fertilization on dinitrogen fixation in soybean, Glycine max. Plant and Soil, 53, 513525.

Russow, R., SiCH, I., NeUE, H.-U., 2000: The formation of the trace gases NO and $\mathrm{N}_{2} \mathrm{O}$ in soils by the coupled processes of nitrification and denitrification: results of kinetic ${ }^{15} \mathrm{~N}$ tracer investigations. Chemosphere, Global Change Science 2, 359-366.

SACHS, L., 1993: Angewandte Statistik. Anwendung statistischer Methoden. 7. neubearbeitete Aufl., Springer-Verlag, Berlin.

SAS-INSTITUTE INC., 1999: The SAS System for Windows, Release 8.01, Cary, Nc, USA. 
SCHERER, H. W., DANZEISEN, L., 1980: Der Einfluss gesteigerter Stickstoffgaben auf die Entwicklung der Wurzelknöllchen, auf die symbiotische Stickstoffassimilation sowie auf das Wachstum und den Ertrag von Ackerbohnen (Vicia faba L.). Z. Pflanzenernaehr. Bodenkd., 143, 464-470.

SCHLEGEL, H.G., 1981: Allgemeine Mikrobiologie. 5. Auflage, Georg Thieme Verlag, Stuttgart, New York.

Schlichting, E., Blume, H.-P. 1966: Bodenkundliches Praktikum. Verlag Paul Parey, Hamburg und Berlin.

SCHMEeR, H., Mengel, K., 1985: Der Einfluss der Strohdüngung auf die Nitratgehalte im Boden im Verlaufe der Wintermonate. Landw. Forsch. 37, Kongressband 1984, 214-229.

SCHMIDTKE, K., 1996: Methodik zur Ermittlung der N-Flächenbilanz beim Anbau von Leguminosen. Mitt. Ges. Pflanzenbauwiss. 9, 43-44.

SCHMIDTKE, K., 1997a: Einfluss von Rotklee (Trifolium pratense L.) in Reinsaat und Gemenge mit Poaceen auf symbiotische $\mathrm{N}_{2}$-Fixierung, bodenbürtige N-Aufnahme und $\mathrm{CaCl}_{2}$-extrahierbare $\mathrm{N}$-Fraktionen im Boden. Diss (agr.) Universität Gießen.

SCHMIDTKE, K., 1997b: Selbstregelung der N-Zufuhr im Ökologischen Landbau - ein Wirkungsmechanismus zum Schutz des Grundwassers? Beiträge zur 4. Wissenschaftstagung zum Ökologischen Landbau 3.-4. März 1997 an der Universität Bonn, U. Köpke \& J.A. Eisele (Hrsg.) 1997, Verlag Dr. Köster, Berlin, 2127.

SCHMIDTKE, K., 1999: N-Flächenbilanz beim Anbau von Futter- und Körner-leguminosen. In: HOFFMANN H. \& S. MÜLLER (Hrsg.): Beiträge zur 5. Wissenschaftstagung zum Ökologischen Landbau "Vom Rand zur Mitte", Verlag Dr. Köster, Berlin, 234-238.

SCHMIDTKE, K., 2001. Umweltgerechter Anbau von Leguminosen - Entwicklung und Anwendung eines Verfahrens zur Quantifizierung der N-Flächenbilanz (Az. 07312). Abschlußbericht des Forschungsvorhabens, gefördert von der Deutschen Bundesstiftung Umwelt, Osnabrück (Dezember 2001), 1-234.

SCHMIDTKE, K., 2004: Methods for estimating legume N balance. Habilitationsschrift Universität Göttingen, in Vorbereitung.

SCHMIDTKE, K., RAUBER, R., 2000: N-Effizienz von Leguminosen im Ackerbau. In: Stickstoffeffizienz von Kulturpflanzen, Deutsche Bundesstiftung Umwelt, Initiativen zum Umweltschutz 12, Erich Schmidt Verlag, Berlin, 48-69.

SCHMIDTKE, K., VABITSCH, A., 2001: Einfluss neuer und älterer Erbsen- und Ackerbohnensorten auf die N-Mineralisation der Ernterückstände und residuale NminMenge im Boden. Abschlußbericht des Forschungsvorhabens gefördert durch die Professor Werner Schulze-Stiftung, Essen (Az. TS 0979/03.037/00), 1-37.

SchmidtKe, K, Neumann, A., Hof, C., Rauber, R., 2004: Soil and atmospheric nitrogen uptake by lentil (Lens culinaris Medik.) and barley (Hordeum vulgare ssp. nudum L.) as monocrops and intercrops. Field Crops Res. 87, 245-256. 
SCHMUDE, D., 1991: Stickstoffdüngung zu Rotkleegras-Gemengen - Einfluss auf Ertrag, Bestandeszusammensetzung, Stickstoffertrag und $\mathrm{N}_{2}$-Fixierung sowie die $\mathrm{N}_{\min }$-Dynamik im Boden. Diss. (agr.) Universität Rostock.

SCHÜLLER, H., 1969: Die CAL-Methode, eine neue Methode zur Bestimmung pflanzenverfügbaren Phosphates in Böden. Z. Pflanzenernaehr. Bodenk. 122, 48-63.

SCHULZE, J., 2004: How are nitrogen fixation rates regulated in legumes? J. Plant Nutr. Soil, 167:125-137.

Schulze, J., Adgo, E., Merbach, W., 1999: Carbon costs associated with $\mathrm{N}_{2}$ fixation in Vicia faba L. and Pisum sativum L. over a 14-day period. Plant biol. 1, 625631.

SchuURman, J. J., Goedewaagen, M. A. J., 1971: Methods for the Examination of Root Systems and Roots. 2. Aufl., Pudoc, Wageningen.

SHAPIRO, S.S., WILK, M.B., 1965: An analysis of variance test for normality (complete samples). Biometrika 52, 591-611.

SHEARER, G., KOHL, D., 1986: $\mathrm{N}_{2}$-fixation in field settings: Estimations based on natural ${ }^{15} \mathrm{~N}$ abundance. Aust. J. Plant Physiol. 13, 699-756.

Smit, A.L., Bengough, A.G., Engels, C., Van NordwiJk, M., Pellerin, S., Van De GEIJN, S.C., 2000: Root Methods. A Handbook, Springer-Verlag Berlin Heidelberg New York.

STEINECK, O., 1983: Der Einfluss der Hauptnährstoffe auf die Wurzelausbildung. Internationales Symposium vom 27.-29. September 1982 veranstaltet von der Bundesanstalt für alpenländische Landwirtschaft, Gumpenstein, S. 99-114.

SteVenson, F.C., KNIGHT, J.D., VAN Kessel, C., 1995: Dinitrogen fixation in pea: Controls at the landscape- and micro-scale. Soil Sci. Soc. Am. J., 59:16031611.

SteVenson, F.C., VAN Kessel, C., 1996: A landscape-scale assessment of the nitrogen and non-nitrogen rotation benefits of pea. Soil Sci. Soc. Am. J. 60:1797-1805.

StREETER, J.G., 1985a: Nitrate inhibition of legume nodule growth and activity - I. Long term studies with a continuous supply of nitrate. Plant Physiol. 77, 321324.

STREETER, J.G., 1985b: Nitrate inhibition of legume nodule growth and activity - II. Short term studies with high nitrate supply. Plant Physiol. 77, 325-328.

STREETER, J.G., 1988: Inhibition of legume nodule formation and $\mathrm{N}_{2}$ fixation by nitrate. CRC Crit. Rev. Plant Sci. 7, 1-23.

STÜLPNAGEL, R., 1982: Schätzung der von Ackerbohnen symbiontisch fixierten Stickstoffmenge im Feldversuch mit der erweiterten Differenzmethode. Z. Acker- u. Pflanzenbau 151, 446-458.

StÜLPNAGEL, R., 1990: A concept to reduce nitrogen losses in a rotation with faba beans (Vicia faba L.). Vortrag auf: European Society of Agronomy, 1. Congress, 5. - 7.12 1990, Paris. 
TA, T.C., FARIS, M.A., 1987a: Species variation in the fixation and transfer of nitrogen from legumes to associated grasses. Plant and Soil 98, 265-274.

TA, T.C., FARIS, M.A., 1987b: Effects of Alfalfa Proportions and Clipping Frequencies on Timothy-Alfalfa Mixtures. II. Nitrogen fixation and Transfer. Agron. J. 79, 820824.

TA, T.C., FARIS, M.A., 1988: Effects of environmental conditions on the fixation and transfer of nitrogen from alfalfa to associated timothy. Plant and Soil 107, 25-30.

TA, T.C., MACDOWALL, F.D.H., FARIS, M.A., 1990: Utilization of carbon and nitrogen reserves of alfalfa roots in supporting $\mathrm{N}_{2}$-fixation and shoot regrowth. Plant and Soil 127, 231-236.

TOMm, O.G., VAN KeSSEL, C., SLINKARD, A.E., 1994: Bi-directional transfer of nitrogen between alfalfa and bromegrass. Short and long term evidence. Plant and Soil, $164,77-86$.

Tomm, G.O., Walley, F.L., van Kessel, C., SlinKard, A.E., 1995: Nitrogen Cycling in an Alfalfa and Bromegrass Sward via Litter fall and Harvest Losses. Agron. J. 87, 1078-1084.

Trimble, M.W., Barnes, D.K., Heichel, G.H., Shaeffer, C.C., 1987: Forage yield and nitrogen partitioning responses of alfalfa to two cutting regimes and three soil nitrogen regimes. Crop. Sci. 27, 909-914.

TUKEY, J.W., 1953: The problem of multiple comparisons. Princeton, NJ: Princeton University. Unpublished report. Zit. in Sachs, L. 1990: Statistische Methoden 2 Planung und Auswertung. Springer Verlag, Berlin.

Turner, G.L., Gault, R.R., Morthorpe, L.A., ChAse, D.L., Bergersen, F.J., 1987:

Differences in the natural abundance of ${ }^{15} \mathrm{~N}$ in the extractable mineral nitrogen of cropped and fallowed surface soils. Aust. J. Agric. Res. 38, 15-25.

Turpin, J.E., HerRidge, D.F., Robertson, M.J., 2002: Nitrogen fixation and soil nitrate interactions in field-grown chickpea (Cicer arietinum) and fababean (Vicia faba). Aust. J. Agric. Res., 53, 599-608.

UNKOVICH, J.M., PATE, J.S., 2000: An appraisal of recent field measurements of symbiotic $\mathrm{N}_{2}$ fixation by annual legumes. Field Crops Res. 65, 211-228.

UNKOVICH, M.J., PATE, J.S., Sanford, P., 1997: Nitrogen fixation by annual legumes in Australian Mediterranean agriculture. Aust. J. Agric. Res. 48, 267-293.

UnKovich, M.J., PATE, J.S., SANFord, P., ARMstrong, E.L., 1994: Potential precision of the $\delta^{15} \mathrm{~N}$ natural abundance method in field estimates of nitrogen fixation by crop and pasture legumes in south-west Australia. Aust. J. Agric. Res.45, 119132.

VALLIS, I., 1978: Nitrogen relationship in grass/legume mixtures. In Plant Relations in Pastures. Ed. J.R.WILSON. pp 190-201. CsIRO Australia.

Van der Ploeg, R.R., Ringe, H., Machaulla, G., Hermsmeyer, D., 1997: Postwar nitrogen use efficiency in West German agriculture and groundwater quality. J. Environ. Oual. 26, 1203-1212. 
VAN Kessel, C., RoskoskI, J.P., 1988: Row spacing effects on $\mathrm{N}_{2}$-fixation, N-yield and soi $\mathrm{N}$ uptake of intercropped cowpea and maize. Plant and Soil 111, 17-23.

VAN NOORDWIJK, M., FLORIS, J., 1979: Loss of dry weight during washing and storage of root samples. Plant and Soil, 53, 239-243.

VAN NOORDWIJK, M., FLORIS, J., DE JAGER, A., 1985: Sampling schemes for estimating root density distribution in cropped fields. Neth. J. agric. Sci. 33, 241-262.

VDLUFA, 1991: VDLUFA Methodenhandbuch Band I. Die Untersuchung von Böden (Hrsg.). 4. Auflage. VDLUFA-Verlag Darmstadt.

VEPRASKAS, M.J., HOYT, G.D., 1988: Comparison of the Trench-Profile and Core Methods for evaluating root distributions in tillage studies. Agron. J. 80, 166-172 (1988).

VESTER, F., 1978: Unsere Welt-ein vernetztes System. Verl. Ernst Klett, Stuttgart.

Vetter, H., Scharafat, S., 1964: Die Wurzelverbreitung landwirtschaftlicher Kulturpflanzen im Unterboden. Z. Acker- und Pflanzenbau 120, 275-298.

Walley, F.L., Tomm, G.O., Matus, A., Slinkard, A.E. Van Kessel, C., 1996: Allocation and Cycling of Nitrogen in an Alfalfa-Bromegrass Sward. Agron. J. 88, 834843.

Werner, D., 1987: Pflanzliche und mikrobielle Symbiosen. Thieme Verlag, Stuttgart und New York.

Werner, D., KROTZKY, A., 1983: Die symbiotische Stickstoff-Fixierung der Leguminosen. Funkt. Biol. Med. 2, 31-39.

West, C.P., WedIN, W.F., 1985: Dinitrogen Fixation in Alfalfa-Orchardgrass Pastures. Agron. J. 77, 89-93.

WILDENHAYN, M., 1990: Auswirkungen der Extensivierung auf Pflanzenkrankheiten und Erträge verschiedener Feldfüchte im Ackerbausystemversuch Reinshof. Dissertation am Institut für Pflanzenbau und Pflanzenzüchtung der Georg-August Universität in Göttingen.

WolF, G. A., 1994: Regulationsmechanismen bei der Epidemiologie von bodenbürtigen Pflanzenpathogenen. Berichte über Landwirtschaft, 209. Sonderheft, Band 7, 85-92. 


\section{Verzeichnis der Anhangstabellen}

Tab. A1: Chemische Kennwerte des Bodens am Versuchsstandort (Mittelwert über die N-Düngungsstufen ${ }^{1}$, IF: lange Fruchtfolge, kF: kurze Fruchtfolge, vgl. Kap. 2.3.2)

Tab. A2: Gewichtungsfaktoren bei der standraumbezogenen Schätzung der Wurzelmassen in Abhängigkeit von der Individuenzahl bei einem Reihenabstand von $28 \mathrm{~cm}$

Tab. A3: Versuchsdaten und Entwicklungsstadien (BBCH; MEIER 1997)

Tab. A4: Mittelwerte der gesamtpflanzlichen Trockenmasse-Akkumulation und TM-Ertragsanteile $\left(\mathrm{dt} \mathrm{ha}^{-1}\right.$ ) beim Anbau von Ackerbohne und Hafer in Rein- und Gemengesaat sowie Ergebnisse der Varianzanalyse zur ersten und zweiten Ernte im Jahr 1998

Tab. A5: Vergleich der Mittelwerte der gesamtpflanzlichen TrockenmasseAkkumulation, der gewichteten Trockenmasse-Harvest-Indizes $\left(\mathrm{H}_{\mathrm{TMBtw}}\right)$ sowie der TM-Ertragsanteile beim Anbau von Ackerbohne und Hafer in Rein- und Gemengesaat zur letzten Ernte (BBCH 87) am 10.08.1998

Tab. A6: Kornerträge (dt TM ha ${ }^{-1}$ ) beim Anbau von Ackerbohne und Hafer in Reinund Gemengesaat (BBCH 87) am 10.08.1998 und am 26.07.1999

Tab. A7: Mittelwerte \pm Standardabweichung der Spross-Trockenmasse ( $\mathrm{dt} \mathrm{ha}^{-1}$ ) und Spross-N-Mengen $\left(\mathrm{kg} \mathrm{ha}^{-1}\right)$ der Kräuter beim Anbau von Ackerbohne und Hafer in Rein- und Gemengesaat zur letzten Ernte im Jahr 1999

Tab. A8: Mittelwerte der gesamtpflanzlichen Trockenmasse-Akkumulation und TM-Ertragsanteile ( $\mathrm{dt} \mathrm{ha}^{-1}$ ) beim Anbau von Ackerbohne und Hafer in Rein- und Gemengesaat sowie Ergebnisse der Varianzanalyse (Ansaatvariante, Düngung) zur ersten und zweiten Ernte im Jahr 1999

Tab. A9: Vergleich der Mittelwerte der gesamtpflanzlichen TrockenmasseAkkumulation, der gewichteten Trockenmasse-Harvest-Indizes $\left(\mathrm{H}_{\text {TMBtw }}\right)$ sowie der TM-Ertragsanteile beim Anbau von Ackerbohne und Hafer in Rein- und Gemengesaat zur letzten Ernte (BBCH 87) am 26.07.1999

Tab. A10: Mittelwerte und Mittelwertvergleich der gesamtpflanzlichen StickstoffAkkumulation $\left(\mathrm{N}_{\mathrm{Bt}} ; \mathrm{kg} \mathrm{ha}^{-1}\right)$, Stickstoff-Ertragsanteile und gewichteter Stickstoff-Harvest-Index $\left(\mathrm{H}_{\mathrm{NBtw}}\right)$ beim Anbau von Ackerbohne und Hafer in Rein- und Gemengesaat zu drei Ernteterminen im Jahr 1998

Tab. A11: Mittelwerte und Mittelwertvergleich der gesamtpflanzlichen StickstoffAkkumulation ( $\mathrm{N}_{\mathrm{B}} ; \mathrm{kg} \mathrm{ha}^{-1}$ ), Stickstoff-Erträge in Korn, Stroh und Wurzel $\left(\mathrm{kg} \mathrm{ha}^{-1}\right)$ und gewichteter Stickstoff-Harvest-Index $\left(\mathrm{H}_{\mathrm{NBtw}}\right)$ beim Anbau von Ackerbohne und Hafer in Rein- und Gemengesaat zu drei Ernteterminen im Jahr 1999 
Tab. A12: Mittelwerte und Mittelwertvergleich ${ }^{1}$ der gewichteten $\delta^{15} \mathrm{~N}$-Werte (\%o) und Anteil Stickstoff aus der Luft $\left(\mathrm{Ndfa}_{\mathrm{w}}\right)$ in der Sprossmasse von Ackerbohne und Hafer in Rein- und Gemengesaat im Jahr 1998 und 1999

Tab. A13: Vergleich der gesamtpflanzlich gewichteten $\delta^{15} \mathrm{~N}$-Mittelwerte beim Anbau von Ackerbohne und Hafer in Rein- und Gemengesaat zu drei Ernteterminen a) 1998 und b) 1999 sowie Ergebnis der zweifaktoriellen Varianzanalyse

Tab. A14: Korrelationskoeffizienten ( $r$ ) zwischen den Ergebnissen der $\delta^{15} \mathrm{~N}$-Methode und der erweiterten Differenzmethode zur Schätzung der symbiotisch fixierten N-Menge beim Anbau von Ackerbohne in Rein- und Gemengesaat mit Hafer im Jahr 1998 und 1999

Tab. A15: Mittelwerte ( $\left.\mathrm{kg} \mathrm{N}_{\min } \mathrm{ha}^{-1}\right)$ und Mittelwertvergleich der $\mathrm{CaCl}_{2}$-extrahierbaren $\mathrm{N}_{\text {min }}$-Mengen im Boden vor der Aussaat von Ackerbohne und Hafer am 20.03.1998 (a) und 15.03.1999 (b)

Tab. A16: Mittelwerte ( $\left.\mathrm{kg} \mathrm{N}_{\min } \mathrm{ha}^{-1}\right)$ und Mittelwertvergleich der $\mathrm{CaCl}_{2}$-extrahierbaren $\mathrm{N}_{\text {min }}$-Mengen im Boden beim Anbau von Ackerbohne und Hafer in Reinund Gemengesaat zu vier Probenahmeterminen im Jahr 1998

Tab. A17: Mittelwerte und Mittelwertvergleich der luft- und bodenbürtigen gesamtpflanzlichen $\mathrm{N}$-Akkumulation $\left(\mathrm{kg} \mathrm{ha}^{-1}\right)$ und gewichtete Anteile Stickstoff aus der Luft ( $\mathrm{Ndfa}_{\mathrm{w}}$ ) beim Anbau von Ackerbohne und Hafer in Rein- und Gemengesaat zu drei Ernteterminen im Jahr 1998

Tab. A18: Mittelwerte ( $\mathrm{kg} \mathrm{N}_{\text {min }} \mathrm{ha}^{-1}$ ) und Mittelwertvergleich der $\mathrm{CaCl}_{2}$-extrahierbaren $\mathrm{N}_{\text {min }}$-Mengen im Boden beim Anbau von Ackerbohne und Hafer in Reinund Gemengesaat zu vier Probenahmeterminen im Jahr 1999

Tab. A19: Mittelwerte und Mittelwertvergleich der luft- und bodenbürtigen gesamtpflanzlichen $\mathrm{N}$-Akkumulation $\left(\mathrm{kg} \mathrm{ha}^{-1}\right)$ und gewichtete Anteile Stickstoff aus der Luft $\left(\mathrm{Ndfa}_{\mathrm{w}}\right)$ beim Anbau von Ackerbohne und Hafer in Rein- und Gemengesaat zu drei Ernteterminen im Jahr 1999

Tab. A20: Ergebnis der Varianzanalyse der gewogenen $\delta^{15} \mathrm{~N}$-Werte sowie multipler Vergleich der gewogenen $\delta^{15} \mathrm{~N}$-Werte in der Sprossmasse von Hafer in Rein- und Gemengesaat zur jeweils letzten Ernte der Jahre 1998 und 1999

Tab. A21: Ergebnis der Varianzanalyse der Transfer-N-Menge und Anteil des Transfer-Stickstoffes an der Gesamt-N-Menge in der Sprossmasse des Hafers zur letzten Ernte im Jahr 1998 und 1999

Tab. A22: Geschätzte Menge an N-Rhizodeposition, die aus der Fixierung stammt (in $\mathrm{kg} \mathrm{N} \mathrm{ha}^{-1}$ ) beim Anbau von Ackerbohne in Rein- und Gemengesaat mit Hafer im Jahr 1998 und 1999 
Tab. A23: Mittelwerte \pm Standardabweichung der Spross-Trockenmassen $\left(\mathrm{dt} \mathrm{ha}^{-1}\right.$ ) und Spross-N-Mengen $\left(\mathrm{kg} \mathrm{ha}^{-1}\right)$ der Kräuter beim Anbau von Luzerne und Knaulgras in Rein- und Gemengesaat zu drei Schnittterminen im Jahr 1998

Tab. A24: Mittelwerte der Trockenmasse-Akkumulation $\left(\mathrm{dt} \mathrm{ha}^{-1}\right)$ sowie der gewichteten Trockenmasse-Harvest-Indizes $\left(\mathrm{H}_{\text {TMBtw }}\right)$ beim Anbau von Luzerne und Knaulgras in Rein- und Gemengesaat sowie Ergebnisse der Varianzanalyse (Ansaatvariante, Düngung) zum ersten und zweiten Schnitt 1998

Tab. A25: Mittelwerte und Mittelwertvergleich der Trockenmasse-Akkumulation $\left(\mathrm{dt} \mathrm{ha}^{-1}\right)$ sowie der gewichteten Trockenmasse-Harvest-Indizes $\left(\mathrm{H}_{\mathrm{TMBtw}}\right)$ beim Anbau von Luzerne und Knaulgras in Rein- und Gemengesaat zum dritten Schnitt am 25.10.1998 ( $=\sum$ 1998)

Tab. A26: Mittelwerte \pm Standardabweichung der Spross-Trockenmassen ( $\mathrm{dt}^{\mathrm{h}} \mathrm{a}^{-1}$ ) und Spross-N-Mengen $\left(\mathrm{kg} \mathrm{ha}^{-1}\right)$ der Kräuter beim Anbau von Luzerne und Knaulgras in Rein- und Gemengesaat zu drei Schnittterminen im Jahr 1999

Tab. A27: Mittelwerte der Trockenmasse-Akkumulation sowie der gewichteten Trockenmasse-Harvest-Indizes ( $\mathrm{H}_{\text {TMBtw }}$ ) beim Anbau von Luzerne und Knaulgras in Rein- und Gemengesaat sowie Ergebnisse der Varianzanalyse (Ansaatvariante, Düngung) zum ersten und zweiten Schnitt im Jahr 1999

Tab. A28: Mittelwerte und Mittelwertvergleich der Trockenmasse-Akkumulation $\left(\mathrm{dt} \mathrm{ha}{ }^{-1}\right)$ sowie der gewichteten Trockenmasse-Harvest-Indizes $\left(\mathrm{H}_{T M B t w}\right)$ beim Anbau von Luzerne und Knaulgras in Rein- und Gemengesaat zum dritten Schnitt am 04.11.1999 $\left(=\sum\right.$ 1999)

Tab. A29: Mittelwerte und Mittelwertvergleich der gesamtpflanzlichen NAkkumulation $\left(\mathrm{N}_{\mathrm{Bt}}, \mathrm{kg} \mathrm{ha}^{-1}\right)$, der Stickstoff-Erträge im Schnittgut, Stoppel und Wurzel $\left(\mathrm{kg} \mathrm{ha}^{-1}\right)$ sowie der gewichteten Stickstoff-Harvest-Indizes $\left(H_{\text {NBtw }}\right)$ beim Anbau von Luzerne und Knaulgras in Rein- und Gemengesaat zu drei Schnittterminen im Jahr 1998

Tab. A30: Mittelwerte und Mittelwertvergleich der gesamtpflanzlichen NAkkumulation ( $\left.\mathrm{N}_{\mathrm{Bt}}, \mathrm{kg} \mathrm{ha}^{-1}\right)$, der Stickstoff-Erträge im Schnittgut, Stoppel und Wurzel $\left(\mathrm{kg} \mathrm{ha}^{-1}\right)$ sowie der gewichteten Stickstoff-Harvest-Indizes $\left(\mathrm{H}_{\text {NBtw }}\right)$ beim Anbau von Luzerne und Knaulgras in Rein- und Gemengesaat zu drei Schnittterminen im Jahr 1999

Tab. A31: Mittelwerte und Mittelwertvergleich ${ }^{1}$ der gewichteten kumulierten $\delta^{15} \mathrm{~N}$ Werte (\%) und Anteil Stickstoff aus der Luft ( $\left.\mathrm{Ndfa}_{\mathrm{w}}\right)$ in der Sprossmasse von Luzerne und Knaulgras in Rein- und Gemengesaat zum dritten Schnitt im Jahr 1998 und 1999 
Tab. A32: Vergleich der gesamtpflanzlich gewichteten $\delta^{15} \mathrm{~N}$-Mittelwerte beim Anbau von Luzerne und Knaulgras in Rein- und Gemengesaat zu drei Schnittterminen a) 1998 und b) 1999 sowie Ergebnis der zweifaktoriellen Varianzanalyse

Tab. A33: Korrelation zwischen den Ergebnissen der $\delta^{15} \mathrm{~N}$-Methode und der erweiterten Differenzmethode zur Schätzung der symbiotisch fixierten $\mathrm{N}$ Menge beim Anbau von Luzerne in Rein- und Gemengesaat mit Knaulgras im Jahr 1998 und 1999

Tab. A34: Mittelwerte ( $\mathrm{kg} \mathrm{N}_{\min } \mathrm{ha}^{-1}$ ) und Mittelwertvergleich der $\mathrm{CaCl}_{2}$-extrahierbaren $\mathrm{N}_{\text {min }}$-Mengen im Boden vor der Aussaat der Luzerne und des Knaulgrases am 24.03.1998 (a) und 06.04.1999 (b)

Tab. A35: Mittelwerte $\left(\mathrm{kg} \mathrm{N}_{\min } \mathrm{ha}^{-1}\right)$ und Mittelwertvergleich der $\mathrm{CaCl}_{2}$-extrahierbaren $\mathrm{N}_{\text {min }}$-Mengen im Boden beim Anbau von Luzerne und Knaulgras in Reinund Gemengesaat zu drei Probenahmeterminen im Jahr 1998

Tab. A36: Mittelwerte und Mittelwertvergleich der luft- und bodenbürtigen gesamtpflanzlichen $\mathrm{N}$-Akkumulation $\left(\mathrm{kg} \mathrm{ha}^{-1}\right)$ und gewichtete Anteile Stickstoff aus der Luft ( $\mathrm{Ndfa}_{w}$ ) beim Anbau von Luzerne und Knaulgras in Rein- und Gemengesaat zu drei Schnittterminen im Jahr 1998

Tab. A37: Mittelwerte ( $\mathrm{kg} \mathrm{N}_{\min } \mathrm{ha}^{-1}$ ) und Mittelwertvergleich der $\mathrm{CaCl}_{2}$-extrahierbaren $\mathrm{N}_{\text {min }}$-Mengen im Boden beim Anbau von Luzerne und Knaulgras in Reinund Gemengesaat zu drei Probenahmeterminen im Jahr 1999

Tab. A38: Mittelwerte und Mittelwertvergleich der luft- und bodenbürtigen gesamtpflanzlichen N-Akkumulation $\left(\mathrm{kg} \mathrm{ha}^{-1}\right)$ und gewichtete Anteile Stickstoff aus der Luft $\left(\mathrm{Ndfa}_{\mathrm{w}}\right)$ beim Anbau von Luzerne und Knaulgras in Rein- und Gemengesaat zu drei Schnittterminen im Jahr 1999

Tab. A39: Ergebnis der Varianzanalyse der gewichteten $\delta^{15} \mathrm{~N}$-Werte sowie multipler Vergleich der gewichteten $\delta^{15} \mathrm{~N}$-Mittelwerte in der Sprossmasse des Knaulgrases in Rein- und Gemengesaat zum dritten Schnitttermin im Jahr 1998 und 1999.

Tab. A40: Vergleich der Werte für die Parameter Transfer-N-Menge und Anteil des Transfer-Stickstoffes an der Gesamt-N-Menge in der Sprossmasse von Knaulgras zum dritten Schnitttermin 1998 und 1999.

Tab. A41: Geschätzte Menge an N-Rhizodeposition die aus der Fixierung stammt $\left(\mathrm{kg} \mathrm{N} \mathrm{ha}^{-1}\right.$ ) beim Anbau von Luzerne in Rein- und Gemengesaat mit Knaulgras im Jahr 1998 und 1999.

Tab. A42: Mittelwerte \pm Standardabweichung der Spross-Trockenmassen ( $\left.\mathrm{dt} \mathrm{ha}^{-1}\right)$ und Spross-N-Mengen (kg ha ${ }^{-1}$ ) der Kräuter beim Anbau der Saatwicke und des Einjährigen Weidelgrases in Rein- und Gemengesaat im Jahr 1998 und 1999 
Tab. A43: Gesamtpflanzliche Trockenmasse-Akkumulation ( $\mathrm{dt} \mathrm{ha}^{-1}$ ), TM-Erträge der Wurzel- und Stoppelmasse $\left(\mathrm{dt} \mathrm{ha}^{-1}\right)$ sowie gewichtete TM-HarvestIndizes beim Anbau der Saatwicke und des Einjährigen Weidelgrases in Rein- und Gemengesaat am 20.10.1998

Tab. A44: Gesamtpflanzliche Trockenmasse-Akkumulation ( $\mathrm{dt} \mathrm{ha}^{-1}$ ), TM-Erträge der Wurzel- und Stoppelmasse $\left(\mathrm{dt} \mathrm{ha}^{-1}\right)$ sowie gewichtete TM-HarvestIndizes beim Anbau der Saatwicke und des Einjährigen Weidelgrases in Rein- und Gemengesaat am 17.10.1999

Tab. A45: Gesamtpflanzliche Stickstoff-Akkumulation $\left(\mathrm{N}_{\mathrm{Bt}}, \mathrm{kg} \mathrm{ha}^{-1}\right)$, StickstoffErträge im Schnittgut, in der Stoppelmasse und den Wurzeln $\left(\mathrm{kg} \mathrm{ha}^{-1}\right)$ sowie der gewichteten Stickstoff-Harvest-Indizes ( $\left.\mathrm{H}_{\mathrm{NBtw}}\right)$ beim Anbau der Saatwicke und des Einjährigen Weidelgrases in Rein- und Gemengesaat am 20.10.1998

Tab. A46: Gesamtpflanzliche Stickstoff-Akkumulation $\left(\mathrm{N}_{\mathrm{Bt}}, \mathrm{kg} \mathrm{ha}^{-1}\right)$, StickstoffErträge im Schnittgut, in der Stoppelmasse und den Wurzeln $\left(\mathrm{kg} \mathrm{ha}^{-1}\right)$ sowie der gewichteten Stickstoff-Harvest-Indizes ( $\left.\mathrm{H}_{\mathrm{NBtw}}\right)$ beim Anbau der Saatwicke und des Einjährigen Weidelgrases in Rein- und Gemengesaat am 17.10.1999

Tab. A47: Mittelwerte und Mittelwertvergleich ${ }^{1}$ der gewichteten $\delta^{15} \mathrm{~N}$-Werte $(\%)$ und Anteil Stickstoff aus der Luft ( $\mathrm{Ndfa}_{w}$ ) in der Sprossmasse von Saatwicke und Einjährigem Weidelgras in Rein- und Gemengesaat 1998 und 1999

Tab. A48: Vergleich der gewichteten gesamtpflanzlich $\delta^{15} \mathrm{~N}$-Mittelwerte beim Anbau von Saatwicke und Einjährigem Weidelgras in Rein- und Gemengesaat am 20.10.1998 (a) und am 17.10.1999 (b) sowie Ergebnis der zweifaktoriellen Varianzanalyse

Tab. A49: Mittelwerte $\left(\mathrm{kg} \mathrm{N}_{\min } \mathrm{ha}^{-1}\right)$ und Mittelwertvergleich der $\mathrm{CaCl}_{2}$-extrahierbaren $\mathrm{N}_{\min }$-Mengen im Boden vor der Aussaat der Saatwicke und des Einjährigen Weidelgrases am 21.07.1998 (a) und 09.07.1999 (b)

Tab. A50: Mittelwerte $\left(\mathrm{kg} \mathrm{N}_{\min } \mathrm{ha}^{-1}\right)$ und Mittelwertvergleiche der $\mathrm{CaCl}_{2}$ extrahierbaren $\mathrm{N}_{\text {min }}$-Mengen im Boden beim Anbau der Saatwicke und des Einjährigen Weidelgrases in Rein- und Gemengesaat zu zwei Probenahmeterminen im Jahr 1998

Tab. A51: Mittelwerte und Mittelwertvergleiche der luft- und bodenbürtigen gesamtpflanzlichen N-Akkumulation $\left(\mathrm{kg} \mathrm{ha}^{-1}\right)$ und gewichtete Anteile Stickstoff aus der Luft $\left(\mathrm{Ndfa}_{\mathrm{w}}\right)$ beim Anbau der Saatwicke und des Einjährigen Weidelgrases in Rein- und Gemengesaat am 20.10.1998

Tab. A52: Mittelwerte $\left(\mathrm{kg} \mathrm{N}_{\min } \mathrm{ha}^{-1}\right)$ und Mittelwertvergleiche der $\mathrm{CaCl}_{2}$ extrahierbaren $\mathrm{N}_{\text {min }}$-Mengen im Boden beim Anbau der Saatwicke und des Einjährigen Weidelgrases in Rein- und Gemengesaat zu zwei Probenahmeterminen im Jahr 1999 
Tab. A53: Mittelwerte und Mittelwertvergleich der luft- und bodenbürtigen gesamtpflanzlichen $\mathrm{N}$-Akkumulation $\left(\mathrm{kg} \mathrm{ha}^{-1}\right)$ und gewichtete Anteile Stickstoff aus der Luft $\left(\mathrm{Ndfa}_{\mathrm{w}}\right)$ beim Anbau der Saatwicke und des Einjährigen Weidelgrases in Rein- und Gemengesaat am 17.10.1999

Tab. A54: Ergebnis der Varianzanalyse der gewichteten $\delta^{15} \mathrm{~N}$-Werte in der Sprossmasse des Einjährigen Weidelgrases in Rein- und Gemengesaat im Jahr 1998 und 1999

Tab. A55: Geschätzte Menge an N-Rhizodeposition, die aus der Fixierung stammt $\left(\mathrm{kg} \mathrm{N} \mathrm{ha}^{-1}\right)$, beim Anbau von Saatwicke in Rein- und Gemengesaat mit Einjährigem Weidelgras im Jahr 1998 und 1999

Tab. A56: Variationskoeffizienten (cv \% $\mathrm{n}=6$ Parzellen/Variante) bei der Wurzelmassenbestimmung von Ackerbohne, Luzerne und Saatwicke in Rein- und Gemengesaat mit Hafer, Knaulgras und Einjährigem Weidelgras.

Tab. A57: Kennwerte des pflanzlichen N-Haushaltes (Spross und Wurzeln) und vereinfachte N-Flächenbilanzsalden für Ackerbohne, Luzerne und Saatwicke in Reinsaat

Tab. A58: Differenz im residualen $\mathrm{N}_{\text {min }}$-Stickstoff $\left(\mathrm{kg} \mathrm{N} \mathrm{ha}^{-1}\right)$ zur Ernte im Boden zwischen den Ansaaten mit Ackerbohne (A), Luzerne (L) und Saatwicke $(S)$ in Rein- und Gemengesaat mit Hafer $(H)$, Knaulgras $(K)$ und $(E)$ Einjährigem Weidelgras und den Reinsaaten der entsprechenden Referenzpflanzen in 1998 und 1999 
8. Anhang

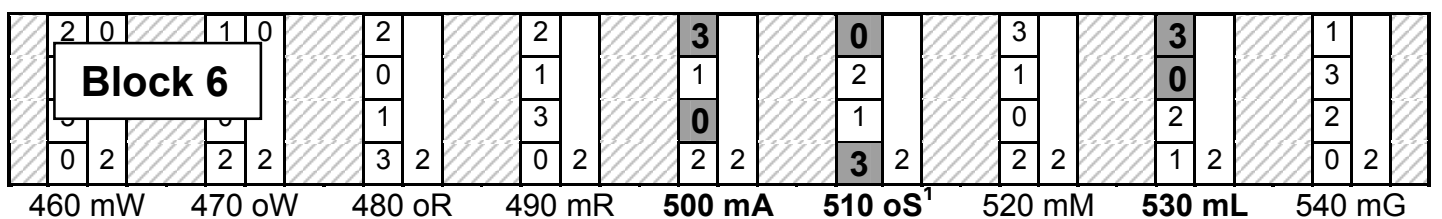

m Ps

WDH

III

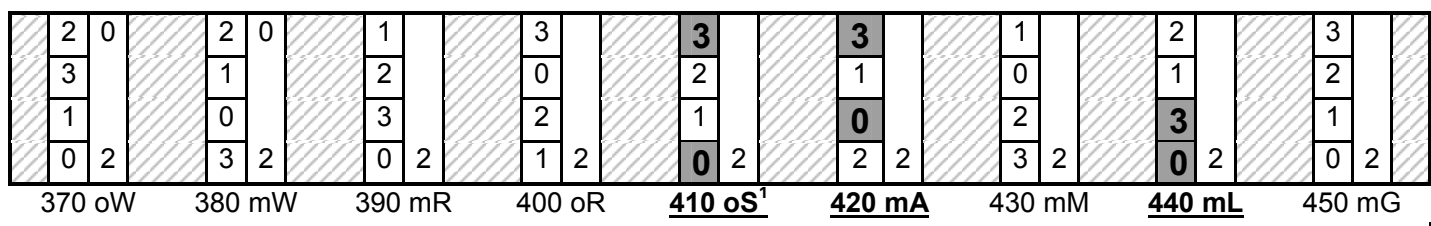

o Ps

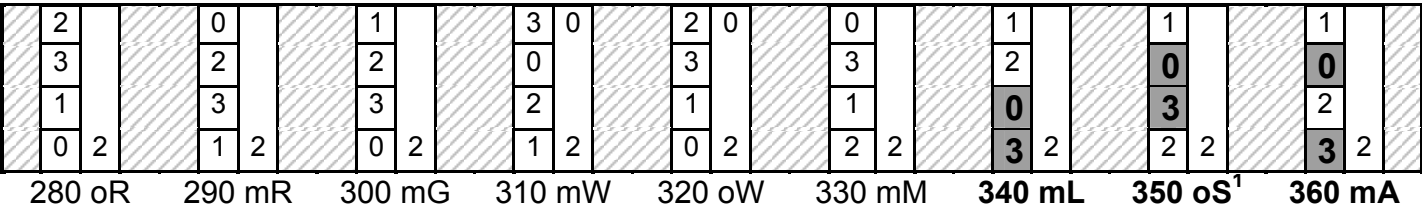

m Ps

WDH

II

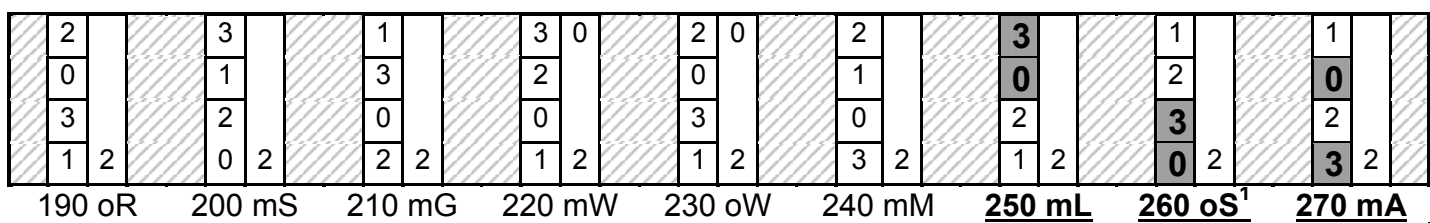

o Ps

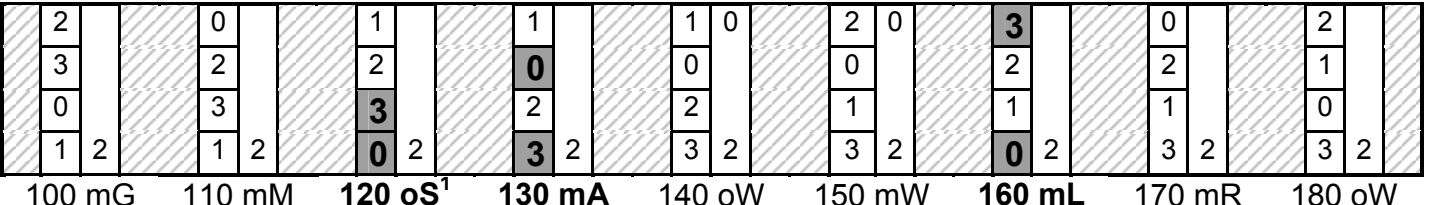

o Ps

WDH

I

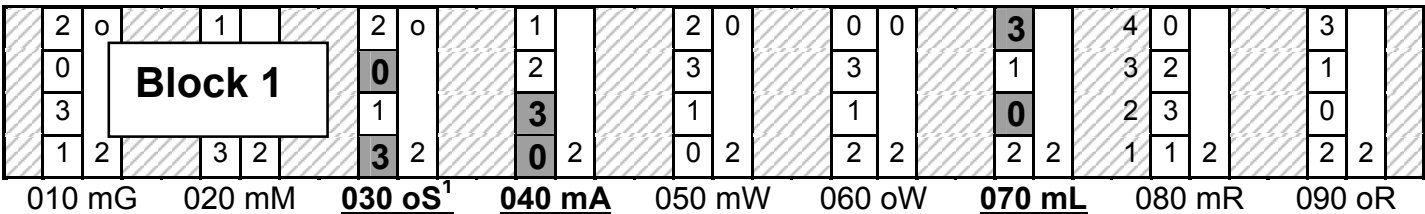

m Ps

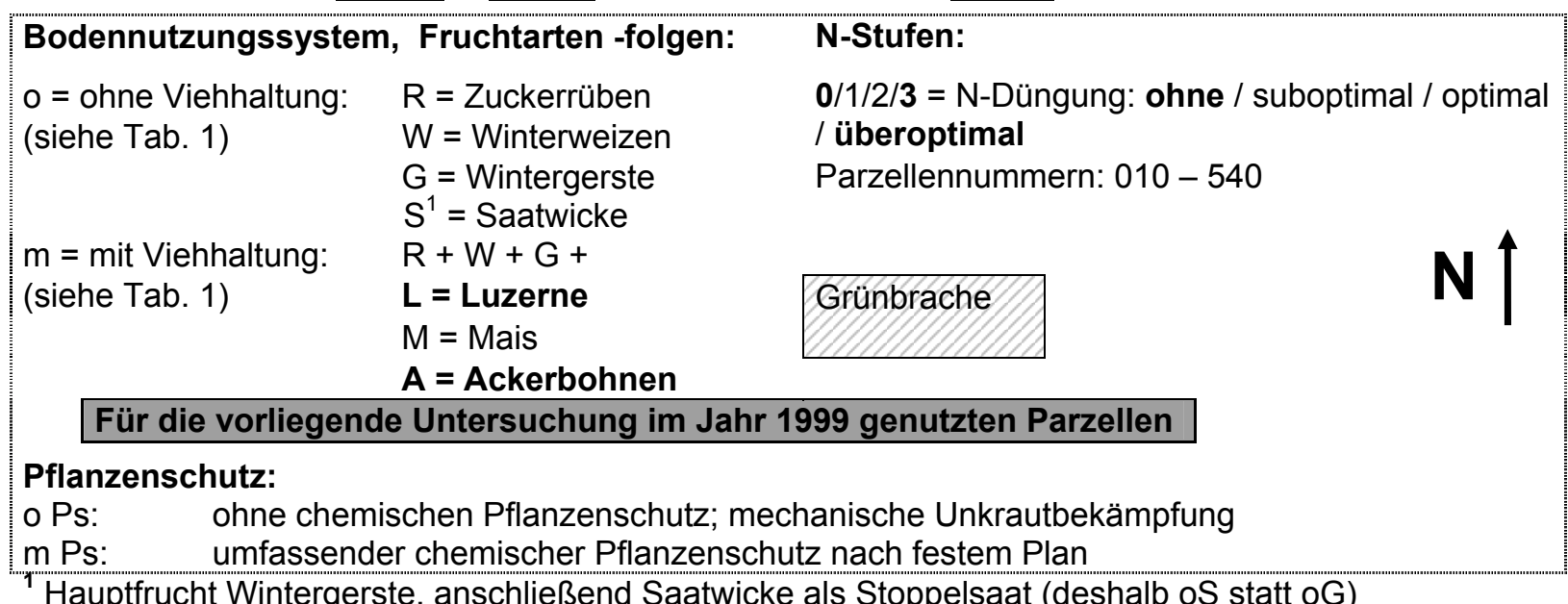

Abb. A1: Lageplan der Prüfglieder im Ackerbau-Systemversuch Göttingen, Feldplan 1999 
Tab. A1: Chemische Kennwerte des Bodens am Versuchsstandort (Mittelwert über die N-Düngungsstufen ${ }^{1}$, IF: lange Fruchtfolge, kF: kurze Fruchtfolge, vgl. Kap. 2.3.2)

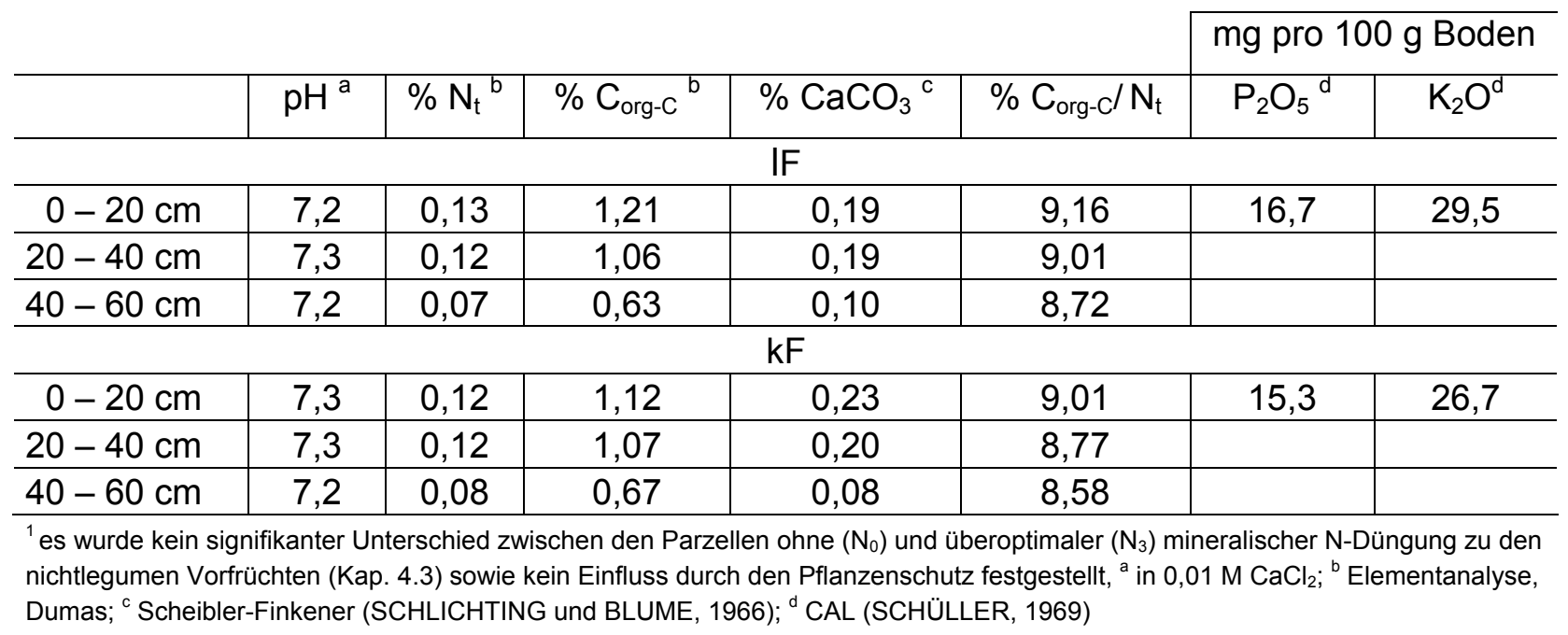

Tab. A2: Gewichtungsfaktoren bei der standraumbezogenen Schätzung der Wurzelmassen in Abhängigkeit von der Individuenzahl bei einem Reihenabstand von $28 \mathrm{~cm}$

\begin{tabular}{c|c|c}
\hline Pflanzen $/ \mathrm{m}^{2}$ & auf der Reihe & $\begin{array}{c}\text { zwischen der } \\
\text { Reihe }\end{array}$ \\
\hline $25 \%$ Ackerbohne (Variante 1A/3H) \\
\hline 10 & 0,059 & 0,941 \\
\hline 11 & 0,065 & 0,935 \\
\hline $75 \%$ Ackerbohne (Variante $3 \mathrm{~A} / 1 \mathrm{H})$ \\
\hline 30 & 0,177 & 0,823 \\
\hline 31 & 0,183 & 0,817 \\
\hline 32 & 0,189 & 0,811 \\
\hline 33 & 0,195 & 0,805 \\
\hline $100 \%$ Ackerbohne (Variante $4 \mathrm{~A})$ \\
\hline 40 & 0,236 & 0,764 \\
\hline 42 & 0,247 & 0,753 \\
\hline 45 & 0,258 & 0,742 \\
\hline
\end{tabular}


Tab. A3: Versuchsdaten und Entwicklungsstadien (BBCH; MEIER 1997)

\begin{tabular}{c|c|c|c|c|c|c|c|c|c|c}
\hline & \multicolumn{3}{|c|}{ Ackerbohne } & \multicolumn{4}{c|}{ Luzerne } & \multicolumn{3}{c}{ Saatwicke } \\
\hline & BBCH & 1998 & 1999 & BBCH & Schnitt & 1998 & 1999 & BBCH & 1998 & 1999 \\
\hline $\mathrm{N}_{\text {min }}$ & & 22.03. & 15.03 & & & 24.03. & 06.04. & & 21.07. & 09.07. \\
\hline Aussaat & 00 & 25.03. & 17.03. & 00 & & 01.04 & 12.04. & 00 & 27.07. & 13.07. \\
\hline $\begin{array}{c}\text { Beikraut- } \\
\text { regulierung }\end{array}$ & & $\begin{array}{c}07.05 .- \\
13.05 .\end{array}$ & 29.04. & & & $\begin{array}{c}13.05 .- \\
20.05 .\end{array}$ & $\begin{array}{c}13.05 .- \\
16.05 .\end{array}$ & & $\begin{array}{c}14.08 .- \\
19.08 .\end{array}$ & 30.07. \\
\hline $\begin{array}{c}\text { Beikraut- } \\
\text { regulierung }\end{array}$ & & & & & & $\begin{array}{c}29.05 .- \\
08.06 .\end{array}$ & & & 20.09. & 18.08 \\
\hline 1. Ernte & 31 & 25.05. & 19.05. & 64 & 1. & 29.06. & 05.07. & & & \\
\hline 2. Ernte & 65 & 22.06. & 09.06. & 64 & 2. & 24.08. & 16.08. & & & \\
\hline $\mathrm{Ba}^{1}$ & & 29.07. & 09.07. & & & & 24.10. & & & \\
\hline $\mathrm{Ba}^{1}$ & & 10.08. & 25.07. & & & & & & & \\
\hline $\mathrm{N}_{\min }$ & & & & & & & & 33 & 03.09. & 24.08. \\
\hline $\mathrm{Endernte}^{1}$ & 87 & 10.08. & 26.07 & 61 & 3. & 25.10. & 04.11. & $61 / 65$ & 20.10. & 17.10. \\
\hline $\mathrm{N}_{\min }$ & & 16.10. & 03.11. & & & & & & & \\
\hline 1
\end{tabular}

${ }^{1}$ Bestandsabfall

Tab. A4: Mittelwerte der gesamtpflanzlichen Trockenmasse-Akkumulation und TM-Ertragsanteile ( $\mathrm{dt} \mathrm{ha}^{-1}$ ) beim Anbau von Ackerbohne und Hafer in Rein- und Gemengesaat sowie Ergebnisse der Varianzanalyse zur ersten und zweiten Ernte im Jahr 1998

\begin{tabular}{|c|c|c|c|c|c|c|c|c|c|c|c|}
\hline Ansaatvariante & \multicolumn{2}{|c|}{$4 \mathrm{~A}$} & \multicolumn{2}{|c|}{$3 \mathrm{~A} / 1 \mathrm{H}$} & \multicolumn{2}{|c|}{$1 \mathrm{~A} / 3 \mathrm{H}$} & \multicolumn{2}{|c|}{$4 \mathrm{H}$} & \multirow{3}{*}{ Ansaatvariante } & \multirow{3}{*}{ Düngung } & \multirow{3}{*}{$\begin{array}{l}\text { Ansaat- } \\
\text { variante } x \\
\text { Düngung }\end{array}$} \\
\hline Düngung & $\mathrm{N}_{0}$ & $\mathrm{~N}_{3}$ & $\mathrm{~N}_{0}$ & $\mathrm{~N}_{3}$ & $\mathrm{~N}_{0}$ & $\mathrm{~N}_{3}$ & $\mathrm{~N}_{0}$ & $\mathrm{~N}_{3}$ & & & \\
\hline 1. Ernte & & & 25.05 & 5.1998 & (BBC & H 31) & & & & & \\
\hline $\mathrm{TM}_{\mathrm{Bt}}$ & 18,5 & 20,7 & 25,0 & 25,5 & 24,4 & 23,9 & 20,7 & 22,3 & * & n.s. & n.s. \\
\hline Stroh-TM & 9,5 & 12,3 & 11,7 & 13,4 & 11,8 & 11,5 & 12,8 & 12,7 & n.s. ${ }^{1}$ & n.s. ${ }^{1}$ & n.s. ${ }^{1}$ \\
\hline Wurzel-TM & 8,9 & 8,4 & 13,3 & 12,1 & 12,6 & 12,4 & 7,9 & 9,6 & $* * *$ & n.s. & n.s. \\
\hline 2. Ernte & & & 22.06 & 6.1998 & (BBC) & H 65) & & & & & \\
\hline $\mathrm{TM}_{\mathrm{Bt}}$ & 68,2 & 69,6 & $82,3^{2}$ & $90,4^{2}$ & $91,2^{2}$ & $104,6^{2}$ & 79,4 & 88,5 & $* * *$ & * & n.s. \\
\hline Stroh-TM & 58,5 & 57,9 & $68,4^{2}$ & $76,9^{2}$ & $78,3^{2}$ & $88,3^{2}$ & 69,5 & 77,7 & $* * *$ & n.s. & n.s. \\
\hline Wurzel-TM & 9,7 & 11,7 & $13,9^{2}$ & $13,5^{2}$ & $12,9^{2}$ & $16,3^{2}$ & 9,9 & 10,8 & * & n.s. & n.s. \\
\hline
\end{tabular}


Tab. A5: Vergleich der Mittelwerte der gesamtpflanzlichen TrockenmasseAkkumulation, der gewichteten Trockenmasse-Harvest-Indizes $\left(\mathrm{H}_{\mathrm{TMBtw}}\right)$ sowie der TM-Ertragsanteile beim Anbau von Ackerbohne und Hafer in Rein- und Gemengesaat zur letzten Ernte (BBCH 87) am 10.08.1998

\begin{tabular}{|c|c|c|c|c|c|c|}
\hline & \multicolumn{4}{|c|}{ Ansaatvariante } & \multirow[b]{2}{*}{ Düngung } & \multirow{2}{*}{$\begin{array}{l}\text { Wechsel- } \\
\text { wirkungen }\end{array}$} \\
\hline 1998 & $4 \mathrm{~A}$ & $3 \mathrm{~A} / 1 \mathrm{H}^{2}$ & $1 \mathrm{~A} / 3 \mathrm{H}^{2}$ & $4 \mathrm{H}$ & & \\
\hline $\mathrm{TM}_{\mathrm{Bt}}$ & $a^{s}$ & $a$ & $a b$ & $b^{*}$ & n.s. & n.s. \\
\hline Korn & $a^{s}$ & $a b$ & $a b$ & $b^{* \star *}$ & n.s. & ** \\
\hline Stroh $^{1}$ & $a^{S}$ & a & $a b$ & $\mathrm{~b}^{\star * *}$ & n.s. & n.s. \\
\hline Wurzel & $a^{s}$ & a & a & a & n.s. & n.s. \\
\hline $\mathrm{H}_{\text {TMBtw }}$ & $a^{S}$ & $a$ & $b$ & $\mathrm{~b}^{\star \star \star}$ & n.s. & n.s. \\
\hline
\end{tabular}

$\mathrm{TM}_{\mathrm{Bt}}=$ gesamtpflanzliche Trockenmasse; $\mathrm{H}_{\mathrm{TMBtw}}=$ gewichteter gesamtpflanzlicher TrockenmasseHarvest-Index; ${ }^{1}$ inklusive Bestandesabfall; nicht gleiche Buchstaben kennzeichnen signifikante Unterschiede; s Scheffé-Test: ${ }^{*}=P<0,05 ;{ }^{* *}=P<0,01 ;{ }^{* *}=P<0,001$; n.s. $=$ nicht signifikant; ${ }^{2} n=5$

Tab. A6: Kornerträge (dt TM ha ${ }^{-1}$ ) beim Anbau von Ackerbohne und Hafer in Reinund Gemengesaat (BBCH 87) am 10.08.1998 und am 26.07.1999

\begin{tabular}{c|c|c|c|c|c|c|c|c}
\cline { 2 - 9 } \multicolumn{2}{c}{1998} & \multicolumn{9}{c|}{$\mathrm{N}_{0}$} & \multicolumn{5}{c}{$\mathrm{N}_{3}$} \\
\cline { 2 - 9 } & $4 \mathrm{~A}$ & $3 \mathrm{~A} / 1 \mathrm{H}$ & $1 \mathrm{~A} / 3 \mathrm{H}$ & $4 \mathrm{H}$ & $4 \mathrm{~A}$ & $3 \mathrm{~A} / 1 \mathrm{H}$ & $1 \mathrm{~A} / 3 \mathrm{H}$ & $4 \mathrm{H}$ \\
\hline Gemengepartner & & $24,7 / 25,8$ & $12,9 / 47,1$ & & & $\begin{array}{c}26,6 / 29, \\
4\end{array}$ & $7,8 / 51,6$ & \\
\hline Kornertrag $\Sigma$ & 54,5 & 50,5 & 60,1 & 56,8 & 49,0 & 56,0 & 59,4 & 64,6 \\
\hline 1999 & & & & & & & \\
\hline Gemengepartner & & $34,9 / 29,5$ & $13,8 / 58,7$ & & & $\begin{array}{c}31,9 / 31, \\
4\end{array}$ & $\begin{array}{c}12,9 / 59, \\
7\end{array}$ \\
\hline Kornertrag $\Sigma$ & 56,9 & 64,4 & 72,5 & 68,3 & 52,8 & 63,3 & 72,6 & 70,2 \\
\hline
\end{tabular}

Tab. A7: Mittelwerte \pm Standardabweichung der Spross-Trockenmasse $\left(\mathrm{dt} \mathrm{ha}^{-1}\right)$ und Spross-N-Mengen ( $\mathrm{kg} \mathrm{ha}^{-1}$ ) der Kräuter beim Anbau von Ackerbohne und Hafer in Rein- und Gemengesaat zur letzten Ernte im Jahr 1999

\begin{tabular}{c|c|c|c|c}
\hline 1999 & \multicolumn{2}{|c|}{ Spross-TM } & \multicolumn{2}{c}{ Spross-N } \\
\hline Ansaatvariante / Düngung & $\mathrm{N}_{0}$ & $\mathrm{~N}_{3}$ & $\mathrm{~N}_{0}$ & $\mathrm{~N}_{3}$ \\
\hline $4 \mathrm{~A}$ & $2,0 \pm 1,5$ & $2,0 \pm 1,4$ & $2,8 \pm 1,1$ & $2,7 \pm 1,2$ \\
\hline $3 \mathrm{~A} / 1 \mathrm{H}$ & $0,6 \pm 0,7$ & $0,9 \pm 1,0$ & $1,1 \pm 1,4$ & $1,6 \pm 1,6$ \\
\hline $1 \mathrm{~A} / 3 \mathrm{H}$ & $0,3 \pm 0,3$ & $0,4 \pm 0,6$ & $0,3 \pm 0,3$ & $0,6 \pm 0,9$ \\
\hline $4 \mathrm{H}$ & 0,0 & $0,3 \pm 0,5$ & 0 & $0,2 \pm 0,3$ \\
\hline
\end{tabular}


Tab. A8: Mittelwerte der gesamtpflanzlichen Trockenmasse-Akkumulation und TM-Ertragsanteile $\left(\mathrm{dt} \mathrm{ha}^{-1}\right.$ ) beim Anbau von Ackerbohne und Hafer in Rein- und Gemengesaat sowie Ergebnisse der Varianzanalyse (Ansaatvariante, Düngung) zur ersten und zweiten Ernte im Jahr 1999

\begin{tabular}{|c|c|c|c|c|c|c|c|c|c|c|c|}
\hline Ansaatvariante & \multicolumn{2}{|c|}{$4 \mathrm{~A}$} & \multicolumn{2}{|c|}{$3 \mathrm{~A} / 1 \mathrm{H}$} & \multicolumn{2}{|c|}{$1 \mathrm{~A} / 3 \mathrm{H}$} & \multicolumn{2}{|c|}{$4 \mathrm{H}$} & \multirow{3}{*}{ Ansaatvariante } & \multirow{3}{*}{ Düngung } & \multirow{3}{*}{$\begin{array}{l}\text { Ansaat- } \\
\text { variante x } \\
\text { Düngung }\end{array}$} \\
\hline Düngung & $\mathrm{N}_{0}$ & $\mathrm{~N}_{3}$ & $\mathrm{~N}_{0}$ & $\mathrm{~N}_{3}$ & $\mathrm{~N}_{0}$ & $\mathrm{~N}_{3}$ & $\mathrm{~N}_{0}$ & $\mathrm{~N}_{3}$ & & & \\
\hline 1. Ernte & \multicolumn{8}{|c|}{ 19.05.1999 (BBCH 31) } & & & \\
\hline $\mathrm{TM}_{\mathrm{Bt}}$ & 20,3 & 23,1 & 24,1 & 23,4 & 21,6 & 22,4 & 16,6 & 19,4 & ** & n.s. & n.s. \\
\hline Stroh-TM & 13,6 & 14,3 & 12,7 & 13,5 & 10,2 & 10,9 & 9,3 & 10,3 & *** & n.s. & n.s. \\
\hline Wurzel-TM & 6,7 & 8,8 & 11,4 & 9,9 & 11,4 & 11,5 & 7,3 & 9,1 & n.s. & n.s. & n.s. \\
\hline 2. Ernte & \multicolumn{8}{|c|}{ 09.06.1999 (BBCH 65) } & & & \\
\hline $\mathrm{TM}_{\mathrm{Bt}}$ & 67,8 & 75,8 & 81,3 & 76,9 & 75,6 & 84,2 & 67,1 & 71,7 & $* * *$ & n.s. & n.s. \\
\hline Stroh-TM & 50.7 & 56,9 & 60,2 & 56,8 & 55,1 & 60,5 & 51,2 & 54,5 & n.s. & n.s. & n.s. \\
\hline Wurzel-TM & 17,1 & 18,9 & 21,1 & 20,1 & 20,5 & 23,7 & 15,9 & 17,2 & n.s. ${ }^{1}$ & n.s. ${ }^{1}$ & n.s. ${ }^{1}$ \\
\hline
\end{tabular}

Tab. A9: Vergleich der Mittelwerte der gesamtpflanzlichen TrockenmasseAkkumulation, der gewichteten Trockenmasse-Harvest-Indizes $\left(\mathrm{H}_{\text {TMBtw }}\right)$ sowie der TM-Ertragsanteile beim Anbau von Ackerbohne und Hafer in Rein- und Gemengesaat zur letzten Ernte (BBCH 87) am 26.07.1999

\begin{tabular}{|c|c|c|c|c|c|c|}
\hline & \multicolumn{4}{|c|}{ Ansaatvariante } & \multirow[b]{2}{*}{ Düngung } & \multirow{2}{*}{$\begin{array}{l}\text { Wechsel- } \\
\text { wirkungen }\end{array}$} \\
\hline 1998 & $4 \mathrm{~A}$ & $3 \mathrm{~A} / 1 \mathrm{H}$ & $1 \mathrm{~A} / 3 \mathrm{H}$ & $4 \mathrm{H}$ & & \\
\hline $\mathrm{TM}_{\mathrm{Bt}}$ & $a b$ & a & a & $b^{* *}$ & n.s. & n.s. \\
\hline Korn & a & $b$ & C & $\mathrm{bc}^{* * *}$ & n.s. & n.s. \\
\hline Stroh $^{1}$ & a & a & $a$ & $b^{* * *}$ & n.s. & n.s. \\
\hline Wurzel $^{2}$ & a & a & a & a & n.s. & n.s. \\
\hline $\mathrm{H}_{\text {TMBtw }}$ & a & $b$ & C & $\mathrm{d}^{*}$ & * & n.s. \\
\hline
\end{tabular}

TM = Trockenmasse; $\mathrm{H}_{\text {TMBtw }}=$ gesamtpflanzlicher Trockenmasse-Harvest-Index; ${ }^{1}$ inklusive Bestandesabfall; nicht gleiche Buchstaben kennzeichnen signifikante Unterschiede; Tukey-Test: * $=P<0,05 ;{ }^{* *}=P<0,01 ;{ }^{* \star}=P<0,001 ;$ n.s. $=$ nicht signifikant 
Tab. A10: Mittelwerte und Mittelwertvergleich der gesamtpflanzlichen StickstoffAkkumulation $\left(\mathrm{N}_{\mathrm{B} t} ; \mathrm{kg} \mathrm{ha}^{-1}\right)$, Stickstoff-Ertragsanteile und gewichteter Stickstoff-Harvest-Index ( $\left.\mathrm{H}_{\mathrm{NBtw}}\right)$ beim Anbau von Ackerbohne und Hafer in Rein- und Gemengesaat zu drei Ernteterminen im Jahr 1998

\begin{tabular}{|c|c|c|c|c|c|c|c|c|c|c|}
\hline \multirow{2}{*}{\begin{tabular}{l|} 
Ansaatvariante \\
Düngung \\
\end{tabular}} & \multicolumn{2}{|c|}{$4 \mathrm{~A}$} & \multicolumn{2}{|c|}{$3 \mathrm{~A} / 1 \mathrm{H}$} & \multicolumn{2}{|c|}{$1 \mathrm{~A} / 3 \mathrm{H}$} & \multicolumn{2}{|c|}{$4 \mathrm{H}$} & \multirow[b]{3}{*}{ Düngung } & \multirow{3}{*}{$\begin{array}{c}\text { Ansaat- } \\
\text { variante x } \\
\text { Düngung }\end{array}$} \\
\hline & $\mathrm{N}_{0}$ & $\mathrm{~N}_{3}$ & $\mathrm{~N}_{0}$ & $\mathrm{~N}_{3}$ & $\mathrm{~N}_{0}$ & $\mathrm{~N}_{3}$ & $\mathrm{~N}_{0}$ & $\mathrm{~N}_{3}$ & & \\
\hline 1. Ernte & \multicolumn{8}{|c|}{ 25.05.1998 (BBCH 31) } & & \\
\hline \multirow{2}{*}{$\mathrm{N}_{\mathrm{Bt}}{ }^{1}$} & \multicolumn{2}{|c|}{$a b^{\top}$} & \multicolumn{2}{|c|}{$a$} & \multicolumn{2}{|c|}{$\mathrm{bc}$} & \multicolumn{2}{|c|}{$C^{* * *}$} & & \\
\hline & 54,3 & 65,7 & 64,6 & 69 & 52,5 & 51,4 & 44,1 & 45,8 & n.s. & n.s. \\
\hline \multirow{2}{*}{$\begin{array}{l}\text { Stroh-N }{ }^{1} \\
\text { Ps }\end{array}$} & \multicolumn{2}{|c|}{$a b^{\top}$} & \multicolumn{2}{|c|}{$\mathrm{a}$} & \multicolumn{2}{|c|}{$\mathrm{b}$} & \multicolumn{2}{|c|}{$b^{* * *}$} & & \\
\hline & 41,8 & 51,9 & 47,1 & 53,3 & 37,9 & 37,1 & 37 & 38,2 & n.s. & n.s. \\
\hline \multirow[t]{2}{*}{ Wurzel-N } & \multicolumn{2}{|c|}{$a b^{\top}$} & \multicolumn{2}{|c|}{$a$} & \multicolumn{2}{|c|}{$a b$} & \multicolumn{2}{|c|}{$\mathrm{b}^{* * *}$} & & \\
\hline & 12,5 & 13,8 & 17,5 & 15,7 & 14,6 & 14,3 & 7,1 & 7,6 & n.s. & n.s. \\
\hline 2. Ernte & \multicolumn{8}{|c|}{ 22.06.1998 (BBCH 65) } & & \\
\hline \multirow[t]{2}{*}{$\overline{N_{B t}}$} & \multicolumn{2}{|c|}{$a b^{S}$} & \multicolumn{2}{|c|}{$a^{2}$} & \multicolumn{2}{|c|}{$b^{2}$} & \multicolumn{2}{|c|}{$\mathrm{C}^{* * *}$} & & \\
\hline & 225,3 & 229,6 & 239,9 & 253,3 & 166,1 & 226,2 & 107 & 174,2 & * & $* *$ \\
\hline \multirow[t]{2}{*}{ Stroh-N } & $a^{c}$ & & $a^{2}$ & & $\mathrm{ab}$ & & $b^{*}$ & ** & & \\
\hline & 206,4 & 206,9 & 216 & 228,6 & 149,7 & 201,8 & 98,8 & 161,4 & * & * \\
\hline Wurzel-N & $a b$ & & $a^{2}$ & & $a b$ & & $b^{*}$ & ** & & \\
\hline & 18,9 & 22,7 & 23,9 & 24,7 & 16,4 & 24,4 & 8,2 & 12,8 & * & n.s. \\
\hline 3. Ernte & & & 10.08 & 3.1998 & (BBCH & $-187)$ & & & & \\
\hline$\overline{N_{B t}}$ & $a^{s}$ & & $b^{2}$ & & $c^{2}$ & & $\mathrm{~d}^{*}$ & ** & & \\
\hline & 380,4 & 340,7 & 286,5 & 301,7 & 211,0 & 225,4 & 137,2 & 182,5 & n.s. & $* *$ \\
\hline Korn-N ${ }^{3}$ & $a^{3}$ & & $b^{2}$ & & bc & & $\mathrm{c}^{*}$ & ** & & \\
\hline & 235,9 & 209,0 & 165,2 & 181,0 & 146,5 & 139,7 & 104,3 & 131,2 & n.s. & $* *$ \\
\hline Ba.-N & 36,0 & 34,0 & 23,0 & 21,3 & 8,4 & 5,3 & 0 & 0 & n.g.v. & n.g.v. \\
\hline Stroh-N & $a^{5}$ & & $a^{2}$ & & $\mathrm{~b}$ & & $b^{*}$ & ** & & \\
\hline & 90,0 & 78,7 & 79,8 & 83,3 & 41,5 & 63,8 & 24,0 & 41,9 & ${ }^{a}$ n.s. & $* *$ \\
\hline Wurzel-N ${ }^{3}$ & $a^{5}$ & & $a b$ & & $\mathrm{ab}$ & & b & 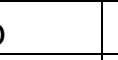 & & \\
\hline & 18,5 & 19,0 & 18,5 & 16,1 & 14,6 & 16,6 & 8,9 & 9,4 & n.s. & n.s. \\
\hline $\mathrm{H}_{\mathrm{NBtw}}$ & $\mathrm{bc}$ & & $c^{2}$ & & $b^{2}$ & & $a^{*}$ & ** & & \\
\hline & \begin{tabular}{|l|}
0,62 \\
\end{tabular} & 0,61 & 0,58 & 0,60 & 0,69 & 0,62 & 0,76 & 0,72 & * & $* *$ \\
\hline
\end{tabular}


Tab. A11: Mittelwerte und Mittelwertvergleich der gesamtpflanzlichen StickstoffAkkumulation ( $\left.\mathrm{N}_{\mathrm{Bt}} ; \mathrm{kg} \mathrm{ha}^{-1}\right)$, Stickstoff-Erträge in Korn, Stroh und Wurzel $\left(\mathrm{kg} \mathrm{ha}^{-1}\right)$ und gewichteter Stickstoff-Harvest-Index $\left(\mathrm{H}_{\mathrm{NBtw}}\right)$ beim Anbau von Ackerbohne und Hafer in Rein- und Gemengesaat zu drei Ernteterminen im Jahr 1999

\begin{tabular}{|c|c|c|c|c|c|c|c|c|c|c|}
\hline Ansaatvariante & \multicolumn{2}{|c|}{$4 \mathrm{~A}$} & \multicolumn{2}{|c|}{$3 \mathrm{~A} / 1 \mathrm{H}$} & \multicolumn{2}{|c|}{$1 \mathrm{~A} / 3 \mathrm{H}$} & \multicolumn{2}{|c|}{$4 \mathrm{H}$} & \multirow{3}{*}{ Düngung } & \multirow{3}{*}{$\begin{array}{l}\text { Ansaat- } \\
\text { variante } x \\
\text { Düngung }\end{array}$} \\
\hline Düngung & $\mathrm{N}_{0}$ & $\mathrm{~N}_{3}$ & $\mathrm{~N}_{0}$ & $\mathrm{~N}_{3}$ & $\mathrm{~N}_{0}$ & $\mathrm{~N}_{3}$ & $\mathrm{~N}_{0}$ & $\mathrm{~N}_{3}$ & & \\
\hline 1. Ernte & \multicolumn{8}{|c|}{ 19.05.1999 (BBCH 31) } & & \\
\hline \multirow[t]{2}{*}{$\mathrm{N}_{\mathrm{Bt}}$} & \multicolumn{2}{|c|}{ a } & \multicolumn{2}{|c|}{ a } & \multicolumn{2}{|c|}{$b$} & \multicolumn{2}{|c|}{$\mathrm{C}^{* * *}$} & & \\
\hline & 80,3 & 88,6 & 81,6 & 83,7 & 61,5 & 64,1 & 37,5 & 45,6 & n.s. & n.s. \\
\hline \multirow[t]{2}{*}{ Stroh-N } & \multicolumn{2}{|c|}{$a$} & \multicolumn{2}{|c|}{ a } & \multicolumn{2}{|c|}{$b$} & \multicolumn{2}{|c|}{$b^{* * *}$} & & \\
\hline & 61,6 & 66,3 & 55,2 & 60,4 & 36,9 & 39,6 & 28,4 & 31,6 & n.s. & n.s. \\
\hline \multirow{2}{*}{$\begin{array}{l}\text { Wurzel-N } \\
\text { wPsA }\end{array}$} & \multicolumn{2}{|c|}{$a b$} & \multicolumn{2}{|c|}{$\mathrm{a}$} & \multicolumn{2}{|c|}{$a b$} & \multicolumn{2}{|c|}{$b^{* * *}$} & & \\
\hline & 18,7 & 22,3 & 26,4 & 23,3 & 24,6 & 24,5 & 9,1 & 14,0 & n.s. & n.s. \\
\hline 2. Ernte & \multicolumn{8}{|c|}{ 09.06.1999 (BBCH 65) } & & \\
\hline \multirow[t]{2}{*}{$\mathrm{N}_{\mathrm{Bt}}$} & \multicolumn{2}{|c|}{ a } & \multicolumn{2}{|c|}{ a } & \multicolumn{2}{|c|}{$b$} & \multicolumn{2}{|c|}{$C^{* * *}$} & & \\
\hline & 218,6 & 236,3 & 221,6 & 216,9 & 163,0 & 172,9 & 86,4 & 125,9 & n.s. & n.s. \\
\hline \multirow[t]{2}{*}{ Stroh-N } & \multicolumn{2}{|c|}{ a } & 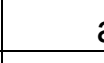 & 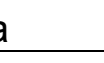 & 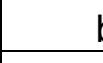 & 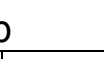 & $b^{*}$ & ** & & \\
\hline & 178,0 & 194,9 & 174,9 & 176,9 & 127,8 & 131,3 & 71,8 & 107,7 & n.s. & n.s. \\
\hline Wurzel-N ${ }^{1}$ & a & $a$ & c & 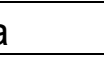 & 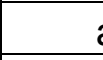 & $a$ & $b^{*}$ & ** & & \\
\hline wPs & 40,6 & 41,4 & 46,7 & 40,0 & 35,2 & 41,6 & 14,6 & 18,2 & n.s. & n.s. \\
\hline 3. Ernte & & & 26.0 & 7.1999 & $(\mathrm{BBCl}$ & 4 87) & & & & \\
\hline $\mathrm{N}_{\mathrm{Bt}}$ & $a$ & $a$ & 1 & 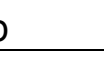 & 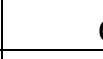 & $c$ & $d^{*}$ & ** & & \\
\hline & 369,5 & 350,9 & 318,9 & 311,7 & 225,9 & 232,8 & 141,3 & 147,8 & n.s. & n.s. \\
\hline Korn- $\mathrm{N}^{3}$ & a & $a$ & 1 & 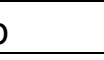 & ( & $c$ & $d^{*}$ & $* *$ & & \\
\hline & 234,8 & 220,6 & 199,0 & 192,3 & 155,1 & 156,2 & 107,9 & 110,9 & n.s. & n.s. \\
\hline Ba.-N & 32,0 & 31,5 & 25,1 & 20,7 & 7,7 & 9,2 & 0 & 0 & n.g.v. & n.g.v. \\
\hline Stroh-N & $a$ & $a$ & & 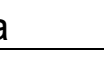 & 1 & 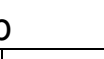 & $c^{*}$ & ** & & \\
\hline WFST & 67,5 & 73,7 & 65,5 & 69,3 & 39,6 & 41,6 & 22,0 & 26,9 & an.s. & n.s. \\
\hline Wurzel-N & a & $T^{\top}$ & 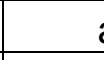 & $a$ & a & $\mathrm{b}$ & $b^{*}$ & & & \\
\hline & 35,2 & 25,1 & 29,3 & 29,4 & 23,5 & 25,8 & 11,4 & 10,0 & n.s. & n.s. \\
\hline $\mathrm{H}_{\mathrm{NBtw}}$ & $\mathrm{b}$ & $c$ & 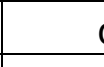 & 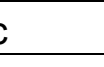 & 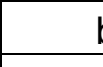 & 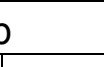 & $a^{*}$ & ** & & \\
\hline & 0,64 & 0,63 & 0,62 & 0,62 & 0,69 & 0,67 & 0,76 & 0,75 & n.s. & n.s. \\
\hline
\end{tabular}

$\mathrm{H}_{\mathrm{NBtw}}=$ gewichteter Stickstoff-Harvest-Index; Ps Einfluss des Pflanzenschutzes nachweisbar; wPsD Wechselwirkung: Pflanzenschutz x Düngung; wPsA Wechselwirkung: Pflanzenschutz x Ansaatvariante; Ba. = Bestandesabfall; ${ }^{\top}$ Tukey-Test: nicht gleiche Buchstaben kennzeichnen signifikante Unterschiede mit: ${ }^{*}=P<0,05$; ${ }^{* *}=P<0,01$; ${ }^{* *}=P<0,001$; n.s. $=$ nicht signifikant; ${ }^{1}$ Originaldaten transformiert (lgx); ${ }^{a}$ Statistik incl. Bestandesabfall; n.g.v. $=$ nicht gesondert verrechnet 
Tab. A12: Mittelwerte und Mittelwertvergleich ${ }^{1}$ der gewichteten $\delta^{15} \mathrm{~N}-$ Werte $(\%)$ und Anteil Stickstoff aus der Luft $\left(\mathrm{Ndfa}_{\mathrm{w}}\right)$ in der Sprossmasse von Ackerbohne und Hafer in Rein- und Gemengesaat im Jahr 1998 und 1999

\begin{tabular}{|c|c|c|c|c|c|c|c|c|c|c|}
\hline & & \multicolumn{4}{|c|}{1998} & \multicolumn{4}{|c|}{1999} \\
\hline & & \multirow[b]{2}{*}{ Ansaatvariante } & \multicolumn{2}{|c|}{$\delta^{15} \mathrm{~N}[\% 0]$} & \multicolumn{2}{|c|}{$\mathrm{Ndfa}_{\mathrm{w}}$} & \multicolumn{2}{|c|}{$\delta^{15} \mathrm{~N}[\%$ ] } & \multicolumn{2}{|c|}{$\mathrm{Ndfa}_{\mathrm{w}}$} \\
\hline & & & $\mathrm{N}_{0}$ & $\mathrm{~N}_{3}$ & $\mathrm{~N}_{0}$ & $\mathrm{~N}_{3}$ & $\mathrm{~N}_{0}$ & $\mathrm{~N}_{3}$ & $\mathrm{~N}_{0}$ & $\mathrm{~N}_{3}$ \\
\hline \multirow[b]{2}{*}{ 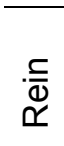 } & \multirow{11}{*}{$\begin{array}{c}\overrightarrow{\mathbb{\pi}} \\
\mathbb{W} \\
\mathbb{\infty}\end{array}$} & $4 \mathrm{~A}$ & 0,73 & 1,16 & 0,70 & 0,66 & 0,90 & 1,01 & 0,78 & 0,73 \\
\hline & & & \multicolumn{2}{|c|}{$\mathrm{S} a \downarrow$} & \multicolumn{2}{|c|}{$\mathrm{a} a \downarrow$} & \multicolumn{2}{|c|}{$a \downarrow$} & \multicolumn{2}{|c|}{$\mathrm{T} a \downarrow$} \\
\hline \multirow{4}{*}{$\begin{array}{l}\mathbb{\Xi} \\
\Phi \\
\mathbb{0} \\
\mathbb{0} \\
0\end{array}$} & & $3 \mathrm{~A}$ & 0,05 & 0,37 & 0,90 & 0,84 & 0,42 & 0,53 & 0,87 & 0,83 \\
\hline & & & \multicolumn{2}{|c|}{$b^{2}$} & \multicolumn{2}{|c|}{$b^{2}$} & \multicolumn{2}{|c|}{ b } & \multicolumn{2}{|c|}{ b } \\
\hline & & $1 \mathrm{H}$ & 2,96 & 3,59 & & & 4,06 & 4,44 & & \\
\hline & & $3 \mathrm{~A}+1 \mathrm{H}$ & 0,99 & 1,56 & 0,63 & 0,56 & 1,43 & 1,75 & 0,69 & 0,57 \\
\hline & & $1 \mathrm{~A}$ & $-0,15$ & $-0,05$ & 0,95 & 0,94 & 0,06 & $-0,01$ & 0,93 & 0,94 \\
\hline$\frac{\bar{c}}{0}$ & & & \multicolumn{2}{|c|}{${ }^{2} \mathrm{c}^{\star \star *}$} & \multicolumn{2}{|c|}{${ }^{2} \mathrm{C}^{\star \star *}$} & \multicolumn{2}{|c|}{$c^{\star \star \star}$} & \multicolumn{2}{|c|}{$\mathrm{C}^{\star * \star}$} \\
\hline$\stackrel{\mathrm{E}}{\mathrm{\sigma}}_{0}$ & & $3 \mathrm{H}$ & 2,76 & 3,97 & & & 4,37 & 4,60 & & \\
\hline \multirow[t]{2}{*}{0} & & $1 \mathrm{~A}+3 \mathrm{H}$ & 1,60 & 3,00 & 0,46 & 0,21 & 2,61 & 2,85 & 0,47 & 0,35 \\
\hline & & $4 \mathrm{H}$ & 3,23 & 4,04 & & & 5,27 & 4,57 & & \\
\hline 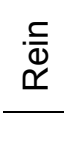 & & Dünqung & $\rightarrow a$ & $b^{* * *}$ & $\rightarrow \mathrm{a}$ & $b^{*}$ & $\rightarrow a$ & $a$ & $\rightarrow a$ & $b^{* *}$ \\
\hline
\end{tabular}

${ }^{1}$ Die Mittelwertvergleiche beziehen sich nur auf den Ackerbohnen-Spross; nicht gleiche Buchstaben kennzeichnen signifikante Unterschiede; ${ }^{\top}$ Tukey- und ${ }^{\mathrm{S}}$ Scheffé-Test: ${ }^{*}=P<0,05 ;{ }^{* *}=P<0,01 ;{ }^{* \star *}=P<0,001 ;{ }^{2} \mathrm{n}=5 ; \downarrow \rightarrow$ Leserichtung

Tab. A13: Vergleich der gesamtpflanzlich gewichteten $\delta^{15} \mathrm{~N}$-Mittelwerte beim Anbau von Ackerbohne und Hafer in Rein- und Gemengesaat zu drei Ernteterminen a) 1998 und b) 1999 sowie Ergebnis der zweifaktoriellen Varianzanalyse

\begin{tabular}{|c|c|c|c|c|c|c|}
\hline & \multicolumn{4}{|c|}{ Ansaatvariante } & \multirow[b]{2}{*}{ Düngung } & \multirow{2}{*}{$\begin{array}{l}\text { Wechsel- } \\
\text { wirkungen }\end{array}$} \\
\hline a) & $4 \mathrm{~A}$ & $3 \mathrm{~A} / 1 \mathrm{H}$ & $1 \mathrm{~A} / 3 \mathrm{H}$ & $4 \mathrm{H}$ & & \\
\hline 1. Ernte & $a^{\top}$ & $b$ & $b$ & $\mathrm{C}^{*}$ & n.s. & n.s. \\
\hline 2. Ernte ${ }^{1}$ & $a^{s}$ & $b^{3}$ & $c^{3}$ & $d^{* * *}$ & $* *$ & n.s. \\
\hline 3. Ernte & $a^{s}$ & $b^{3}$ & $c^{3}$ & $d^{* *}$ & *** & $* * *$ \\
\hline \multicolumn{7}{|l|}{ b) } \\
\hline 1. Ernte $^{2}$ & $a^{\top}$ & $b$ & C & $d^{*}$ & * & n.s. \\
\hline 2. Ernte ${ }^{1}$ & $a^{\top}$ & $b$ & $\mathrm{C}$ & $d^{* *}$ & n.s. & n.s. \\
\hline 3. Ernte ${ }^{\mathrm{wPs} A D}$ & $a^{\top}$ & $b$ & C & $\mathrm{d}^{* * *}$ & n.s. & ** \\
\hline
\end{tabular}

wPsAD: Wechselwirkung: Pflanzenschutz $\times$ Düngung $\times$ Ansaatvariante; nicht gleiche Buchstaben kennzeichnen signifikante Unterschiede; 'Tukey- und ${ }^{\mathrm{S}}$ Scheffé-Test: ${ }^{*}=P<0,05 ;{ }^{* *}=P<0,01 ;{ }^{* * *}=P<0,001$ n.s. $=$ nicht signifikant; ${ }^{1}$ Originaldaten transformiert $(\operatorname{lgx}) ;{ }^{2}$ Originaldaten transformiert (Wurzelx); ${ }^{3} \mathrm{n}=5$ 
Tab. A14: Korrelationskoeffizienten ( $r$ ) zwischen den Ergebnissen der $\delta^{15} \mathrm{~N}-\mathrm{Me}-$ thode und der erweiterten Differenzmethode zur Schätzung der symbiotisch fixierten N-Menge beim Anbau von Ackerbohne in Rein- und Gemengesaat mit Hafer im Jahr 1998 und 1999

\begin{tabular}{c|c|c|c}
\hline & $\mathrm{r}$ & $P$ & $\mathrm{n}$ \\
\hline 1998 & & & \\
\hline 1. Ernte am 25.05. & 0,67 & 0,0001 & 36 \\
\hline 2. Ernte am 22.06. & 0,80 & 0,0001 & 34 \\
\hline 3. Ernte am 10.08. & 0,94 & 0,0001 & 34 \\
\hline 1999 & & & \\
\hline 1. Ernte am 19.05. & 0,78 & 0,0001 & 36 \\
\hline 2. Ernte am 09.06. & 0,71 & 0,0001 & 36 \\
\hline 3. Ernte am 26.07. & 0,91 & 0,0001 & 36 \\
\hline
\end{tabular}

Tab. A15: Mittelwerte $\left(\mathrm{kg} \mathrm{N}_{\min } \mathrm{ha}^{-1}\right)$ und Mittelwertvergleich der $\mathrm{CaCl}_{2}$-extrahierbaren $\mathrm{N}_{\text {min }}$-Mengen im Boden vor der Aussaat von Ackerbohne und Hafer am 20.03.1998 (a) und 15.03.1999 (b)

\begin{tabular}{|c|c|c|c|c|c|c|c|c|}
\hline Jahr & \multicolumn{4}{|c|}{1998} & \multicolumn{2}{|l|}{ b) } & \multicolumn{2}{|c|}{999} \\
\hline Düngung & $\mathrm{N}_{0}$ & $\mathrm{~N}_{3}$ & Ps & W & $\mathrm{N}_{0}$ & $\mathrm{~N}_{3}$ & Ps & W \\
\hline \multirow[t]{2}{*}{$0-125 \mathrm{~cm}$} & 92,2 & 172,3 & & & 72,3 & 102,7 & & \\
\hline & $a^{1}$ & $b^{* *}$ & n.s. & n.s. & $a$ & $b^{*}$ & n.s. & n.s. \\
\hline \multirow[t]{2}{*}{$0-50 \mathrm{~cm}$} & 47,0 & 54,6 & & & 30,0 & 36,2 & & \\
\hline & $a^{1}$ & $a$ & n.s. & n.s. & $a$ & $b^{* *}$ & n.s. & n.s. \\
\hline \multirow[t]{2}{*}{$50-125 \mathrm{~cm}$} & 45,2 & 117,7 & & & 42,3 & 66,5 & & \\
\hline & $a^{2}$ & $b^{* *}$ & n.s. & n.s. & a & $b^{*}$ & n.s. & n.s. \\
\hline
\end{tabular}


Tab. A16: Mittelwerte $\left(\mathrm{kg} \mathrm{N}_{\min } \mathrm{ha}^{-1}\right)$ und Mittelwertvergleich der $\mathrm{CaCl}_{2}$-extrahierbaren $\mathrm{N}_{\text {min }}$-Mengen im Boden beim Anbau von Ackerbohne und Hafer in Rein- und Gemengesaat zu vier Probenahmeterminen im Jahr 1998

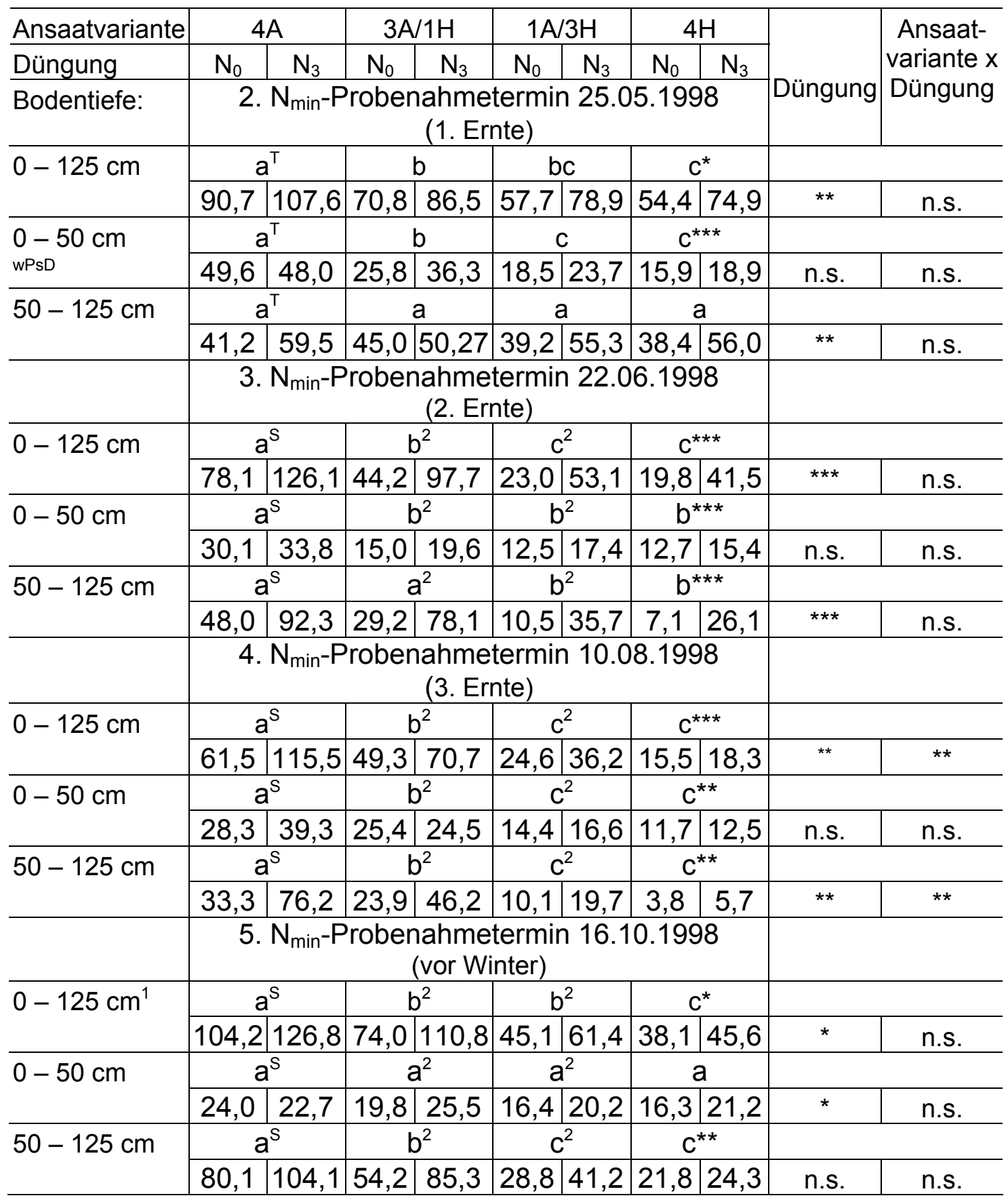

wPsD = Wechselwirkung: Pflanzenschutz x Düngung; ${ }^{\top}$ Tukey- und ${ }^{\mathrm{S} S c h e f f e ́-T e s t: ~ n i c h t ~ g l e i c h e ~ B u c h s t a b e n ~ k e n n z e i c h-~}$ nen signifikante Unterschiede mit: ${ }^{*}=P<0,05 ;{ }^{* *}=P<0,01 ;{ }^{* * *}=P<0,001$; n.s. $=$ nicht signifikant; ${ }^{1}$ Originaldaten transformiert (Wurzelx); ${ }^{2} \mathrm{n}=5$ 
Tab. A17: Mittelwerte und Mittelwertvergleich der luft- und bodenbürtigen gesamtpflanzlichen N-Akkumulation $\left(\mathrm{kg} \mathrm{ha}^{-1}\right)$ und gewichtete Anteile Stickstoff aus der Luft (Ndfa ${ }_{w}$ ) beim Anbau von Ackerbohne und Hafer in Rein- und Gemengesaat zu drei Ernteterminen im Jahr 1998

\begin{tabular}{|c|c|c|c|c|c|c|c|c|c|c|}
\hline \multirow{2}{*}{$\begin{array}{l}\text { Ansaatvariante } \\
\text { Düngung }\end{array}$} & \multicolumn{2}{|c|}{$4 \mathrm{~A}$} & \multicolumn{2}{|c|}{$3 \mathrm{~A} / 1 \mathrm{H}$} & \multicolumn{2}{|c|}{$1 \mathrm{~A} / 3 \mathrm{H}$} & \multicolumn{2}{|c|}{$4 \mathrm{H}$} & \multirow{3}{*}{ Düngung } & \multirow{3}{*}{$\begin{array}{c}\text { Ansaat- } \\
\text { variante } x \\
\text { Düngung }\end{array}$} \\
\hline & $\mathrm{N}_{0}$ & $\mathrm{~N}_{3}$ & $\mathrm{~N}_{0}$ & $\mathrm{~N}_{3}$ & $\mathrm{~N}_{0}$ & $\mathrm{~N}_{3}$ & $\mathrm{~N}_{0}$ & $\mathrm{~N}_{3}$ & & \\
\hline 1. Ernte & \multicolumn{8}{|c|}{ 25.05.1998 (BBCH 31) } & & \\
\hline \multirow[t]{2}{*}{ Boden-N } & \multicolumn{2}{|c|}{$a^{\top}$} & \multicolumn{2}{|c|}{$a b$} & \multicolumn{2}{|c|}{$a b$} & \multicolumn{2}{|c|}{$\mathrm{b}^{* * *}$} & & \\
\hline & 35,3 & 46,0 & 55,1 & 53,1 & 48,6 & 41,8 & 44,1 & 45,8 & n.s. & * \\
\hline \multirow[t]{2}{*}{ Luft-N } & \multicolumn{2}{|c|}{$a^{\top}$} & \multicolumn{2}{|c|}{$a b$} & \multicolumn{2}{|c|}{$\mathrm{b}^{* * *}$} & & & \\
\hline & 19,0 & 19,7 & 9,5 & 15,9 & 3,9 & 9,6 & 0 & 0 & n.s. & n.s. \\
\hline \multirow{2}{*}{$\begin{array}{l}\mathrm{Ndfa}_{\mathrm{w}} \\
\mathrm{Ps}\end{array}$} & \multicolumn{2}{|c|}{$a^{\top}$} & \multicolumn{2}{|c|}{$a b$} & \multicolumn{2}{|c|}{$b^{* * *}$} & & & \\
\hline & 0,35 & 0,30 & 0,15 & 0,23 & 0,07 & 0,19 & 0 & 0 & n.s. & n.s. \\
\hline 2. Ernte & \multicolumn{8}{|c|}{ 22.06.1998 (BBCH 65) } & & \\
\hline \multirow[t]{2}{*}{ Boden- $N^{1}$} & \multicolumn{2}{|c|}{$a^{s}$} & \multicolumn{2}{|c|}{$a^{2}$} & \multicolumn{2}{|c|}{$a^{2}$} & \multicolumn{2}{|c|}{$b^{* * *}$} & & \\
\hline & 66,4 & 69,8 & 106,1 & 121,8 & 117,4 & 162,8 & 107,0 & 174,2 & * & $* *$ \\
\hline \multirow[t]{2}{*}{ Luft-N } & \multicolumn{2}{|c|}{$a^{s}$} & \multicolumn{2}{|c|}{$a^{2}$} & $b^{2}$ & $* * *$ & & & & \\
\hline & 158,9 & 159,8 & 133,8 & 131,5 & 48,7 & 63,4 & 0 & 0 & n.s. & n.s. \\
\hline $\mathrm{Ndfa}_{\mathrm{w}}$ & a & s & $b$ & )$^{2}$ & $c^{2}$ & $* * *$ & & & & \\
\hline & 0,71 & 0,70 & 0,56 & 0,52 & 0,29 & 0,28 & 0 & 0 & n.s. & n.s. \\
\hline 3. Ernte & & & 10.08 & 3.1998 & $(\mathrm{BBC}$ & H 87) & & & & \\
\hline Boden-N & $\mathrm{b}$ & $s$ & $b$ & )$^{2}$ & a & $b^{2}$ & $a^{*}$ & $\star * \star *$ & & \\
\hline & 118,2 & 119,5 & 103,9 & 123,7 & 97,5 & 175,6 & 137,3 & 182,5 & * & $* * *$ \\
\hline Luft-N & $a$ & s & $b$ & )$^{2}$ & $c^{2}$ & $* * *$ & & & & \\
\hline & 262,2 & 221,2 & 182,6 & 178,1 & 113,4 & 49,8 & 0 & 0 & $* *$ & n.s. \\
\hline $\mathrm{Ndfa}_{\mathrm{w}}$ & a & $s$ & $b$ & 2 & $c^{2}$ & & & & & \\
\hline & 0,69 & 0,65 & 0,64 & 0,59 & 0,54 & 0,22 & 0 & 0 & $* *$ & $* * *$ \\
\hline
\end{tabular}

$\mathrm{Ndfa}_{\mathrm{w}}=$ gewichteter Anteil Stickstoff aus der Luft; Ps Einfluss des Pflanzenschutzes nachweisbar; ${ }^{\mathrm{T}}$ Tukey- und ${ }^{\mathrm{S}}$ SchefféTest: nicht gleiche Buchstaben kennzeichnen signifikante Unterschiede mit: ${ }^{*}=P<0,05 ;{ }^{* \star}=P<0,01 ;{ }^{* \star *}=P<0,001$; n.s. = nicht signifikant $;{ }^{1}$ Originaldaten transformiert (Wurzelx), ${ }^{2} n=5$ 
Tab. A18: Mittelwerte $\left(\mathrm{kg} \mathrm{N}_{\min } \mathrm{ha}^{-1}\right)$ und Mittelwertvergleich der $\mathrm{CaCl}_{2}$-extrahierbaren $\mathrm{N}_{\text {min }}$-Mengen im Boden beim Anbau von Ackerbohne und Hafer in Rein- und Gemengesaat zu vier Probenahmeterminen im Jahr 1999

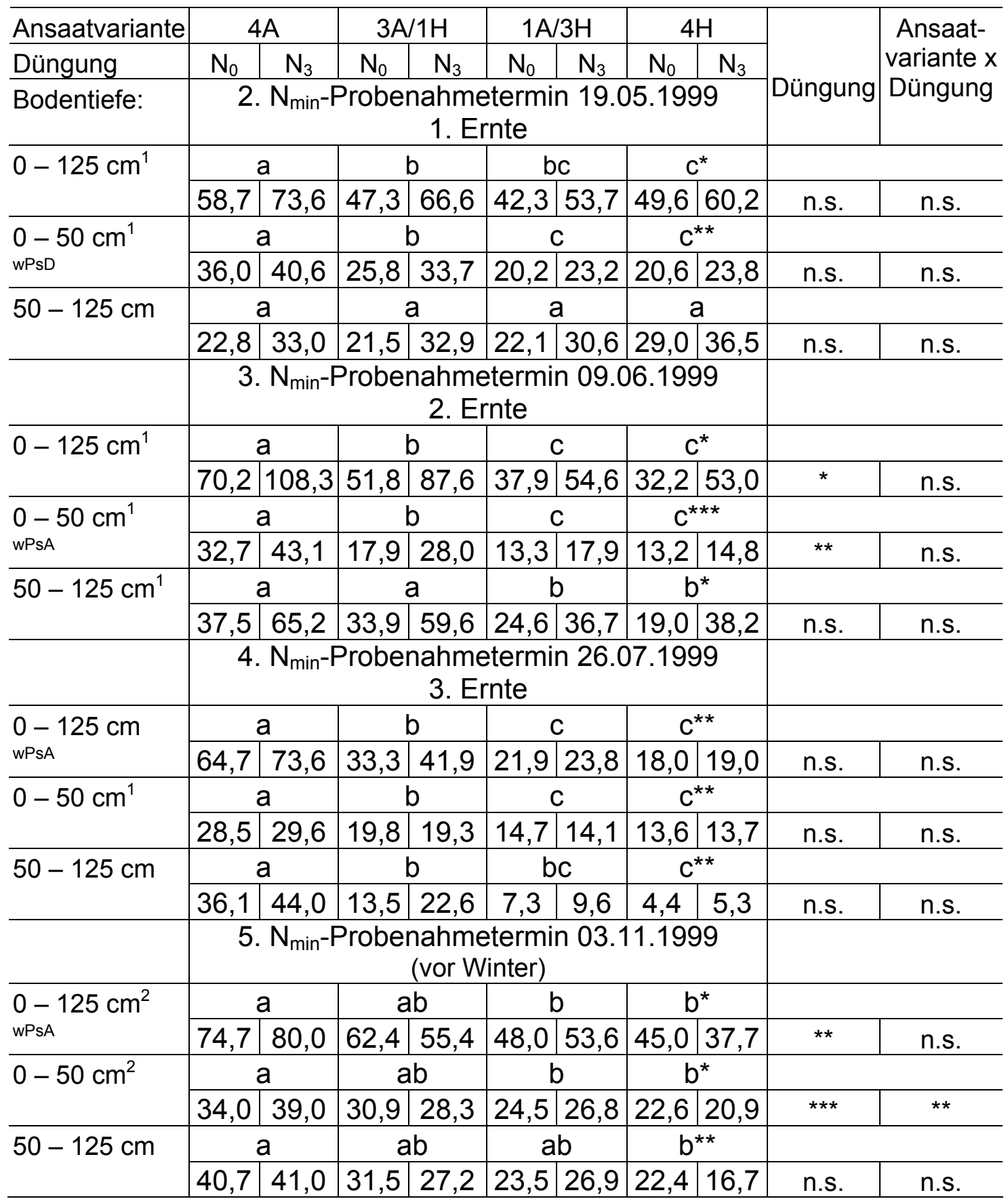

wPsA = Wechselwirkung: Pflanzenschutz $\times$ Ansaatvariante; wPsD = Wechselwirkung: Pflanzenschutz $\times$ Düngung; Tukey-Test: nicht gleiche Buchstaben kennzeichnen signifikante Unterschiede mit: ${ }^{*}=P<0,05 ;{ }^{* *}=P<0,01$; $^{* *}=P<$ 0,$001 ;$ n.s. $=$ nicht signifikant; ${ }^{1}$ Originaldaten transformiert $(\operatorname{lgx}) ;{ }^{2}$ Originaldaten transformiert (Wurzelx) 
Tab. A19: Mittelwerte und Mittelwertvergleich der luft- und bodenbürtigen gesamtpflanzlichen N-Akkumulation $\left(\mathrm{kg} \mathrm{ha}^{-1}\right)$ und gewichtete Anteile Stickstoff aus der Luft $\left(\mathrm{Ndfa}_{\mathrm{w}}\right)$ beim Anbau von Ackerbohne und Hafer in Rein- und Gemengesaat zu drei Ernteterminen im Jahr 1999

\begin{tabular}{|c|c|c|c|c|c|c|c|c|c|c|}
\hline Ansaatvariante & \multicolumn{2}{|c|}{$4 \mathrm{~A}$} & \multicolumn{2}{|c|}{$3 \mathrm{~A} / 1 \mathrm{H}$} & \multicolumn{2}{|c|}{$1 \mathrm{~A} / 3 \mathrm{H}$} & \multicolumn{2}{|c|}{$4 \mathrm{H}$} & \multirow{3}{*}{ Düngung } & \multirow{3}{*}{$\begin{array}{l}\text { Ansaat- } \\
\text { variante } x \\
\text { Düngung }\end{array}$} \\
\hline Düngung & $\mathrm{N}_{0}$ & $\mathrm{~N}_{3}$ & $\mathrm{~N}_{0}$ & $\mathrm{~N}_{3}$ & $\mathrm{~N}_{0}$ & $\mathrm{~N}_{3}$ & $\mathrm{~N}_{0}$ & $\mathrm{~N}_{3}$ & & \\
\hline 1. Ernte & \multicolumn{8}{|c|}{ 19.05.1999 (BBCH 31) } & & \\
\hline \multirow[t]{2}{*}{ Boden-N } & \multicolumn{2}{|c|}{$a$} & \multicolumn{2}{|c|}{$a$} & \multicolumn{2}{|c|}{ a } & \multicolumn{2}{|c|}{$a$} & & \\
\hline & \begin{tabular}{|l|}
36,3 \\
\end{tabular} & 45,2 & 41,9 & 49,3 & 41,0 & 49,0 & 37,5 & 45,6 & n.s. & n.s. \\
\hline \multirow{2}{*}{$\begin{array}{l}\text { Luft-N } \\
\text { wPsA }\end{array}$} & \multicolumn{2}{|c|}{ a } & \multicolumn{2}{|c|}{ a } & \multicolumn{2}{|c|}{$b^{* * *}$} & & & \\
\hline & 43,9 & 43,4 & 39,8 & 34,4 & 20,5 & 15,1 & 0 & 0 & n.s. & n.s. \\
\hline \multirow[t]{2}{*}{$\mathrm{Ndfa}_{\mathrm{w}}$} & \multicolumn{2}{|c|}{$a$} & \multicolumn{2}{|c|}{$b$} & \multicolumn{2}{|c|}{$c^{*}$} & & & \\
\hline & 0,56 & 0,49 & 0,49 & 0,41 & 0,33 & 0,24 & 0 & 0 & n.s. & n.s. \\
\hline 2. Ernte & \multicolumn{8}{|c|}{ 09.06.1999 (BBCH 65) } & & \\
\hline \multirow[t]{2}{*}{ Boden-N } & \multicolumn{2}{|c|}{$\mathrm{b}$} & \multicolumn{2}{|c|}{$a b$} & \multicolumn{2}{|c|}{ a } & \multicolumn{2}{|c|}{$a b^{*}$} & & \\
\hline & 81,4 & 82,0 & 110,9 & 96,6 & 107,0 & 116,8 & 86,4 & 125,9 & n.s. & n.s. \\
\hline \multirow[t]{2}{*}{ Luft-N } & \multicolumn{2}{|c|}{$a$} & \multicolumn{2}{|c|}{$a$} & \multicolumn{2}{|c|}{$b^{* * *}$} & 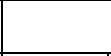 & 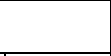 & & \\
\hline & 137,3 & 154,3 & 110,7 & 120,3 & 56,0 & 56,1 & 0 & 0 & n.s. & n.s. \\
\hline $\mathrm{Ndfa}_{\mathrm{w}}$ & $a$ & $a$ & & $\mathrm{~b}$ & $\mathrm{c}$ & * & & & & \\
\hline & 0,63 & 0,65 & 0,50 & 0,56 & 0,35 & 0,33 & 0 & 0 & n.s. & n.s. \\
\hline 3. Ernte & & & 26.07 & 7.1999 & $(\mathrm{BBCl}$ & $-187)$ & & & & \\
\hline Boden-N & $b$ & & & $a b$ & a & $b$ & $a^{*}$ & $k * *$ & & \\
\hline & 87,3 & 101,3 & 85,5 & 134,1 & 101,1 & 139,8 & 141,3 & 147,8 & ** & n.s. \\
\hline Luft-N & a & & & b & $c^{*}$ & ** & & & & \\
\hline & 282,2 & 249,5 & 233,4 & 177,6 & 124,8 & 93,0 & 0 & 0 & n.s. & n.s. \\
\hline $\mathrm{Ndfa}_{\mathrm{w}}$ & a & & & $\mathrm{b}$ & $c^{*}$ & ** & & & & \\
\hline & 0,76 & 0,71 & 0,73 & 0,57 & 0,55 & 0,40 & 0 & 0 & n.s. & n.s. \\
\hline
\end{tabular}

$\mathrm{Ndfa}_{\mathrm{w}}=$ gewichteter Anteil Stickstoff aus der Luft; Ps = Einfluss des Pflanzenschutzes nachweisbar; wPsA = Wechselwirkung: Pflanzenschutz $x$ Ansaatvariante; Tukey-Test: nicht gleiche Buchstaben kennzeichnen signifikante Unterschiede mit: ${ }^{*}=P<0,05 ;{ }^{* *}=P<0,01 ;{ }^{* * *}=P<0,001 ;$ n.s. $=$ nicht signifikant; ${ }^{1}$ Originaldaten transformiert (Igx)

Tab. A20: Ergebnis der Varianzanalyse der gewogenen $\delta^{15} \mathrm{~N}$-Werte sowie multipler Vergleich der gewogenen $\delta^{15} \mathrm{~N}$-Werte in der Sprossmasse von Hafer in Rein- und Gemengesaat zur jeweils letzten Ernte der Jahre 1998 und 1999

\begin{tabular}{|c|c|c|c|c|c|c|c|c|c|c|c|}
\hline \multicolumn{3}{|c|}{ Varianzanalyse } & \multirow{2}{*}{\multicolumn{3}{|c|}{$\begin{array}{l}\text { Scheffé-/Tukey-Test } \\
\text { Ansaatvariante }\end{array}$}} & \multicolumn{6}{|c|}{ Düngung } \\
\hline & \multicolumn{2}{|l|}{ Ansaat- } & & & & \multicolumn{2}{|c|}{$3 \mathrm{~A} / 1 \mathrm{H}$} & \multicolumn{2}{|c|}{$1 \mathrm{~A} / 3 \mathrm{H}$} & \multicolumn{2}{|c|}{$4 \mathrm{H}$} \\
\hline & variante & Düngung & $3 \mathrm{~A} / 1 \mathrm{H}$ & $1 \mathrm{~A} / 3 \mathrm{H}$ & $4 \mathrm{H}$ & $\mathrm{N}_{0}$ & $\mathrm{~N}_{3}$ & $\mathrm{~N}_{0}$ & $\mathrm{~N}_{3}$ & $\mathrm{~N}_{0}$ & $\mathrm{~N}_{3}$ \\
\hline 1998 & ** & *** & $a^{\mathrm{S2}^{2}}$ & $a^{2}$ & $b^{*}$ & $a^{2}$ & a & $a^{2}$ & $b^{*}$ & $a$ & $a$ \\
\hline \multicolumn{12}{|l|}{ Wechselw. $^{1}$} \\
\hline 1999 & ** & n.s. & $a^{\top}$ & $a b$ & $b^{* *}$ & $a$ & $a$ & $a$ & $a$ & $a$ & $a$ \\
\hline Wechselw. $^{1}$ & & * & & & & & & & & & \\
\hline
\end{tabular}

Varianzanalyse: n.s. $=$ nicht signifikant, ${ }^{*}=P<0,05,{ }^{* *} P=0,01,{ }^{* * *}=P<0,001$; Mittelwertvergleich: nicht gleiche Buchstaben kennzeichnen signifikante Unterschiede; ${ }^{\mathrm{S}}$ Scheffé- und ${ }^{\top}$ Tukey-Test: ${ }^{*}=P<0,05,{ }^{*}=P<0,01,{ }^{1}$ Wechselwirkung: Ansaatvariante $x$ Düngung; ${ }^{2} \mathrm{n}=5$ 
Tab. A21: Ergebnis der Varianzanalyse der Transfer-N-Menge und Anteil des Transfer-Stickstoffes an der Gesamt-N-Menge in der Sprossmasse des Hafers zur letzten Ernte im Jahr 1998 und 1999

\begin{tabular}{|c|c|c|c|}
\hline 1998 & $\begin{array}{l}\text { Ansaat- } \\
\text { variante }\end{array}$ & Düngung & $\begin{array}{c}\text { Ansaatvariante } \\
\text { x Düngung }\end{array}$ \\
\hline${ }^{\mathrm{a}} \mathrm{N}-$ Transfer $^{1}$ & n.s. & n.s. & n.s. \\
\hline${ }^{\mathrm{b}}$ Anteil $\mathrm{N}_{\text {trans }}{ }^{1}$ & n.s. & n.s. & n.s. \\
\hline \multicolumn{4}{|l|}{1999} \\
\hline $\mathrm{N}^{\mathrm{N}}$ Transfer $^{1}$ & n.s. & * & n.s. \\
\hline Anteil $\mathrm{N}_{\text {trans }}{ }^{1}$ & n.s. & * & n.s. \\
\hline
\end{tabular}

Tab. A22: Geschätzte Menge an N-Rhizodeposition, die aus der Fixierung stammt (in $\mathrm{kg} \mathrm{N} \mathrm{ha}^{-1}$ ) beim Anbau von Ackerbohne in Rein- und Gemengesaat mit Hafer im Jahr 1998 und 1999

\begin{tabular}{r|c|c|c}
\hline 1998 & $4 \mathrm{~A}$ & $3 \mathrm{~A} / 1 \mathrm{H}$ & $1 \mathrm{~A} / \mathrm{H}$ \\
\hline $\mathrm{N}_{0}$ & 28,3 & 13,2 & 4,7 \\
\hline $\mathrm{N}_{3}$ & 23,9 & 11,9 & 1,5 \\
\hline 1999 & \multicolumn{3}{|c}{} \\
\hline $\mathrm{N}_{0}$ & 30,5 & 17,4 & 5,6 \\
\hline $\mathrm{N}_{3}$ & 26,9 & 13,6 & 4,3 \\
\hline
\end{tabular}

Tab. A23: Mittelwerte \pm Standardabweichung der Spross-Trockenmassen ( $\left.\mathrm{dt} \mathrm{ha}^{-1}\right)$ und Spross-N-Mengen $\left(\mathrm{kg} \mathrm{ha}^{-1}\right)$ der Kräuter beim Anbau von Luzerne und Knaulgras in Rein- und Gemengesaat zu drei Schnittterminen im Jahr 1998

\begin{tabular}{c|c|c|c|c|c}
\hline \multirow{4}{*}{ Schnitt } & & \multicolumn{2}{|c|}{ Spross-TM. } & \multicolumn{2}{c}{ Spross-N } \\
\cline { 2 - 6 } & $\begin{array}{c}\text { Düngung An- } \\
\text { saatvariante }\end{array}$ & $\mathrm{N}_{0}$ & $\mathrm{~N}_{3}$ & $\mathrm{~N}_{0}$ & $\mathrm{~N}_{3}$ \\
\hline \multirow{6}{*}{1} & $4 \mathrm{~L}$ & $0,6 \pm 0,4$ & $0,6 \pm 0,3$ & $2,4 \pm 1,7$ & $2,7 \pm 1,8$ \\
\cline { 2 - 6 } & $3 \mathrm{~L} / 1 \mathrm{~K}$ & $0,8 \pm 0,5$ & $1,0 \pm 0,8$ & $3,1 \pm 2,3$ & $3,9 \pm 2,9$ \\
\cline { 2 - 6 } & $1 \mathrm{~L} / 3 \mathrm{~K}$ & $1,1 \pm 1,1$ & $0,9 \pm 0,6$ & $3,6 \pm 3,9$ & $2,8 \pm 2,1$ \\
\cline { 2 - 6 } & $4 \mathrm{~K}$ & $0,7 \pm 0,6$ & $1,1 \pm 1,1$ & $2,3 \pm 2,3$ & $3,6 \pm 3,6$ \\
\hline \multirow{4}{*}{2} & $4 \mathrm{~L}$ & $0,4 \pm 0,7$ & $0,5 \pm 0,5$ & $0,3 \pm 0,4$ & $1,4 \pm 1,3$ \\
\cline { 2 - 6 } & $3 \mathrm{~L} / 1 \mathrm{~K}$ & $1,0 \pm 1,5$ & $0,5 \pm 0,5$ & $2,5 \pm 3,9$ & $1,3 \pm 1,5$ \\
\cline { 2 - 6 } & $1 \mathrm{~L} / 3 \mathrm{~K}$ & $0,4 \pm 0,5$ & $0,3 \pm 0,2$ & $0,9 \pm 1,3$ & $0,9 \pm 0,5$ \\
\cline { 2 - 6 } & $4 \mathrm{~K}$ & $0,4 \pm 0,6$ & $0,3 \pm 0,5$ & $1,3 \pm 1,5$ & $0,9 \pm 1,5$ \\
\hline \multirow{4}{*}{3} & $4 \mathrm{~L}$ & 0 & 0 & 0 & 0 \\
\cline { 2 - 6 } & $3 \mathrm{~L} / 1 \mathrm{~K}$ & 0 & $0,1 \pm 0,2$ & 0 & $0,4 \pm 0,9$ \\
\cline { 2 - 6 } & $1 \mathrm{~L} / 3 \mathrm{~K}$ & 0 & 0 & 0 & 0 \\
\cline { 2 - 6 } & $4 \mathrm{~K}$ & 0 & 0 & 0 & 0 \\
\hline
\end{tabular}


Tab. A24: Mittelwerte der Trockenmasse-Akkumulation ( $\left.\mathrm{dt} \mathrm{ha}^{-1}\right)$ sowie der gewichteten Trockenmasse-Harvest-Indizes $\left(\mathrm{H}_{\mathrm{TMBtw}}\right)$ beim Anbau von Luzerne und Knaulgras in Rein- und Gemengesaat sowie Ergebnisse der Varianzanalyse (Ansaatvariante, Düngung) zum ersten und zweiten Schnitt 1998

\begin{tabular}{|c|c|c|c|c|c|c|c|c|c|c|c|}
\hline Ansaatvariante & \multicolumn{2}{|c|}{$4 \mathrm{~L}$} & \multicolumn{2}{|c|}{$3 \mathrm{~L} / 1 \mathrm{~K}$} & \multicolumn{2}{|c|}{$1 \mathrm{~L} / 3 \mathrm{~K}$} & \multicolumn{2}{|c|}{$4 \mathrm{~K}$} & \multirow{3}{*}{$\begin{array}{l}\text { Ansaat- } \\
\text { variante }\end{array}$} & \multirow{3}{*}{ Düngung } & \multirow{3}{*}{$\begin{array}{l}\text { Ansaat- } \\
\text { variante } x \\
\text { Düngung }\end{array}$} \\
\hline Düngung & $\mathrm{N}_{0}$ & $\mathrm{~N}_{3}$ & $\mathrm{~N}_{0}$ & $\mathrm{~N}_{3}$ & $\mathrm{~N}_{0}$ & $\mathrm{~N}_{3}$ & $\mathrm{~N}_{0}$ & $\mathrm{~N}_{3}$ & & & \\
\hline 1. Schnitt & & & & 29.06 & .1998 & & & & & & \\
\hline $\mathrm{TM}_{\mathrm{Bt}}$ & 29,0 & 27,1 & 34,6 & 28,7 & 29,8 & 31,8 & 26,5 & 31,8 & n.s. & n.s. & n.s. \\
\hline Schnittgut-TM & 14,8 & 15,6 & 18,0 & 15,2 & 12,3 & 13,7 & 9,2 & 10,5 & $* * *$ & n.s. & n.s. \\
\hline Ernterest-TM & 14,2 & 11,5 & 16,6 & 13,5 & 17,5 & 18,1 & 17,3 & 21,3 & $* * *$ & n.s. & n.s. \\
\hline Stoppel-TM & 3,4 & 4,4 & 6,7 & 6,5 & 9,8 & 9,3 & 10,2 & 10,1 & n.g.v. & n.g.v. & n.g.v. \\
\hline Wurzel-TM & 10,8 & 7,1 & 9,9 & 7,0 & 7,7 & 8,8 & 7,1 & 11,2 & n.s. & n.s. & ** \\
\hline${ }^{\mathrm{wPsD}} \mathrm{H}_{\mathrm{TMBtw}}$ & 0,51 & 0,58 & 0,52 & 0,53 & 0,41 & 0,43 & 0,35 & 0,33 & $* * *$ & n.s. & n.s. \\
\hline 2. Schnitt ${ }^{\text {kum }}$ & & & & 24.08 & 3.1998 & & & & & & \\
\hline $\mathrm{TM}_{\mathrm{Bt}}$ & 69,5 & 58,8 & 79,6 & 70,8 & 69,0 & 71,9 & 64,6 & 67,5 & ** & n.s. & n.s. \\
\hline Schnittgut-TM & 33,4 & 35,1 & 39,8 & 38,7 & 27,0 & 36,7 & 28,3 & 31,8 & $* * *$ & * & n.s. \\
\hline Ernterest-TM & 36,1 & 23,7 & 39,8 & 32,1 & 42,0 & 35,2 & 36,3 & 35,7 & ** & * & n.s. \\
\hline Stoppel-TM & 4,2 & 4,5 & 12,7 & 12,6 & 17,6 & 15,8 & 17,3 & 14,3 & n.g.v. & n.g.v. & n.g.v. \\
\hline Wurzel-TM & 31,9 & 19,2 & 27,1 & 19,5 & 24,4 & 19,4 & 19,0 & 21,4 & n.s. & * & * \\
\hline $\mathrm{H}_{\text {TMBtw }}$ & 0,48 & 0,60 & 0,50 & 0,55 & 0,39 & 0,51 & 0,44 & 0,47 & $* * *$ & * & n.s. \\
\hline
\end{tabular}

Tab. A25: Mittelwerte und Mittelwertvergleich der Trockenmasse-Akkumulation $\left(\mathrm{dt} \mathrm{ha}^{-1}\right)$ sowie der gewichteten Trockenmasse-Harvest-Indizes $\left(\mathrm{H}_{\mathrm{TMBtw}}\right)$ beim Anbau von Luzerne und Knaulgras in Rein- und Gemengesaat zum dritten Schnitt am 25.10.1998 (= $\sum$ 1998)

\begin{tabular}{|c|c|c|c|c|c|c|c|c|c|}
\hline \multirow{2}{*}{$\begin{array}{l}\text { Ansaatvariante } \\
\text { Düngung }\end{array}$} & \multicolumn{2}{|c|}{$4 \mathrm{~L}$} & $3 \mathrm{~L} / 1 \mathrm{~K}$ & \multicolumn{2}{|c|}{$1 \mathrm{~L} / 3 \mathrm{~K}$} & \multicolumn{2}{|c|}{$4 \mathrm{~K}$} & \multirow{3}{*}{ Düngung } & \multirow{3}{*}{$\begin{array}{l}\text { Ansaat- } \\
\text { variante } x \\
\text { Düngung }\end{array}$} \\
\hline & $\mathrm{N}_{0}$ & $\mathrm{~N}_{3}$ & $\mathrm{~N}_{3}$ & $\mathrm{~N}_{0}$ & $\mathrm{~N}_{3}$ & $\mathrm{~N}_{0}$ & $\mathrm{~N}_{3}$ & & \\
\hline 3. Schnitt & \multicolumn{7}{|c|}{25.10 .1998} & & \\
\hline \multirow[t]{2}{*}{$\mathrm{TM}_{\mathrm{Bt}}$} & \multicolumn{2}{|c|}{$a b$} & $a$ & \multicolumn{2}{|c|}{$a b$} & \multicolumn{2}{|c|}{$b^{*}$} & & \\
\hline & 84,1 & 79,5 & \begin{tabular}{|l|l|}
94,3 & 92,9 \\
\end{tabular} & 78,6 & 87,4 & 75,6 & 82,3 & n.s. & n.s. \\
\hline \multirow[t]{2}{*}{ Schnittgut-TM } & \multicolumn{2}{|c|}{$a b$} & $a$ & \multicolumn{2}{|c|}{$b$} & \multicolumn{2}{|c|}{$b^{* * *}$} & & \\
\hline & 42,2 & 45,1 & \begin{tabular}{|l|l|}
48,1 & 47,8 \\
\end{tabular} & 32,7 & 43,3 & 32,7 & 37,7 & * & n.s \\
\hline \multirow[t]{2}{*}{ Ernterest-TM } & \multicolumn{2}{|c|}{$a$} & $a$ & \multicolumn{2}{|c|}{$a$} & \multicolumn{2}{|c|}{7} & & \\
\hline & 41,9 & 34,4 & \begin{tabular}{|l|l|}
46,3 & 45,1 \\
\end{tabular} & 45,9 & 44,1 & 42,9 & 44,6 & n.s. & n.s. \\
\hline \multirow[t]{2}{*}{ Stoppel-TM } & \multicolumn{2}{|c|}{ n.g.v. } & n.g.v. & \multicolumn{2}{|c|}{ n.g.v. } & \multicolumn{2}{|c|}{ n.g.v. } & & \\
\hline & 4,8 & 5,2 & \begin{tabular}{l|l}
13,6 & 15,7 \\
\end{tabular} & 14,7 & 17,3 & 18,5 & 20,5 & n.g.v. & n.g.v. \\
\hline \multirow[t]{2}{*}{ Wurzel-TM } & \multicolumn{2}{|c|}{ a } & $a b$ & \multicolumn{2}{|c|}{$a b$} & \multicolumn{2}{|c|}{$\mathrm{b}^{*}$} & & \\
\hline & 37,1 & 29,2 & \begin{tabular}{|l|l|}
32,7 & 29,4 \\
\end{tabular} & 31,2 & 26,8 & 24,4 & 24,1 & n.s. & n.s. \\
\hline \multirow[t]{2}{*}{$\mathrm{H}_{\text {TMBtw }}$} & \multicolumn{2}{|c|}{$a$} & $a b$ & \multicolumn{2}{|c|}{ 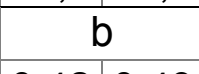 } & $b^{*}$ & & & \\
\hline & 0,50 & 0,57 & $0,51 \quad 0,51$ & 0,42 & 0,49 & 0,43 & 0,46 & n.s. & n.s. \\
\hline
\end{tabular}

TM = Trockenmasse; $\mathrm{H}_{\text {TMBtw }}=$ gewichteter gesamtpflanzlicher Trockenmasse-Harvest-Index; nicht gleiche Buchstaben kennzeichnen signifikante Unterschiede; Tukey-Test: ${ }^{*}=P<0,05 ;{ }^{* *}=P<0,01$; ${ }^{* *}=P<0,001$; n.s. $=$ nicht signifikant; n.g.v. $=$ nicht gesondert verrechnet. 
Tab. A26: Mittelwerte \pm Standardabweichung der Spross-Trockenmassen $\left(\mathrm{dt} \mathrm{ha}^{-1}\right)$ und Spross-N-Mengen $\left(\mathrm{kg} \mathrm{ha}^{-1}\right)$ der Kräuter beim Anbau von Luzerne und Knaulgras in Rein- und Gemengesaat zu drei Schnittterminen im Jahr 1999

\begin{tabular}{c|c|c|c|c|c}
\hline \multirow{4}{*}{ Schnitt } & & \multicolumn{2}{|c|}{ Spross-TM } & \multicolumn{2}{c}{ Spross-N } \\
\cline { 2 - 6 } & $\begin{array}{c}\text { Düngung } \\
\text { Ansaatvariante }\end{array}$ & $\mathrm{N}_{0}$ & $\mathrm{~N}_{3}$ & $\mathrm{~N}_{0}$ & $\mathrm{~N}_{3}$ \\
\hline \multirow{4}{*}{} & $4 \mathrm{~L}$ & $2,8 \pm 2,1$ & $3,8 \pm 2,4$ & $6,3 \pm 5,6$ & $8,7 \pm 6,4$ \\
\cline { 2 - 6 } & $3 \mathrm{~L} / 1 \mathrm{~K}$ & $3,8 \pm 2,3$ & $3,3 \pm 1,6$ & $8,9 \pm 5,6$ & $7,6 \pm 3,6$ \\
\cline { 2 - 6 } & $1 \mathrm{~L} / 3 \mathrm{~K}$ & $3,5 \pm 2,3$ & $3,2 \pm 0,7$ & $8,9 \pm 7,0$ & $7,3 \pm 3,0$ \\
\cline { 2 - 6 } & $4 \mathrm{~K}$ & $3,5 \pm 3,1$ & $3,5 \pm 3,1$ & $7,5 \pm 7,3$ & $6,9 \pm 4,9$ \\
\hline \multirow{4}{*}{2} & $4 \mathrm{~L}$ & $0,3 \pm 0,2$ & $0,3 \pm 0,5$ & $0,4 \pm 0,3$ & $0,7 \pm 1,3$ \\
\cline { 2 - 6 } & $3 \mathrm{~L} / 1 \mathrm{~K}$ & $0,2 \pm 0,2$ & $0,6 \pm 0,5$ & $0,3 \pm 0,4$ & $0,7 \pm 0,6$ \\
\cline { 2 - 6 } & $1 \mathrm{~L} / 3 \mathrm{~K}$ & $0,4 \pm 0,5$ & $0,8 \pm 0,9$ & $1,0 \pm 1,2$ & $1,7 \pm 1,9$ \\
\cline { 2 - 6 } & $4 \mathrm{~K}$ & $1,0 \pm 1,0$ & $0,6 \pm 0,6$ & $2,6 \pm 2,3$ & $1,4 \pm 1,6$ \\
\hline \multirow{4}{*}{3} & $4 \mathrm{~L}$ & 0 & 0 & 0 & 0 \\
\cline { 2 - 6 } & $3 \mathrm{~L} / 1 \mathrm{~K}$ & $0,1 \pm 0,1$ & $0,1 \pm 0,2$ & $0,1 \pm 0,2$ & $0,2 \pm 0,3$ \\
\cline { 2 - 6 } & $1 \mathrm{~L} / 3 \mathrm{~K}$ & $0,2 \pm 0,3$ & $0,2 \pm 0,3$ & $0,4 \pm 0,5$ & $0,2 \pm 0,3$ \\
\cline { 2 - 6 } & $4 \mathrm{~K}$ & $0,5 \pm 0,5$ & $0,4 \pm 0,4$ & $1,1 \pm 0,9$ & $0,9 \pm 1,0$ \\
\hline
\end{tabular}

Tab. A27: Mittelwerte der Trockenmasse-Akkumulation sowie der gewichteten Trockenmasse-Harvest-Indizes $\left(\mathrm{H}_{\text {TMBtw }}\right)$ beim Anbau von Luzerne und Knaulgras in Rein- und Gemengesaat sowie Ergebnisse der Varianzanalyse (Ansaatvariante, Düngung) zum ersten und zweiten Schnitt im Jahr 1999

\begin{tabular}{|c|c|c|c|c|c|c|c|c|c|c|c|}
\hline Ansaatvariante & \multicolumn{2}{|c|}{$4 \mathrm{~L}$} & \multicolumn{2}{|c|}{$3 \mathrm{~L} / 1 \mathrm{~K}$} & \multicolumn{2}{|c|}{$1 \mathrm{~L} / 3 \mathrm{~K}$} & \multicolumn{2}{|c|}{$4 \mathrm{~K}$} & \multirow{3}{*}{$\begin{array}{l}\text { Ansaat- } \\
\text { variante }\end{array}$} & \multirow{3}{*}{ Düngung } & \multirow{3}{*}{$\begin{array}{c}\text { Ansaat- } \\
\text { variante } x \\
\text { Düngung }\end{array}$} \\
\hline Düngung & $\mathrm{N}_{0}$ & $\mathrm{~N}_{3}$ & $\mathrm{~N}_{0}$ & $\mathrm{~N}_{3}$ & $\mathrm{~N}_{0}$ & $\mathrm{~N}_{3}$ & $\mathrm{~N}_{0}$ & $\mathrm{~N}_{3}$ & & & \\
\hline 1. Schnitt & & & & 05.07 & .1999 & & & & & & \\
\hline $\mathrm{TM}_{\mathrm{Bt}}$ & 85,2 & 71,4 & 73,6 & 70,3 & 61,1 & 65,0 & 56,4 & 69,7 & $* * *$ & n.s. & ** \\
\hline${ }^{P s}$ Schnittgut-TM & 44,6 & 41,0 & 41,1 & 40,6 & 35,9 & 35,1 & 26,8 & 33,6 & $* * *$ & n.s. & * \\
\hline Ernterest-TM & 40,6 & 30,4 & 32,5 & 29,7 & 25,2 & 29,9 & 29,6 & 36,1 & n.s. & n.s. & * \\
\hline Stoppel-TM & 6,7 & 6,4 & 6,7 & 7,2 & 10,5 & 11,9 & 15,3 & 17,5 & n.g.v. & n.g.v. & n.g.v. \\
\hline Wurzel-TM & 33,9 & 24,0 & 25,8 & 22,5 & 14,7 & 18,0 & 14,3 & 18,6 & $* * *$ & n.s. & n.s. \\
\hline $\mathrm{H}_{\text {TMBtw }}$ & 0,52 & 0,57 & 0,56 & 0,58 & 0,59 & 0,54 & 0,48 & 0,48 & ** & n.s. & n.s. \\
\hline 2. Schnitt ${ }^{\text {kum }}$ & & & & 16.0 & 3.1999 & & & & & & \\
\hline $\mathrm{TM}_{\mathrm{Bt}}$ & 148,6 & 122,3 & 124,5 & 116,0 & 115,5 & 103,1 & 80,3 & 101,9 & ** & n.s. & $* * *$ \\
\hline Schnittgut-TM & 81,7 & 75,5 & 70,9 & 73,2 & 64,1 & 59,0 & 40,6 & 49,0 & $* * *$ & n.s. & ** \\
\hline Ernterest-TM & 66,9 & 46,8 & 53,6 & 42,8 & 51,4 & 44,1 & 39,7 & 52,9 & * & n.s. & n.s. \\
\hline Stoppel-TM & 6,1 & 6,3 & 7,2 & 7,4 & 10,9 & 11,4 & 17,0 & 19,1 & n.g.v. & n.g.v. & n.g.v. \\
\hline Wurzel-TM & 60,8 & 40,5 & 46,4 & 35,4 & 40,5 & 32,7 & 22,7 & 33,8 & $* * *$ & $* * *$ & n.s. \\
\hline $\mathrm{H}_{\text {TMBtw }}$ & 0,55 & 0,62 & 0,57 & 0,63 & 0,55 & 0,57 & 0,51 & 0,48 & $* * *$ & n.s. & n.s. \\
\hline
\end{tabular}

Ps = Einfluss des Pflanzenschutzes nachweisbar; TM = Trockenmasse; $\mathrm{TM}_{\mathrm{Bt}}=$ gesamtpflanzliche Trockenmasse; $\mathrm{kum}=$ kumulierte Trockenmasse; n.s. = nicht signifikant; ${ }^{*}=P<0,05 ;{ }^{* *}=P<0,01$; ${ }^{* *}=P<0,001$; n.g.v. = nicht gesondert verrechnet 
Tab. A28: Mittelwerte und Mittelwertvergleich der Trockenmasse-Akkumulation $\left(\mathrm{dt} \mathrm{ha}^{-1}\right)$ sowie der gewichteten Trockenmasse-Harvest-Indizes $\left(\mathrm{H}_{\mathrm{TMBtw}}\right)$ beim Anbau von Luzerne und Knaulgras in Rein- und Gemengesaat zum dritten Schnitt am 04.11.1999 $\left(=\sum\right.$ 1999)

\begin{tabular}{|c|c|c|c|c|c|c|c|c|c|c|}
\hline \multirow{2}{*}{$\begin{array}{l}\text { Ansaatvariante } \\
\text { Düngung }\end{array}$} & \multicolumn{2}{|c|}{$4 \mathrm{~L}$} & \multicolumn{2}{|c|}{$3 \mathrm{~L} / 1 \mathrm{~K}$} & \multicolumn{2}{|c|}{$1 \mathrm{~L} / 3 \mathrm{~K}$} & \multicolumn{2}{|c|}{$4 \mathrm{~K}$} & \multirow[b]{3}{*}{ Düngung } & \multirow{3}{*}{$\begin{array}{l}\text { Ansaat- } \\
\text { variante } x \\
\text { Düngung }\end{array}$} \\
\hline & $\mathrm{N}_{0}$ & $\mathrm{~N}_{3}$ & $\mathrm{~N}_{0}$ & $\mathrm{~N}_{3}$ & $\mathrm{~N}_{0}$ & $\mathrm{~N}_{3}$ & $\mathrm{~N}_{0}$ & $\mathrm{~N}_{3}$ & & \\
\hline 3. Schnitt & \multicolumn{8}{|c|}{04.11 .1999} & & \\
\hline \multirow[t]{2}{*}{$\mathrm{TM}_{\mathrm{Bt}}$} & \multicolumn{2}{|c|}{$a$} & \multicolumn{2}{|c|}{$a b$} & \multicolumn{2}{|c|}{$b$} & \multicolumn{2}{|c|}{$C^{* * *}$} & & \\
\hline & 176,6 & 150,1 & 152,3 & 141,8 & 141,1 & 130,5 & 97,4 & 104,2 & * & * \\
\hline \multirow[t]{2}{*}{ Schnittgut-TM } & \multicolumn{2}{|c|}{$a$} & \multicolumn{2}{|c|}{$a$} & \multicolumn{2}{|c|}{$b$} & \multicolumn{2}{|c|}{$C^{* * *}$} & & \\
\hline & 102,1 & 94,7 & 88,5 & 93,7 & 84,3 & 75,6 & 46,8 & 55,4 & n.s. & ** \\
\hline \multirow[t]{2}{*}{ Ernterest-TM $^{1}$} & \multicolumn{2}{|c|}{ a } & \multicolumn{2}{|c|}{ a } & \multicolumn{2}{|r|}{ a } & \multicolumn{2}{|c|}{ a } & & \\
\hline & 74,5 & 55,3 & 63,8 & 48,1 & 56,9 & 54,9 & 50,5 & 48,8 & $* * *$ & n.s. \\
\hline \multirow[t]{2}{*}{ Stoppel-TM } & \multicolumn{2}{|c|}{ n.g.v. } & \multicolumn{2}{|c|}{ n.g.v. } & \multicolumn{2}{|c|}{ n.g.v. } & \multicolumn{2}{|c|}{ n.g.v. } & & \\
\hline & 8,0 & 7,7 & 7,3 & 8,7 & 11,9 & 13,0 & 24,6 & 23,7 & n.g.v. & n.g.v. \\
\hline \multirow[t]{2}{*}{ Wurzel } & \multicolumn{2}{|c|}{$d$} & \multicolumn{2}{|c|}{$a$} & \multicolumn{2}{|c|}{$a b$} & \multicolumn{2}{|c|}{$b^{* * *}$} & & \\
\hline & 64,3 & 46,2 & 54,9 & 38,5 & 44,1 & 41,3 & 25,9 & 25,1 & $* * *$ & n.s. \\
\hline \multirow{2}{*}{$\begin{array}{l}\mathrm{H}_{\text {TMBtw }} \\
\text { wPsA }\end{array}$} & \multicolumn{2}{|c|}{ a } & \multicolumn{2}{|c|}{$a$} & & $a b$ & & $\star \star \star *$ & & \\
\hline & 0,58 & 0,63 & 0,58 & 0,66 & 0,60 & 0,58 & 0,48 & 0,53 & $* * *$ & n.s. \\
\hline
\end{tabular}

wPsA = Wechselwirkung: Pflanzenschutz x Ansaatvariante; TM = Trockenmasse; $\mathrm{H}_{\mathrm{TMBtw}}=$ gewichteter gesamtpflanzlicher Trockenmasse-Harvest-Index; ${ }^{1}$ inklusive Bestandesabfall; nicht gleiche Buchstaben kennzeichnen signifikante Unterschiede; Tukey-Test: ${ }^{*}=P<0,05 ;{ }^{* *}=P<0,01$; ${ }^{* *}=P<0,001$; n.s. = nicht signifikant; n.g.v. = nicht gesondert verrechnet. 
Tab. A29: Mittelwerte und Mittelwertvergleich der gesamtpflanzlichen N-Akkumulation $\left(\mathrm{N}_{\mathrm{Bt}}\right.$, $\left.\mathrm{kg} \mathrm{ha}^{-1}\right)$, der Stickstoff-Erträge im Schnittgut, Stoppel und Wurzel $\left(\mathrm{kg} \mathrm{ha}^{-1}\right)$ sowie der gewichteten Stickstoff-Harvest-Indizes $\left(\mathrm{H}_{\mathrm{NBtw}}\right)$ beim Anbau von Luzerne und Knaulgras in Rein- und Gemengesaat zu drei Schnittterminen im Jahr 1998

\begin{tabular}{|c|c|c|c|c|c|c|c|c|c|c|}
\hline \multirow{2}{*}{$\frac{\text { Ansaatvariante }}{\text { Düngung }}$} & \multicolumn{2}{|c|}{$4 \mathrm{~L}$} & \multicolumn{2}{|c|}{$3 \mathrm{~L} / 1 \mathrm{~K}$} & \multicolumn{2}{|c|}{$1 \mathrm{~L} / 3 \mathrm{~K}$} & \multicolumn{2}{|c|}{$4 \mathrm{~K}$} & \multirow[b]{3}{*}{ Düngung } & \multirow{3}{*}{$\begin{array}{l}\text { Ansaat- } \\
\text { variante } x \\
\text { Düngung }\end{array}$} \\
\hline & $\mathrm{N}_{0}$ & $\mathrm{~N}_{3}$ & $\mathrm{~N}_{0}$ & $\mathrm{~N}_{3}$ & $\mathrm{~N}_{0}$ & $\mathrm{~N}_{3}$ & $\mathrm{~N}_{0}$ & $\mathrm{~N}_{3}$ & & \\
\hline 1. Schnitt & \multicolumn{8}{|c|}{29.06 .1998} & & \\
\hline \multirow[t]{2}{*}{$\mathrm{N}_{\mathrm{Bt}}$} & \multicolumn{2}{|c|}{ a } & \multicolumn{2}{|c|}{$a$} & \multicolumn{2}{|c|}{$\mathrm{b}$} & \multicolumn{2}{|c|}{$C^{* *}$} & & \\
\hline & 89,5 & 79,9 & 93,6 & 79,3 & 62,0 & 70,8 & 40,7 & 53,8 & n.s. & * \\
\hline \multirow[t]{2}{*}{ Schnittgut-N } & \multicolumn{2}{|c|}{ a } & \multicolumn{2}{|c|}{$a$} & \multicolumn{2}{|c|}{$\mathrm{b}$} & \multicolumn{2}{|c|}{$c^{* *}$} & & \\
\hline & 61,7 & 58,3 & 64,7 & 56,9 & 37,2 & 44,3 & 20,2 & 27,2 & n.s. & n.s. \\
\hline \multirow[t]{2}{*}{ Ernterest-N } & \multicolumn{2}{|c|}{ a } & \multicolumn{2}{|c|}{ a } & \multicolumn{2}{|c|}{ a } & \multicolumn{2}{|c|}{ a } & & \\
\hline & 27,8 & 21,6 & 28,9 & 22,4 & 24,8 & 26,5 & 20,5 & 26,6 & n.s. & * \\
\hline Stoppel-N & 5,9 & 7,9 & 11,7 & 11,7 & 15,0 & 14,9 & 13,6 & 14,5 & n.g.v. & n.g.v. \\
\hline \multirow[t]{2}{*}{ Wurzel-N $^{1}$} & \multicolumn{2}{|c|}{$a$} & $a$ & $b$ & 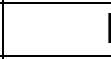 & $b$ & $b^{\prime}$ & & & \\
\hline & 21,9 & 13,7 & 17,2 & 10,7 & 9,8 & 11,6 & 6,9 & 12,1 & n.s. & ** \\
\hline $\mathrm{H}_{\mathrm{NBtw}}$ & a & 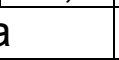 & $a$ & $a$ & 1 & $b$ & $c^{*}$ & & & \\
\hline & 0,69 & 0,73 & 0,69 & 0,72 & 0,60 & 0,63 & 0,50 & 0,51 & n.s. & n.s. \\
\hline 2. Schnitt ${ }^{\text {kum }}$ & & & & & & 4.08 .19 & & & & \\
\hline $\mathrm{N}_{\mathrm{Bt}}^{\text {kum }}$ & a & $a$ & $k$ & $b$ & 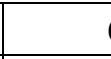 & C & $d^{\prime}$ & & & \\
\hline & 194,5 & 169,2 & 175,1 & 151,2 & 105,0 & 124,2 & 80,1 & 95,0 & n.s. & ** \\
\hline Schnittgut- $N^{\text {kum }}$ & $a$ & 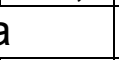 & $a$ & 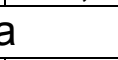 & 1 & $b$ & $c^{*}$ & & & \\
\hline & 120,8 & 121,8 & 117,5 & 112,5 & 65,4 & 90,0 & 53,0 & 64,7 & * & * \\
\hline Ernterest-N & a & $a$ & $k$ & 0 & & C & $C^{\prime}$ & & & \\
\hline & 73,7 & 47,4 & 57,6 & 38,7 & 39,6 & 34,2 & 27,1 & 30,3 & * & ** \\
\hline Stoppel-N & 6,9 & 7,2 & 14,6 & 13,4 & 14,1 & 14,5 & 12,8 & 13,0 & n.g.v. & n.g.v. \\
\hline Wurzel-N & a & $a$ & $k$ & $b$ & ( & C & $C^{\prime}$ & & & \\
\hline & 66,8 & 40,2 & 43,0 & 25,3 & 25,5 & 19,7 & 14,3 & 17,3 & * & ** \\
\hline $\mathrm{H}_{\mathrm{NBtw}}{ }^{\text {kum }}$ & 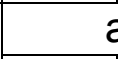 & $a$ & a & a & 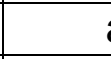 & a & a & & & \\
\hline & 0,62 & 0,72 & 0,67 & 0,74 & 0,62 & 0,72 & 0,66 & 0,68 & * & n.s. \\
\hline 3. Schnitt ${ }^{\text {kum }}$ & & & & & & 5.10 .19 & & & & \\
\hline $\mathrm{N}_{\mathrm{Bt}}{ }^{\text {kum }}$ & $a$ & $a$ & $a$ & $a$ & 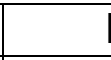 & $b$ & $C^{\prime}$ & & & \\
\hline & 243,8 & 226,6 & 223,7 & 208,7 & 130,9 & 154,5 & 104,1 & 126,9 & n.s. & * \\
\hline Schnittgut- $N^{\text {kum }}$ & $a$ & $a$ & $a$ & 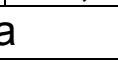 & 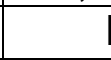 & $b$ & $\mathrm{C}^{*}$ & & & \\
\hline & 153,7 & 160,7 & 146,4 & 144,4 & 82,0 & 110,1 & 65,3 & 82,2 & * & n.s \\
\hline Ernterest-N & a & $a$ & $a$ & 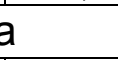 & 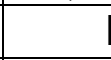 & $b$ & $b^{*}$ & & & \\
\hline & 90,1 & 65,9 & 77,3 & 64,3 & 48,9 & 44,4 & 38,8 & 44,7 & n.s. & n.s. \\
\hline Stoppel-N & 11,6 & 14,4 & 23,9 & 24,8 & 19,7 & 22,1 & 22,0 & 26,3 & n.g.v. & n.g.v. \\
\hline Wurzel-N & a & $a$ & $\mathrm{k}$ & $b$ & (n) & $c$ & $\mathrm{C}^{*}$ & & & \\
\hline & 78,5 & 51,5 & 53,4 & 39,5 & 29,2 & 22,3 & 16,8 & 18,4 & n.s. & * \\
\hline $\mathrm{H}_{\mathrm{NBtw}}{ }^{\text {kum }}$ & a & $a$ & $a$ & a & ( & a & a & & & \\
\hline & 0,63 & 0,71 & 0,65 & 0,69 & 0,63 & 0,71 & 0,63 & 0,65 & ** & n.s. \\
\hline
\end{tabular}

$\mathrm{H}_{\mathrm{NBtw}}=$ gewichteter gesamtpflanzlicher Stickstoff-Harvest-Index; nicht gleiche Buchstaben kennzeichnen signifikante Unterschiede; Tukey-Test: ${ }^{*}=P<0,05 ;{ }^{* *}=P<0,01 ;{ }^{* * *}=P<0,001$; n.s. $=$ nicht signifikant; ${ }^{1}$ Originaldaten transformiert $(\lg \mathrm{x})$; n.g.v. = nicht gesondert verrechnet; kum kumulierte Werte 
Tab. A30: Mittelwerte und Mittelwertvergleich der gesamtpflanzlichen N-Akkumulation $\left(\mathrm{N}_{\mathrm{Bt}}\right.$, $\left.\mathrm{kg} \mathrm{ha}^{-1}\right)$, der Stickstoff-Erträge im Schnittgut, Stoppel und Wurzel $\left(\mathrm{kg} \mathrm{ha}^{-1}\right)$ sowie der gewichteten Stickstoff-Harvest-Indizes $\left(\mathrm{H}_{\mathrm{NBtw}}\right)$ beim Anbau von Luzerne und Knaulgras in Rein- und Gemengesaat zu drei Schnittterminen im Jahr 1999

\begin{tabular}{|c|c|c|c|c|c|c|c|c|c|c|}
\hline \multirow{2}{*}{$\begin{array}{l}\text { Ansaatvariante } \\
\text { Düngung }\end{array}$} & \multicolumn{2}{|c|}{$4 \mathrm{~L}$} & \multicolumn{2}{|c|}{$3 \mathrm{~L} / 1 \mathrm{~K}$} & \multicolumn{2}{|c|}{$1 \mathrm{~L} / 3 \mathrm{~K}$} & \multicolumn{2}{|c|}{$4 \mathrm{~K}$} & \multirow[b]{3}{*}{ Düngung } & \multirow{3}{*}{$\begin{array}{l}\text { Ansaat- } \\
\text { variante } x \\
\text { Düngung }\end{array}$} \\
\hline & $\mathrm{N}_{0}$ & $\mathrm{~N}_{3}$ & $\mathrm{~N}_{0}$ & $\mathrm{~N}_{3}$ & $\mathrm{~N}_{0}$ & $\mathrm{~N}_{3}$ & $\mathrm{~N}_{0}$ & $\mathrm{~N}_{3}$ & & \\
\hline 1. Schnitt & \multicolumn{8}{|c|}{ 05.07.1999 } & & \\
\hline \multirow{2}{*}{$\begin{array}{l}\mathrm{N}_{\mathrm{Bt}} \\
\mathrm{Ps}\end{array}$} & \multicolumn{2}{|c|}{ a } & \multicolumn{2}{|c|}{$a$} & \multicolumn{2}{|c|}{ b } & \multicolumn{2}{|c|}{$C^{* * *}$} & & \\
\hline & \multicolumn{2}{|c|}{\begin{tabular}{|l|l|}
229,1 & 179,3 \\
\end{tabular}} & \multicolumn{2}{|c|}{\begin{tabular}{|l|l|}
189,7 & 178,1 \\
\end{tabular}} & \multicolumn{2}{|c|}{\begin{tabular}{|l|l|}
128,5 & 123,6 \\
\end{tabular}} & 79,6 & 97,6 & n.s. & ** \\
\hline \multirow[t]{2}{*}{ Schnittgut-N } & \multicolumn{2}{|c|}{$a$} & \multicolumn{2}{|c|}{ a } & \multicolumn{2}{|c|}{$\mathrm{b}$} & \multicolumn{2}{|c|}{$C^{\star * *}$} & & \\
\hline & 137,1 & 116,6 & 123,1 & 120,1 & 90,1 & 82,8 & 49,9 & 63,3 & n.s. & ** \\
\hline \multirow[t]{2}{*}{ Ernterest-N ${ }^{1}$} & \multicolumn{2}{|c|}{$\mathrm{a}$} & \multicolumn{2}{|c|}{$a$} & $k$ & 0 & $b^{*}$ & $* *$ & & \\
\hline & 92,0 & 62,7 & 66,6 & 58,0 & 38,4 & 40,8 & 29,7 & 34,3 & n.s. & n.s. \\
\hline Stoppel-N & 10,0 & 9,2 & 9,0 & 9,5 & 11,3 & 12,3 & 15,3 & 17,1 & n.g.v. & n.g.v. \\
\hline Wurzel-N ${ }^{1}$ & a & $a$ & a & & $\mathrm{k}$ & 0 & $b^{*}$ & $* *$ & & \\
\hline & 82,0 & 53,5 & 57,6 & 48,5 & 27,1 & 28,5 & 14,4 & 17,2 & n.s. & n.s. \\
\hline $\mathrm{H}_{\mathrm{NBtw}}$ & $a$ & $a$ & a & & $a$ & $a$ & a & & & \\
\hline & 0,60 & 0,65 & 0,65 & 0,67 & 0,70 & 0,67 & 0,63 & 0,65 & n.s. & n.s. \\
\hline 2. Schnitt ${ }^{\text {kum }}$ & & & & & & 5.08 .19 & & & & \\
\hline $\mathrm{N}_{\mathrm{Bt}}{ }^{\text {kum }}$ & a & & $b$ & & c & $C$ & $d^{*}$ & & & \\
\hline & 377,0 & 314,3 & 316,1 & 301,8 & 252,1 & 210,4 & 103,7 & 121,3 & n.s. & ** \\
\hline Schnittgut-N ${ }^{\text {kum }}$ & $a$ & & $\mathrm{~b}$ & & $\mathrm{c}$ & $c$ & d & & & \\
\hline & 248,9 & 222,4 & 214,3 & 223,0 & 173,4 & 148,0 & 73,5 & 90,4 & n.s. & $* * *$ \\
\hline Ernterest-N & $a$ & & $\mathrm{~b}$ & & c & $c$ & d & & & \\
\hline & 128,1 & 91,9 & 101,8 & 78,8 & 78,7 & 62,4 & 30,2 & 30,9 & * & n.s. \\
\hline Stoppel-N & 8,5 & 9,6 & 11,3 & 10,6 & 11,5 & 10,6 & 11,5 & 12,7 & n.g.v. & n.g.v. \\
\hline Wurzel-N & a & & b & & $c$ & $c$ & d & & & \\
\hline & 119,6 & 82,3 & 90,5 & 68,2 & 67,2 & 51,8 & 18,7 & 18,2 & * & * \\
\hline $\mathrm{H}_{\mathrm{NBtw}}{ }^{\text {kum }}$ & a & $a$ & a & & a & $a$ & a & & & \\
\hline & 0,66 & 0,71 & 0,68 & 0,74 & 0,69 & 0,70 & 0,71 & 0,75 & n.s. & n.s. \\
\hline 3. Schnitt ${ }^{\text {kum }}$ & & & & & & 4.11 .19 & & & & \\
\hline $\mathrm{N}_{\mathrm{Bt}}^{\text {kum }}$ & a & & $b$ & & $c$ & $C$ & $d^{*}$ & & & \\
\hline & 472,4 & 404,3 & 412,0 & 390,4 & 335,5 & 297,3 & 127,2 & 145,3 & * & ** \\
\hline Schnittgut-N $^{\text {kum }}$ & a & & $\mathrm{b}$ & & c & $c$ & d & & & \\
\hline & 304,0 & 276,4 & 263,1 & 281,4 & 228,4 & 195,3 & 88,4 & 105,9 & n.s. & $* * *$ \\
\hline Ernterest- $\mathrm{N}^{\mathrm{a}}$ & a & & al & & $k$ & o & $c^{*}$ & & & \\
\hline & 168,4 & 127,9 & 148,9 & 109,0 & 107,1 & 102,0 & 38,8 & 39,4 & ** & n.s. \\
\hline Ba-N $N^{\text {kum, b }}$ & 7,0 & 4,3 & 5,5 & 3,4 & 2,7 & 2,3 & 0 & 0 & n.g.v. & n.g.v. \\
\hline Stoppel-N & 17,8 & 17,8 & 17,5 & 19,2 & 18,8 & 19,9 & 18,9 & 20,8 & n.g.v. & n.g.v. \\
\hline Wurzel-N & $a$ & a & al & $b$ & $\mathrm{k}$ & o & $C^{*}$ & $* *$ & & \\
\hline & 143,6 & 105,8 & 125,9 & 86,4 & 85,6 & 79,8 & 19,9 & 18,6 & $* *$ & n.s. \\
\hline $\mathrm{H}_{\mathrm{NBtw}}{ }^{\mathrm{kum}}$ & $a$ & 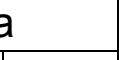 & a & 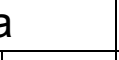 & a & 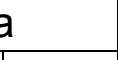 & a & 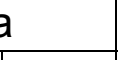 & & \\
\hline wPs & 0,64 & 0,68 & \begin{tabular}{|l|}
0,64 \\
\end{tabular} & 0,72 & 0,68 & 0,66 & \begin{tabular}{|l|}
0,69 \\
\end{tabular} & 0,73 & ** & n.s. \\
\hline
\end{tabular}

$\mathrm{Ps}=$ Einfluss des Pflanzenschutz nachweisbar; wPsA = Wechselwirkung: Pflanzenschutz $\times$ Ansaatvariante; $\mathrm{H}_{\mathrm{NBtw}}=\mathrm{gewichteter}$ gesamtpflanzlicher Stickstoff-Harvest-Index; $a=$ inklusive Bestandesabfall; $b=$ Bestandesabfall; nicht gleiche Buchstaben kennzeichnen signifikante Unterschiede; Tukey-Test: ${ }^{*}=P<0,05 ;{ }^{* *}=P<0,01 ;{ }^{* * *}=P<0,001$; n.s. $=$ nicht signifikant; ${ }^{1}$ Originaldaten transformiert $(\lg x)$; n.g.v. = nicht gesondert verrechnet; kum kumulierte Werte 
Tab. A31: Mittelwerte und Mittelwertvergleich ${ }^{1}$ der gewichteten kumulierten $\delta^{15} \mathrm{~N}$ Werte (\%) und Anteil Stickstoff aus der Luft $\left(\mathrm{Ndfa}_{\mathrm{w}}\right)$ in der Sprossmasse von Luzerne und Knaulgras in Rein- und Gemengesaat zum dritten Schnitt im Jahr 1998 und 1999

\begin{tabular}{|c|c|c|c|c|c|c|c|c|c|c|}
\hline & & \multicolumn{4}{|c|}{1998} & \multicolumn{4}{|c|}{1999} \\
\hline & & \multirow[b]{2}{*}{ Ansaatvariante } & \multicolumn{2}{|c|}{$\delta^{15} \mathrm{~N}[\%]$} & \multicolumn{2}{|c|}{$\mathrm{Ndfa}_{\mathrm{w}}$} & \multicolumn{2}{|c|}{$\delta^{15} \mathrm{~N}[\% \circ]$} & \multicolumn{2}{|c|}{$\mathrm{Ndfa}_{\mathrm{w}}$} \\
\hline & & & $\mathrm{N}_{0}$ & $\mathrm{~N}_{3}$ & $\mathrm{~N}_{0}$ & $\mathrm{~N}_{3}$ & $\mathrm{~N}_{0}$ & $\mathrm{~N}_{3}$ & $\mathrm{~N}_{0}$ & $\mathrm{~N}_{3}$ \\
\hline \multirow[b]{2}{*}{$\begin{array}{l}\text { वे } \\
\widetilde{\alpha}\end{array}$} & \multirow{12}{*}{$\begin{array}{l}\overrightarrow{\mathbb{N}} \\
\mathbb{N} \\
\hat{c}\end{array}$} & $4 \mathrm{~L}$ & 2,03 & 2,18 & 0,56 & 0,44 & 1,08 & 1,10 & 0,69 & 0,68 \\
\hline & & & \multicolumn{2}{|c|}{$a \downarrow$} & \multicolumn{2}{|c|}{$a \downarrow$} & \multicolumn{2}{|c|}{$a \downarrow$} & \multicolumn{2}{|c|}{$a \downarrow$} \\
\hline \multirow{4}{*}{ 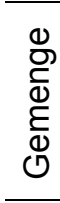 } & & $3 \mathrm{~L}$ & 1,62 & 1,65 & 0,63 & 0,56 & 0,98 & 1,04 & 0,71 & 0,69 \\
\hline & & & & $b$ & & $b$ & & a & & $a$ \\
\hline & & $1 \mathrm{~K}$ & 3,29 & 3,28 & & & 2,65 & 2,83 & & \\
\hline & & $3 L+1 K$ & 2,43 & 2,43 & 0,48 & 0,39 & 1,11 & 1,19 & 0,68 & 0,66 \\
\hline \multirow{4}{*}{$\begin{array}{l}\mathscr{D} \\
\stackrel{\Xi}{\Phi} \\
\bar{\Phi} \\
0\end{array}$} & & $1 \mathrm{~L}$ & 1,42 & 1,54 & 0,67 & 0,59 & 0,94 & 0,97 & 0,72 & 0,71 \\
\hline & & & \multicolumn{2}{|c|}{$\mathrm{b}^{* * *}$} & \multicolumn{2}{|c|}{$b^{* * *}$} & \multicolumn{2}{|c|}{$a$} & \multicolumn{2}{|c|}{$a$} \\
\hline & & $3 \mathrm{~K}$ & 4,18 & 3,84 & & & 3,37 & 3,60 & & \\
\hline & & $1 \mathrm{~L}+3 \mathrm{~K}$ & 3,45 & 3,27 & 0,29 & 0,21 & 1,62 & 1,91 & 0,57 & 0,49 \\
\hline \multirow{2}{*}{$\begin{array}{l}\cdot \frac{c}{\mathbb{Q}} \\
\widetilde{\alpha}\end{array}$} & & $4 \mathrm{~K}$ & 5,02 & 4,25 & & & 4,22 & 4,11 & & \\
\hline & & Düngung & $\rightarrow a$ & a & $\rightarrow a$ & $b^{*}$ & $\rightarrow a$ & a & $\rightarrow a$ & a \\
\hline
\end{tabular}

\footnotetext{
${ }^{1}$ Die Mittelwertvergleiche beziehen sich nur auf den Luzerne-Spross; nicht gleiche Buchstaben kennzeichnen signifikante
} Unterschiede; ${ }^{*}=P<0,05 ;{ }^{* *}=P<0,01 ;{ }^{* * *}=P<0,001 ; \downarrow \rightarrow$ Leserichtung

Tab. A32: Vergleich der gesamtpflanzlich gewichteten $\delta^{15} \mathrm{~N}$-Mittelwerte beim Anbau von Luzerne und Knaulgras in Rein- und Gemengesaat zu drei Schnittterminen a) 1998 und b) 1999 sowie Ergebnis der zweifaktoriellen Varianzanalyse

\begin{tabular}{|c|c|c|c|c|c|c|}
\hline & \multicolumn{4}{|c|}{ Ansaatvariante } & \multirow[b]{2}{*}{ Düngung } & \multirow{2}{*}{$\begin{array}{l}\text { Wechsel- } \\
\text { wirkungen }\end{array}$} \\
\hline a) & $4 \mathrm{~L}$ & $3 \mathrm{~L} / 1 \mathrm{~K}$ & $1 \mathrm{~L} / 3 \mathrm{~K}$ & $4 \mathrm{~K}$ & & \\
\hline 1. Schnitt ${ }^{1}$ & $a$ & $a b$ & $\mathrm{~b}$ & $C^{* * *}$ & n.s. & n.s. \\
\hline 2. Schnitt ${ }^{1}$ & $a$ & $a$ & $\mathrm{~b}$ & $\mathrm{C}^{* * *}$ & n.s. & $* *$ \\
\hline 3. Schnitt ${ }^{2}$ & $a$ & $a$ & $b$ & $C^{* * *}$ & n.s. & ** \\
\hline \multicolumn{7}{|l|}{ b) } \\
\hline 1. Schnitt & $a$ & $a$ & $b$ & $C^{\star \star \star}$ & n.s. & n.s. \\
\hline 2. Schnitt & $a$ & $a$ & $\mathrm{~b}$ & $C^{\star * \star}$ & n.s. & n.s. \\
\hline 3. Schnitt & $a$ & $a$ & $b$ & $C^{* * *}$ & n.s. & ** \\
\hline
\end{tabular}

nicht gleiche Buchstaben kennzeichnen signifikante Unterschiede; Tukey-Test: ${ }^{*}=P<0,05 ;{ }^{* *}=P<0,01$; ${ }_{* \star *}=P<0,001$ n.s. $=$ nicht signifikant; ${ }^{1}$ Originaldaten transformiert $(\operatorname{lgx}) ;{ }^{2}$ Originaldaten transformiert (1/Wurzelx) 
Tab. A33: Korrelation zwischen den Ergebnissen der $\delta^{15} \mathrm{~N}$-Methode und der erweiterten Differenzmethode zur Schätzung der symbiotisch fixierten $\mathrm{N}$ Menge beim Anbau von Luzerne in Rein- und Gemengesaat mit Knaulgras im Jahr 1998 und 1999

\begin{tabular}{c|c|c|c}
\hline & $\mathrm{r}$ & $P$ & $\mathrm{n}$ \\
\hline 1998 & & & \\
\hline 1. Schnitt am 29.06. & 0,46 & 0,0047 & 36 \\
\hline 2. Schnitt am 24.08. & 0,78 & 0,0001 & 36 \\
\hline 3. Schnitt am 25.10. & 0,93 & 0,0001 & 36 \\
\hline 1999 & & & \\
\hline 1. Schnitt am 05.07. & 0,91 & 0,0001 & 36 \\
\hline 2. Schnitt am 16.08. & 0,98 & 0,0001 & 36 \\
\hline 3. Schnitt am 04.11. & 0,97 & 0,0001 & 36 \\
\hline \multicolumn{4}{|l}{}
\end{tabular}

Tab. A34: Mittelwerte $\left(\mathrm{kg} \mathrm{N}_{\min } \mathrm{ha}^{-1}\right)$ und Mittelwertvergleich der $\mathrm{CaCl}_{2}$-extrahierbaren $\mathrm{N}_{\text {min }}$-Mengen im Boden vor der Aussaat der Luzerne und des Knaulgrases am 24.03.1998 (a) und 06.04.1999 (b)

\begin{tabular}{|c|c|c|c|c|c|c|c|c|}
\hline Jahr & \multicolumn{4}{|c|}{1998} & \multicolumn{4}{|c|}{1999} \\
\hline Düngung & $\mathrm{N}_{0}$ & $\mathrm{~N}_{3}$ & Ps & W & $\mathrm{N}_{0}$ & $\mathrm{~N}_{3}$ & Ps & $W$ \\
\hline \multirow[t]{2}{*}{$0-125 \mathrm{~cm}$} & $a$ & $b^{* *}$ & & & $a$ & $a$ & & \\
\hline & 60,7 & 81,9 & n.s. & n.s. & 70,3 & 82,3 & n.s. & n.s. \\
\hline \multirow[t]{2}{*}{$0-50 \mathrm{~cm}$} & a & a & & & a & $a$ & & \\
\hline & 27,2 & 31,2 & n.s. & n.s. & 39,9 & 42,0 & n.s. & n.s. \\
\hline \multirow[t]{2}{*}{$50-125 \mathrm{~cm}$} & $a$ & $b^{* *}$ & & & $a$ & $a$ & & \\
\hline & 33,5 & 50,7 & n.s. & n.s. & 37,4 & 40,3 & n.s. & n.s \\
\hline
\end{tabular}

Ps: Wirkung Pflanzenschutz; W: Wechselwirkung Pflanzenschutz x Düngung; Tukey-

Test: nicht gleiche Buchstaben kennzeichnen signifikante Unterschiede mit: ${ }^{*}=P<0,05$;

${ }^{* *}=P<0,01 ;$ n.s. $=$ nicht signifikant 
Tab. A35: Mittelwerte $\left(\mathrm{kg} \mathrm{N}_{\min } \mathrm{ha}^{-1}\right)$ und Mittelwertvergleich der $\mathrm{CaCl}_{2}$-extrahierbaren $\mathrm{N}_{\text {min }}$-Mengen im Boden beim Anbau von Luzerne und Knaulgras in Rein- und Gemengesaat zu drei Probenahmeterminen im Jahr 1998

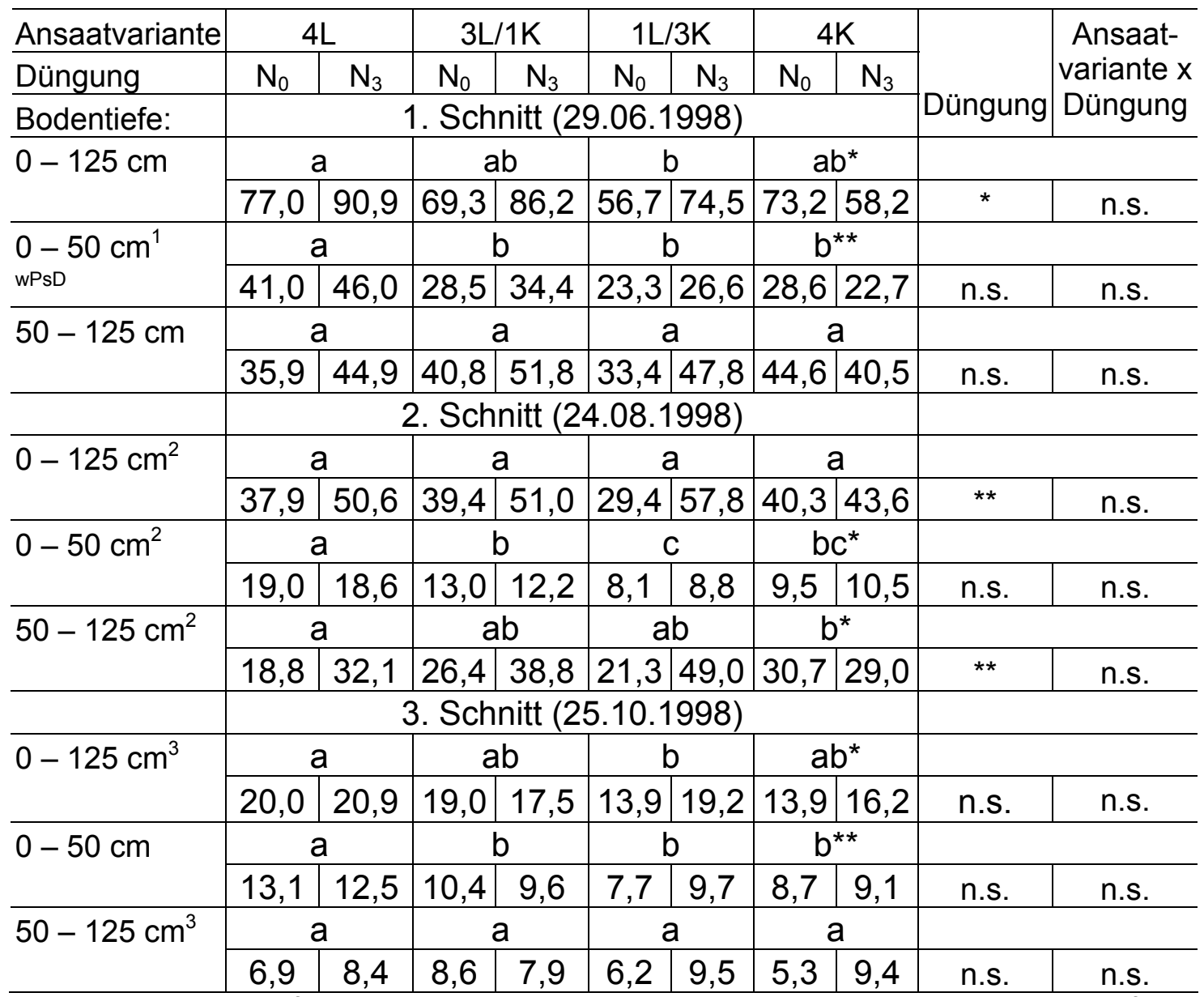


Tab. A36: Mittelwerte und Mittelwertvergleich der luft- und bodenbürtigen gesamtpflanzlichen N-Akkumulation $\left(\mathrm{kg} \mathrm{ha}^{-1}\right)$ und gewichtete Anteile Stickstoff aus der Luft ( $\mathrm{Ndfa}_{w}$ ) beim Anbau von Luzerne und Knaulgras in Reinund Gemengesaat zu drei Schnittterminen im Jahr 1998

\begin{tabular}{|c|c|c|c|c|c|c|c|c|c|c|}
\hline \multirow{2}{*}{$\begin{array}{l}\text { Ansaatvariante } \\
\text { Düngung }\end{array}$} & \multicolumn{2}{|c|}{$4 \mathrm{~L}$} & \multicolumn{2}{|c|}{$3 \mathrm{~L} / 1 \mathrm{~K}$} & \multicolumn{2}{|c|}{$1 \mathrm{~L} / 3 \mathrm{~K}$} & \multicolumn{2}{|c|}{$4 \mathrm{~K}$} & \multirow{3}{*}{ Düngung } & \multirow{3}{*}{$\begin{array}{l}\text { Ansaat- } \\
\text { variante } x \\
\text { Düngung }\end{array}$} \\
\hline & $\mathrm{N}_{0}$ & $\mathrm{~N}_{3}$ & $\mathrm{~N}_{0}$ & $\mathrm{~N}_{3}$ & $\mathrm{~N}_{0}$ & $\mathrm{~N}_{3}$ & $\mathrm{~N}_{0}$ & $\mathrm{~N}_{3}$ & & \\
\hline 1. Schnitt & \multicolumn{8}{|c|}{29.06 .1998} & & \\
\hline \multirow[t]{2}{*}{ Boden-N } & \multicolumn{2}{|c|}{$a$} & \multicolumn{2}{|c|}{$a b$} & \multicolumn{2}{|c|}{$a b$} & \multicolumn{2}{|c|}{$b^{*}$} & & \\
\hline & 35,6 & 44,9 & 43,3 & 45,3 & 36,9 & 52,3 & 40,7 & 53,8 & $* *$ & n.s. \\
\hline \multirow[t]{2}{*}{ Luft-N } & \multicolumn{2}{|c|}{$a$} & \multicolumn{2}{|c|}{$a$} & \multicolumn{2}{|c|}{$b^{* * *}$} & & & & \\
\hline & 54,0 & 35,0 & 50,3 & 34,0 & 25,1 & 18,5 & 0 & 0 & * & n.s. \\
\hline \multirow[t]{2}{*}{$\mathrm{Ndfa}_{\mathrm{w}}$} & \multicolumn{2}{|c|}{$a$} & \multicolumn{2}{|c|}{$a$} & \multicolumn{2}{|c|}{$b^{* * *}$} & & & & \\
\hline & 0,60 & 0,44 & 0,54 & 0,43 & 0,40 & 0,26 & 0 & 0 & * & n.s. \\
\hline 2. Schnitt & \multicolumn{8}{|c|}{24.08 .1998} & & \\
\hline \multirow[t]{2}{*}{ Boden-N } & \multicolumn{2}{|c|}{ a } & \multicolumn{2}{|r|}{ a } & \multicolumn{2}{|c|}{ a } & \multicolumn{2}{|c|}{$a$} & & \\
\hline & 68,2 & 87,0 & 77,9 & 86,9 & 66,9 & 88,8 & 80,1 & 95,0 & ** & n.s. \\
\hline \multirow[t]{2}{*}{ Luft-N } & \multicolumn{2}{|c|}{$a$} & \multicolumn{2}{|c|}{$b$} & \multicolumn{2}{|c|}{$C^{* * *}$} & & & & \\
\hline & 126,3 & 82,2 & 97,3 & 64,3 & 38,1 & 35,4 & 0 & 0 & $* *$ & $* *$ \\
\hline \multirow{2}{*}{$\begin{array}{l}\mathrm{Ndfa}_{\mathrm{w}} \\
\mathrm{wPsD}\end{array}$} & & $a$ & & $b$ & $c^{*}$ & * & & & & \\
\hline & 0,65 & 0,49 & 0,56 & 0,43 & 0,36 & 0,29 & 0 & 0 & $* * *$ & n.s. \\
\hline 3. Schnitt & & & & 25.10 & .1998 & & & & & \\
\hline Boden-N & 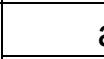 & $a$ & & $a$ & $a$ & $a$ & a & & & \\
\hline & 91,8 & 117,9 & 101,9 & 117,4 & 86,1 & 115,3 & 104,1 & 126,9 & $* *$ & n.s. \\
\hline Luft-N & 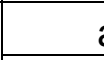 & $a$ & & $b$ & $c^{*}$ & ** & & & & \\
\hline & 152,0 & 108,8 & 121,8 & 91,3 & 44,9 & 39,2 & 0 & 0 & * & * \\
\hline $\mathrm{Ndfa}_{\mathrm{w}}$ & & $a$ & & $b$ & $c^{*}$ & * & & & & \\
\hline & 0,62 & 0,48 & 0,54 & 0,44 & 0,34 & 0,25 & 0 & 0 & $* *$ & n.s. \\
\hline
\end{tabular}

$\mathrm{Ndfa}_{\mathrm{w}}=$ gewichteter Anteil Stickstoff aus der Luft; wPsD = Wechselwirkung: Pflanzenschutz $x$ Düngung; Tukey-Test: nicht gleiche Buchstaben kennzeichnen signifikante Unterschiede mit: ${ }^{*}=P<0,05 ;{ }^{* *}=P<0,01$; ${ }^{* *}=P<0,001$; n.s. $=$ nicht signifikant 
Tab. A37: Mittelwerte $\left(\mathrm{kg} \mathrm{N}_{\min } \mathrm{ha}^{-1}\right)$ und Mittelwertvergleich der $\mathrm{CaCl}_{2}$-extrahierbaren $\mathrm{N}_{\text {min }}$-Mengen im Boden beim Anbau von Luzerne und Knaulgras in Rein- und Gemengesaat zu drei Probenahmeterminen im Jahr 1999

\begin{tabular}{|c|c|c|c|c|c|c|c|c|c|c|}
\hline \multirow{2}{*}{$\begin{array}{l}\text { Ansaatvariante } \\
\text { Düngung }\end{array}$} & \multicolumn{2}{|c|}{$4 \mathrm{~L}$} & \multicolumn{2}{|c|}{$3 \mathrm{~L} / 1 \mathrm{~K}$} & \multicolumn{2}{|c|}{$1 \mathrm{~L} / 3 \mathrm{~K}$} & \multicolumn{2}{|c|}{$4 \mathrm{~K}$} & \multirow[b]{3}{*}{ Düngung } & \multirow{2}{*}{$\begin{array}{l}\text { Ansaat- } \\
\text { variante } x\end{array}$} \\
\hline & $\mathrm{N}_{0}$ & $\mathrm{~N}_{3}$ & $\mathrm{~N}_{0}$ & $\mathrm{~N}_{3}$ & & $\mathrm{~N}_{3}$ & $\mathrm{~N}_{0}$ & $\mathrm{~N}_{3}$ & & \\
\hline Bodentiefe: & \multicolumn{8}{|c|}{ 1. Schnitt (05.07.1999) } & & \\
\hline \multirow[t]{2}{*}{$0-125 \mathrm{~cm}^{1}$} & \multicolumn{2}{|c|}{$A$} & \multicolumn{2}{|c|}{$a b$} & \multicolumn{2}{|c|}{$a b$} & \multicolumn{2}{|c|}{$b^{*}$} & & \\
\hline & 51,9 & 55,4 & 47,4 & 53,6 & 48,4 & 53,1 & 42,1 & 42,6 & n.s. & n.s. \\
\hline \multirow[t]{2}{*}{$0-50 \mathrm{~cm}^{1}$} & \multicolumn{2}{|c|}{$a$} & \multicolumn{2}{|c|}{$a b$} & \multicolumn{2}{|c|}{$b$} & \multicolumn{2}{|c|}{$\mathrm{C}^{*}$} & & \\
\hline & 23,0 & 21,9 & 19,6 & 20,3 & 19,7 & 19,1 & 17,0 & 14,5 & n.s. & n.s. \\
\hline \multirow[t]{3}{*}{$50-125 \mathrm{~cm}^{1}$} & \multicolumn{2}{|c|}{$a$} & \multicolumn{2}{|c|}{$a$} & \multicolumn{2}{|c|}{$a$} & \multicolumn{2}{|c|}{$\mathrm{a}$} & & \\
\hline & 28,9 & 33,5 & 27,8 & 33,4 & 28,7 & 34,0 & 25,1 & 23,6 & n.s. & n.s. \\
\hline & \multicolumn{8}{|c|}{ 2. Schnitt (16.08.1999) } & & \\
\hline \multirow{2}{*}{$\begin{array}{l}0-125 \mathrm{~cm}^{2} \\
\text { wPsA }\end{array}$} & \multicolumn{2}{|c|}{$a b$} & \multicolumn{2}{|c|}{$a b$} & \multicolumn{2}{|c|}{$\mathrm{a}$} & \multicolumn{2}{|c|}{$b^{* *}$} & & \\
\hline & 23,6 & 25,2 & 22,8 & 26,3 & 20,2 & 22,1 & 28,9 & 24,7 & n.s. & n.s. \\
\hline \multirow[t]{2}{*}{$0-50 \mathrm{~cm}^{2}$} & \multicolumn{2}{|c|}{$a$} & \multicolumn{2}{|c|}{$a$} & $\mathrm{~b}$ & & $b^{* *}$ & $k * *$ & & \\
\hline & 14,0 & 13,9 & 13,7 & 14,3 & 9,2 & 9,6 & 8,3 & 9,3 & n.s. & n.s. \\
\hline $50-125 \mathrm{~cm}^{1}$ & a & & a & $a$ & a & & $b^{*}$ & $k * *$ & & \\
\hline & 9,7 & 11,3 & 9,1 & 12,0 & 11,0 & 12,5 & 20,6 & 13,8 & n.s. & n.s. \\
\hline & & & 3. Sch & nitt $(0$ & 4.11 .1 & 999) & & & & \\
\hline $0-125 \mathrm{~cm}$ & a & & $a$ & $a$ & a & & a & 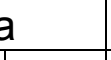 & & \\
\hline & 19,7 & 19,2 & 17,8 & 20,5 & 16,8 & 16,8 & 16,1 & 15,3 & n.s. & n.s. \\
\hline $0-50 \mathrm{~cm}^{3}$ & a & & a & $a$ & a & & $b^{*}$ & ** & & \\
\hline & 14,2 & 14,0 & 13,0 & 13,7 & 11,6 & 11,6 & 7,7 & 10,8 & n.s. & n.s. \\
\hline $50-125 \mathrm{~cm}^{2}$ & a & & a & $a$ & a & & a & $a$ & & \\
\hline & 5,5 & 5,2 & 4,8 & 6,8 & 5,2 & 5,2 & 8,4 & 9,2 & n.s. & n.s. \\
\hline
\end{tabular}


Tab. A38: Mittelwerte und Mittelwertvergleich der luft- und bodenbürtigen gesamtpflanzlichen N-Akkumulation $\left(\mathrm{kg} \mathrm{ha}^{-1}\right)$ und gewichtete Anteile Stickstoff aus der Luft $\left(\mathrm{Ndfa}_{\mathrm{w}}\right)$ beim Anbau von Luzerne und Knaulgras in Reinund Gemengesaat zu drei Schnittterminen im Jahr 1999

\begin{tabular}{|c|c|c|c|c|c|c|c|c|c|c|}
\hline Ansaatvariante & \multicolumn{2}{|c|}{$4 \mathrm{~L}$} & \multicolumn{2}{|c|}{$3 \mathrm{~L} / 1 \mathrm{~K}$} & \multicolumn{2}{|c|}{$1 \mathrm{~L} / 3 \mathrm{~K}$} & \multicolumn{2}{|c|}{$4 \mathrm{~K}$} & \multirow{3}{*}{ Düngung } & \multirow{3}{*}{$\begin{array}{l}\text { Ansaat- } \\
\text { variante } x \\
\text { Düngung }\end{array}$} \\
\hline Düngung & $\mathrm{N}_{0}$ & $\mathrm{~N}_{3}$ & $\mathrm{~N}_{0}$ & $\mathrm{~N}_{3}$ & $\mathrm{~N}_{0}$ & $\mathrm{~N}_{3}$ & $\mathrm{~N}_{0}$ & $\mathrm{~N}_{3}$ & & \\
\hline 1. Schnitt & \multicolumn{8}{|c|}{05.07 .1999} & & \\
\hline \multirow[t]{2}{*}{ Boden-N ${ }^{1}$} & \multicolumn{2}{|c|}{$a$} & \multicolumn{2}{|c|}{ a } & \multicolumn{2}{|c|}{$a$} & \multicolumn{2}{|c|}{$b^{* *}$} & & \\
\hline & 66,2 & 63,9 & 65,7 & 69,8 & 67,6 & 76,0 & 79,6 & 97,7 & n.s. & n.s. \\
\hline \multirow{2}{*}{$\begin{array}{l}\text { Luft-N } \\
\text { Ps }\end{array}$} & \multicolumn{2}{|c|}{$a$} & \multicolumn{2}{|c|}{$\mathrm{b}$} & \multicolumn{2}{|c|}{$c^{*}$} & & & \\
\hline & 162,9 & 115,4 & 124,0 & 108,3 & 60,9 & 47,6 & 0 & 0 & ** & n.s. \\
\hline \multirow[t]{2}{*}{$\mathrm{Ndfa}_{w}{ }^{2}$} & \multicolumn{2}{|c|}{$a$} & \multicolumn{2}{|c|}{$a$} & \multicolumn{2}{|c|}{$b^{* * *}$} & & & \\
\hline & 0,71 & 0,64 & 0,65 & 0,61 & 0,47 & 0,38 & 0 & 0 & * & n.s. \\
\hline 2. Schnitt & \multicolumn{8}{|c|}{16.08 .1999} & & \\
\hline \multirow[t]{2}{*}{ Boden-N } & \multicolumn{2}{|c|}{$a$} & \multicolumn{2}{|c|}{ a } & \multicolumn{2}{|c|}{$a$} & \multicolumn{2}{|c|}{$b^{*}$} & & \\
\hline & 90,5 & 92,1 & 88,6 & 99,0 & 96,6 & 105,8 & 103,7 & 121,3 & n.s. & n.s. \\
\hline \multirow[t]{2}{*}{ Luft-N } & \multicolumn{2}{|c|}{$a$} & \multicolumn{2}{|c|}{$\mathrm{b}$} & \multicolumn{2}{|c|}{$c^{* * *}$} & & & & \\
\hline & 286,5 & 222,2 & 227,6 & 202,8 & 155,5 & 104,7 & 0 & 0 & ** & n.s. \\
\hline $\mathrm{Ndfa}_{\mathrm{w}}$ & a & $a$ & a & & $b^{* *}$ & $* *$ & & & & \\
\hline & 0,76 & 0,71 & 0,72 & 0,67 & 0,62 & 0,50 & 0 & 0 & * & n.s. \\
\hline 3. Schnitt & & & & 04.11 & 1999 & & & & & \\
\hline Boden-N & $\mathrm{bc}$ & $c$ & C & & $\mathrm{ab}$ & $\mathrm{b}$ & $a^{*}$ & & & \\
\hline & 111,7 & 114,5 & 101,3 & 114,6 & 118,8 & 138,0 & 127,2 & 145,3 & n.s. & n.s. \\
\hline Luft-N & a & $a$ & a & & $b^{* *}$ & & & & & \\
\hline & 360,7 & 289,8 & 310,7 & 275,8 & 216,8 & 159,3 & 0 & 0 & ** & n.s. \\
\hline $\mathrm{Ndfa}_{\mathrm{w}}$ & a & $a$ & a & & $b^{* *}$ & & & & & \\
\hline & 0,76 & 0,72 & 0,75 & 0,71 & 0,65 & 0,54 & 0 & 0 & $* *$ & n.s. \\
\hline
\end{tabular}

$\mathrm{Ndfa}_{w}=$ gewichteter Anteil Stickstoff aus der Luft; Ps = Einfluss des Pflanzenschutzes nachweisbar; Tukey-Test: nicht gleiche Buchstaben kennzeichnen signifikante Unterschiede mit: ${ }^{*}=P<0,05$; ${ }^{* *}=P<0,01$; ${ }^{* * *}=P<0,001$; n.s. $=$ nicht signifikant; ${ }^{1}$ Originaldaten transformiert (Wurzelx), ${ }^{2}$ Originaldaten transformiert (arcsin $\mathrm{x}$ )

Tab. A39: Ergebnis der Varianzanalyse der gewichteten $\delta^{15} \mathrm{~N}$-Werte sowie multipler Vergleich der gewichteten $\delta^{15} \mathrm{~N}$-Mittelwerte in der Sprossmasse des Knaulgrases in Rein- und Gemengesaat zum dritten Schnitttermin im Jahr 1998 und 1999.

\begin{tabular}{|c|c|c|c|c|c|c|c|c|c|c|c|}
\hline \multicolumn{3}{|c|}{ Varianzanalyse } & \multirow{2}{*}{\multicolumn{3}{|c|}{$\begin{array}{l}\text { Tukey-Test } \\
\text { Ansaatvariante }\end{array}$}} & \multicolumn{6}{|c|}{ Düngung } \\
\hline & Ansaat- & & & & & $3 \mathrm{~L}$ & & & $3 \mathrm{~K}$ & & \\
\hline & Variante & Düngung & $3 \mathrm{~L} / 1 \mathrm{~K}$ & $1 \mathrm{~L} / 3 \mathrm{~K}$ & $4 \mathrm{~K}$ & $\mathrm{~N}_{0}$ & $\mathrm{~N}_{3}$ & $\mathrm{~N}_{0}$ & $\mathrm{~N}_{3}$ & $\mathrm{~N}_{0}$ & $\mathrm{~N}_{3}$ \\
\hline 1998 & $* * *$ & * & $\mathrm{A}$ & $\mathrm{A}$ & $\mathrm{B}^{\star \star \star}$ & $a$ & $a$ & $a$ & $a$ & $a$ & $a$ \\
\hline Wechselw. $^{1}$ & & S. & & & & & & & & & \\
\hline 1999 & 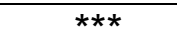 & n.s. & $A$ & $B$ & $\mathrm{C}^{* * *}$ & $a$ & $a$ & $a$ & $a$ & $a$ & $a$ \\
\hline $\begin{array}{l}\text { Wechselw. }^{1} \\
\text { Varianzanalyse: } n . s\end{array}$ & & & & & & & & & & & \\
\hline
\end{tabular}


Tab. A40: Vergleich der Werte für die Parameter Transfer-N-Menge und Anteil des Transfer-Stickstoffes an der Gesamt-N-Menge in der Sprossmasse von Knaulgras zum dritten Schnitttermin 1998 und 1999.

\begin{tabular}{|c|c|c|c|}
\hline 1998 & $\begin{array}{l}\text { Ansaat- } \\
\text { variante }\end{array}$ & Düngung & $\begin{array}{c}\text { Ansaatvariante } \\
\text { x Düngung }\end{array}$ \\
\hline${ }^{\mathrm{a}} \mathrm{N}-\mathrm{Transfer}$ & ** & * & n.s. \\
\hline${ }^{\mathrm{b}}$ Anteil $\mathrm{N}_{\text {trans }}$ & $* * *$ & ** & n.s. \\
\hline \multicolumn{4}{|l|}{1999} \\
\hline N-Transfer & n.s. & n.s. & n.s. \\
\hline Anteil $N_{\text {trans }}$ & $* * *$ & n.s. & n.s. \\
\hline
\end{tabular}

Tab. A41: Geschätzte Menge an N-Rhizodeposition die aus der Fixierung stammt $\left(\mathrm{kg} \mathrm{N} \mathrm{ha}^{-1}\right.$ ) beim Anbau von Luzerne in Rein- und Gemengesaat mit Knaulgras im Jahr 1998 und 1999.

\begin{tabular}{r|c|c|c}
\hline 1998 & $4 \mathrm{~L}$ & $3 \mathrm{~L} / 1 \mathrm{~K}$ & $1 \mathrm{~L} / 3 \mathrm{~K}$ \\
\hline $\mathrm{N}_{0}$ & 11,6 & 9,3 & 3,4 \\
\hline $\mathrm{N}_{3}$ & 8,3 & 7,0 & 3,0 \\
\hline 1999 & \multicolumn{3}{|c}{} \\
\hline $\mathrm{N}_{0}$ & 27,5 & 23,7 & 16,5 \\
\hline $\mathrm{N}_{3}$ & 22,1 & 21,0 & 12,2 \\
\hline
\end{tabular}

Tab. A42: $\quad$ Mittelwerte \pm Standardabweichung der Spross-Trockenmassen $\left(\mathrm{dt} \mathrm{ha}^{-1}\right)$ und Spross-N-Mengen $\left(\mathrm{kg} \mathrm{ha}^{-1}\right)$ der Kräuter beim Anbau der Saatwicke und des Einjährigen Weidelgrases in Rein- und Gemengesaat im Jahr 1998 und 1999

\begin{tabular}{c|c|c|c|c}
\hline 1998 & \multicolumn{2}{|c|}{ Spross-TM } & \multicolumn{2}{c}{ Spross-N } \\
\hline $\begin{array}{c}\text { Düngung } \\
\text { Ansaatvariante }\end{array}$ & $\mathrm{N}_{0}$ & $\mathrm{~N}_{3}$ & $\mathrm{~N}_{0}$ & $\mathrm{~N}_{3}$ \\
\hline $4 \mathrm{~S}$ & $0,9 \pm 1,3$ & $2,1 \pm 1,4$ & $3,0 \pm 3,8$ & $6,5 \pm 3,8$ \\
\hline $3 \mathrm{~S} / 1 \mathrm{E}$ & $1,7 \pm 1,2$ & $1,1 \pm 1,0$ & $5,0 \pm 3,8$ & $3,3 \pm 3,1$ \\
\hline $1 \mathrm{~S} / 3 \mathrm{E}$ & $0,3 \pm 0,2$ & $0,4 \pm 0,4$ & $0,9 \pm 0,7$ & $1,3 \pm 1,2$ \\
\hline $4 \mathrm{E}$ & $0,1 \pm 0,1$ & $0,1 \pm 0,1$ & $0,3 \pm 0,3$ & $0,2 \pm 0,3$ \\
\hline 1999 & \multicolumn{5}{|c}{} \\
\hline $4 \mathrm{~S}$ & $0,1 \pm 0,2$ & $0,4 \pm 0,3$ & $0,5 \pm 0,7$ & $0,4 \pm 0,5$ \\
\hline $3 \mathrm{~S} / 1 \mathrm{E}$ & $0,1 \pm 0,1$ & 0 & $0,2 \pm 0,3$ & 0 \\
\hline $1 \mathrm{~S} / 3 \mathrm{E}$ & $0,4 \pm 0,5$ & $0,4 \pm 0,4$ & $1,8 \pm 1,6$ & $0,6 \pm 0,9$ \\
\hline $4 \mathrm{E}$ & $0,7 \pm 0,6$ & $0,2 \pm 0,3$ & $1,3 \pm 1,5$ & $1,4 \pm 1,2$ \\
\hline \multicolumn{5}{|l}{}
\end{tabular}


Tab. A43: Gesamtpflanzliche Trockenmasse-Akkumulation ( $\mathrm{dt} \mathrm{ha}^{-1}$ ), TM-Erträge der Wurzel- und Stoppelmasse $\left(\mathrm{dt} \mathrm{ha}^{-1}\right)$ sowie gewichtete TM-Harvest-Indizes beim Anbau der Saatwicke und des Einjährigen Weidelgrases in Rein- und Gemengesaat am 20.10.1998

\begin{tabular}{|c|c|c|c|c|c|c|c|c|c|c|}
\hline \multirow{2}{*}{$\begin{array}{l}\text { Ansaatvariante } \\
\text { Düngung }\end{array}$} & \multicolumn{2}{|c|}{$4 S$} & \multicolumn{2}{|c|}{$3 S / 1 E$} & \multicolumn{2}{|c|}{$1 \mathrm{~S} / 3 \mathrm{E}$} & \multicolumn{2}{|c|}{$4 \mathrm{E}$} & \multirow[b]{3}{*}{ Düngung } & \multirow{3}{*}{$\begin{array}{c}\text { Ansaat- } \\
\text { variante x } \\
\text { Düngung }\end{array}$} \\
\hline & $\mathrm{N}_{0}$ & $\mathrm{~N}_{3}$ & $\mathrm{~N}_{0}$ & $\mathrm{~N}_{3}$ & $\mathrm{~N}_{0}$ & $\mathrm{~N}_{3}$ & $\mathrm{~N}_{0}$ & $\mathrm{~N}_{3}$ & & \\
\hline Ernte & \multicolumn{8}{|c|}{20.10 .1998} & & \\
\hline \multirow[t]{2}{*}{$\mathrm{TM}_{\mathrm{Bt}}$} & \multicolumn{2}{|c|}{ a } & \multicolumn{2}{|c|}{$a$} & \multicolumn{2}{|c|}{$b$} & \multicolumn{2}{|c|}{$b^{* * *}$} & & \\
\hline & 23,3 & 18,8 & 23,4 & 27,1 & 32,7 & 36,6 & 32,8 & 41,9 & * & ** \\
\hline \multirow{2}{*}{$\begin{array}{l}\text { Schnittgut-TM } \\
\text { wPsD }\end{array}$} & \multicolumn{2}{|c|}{$\mathrm{b}$} & \multicolumn{2}{|c|}{$\mathrm{b}$} & \multicolumn{2}{|c|}{$a b$} & \multicolumn{2}{|c|}{$a^{* * *}$} & & \\
\hline & 13,7 & 11,6 & 11,8 & 13,5 & 15,2 & 17,7 & 13,8 & 20,6 & n.s. & ** \\
\hline \multirow[t]{2}{*}{ Ernterest-TM } & \multicolumn{2}{|c|}{$a$} & \multicolumn{2}{|c|}{$a$} & \multicolumn{2}{|c|}{$b$} & \multicolumn{2}{|c|}{$b^{* * *}$} & & \\
\hline & 9,6 & 7,2 & 11,6 & 13,6 & 17,5 & 18,9 & 19,0 & 21,3 & n.s. & n.s. \\
\hline \multirow[t]{2}{*}{ Stoppel-TM } & \multicolumn{2}{|c|}{ n.g.v. } & \multicolumn{2}{|c|}{ n.g.v. } & \multicolumn{2}{|c|}{ n.g.v. } & \multicolumn{2}{|c|}{ n.g.v. } & & \\
\hline & 3,9 & 2,9 & 4,3 & 6,1 & 5,6 & 7,2 & 5,5 & 7,6 & n.g.v. & n.g.v. \\
\hline \multirow[t]{2}{*}{ Wurzel-TM } & \multicolumn{2}{|c|}{$\mathrm{C}$} & \multicolumn{2}{|c|}{$\mathrm{bc}$} & \multicolumn{2}{|c|}{$a b$} & \multicolumn{2}{|c|}{$a^{* * *}$} & & \\
\hline & 5,7 & 4,3 & 7,3 & 7,5 & 11,9 & 11,7 & 13,5 & 13,7 & n.s. & n.s. \\
\hline \multirow[t]{2}{*}{$\mathrm{H}_{\text {TMBtw }}$} & \multicolumn{2}{|c|}{$a$} & \multicolumn{2}{|c|}{$a b$} & $b$ & p & $b^{*}$ & $* *$ & & \\
\hline & 0,59 & 0,62 & 0,50 & 0,50 & 0,47 & 0,48 & 0,42 & 0,49 & n.s. & n.s. \\
\hline
\end{tabular}

Tab. A44: Gesamtpflanzliche Trockenmasse-Akkumulation ( $\mathrm{dt} \mathrm{ha}^{-1}$ ), TM-Erträge der Wurzel- und Stoppelmasse $\left(\mathrm{dt} \mathrm{ha}^{-1}\right)$ sowie gewichtete TM-Harvest-Indizes beim Anbau der Saatwicke und des Einjährigen Weidelgrases in Rein- und Gemengesaat am 17.10.1999

\begin{tabular}{|c|c|c|c|c|c|c|c|c|c|c|}
\hline \multirow{2}{*}{\begin{tabular}{l|} 
Ansaatvariante \\
Düngung
\end{tabular}} & \multicolumn{2}{|c|}{$4 S$} & \multicolumn{2}{|c|}{$3 \mathrm{~S} / 1 \mathrm{E}$} & \multicolumn{2}{|c|}{$1 \mathrm{~S} / 3 \mathrm{E}$} & \multicolumn{2}{|c|}{ 4E } & \multirow[b]{3}{*}{ Düngung } & \multirow{2}{*}{$\begin{array}{l}\text { Ansaat- } \\
\text { variante } x\end{array}$} \\
\hline & $\mathrm{N}_{0}$ & $\mathrm{~N}_{3}$ & $\mathrm{~N}_{0}$ & $\mathrm{~N}_{3}$ & $\mathrm{~N}_{0}$ & $\mathrm{~N}_{3}$ & $\mathrm{~N}_{0}$ & $\mathrm{~N}_{3}$ & & \\
\hline Ernte & \multicolumn{8}{|c|}{ 17.10.1999 } & & \\
\hline \multirow[t]{2}{*}{$\mathrm{TM}_{\mathrm{Bt}}$} & \multicolumn{2}{|c|}{$a$} & \multicolumn{2}{|c|}{ a } & \multicolumn{2}{|c|}{$a b$} & \multicolumn{2}{|c|}{$b^{* * *}$} & & \\
\hline & 57,5 & 48,7 & 51,7 & 48,7 & 44,8 & 46,0 & 32,5 & 38,7 & n.s. & n.s. \\
\hline \multirow[t]{2}{*}{ Schnittgut-TM } & \multicolumn{2}{|c|}{ a } & \multicolumn{2}{|c|}{$a$} & \multicolumn{2}{|c|}{ a } & \multicolumn{2}{|c|}{$b^{* * *}$} & & \\
\hline & 51,5 & 41,4 & 44,1 & 40,7 & 36,7 & 36,8 & 16,0 & 19,1 & n.s. & n.s. \\
\hline \multirow[t]{2}{*}{ Ernterest-TM $^{1}$} & \multicolumn{2}{|c|}{$\mathrm{a}$} & \multicolumn{2}{|c|}{$a$} & \multicolumn{2}{|c|}{$\mathrm{a}$} & \multicolumn{2}{|c|}{$b^{* * *}$} & & \\
\hline & 6,0 & 7,3 & 7,6 & 8,0 & 8,1 & 9,2 & 16,5 & 19,6 & n.s. & n.s. \\
\hline \multirow[t]{2}{*}{ Stoppel-TM } & \multicolumn{2}{|c|}{ n.g.v. } & \multicolumn{2}{|c|}{ n.g.v. } & \multicolumn{2}{|c|}{ n.g.v. } & \multicolumn{2}{|c|}{ n.g.v. } & & \\
\hline & 2,1 & 2,1 & 2,1 & 3,7 & 4,3 & 4,2 & 7,6 & 10,7 & n.g.v. & n.g.v. \\
\hline \multirow{2}{*}{$\begin{array}{l}\text { Wurzel-TM } \\
\text { Ps; wPsA } \\
\end{array}$} & \multicolumn{2}{|c|}{ a } & \multicolumn{2}{|c|}{$a$} & \multicolumn{2}{|c|}{$u$} & \multicolumn{2}{|c|}{$b^{* * *}$} & & \\
\hline & 3,9 & 5,2 & 5,5 & 4,3 & 3,8 & 5,0 & 8,9 & 8,9 & n.s. & n.s. \\
\hline \multirow[t]{2}{*}{$\mathrm{H}_{\text {TMBtw }}$} & \multicolumn{2}{|c|}{ a } & \multicolumn{2}{|c|}{ a } & $a$ & & $b^{\prime}$ & & & \\
\hline & 0,90 & 0,85 & 0,85 & 0,84 & 0,82 & 0,80 & 0,49 & 0,49 & n.s. & n.s. \\
\hline
\end{tabular}

Ps = Einfluss des Pflanzenschutzes nachweisbar; wPsA = Wechselwirkung: Pflanzenschutz $\times$ Ansaatvariante; $\mathrm{TM}=$ Trockenmasse; $\mathrm{H}_{\text {TMBtw }}=$ gewichteter gesamtpflanzlicher Trockenmasse-Harvest-Index; ${ }^{1}$ Originaldaten transformiert (lg $\mathrm{x})$; nicht gleiche Buchstaben kennzeichnen signifikante Unterschiede; Tukey-Test: ${ }^{*}=P<0,05$; ${ }^{* *}=P<0,01$; ${ }^{* *}=P<$ 0,$001 ;$ n.s. $=$ nicht signifikant; n.g.v. $=$ nicht gesondert verrechnet 
Tab. A45: Gesamtpflanzliche Stickstoff-Akkumulation $\left(\mathrm{N}_{\mathrm{Bt}}, \mathrm{kg} \mathrm{ha}^{-1}\right)$, StickstoffErträge im Schnittgut, in der Stoppelmasse und den Wurzeln $\left(\mathrm{kg} \mathrm{ha}^{-1}\right)$ sowie der gewichteten Stickstoff-Harvest-Indizes ( $\left.\mathrm{H}_{\mathrm{NB} \text { tw }}\right)$ beim Anbau der Saatwicke und des Einjährigen Weidelgrases in Rein- und Gemengesaat am 20.10.1998

\begin{tabular}{|c|c|c|c|c|c|c|c|c|c|c|}
\hline Ansaatvariante & \multicolumn{2}{|c|}{$4 S$} & \multicolumn{2}{|c|}{$3 \mathrm{~S} / 1 \mathrm{E}$} & \multicolumn{2}{|c|}{$1 \mathrm{~S} / 3 \mathrm{E}$} & \multicolumn{2}{|c|}{$4 \mathrm{E}$} & \multirow[b]{3}{*}{ Düngung } & \multirow{3}{*}{$\begin{array}{c}\text { Ansaat- } \\
\text { variante x } \\
\text { Düngung }\end{array}$} \\
\hline Düngung & $\mathrm{N}_{0}$ & $\mathrm{~N}_{3}$ & $\mathrm{~N}_{0}$ & $\mathrm{~N}_{3}$ & $\mathrm{~N}_{0}$ & $\mathrm{~N}_{3}$ & $\mathrm{~N}_{0}$ & $\mathrm{~N}_{3}$ & & \\
\hline Ernte & \multicolumn{8}{|c|}{20.10 .1998} & & \\
\hline \multirow{2}{*}{$\begin{array}{l}\mathrm{N}_{\mathrm{Bt}} \\
\mathrm{wPsD} \\
\end{array}$} & \multicolumn{2}{|c|}{ a } & \multicolumn{2}{|c|}{ a } & \multicolumn{2}{|c|}{ a } & \multicolumn{2}{|c|}{$a$} & & \\
\hline & 78,7 & 63,0 & 63,5 & 71,6 & 66,3 & 75,4 & 71,0 & 87,6 & n.s. & n.s. \\
\hline \multirow[t]{2}{*}{ Schnittgut-N } & \multicolumn{2}{|c|}{$a$} & \multicolumn{2}{|c|}{$b$} & \multicolumn{2}{|c|}{$b$} & \multicolumn{2}{|c|}{$a^{* *}$} & & \\
\hline & 57,0 & 45,7 & 45,2 & 49,3 & 44,1 & 51,7 & 52,8 & 62,0 & n.s. & n.s. \\
\hline \multirow[t]{2}{*}{ Ernterest-N } & \multicolumn{2}{|c|}{ a } & \multicolumn{2}{|c|}{$a$} & \multicolumn{2}{|c|}{ a } & \multicolumn{2}{|c|}{$a$} & & \\
\hline & 21,7 & 17,3 & 18,3 & 22,3 & 22,2 & 23,7 & 18,2 & 25,6 & * & ** \\
\hline Stoppel-N & 9,6 & 7,6 & 8,7 & 11,5 & 8,7 & 11,4 & 6,5 & 11,1 & n.g.v. & n.g.v. \\
\hline \multirow[t]{2}{*}{ Wurzel-N ${ }^{1}$} & \multicolumn{2}{|c|}{ a } & \multicolumn{2}{|c|}{ a } & \multicolumn{2}{|c|}{$a$} & \multicolumn{2}{|c|}{ a } & & \\
\hline & 12,1 & 9,6 & 9,6 & 10,8 & 13,5 & 12,3 & 11,7 & 14,5 & n.s. & n.s. \\
\hline \multirow{2}{*}{$\mathrm{H}_{\mathrm{NBtw}}$} & \multicolumn{2}{|c|}{ a } & \multicolumn{2}{|c|}{ a } & \multicolumn{2}{|c|}{ a } & \multicolumn{2}{|c|}{ a } & & \\
\hline & 0,72 & 0,73 & 0,71 & 0,69 & 0,67 & 0,69 & 0,74 & 0,71 & n.s. & n.s. \\
\hline
\end{tabular}

wPsD = Wechselwirkung: Düngung $\times$ Pflanzenschutz; $\mathrm{H}_{\mathrm{NBtw}}=$ gewichteter gesamtpflanzlicher Stickstoff-Harvest-Index; nicht gleiche Buchstaben kennzeichnen signifikante Unterschiede; Tukey-Test: ${ }^{*}=P<0,05 ;{ }^{* *}=P<0,01$; ${ }^{* *}=P<0,001$; n.s. $=$ nicht signifikant; ${ }^{1}$ Originaldaten transformiert $(\lg \mathrm{x}) ; \mathrm{n} . \mathrm{g.v} . \mathrm{=}$ nicht gesondert verrechnet

Tab. A46: Gesamtpflanzliche Stickstoff-Akkumulation $\left(\mathrm{N}_{\mathrm{Bt}}, \mathrm{kg} \mathrm{ha}^{-1}\right)$, StickstoffErträge im Schnittgut, in der Stoppelmasse und den Wurzeln $\left(\mathrm{kg} \mathrm{ha}^{-1}\right)$ sowie der gewichteten Stickstoff-Harvest-Indizes $\left(\mathrm{H}_{\mathrm{NBtw}}\right)$ beim Anbau der Saatwicke und des Einjährigen Weidelgrases in Rein- und Gemengesaat am 17.10.1999

\begin{tabular}{|c|c|c|c|c|c|c|c|c|c|c|}
\hline Ansaatvariante & \multicolumn{2}{|c|}{$4 S$} & \multicolumn{2}{|c|}{$3 \mathrm{~S} / 1 \mathrm{E}$} & \multicolumn{2}{|c|}{$1 \mathrm{~S} / 3 \mathrm{E}$} & \multicolumn{2}{|c|}{$4 \mathrm{E}$} & \multirow[b]{3}{*}{ Düngung } & \multirow{3}{*}{$\begin{array}{l}\text { Ansaat- } \\
\text { variante } x \\
\text { Düngung }\end{array}$} \\
\hline Düngung & $\mathrm{N}_{0}$ & $\mathrm{~N}_{3}$ & $\mathrm{~N}_{0}$ & $\mathrm{~N}_{3}$ & $\mathrm{~N}_{0}$ & $\mathrm{~N}_{3}$ & $\mathrm{~N}_{0}$ & $\mathrm{~N}_{3}$ & & \\
\hline Ernte & \multicolumn{8}{|c|}{ 17.10.1999 } & & \\
\hline \multirow{2}{*}{$\overline{\mathrm{N}_{\mathrm{Bt}}}$} & \multicolumn{2}{|c|}{$a$} & \multicolumn{2}{|c|}{$a$} & \multicolumn{2}{|c|}{ a } & \multicolumn{2}{|c|}{$b^{* * *}$} & & \\
\hline & \multicolumn{2}{|c|}{\begin{tabular}{l|l|}
155,5 & 137,7 \\
\end{tabular}} & \multicolumn{2}{|c|}{\begin{tabular}{|l|l|}
141,3 & 133,9 \\
\end{tabular}} & \multicolumn{2}{|c|}{\begin{tabular}{|l|l|}
115,3 & 122,2 \\
\end{tabular}} & 50,8 & 53,4 & n.s. & n.s. \\
\hline \multirow[t]{2}{*}{ Schnittgut-N } & \multicolumn{2}{|c|}{$a$} & \multicolumn{2}{|c|}{$a b$} & \multicolumn{2}{|c|}{$\mathrm{b}$} & \multicolumn{2}{|c|}{$\mathrm{C}^{* *}$} & & \\
\hline & 143,0 & 121,0 & 126,4 & 116,3 & 102,4 & 106,4 & 35,6 & 35,0 & n.s & n.s. \\
\hline \multirow[t]{2}{*}{ Ernterest-N ${ }^{1}$} & \multicolumn{2}{|c|}{$\mathrm{a}$} & \multicolumn{2}{|c|}{$\mathrm{a}$} & \multicolumn{2}{|c|}{$a$} & \multicolumn{2}{|c|}{$a$} & & \\
\hline & 12,5 & 16,7 & 14,9 & 17,6 & 12,9 & 15,8 & 15,2 & 18,4 & n.s. & n.s. \\
\hline Stoppel-N & 4,1 & 3,5 & 4,0 & 7,2 & 6,2 & 7,3 & 7,6 & 10,1 & n.g.v. & n.g.v. \\
\hline \multirow{2}{*}{$\begin{array}{l}\text { Wurzel-N }{ }^{1} \\
\text { Ps }\end{array}$} & \multicolumn{2}{|c|}{$a$} & \multicolumn{2}{|c|}{ a } & \multicolumn{2}{|c|}{ a } & \multicolumn{2}{|c|}{$a$} & & \\
\hline & 8,4 & 13,2 & 10,1 & 10,4 & 6,7 & 8,5 & 7,6 & 8,3 & n.s. & n.s. \\
\hline \multirow[t]{2}{*}{$\mathrm{H}_{\mathrm{NBtw}}$} & \multicolumn{2}{|c|}{$a$} & \multicolumn{2}{|c|}{$t$} & \multicolumn{2}{|c|}{$a$} & \multicolumn{2}{|c|}{$b^{* *}$} & & \\
\hline & 0,92 & 0,88 & 0,89 & 0,87 & 0,89 & 0,87 & 0,70 & 0,66 & n.s. & n.s. \\
\hline
\end{tabular}

$\mathrm{Ps}=$ Einfluss des Pflanzenschutzes nachweisbar; $\mathrm{H}_{\mathrm{NBtw}}=$ gewichteter gesamtpflanzlicher Stickstoff-Harvest-Index; nicht gleiche Buchstaben kennzeichnen signifikante Unterschiede; Tukey-Test: ${ }^{*}=P<0,05 ;{ }^{* *}=P<0,01 ;{ }^{* * *}=P<0,001$; n.s. $=$ nicht signifikant; ${ }^{1}$ Originaldaten transformiert $(\lg \mathrm{x})$; n.g.v. $=$ nicht gesondert verrechnet 
Tab. A47: Mittelwerte und Mittelwertvergleich ${ }^{1}$ der gewichteten $\delta^{15} \mathrm{~N}-$ Werte (\%o) und Anteil Stickstoff aus der Luft $\left(\mathrm{Ndfa}_{\mathrm{w}}\right)$ in der Sprossmasse von Saatwicke und Einjährigem Weidelgras in Rein- und Gemengesaat 1998 und 1999

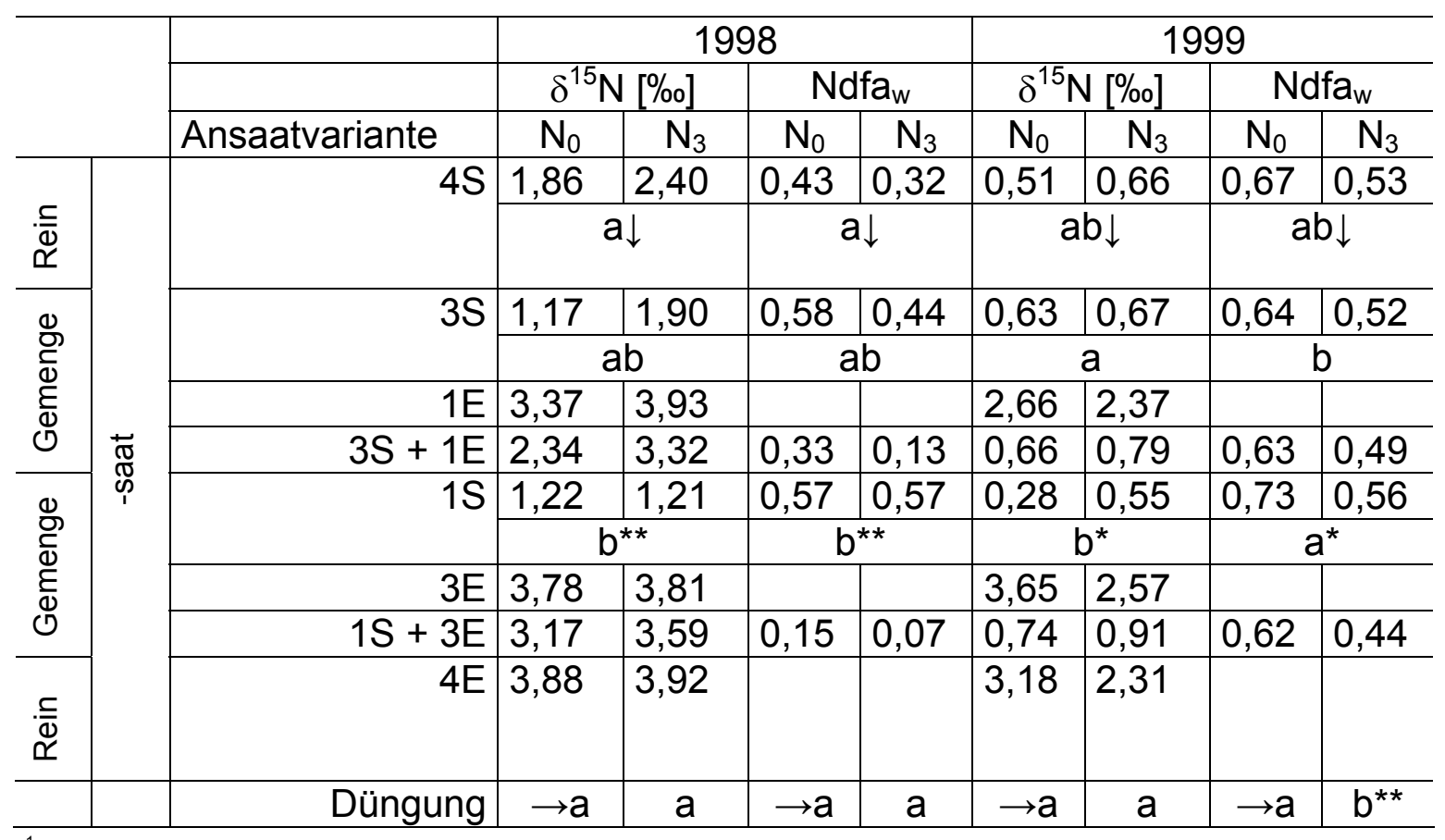

${ }^{1}$ Die Mittelwertvergleiche beziehen sich nur auf den Spross der Saatwicke; nicht gleiche Buchstaben kennzeichnen signifikante Unterschiede; ${ }^{*}=P<0,05 ;{ }^{* *}=P<0,01 ; \downarrow \rightarrow$ Leserichtung

Tab. A48: Vergleich der gewichteten gesamtpflanzlich $\delta^{15} \mathrm{~N}$-Mittelwerte beim Anbau von Saatwicke und Einjährigem Weidelgras in Rein- und Gemengesaat am 20.10.1998 (a) und am 17.10.1999 (b) sowie Ergebnis der zweifaktoriellen Varianzanalyse

\begin{tabular}{|c|c|c|c|c|c|c|}
\hline & \multicolumn{4}{|c|}{ Ansaatvariante } & \multirow[b]{2}{*}{ Düngung } & \multirow{2}{*}{$\begin{array}{l}\text { Wechsel- } \\
\text { wirkungen? }\end{array}$} \\
\hline a) & $4 S$ & $3 \mathrm{~S} / 1 \mathrm{E}$ & $1 \mathrm{~S} / 3 \mathrm{E}$ & $4 \mathrm{E}$ & & \\
\hline Ernte & $a$ & $\mathrm{~b}$ & $\mathrm{C}$ & $\mathrm{d}^{* *}$ & n.s. & * \\
\hline \multicolumn{7}{|l|}{ b) } \\
\hline Ernte $^{1}$ & a & a & a & $b^{* * *}$ & n.s. & n.s. \\
\hline
\end{tabular}


Tab. A49: $\quad$ Mittelwerte ( $\mathrm{kg} \mathrm{N}_{\min }$ ha ${ }^{-1}$ ) und Mittelwertvergleich der $\mathrm{CaCl}_{2}$-extrahierbaren $\mathrm{N}_{\text {min- }}$-Mengen im Boden vor der Aussaat der Saatwicke und des Einjährigen Weidelgrases am 21.07.1998 (a) und 09.07.1999 (b)

\begin{tabular}{|c|c|c|c|c|c|c|c|c|}
\hline Jahr & \multicolumn{4}{|c|}{1998} & \multicolumn{4}{|c|}{1999} \\
\hline Düngung & $\mathrm{N}_{0}$ & $\mathrm{~N}_{3}$ & Ps & $W$ & $\mathrm{~N}_{0}$ & $\mathrm{~N}_{3}$ & Ps & W \\
\hline \multirow[t]{2}{*}{$0-125 \mathrm{~cm}$} & $a$ & $b^{* *}$ & & & $a$ & $a$ & & \\
\hline & 28,8 & 76,2 & n.s. & n.s. & 25,5 & 36,8 & n.s. & n.s. \\
\hline \multirow[t]{2}{*}{$0-50 \mathrm{~cm}$} & $a$ & $b^{* *}$ & & & $a$ & $a$ & & \\
\hline & 22,4 & 67,0 & n.s. & n.s. & 17,3 & 25,4 & n.s. & \\
\hline \multirow[t]{2}{*}{$50-125 \mathrm{~cm}$} & $a$ & $b^{* *}$ & & & $a$ & $a$ & & \\
\hline & $6,4^{1}$ & 9,2 & n.s. & * & 8,3 & 11,3 & n.s. & 1 \\
\hline
\end{tabular}

Ps: Wirkung Pflanzenschutz; W: Wechselwirkung Pflanzenschutz x Düngung; Tukey-

Test: nicht gleiche Buchstaben kennzeichnen signifikante Unterschiede mit: ${ }^{*}=P<0,05$;

${ }^{* *}=P<0,01 ;$ n.s. $=$ nicht signifikant ${ }^{1}$ Originaldaten transformiert $(1 /$ Wurzel $\mathrm{x})$

Tab. A50: Mittelwerte ( $\mathrm{kg} \mathrm{N}_{\min } \mathrm{ha}^{-1}$ ) und Mittelwertvergleiche der $\mathrm{CaCl}_{2}$-extrahierbaren $\mathrm{N}_{\text {min }}$-Mengen im Boden beim Anbau der Saatwicke und des Einjährigen Weidelgrases in Rein- und Gemengesaat zu zwei Probenahmeterminen im Jahr 1998

\begin{tabular}{|c|c|c|c|c|c|c|c|c|c|c|}
\hline \multirow{2}{*}{$\begin{array}{l}\text { Ansaatvariante } \\
\text { Düngung }\end{array}$} & \multicolumn{2}{|c|}{$4 S$} & \multicolumn{2}{|c|}{$3 \mathrm{~S} / 1 \mathrm{E}$} & \multicolumn{2}{|c|}{$1 \mathrm{~S} / 3 \mathrm{E}$} & \multicolumn{2}{|c|}{$4 \mathrm{E}$} & \multirow{3}{*}{ Düngung } & \multirow{3}{*}{$\begin{array}{c}\text { Ansaat- } \\
\text { variante } x \\
\text { Düngung }\end{array}$} \\
\hline & $\mathrm{N}_{0}$ & $\mathrm{~N}_{3}$ & $\mathrm{~N}_{0}$ & $\mathrm{~N}_{3}$ & $\mathrm{~N}_{0}$ & $\mathrm{~N}_{3}$ & $\mathrm{~N}_{0}$ & $\mathrm{~N}_{3}$ & & \\
\hline Bodentiefe: & \multicolumn{8}{|c|}{ 1. Probenahmetermin (03.09.1998) } & & \\
\hline \multirow[t]{2}{*}{$0-125 \mathrm{~cm}^{1}$} & \multicolumn{2}{|c|}{ a } & \multicolumn{2}{|c|}{ a } & \multicolumn{2}{|c|}{$\mathrm{b}$} & \multicolumn{2}{|c|}{$b^{* *}$} & & \\
\hline & 52,4 & 75,2 & 50,6 & 73,2 & 44,9 & 48,0 & 43,3 & 53,5 & n.s. & n.s. \\
\hline \multirow[t]{2}{*}{$0-50 \mathrm{~cm}^{2}$} & \multicolumn{2}{|c|}{ a } & \multicolumn{2}{|c|}{ a } & \multicolumn{2}{|c|}{$b$} & \multicolumn{2}{|c|}{$b^{* *}$} & & \\
\hline & 41,8 & 60,8 & 40,4 & 58,5 & 34,9 & 37,8 & 34,2 & 40,8 & n.s. & n.s. \\
\hline \multirow[t]{2}{*}{$50-125 \mathrm{~cm}$} & \multicolumn{2}{|c|}{$a$} & \multicolumn{2}{|c|}{$a$} & \multicolumn{2}{|c|}{$b$} & \multicolumn{2}{|c|}{$a b$} & & \\
\hline & 10,6 & 14,4 & 10,2 & 14,7 & 10,0 & 10,2 & 9,1 & 12,7 & n.s. & n.s. \\
\hline Ernte & \multicolumn{8}{|c|}{ 2. Probenahmetermin (20.10.1998) } & & \\
\hline \multirow[t]{2}{*}{$0-125 \mathrm{~cm}^{1}$} & \multicolumn{2}{|c|}{ a } & \multicolumn{2}{|c|}{$b$} & \multicolumn{2}{|c|}{ bc } & \multicolumn{2}{|c|}{$c^{* *}$} & & \\
\hline & 43,2 & 73,7 & 31,9 & 46,6 & 23,9 & 43,0 & 22,6 & 35,2 & * & n.s. \\
\hline \multirow[t]{2}{*}{$0-50 \mathrm{~cm}^{2}$} & \multicolumn{2}{|c|}{ a } & \multicolumn{2}{|c|}{$b$} & \multicolumn{2}{|c|}{ C } & \multicolumn{2}{|c|}{$\mathrm{C}^{* *}$} & & \\
\hline & 16,6 & 23,4 & 14,1 & 14,4 & 9,5 & 12,0 & 9,6 & 13,6 & n.s. & n.s. \\
\hline \multirow{2}{*}{$\begin{array}{l}50-125 \mathrm{~cm}^{2} \\
\text { Ps }\end{array}$} & \multicolumn{2}{|c|}{$a$} & b & & $\mathrm{bc}$ & & $C^{*}$ & & & \\
\hline & 26,6 & 50,3 & 17,8 & 32,2 & 14,4 & 31,0 & 13,0 & 21,6 & * & n.s. \\
\hline
\end{tabular}

Ps = Einfluss des Pflanzenschutzes nachweisbar; Tukey-Test: nicht gleiche Buchstaben kennzeichnen signifikante Unterschiede mit: ${ }^{*}=P<0,05 ;{ }^{* *}=P<0,01 ;{ }^{* *}=P<0,001$; n.s. $=$ nicht signifikant; ${ }^{1}$ Originaldaten transformiert $(\operatorname{lgx}) ;{ }^{2}$ Originaldaten transformiert (1/Wurzel $x)$ 
Tab. A51: Mittelwerte und Mittelwertvergleiche der luft- und bodenbürtigen gesamtpflanzlichen N-Akkumulation ( $\left.\mathrm{kg} \mathrm{ha}^{-1}\right)$ und gewichtete Anteile Stickstoff aus der Luft ( $\left.\mathrm{Ndfa}_{w}\right)$ beim Anbau der Saatwicke und des Einjährigen Weidelgrases in Rein- und Gemengesaat am 20.10.1998

\begin{tabular}{|c|c|c|c|c|c|c|c|c|c|c|}
\hline Ansaatvariante & \multicolumn{2}{|c|}{$4 S$} & \multicolumn{2}{|c|}{$3 S / 1 E$} & \multicolumn{2}{|c|}{$1 S / 3 E$} & \multicolumn{2}{|c|}{$4 \mathrm{E}$} & \multirow{3}{*}{ Düngung } & \multirow{3}{*}{$\begin{array}{c}\text { Ansaat- } \\
\text { variante } x \\
\text { Düngung }\end{array}$} \\
\hline Düngung & $\mathrm{N}_{0}$ & $\mathrm{~N}_{3}$ & $\mathrm{~N}_{0}$ & $\mathrm{~N}_{3}$ & $\mathrm{~N}_{0}$ & $\mathrm{~N}_{3}$ & $\mathrm{~N}_{0}$ & $\mathrm{~N}_{3}$ & & \\
\hline Ernte & \multicolumn{8}{|c|}{20.10 .1998} & & \\
\hline \multirow{2}{*}{$\begin{array}{l}\text { Boden-N } \\
\text { wPsD }\end{array}$} & \multicolumn{2}{|c|}{$a$} & \multicolumn{2}{|c|}{$a$} & \multicolumn{2}{|c|}{$a b$} & \multicolumn{2}{|c|}{$b^{* * *}$} & & \\
\hline & 42,8 & 40,6 & 41,1 & 61,0 & 55,6 & 70,5 & 71,0 & 87,6 & n.s. & n.s. \\
\hline \multirow[t]{2}{*}{ Luft-N ${ }^{1}$} & \multicolumn{2}{|c|}{$a$} & \multicolumn{2}{|c|}{$a b$} & \multicolumn{2}{|c|}{$b^{* * *}$} & & & & \\
\hline & 35,9 & 22,4 & 22,4 & 10,6 & 10,7 & 4,9 & - & - & n.s. & n.s. \\
\hline \multirow[t]{2}{*}{$\mathrm{Ndfa}_{\mathrm{w}}{ }^{1}$} & \multicolumn{2}{|c|}{ a } & \multicolumn{2}{|c|}{$b$} & \multicolumn{2}{|c|}{$\mathrm{C}^{* * *}$} & & & & \\
\hline & 0,46 & 0,36 & 0,35 & 0,15 & 0,16 & 0,07 & - & - & * & n.s. \\
\hline
\end{tabular}

$\mathrm{wPsD}=$ Wechselwirkung: Pflanzenschutz $\times$ Düngung; $\mathrm{Ndfa}_{\mathrm{w}}=$ gewichteter Anteil Stickstoff aus der Luft; Tukey-Test: nicht gleiche Buchstaben kennzeichnen signifikante Unterschiede mit: ${ }^{*}=P<0,05 ;{ }^{* *}=P<0,01 ;{ }^{* *}=P<0,001$; n.s. $=$ nicht signifikant; ${ }^{1}$ Originaldaten transformiert (Wurzelx), ${ }^{2}$ Originaldaten transformiert (arcsin $\left.\mathrm{x}\right)$;

Tab. A52: Mittelwerte ( $\mathrm{kg} \mathrm{N}_{\min } \mathrm{ha}^{-1}$ ) und Mittelwertvergleiche der $\mathrm{CaCl}_{2}$-extrahierbaren $\mathrm{N}_{\text {min }}$-Mengen im Boden beim Anbau der Saatwicke und des Einjährigen Weidelgrases in Rein- und Gemengesaat zu zwei Probenahmeterminen im Jahr 1999

\begin{tabular}{|c|c|c|c|c|c|c|c|c|c|c|}
\hline \multirow{2}{*}{$\begin{array}{l}\text { Ansaatvariante } \\
\text { Düngung }\end{array}$} & \multicolumn{2}{|c|}{$4 S$} & \multicolumn{2}{|c|}{$3 S / 1 E$} & \multicolumn{2}{|c|}{$1 \mathrm{~S} / 3 \mathrm{E}$} & \multicolumn{2}{|c|}{$4 \mathrm{E}$} & \multirow[b]{3}{*}{ Düngung } & \multirow{2}{*}{$\begin{array}{l}\text { Ansaat- } \\
\text { variante x }\end{array}$} \\
\hline & $\mathrm{N}_{0}$ & $\mathrm{~N}_{3}$ & $\mathrm{~N}_{0}$ & $\mathrm{~N}_{3}$ & $\mathrm{~N}_{0}$ & $\mathrm{~N}_{3}$ & $\mathrm{~N}_{0}$ & $\mathrm{~N}_{3}$ & & \\
\hline Bodentiefe: & \multicolumn{8}{|c|}{ 1. Probenahmetermin (24.08.1999) } & & \\
\hline \multirow{2}{*}{$0-125 \mathrm{~cm}^{1}$} & \multicolumn{2}{|c|}{ a } & \multicolumn{2}{|c|}{$a$} & \multicolumn{2}{|c|}{$a$} & \multicolumn{2}{|c|}{ a } & & \\
\hline & 35,5 & 46,8 & 35,9 & 55,4 & 37,5 & 47,8 & 38,1 & 51,9 & * & n.s. \\
\hline \multirow[t]{2}{*}{$0-50 \mathrm{~cm}^{1}$} & \multicolumn{2}{|c|}{ a } & \multicolumn{2}{|c|}{ a } & \multicolumn{2}{|c|}{ a } & \multicolumn{2}{|c|}{$\mathrm{a}$} & & \\
\hline & 25,2 & 32,3 & 26,8 & 40,5 & 26,2 & 33,8 & 28,5 & 38,6 & * & n.s. \\
\hline \multirow[t]{2}{*}{$50-125 \mathrm{~cm}^{2}$} & \multicolumn{2}{|c|}{ a } & \multicolumn{2}{|c|}{$\mathrm{a}$} & \multicolumn{2}{|c|}{ a } & \multicolumn{2}{|c|}{$\mathrm{a}$} & & \\
\hline & 10,3 & 14,5 & 9,1 & 14,9 & 11,3 & 14,0 & 9,6 & 13,3 & * & n.s. \\
\hline Ernte & \multicolumn{8}{|c|}{ 2. Probenahmetermin (17.10.1999) } & & \\
\hline \multirow[t]{2}{*}{$0-125 \mathrm{~cm}^{3}$} & \multicolumn{2}{|c|}{ a } & \multicolumn{2}{|c|}{ a } & \multicolumn{2}{|c|}{$a b$} & \multicolumn{2}{|c|}{$b^{* *}$} & & \\
\hline & 29,2 & 36,8 & 27,7 & 39,3 & 24,1 & 35,0 & 15,2 & 21,6 & * & n.s. \\
\hline \multirow[t]{2}{*}{$0-50 \mathrm{~cm}$} & \multicolumn{2}{|c|}{ a } & \multicolumn{2}{|c|}{ a } & \multicolumn{2}{|c|}{$b$} & \multicolumn{2}{|c|}{$C^{*}$} & & \\
\hline & 19,1 & 20,9 & 18,5 & 21,2 & 12,2 & 17,9 & 7,5 & 9,5 & * & n.s. \\
\hline \multirow{2}{*}{$\begin{array}{l}50-125 \mathrm{~cm}^{3} \\
\text { WPsD }\end{array}$} & \multicolumn{2}{|c|}{$a b$} & & $a$ & a & $a$ & $\mathrm{~b}$ & )$^{*}$ & & \\
\hline & 10,1 & 15,9 & 9,2 & 18,1 & 11,9 & 17,1 & 7,7 & 12,1 & $* * \star$ & n.s. \\
\hline
\end{tabular}

wPsD = Wechselwirkung: Pflanzenschutz $\times$ Düngung; Tukey-Test: nicht gleiche Buchstaben kennzeichnen signifikante Unterschiede mit: ${ }^{*}=P<0,05 ;{ }^{* *}=P<0,01 ;{ }^{* *}=P<0,001$; n.s. $=$ nicht signifikant; ${ }^{1}$ Originaldaten transformiert $(1 /$ Wurzel $\mathrm{x}) ;{ }^{2}$ Originaldaten transformiert $(\lg \mathrm{x}) ;{ }^{3}$ Originaldaten transformiert (Wurzel $\left.\mathrm{x}\right)$ 
Tab. A53: Mittelwerte und Mittelwertvergleich der luft- und bodenbürtigen gesamtpflanzlichen N-Akkumulation $\left(\mathrm{kg} \mathrm{ha}^{-1}\right)$ und gewichtete Anteile Stickstoff aus der Luft $\left(\mathrm{Ndfa}_{w}\right)$ beim Anbau der Saatwicke und des Einjährigen Weidelgrases in Rein- und Gemengesaat am 17.10.1999

\begin{tabular}{|c|c|c|c|c|c|c|c|c|c|c|}
\hline Ansaatvariante & \multicolumn{2}{|c|}{$4 S$} & \multicolumn{2}{|c|}{$3 \mathrm{~S} / 1 \mathrm{E}$} & \multicolumn{2}{|c|}{$1 \mathrm{~S} / 3 \mathrm{E}$} & \multicolumn{2}{|c|}{$4 \mathrm{E}$} & \multirow{3}{*}{ Düngung } & \multirow{3}{*}{$\begin{array}{l}\text { Ansaat- } \\
\text { variante } x \\
\text { Düngung }\end{array}$} \\
\hline Düngung & $\mathrm{N}_{0}$ & $\mathrm{~N}_{3}$ & $\mathrm{~N}_{0}$ & $\mathrm{~N}_{3}$ & $\mathrm{~N}_{0}$ & $\mathrm{~N}_{3}$ & $\mathrm{~N}_{0}$ & $\mathrm{~N}_{3}$ & & \\
\hline Ernte & \multicolumn{8}{|c|}{ 17.10.1999 } & & \\
\hline \multirow{2}{*}{$\begin{array}{l}\text { Boden-N } \\
\text { Ps }\end{array}$} & \multicolumn{2}{|c|}{ a } & \multicolumn{2}{|c|}{ a } & \multicolumn{2}{|c|}{ a } & \multicolumn{2}{|c|}{ a } & & \\
\hline & 48,5 & 63,3 & 50,0 & 65,4 & 42,0 & 63,9 & 50,8 & 53,4 & n.s. & n.s. \\
\hline \multirow[t]{2}{*}{ Luft-N } & \multicolumn{2}{|c|}{$a$} & \multicolumn{2}{|c|}{$a b$} & \multicolumn{2}{|c|}{$b^{* *}$} & & & & \\
\hline & 107,0 & 74,4 & 91,3 & 68,4 & 73,3 & 58,3 & 0 & 0 & * & n.s. \\
\hline \multirow[t]{2}{*}{$\mathrm{Ndfa}_{\mathrm{w}}$} & \multicolumn{2}{|c|}{ a } & \multicolumn{2}{|c|}{$d$} & \multicolumn{2}{|c|}{ a } & & & & \\
\hline & 0,69 & 0,54 & 0,65 & 0,51 & 0,64 & 0,48 & 0 & 0 & ** & n.s. \\
\hline
\end{tabular}

Ps = Einfluss des Pflanzenschutzes nachweisbar; Ndfa $_{w}=$ gewichteter Anteil Stickstoff aus der Luft; Tukey-Test: nicht gleiche Buchstaben kennzeichnen signifikante Unterschiede mit: ${ }^{*}=P<0,05 ;{ }^{* *}=P<0,01 ;{ }^{* * *}=P<0,001$; n.s. $=$ nicht signifikant; ${ }^{1}$ Originaldaten transformiert (Wurzelx), ${ }^{2}$ Originaldaten transformiert $(\arcsin \mathrm{x})$

Tab. A54: Ergebnis der Varianzanalyse der gewichteten $\delta^{15} \mathrm{~N}-$ Werte in der Sprossmasse des Einjährigen Weidelgrases in Rein- und Gemengesaat im Jahr 1998 und 1999

\begin{tabular}{l|c|c|c}
\hline & $\begin{array}{c}\text { Ansaat- } \\
\text { variante }\end{array}$ & Düngung & $\begin{array}{c}\text { Ansaatvariante } \\
\text { x Düngung }\end{array}$ \\
\hline 1998 & n.s. & n.s. & n.s. \\
\hline 1999 & n.s. & n.s. & n.s. \\
\hline n.s. = nicht signifikant
\end{tabular}

Tab. A55: Geschätzte Menge an N-Rhizodeposition, die aus der Fixierung stammt $\left(\mathrm{kg} \mathrm{N} \mathrm{ha}^{-1}\right)$, beim Anbau von Saatwicke in Rein- und Gemengesaat mit Einjährigem Weidelgras im Jahr 1998 und 1999

\begin{tabular}{r|c|c|c}
\hline 1998 & $4 \mathrm{~S}$ & $3 \mathrm{~S} / 1 \mathrm{E}$ & $1 \mathrm{~S} / 3 \mathrm{E}$ \\
\hline $\mathrm{N}_{0}$ & 6,4 & 4,0 & 1,9 \\
\hline $\mathrm{N}_{3}$ & 4,0 & 1,9 & 0,9 \\
\hline 1999 & \multicolumn{3}{|c}{} \\
\hline $\mathrm{N}_{0}$ & 19,2 & 16,3 & 13,1 \\
\hline $\mathrm{N}_{3}$ & 13,3 & 12,3 & 10,4 \\
\hline
\end{tabular}


Tab. A56: Variationskoeffizienten (cv $\% \quad n=6$ Parzellen/Variante) bei der Wurzelmassenbestimmung von Ackerbohne, Luzerne und Saatwicke in Rein- und Gemengesaat mit Hafer, Knaulgras und Einjährigem Weidelgras.

\begin{tabular}{|c|c|c|c|c|}
\hline \multirow{2}{*}{$\begin{array}{l}\text { Tiefenstufe in cm } \\
\text { Düngungsstufe }\end{array}$} & \multicolumn{2}{|c|}{$0-25$} & \multicolumn{2}{|c|}{$25-50$} \\
\hline & $N_{0}$ & $\mathrm{~N}_{3}$ & $\mathrm{~N}_{0}$ & $\mathrm{~N}_{3}$ \\
\hline Ackerbohne & \multicolumn{4}{|c|}{1998} \\
\hline $4 \mathrm{~A}$ & 6.7 & 11.8 & 98.5 & 57.0 \\
\hline $3 \mathrm{~A} / 1 \mathrm{H}$ & 18.2 & 17.8 & 30.3 & 52.2 \\
\hline $1 \mathrm{~A} / 3 \mathrm{H}$ & 17.0 & 14.8 & 81.4 & 73.9 \\
\hline $\mathrm{H}$ & 19.7 & 4.6 & 25.3 & 22.1 \\
\hline & \multicolumn{4}{|c|}{1999} \\
\hline $4 \mathrm{~A}$ & 27.6 & 35.2 & 77.5 & 57.9 \\
\hline $3 \mathrm{~A} / 1 \mathrm{H}$ & 13.5 & 17.5 & 30.1 & 32.0 \\
\hline $1 \mathrm{~A} / 3 \mathrm{H}$ & 6.7 & 54.4 & 61.0 & 40.8 \\
\hline $\mathrm{H}$ & 18.4 & 5.0 & 8.6 & 34.8 \\
\hline Luzerne & \multicolumn{4}{|c|}{1998} \\
\hline $4 \mathrm{~L}$ & 16.3 & 22.2 & 66.4 & 73.0 \\
\hline $3 \mathrm{~L} / 1 \mathrm{~K}$ & 26.3 & 38.4 & 68.5 & 64.8 \\
\hline $1 \mathrm{~L} / 3 \mathrm{~K}$ & 11.2 & 17.2 & 47.3 & 67.3 \\
\hline $\mathrm{K}$ & 23.9 & 22.8 & 40.4 & 21.4 \\
\hline & \multicolumn{4}{|c|}{1999} \\
\hline $4 \mathrm{~L}$ & 32.5 & 33.3 & 45.0 & 47.8 \\
\hline $3 \mathrm{~L} / 1 \mathrm{~K}$ & 61.0 & 19.4 & 45.6 & 50.6 \\
\hline $1 \mathrm{~L} / 3 \mathrm{~K}$ & 20.6 & 58.9 & 24.8 & 55.4 \\
\hline $\mathrm{K}$ & 36.9 & 35.6 & \begin{tabular}{|l|l|}
44.7 \\
\end{tabular} & 74.9 \\
\hline atwicke & \multicolumn{4}{|c|}{1998} \\
\hline $4 \mathrm{~S}$ & 32.5 & 43.5 & 99.6 & 9.2 \\
\hline $3 \mathrm{~S} / 1 \mathrm{E}$ & 27.6 & 53.4 & 61.4 & 58.4 \\
\hline $1 \mathrm{~S} / 3 \mathrm{E}$ & 37.9 & 60.7 & 41.2 & 37.3 \\
\hline$E$ & 46.0 & 38.7 & 34.2 & 51.1 \\
\hline & \multicolumn{4}{|c|}{1999} \\
\hline $4 S$ & 32.6 & 56.6 & 29.6 & 36.7 \\
\hline $3 \mathrm{~S} / 1 \mathrm{E}$ & 29.7 & 64.7 & 21.9 & 79.9 \\
\hline $1 \mathrm{~S} / 3 \mathrm{E}$ & 67.1 & 61.8 & 52.8 & 58.9 \\
\hline$E$ & 59.7 & 26.1 & 35.7 & 58.3 \\
\hline
\end{tabular}


Tab. A57: Kennwerte des pflanzlichen N-Haushaltes (Spross und Wurzeln) und vereinfachte N-Flächenbilanzsalden für Ackerbohne, Luzerne und Saatwicke in Reinsaat

\begin{tabular}{|c|c|c|c|c|c|c|}
\hline & $\begin{array}{c}{ }^{1} \mathrm{~N}_{\mathrm{Bt}} \\
{\left[\mathrm{kg} \mathrm{ha}^{-1}\right]}\end{array}$ & ${ }^{2} \mathrm{H}_{\mathrm{NBt}}$ & ${ }^{3} \mathrm{Ndfa}$ & $\begin{array}{c}{ }^{4} \mathrm{~S} \\
{\left[\mathrm{~kg} \mathrm{~N} \mathrm{ha}^{-1}\right]}\end{array}$ & $\begin{array}{l}{ }^{5} \text { Schätz- } \\
\text { methoden }\end{array}$ & $\begin{array}{c}\text { Angaben bzw. } \\
\text { Berechnungen aus }\end{array}$ \\
\hline \multirow{11}{*}{ 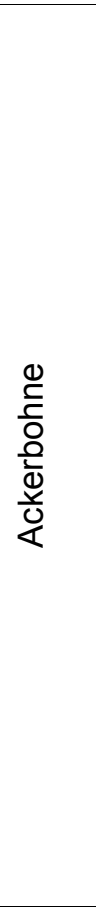 } & $\begin{array}{l}190 \text { bis } \\
293\end{array}$ & n.e. & $\begin{array}{l}0,57 \text { bis } \\
0,74\end{array}$ & -9 bis +92 & ${ }^{15} \mathrm{~N}-$ IDM & AMANUEL et al. 2000 \\
\hline & $\begin{array}{l}230 \text { bis } \\
418\end{array}$ & $\begin{array}{l}0,72 \text { bis } \\
0,75\end{array}$ & $\begin{array}{l}0,69 \text { bis } \\
0,76\end{array}$ & $\begin{array}{l}-7,5 \text { bis } \\
6,7\end{array}$ & N-DM & HAUSER 1987 \\
\hline & $\begin{array}{l}173 \text { bis } \\
292\end{array}$ & $\begin{array}{l}0,56 \text { bis } \\
0,73\end{array}$ & $\begin{array}{l}0,28 \text { bis } \\
0,34\end{array}$ & $\begin{array}{l}-141 \text { bis - } \\
50\end{array}$ & ${ }^{15} \mathrm{~N}-$ IDM & HAYNES et al. 1993 \\
\hline & $\begin{array}{l}258 \text { bis } \\
288\end{array}$ & 0,74 & 0,80 & +22 bis +25 & ${ }^{15} \mathrm{~N}-$ IDN & HUBER 1988, 1989 \\
\hline & $\begin{array}{l}140,9 \text { bis } \\
320\end{array}$ & $\begin{array}{l}0,42 \text { bis } \\
0,78\end{array}$ & $\begin{array}{l}0,44 \text { bis } \\
0,87\end{array}$ & $\begin{array}{l}-24,8 \text { bis } \\
+75,6\end{array}$ & $\delta^{15} \mathrm{~N}$ & JOST 2003 \\
\hline & $\begin{array}{l}240,5 \text { bis } \\
298,4\end{array}$ & $\begin{array}{l}75,1 \text { bis } \\
77,1\end{array}$ & $\begin{array}{l}0,66 \text { bis } \\
0,87\end{array}$ & $\begin{array}{l}-31,5 \text { bis } \\
+26,2\end{array}$ & $\delta^{15} \mathrm{~N}$ & RÄTZ 1998 \\
\hline & $\begin{array}{l}307 \text { bis } \\
505\end{array}$ & $\begin{array}{l}0,58 \text { bis } \\
0,65\end{array}$ & $\begin{array}{l}0,75 \text { bis } \\
0,81\end{array}$ & $\begin{array}{l}+29 \text { bis } \\
+115\end{array}$ & $\delta^{15} \mathrm{~N}$ & $\begin{array}{l}\text { SCHMIDTKE 1996, } \\
\text { KILIAN } 1994\end{array}$ \\
\hline & $\begin{array}{l}303,5 \text { bis } \\
383,7\end{array}$ & $\begin{array}{l}0,72 \text { bis } \\
0,73\end{array}$ & $\begin{array}{l}0,75 \text { bis } \\
0,92\end{array}$ & $\begin{array}{l}+7,5 \text { bis } \\
+70,4\end{array}$ & $\delta^{15} \mathrm{~N}$ & SCHMIDTKE 1999 \\
\hline & $\begin{array}{l}171,3 \text { bis } \\
391,7\end{array}$ & $\begin{array}{l}0,28 \text { bis } \\
0,76\end{array}$ & $\begin{array}{l}0,50 \text { bis } \\
0,86\end{array}$ & $\begin{array}{l}-0,3 \text { bis } \\
+44,0\end{array}$ & $\delta^{15} \mathrm{~N}$ & SCHMIDTKE et al. 2000 \\
\hline & $\begin{array}{l}107 \text { bis } \\
161\end{array}$ & $\begin{array}{l}0,17 \text { bis } \\
0,78\end{array}$ & n.e. & -20 bis +57 & $\begin{array}{l}\text { AV u. } \\
{ }^{15} \mathrm{~N}-\text { IDN }\end{array}$ & SMITH et al. 1997 \\
\hline & $\begin{array}{l}284 \text { bis } \\
340\end{array}$ & $\begin{array}{l}0,35 \text { bis } \\
0,43\end{array}$ & $\begin{array}{l}0,69 \text { bis } \\
0,88\end{array}$ & $\begin{array}{l}+79 \text { bis } \\
+157\end{array}$ & $\delta^{15} \mathrm{~N}$ & TURPIN et al. 2002 \\
\hline \multirow{6}{*}{ 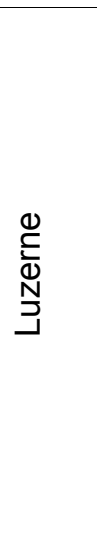 } & $\begin{array}{l}478 \text { bis } \\
539^{6}\end{array}$ & $\begin{array}{l}0,66 \text { bis } \\
0,74\end{array}$ & $\begin{array}{l}0,74 \text { bis } \\
0,77\end{array}$ & $\begin{array}{l}+7 \text { bis } \\
+40\end{array}$ & N-DM & $\begin{array}{l}\text { MANZKE } 1990 \\
\text { SCHREIBER } 1992 \\
\text { CLAUPEIN1994 }\end{array}$ \\
\hline & $\begin{array}{l}280 \text { bis } \\
486^{7}\end{array}$ & $\begin{array}{l}0,37 \text { bis } \\
0,74\end{array}$ & $\begin{array}{l}0,28 \text { bis } \\
0,62\end{array}$ & -107 bis +51 & ${ }^{15} \mathrm{~N}-$ IDM & KELNER et al. 1995 \\
\hline & $\begin{array}{l}274 \text { bis } \\
666^{8}\end{array}$ & $\begin{array}{l}0,33 \text { bis } \\
0,52\end{array}$ & $\begin{array}{l}0,62 \text { bis } \\
0,78\end{array}$ & $\begin{array}{l}+83 \text { bis } \\
+181\end{array}$ & ${ }^{15} \mathrm{~N}-$ IDM & KELNER et al. 1997 \\
\hline & $\begin{array}{l}320^{8} \text { bis } \\
425\end{array}$ & $\begin{array}{l}0,43 \text { bis } \\
0,86\end{array}$ & \begin{tabular}{|l}
0,59 bis \\
0,75
\end{tabular} & -59 bis +66 & $\begin{array}{l}{ }^{15} \mathrm{~N}-\text { IDM } \\
\text { DM, } \delta^{15} \mathrm{~N}-\end{array}$ & WALLEY et al. 1996 \\
\hline & $\begin{array}{l}126,7^{9} \text { bis } \\
598,6\end{array}$ & $\begin{array}{l}0,54 \text { bis } \\
0,83\end{array}$ & $\begin{array}{l}0,34 \text { bis } \\
0,80\end{array}$ & $\begin{array}{l}+114,8 \text { bis } \\
-191,1\end{array}$ & $\delta^{15} \mathrm{~N}$ & JUNG $2003^{11}$ \\
\hline & $\begin{array}{l}355,1 \text { bis } \\
363,1^{7}\end{array}$ & $\begin{array}{l}0,76 \text { bis } \\
0,80\end{array}$ & $\begin{array}{l}0,58 \text { bis } \\
0,68\end{array}$ & $\begin{array}{l}-16,6 \text { bis } \\
-71,9\end{array}$ & $\delta^{15} \mathrm{~N}$ & SCHMIDTKE 1999 \\
\hline 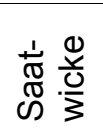 & 70 bis $88^{10}$ & $\begin{array}{l}0,45 \text { bis } \\
0,47\end{array}$ & $\begin{array}{l}0,39 \text { bis } \\
0,56\end{array}$ & -9 bis +18 & N-DM & MANZKE 1990 \\
\hline
\end{tabular}

${ }^{1} \mathrm{~N}_{\mathrm{Bt}}=$ gesamtpflanzlicher Stickstoff; ${ }^{2} \mathrm{H}_{\mathrm{NBt}}=$ Stickstoff-Harvest-Index; ${ }^{3} \mathrm{Ndfa}=$ Anteil Stickstoff aus der Luft; ${ }^{4} \mathrm{~S}=$ vereinfachter N-Flächenbilanzsaldo; ${ }^{5}$ Schätzmethoden für Ndfa: N-DM = N-Differenzmethode; ${ }^{15} \mathrm{~N}$-IDM $={ }^{15} \mathrm{~N}$-Isotopenverdünngungsmethode; $\delta^{15} \mathrm{~N}=$ Delta- ${ }^{15} \mathrm{~N}$-Methode; $\mathrm{AV}=\mathrm{A}$ value Methode $;{ }^{6} 1$. Hauptnutzungsjahr; ${ }^{7}$ Ansaatjahr; ${ }^{8}$ Ansaatjahr, 1. und 2. Hauptnutzungsjahr, ${ }^{9}$ erstes Hauptnutzungsjahr; ${ }^{10}$ Stoppelansaat; ${ }^{11}$ ohne Dasselsbruch 
Tab. A58: Differenz im residualen $\mathrm{N}_{\text {min }}$-Stickstoff $\left(\mathrm{kg} \mathrm{N} \mathrm{ha}^{-1}\right)$ zur Ernte im Boden zwischen den Ansaaten mit Ackerbohne (A), Luzerne (L) und Saatwicke $(S)$ in Rein- und Gemengesaat mit Hafer $(H)$, Knaulgras $(K)$ und $(E)$ Einjährigem Weidelgras und den Reinsaaten der entsprechenden Referenzpflanzen in 1998 und 1999

\begin{tabular}{|c|c|c|c|c|c|c|}
\hline Düngung & $\mathrm{N}_{0}$ & $\mathrm{~N}_{3}$ & $\mathrm{~N}_{0}$ & $\mathrm{~N}_{3}$ & $\mathrm{~N}_{0}$ & $\mathrm{~N}_{3}$ \\
\hline Ackerbohne & \multicolumn{2}{|c|}{$4 \mathrm{~A}$} & \multicolumn{2}{|c|}{$3 \mathrm{~A} / 1 \mathrm{H}$} & \multicolumn{2}{|c|}{$1 \mathrm{~A} / 3 \mathrm{H}$} \\
\hline Bodentiefe: & \multicolumn{6}{|c|}{ Ernte (10.08.1998) } \\
\hline $0-125 \mathrm{~cm}$ & 46,0 & 97,2 & 33,8 & 52,4 & 9,1 & 17,9 \\
\hline $0-50 \mathrm{~cm}$ & 16,6 & 26,8 & 13,7 & 12,0 & 2,7 & 4,1 \\
\hline $50-125 \mathrm{~cm}$ & 29,5 & 70,5 & 20,1 & 40,5 & 6,3 & 14,0 \\
\hline Bodentiefe: & \multicolumn{6}{|c|}{ Ernte (26.07.1999) } \\
\hline $0-125 \mathrm{~cm}$ & 46,7 & 54,6 & 15,3 & 22,9 & 3,9 & 4,8 \\
\hline $0-50 \mathrm{~cm}$ & 14,9 & 15,9 & 6,2 & 5,6 & 1,1 & 0,4 \\
\hline $50-125 \mathrm{~cm}$ & 31,7 & 38,7 & 9,1 & 17,3 & 2,9 & 4,3 \\
\hline Luzerne & & & & $1 \mathrm{~K}$ & & $3 \mathrm{~K}$ \\
\hline Bodentiefe: & \multicolumn{6}{|c|}{ 3. Schnitt (25.10.1998) } \\
\hline $0-125 \mathrm{~cm}$ & 6,1 & 4,7 & 5,1 & 1,3 & 0,0 & 3,0 \\
\hline $0-50 \mathrm{~cm}$ & 4,4 & 3,4 & 1,7 & 0,5 & $-1,0$ & 0,6 \\
\hline $50-125 \mathrm{~cm}$ & 1,6 & $-1,0$ & 3,3 & $-1,5$ & 0,9 & 0,1 \\
\hline Bodentiefe: & \multicolumn{6}{|c|}{ 3. Schnitt (04.11.1999) } \\
\hline $0-125 \mathrm{~cm}$ & 3,6 & 3,9 & 1,7 & 5,2 & 0,7 & 1,5 \\
\hline $0-50 \mathrm{~cm}$ & 6,5 & 3,2 & 5,3 & 2,9 & 3,9 & 0,8 \\
\hline $50-125 \mathrm{~cm}$ & $-2,9$ & $-4,0$ & $-3,6$ & $-2,4$ & $-3,2$ & $-4,0$ \\
\hline Saatwicke & & $\mathrm{S}$ & & $11 \mathrm{E}$ & $1 \mathrm{~S}$ & \\
\hline Bodentiefe: & \multicolumn{6}{|c|}{ Ernte (20.10.1998) } \\
\hline$\underline{0-125 \mathrm{~cm}}$ & 20,6 & 38,5 & 9,3 & 11,4 & 1,3 & 7,8 \\
\hline $0-50 \mathrm{~cm}$ & 7,0 & 9,8 & 4,5 & 0,8 & $-0,1$ & $-1,6$ \\
\hline $50-125 \mathrm{~cm}$ & 13,6 & 28,7 & 4,8 & 10,6 & 1,4 & 9,4 \\
\hline Bodentiefe: & \multicolumn{6}{|c|}{ Ernte (17.10.1999) } \\
\hline $0-125 \mathrm{~cm}$ & 14,0 & 15,2 & 12,5 & 17,7 & 8,9 & 13,4 \\
\hline $0-50 \mathrm{~cm}$ & 11,6 & 11,4 & 11,0 & 11,7 & 4,7 & 8,4 \\
\hline $50-125 \mathrm{~cm}$ & 2,4 & 3,8 & 1,5 & 6,0 & 4,2 & 5,0 \\
\hline
\end{tabular}


Herzlich danken möchte ich,

Herrn Prof. Dr. Rolf Rauber für die Überlassung des Themas und die Gewährung großer Freiheiten bei der Bearbeitung dieser Fragestellung,

Herrn Prof. Dr. Norbert Claassen für die Übernahme des Korreferates und die Bereitstellung von Analysengeräte für die bodenchemischen Untersuchungen,

insbesondere Herrn Prof. Dr. Knut Schmidtke der maßgeblich an der Idee, der Planung, der Durchführung sowie an der Fertigstellung der Arbeit beteiligt war und einfach nie müde wurde zu Helfen,

Dr. Karin Reiter für die große Hilfe bei der Probenentnahme auf dem Feld, für die nicht endende Diskussionsbereitschaft bei der Interpretation der Daten und die Überarbeitung des Manuskriptes sowie für den Glauben daran, dass auch diese Arbeit einmal fertig wird,

Claudia Torborg für die Bereitschaft mir zahllose einsame Stunden im Feld und bei der Aufarbeitung der Proben zu ersparen,

Dr. Britta Jost, für die Korrekturen am Manuskript, die Hilfe bei der Literaturbeschaffung und beim Fertigstellen der Arbeit,

Dr. Joachim Schulze für die wertvollen Hinweise bei der Interpretation der Daten,

Herrn Konrad Johne für die Einweisung in die Geheimnisse des Ackerbau-Systemversuchs-Göttingen

dem Feldpersonal des Instituts für Pflanzenbau und Pflanzenzüchtung ohne deren Unterstützung bei der Probenentnahme und Probenaufbereitung auch diese Arbeit nicht möglich gewesen wäre,

den Kollegen(innen) Christiane Münter, Dr. Rüdiger Jung, Dr. Philipp Lehne, Thomas Seibold für die bereitwillige Hilfe und das angenehme Klima in der Arbeitsgruppe,

Frau Nina Hoffmann und Frau Gabi Kolle für die stets zuverlässige Durchführung der Laborarbeiten,

den Herren Reinhard Langel und August Reineking vom Isotopenlabor für die Isotopenanalysen,

mehr als anderen Antrin Fricke, die mir nicht nur bei der Literaturverwaltung behilflich war, sondern mich stets ertragen hat. 


\section{Lebenslauf}

Persönliche Daten: Johann Anthes

geb. 12.02.1967

ledig, ein Kind

\section{Berufspraxis}

$04 / 2004$

selbstständig: Landwirtschaftlicher Sachverständiger und Berater im Ökologischen Landbau

11/2000 - 04/2003 Bereichsleiter Tier auf der Hessischen Staatsdomäne Frankenhausen, Lehr- und Versuchsbetrieb für ökologischen Landbau der Universität - Kassel.

04/1997 - 12/2000 Inspekteur (Vo (EWG) Nr. 2092/91) im Auftrag der Gesellschaft für Ressourcenschutz in Göttingen

02/1991 - 06/1997 Betriebsleiter (3er GbR) 320 ha Bioland-Gemischtbetrieb.

10/1989 - 09/1990 Einjähriges Praktikum auf Bioland Milchviehbetrieb in Hohenstein (Taunus).

\section{Studium und Schule}

04/1997 - 12/2000 Wissenschaftliche Mitarbeit am Institut für Pflanzenbau und Pflanzenzüchtung der Georg-August Universität Göttingen,

10/1986 - 04/1993 Studium der Agrarwissenschaften an der Universität Gießen, Fachrichtung Pflanzenproduktion.

Diplomarbeit am Institut für Biologische Produktionssysteme: Thema: Einfluss des Bodens auf Ertragsparameter eines Luzerne-Gras-Gemenges und Wirkungen auf N-Fraktionen im Boden.

06/1973 - 05/1986 Besuch der Gymnasialen Oberstufe in Rüsselsheim, Abitur, Besuch der Integrierten Gesamtschule und Grundschule in Kelsterbach 